University of Rhode Island

DigitalCommons@URI

Open Access Dissertations

2020

\title{
DISTRIBUTION OF NOVEL AND LEGACY ORGANIC POLLUTANTS IN SEABIRDS FROM CONTRASTING MARINE ENVIRONMENTS
}

Anna Ruth Robuck

University of Rhode Island, annarobuck@gmail.com

Follow this and additional works at: https://digitalcommons.uri.edu/oa_diss

\section{Recommended Citation}

Robuck, Anna Ruth, "DISTRIBUTION OF NOVEL AND LEGACY ORGANIC POLLUTANTS IN SEABIRDS FROM CONTRASTING MARINE ENVIRONMENTS" (2020). Open Access Dissertations. Paper 1227.

https://digitalcommons.uri.edu/oa_diss/1227

This Dissertation is brought to you for free and open access by DigitalCommons@URI. It has been accepted for inclusion in Open Access Dissertations by an authorized administrator of DigitalCommons@URI. For more information, please contact digitalcommons-group@uri.edu. 


\author{
DISTRIBUTION OF NOVEL AND LEGACY ORGANIC \\ POLLUTANTS IN SEABIRDS FROM CONTRASTING \\ MARINE ENVIRONMENTS \\ BY \\ ANNA RUTH ROBUCK
}

A DISSERTATION SUBMITTED IN PARTIAL FULFILLMENT OF THE

REQUIREMENTS FOR THE DEGREE OF

DOCTOR OF PHILOSOPHY

IN

OCEANOGRAPHY

UNIVERSITY OF RHODE ISLAND

2020 


\section{DOCTOR OF PHILOSOPHY}

OF

ANNA RUTH ROBUCK

\section{APPROVED:}

Dissertation Committee:

Major Professor Rainer Lohmann

Mark G. Cantwell

Candace Oviatt

Gavino Puggioni

Nasser H. Zawia

DEAN OF THE GRADUATE SCHOOL

UNIVERSITY OF RHODE ISLAND

2020 


\begin{abstract}
Despite their ubiquity in the environment, large data gaps exist surrounding the distribution, biotic accumulation, and possible impacts of many high priority organic pollutants, particularly those considered emerging contaminants. Emerging pollutants, or contaminants of emerging concern (CECs), represent a dynamic and rapidly evolving group of substances, some of which have been extant for decades. Chapter 1 provides background about CECs covered in this dissertation, and highlights key data gaps explored within this work.
\end{abstract}

The first three manuscripts of this dissertation (Chapters $2-5$ ) focus on per- and polyfluoroalkyl substances, or PFAS. PFAS are a family of CECs that demonstrate amphiphilic or hydrophilic environmental behaviors, and thus are ubiquitously distributed in aqueous and biological matrices. PFAS are associated with adverse effects in humans and wildlife at very low concentrations, and thus their biological behavior and impacts are under a great deal of scrutiny. Little work over the past two decades has evaluated these chemicals in marine food webs along the US East Coast, despite the proximity of this region to major human population centers and PFAS point sources. Tandem mass spectrometry and high-resolution mass spectrometry techniques were applied to tissue samples collected as part of a large-scale necropsy program to derive PFAS measurements in seabirds from the US East Coast. Names, Chemical Abstracts Service (CAS) number, and potential sources of each PFAS measured in this work are provided in Appendix B. 
Chapter Two measured PFAS in seabird juveniles from three habitats along the US East Coast representing a range of exposure potential. Seabirds collected downstream from a major fluoropolymer production site contained the highest concentrations of legacy and novel PFAS, surpassing toxicity reference values established in controlled studies for avifauna. The novel PFAS Nafion by-product 2 (Nafion BP2) was detected in seabirds from all habitats, marking the first identification of this compound in biota beyond the industrially influenced Cape Fear region in North Carolina. Perfluorooctanesulfonic acid (PFOS) and perfluorononanoic acid (PFNA) were associated with decreased phospholipid levels, marking the first time this trend has been observed in a wild population. Chapter Three examined novel and legacy PFAS in Great Shearwaters from Massachusetts Bay over 2010 - 2019. PFOS and perfluorooctanesulfonamide (FOSA) decreased over the time series, while perfluoroalkyl carboxylic acids (PFCAs) and the novel PFAS 7:3 fluorotelomer carboxylic acid (7:3 FTCA) showed no clear trends over time. Multiple PFAS were significantly associated with morphometric variables, with increased PFAS levels associated with reduced organ weights and reduced fat depth in Great Shearwaters. Perfluorohexanesulfonic acid (PFHxS) and PFNA were negatively associated with mass of ingested plastic, marking the first time any relationship has been identified between plastic consumption and PFAS levels in a seabird. This relationship likely reflects the amphiphilic nature of PFAS that reduces the likelihood of sorption to plastic items in aqueous matrices, as well as the divergent exposure pathways for these two pollutant groups, as seabirds are primarily exposed to PFAS via diet as opposed to direct ingestion of plastics via discovery in the environment. Chapter Four assessed PFAS in eight 
tissues obtained from juvenile seabirds collected from three habitats along the US East Coast, the same habitats assessed in Chapter Two. These tissue-specific measurements suggested blood, liver, kidney, and lungs are primary reservoirs of PFAS in seabirds. Novel per- and polyfluoroalkyl ether acids (PFEAs) preferred blood compared to liver, suggesting reduced compatibility with the binding environment of liver fatty acid binding protein that drives PFAS uptake in liver. These results were also the first to measure novel PFEAs in brain tissue, indicating these compounds are capable of surpassing the highly selective blood-brain barrier in vertebrate wildlife.

Chapter Five focuses on microplastics, another type of CEC. Microplastics are a pervasive pollutant in aquatic ecosystems and ingestion of plastics is associated with an increased risk or mortality in seabirds. Chapter Five examined plastic ingestion in 175 Great Shearwaters from $2010-2019$, collected at multiple locations relevant to their annual cycle across the North and South Atlantic Oceans. Each bird contained an average of 8 plastic fragments, and more than $50 \%$ of individuals contained greater than $0.1 \mathrm{~g}$ plastic, significantly surpassing the ecological threshold suggesting no more than $10 \%$ of a given population should contain greater than $0.1 \mathrm{~g}$ ingested plastic. Breeding adults from the South Atlantic ingested plastic less frequently ( $\sim 60 \%)$, while $98 \%$ of juveniles from Massachusetts Bay were found to contain ingested plastics. Each plastic fragment was assessed using Fourier-transform ion spectroscopy to discern polymer identity. We found low- and high-density polyethylene fragments dominated within all years and locations. Breeding adults contained a higher proportion of larger polypropylene fragments compared to juveniles and non-breeding adults; breeding adults in Massachusetts Bay likewise ingested marginally larger plastic items compared 
to Massachusetts Bay juveniles but this was outside the limits of significance defined within the study. The abundance of larger plastic items in breeding and non-breeding adults is likely indicative of an increased use of remote, pelagic habitats subject to reduced inputs of smaller, land-derived plastics due to size-mediated distribution in the pelagic environment. Different signatures of polymer type and size between breeding and non-breeding mature birds likewise suggests rapid turnover of ingested plastics commensurate with migratory stage and location.

Chapter Six of this study leverages the vast amount of necropsy data derived in support of the included contaminant studies to correct the record regarding plumage and age characteristics of Great Shearwaters. Necropsy efforts demonstrated Massachusetts Bay is dominated by juvenile Great Shearwaters, with juveniles making up over $90 \%$ of collected birds considering the entire necropsy sample set. Nape plumage has been previously associated with individual age, with the suggestion that mature individuals possessed a completely white nape while the nape area of younger individuals was interrupted by variable amounts of brown or gray. Necropsy data collected herein suggest nape plumage is not a reliable indicator of age. Instead this data suggests nape plumage may be associated with sexual behaviors, as completely white napes were more abundant in females of all ages.

These results highlight the utility of necropsy efforts to explore multiple complimentary scientific questions within a given species, and raise further questions about Great Shearwater demographics, PFAS, and plastic ingestion. The abundance of juvenile birds in the bycatch sample set and overall Massachusetts Bay region raises questions about the impact of commercial fishing activities on Great Shearwater 
population, as fishing activities remove hundreds to thousands of pre-reproductive individuals from the population each year. This work also demonstrates the ability of several novel PFAS to bioaccumulate in seabirds, and indicates the need for significantly more research describing the bioaccumulation potential of new PFAS formulations in a wider range of wildlife. Additionally, these data suggest potential impacts of PFAS at environmentally relevant, ongoing exposure levels, with uncertain population-scale impacts of reduced organ weights, altered fat storage or expression, and morphological alterations. Further research should evaluate the effects of legacy and novel PFAS in marine biota with a focus on potential implications for individual and population health considering the importance of body condition and lipid stores on bird migratory and reproductive ability. Plastic ingestion in Great Shearwaters likewise merits additional investigation; over $50 \%$ of individuals evaluated in the study contained more than $0.1 \mathrm{~g}$ of ingested plastic, surpassing a threshold established as problematic for seabird plastic ingestion. Further work should evaluate trends of plastic ingestion over time using harmonized methods to leverage the Great Shearwater as a sentinel for specific Atlantic habitats and monitor for potential impacts.

Within a management context, these results provide justification for continued investigation and potential phase-out or regulation of novel PFAS akin to some legacy formulations, considering their potential for long-range transport and bioaccumulation within offshore ocean biota demonstrated within this work. Additionally, the demonstrated abundance of long-chain PFCAs in seabirds demonstrates the importance of considering the downstream environmental transformations and impacts of PFAS currently in use. Long-chain PFCAs are rarely manufactured purposefully, but may 
form as production by-products from creation of other PFCAs or may form in the environment or in biota via oxidation of precursor compounds. The abundant downstream bioaccumulation of these inadvertently created compounds suggests the total environmental life-cycle of a given PFAS should be evaluated to prevent formation of bioaccumulative and ubiquitous terminal end products like long-chain PFCAs or 7:3 FTCA. This work additionally underscores the importance of policies and practices that support waste management and effective recycling, considering upwards of $90 \%$ of plastic items found within seabirds were recyclable polymers that likely ended up in the environment due to waste mismanagement. 


\section{ACKNOWLEDGMENTS}

Funding for this dissertation research was provided by the University of Rhode Island (2015 - 2017) and the U.S. National Oceanic and Atmospheric Administration (NOAA) Dr. Nancy Foster Scholarship program (2017 - 2020) (NA17NOS4290028). Support was also provided by the NIEHS Superfund Research Program (P42ES027706), the Robert and Patricia Switzer Foundation (2019), and the Oak Ridge Institute for Science and Education Program (2019 - 2020).

My biggest thanks go to my Mom and Dad. This would have been impossible without you, and there aren't really words to describe what you mean to me. I'm also very grateful to my siblings and nephew Markie for all the support they provided before and during this process. Likewise, I'm so thankful for my lovely best girl Gypsy, who was the primary source of any sanity retained during this process. Thank you to my dear friends Shelby, Carolyn, Cherie, Brigid, Tiffany, Debbi, and Landis, for unwavering love and listening skills, despite me often taking months to return calls sometimes. Thank you to Mike, Paula, and Dennis for saving the day so many times personally and professionally, your love and support has made the last few years so much brighter.

I want to thank my major professor Rainer Lohmann for the tremendous amount of support provided over our tenure together. You provided a unique blend of personal and scientific independence, mentorship, and goal-setting, which allowed me to learn so much during this experience. Thank you for all you have done and continue to do to support my career, even at times when I had doubts about my own path and abilities.

I also want to offer immense thanks to Mark Cantwell, aka "Lab Dad". Your mentorship and unwavering support have made such a difference in the last few years. 
You saved my morale and experiments essentially every day along the way, and I can't imagine getting through this process without your support

Thank you to Dave Wiley, who indelibly changed my path by betting on my scientific star and supporting me in pursuit of the Nancy Foster Scholarship, while barely knowing me nonetheless. I'm so glad you were impressed instead of freaked out when I gave a bird mouth to mouth. Thank you for the inspiration, support, humor, and creativity you offered throughout my degree progress, and for always being on "Team Anna". I will steal glasses for you any time.

I would also like to thank my committee members Candace Oviatt and Gavino Puggioni - your classes were formative in my development as a well-rounded environmental scientist, and I value the time and perspective you have invested to help me achieve the $\mathrm{PhD}$ despite how much my dissertation has changed over the course of this process. I'd also like to thank my Defense Chair Angela Slitt, who has supported my interests in toxicology and lipidomics at every step of the way, while modeling good humor and kindness.

Thank you to the NOAA Nancy Foster Scholarship and all those who make the opportunity a reality, including Kate Thompson, Seaberry Nachbar, Claire Fackler, Liz Weinberg, Kathy Broughton, Steve Gittings, and so many others. This opportunity changed my personal and professional trajectory in every way imaginable, and I am thankful for the time and energy you all put into the program to provide financial support, professional development, and personal support to the Scholars. Also thanks and huzzah to my fellow Scholars! I am so proud and thankful to count you as friends and colleagues. 
Resounding thanks to all the folks at Stellwagen Bank National Marine Sanctuary for supporting me and helping me grow as a professional and person during my years as a Scholar - Pete DeCola, Alice Stratton, Bibi Stokes, Ben Haskell, Anne-Marie Runfola, and Anne Smrcina. Pete, particular thanks to you for supporting the Massachusetts Bay water column study, it was a highlight of my $\mathrm{PhD}$ career to plan and execute "my own" water quality cruise with your aid. Also at SBNMS, special thanks to Amy Meloski, Dave Slocum, Peter Hong, and Tammy Silva, for lots of field work help and friendship over the years. Also, a special thanks to the most dedicated SBNMS volunteer in existence, Kevin Powers - one of the highlights of my dissertation journey was meeting you every day at WHOI for almost three weeks (!) to cut up birds and shoot the breeze. I would never have the comprehensive sample set that I was able to amass without your dedication at every step.

Beyond the folks above, the success of this work was facilitated by a veritable village. I am enormously grateful to the many collaborators, administrators, and friends who made this work possible in big and small ways. Thank you to David Smith and Meredith Clark for abundant help navigating the PhD process at GSO. Thank you to Michaela Cashman, Julia Sullivan, Melanie Hedgespeth, and Dave Katz, my EPA PFAS PFurious team-I could never have done this without your thoughtful support and good humor. Also thanks to Christine Gardiner, who was a source of assistance and friendship at some of the most challenging points of this process. Thank you as well to Gwen Emery, Lindsay Agvent, Tom Garrow, the entire Lohmann lab past and present, Heidi Pickard, Charlotte Wagner, the Sunderland lab, as well as the entire STEEP team. I 
would particularly like to thank Judith Swift within URI STEEP for her continued support and coaching on public engagement and interview skills.

Another round of thanks to the $\mathrm{NC}$ folks who taught me so much and also reminded me that hobbies were a thing at a really bleak point in this process -- James McCord, Mark Strynar, Theresa Guillette, Kim McKeever, and Hannah Liberatore.

Last but far from least, thank you to Northeast Fisheries Science Center, Woods Hole Oceanographic Institute, NC Audubon, the Wildlife Clinic of Rhode Island, and University of Cape Town folks -- Gina Shields, Johanna Pedersen, Stephanie DePasquale, Michael Moore, Peter Ryan, Vonica Perold, Maelle Connan, Lindsay Addison, Marissa Sprouls, and the entire WRARI staff - who were so generous with their time and support to help me find, store, and necropsy the birds used in this work. Particular thanks to Michael Moore, who went above and beyond during the COVID19 pandemic to secure access to WHOI MRF for me so I could finish one more year of necropsies. 


\section{DEDICATION}

I wish to dedicate this effort to all those dear to me and my family that we lost during my PhD journey - Big R, Gramma Anne, Aunt Patti, Uncle Joey, Aunt Jeannie, Aunt Beenie, and Ben Privott. Each of these folks shaped me into the person I am today. I strive each day, while "vertical and ventilating", to professionally and personally emulate a fraction of the grace, boldness, humility, humor, and kindness you each so artfully modeled to me and so many others.

I also dedicate this to my wider family, past and present. We are a family of immigrants, coal miners, bootleggers, engineers, mill workers, mechanics, highway construction workers, typists, lumberjacks, stay-at-home moms, nurses, teachers, farmers, and many other industrious enterprises. So many brilliant folks in my family worked hard in any way they could to make things a little better and more stable for our family. I am here today because of all those sacrifices along the way. 


\section{PREFACE}

This dissertation is written and organized in the manuscript format according to URI Graduate School guidelines for dissertation preparation. It is a compilation of five manuscripts, one of which has been published, one of which is in review, and two of which are in some stage of submission. The first manuscript (Chapter 2) was published in Environmental Science and Technology in August 2020. The second manuscript (Chapter 3) is formatted for Environmental Science and Technology. The third manuscript (Chapter 4) is in review at Environmental Science and Technology Letters. The abstract of the fourth manuscript (Chapter 5) has been accepted to a special issue on plastic ingestion in Frontiers in Marine Science and will be submitted for full review in March 2021. The fifth manuscript (Chapter 6) is intended for Avian Biology and pending immediate submission. Appendix A contains co-author and collaborative works completed over the course of the dissertation. 


\section{TABLE OF CONTENTS}

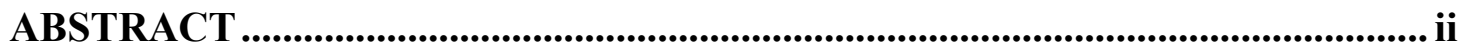

ACKNOWLEDGMENTS ............................................................................................ viii

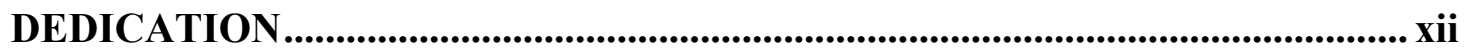

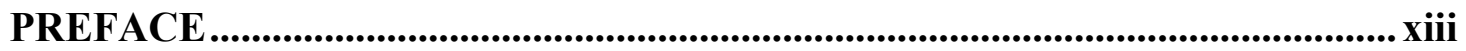

TABLE OF CONTENTS............................................................................................. xiv

LIST OF TABLES ................................................................................................

LIST OF FIGURES ....................................................................................................... $\mathrm{xx}$

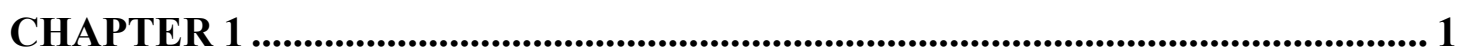

INTRODUCTION

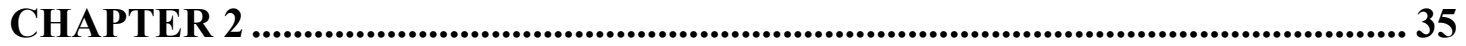

LEGACY AND NOVEL PER- AND POLYFLUOROALKYL SUBSTANCES IN JUVENILE SEABIRDS FROM THE U.S. ATLANTIC COAST .......................... 35

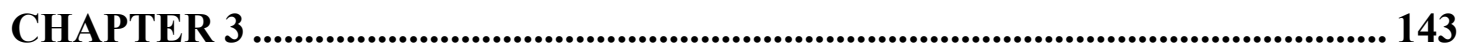

TIME TRENDS OF PER- AND POLYFLUOROALKYL SUBSTANCES FROM 2010 - 2019 IN A TRANS-EQUATORIAL MIGRATORY SEABIRD ............... 143

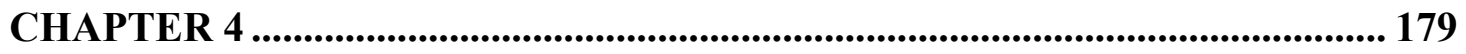

TISSUE-SPECIFIC DISTRIBUTION OF LEGACY AND NOVEL PER- AND POLYFLUOROALKYL SUBSTANCES IN JUVENILE SEABIRDS ................ 179

CHAPTER 5 ............................................................................................................... 224

PLASTIC INGESTION IN THE GREAT SHEARWATER, AN ENDEMIC

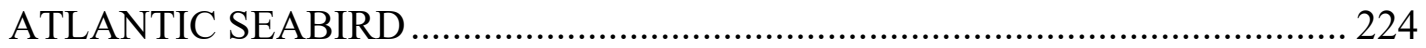

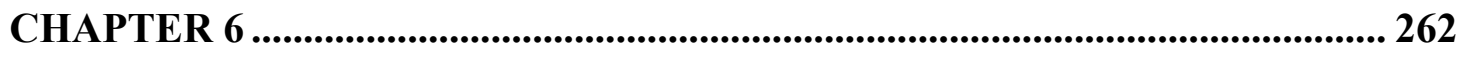

NAPE COLORATION IS NOT A RELIABLE MARKER OF AGE IN GREAT

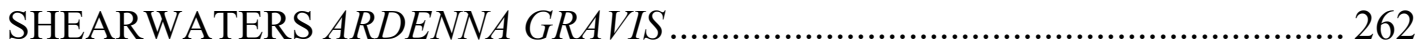

CHAPTER 7 .................................................................................................................... 286

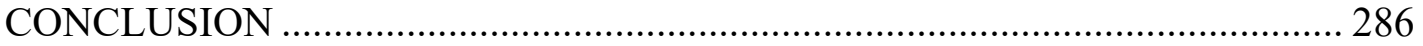




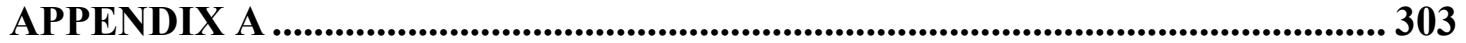

COLLABORATIVE CONTRIBUTIONS ........................................................ 303

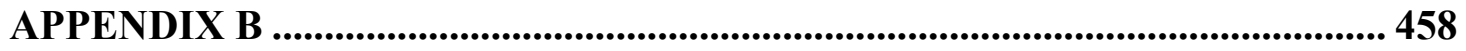

TABLE OF PFAS ACRONYMS ......................................................................... 458 


\section{LIST OF TABLES}

TABLE

PAGE

Table 2.1. Detection frequency of quantifiable and semi-quantitative analytes in seabird juveniles from each habitat and as a total sample set across all habitats combined. Mass. Bay = massachusetts bay, narra. Bay = narragansett bay, and cfre = cape fear river estuary. Family names are from buck et al. 2011. Compounds highlighted in gray are those compounds detected above reporting levels in at least $97 \%$ of individuals via lc-ms/ms. ${ }^{a}$ detection based on raw abundances in comparison to blank raw abundances due to lack of authentic standards. ${ }^{b}$ low recovery $(14 \%)$ related to sample preparation.

Table S2.1. Table presenting all compounds assessed in this study, method of instrumental analysis applied, method used to report concentrations, quantification approach used based on availability of native standard, and summary of detections in liver extracts. Family designation based on Buck et al. 2011 and Strynar et al. 2015.

Table S2.2. Table containing relevant life history details of seabird species included in this study. Diet and life history sources include species profiles on the Cornell Lab of Ornithology All About Birds data portal -- https://www.allaboutbirds.org/guide/ -- and all references therein -- were referenced for diet information, in addition to personal communications from colony monitoring technicians. Age examples are from https//www.pwrc.usgs.gov/BBL/longevity/longvrec.cfm.

Table S2.3. Table containing relevant morphometric and collection details describing each individual included in this study. "NE" designates "Not Examined", "CBD" designates "Couldn't Be Determined"

Table S2.4. Table presenting details about all native analytes measured in this study via $\mathrm{LC}-\mathrm{MS} / \mathrm{MS}$.

Table S2.5. Table presenting details about all native analytes measured in this study via LC-HRMS.

Table S2.6. LC-MS/MS Method recovery as a percentage, following: \% Recovery $=(($ Pre-extraction spiked sample $) /($ Post-extraction spiked sample $)) \times 100117$

Table S2.7. Table of targeted native analytes, associated instrument parameters, and quantification parameters targeted with LC-MS/MS analysis. 
Table S2.8. Table of target mass-labeled compounds, associated abbreviations, and concentrations of purchased stock solutions used in both targeted and HRMS analysis.

Table S2.9. Table of target mass-labeled compounds, associated instrument parameters, and quantification parameters used for targeted LC-MS/MS analysis... 120

Table S2.10. Table presenting mobile phase gradient ratios used during sample analysis via $\mathrm{LC}-\mathrm{MS} / \mathrm{MS}$.

Table S2.11. Table presenting mobile phase gradient ratios used during sample analysis via HRMS.

Table S2.12. Raw abundances of instrumental and process blanks observed in HRMS analysis. Estimated MDLs are calculated as the mean process blank area plus three times the standard deviation. The estimated MDL for PFO4DA was calculated using instrumental blank values due to limited detection in process blanks, precluding calculation of standard deviation using process blank values. HFPO-DA was analyzed via both HRMS and LC-MS/MS, but high instrumental and process blanks values associated with HRMS prevented quantification of HFPO-DA using this technique. Detections of HFPO-DA reported in this study are derived from LC-MS/MS measurements. Quantification limits for HRMS were constrained by the linear dynamic range of the curve.

Table S2.13. Method accuracy and precision as relative standard deviation (RSD); both metrics were calculated using five spiked replicate samples consisting of organic chicken liver spiked with 2 ng native stock and $10 \mathrm{ng}$ of isotopically labeled surrogate stock. 124

Table S2.14. Table presenting the degree of ion enhancement or suppression associated with matrix interference calculated using: \% Matrix Effects $=(($ Post Extraction Spike in Chicken Matrix)/(Post Extraction Spike in Solvent)-1) $\times 100 . .125$

Table S2.15. Method reporting limits for LC-MS/MS analysis calculated using the standard deviation of spiked replicate samples multiplied by the Student's t-value appropriate for a single-tailed 99th percentile, according to: 『MDL》_s $=\mathrm{t} \_((\mathrm{n}-1,1-$

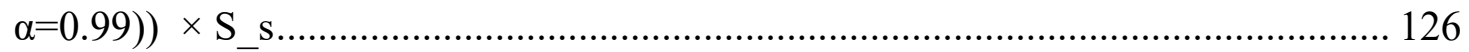

Table S2.16. Detection of PFEAs, based on compariston to estimated method detection limit (mean process blank abundance $+3 *$ std. dev.) or the lowest curve point.

Table S2.17. PFAS concentrations observed in this study. Responses not detected or outside the linear dynamic range of the curve were labeled as "nd" and assigned a value of zero. Observations below method detection limits but with clear signal, clear qualifier ions, and detected in at least $50 \%$ of samples in one habitat were replaced 
with the half the method detection limit for statistical analyses, and included as such in calculation of summed concentrations.

Table S2.18. Table providing surface water concentrations, mean liver concentrations, and calculation of bioconcentration factors $(\mathrm{BCFs})$. 129

Table S2.20. Summary statistics describing stable isotope measurements of bird liver and muscle. Trophic levels were calculated following methods of Fisk et al. 2001 and Hobson et al. 1994. 131

Table S2.21. Measured 15N ratios alongside estimates of trophic level (TL), assuming a copepod primary consumer after Fisk et al. 2001. 132

Table S2.22. Definition of correlation coefficient significance and descriptors, from https://dx.doi.org/10.1016\%2Fj.tjem.2018.08.001. 133

Table S2.23. Results of Spearman correlation analysis assessing relationships between each PFAS and stable isotope ratios. Statistically significant ( $\mathrm{p} \quad 0.05$ ) relationships are highlighted in orange. Non-statistically significant relationships with $0.05<$ p.value $<0.1$ are highlighted in yellow for illustrative purposes. 134

Table 3.1. Detection frequency, arithmetic mean, standard error, and range of concentrations included in this study. PFBA and PFBS were not reported, or "nr" due to chromatographic imprecision. Family designations are after Buck et al. 2011.... 175

Table 3.2. Table presenting significant associations identified via Spearman rho correlation analysis between continuous biological covariates and PFAS concentrations. Associations above the pre-determined significance level $(p=0.05)$ but below $\mathrm{p}=0.1$ are highlighted in orange. 178

Table S.4.1. Table presenting summary statistics of the sum PFAS in each tissue, stratified by habitat. 216

Table S4.2. Table presenting summary statistics of the each PFAS identified in at least one tissue in eight different tissues, stratified by habitat.

Table S4.3. Table presenting non-zero and non-infinite pairwise ratios in each tissue, stratified by habitat. 218

Table S4.4. Table presenting all analytes included in this study. Semi-quantitative analytes lacked a matched native and internal standard. Quantitative analytes were measured using at least one exact matched standard.

Table S4.5. Table presenting proportions of each PFAS in each tissue, stratified by habitat. 
Table S4.6. Table presenting proportions of each PFAS family in each tissue, stratified by CFRE and all habitats combined.

Table S4.7. Percent difference of sigma probabilities between the lowest energy conformer of each PFEA anion, compared to an appropriate low energy PFAA anion analogue. Negative sigma values correspond to a positive surface charge density, while positive values correspond to a negative surface charge density. 222

Table 5.1. Summary statistics describing accumulated ingested plastics by mass and count, in different regions and years.

Table 5.2. Frequency of occurrence (FO) of ingested plastic by location, year, and age.

Table 6.1: Age classes and associated criteria from Pyle (2008) and Powers et al. (2020).

Table 6.2. Confusion matrix presenting counts of known age (HY or Mature) birds, compared to the age class as predicted according to Pyle's nape classification system, under the "Inferred from nape" heading. 


\section{LIST OF FIGURES}

\section{FIGURE}

PAGE

Figure 2.1. Measured concentrations of A) PFAS in juvenile seabird livers measured via LC-MS/MS, B) two emerging PFAS measured via targeted HRMS, alongside Binset) ratios of emerging PFEAs to PFOS in CFRE chicks. Nafion BP2 concentrations positively identified in non-CFRE chicks but below the linear dynamic curve range are graphed in panel B as half the reporting limit; grey arrows are used to distinguish these data points. 74

Figure 2.2. The composition of PFAS in liver tissue, presented by individual and grouped by habitat. 75

Figure 2.3. $\sum{ }_{19} \mathrm{PFAS}$ presented in A) as a boxplot, with the dark line representing the median, box limits representing the first and third quartiles, whiskers denoting 1.5 times the interquartile range, and crosses denoting outliers. The asterisk indicates a statistically significant difference between habitat mean $\sum_{19}$ PFAS compared via Wilcoxon rank sum test using the CFRE as the reference group, while B) presents ratios of $\sum$ PFCAs to $\sum$ PFSAs in each individual. Ratios above 1 indicate PFCA dominance, while ratios below 1 indicate PFSA dominance. (Small figure, 300 word equivalent)

Figure 2.4. A) presents log-transformed concentrations of PFOS vs phospholipid (PL) grouped by habitat, while B) displays log-transformed PFOS and phospholipid (PL), assessed as a total sample set $(n=31)$. Text annotation presents $R, R_{s}{ }^{2}$, and $p$-value derived from Spearman rank correlation analysis.

Figure S2.1. Map of the US Atlantic East Coast. Insets provide further detail about collection locations of from each habitat. Great shearwaters were collected as bycatch in Massachusetts Bay and off Cape Cod, designated as NOAA Fisheries Stat Areas 514 and 521. Stellwagen Bank National Marine Sanctuary, a key foraging area for Great Shearwaters, is outlined in white and indicated as "SB" in the top right inset. Herring Gull chicks came from nests located in Narragansett, RI and Newport, RI. The bottom inset shows the Cape Fear River and Estuary system, located in southeastern NC. A Chemours facility is located near Fayetteville, NC, designated on the map as a white star. The red box indicates the lower river and estuary portion of the system; CFRE chicks were hatched on dredge islands near the mouth of the estuary.............. 88

Figure S2.2. Concentrations of PFUnDA by habitat. Habitat mean concentrations of PFUnDA presented as boxplots, with the dark line representing the median, box limits representing the first and third quartiles, whiskers denoting 1.5 times the interquartile range, and crosses denoting outliers. The points reflect measured observations contributing to the summary statistics presented by the boxplot. The asterisks indicate 
a statistically significant difference between habitat mean PFUnDA compared via the Wilcoxon rank sum test, using the CFRE group as the reference group. 103

Figure S2.3. Observed concentrations of PFOS versus concentrations of PFNA, PFDA, and PFUnDA in A) all individuals across three habitats, and B) in only individuals from Narragansett Bay and Massachusetts Bay with two outlier individuals removed. All concentrations were measured via UPLC-MS/MS. 104

Figure S2.4. Concentrations of $\mathrm{C} 8$ - C14 PFCAs as a function of PFDA measured via LC-MS/MS in A) all individuals across three habitats, and B) in only individuals from Narragansett Bay and Massachusetts Bay. Associations between PFCAs as approximated by linear regression slightly decrease without CFRE data included, likely due to the truncation of the data range as a result of more similar concentrations observed in habitats removed from point sources and inclusion of MDL/2 values... 105

Figure S2.5. Observed concentrations of emerging PFEAs versus legacy PFAS in CFRE chicks with A) displaying concentrations of PFDA vs PFEAs and other longchain PFCAs, and B) displaying concentrations of PFOS vs PFEAs. PFCA and PFSA concentrations were measured via UPLC-MS/MS while PFEAs were measured via HRMS. 106

Figure S2.6. $\delta 15 \mathrm{~N}$ vs concentrations of PFAS. Measured $\delta^{15} \mathrm{~N}$ in seabird liver and muscle did not relate to concentrations of individual PFAS, or ${ }_{19} \sum$ PFAS in seabird liver across any habitat. No correlation was found when the total sample set $(n=31)$ was assessed in the same way. Muscle $\delta^{15} \mathrm{~N}$ ratios are presented here; facet plots include only those compounds found in $>40 \%$ of the sample set.

Figure S2.7. $\delta 13 \mathrm{C}$ vs concentrations of PFAS. Measured $\delta^{13} \mathrm{C}$ in seabird liver and muscle did not relate to concentrations of individual PFAS, or $19 \sum$ PFAS in seabird liver from Massachusetts Bay or Narragansett Bay. No correlation was found when the total sample set $(\mathrm{n}=31)$ was assessed in the same way. PFDA, PFNA, PFOS, PFUnDA, and ${ }_{19} \sum$ PFAS were moderately associated $(\mathrm{p}<0.05)$ with $\delta^{13} \mathrm{C}$ ratios, only in CFRE chicks. See SI text for further discussion of this limited phenomenon. Muscle $\delta^{13} \mathrm{C}$ ratios are presented here; facet plots include only those compounds found in $>40 \%$ of the sample set. 108

Figure S2.8. $\delta^{13} \mathrm{C}$ in key organic and inorganic matrices. 109

Figure S2.9. Log BAFs for 10 compounds across three habitats. BAFs were calculated by dividing mean liver concentrations observed in each habitat by observed or estimated surface water concentrations.

Figure S2.10. Chromatograms of Nafion BP2 in native standard and Great Shearwater liver. Extracted ion chromatogram (EIC) of Nafion BP2, $[\mathrm{M}-\mathrm{H}]$ ion with $\mathrm{m} / \mathrm{z}=$ $462.9326 \pm 5 \mathrm{ppm}$. The figure shows the authentic standard (top) compared to the EIC observed in a Great Shearwater liver from Massachusetts Bay (bottom). Nafion BP2 
was identified in two Great Shearwater juveniles and one Herring Gull chick outside of the CFRE system. Positive identification of Nafion BP2 in these non-CFRE samples was confirmed by comparison to authentic standards, distinctive peak shape, accurate mass, in-source fragmentation patterns, $\mathrm{MS}^{\mathrm{n}}$ data when available, retention time, and sample preparation and analysis information.

Figure 3.1. Mean concentrations of A) Nafion BP2 and B) 7:3 FTCA in ng/g wet weight over time, with bars representing standard error of the mean.

Figure 3.2. Concentrations of six PFAS over 2010 - 2019; the blue line represents a linear model using year as the sole predictor to deduce any time trends.

Figure 4.1. Detection frequencies by habitat and tissue type, including 15 compounds that were found at least two samples. See SI for compound abbreviation definitions.

Figure 4.2. Mean percentages of three subclasses of PFAS observed in eight tissues, averaged across all habitats (left) and solely considering CFRE individuals (right). Percentages reported reflect the concentration of the given PFAS group, divided by the total concentration (in $\mathrm{ng} / \mathrm{g}$ ) observed in a given tissue. The proportion of PFSA in each tissue is majorly dominated by PFOS. PFEA proportions are driven by Nafion BP2. Proportions stratified by habitat and compound are presented in the SI, along with relative mass weighted proportions.

Figure 4.3. Mean concentrations and standard errors of each compound, observed in eight tissues, stratified by habitat as indicated by point and line color. 205

Figure 4.4. Ratios of PFAS concentrations in six tissues from CFRE individuals, with A) presenting concentrations of PFO5DoDA divided by PFUnDA and B) presenting concentrations of Nafion BP2 divided by concentrations of PFO5DoDA. The blue line within each box plot indicates the arithmetic mean while the black line indicates the median. The box hinges represent the first and third quartiles, and the whiskers indicate 1.5 times the interquartile range. Outliers are indicated by black dots. The red line indicates the mean of each concentration ratio including all tissues except brain. Common letters above each boxplot indicate tissues that share statistically indistinguishable concentration ratios as determined using Tukey's post-hoc test. .. 206

Figure S4.1. Map of the US Atlantic East Coast. Insets provide further detail about collection locations of from each habitat. Great shearwaters were collected as bycatch in Massachusetts Bay and off Cape Cod, designated as NOAA Fisheries Stat Areas 514 and 521. Stellwagen Bank National Marine Sanctuary, a key foraging area for Great shearwaters, is outlined in white and indicated as "SB" in the top right inset. Herring gull chicks came from nests located in Narragansett, RI and Newport, RI. The bottom inset shows the Cape Fear River and Estuary system, located in southeastern NC. A Chemours facility is located near Fayetteville, NC, designated on the map as a 
white star. The red box indicates the lower river and estuary portion of the system; CFRE chicks were hatched on dredge islands near the mouth of the estuary.....

Figure S4.2. RBBs by habitat. Relative body burdens (RBBs) of each PFAS by tissue compartment, stratified by habitat. 213

Figure S4.3. TBRs by Habitat. Tissue-blood ratios of each PFAS by tissue compartment; point color denotes habitat, while the black bars delineate the standard error of the mean.

Figure S4.4. Proportions by Tissue. Mean percentages of three subclasses of PFAS observed in eight tissues, averaged across all habitats (left) and solely considering CFRE individuals (right). Percentages reported reflect the concentration of the given PFAS group, divided by the total concentration (in $\mathrm{ng} / \mathrm{g}$ ) observed in a given tissue. The proportion of PFSA in each tissue is majorly dominated by PFOS. PFEA proportions are driven by Nafion BP2. Proportions stratified by habitat and compound are presented in the SI, along with relative mass weighted proportions. 215

Figure 5.1. Map of Great Shearwater migratory routes (black lines), and sample collection locations (marked by colored points). Surface plastic density is indicated by heatmap gradients in pink and blue. No samples were collected off the Patagonian Shelf (marked by the yellow dot), but this location is heavily used by both adult and juvenile Great Shearwaters as a staging location before and after trans-equatorial migration.

Figure 5.2. Proportions of each plastic morphology, as a percentage of the total count of plastic items measured within a given year and location. The summary of the Gough Island dataset presented herein omits one clear outlier that possessed 194 plastic pieces of greater category diversity than observed within other mature birds from Gough Island.

Figure 5.3. A) Length and width and B) surface area and volume of plastic fragments, with colors indicating collection region. $95 \%$ of the data is displayed, with the upper $5 \%$ removed. Error bars present the standard error of the mean. 259

Figure 5.4. Polymer composition by A) year and B) age presented by location. ..... 260

Figure 6.1: Examples of each nape class as observed in live birds. The diagram inset is reproduced from Pyle 2008. 279

Figure 6.2. Variation in nape class (as defined in Fig. 1) by age class defined following either (A) Pyle (2008) or (B)Powers et al. (2020). Both panels include observations from all locations.

Figure 6.3. Nape classification of Massachusetts Bay bycatch individuals by year. 283 
Figure 6.4. Nape classification by age and location............................................ 284

Figure 6.5. Nape classification by sex, age, and location. .................................... 285 


\section{CHAPTER 1}

\section{INTRODUCTION}

If we are going to live so intimately with these chemicals eating and drinking them, taking them into the very marrow of our bones - we had better know something about their nature and their power." - Rachel Carson, Silent Spring

The environmental distribution and persistence of human-created pollutants represents a pervasive global problem, with various anthropogenic pollutants found in the deepest depths of the ocean to the highest polar latitudes ${ }^{1-5}$. Despite their ubiquity in the environment, large data gaps exist surrounding the distribution, biotic accumulation, and possible impacts of many high priority organic pollutants, particularly those considered emerging contaminants. Emerging pollutants, or contaminants of emerging concern (CECs), represent a dynamic and rapidly evolving group of substances. The definition of what constitutes an emerging pollutant is somewhat tenuous as it relies heavily on human perspective and knowledge; today we understand emerging pollutants as those substances that are newly discovered or measurable, or lack data describing basic elements of their behavior, distribution, or effects $^{6,7}$.

The emerging pollutants explored herein have been extant for decades, but have only recently become widely measurable due to advances in analytical instrumentation or observational techniques, and have thus gained research traction due to increased awareness of their environmental ubiquity and potential impacts. 
This dissertation contributes data and interpretation to describe the environmental distribution and biological occurrence of emerging contaminants in biotic and abiotic systems from the US Atlantic Coast, focusing on seabirds as sentinels of emerging contaminant dynamics in marine food webs. Seabirds serve as effective environmental indicators representing the health and condition of coastal or oceanic habitats in which they live, as their upper trophic level position, fast metabolism, and life history choices intimately connect them to the conditions of a given habitat ${ }^{8-10}$. Previous research indicates seabirds are particularly vulnerable to bioaccumulation of organic contaminants; polychlorinated biphenyls, pesticides, and flame retardants have all been documented in seabirds worldwide ${ }^{11-14}$.

The dissertation specifically focuses on per- and polyfluoroalkyl substances (PFAS), while also providing data on active pharmaceutical ingredients (APIs) and microplastics (MPs) in lesser detail. Each of these pollutant families have demonstrated environmental persistence, bioaccumulative capacity, and toxic potential in humans and wildlife, yet are considered emerging contaminants due to a limited knowledge or understanding 14-21.

PFAS. Per- and polyfluoroalkyl substances (PFAS) are a group of over 9,000 humancreated chemicals or byproducts that have been manufactured since the $1940 \mathrm{~s}^{22,23}$. Various sub-groups of PFAS exist under the large PFAS umbrella, including perfluoroalkyl sulfonic acids (PFSAs), perfluoroalkyl carboxylic acid (PFCAs), perfluoroalkyl ether acids (PFEAs), and the so-called "precursor" compounds that readily transform into PFCAs, PFSAs, or other stable PFAS under environmental conditions $^{23}$. Perfluoroalkyl acids, or PFAAs, refers to both PFSAs and PFCAs. These 
sub-groups within the wider PFAS class vary based on molecular structure and functional group but all are considered PFAS as they include at least one least one perfluoroalkyl moiety $\left(\mathrm{C}_{\mathrm{n}} \mathrm{F}_{2 \mathrm{n}+1}-\right)$ associated with a minimum of three carbon fluorine (C-F) bonds ${ }^{22,23}$. PFAS are often referred to by chain length, or how many carbons make up the backbone of the molecule. PFCAs are considered long-chain with seven or more alkyl carbons, while PFSAs are considered long-chain with six or more alkyl carbons ${ }^{23}$.

Reliance on the C-F bond defines PFAS while also making them remarkably persistent in the environment ${ }^{24,25}$. This bond is one of the strongest bonds in organic chemistry, and its prolific use in PFAS molecules renders powerless an arsenal of chemical and environmental mechanisms that degrade many other pollutants $\left({ }^{24,26}\right)$. Some PFAS are capable of breaking down to some extent via in situ or in vivo oxidation, but because of the stability of the C-F bond, they often degrade into other, more stable PFAS structures ${ }^{27,28}$. For example, a sub-group of PFAS called fluorotelomer alcohols (FTOHs) transform in the environment into the more persistent and toxic PFCA sub-group ${ }^{28,29}$. PFAS are also chemically unique in that they are amphiphilic or "both loving", allowing many PFAS to associate with both water and $\mathrm{oil}^{26}$. This property means PFAS readily migrate within and beyond the water cycle ${ }^{30-}$ 32 while also accumulating in living organisms in ways distinct from hydrophobic organic contaminants ${ }^{33-35}$.

Sources and environmental occurrence of PFAS. PFAS are solely created by human industrial processes ${ }^{23,26,36}$. Major primary sources of PFAS include creation of PFAS via industrial activity ${ }^{37,38}$, and use of aqueous firefighting foams (AFFFs) at airports, 
military bases, and oil and gas operations ${ }^{31,39,40}$, and the intermediate use of PFAS during production of consumer and industrial goods ${ }^{41}$. Due to their unique and attractive chemical qualities, PFAS can be found in hundreds of household or consumer goods including food packaging, take-out food containers, non-stick cookware, paper goods, cleaning products, cosmetics, fire-fighting foams, carpets, ski wax, textiles, waterproof clothing and coatings, paints, and many more ${ }^{41,42}$. PFAS also play a central role in many industrial and workplace processes including electroplating, electronics creation, production of medical equipment, oil recovery, textile manufacture, plastics manufacturing, specialty chemical production, fuel cell production, and membrane manufacture ${ }^{43}$.

PFAS do not remain confined to products or sites where they are used or discharged - their persistence coupled to high mobility allows them to leach into the environment readily, where they can be subsequently transported via water, air, or solid materials ${ }^{44,45}$. PFAS display a wide range of environmental behaviors based on their unique chemical characteristics and subsequent speciation in the environment as zwitterionic, cationic, or anionic compounds ${ }^{46,47}$. The most well-studied compounds are PFAAs which are primarily anionic at environmentally relevant $\mathrm{pHs}$ and therefore amphiphilic or hydrophilic in behavior ${ }^{47}$.

Once in the environment, PFAS travel in regional and global biogeochemical cycles based on their individual physico-chemical characteristics. PFAS have been detected in air $^{32}$, water ${ }^{32}$, ice from the polar regions ${ }^{48}$, mountaintop glaciers ${ }^{49}$, river water $^{37,50}$, lake water ${ }^{32}$, ocean water ${ }^{25}$, sediments ${ }^{51}$ and soils ${ }^{52}$. PFAS measurements in remote polar regions indicate their ability for long-range transport via both 
atmospheric and water-borne pathways, the two primary routes of environmental transport for these compounds ${ }^{28,29}$.

Regardless of specific entry point into the environment, aquatic systems possess the greatest exposure potential for these compounds; the ocean is the ultimate sink for PFAS, as atmospherically transported PFAS oxidize or wash out of the gas phase into the water cycle, while water-borne PFAS in rain, rivers, and streams flow downstream to the ocean ${ }^{44,45,53}$. PFAS are a suitable tracer indicating the age of ocean water -- any water mass that entered Atlantic Meridional Overturning Circulation after PFAS proliferation now carries PFAS, while older water masses possess significantly lower PFAS concentrations $^{25}$.

Human Exposure. Their environmental ubiquity also means PFAS are distributed pervasively in the biosphere. Humans are primarily exposed to PFAS via food, drinking water, and dust; dermal and inhalation exposure are more minor contributors to average adult PFAS exposure ${ }^{54}$. Occupational PFAS exposure may exist for individuals working in an industry that produces or uses PFAS, or for those who work with AFFF, where inhalation is likely a significant source of exposure for those occupationally exposed ${ }^{54}$. Seafood is a significant contributor of PFAS in human diet, and the European Food Safety Administration has previously suggested up to $86 \%$ of human dietary PFAS stems from seafood sources ${ }^{54-56}$. PFAS contamination of drinking water is associated with proximity to industrial sources, AFFF-impacted sites, airports, and wastewater treatment plants, though ambient levels of PFAS are detected in drinking water across the coutnry ${ }^{31,57,58}$. Children may be more exposed to PFAS via maternal transfer of PFAS in utero, through breast milk, as well as through 
dermal and inhalation exposure through increased proximity to and contact with PFAS-containing textiles and carpets ${ }^{54}$.

Wildlife Exposure. Exposure of wildlife to PFAS has materialized as a pervasive and poorly understood global challenge. In parallel to humans, wildlife exposure is an iterative series of events, whereby multiple human sources introduce PFAS into surrounding abiotic matrices, on a site- and use-specific basis. Wildlife are externally exposed to PFAS based on specific life histories and behaviors that dictate their interactions with ambient environmental exposures via air, water, soil/sediment use, and diet ${ }^{59}$. Wildlife may also be particularly exposed to PFAS via use of industrial or AFFF-impacted habitat $34,60,61$.

Since the first report on the global distribution and related exposure of perfluorooctane sulfonic acid (PFOS) in wildlife in 2001, concentrations of PFOS and other PFAAs have been measured in fish ${ }^{62}$, invertebrates like insects or crustaceans ${ }^{60}$, shellfish, marine mammals ${ }^{63}$, birds ${ }^{64}$, reptiles ${ }^{65}$, domestic animals ${ }^{66}$, animal food products $^{66}$, plants $^{67}$, plant food products ${ }^{68}$, and humans ${ }^{69}$. These studies include investigations in wildlife from landscapes and aquatic habitats adjacent to human population centers to remote polar regions, including the Arctic ${ }^{70-73}$ Great Lakes ${ }^{64,74-}$ ${ }^{76}$, North Atlantic Ocean ${ }^{30,77-79}$, Pacific Ocean ${ }^{11,80,81}$, Antarctica ${ }^{82,83}$ well as in coastal regions and riverine systems across the globe $\mathrm{g}^{50,84-86}$.

Trends in PFAS occurrence and exposure over time and space. In recent years, a growing number of field-based studies have assessed geographic variation and temporal trends of PFAS concentrations in wildlife from multiple taxa $a^{64,65,70,71,87-90}$. While the reported spatiotemporal trends of PFAA concentrations in wildlife vary 
among compounds, species, and geographic location, the available data indicate that decreases for the majority of PFAAs in exposed wildlife has been relatively slow, if present at all. Some decreases in PFOS and FOSA have been documented following phase out of their production and use in the early $2000 \mathrm{~s}^{45,79,90}$. Data from other species or locations suggest sustained or increased PFOS in wildlife, as well as increased concentrations of PFCAs over time, which has been attributed to continued emissions of long-chain PFCAs, PFCA precursors, or PFOS precursors ${ }^{70,89}$.

PFAS in seabirds. PFAS were first documented in seabirds from around the globe in the late $1990 \mathrm{~s}^{91-93}$, and continued research over the past thirty years has established PFAS in multiple species of seabirds from divergent environments, including guillemots from the Arctic ${ }^{72,94}$, herring gulls from the Great Lakes ${ }^{64,95-97}$, albatross from Midway Atoll ${ }^{80}$, great skuas from the $\mathrm{UK}^{98}$, cormorants from San Francisco $\mathrm{Bay}^{81}$, coastal and pelagic seabirds from British Columbia ${ }^{11}$, and multiple species of petrels from the South Atlantic and Indian Oceans ${ }^{99}$. The range of extant studies have provided discrete "snapshots" of PFAS levels in seabirds, as well as assessment of temporal trends dating back as far as $1968^{72}$. Trends identified in seabirds to date align with PFAS trends observed in other environmental matrices, including some decreases in PFOS and FOSA ${ }^{64}$ and increases of long-chain PFCAs over time ${ }^{11}$. Wildlife exposure at contaminated sites. Increased public interest in PFAS and improved analytical capacity have resulted in identification of a growing number of PFAS-impacted sites, with reports of numerous contaminated sites across the United States, Europe, Australia and Asia. Localized PFAS contamination may be related to releases from fire-fighting activities involving the use of AFFF, releases from 
industrial sites manufacturing or using PFAS, or application of sewage sludge containing high levels of PFAS ${ }^{52,100-103}$. Habitat subject to high levels of concerted PFAS inputs may pose a risk to wildlife using the contaminated area, but abundant data gaps exist regarding the magnitude of wildlife exposure related to contaminated sites and potentially related individual or population level impacts.

Manufacturing activities are increasingly associated with concerted areas of PFAS contamination. Regulation and phase-outs have caused a geographical shift in production beyond North America and Europe, alongside a departure from long-chain aliphatic chemistries to replacements with fewer than seven fluorinated carbons, polyfluorinated structures, and/or modified iterations of legacy PFAS, such as perfluoroalkyl ether acids (PFEAs) incorporating different numbers of ether linkages, or legacy PFAS analogues substituting chlorine in previously fluorinated positions ${ }^{36,104,105}$. This has resulted in an evolving and poorly understood landscape of PFAS exposure for wildlife surrounding production facilities, including both legacy PFAAs and novel compounds.

PFAS manufacturing and use sites in China, Europe and the US have been documented and observed to cause elevated levels of PFAS contamination in adjacent habitats, downstream environments, and regional wildlife $36,38,52,106,107$. Zhou et al. found PFOS was the predominant compound in fish from Lake Tangxun, adjacent to a major fluorochemical production facility in Wuhan, China, with a mean PFOS value of $263 \mathrm{ng} / \mathrm{g}$ ww in crucian carp muscle and a mean value of $348 \mathrm{ng} / \mathrm{g}$ ww in sharpbelly muscle ${ }^{107}$. Suspect and non-target screening subsequently revealed a suite of 330 novel fluorinated structures belonging to 10 different chemical classes in fish liver 
from the same region ${ }^{108}$. Songbirds near a fluorochemical production site in Antwerp, Belgium contain some of the highest PFOS concentrations identified to date in wildlife, with up to $48,056 \mathrm{ng} / \mathrm{g}$ in eggs of great tits nesting proximate to the production site ${ }^{109-111}$. Songbirds nesting adjacent to manufacturing facilities and disposal sites in Minnesota contained significant concentrations of PFOS (geo. mean $=$ $270 \mathrm{ng} / \mathrm{g}$ egg), and were found to be impacted by PFOS at lower levels than suggested by lab-based studies, with decreased hatching success estimated to occur at 150-250 ng/g egg ${ }^{112,113}$. Chlorinated polyfluoroalkyl ether sulfonic acids (Cl-PFESAs, often referred to under the trade name F53B) are PFOS alternatives widely used in China, and have been identified beyond point sources in coastal biota, with the compound 6:2 Cl-PFESA displaying a trophic magnification factor (TMF) $>1$ in a study involving marine biota samples from the Bohai Sea ${ }^{106}$. In the US, production of PFEAs in North Carolina has resulted in downstream PFEA contamination in fish and humans, along with identification of associations between immune and liver function and elevated PFAS levels in fish ${ }^{34,114}$.

Health Effects in Humans. PFAS are not innocuous, and the environmental ubiquity of this group of emerging pollutants does not translate to a lack of harm or risk. Potential human health and ecosystem impacts were first identified by industry in the 1960s, but it took almost four decades before that information was communicated to the public and action was taken to reduce, minimize, or eliminate some PFAS. To date, most identified human health impacts fall into four general categories: developmental effects, hormonal and immune disruption, potential carcinogenicity, and changes in blood lipid levels ${ }^{54,115}$. 
Many identified effects are associated with environmentally relevant levels of exposure, while some, like increased risk for some cancers, are associated only with high levels of PFAS exposure ${ }^{115}$. Animal studies offer additional insight about potential associations between PFAS and certain health effects such as liver degeneration, decreased immune response, and impaired mammary gland development, but should be interpreted with caution beyond the study species as adverse effects may be taxa-specific ${ }^{115,116}$. Research detailing the effects of some PFAS on human health is rapidly expanding, yet uncertainties remain regarding how mixtures of PFAS or novel PFAS like Gen X and other PFEAs may impact human health. Existing studies suggest novel PFAS may impart similar impacts to the longchain PFAAs they seek to replace ${ }^{117,118}$.

Health Effects in Wildiffe. Most wildlife research to date focuses on the occurrence of PFOA and PFOS in wildlife, with some attention to fluorotelomer-based substances, long-chain PFCAs or emerging PFAS ${ }^{59}$. Existing data suggest these PFAS are widely abundant in wildlife at every level of biological organization and readily bioaccumulate and biomagnify in ways divergent from legacy persistent organic pollutants ${ }^{19,77,119}$, due to their preference for aqueous and protein-based matrices like blood and liver ${ }^{120}$. Traditional modeling based on the octanol-water partitioning coefficient, or $\mathrm{K}_{\text {ow }}$, does not adequately reflect the internal behavior of PFAS ${ }^{121}$. Current modeling suggests a protein-partitioning model, in tandem with a phospholipid-based partitioning model may explain the observed distribution of PFAS, but more empirical measurements and mechanistic details are required to more fully apply these models within realistic food web scenarios ${ }^{33,122-124}$. Long-chain 
compounds have been identified as particularly bioaccumulative in multiple tissues of wildlife $94,125-127$.

Laboratory data suggest PFAS may be associated with adverse impacts on growth, development, reproduction, and metabolism based on rodent, fish, and invertebrate studies ${ }^{128,129}$. Most laboratory toxicity studies describing PFAS impacts rely upon freshwater receptors or other lab organisms that may poorly reflect marine wildlife endpoints and complex food webs ${ }^{129}$. Additionally research identifying impacts of PFAS on wildlife species and food webs stands as a significant data gap and persistent management challenge. As of 2020, roughly 40 studies have investigated the impacts of PFAS in free-living wildlife including birds, mammals, reptiles, fish, and invertebrates ${ }^{129}$. These studies have found impacts on reproductive success exposure ${ }^{112,113}$, changes in a suite of enzymes and signaling pathways transmission $^{130,131}$, as well as associations between immune endpoints and morphometric measurements in diverse taxa $\mathrm{a}^{132}$.

In birds, PFAS have been associated with altered metabolism, altered endocrine function, reproductive failure, decreased egg pipping ability, pathological changes in the liver, brain asymmetry, and other physiological changes indicative of altered immune function ${ }^{129,133-136}$. These studies primarily rely on PFAS measurements in blood or egg paired with measurements of specific biomarkers in wildlife to arrive at these noted effects. Some of these studies note PFAS impacts at environmentally relevant levels of exposure. Significantly more research is required to more fully describe if or how PFAS may be chronically or acutely impacting wildlife or wildlife populations at ambient concentrations. 
PFAS data gaps addressed in this work. While abundant data gaps exist related to the distribution and impacts of PFAS in natural and human environment, this dissertation builds upon existing knowledge by exploring specific and timely questions addressing the occurrence and bioaccumulation of legacy and novel PFAS in seabirds.

Manufacturing activities continue to introduce new PFAS formulations into the environment ${ }^{52}$, with little information provided to the public about their structures, human health risks, or environmental toxicity under protections as confidential business information. Continued research is vital to better describe the environmental and biological occurrence of PFAS adjacent to manufacturing activities, as well describe novel PFAS in the wider environment and biosphere. Additionally, exploration of legacy PFAS has focused on only a handful of PFAAs in select matrices and food webs ${ }^{23,36,137-139}$, with scant investigation of PFAS levels in marine food webs along the US East Coast to date.

While some trends of PFAS have been identified over time, studies documenting changes in PFAA concentrations over the last decade (2010 - 2019) are limited; the existing data offer conflicting evidence about current trends ${ }^{11,88-90}$. Additionally, studies exploring trends of PFAS beyond PFAAs are limited, and temporal and spatial understanding has not been achieved for compounds beyond PFAAs.

This work addresses these data gaps by measuring legacy PFAAs as well as five novel PFAS in seabirds across three US East Coast habitats including a habitat adjacent to a PFAS manufacturing site. This work further explores PFAS temporal trends by measuring legacy and novel PFAS over a ten-year time series in the Great Shearwater, a seabird from Massachusetts Bay. 
Additionally, little work has assessed how novel PFAS partition between different tissues in homeothermic vertebrates, yet such data are vital to predict bioaccumulation and those tissues likely to be most impacted by PFAS. This work entails a tissuespecific study and subsequent commentary on bioaccumulation mechanisms for novel PFEAs and PFAAs to further expand our understanding of PFAS bioaccumulation potential.

Microplastics and seabirds. Plastic pollution in natural environments has emerged as a significant global problem over recent decades. Mismanaged plastic materials are transported to aquatic systems via point and non-point source pathways, and fragment into smaller and smaller pieces under continued exposure to environmental conditions, resulting in the proliferation of microplastics, or small plastic pieces generally $>5 \mathrm{~mm}$ in size ${ }^{140,141}$. Seabirds have been documented to ingest small plastic fragments dating back to the $1960 \mathrm{~s}-1980 \mathrm{~s}^{142}$, but are increasingly at risk of ingesting plastic items due to increasing concentrations of plastic pieces in ocean environments related to exponential growth in the production and use of plastics ${ }^{141,143,144}$.

Seabirds in the order Procellariiformes are particularly vulnerable to plastic ingestion, as 91/144 Procellariiform species have been documented to ingest plastic, either directly from the environment or through the consumption of prey containing plastic items ${ }^{145-147}$. This group, which includes Great Shearwaters, is thought to be especially prone to plastic ingestion due to reduced specialization of diet, wideranging foraging behaviors, and reliance on olfactory cues in prey location ${ }^{148-150}$.

Accumulation of ingested plastics significantly increases risk of mortality in seabirds ${ }^{151}$, while the sublethal impacts of plastic ingestion are more poorly 
understood due to the range of factors that might influence the magnitude of harm. These factors include the type and size of plastic ingested, the duration of time the item has already been in the environment, other diet items consumed, species-specific in vivo clearance rates, and exposure to other environmental stressors or cocontaminants $^{152-156}$.

Cohesive understanding of potential harm from plastic ingestion is also limited by patchy data describing the occurrence of ingested plastics over time, between different locations, and between life stages. Existing work often relies upon opportunistic samples of convenience from readily accessible species and therefore often depicts plastic ingestion at only a discrete time and place. Great Shearwaters have been previously assessed for plastic ingestion in such discrete studies across their breeding and non-breeding range ${ }^{149,157-159}$. Additionally, existing work in Great Shearwaters has focused on the count and mass of ingested plastics, without further detail about the nature of the plastic items ingested or relationships to other physiological variables. With this work, we expand upon previous research in this species by offering a thorough characterization of the size of ingested plastic pieces, polymer types, temporal trends, and relationships to bird body condition by assessing Great Shearwaters across both their breeding and non-breeding range.

Summary. Overall, this dissertation contributes data that describes the occurrence of emerging contaminants including legacy and novel PFAS and microplastics in marine habitats over space and time, primarily using seabirds as sentinels of PFAS and plastic accumulation. We also contribute insight regarding the internal toxicokinetics of PFAS through tissue specific measurements in juvenile seabirds to better constrain the 
internal behavior of PFAS in homeothermic organisms. We further used the large body of necropsy data derived in support of contaminant studies to improve plumage and demographic characterization of the Great Shearwater in the SW Gulf of Maine. 


\section{LITERATURE CITED}

(1) Jamieson, A. J.; Malkocs, T.; Piertney, S. B.; Fujii, T.; Zhang, Z.

Bioaccumulation of Persistent Organic Pollutants in the Deepest Ocean Fauna. Nat. Ecol. Evol. 2017, 1 (3), 24-27. https://doi.org/10.1038/s41559-016-0051.

(2) Wania, F.; Mackay, D. Global Fractionation and Cold Condensation of Low Volatility Organochlorine Compounds in Polar-Regions. Ambio 1993, 22 (1), $10-18$.

(3) Lohmann, R.; Breivik, K.; Dachs, J.; Muir, D. Global Fate of POPs: Current and Future Research Directions. Environ. Pollut. 2007, 150 (1), 150-165. https://doi.org/10.1016/j.envpol.2007.06.051.

(4) Bogdal, C.; Schmid, P.; Zennegg, M.; Anselmetti, F. S.; Scheringer, M.; Hungerb??hler, K. Blast from the Past: Melting Glaciers as a Relevant Source for Persistent Organic Pollutants. Environ. Sci. Technol. 2009, 43 (21), 81738177. https://doi.org/10.1021/es901628x.

(5) Corsolini, S. Industrial Contaminants in Antarctic Biota. J. Chromatogr. A 2009, 1216 (3), 598-612. https://doi.org/10.1016/j.chroma.2008.08.012.

(6) Richardson, S. D.; Kimura, S. Y. Water Analysis: Emerging Contaminants and Current Issues. Anal. Chem. 2016, 88, 546-582.

https://doi.org/10.1021/ac500508t.

(7) Sauvé, S.; Desrosiers, M. A Review of What Is an Emerging Contaminant. Chem. Cent. J. 2014, 8 (1), 1-7. https://doi.org/10.1186/1752-153X-8-15.

(8) Thompson, D. R.; Furness, R. W.; Monteiro, L. R. Seabirds as Biomonitors of Mercury Inputs to Epipelagic and Mesopelagic Marine Food Chains. Sci. Total Environ. 1998, 213 (1-3), 299-305. https://doi.org/10.1016/S00489697(98)00103-X.

(9) Einoder, L. D. A Review of the Use of Seabirds as Indicators in Fisheries and Ecosystem Management. Fish. Res. 2009, 95 (1), 6-13.

https://doi.org/10.1016/j.fishres.2008.09.024.

(10) Durant, J. M.; Hjermann, D.; Frederiksen, M.; Charrassin, J. B.; Le Maho, Y.; Sabarros, P. S.; Crawford, R. J. M.; Stenseth, N. C. Pros and Cons of Using Seabirds as Ecological Indicators. Clim. Res. 2009, 39 (2), 115-129. https://doi.org/10.3354/cr00798.

(11) Miller, A.; Elliott, J. E.; Elliott, K. H.; Lee, S.; Cyr, F. Temporal Trends of Perfluoroalkyl Substances (PFAS) in Eggs of Coastal and Offshore Birds: Increasing PFAS Levels Associated with Offshore Bird Species Breeding on 
the Pacific Coast of Canada and Wintering near Asia. Environ. Toxicol. Chem. 2015, 34 (8), 1799-1808. https://doi.org/10.1002/etc.2992.

(12) Lavers, J. L.; Bond, A. L. Contaminants in Indigenous Harvests of Apex Predators: The Tasmanian Short-Tailed Shearwater as a Case Study. Ecotoxicol. Environ. Saf. 2013, 95, 78-82. https://doi.org/10.1016/j.ecoenv.2013.05.021.

(13) Costantini, D.; Sebastiano, M.; Müller, M. S.; Eulaers, I.; Ambus, P.; Malarvannan, G.; Covaci, A.; Massa, B.; Dell, G.; Dell'Omo, G. Individual Variation of Persistent Organic Pollutants in Relation to Stable Isotope Ratios, Sex, Reproductive Phase and Oxidative Status in Scopoli 's Shearwaters (Calonectris Diomedea) from the Southern Mediterranean. Sci. Total Environ. 2017, 598, 179-187. https://doi.org/10.1016/j.scitotenv.2017.04.014.

(14) Chen, D.; Hale, R. C. A Global Review of Polybrominated Diphenyl Ether Flame Retardant Contamination in Birds. Environ. Int. 2010, 36 (7), 800-811. https://doi.org/10.1016/j.envint.2010.05.013.

(15) McDonald, T. A. A Perspective on the Potential Health Risks of PBDEs. Chemosphere 2002, 46 (5), 745-755. https://doi.org/10.1016/S00456535(01)00239-9.

(16) Liu, G.; Dhana, K.; Furtado, J. D. J. D.; Rood, J.; Zong, G.; Liang, L.; Qi, L. L.; Bray, G. A. G. A.; DeJonge, L.; Coull, B.; Grandjean, P.; Sun, Q.

Perfluoroalkyl Substances and Changes in Body Weight and Resting Metabolic Rate in Response to Weight-Loss Diets: A Prospective Study. PLoS Med. 2018, 15 (2), 1-21. https://doi.org/10.1371/journal.pmed.1002502.

(17) Blévin, P.; Tartu, S.; Ellis, H. I.; Chastel, O.; Bustamante, P.; Parenteau, C.; Herzke, D.; Angelier, F.; Gabrielsen, G. W. Contaminants and Energy Expenditure in an Arctic Seabird: Organochlorine Pesticides and Perfluoroalkyl Substances Are Associated with Metabolic Rate in a Contrasted Manner. Environ. Res. 2017, 157 (February), 118-126. https://doi.org/10.1016/j.envres.2017.05.022.

(18) Kelly, B. C.; Ikonomou, M. G.; Blair, J. D.; Morin, A. E.; Gobas, F. A. P. C. F. a P. C. Food Web-Specific Biomagnification of Persistent Organic Pollutants. Science (80-. ). 2007, 317 (5835), 236-239. https://doi.org/10.1126/science.1138275.

(19) Haukås, M.; Berger, U.; Hop, H.; Gulliksen, B.; Gabrielsen, G. W. Bioaccumulation of Per- and Polyfluorinated Alkyl Substances (PFAS) in Selected Species from the Barents Sea Food Web. Environ. Pollut. 2007, 148 (1), 360-371. https://doi.org/10.1016/j.envpol.2006.09.021.

(20) Li, Q. Q.; Obbard, J. P.; Loganath, A.; Chong, Y. S.; Tan, J.; Bayen, S. 
Persistent Organic Pollutants and Adverse Health Effects in Humans. $J$. Toxicol. Environ. Heal. Part A 2006, 69 (21), 1987-2005. https://doi.org/10.1016/S1474-8177(07)07016-7.

(21) Kuzyk, Z. Z. a; Burgess, N. M.; Stow, J. P.; Fox, G. a. Biological Effects of Marine PCB Contamination on Black Guillemot Nestlings at Saglek, Labrador: Liver Biomarkers. Ecotoxicology 2003, 12 (1-4), 183-197. https://doi.org/10.1023/A:1022550709962.

(22) OECD. Toward a New Comprehensive Global Database of Per- and Polyfluoroalkyl Substances (PFASs). Ser. Risk Manag. 2018, No. 39, 1-24.

(23) Buck, R. C.; Franklin, J.; Berger, U.; Conder, J. M.; Cousins, I. T.; Voogt, P. De; Jensen, A. A.; Kannan, K.; Mabury, S. A.; van Leeuwen, S. P. J. Perfluoroalkyl and Polyfluoroalkyl Substances in the Environment: Terminology, Classification, and Origins. Integr. Environ. Assess. Manag. 2011, 7 (4), 513-541. https://doi.org/10.1002/ieam.258.

(24) Wang, Z.; Cousins, I. T.; Scheringer, M.; Hungerbuehler, K. Hazard Assessment of Fluorinated Alternatives to Long-Chain Perfluoroalkyl Acids (PFAAs) and Their Precursors: Status Quo, Ongoing Challenges and Possible Solutions. Environ. Int. 2015, 75, 172-179.

https://doi.org/10.1016/j.envint.2014.11.013.

(25) Yamashita, N.; Taniyasu, S.; Petrick, G.; Wei, S.; Gamo, T.; Lam, P. K. S.; Kannan, K. Perfluorinated Acids as Novel Chemical Tracers of Global Circulation of Ocean Waters. Chemosphere 2008, 70 (7), 1247-1255. https://doi.org/10.1016/j.chemosphere.2007.07.079.

(26) Kissa, E. Fluorinated Surfactants and Repellents (2nd Edition Revised and Expanded) (Surfactant Science Series 97); Marcel Dekker, Ed.; New York, NY, 2001.

(27) Martin, J. W.; Asher, B. J.; Beesoon, S.; Benskin, J. P.; Ross, M. S. PFOS or PreFOS? Are Perfluorooctane Sulfonate Precursors (PreFOS) Important Determinants of Human and Environmental Perfluorooctane Sulfonate (PFOS) Exposure? J. Environ. Monit. JEM 2010, 12 (11), 1979-2004. https://doi.org/10.1039/c0em00295j.

(28) Ellis, D. A.; Martin, J. W.; Silva, A. O. D. E.; Hurley, M. D.; De Silva, A. O.; Mabury, S. A.; Hurley, M. D.; Sulbaek Andersen, M. P.; Wallington, T. J. Degradation of Fluorotelomer Alcohols: A Likely Atmospheric Source of Perfluorinated Carboxylic Acids. Environ. Sci. Technol. 2004, 38 (12), 33163321. https://doi.org/10.1021/es049860w.

(29) Wallington, T. J.; Hurley, M. D.; Xia, J.; Wuebbles, D. J.; Sillman, S.; Ito, A.; 
Penner, J. E.; Ellis, D. A.; Martin, J.; Mabury, S. A.; Nielsen, O. J.; Sulbaek Andersen, M. P. Formation of C7F15COOH (PFOA) and Other Perfluorocarboxylic Acids during the Atmospheric Oxidation of 8:2 Fluorotelomer Alcohol. Environ. Sci. Technol. 2006, 40 (3), 924-930. https://doi.org/10.1021/es051858x.

(30) Yeung, L. W. Y.; Dassuncao, C.; Mabury, S.; Sunderland, E. M.; Zhang, X.; Lohmann, R. Vertical Profiles, Sources, and Transport of PFASs in the Arctic Ocean. Environ. Sci. Technol. 2017, 51 (12), 6735-6744. https://doi.org/10.1021/acs.est.7b00788.

(31) Hu, X. C.; Andrews, D. Q.; Lindstrom, A. B.; Bruton, T. A.; Schaider, L. A.; Grandjean, P.; Lohmann, R.; Carignan, C. C.; Blum, A.; Balan, S. A.; Higgins, C. P.; Sunderland, E. M. Detection of Poly- and Perfluoroalkyl Substances (PFASs) in U.S. Drinking Water Linked to Industrial Sites, Military Fire Training Areas, and Wastewater Treatment Plants. Environ. Sci. Technol. Lett. 2016, 3 (10), 344-350. https://doi.org/10.1021/acs.estlett.6b00260.

(32) Kim, S. K.; Kannan, K. Perfluorinated Acids in Air, Rain, Snow, Surface Runoff, and Lakes: Relative Importance of Pathways to Contamination of Urban Lakes. Environ. Sci. Technol. 2007, 41 (24), 8328-8334. https://doi.org/10.1021/es072107t.

(33) Martin, J. W.; Mabury, S. A.; Solomon, K. R.; Muir, D. C. G. Dietary Accumulation of Perfluorinated Acids in Juvenile Rainbow Trout ( Oncorhynchus Mykiss ). Environ. Toxicol. Chem. 2003, 22 (1), 189-195. https://doi.org/10.1002/etc.5620220125.

(34) Guillette, T. C.; McCord, J.; Guillette, M.; Polera, M. E.; Rachels, K. T.; Morgeson, C.; Kotlarz, N.; Knappe, D. R. U.; Reading, B. J.; Strynar, M.; Belcher, S. M. Per- and Polyfluoroalkyl Substances Exposure in Cape Fear River Striped Bass (Morone Saxatilis) Is Associated with Biomarkers of Altered Immune and Liver Function Research. Environ. Int. 2019, 2 (1), 105358. https://doi.org/10.1016/j.envint.2019.105358.

(35) Lynch, K. M.; Fair, P. A.; Houde, M.; Muir, D. C. G.; Kannan, K.; Bossart, G. D.; Bartell, S. M.; Gribble, M. O. Temporal Trends in Per- and Polyfluoroalkyl Substances in Bottlenose Dolphins (Tursiops Truncatus) of Indian River Lagoon, Florida and Charleston, South Carolina. Environ. Sci. Technol. 2019, 53 (24), 14194-14203. https://doi.org/10.1021/acs.est.9b04585.

(36) Wang, Z.; Dewitt, J. C.; Higgins, C. P.; Cousins, I. T. A Never-Ending Story of Per- and Polyfluoroalkyl Substances (PFASs)? Environ. Sci. Technol. 2017, 51 (5), 2508-2518. https://doi.org/10.1021/acs.est.6b04806.

(37) Strynar, M.; Dagnino, S.; McMahen, R.; Liang, S.; Lindstrom, A.; Andersen, 
E.; McMillan, L.; Thurman, M.; Ferrer, I.; Ball, C. Identification of Novel Perfluoroalkyl Ether Carboxylic Acids (PFECAs) and Sulfonic Acids (PFESAs) in Natural Waters Using Accurate Mass Time-of-Flight Mass Spectrometry (TOFMS). Environ. Sci. Technol. 2015, 49 (19), 11622-11630. https://doi.org/10.1021/acs.est.5b01215.

(38) Newton, S.; McMahen, R.; Stoeckel, J. A.; Chislock, M.; Lindstrom, A.; Strynar, M. Novel Polyfluorinated Compounds Identified Using High Resolution Mass Spectrometry Downstream of Manufacturing Facilities near Decatur, Alabama. Environ. Sci. Technol. 2017, 51, 1544-1552. https://doi.org/10.1021/acs.est.6b05330.

(39) Barzen-Hanson, K. A.; Roberts, S. C.; Choyke, S.; Oetjen, K.; Mcalees, A.; Riddell, N.; McCrindle, R.; Ferguson, P. L.; Higgins, C. P.; Field, J. A. Discovery of 40 Classes of Per- and Polyfluoroalkyl Substances in Historical Aqueous Film-Forming Foams (AFFFs) and AFFF-Impacted Groundwater. Environ. Sci. Technol. 2017, 51, 2047-2057. https://doi.org/10.1021/acs.est.6b05843.

(40) Moody, C. A.; Field, J. A. Perfluorinated Surfactants and the Environmental Implications of Their Use in Fire-Fighting Foams. Environ. Sci. Technol. 2000, 34 (18), 3864-3870. https://doi.org/10.1021/es991359u.

(41) Glüge, J.; Scheringer, M.; Cousins, I. T.; Dewitt, J. C.; Herzke, D.; Lohmann, R.; Ng, C. A.; Trier, X. An Overview of the Uses of Per- and Polyfluoroalkyl Substances ( PFAS ) 1 Introduction 2 Methods.

(42) Schaider, L. A.; Balan, S. A.; Blum, A.; Andrews, D. Q.; Strynar, M. J.; Dickinson, M. E.; Lunderberg, D. M.; Lang, J. R.; Peaslee, G. F. Fluorinated Compounds in U.S. Fast Food Packaging. 2018. https://doi.org/10.1021/acs.estlett.6b00435.Fluorinated.

(43) Glüge, J.; Scheringer, M.; Cousins, I. T.; DeWitt, J. C.; Goldenman, G.; Herzke, D.; Lohmann, R.; Ng, C. A.; Trier, X.; Wang, Z. An Overview of the Uses of Per- and Polyfluoroalkyl Substances (PFAS). Environ. Sci. Process. Impacts 2020. https://doi.org/10.1039/d0em00291g.

(44) Armitage, J. M.; MacLeod, M.; Cousins, I. T. Modeling the Global Fate and Transport of Perfluorooctanoic Acid (PFOA) and Perfluorooctanoate (PFO) Emitted from Direct Sources Using a Multispecies Mass Balance Model (Environmental Science and Technology (2009) 43, 1134-1140)). Environ. Sci. Technol. 2009, 43 (16), 6438-6439. https://doi.org/10.1021/es901832b.

(45) Armitage, J. M.; Schenker, U.; Scheringer, M.; Martin, J. W.; Macleod, M.; Cousins, I. T. Modeling the Global Fate and Transport of Perfluorooctane Sulfonate (PFOS) and Precursor Compounds in Relation to Temporal Trends in 
Wildlife Exposure. Environ. Sci. Technol. 2009, 43 (24), 9274-9280.

https://doi.org/10.1021/es901448p.

(46) Xiao, F.; Hanson, R. A.; Golovko, S. A.; Golovko, M. Y.; Arnold, W. A. PFOA and PFOS Are Generated from Zwitterionic and Cationic Precursor Compounds During Water Disinfection with Chlorine or Ozone. Environ. Sci. Technol. Lett. 2018, acs.estlett.8b00266. https://doi.org/10.1021/acs.estlett.8b00266.

(47) Vierke, L.; Berger, U.; Cousins, I. T. Estimation of the Acid Dissociation Constant of Perfluoroalkyl Carboxylic Acids through an Experimental Investigation of Their Water-to-Air Transport. Environ. Sci. Technol. 2013, 47 (19), 11032-11039. https://doi.org/10.1021/es402691z.

(48) Pickard, H. M.; Criscitiello, A. S.; Spencer, C.; Sharp, M. J.; Muir, D. C. G.; De Silva, A. O.; Young, C. J. Continuous Non-Marine Inputs of per- and Polyfluoroalkyl Substances to the High Arctic: A Multi-Decadal Temporal Record. Atmos. Chem. Phys. 2018, 18 (7), 5045-5058. https://doi.org/10.5194/acp-18-5045-2018.

(49) Kwok, K. Y.; Yamazaki, E.; Yamashita, N.; Taniyasu, S.; Murphy, M. B.; Horii, Y.; Petrick, G.; Kallerborn, R.; Kannan, K.; Murano, K.; Lam, P. K. S. Transport of Perfluoroalkyl Substances (PFAS) from an Arctic Glacier to Downstream Locations: Implications for Sources. Sci. Total Environ. 2013, 447, 46-55. https://doi.org/10.1016/j.scitotenv.2012.10.091.

(50) Nakayama, S.; Strynar, M. J.; Helfant, L.; Egeghy, P.; Ye, X.; Lindstrom, A. B. Perfluorinated Compounds in the Cape Fear Drainage Basin in North Carolina. Environ. Sci. Technol. 2007, 41, 5271-5276. https://doi.org/10.1021/es070792y.

(51) Munoz, G.; Budzinski, H.; Babut, M.; Lobry, J.; Selleslagh, J.; Tapie, N.; Labadie, P. Temporal Variations of Perfluoroalkyl Substances Partitioning between Surface Water, Suspended Sediment, and Biota in a Macrotidal Estuary. Chemosphere 2019, 233, 319-326. https://doi.org/10.1016/j.chemosphere.2019.05.281.

(52) Washington, J. W.; Rosal, C. G.; McCord, J. P.; Strynar, M. J.; Lindstrom, A. B.; Bergman, E. L.; Goodrow, S. M.; Tadesse, H. K.; Pilant, A. N.; Washington, B. J.; Davis, M. J.; Stuart, B. G.; Jenkins, T. M. Nontargeted Mass-Spectral Detection of Chloroperfluoropolyether Carboxylates in New Jersey Soils. Science 2020, 368 (6495), 1103-1107. https://doi.org/10.1126/science.aba7127.

(53) Paul, A. G.; Jones, K. C.; Sweetman, A. J. A First Global Production, Emission, and Environmental Inventory for Perfluorooctane Sulfonate. Environ. Sci. Technol. 2009, 43 (2), 386-392. https://doi.org/10.1021/es802216n. 
(54) Sunderland, E. M.; Hu, X. C.; Dassuncao, C.; Tokranov, A. K.; Wagner, C. C.; Allen, J. G. A Review of the Pathways of Human Exposure to Poly- and Perfluoroalkyl Substances (PFASs) and Present Understanding of Health Effects. J. Expo. Sci. Environ. Epidemiol. 2019, 29 (2), 131-147. https://doi.org/10.1038/s41370-018-0094-1.

(55) Hu, X. C.; Dassuncao, C.; Zhang, X.; Grandjean, P.; Weihe, P.; Webster, G. M.; Nielsen, F.; Sunderland, E. M. Can Profiles of Poly- and Perfluoroalkyl Substances (PFASs) in Human Serum Provide Information on Major Exposure Sources? Environ. Heal. A Glob. Access Sci. Source 2018, 17 (1), 1-15. https://doi.org/10.1186/s12940-018-0355-4.

(56) Dassuncao, C.; Hu, X. C.; Nielsen, F.; Weihe, P.; Grandjean, P.; Sunderland, E. M. Shifting Global Exposures to Poly- and Perfluoroalkyl Substances (PFASs) Evident in Longitudinal Birth Cohorts from a Seafood-Consuming Population. Environ. Sci. Technol. 2018, 52 (6), 3738-3747. https://doi.org/10.1021/acs.est.7b06044.

(57) Zhang, X.; Lohmann, R.; Dassuncao, C.; Hu, X. C.; Weber, A. K.; Vecitis, C. D.; Sunderland, E. M. Source Attribution of Poly- and Perfluoroalkyl Substances (PFASs) in Surface Waters from Rhode Island and the New York Metropolitan Area. Environ. Sci. Technol. Lett. 2016, 3 (9), 316-321. https://doi.org/10.1021/acs.estlett.6b00255.

(58) Andrews, D. Q.; Naidenko, O. V. Population-Wide Exposure to Per- and Polyfluoroalkyl Substances from Drinking Water in the United States. Environ. Sci. Technol. Lett. 2020. https://doi.org/10.1021/acs.estlett.0c00713.

(59) Reiner, J. J. L.; Place, B. L. Perfluorinated Alkyl Acids in Wildlife. In Toxicological Effects of Perfluoroalkyl and Polyfluoralkyl Substances; DeWitt, J. C., Ed.; Humana Press, 2015; pp 127-150. https://doi.org/https://doi.org/10.1007/978-3-319-15518-0_5.

(60) Langberg, H. A.; Breedveld, G. D.; Grønning, H. M.; Kvennås, M.; Jenssen, B. M.; Hale, S. E. Bioaccumulation of Fluorotelomer Sulfonates and Perfluoroalkyl Acids in Marine Organisms Living in Aqueous Film-Forming Foam Impacted Waters. Environ. Sci. Technol. 2019, 53 (18), 10951-10960. https://doi.org/10.1021/acs.est.9b00927.

(61) Lanza, H. A.; Cochran, R. S.; Mudge, J. F.; Olson, A. D.; Blackwell, B. R.; Maul, J. D.; Salice, C. J.; Anderson, T. A. Temporal Monitoring of Perfluorooctane Sulfonate Accumulation in Aquatic Biota Downstream of Historical Aqueous Film Forming Foam Use Areas. Environ. Toxicol. Chem. 2017, 36 (8), 2022-2029. https://doi.org/10.1002/etc.3726.

(62) White, N. D.; Vena, J. E.; Kannan, K.; Karthikraj, R.; Wolf, B.; Fair, P. A.; 
Arnott, S. A. Perfluoroalkyl Substances (PFASs) in Edible Fish Species from Charleston Harbor and Tributaries, South Carolina, United States: Exposure and Risk Assessment. Environ. Res. 2019, 171 (November 2018), 266-277. https://doi.org/10.1016/j.envres.2019.01.021.

(63) Dassuncao, C.; Hu, X. C.; Zhang, X.; Bossi, R.; Dam, M.; Mikkelsen, B.; Sunderland, E. M. Temporal Shifts in Poly- and Perfluoroalkyl Substances (PFASs) in North Atlantic Pilot Whales Indicate Large Contribution of Atmospheric Precursors. Environ. Sci. Technol. 2017, 51 (8), 4512-4521. https://doi.org/10.1021/acs.est.7b00293.

(64) Gebbink, W. A.; Letcher, R. J.; Hebert, C. E.; Chip Weseloh, D. V. Twenty Years of Temporal Change in Perfluoroalkyl Sulfonate and Carboxylate Contaminants in Herring Gull Eggs from the Laurentian Great Lakes. $J$. Environ. Monit. JEM 2011, 13 (12), 3365-3372. https://doi.org/10.1039/c1em10663e.

(65) Bangma, J. T.; Ragland, J. M.; Rainwater, T. R.; Bowden, J. A.; Gibbons, J. W.; Reiner, J. L. Perfluoroalkyl Substances in Diamondback Terrapins (Malaclemys Terrapin) in Coastal South Carolina. Chemosphere 2019, 215, 305-312. https://doi.org/10.1016/j.chemosphere.2018.10.023.

(66) Kowalczyk, J.; Ehlers, S.; Oberhausen, A.; Tischer, M.; Fürst, P.; Schafft, H.; Lahrssen-Wiederholt, M. Absorption, Distribution, and Milk Secretion of the Perfluoroalkyl Acids PFBS, PFHxS, PFOS, and PFOA by Dairy Cows Fed Naturally Contaminated Feed. J. Agric. Food Chem. 2013, 61 (12), 2903-2912. https://doi.org/10.1021/jf304680j.

(67) Scher, D. P.; Kelly, J. E.; Huset, C. A.; Barry, K. M.; Hoffbeck, R. W.; Yingling, V. L.; Messing, R. B. Occurrence of Perfluoroalkyl Substances (PFAS) in Garden Produce at Homes with a History of PFAS-Contaminated Drinking Water. Chemosphere 2018, 196, 548-555. https://doi.org/10.1016/j.chemosphere.2017.12.179.

(68) Ghisi, R.; Vamerali, T.; Manzetti, S. Accumulation of Perfluorinated Alkyl Substances (PFAS) in Agricultural Plants: A Review. Environ. Res. 2019, 169 (October 2018), 326-341. https://doi.org/10.1016/j.envres.2018.10.023.

(69) Sunderland, E. M.; Hu, X. C.; Dassuncao, C.; Tokranov, A. K.; Wagner, C. C.; Allen, J. G. A Review of the Pathways of Human Exposure to Poly- and per Fl Uoroalkyl Substances ( PFASs ) and Present Understanding of Health Effects. 2018. https://doi.org/10.1038/s41370-018-0094-1.

(70) Muir, D.; Bossi, R.; Carlsson, P.; Evans, M.; De Silva, A.; Halsall, C.; Rauert, C.; Herzke, D.; Hung, H.; Letcher, R.; Rigét, F.; Roos, A. Levels and Trends of Poly- and Perfluoroalkyl Substances in the Arctic Environment - An Update. 
Emerg. Contam. 2019, 5, 240-271.

https://doi.org/10.1016/j.emcon.2019.06.002.

(71) Martin, J. W.; Smithwick, M. M.; Braune, B. M.; Hoekstra, P. F.; Muir, D. C. G.; Mabury, S. A. Identification of Long-Chain Perfluorinated Acids in Biota from the Canadian Arctic. Environ. Sci. Technol. 2004, 38 (2), 373-380. https://doi.org/10.1021/es034727+.

(72) Braune, B. M.; Letcher, R. J. Perfluorinated Sulfonate and Carboxylate Compounds in Eggs of Seabirds Breeding in the Canadian Arctic: Temporal Trends (1975-2011) and Interspecies Comparison. Environ. Sci. Technol. 2013, 47 (1), 616-624. https://doi.org/10.1021/es303733d.

(73) Routti, H.; Aars, J.; Fuglei, E.; Hanssen, L.; Lone, K.; Polder, A.; Pedersen, Å. Ø.; Tartu, S.; Welker, M.; Yoccoz, N. G. Emission Changes Dwarf the In Fl Uence of Feeding Habits on Temporal Trends of Per- and Poly Fl Uoroalkyl Substances in Two Arctic Top Predators. 2017. https://doi.org/10.1021/acs.est.7b03585.

(74) Martin, J. W.; Whittle, D. M.; Muir, D. C. G.; Mabury, S. A. Perfluoroalkyl Contaminants in a Food Web from Lake Ontario. Environ. Sci. Technol. 2004, 38 (20), 5379-5385. https://doi.org/10.1021/es049331s.

(75) Wu, Y.; Simon, K. L.; Best, D. A.; Bowerman, W.; Venier, M. Novel and Legacy Per- and Polyfluoroalkyl Substances in Bald Eagle Eggs from the Great Lakes Region. Environ. Pollut. 2020, 260, 113811. https://doi.org/10.1016/j.envpol.2019.113811.

(76) Furdui, V. I.; Stock, N. L.; Ellis, D. A.; Butt, C. M.; Whittle, D. M.; Crozier, P. W.; Reiner, E. J.; Muir, D. C. G. G.; Mabury, S. A. Spatial Distribution of Perfluoroalkyl Contaminants in Lake Trout from the Great Lakes.

Environ.Sci.Technol. 2007, 41 (5), 1554-1559.

https://doi.org/10.1021/es0620484.

(77) Zhang, X.; Lohmann, R.; Sunderland, E. M. Poly- and Perfluoroalkyl Substances (PFAS) in Seawater and Plankton from the Northwestern Atlantic Margin. Environ. Sci. Technol. 2019, 53 (21), 12348-12356. https://doi.org/10.1021/acs.est.9b03230.

(78) Zhang, X.; Lohmann, R.; Dassuncao, C.; Hu, X. C.; Weber, A. K.; Vecitis, C. D.; Sunderland, E. M. Source Attribution of Poly- and Perfluoroalkyl Substances (PFASs) in Surface Waters from Rhode Island and the New York Metropolitan Area. Environ. Sci. Technol. Lett. 2016, 3 (9), 316-321. https://doi.org/10.1021/acs.estlett.6b00255.

(79) Dassuncao, C.; Hu, X. C.; Zhang, X.; Bossi, R.; Dam, M.; Mikkelsen, B.; 
Sunderland, E. M. Temporal Shifts in Poly- and Perfluoroalkyl Substances (PFASs) in North Atlantic Pilot Whales Indicate Large Contribution of Atmospheric Precursors. Environ. Sci. Technol. 2017, 51 (8), 4512-4521. https://doi.org/10.1021/acs.est.7b00293.

(80) Chu, S.; Wang, J.; Leong, G.; Woodward, L. A.; Letcher, R. J.; Li, Q. X. Perfluoroalkyl Sulfonates and Carboxylic Acids in Liver, Muscle and Adipose Tissues of Black-Footed Albatross (Phoebastria Nigripes) from Midway Island, North Pacific Ocean. Chemosphere 2015, 138, 60-66. https://doi.org/10.1016/j.chemosphere.2015.05.043.

(81) Sedlak, M. D.; Benskin, J. P.; Wong, A.; Grace, R.; Greig, D. J. Per- and Polyfluoroalkyl Substances (PFASs) in San Francisco Bay Wildlife: Temporal Trends, Exposure Pathways, and Notable Presence of Precursor Compounds. Chemosphere 2017, 185, 1217-1226.

https://doi.org/10.1016/j.chemosphere.2017.04.096.

(82) Tao, L.; Kannan, K.; Kajiwara, N.; Costa, M. M.; Fillmann, G.; Takahashi, S.; Tanabe, S. Perfluorooctanesulfonate and Related Fluorochemicals in Albatrosses, Elephant Seals, Penguins, and Polar Skuas from the Southern Ocean. Environ. Sci. Technol. 2006, 40 (24), 7642-7648. https://doi.org/10.1021/es061513u.

(83) Routti, H.; Krafft, B. A.; Herzke, D.; Eisert, R.; Oftedal, O. Perfluoroalkyl Substances Detected in the World's Southernmost Marine Mammal, the Weddell Seal (Leptonychotes Weddellii). Environ. Pollut. 2015, 197, 62-67. https://doi.org/10.1016/j.envpol.2014.11.026.

(84) Loi, E. I. H.; Yeung, L. W. Y.; Taniyasu, S.; Lam, P. K. S.; Kannan, K.; Yamashita, N. Trophic Magnification of Poly- and Perfluorinated Compounds in a Subtropical Food Web. Environ. Sci. Technol. 2011, 45 (13), 5506-5513. https://doi.org/10.1021/es200432n.

(85) Penland, T. N.; Cope, W. G.; Kwak, T. J.; Strynar, M. J.; Grieshaber, C. A.; Heise, R. J.; Sessions, F. W. Trophodynamics of Per-and Polyfluoroalkyl Substances in the Food Web of a Large Atlantic Slope River. Environ. Sci. Technol. 2020. https://doi.org/10.1021/acs.est.9b05007.

(86) Munoz, G.; Budzinski, H.; Babut, M.; Drouineau, H.; Lauzent, M.; Menach, K. Le; Lobry, J.; Selleslagh, J.; Simonnet-Laprade, C.; Labadie, P. Evidence for the Trophic Transfer of Perfluoroalkylated Substances in a Temperate Macrotidal Estuary. Environ. Sci. Technol. 2017, 51 (15), 8450-8459. https://doi.org/10.1021/acs.est.7b02399.

(87) Smithwick, M.; Solomon, K. R.; Muir, D. G. G. C. G.; Mabury, S. A.; Martin, J. W.; Sonne, C.; Dietz, R.; Born, E. W.; Letcher, R. J.; Solomon, K. R.; 
Martin, J. W.; Sonne, C.; Born, E. W.; Letcher, R. J.; Dietz, R. Perflouroalkyl Contaminants in Liver Tissue from East Greenland Polar Bears (Ursus Maritimus). Environ. Toxicol. Chem. 2005, 24 (4), 981-986. https://doi.org/10.1897/04-258R.1.

(88) Jouanneau, W.; Bårdsen, B.-J.; Herzke, D.; Johnsen, T. V.; Eulaers, I.; Bustnes, J. O. Spatiotemporal Analysis of Perfluoroalkyl Substances in White-Tailed Eagle (Haliaeetus Albicilla) Nestlings from Northern Norway-A Ten-Year Study. Environ. Sci. Technol. 2020, 54 (8), 5011-5020. https://doi.org/10.1021/acs.est.9b06818.

(89) Sun, J.; Bossi, R.; Bustnes, J. O.; Helander, B.; Boertmann, D.; Dietz, R.; Herzke, D.; Jaspers, V. L. B.; Labansen, A. L.; Lepoint, G.; Schulz, R.; Sonne, C.; Thorup, K.; Tøttrup, A. P.; Zubrod, J. P.; Eens, M.; Eulaers, I. White-Tailed Eagle (Haliaeetus Albicilla) Body Feathers Document Spatiotemporal Trends of Perfluoroalkyl Substances in the Northern Environment. Environ. Sci. Technol. 2019. https://doi.org/10.1021/acs.est.9b03514.

(90) Route, W. T.; Key, R. L.; Russell, R. E.; Lindstrom, A. B.; Strynar, M. J. Spatial and Temporal Patterns in Concentrations of Perfluorinated Compounds in Bald Eagle Nestlings in the Upper Midwestern United States. Environ. Sci. Technol. 2014, 48 (12), 6653-6660. https://doi.org/10.1021/es501055d.

(91) Giesy, J. P.; Kannan, K. Perfluorochemical Surfactants in the Environment. Environ. Sci. Technol. 2002, 36 (7), 146A-152A. https://doi.org/10.1021/es022253t.

(92) Kannan, K.; Franson, J. C.; Bowerman, W. W.; Hansen, K. J.; Jones, P. D.; Giesy, J. P. Perfluorooctane Sulfonate in Fish-Eating Water Birds Including Bald Eagles and Albatrosses. Environ. Sci. Technol. 2001, 35 (15), 3065-3070. https://doi.org/10.1021/es001935i.

(93) Giesy, J. P.; Kannan, K. Global Distribution of Perfluorooctane Sulfonate in Wildlife Global Distribution of Perfluorooctane Sulfonate in Wildlife. Environ. Sci. Technol. 2001, 35 (March), 1339-1342. https://doi.org/10.1021/es001834k.

(94) Holmström, K. E.; Berger, U. Tissue Distribution of Perfluorinated Surfactants in Common Guillemot (Uria Aalge) from the Baltic Sea. Environ. Sci. Technol. 2008, 42 (16), 5879-5884. https://doi.org/10.1021/es800529h.

(95) Gebbink, W. A.; Letcher, R. J.; Burgess, N. M.; Champoux, L.; Elliott, J. E.; Hebert, C. E.; Martin, P.; Wayland, M.; Weseloh, D. V. C.; Wilson, L. Perfluoroalkyl Carboxylates and Sulfonates and Precursors in Relation to Dietary Source Tracers in the Eggs of Four Species of Gulls (Larids) from Breeding Sites Spanning Atlantic to Pacific Canada. Environ. Int. 2011, 37 (7), 1175-1182. https://doi.org/10.1016/j.envint.2011.04.003. 
(96) Gebbink, W. A.; Hebert, C. E.; Letcher, R. J. Perfluorinated Carboxylates and Sulfonates and Precursor Compounds in Herring Gull Eggs from Colonies Spanning the Laurentian Great Lakes of North America. Environ. Sci. Technol. 2009, 43 (19), 7443-7449. https://doi.org/10.1021/es901755q.

(97) Gebbink, W. A.; Letcher, R. J. Comparative Tissue and Body Compartment Accumulation and Maternal Transfer to Eggs of Perfluoroalkyl Sulfonates and Carboxylates in Great Lakes Herring Gulls. Environ. Pollut. 2012, 162, 40-47. https://doi.org/10.1016/j.envpol.2011.10.011.

(98) Leat, E. H. K.; Bourgeon, S.; Eze, J. I.; Muir, D. C. G.; Williamson, M.; Bustnes, J. O.; Furness, R. W.; Borgå, K. Perfluoroalkyl Substances in Eggs and Plasma of an Avian Top Predator, Great Skua (Stercorarius Skua), in the North Atlantic. Environ. Toxicol. Chem. 2013, 32 (3), 569-576. https://doi.org/10.1002/etc.2101.

(99) Roscales, J. L.; Vicente, A.; Ryan, P. G.; González-Solís, J.; Jiménez, B. Spatial and Interspecies Heterogeneity in Concentrations of Perfluoroalkyl Substances (PFASs) in Seabirds of the Southern Ocean. Environ. Sci. Technol. 2019, 53 (16), 9855-9865. https://doi.org/10.1021/acs.est.9b02677.

(100) Strynar, M.; Dagnino, S.; McMahen, R.; Liang, S.; Lindstrom, A.; Andersen, E.; McMillan, L.; Thurman, M.; Ferrer, I.; Ball, C. Identification of Novel Perfluoroalkyl Ether Carboxylic Acids (PFECAs) and Sulfonic Acids (PFESAs) in Natural Waters Using Accurate Mass Time-of-Flight Mass Spectrometry (TOFMS). Environ. Sci. Technol. 2015, 49 (19), 11622-11630. https://doi.org/10.1021/acs.est.5b01215.

(101) Ahrens, L.; Norström, K.; Viktor, T.; Cousins, A. P.; Josefsson, S. Stockholm Arlanda Airport as a Source of Per- and Polyfluoroalkyl Substances to Water, Sediment and Fish. Chemosphere 2015, 129, 33-38. https://doi.org/10.1016/j.chemosphere.2014.03.136.

(102) Lindstrom, A. B.; Strynar, M. J.; Delinsky, A. D.; Nakayama, S. F.; Mcmillan, L.; Libelo, E. L.; Neill, M.; Thomas, L. Application of WWTP Biosolids and Resulting Perfluorinated Compound Contamination of Surface and Well Water in Decatur ,. 2011, 8015-8021. https://doi.org/10.1021/es1039425.

(103) Letcher, R. J.; Chu, S.; Smyth, S. A. Side-Chain Fluorinated Polymer Surfactants in Biosolids from Wastewater Treatment Plants. J. Hazard. Mater. 2020, 388 (January), 122044. https://doi.org/10.1016/j.jhazmat.2020.122044.

(104) Wang, Z.; Cousins, I. T.; Scheringer, M.; Hungerbühler, K. Fluorinated Alternatives to Long-Chain Perfluoroalkyl Carboxylic Acids (PFCAs), Perfluoroalkane Sulfonic Acids (PFSAs) and Their Potential Precursors. Environ. Int. 2013, 60 (2013), 242-248. 
https://doi.org/10.1016/j.envint.2013.08.021.

(105) Liu, Y.; Richardson, E. S.; Derocher, A. E.; Lunn, N. J.; Lehmler, H. J.; Li, X.; Zhang, Y.; Cui, J. Y.; Cheng, L.; Martin, J. W. Hundreds of Unrecognized Halogenated Contaminants Discovered in Polar Bear Serum. Angew. Chemie Int. Ed. 2018, 57 (50), 16401-16406. https://doi.org/10.1002/anie.201809906.

(106) Liu, Y.; Ruan, T.; Lin, Y.; Liu, A.; Yu, M.; Liu, R.; Meng, M.; Wang, Y.; Liu, J.; Jiang, G. Chlorinated Polyfluoroalkyl Ether Sulfonic Acids in Marine Organisms from Bohai Sea, China: Occurrence, Temporal Variations, and Trophic Transfer Behavior. Environ. Sci. Technol. 2017, 51 (8), 4407-4414. https://doi.org/10.1021/acs.est.6b06593.

(107) Zhou, Z.; Liang, Y.; Shi, Y.; Xu, L.; Cai, Y. Occurrence and Transport of Perfluoroalkyl Acids (PFAAs), Including Short-Chain PFAAs in Tangxun Lake, China. Environ. Sci. Technol. 2013, 47 (16), 9249-9257. https://doi.org/10.1021/es402120y.

(108) Liu, Y.; Qian, M.; Ma, X.; Zhu, L.; Martin, J. W.; Qian, M.; Ma, X.; Zhu, L.; Martin, J. W. Nontarget Mass Spectrometry Reveals New Perfluoroalkyl Substances in Fish from the Yangtze River and Tangxun Lake, China. Environ. Sci. Technol. 2018, 52, 5830-5840. https://doi.org/10.1021/acs.est.8b00779.

(109) Lopez-Antia, A.; Groffen, T.; Lasters, R.; AbdElgawad, H.; Sun, J.; Asard, H.; Bervoets, L.; Eens, M. Perfluoroalkyl Acids (PFAAs) Concentrations and Oxidative Status in Two Generations of Great Tits Inhabiting a Contamination Hotspot. Environ. Sci. Technol. 2019, 53, 1617-1626. https://doi.org/10.1021/acs.est.8b05235.

(110) Groffen, T.; Lopez-Antia, A.; Hollander, W. D.; Prinsen, E.; Eens, M. Perfluoroalkylated Acids in the Eggs of Great Tits ( Parus Major ) near a Flurochemical Plant in Flanders, Belgium. Environ. Pollut. 2017, 228, 140148. https://doi.org/10.1016/j.envpol.2017.05.007.

(111) Groffen, T.; Lasters, R.; Lopez-Antia, A.; Prinsen, E.; Bervoets, L.; Eens, M. Limited Reproductive Impairment in a Passerine Bird Species Exposed along a Perfluoroalkyl Acid (PFAA) Pollution Gradient. Sci. Total Environ. 2019, 652 (2019), 718-728. https://doi.org/10.1016/j.scitotenv.2018.10.273.

(112) Custer, C. M.; Custer, T. W.; Dummer, P. M.; Etterson, M. A.; Mckann, P. C. Exposure and Effects of Perfluoroalkyl Substances in Tree Swallows Nesting in Minnesota and Wisconsin, USA. 2014, 2000, 120-138. https://doi.org/10.1007/s00244-013-9934-0.

(113) Custer, C. M.; Custer, T. W.; Schoenfuss, H. L.; Poganski, B. H.; Solem, L. Exposure and Effects of Perfluoroalkyl Compounds on Tree Swallows Nesting 
at Lake Johanna in East Central Minnesota, USA. Reprod. Toxicol. 2012, 33

(4), 556-562. https://doi.org/10.1016/j.reprotox.2011.01.005.

(114) Kotlarz, N.; McCord, J.; Collier, D.; Lea, C.; Strynar, M.; Lindstron, A.; Wilkie, A.; Islam, J.; Matney, K.; Tarte, P.; Polera, M.; Burdette, K.; DeWitt, J.; May, K.; Smart, R.; Knappe, D.; Hoppin, J. A. Measurement of Novel, Drinking Water-Associated PFAS in Blood from Adults and Children in Wilmington, North Carolina. Env. Heal. Perspect 2020, 128 (July), 1-12.

(115) Fenton, S. E.; Ducatman, A.; Boobis, A.; DeWitt, J. C.; Lau, C.; Ng, C.; Smith, J. S.; Roberts, S. M. Per- and Polyfluoroalkyl Substance Toxicity and Human Health Review: Current State of Knowledge and Strategies for Informing Future Research. Environ. Toxicol. Chem. 2020, 0-2. https://doi.org/10.1002/etc.4890.

(116) Pizzurro, D. M.; Seeley, M.; Kerper, L. E.; Beck, B. D. Interspecies Differences in Perfluoroalkyl Substances (PFAS)Toxicokinetics and Application to HealthBased Criteria. Regul. Toxicol. Pharmacol. 2019, 106 (May), 239-250. https://doi.org/10.1016/j.yrtph.2019.05.008.

(117) Blake, B. E.; Cope, H. A.; Hall, S. M.; Keys, R. D.; Mahler, B. W.; McCord, J.; Scott, B.; Stapleton, H. M.; Strynar, M. J.; Elmore, S. A.; Fenton, S. E. Evaluation of Maternal, Embryo, and Placental Effects in CD-1 Mice Following Gestational Exposure to Perfluorooctanoic Acid (PFOA) or Hexafluoropropylene Oxide Dimer Acid (HFPO-DA or GenX). Environ. Health Perspect. 2020, 128 (2), 1-17. https://doi.org/10.1289/EHP6233.

(118) Gomis, M. I.; Vestergren, R.; Borg, D.; Cousins, I. T. Comparing the Toxic Potency in Vivo of Long-Chain per Fl Uoroalkyl Acids and Fl Uorinated Alternatives. 2018, 113 (November 2017), 1-9. https://doi.org/10.1016/j.envint.2018.01.011.

(119) Kelly, B. C.; Ikonomou, M. G.; Blair, J. D.; Surridge, B.; Hoover, D.; Grace, R.; Gobas, F. A. P. C. Perfluoroalkyl Contaminants in an Arctic Marine Food Web: Trophic Magnification and Wildlife Exposure. Environ. Sci. Technol. 2009, 43 (11), 4037-4043. https://doi.org/10.1021/es9003894.

(120) Ng, C. A.; Hungerbühler, K. Bioaccumulation of Perfluorinated Alkyl Acids: Observations and Models. Environ. Sci. Technol. 2014, 48 (9), 4637-4648. https://doi.org/10.1021/es404008g.

(121) Armitage, J. M.; Arnot, J. A.; Wania, F.; Mackay, D. Development and Evaluation of a Mechanistic Bioconcentration Model for Ionogenic Organic Chemicals in Fish. Environ. Toxicol. Chem. 2013, 32 (1), 115-128. https://doi.org/10.1002/etc.2020. 
(122) Fujii, Y.; Kato, Y.; Sakamoto, K.; Matsuishi, T.; Kouji, H. H.; Koizumi, A.; Kimura, O.; Endo, T.; Haraguchi, K. Tissue-Specific Bioaccumulation of LongChain Perfluorinated Carboxylic Acids and Halogenated Methylbipyrroles in Dall's Porpoises (Phocoenoides Dalli) and Harbor Porpoises (Phocoena Phocoena) Stranded in Northern Japan. Sci. Total Environ. 2018, 616-617, 554-563. https://doi.org/10.1016/j.scitotenv.2017.10.033.

(123) Ng, C. A.; Hungerbühler, K. Bioconcentration of Perfluorinated Alkyl Acids: How Important Is Specific Binding? Environ. Sci. Technol. 2013, 47 (13), 7214-7223. https://doi.org/10.1021/es400981a.

(124) Armitage, J. M.; Arnot, J. A.; Wania, F. Potential Role of Phospholipids in Determining the Internal Tissue Distribution of Perfluoroalkyl Acids in Biota. Environ. Sci. Technol. 2012, 46 (22), 12285-12286. https://doi.org/10.1021/es304430r.

(125) Dassuncao, C.; Pickard, H.; Pfohl, M.; Tokranov, A. K.; Li, M.; Mikkelsen, B.; Slitt, A.; Sunderland, E. M. Phospholipid Levels Predict the Tissue Distribution of Poly- and Perfluoroalkyl Substances in a Marine Mammal. Environ. Sci. Technol. Lett. 2019, 6 (3), acs.estlett.9b00031. https://doi.org/10.1021/acs.estlett.9b00031.

(126) Greaves, A. K.; Letcher, R. J.; Sonne, C.; Dietz, R.; Born, E. W. TissueSpecific Concentrations and Patterns of Perfluoroalkyl Carboxylates and Sulfonates in East Greenland Polar Bears. Environ. Sci. Technol. 2012, 46 (21), 11575-11583. https://doi.org/10.1021/es303400f.

(127) Ahrens, L.; Siebert, U.; Ebinghaus, R. Total Body Burden and Tissue Distribution of Polyfluorinated Compounds in Harbor Seals (Phoca Vitulina) from the German Bight. Mar. Pollut. Bull. 2009, 58 (4), 520-525. https://doi.org/10.1016/j.marpolbul.2008.11.030.

(128) Ahrens, L.; Bundschuh, M.; Hrens, L. U. T. Z. A.; Undschuh, M. I. B. Fate and Effects of Poly- and Perfluoroalkyl Substances in the Aquatic Environment: A Review. Environ. Toxicol. Chem. 2014, 33 (9), 1921-1929. https://doi.org/10.1002/etc.2663.

(129) Ankley, G. T.; Cureton, P.; Hoke, R. A.; Houde, M.; Kumar, A.; Kurias, J.; Lanno, R.; McCarthy, C.; Newsted, J.; Salice, C. J.; Sample, B. E.; Sepúlveda, M. S.; Steevens, J.; Valsecchi, S. Assessing the Ecological Risks of Per- and Polyfluoroalkyl Substances: Current State-of-the Science and a Proposed Path Forward. Environ. Toxicol. Chem. 2020, 00 (00), 1-42. https://doi.org/10.1002/etc.4869.

(130) Eggers Pedersen, K.; Basu, N.; Letcher, R.; Greaves, A. K.; Sonne, C.; Dietz, R.; Styrishave, B. Brain Region-Specific Perfluoroalkylated Sulfonate (PFSA) 
and Carboxylic Acid (PFCA) Accumulation and Neurochemical Biomarker Responses in East Greenland Polar Bears (Ursus Maritimus). Environ. Res. 2015, 138, 22-31. https://doi.org/10.1016/j.envres.2015.01.015.

(131) Kurtz, A. E.; Reiner, J. L.; West, K. L.; Jensen, B. A. Perfluorinated Alkyl Acids in Hawaiian Cetaceans and Potential Biomarkers of Effect: Peroxisome Proliferator-Activated Receptor Alpha and Cytochrome P450 4A. Environ. Sci. Technol. 2019, acs.est.8b05619. https://doi.org/10.1021/acs.est.8b05619.

(132) Dietz, R.; Letcher, R. J.; Desforges, J. P.; Eulaers, I.; Sonne, C.; Wilson, S.; Andersen-Ranberg, E.; Basu, N.; Barst, B. D.; Bustnes, J. O.; Bytingsvik, J.; Ciesielski, T. M.; Drevnick, P. E.; Gabrielsen, G. W.; Haarr, A.; Hylland, K.; Jenssen, B. M.; Levin, M.; McKinney, M. A.; Nørregaard, R. D.; Pedersen, K. E.; Provencher, J.; Styrishave, B.; Tartu, S.; Aars, J.; Ackerman, J. T.; RosingAsvid, A.; Barrett, R.; Bignert, A.; Born, E. W.; Branigan, M.; Braune, B.; Bryan, C. E.; Dam, M.; Eagles-Smith, C. A.; Evans, M.; Evans, T. J.; Fisk, A. T.; Gamberg, M.; Gustavson, K.; Hartman, C. A.; Helander, B.; Herzog, M. P.; Hoekstra, P. F.; Houde, M.; Hoydal, K.; Jackson, A. K.; Kucklick, J.; Lie, E.; Loseto, L.; Mallory, M. L.; Miljeteig, C.; Mosbech, A.; Muir, D. C. G.; Nielsen, S. T.; Peacock, E.; Pedro, S.; Peterson, S. H.; Polder, A.; Rigét, F. F.; Roach, P.; Saunes, H.; Sinding, M. H. S.; Skaare, J. U.; Søndergaard, J.; Stenson, G.; Stern, G.; Treu, G.; Schuur, S. S.; Víkingsson, G. Current State of Knowledge on Biological Effects from Contaminants on Arctic Wildlife and Fish. Sci. Total Environ. 2019, 696. https://doi.org/10.1016/j.scitotenv.2019.133792.

(133) O’Brien, J. M.; Carew, A. C.; Chu, S.; Letcher, R. J.; Kennedy, S. W. Perfluorooctane Sulfonate (PFOS) Toxicity in Domestic Chicken (Gallus Gallus Domesticus) Embryos in the Absence of Effects on Peroxisome Proliferator Activated Receptor Alpha (PPAR $\alpha$ )-Regulated Genes. Comp. Biochem. Physiol. - C Toxicol. Pharmacol. 2009, 149 (4), 524-530. https://doi.org/10.1016/j.cbpc.2008.11.009.

(134) O’Brien, J. M.; Crump, D.; Mundy, L. J.; Chu, S.; McLaren, K. K.; Vongphachan, V.; Letcher, R. J.; Kennedy, S. W. Pipping Success and Liver MRNA Expression in Chicken Embryos Exposed in Ovo to C8and C11perfluorinated Carboxylic Acids and C10perfluorinated Sulfonate. Toxicol. Lett. 2009, 190 (2), 134-139. https://doi.org/10.1016/j.toxlet.2009.07.004.

(135) Nordén, M.; Berger, U.; Engwall, M. Developmental Toxicity of PFOS and PFOA in Great Cormorant (Phalacrocorax Carbo Sinensis), Herring Gull (Larus Argentatus) and Chicken (Gallus Gallus Domesticus). Environ. Sci. Pollut. Res. 2016, 23 (11), 10855-10862. https://doi.org/10.1007/s11356-016-6285-1.

(136) Molina, E. D.; Balander, R.; Fitzgerald, S. D.; Giesy, J. P.; Kannan, K.; Mitchell, R.; Bursian, S. J. Effects of Air Cell Injection of Perfluorooctane Sulfonate before Incubation on Development of the White Leghorn Chicken 
(Gallus Domesticus) Embryo. Environ. Toxicol. Chem. 2006, 25 (1), 227-232. https://doi.org/10.1897/04-414R.1.

(137) Gomis, M. I.; Wang, Z.; Scheringer, M.; Cousins, I. T. A Modeling Assessment of the Physicochemical Properties and Environmental Fate of Emerging and Novel Per- and Polyfluoroalkyl Substances. Sci. Total Environ. 2015, 505, $981-$ 991. https://doi.org/10.1016/j.scitotenv.2014.10.062.

(138) Kim, M.; Li, L. Y.; Grace, J. R.; Yue, C. Selecting Reliable Physicochemical Properties of Perfluoroalkyl and Polyfluoroalkyl Substances (PFASs) Based on Molecular Descriptors. Environ. Pollut. 2015, 196 (May 2009), 462-472. https://doi.org/10.1016/j.envpol.2014.11.008.

(139) Conder, J. M.; Hoke, R. a; Wolf, W. De; Russell, M. H.; Buck, R. C. Supporting Information For: Are PFCAs Bioaccumulative? A Critical Review and Comparison with Persistent Lipophilic Compounds. Environ. Sci. Technol. 2008, 42 (4), 1-31. https://doi.org/10.1021/es070895g.

(140) Geyer, R.; Jambeck, J. R.; Law, K. L. Production, Use, and Fate of All Plastics Ever Made. Sci. Adv. 2017, 3 (July), 25-29.

(141) Jambeck, J. R.; Geyer, R.; Wilcox, C.; Siegler, T. R.; Perryman, M.; Andrady, A.; Narayan, R.; Law, K. L. Plastic Waste Inputs from Land into the Ocean. Science (80-. ). 2015, 347 (6223).

(142) Ryan, P. G. The Effects of Ingested Plastic and Other Marine Debris on Seabirds. In Proceedings of the Second International Conference on Marine Debris; Shomura, R. S., Godfrey, M. L., Eds.; U.S. Department of Commerce, 1989; pp 623-634.

(143) Wilcox, C.; Van Sebille, E.; Hardesty, B. D.; Sebille, E. Van; Denise, B. Threat of Plastic Pollution to Seabirds Is Global, Pervasive, and Increasing. Proc. Natl. Acad. Sci. 2015, 112 (38), 1-6. https://doi.org/10.1073/pnas.1502108112.

(144) Wilcox, C.; Hardesty, B. D.; Law, K. L. Abundance of Floating Plastic Particles Is Increasing in the Western North Atlantic Ocean. Environ. Sci. Technol. 2020, 54 (2), 790-796. https://doi.org/10.1021/acs.est.9b04812.

(145) Hammer, S.; Nager, R. G.; Johnson, P. C. D.; Furness, R. W.; Provencher, J. F. Plastic Debris in Great Skua ( Stercorarius Skua) Pellets Corresponds to Seabird Prey Species. Mar. Pollut. Bull. 2016, 103 (1-2), 206-210. https://doi.org/10.1016/j.marpolbul.2015.12.018.

(146) Kühn, S.; van Franeker, J. A. Quantitative Overview of Marine Debris Ingested by Marine Megafauna. Mar. Pollut. Bull. 2020, 151 (October 2019). https://doi.org/10.1016/j.marpolbul.2019.110858. 
(147) Provencher, J. F.; Ammendolia, J.; Rochman, C. M.; Mallory, M. L. Assessing Plastic Debris in Aquatic Food Webs: What We Know and Don't Know about Uptake and Trophic Transfer. Environ. Rev. 2019, 27 (3), 304-317. https://doi.org/10.1139/er-2018-0079.

(148) Roman, L.; Bell, E.; Wilcox, C.; Hardesty, B. D.; Hindell, M. Ecological Drivers of Marine Debris Ingestion in Procellariiform Seabirds. Sci. Rep. 2019, 9 (1), 1-8. https://doi.org/10.1038/s41598-018-37324-w.

(149) Ryan, P. G. The Incidence and Characteristics of Plastic Particles Ingested by Seabirds. Mar. Environ. Res. 1987, 23 (3), 175-206. https://doi.org/10.1016/0141-1136(87)90028-6.

(150) Savoca, M. S.; Wohlfeil, M. E.; Ebeler, S. E.; Nevitt, G. A. Marine Plastic Debris Emits a Keystone Infochemical for Olfactory Foraging Seabirds. Sci. $A d v$. 2016, 2 (11), 1-9. https://doi.org/10.1126/sciadv.1600395.

(151) Roman, L.; Hardesty, B. D.; Hindell, M. A.; Wilcox, C. A Quantitative Analysis Linking Seabird Mortality and Marine Debris Ingestion. Sci. Rep. 2019, 9 (1), 1-7. https://doi.org/10.1038/s41598-018-36585-9.

(152) Carbery, M.; O’Connor, W.; Palanisami, T. Trophic Transfer of Microplastics and Mixed Contaminants in the Marine Food Web and Implications for Human Health. Environ. Int. 2018, No. March. https://doi.org/10.1016/j.envint.2018.03.007.

(153) Rochman, C. M.; Hoh, E.; Hentschel, B. T.; Kaye, S. Long-Term Field Measurement of Sorption of Organic Contaminants to Five Types of Plastic Pellets: Implications for Plastic Marine Debris. 2013. https://doi.org/10.1021/es303700s.

(154) Senko, J.; Nelms, S.; Reavis, J.; Witherington, B.; Godley, B.; Wallace, B. Understanding Individual and Population-Level Effects of Plastic Pollution on Marine Megafauna. Endanger. Species Res. 2020, 43 (January), 234-252. https://doi.org/10.3354/esr01064.

(155) Ryan, P. G.; Moore, C. J.; van Franeker, J. A.; Moloney, C. L. Monitoring the Abundance of Plastic Debris in the Marine Environment. Philos. Trans. R. Soc. B Biol. Sci. 2009, 364 (1526), 1999-2012. https://doi.org/10.1098/rstb.2008.0207.

(156) Kühn, S.; van Oyen, A.; Bravo Rebolledo, E. L.; Ask, A. V.; van Franeker, J. A. Polymer Types Ingested by Northern Fulmars (Fulmarus Glacialis) and Southern Hemisphere Relatives. Environ. Sci. Pollut. Res. 2020. https://doi.org/10.1007/s11356-020-10540-6. 
(157) Moser, Mary L., Lee, D. S. A Fourteen-Year Survey of Plastic Ingestion by Western North Atlantic Seabirds Author ( s ): Mary L . Moser and David S . Lee Published by: Waterbird Society Stable URL:

Http://Www.Jstor.Org/Stable/1521357 REFERENCES Linked References Are Available on J. 2016, 15 (1), 83-94.

(158) Bond, A. L.; Provencher, J. F.; Daoust, P. Y.; Lucas, Z. N. Plastic Ingestion by Fulmars and Shearwaters at Sable Island, Nova Scotia, Canada. Mar. Pollut. Bull. 2014, 87 (1), 68-75. https://doi.org/10.1016/j.marpolbul.2014.08.010.

(159) Haman, K. H.; Norton, T. M.; Ronconi, R. A.; Nemeth, N. M.; Thomas, A. C.; Courchesne, S. J.; Segars, A.; Keel, M. K. Great Shearwater(Puffinus Gravis) Mortality Events Along the Eastern Coast of the United States. J. Wildl. Dis. 2013, 49 (2), 235-245. https://doi.org/10.7589/2012-04-119. 


\title{
CHAPTER 2
}

\section{LEGACY AND NOVEL PER- AND POLYFLUOROALKYL SUBSTANCES IN JUVENILE SEABIRDS FROM THE U.S. ATLANTIC COAST}

\author{
This manuscript has been published and is formatted for the journal \\ Environmental Science and Technology, DOI: \\ https://dx.doi.org/10.1021/acs.est.0c01951
}

\begin{abstract}
Anna R. Robuck ${ }^{\dagger *}$, Mark G. Cantwell ${ }^{\ddagger}$, James McCord ${ }^{\perp}$, Lindsay M. Addison ${ }^{\#}$, Marisa Pfohl ${ }^{\S}$, Mark J. Strynar ${ }^{\perp}$, Richard McKinney Wiley", and Rainer Lohmann ${ }^{\dagger}$
\end{abstract}

${ }^{\dagger}$ University of Rhode Island Graduate School of Oceanography, Narragansett, RI 02882

tUS Environmental Protection Agency, Center for Environmental Measurement and Modeling, Atlantic Coastal Environmental Sciences Division, Narragansett, RI 02882

${ }^{\perp}$ US Environmental Protection Agency, Center for Environmental Measurement and Modeling, Durham, NC 27709

\# Audubon North Carolina, Wilmington Coastal Office, Wilmington, NC 28411

$\S$ University of Rhode Island, Biomedical and Pharmaceutical Sciences, Kingston, RI 02881 
" National Oceanic and Atmospheric Administration Stellwagen Bank National Marine Sanctuary, Scituate, MA 02066

*Corresponding author: annarobuck@gmail.com

KEYWORDS: PFAS, PFEAS, PFESA, PFECA, PFOS, bioaccumulation, seabirds

\section{ABSTRACT}

Per- and polyfluoroalkyl substances (PFAS) are anthropogenic, globally distributed chemicals. Legacy PFAS, including perfluorooctane sulfonate (PFOS), have been regularly detected in marine fauna but little is known about their current levels or the presence of novel PFAS in seabirds. We measured 36 emerging and legacy PFAS in livers from 31 juvenile seabirds from Massachusetts Bay, Narragansett Bay, and the Cape Fear River Estuary (CFRE), USA. PFOS was the major legacy perfluoroalkyl acid present, making up 58\% of concentrations observed across all habitats (range: 11 - $280 \mathrm{ng} / \mathrm{g}$ ). Novel PFAS were confirmed in chicks hatched downstream of a fluoropolymer production site in the CFRE - a perfluorinated ether sulfonic acid (Nafion byproduct-2; range: 1 $110 \mathrm{ng} / \mathrm{g})$ and two perfluorinated ether carboxylic acids $\left(\mathrm{PFO}_{4} \mathrm{DA}\right.$ and $\mathrm{PFO}_{5}$ DoDA; $\mathrm{PFO}_{5}$ DoDA range: 5 - $30 \mathrm{ng} / \mathrm{g}$ ). PFOS was inversely associated with phospholipid content in livers from CFRE and Massachusetts Bay individuals, while $\delta^{13} \mathrm{C}$, an indicator of marine vs. terrestrial foraging, was 
positively correlated with some long-chain PFAS in CFRE chick livers. These results detail concentrations of legacy and novel PFAS across different marine ecosystems along the US Atlantic East Coast. There is also an indication that seabird phospholipid dynamics are negatively impacted by PFAS, which should be further explored given the importance of lipids for seabirds.

\section{INTRODUCTION}

Per- and polyfluoroalkyl substances (PFAS) are anthropogenic organic contaminants used extensively in a variety of commercial, industrial, and military applications globally. ${ }^{1}$ Continued, widespread use of PFAS with diverse formulae has resulted in the detection of multiple PFAS into the global environment ${ }^{2-4}$. Industrial production has shifted away from long-chain chemistries to replacements with fewer than seven fluorinated carbons, polyfluorinated structures, and/or structurally modified iterations of perfluoroalkyl acids (PFAAs) such as perfluoroalkyl ether acids (PFEAs) incorporating different numbers of ether linkages ${ }^{5,6}$. PFEAs include both perfluoroalkyl ether carboxylic acids (PFECAs) and perfluoroalkyl ether sulfonic acids (PFESAs).

Both legacy PFAS and new formulations may be released to the environment via both direct or indirect discharges, or some interplay of the two ${ }^{1,7-9}$. The continued inputs of PFAS act in tandem with their extreme environmental persistence to sustain the proliferation of these compounds within terrestrial ${ }^{10}$, marine $^{11}$, estuarine ${ }^{12}$, freshwater ${ }^{13}$, cryosphere ${ }^{14}$, and atmospheric compartments ${ }^{14,15}$ worldwide. PFAS compounds can permeate biota within these 
compartments, including marine invertebrates ${ }^{16}$, fish $^{17}$, birds ${ }^{18-21}$, and marine mammals ${ }^{22}$. This environmental and biological ubiquity presents challenges for ecological health, as substantial evidence suggests a variety of legacy and new PFAS have the potential for adverse effects across multiple taxa ${ }^{23,24}$.

Toxicology and field studies suggest associations between PFAS concentrations and reproductive parameters, morphometric characteristics, and metabolic processes in avifauna ${ }^{25-31}$. Controlled studies across multiple taxa, including birds, also suggest variable associations between PFAS and lipid production, metabolism, and storage pathways ${ }^{32-34}$. Yet comprehensive assessments of PFAS exposure or potential toxicity in wild avifauna are stymied by a lack of foundational data detailing the environmental occurrence of diverse PFAS across multiple habitats and bird taxa. Transfer of PFAS via trophic interactions is likewise still under investigation, with habitat- and food webspecific trends apparent ${ }^{35-37}$. Furthermore, there is a dearth of data detailing PFAS concentrations in avifauna near industrial point sources, or potential impacts related to chronic, elevated exposure from such direct or substantial discharges ${ }^{29,30,38}$. This stands as a significant data gap considering that effective protection of endangered species and habitat surrounding contaminated sites relies on understanding exposure and impacts in bird species and other keystone wildlife.

The oceans are thought to be the final sink for legacy and novel PFAS compounds, receiving inputs via rivers/estuaries and atmospheric deposition ${ }^{2}$. 
Long-lived seabirds present an opportunity to assess and compare PFAS detection and trends across a range of coastal and oceanic habitats. In their given marine habitats, seabirds act as integrative sentinels due to their generally predictable life histories and foraging strategies, long life span, top predator trophic position, sensitivity to environmental stressors on observable time scales, and physiological interconnectivity to both air and water ${ }^{39,40}$. They assimilate resources and related environmental conditions, and demonstrate organismal and population level responses. This responsiveness allows seabird population and individual condition to be utilized as indicators reflecting chemical contamination and/or overall ecosystem health or stress ${ }^{20,41-43}$.

We used seabirds as sentinels to assess and contrast patterns and magnitude of PFAS exposure in three marine regions. We targeted 36 PFAS in the livers of 31 juvenile seabirds found dead in 2017 in three coastal and pelagic marine habitats with variable PFAS exposure potential. The main objectives of this study were to a) measure and compare legacy and emerging PFAS in different seabirds from marine habitats subject to variable direct or indirect PFAS discharges, and b) ascertain any association between stable isotope signatures approximating trophic habits, phospholipid levels, and PFAS concentrations.

\section{MATERIALS AND METHODS}

\section{Chemicals and Reagents}

A total of 36 PFAS were assessed in juvenile seabird livers using target and suspect screening, and 27 PFAS quantified (Table S1), including $\mathrm{C}_{4}-\mathrm{C}_{14}$ 
perfluorocarboxylates (PFCAs), $\mathrm{C}_{4}-\mathrm{C}_{10}$ perfluorosulfonates (PFSAs), three perfluoroalkyl ether carboxylic acids (PFECAs), one perfluoroalkyl ether sulfonate (PFESA), three fluorotelomer sulfonates (FTS), and three sulfonamide precursors (Tables S1,S4-S5). More details about target and suspect analytes, chemicals, and reagents can be found in the Supplementary Information (SI).

\section{Sample Collection}

Liver tissue was obtained from six species of deceased juvenile seabirds. Juvenile Great Shearwaters (Ardenna gravis) originated from Massachusetts Bay $(\mathrm{n}=10)$ and Herring Gull chicks (Larus argentatus smithsonianus) from Narragansett Bay $(\mathrm{n}=10)$. Royal Tern (Thalasseus maximus), Sandwich Tern (Thalasseus sandvicensis), Laughing Gull (Leucophaeus atricilla), and Brown Pelican (Pelecanus occidentalis) chicks originated from the Cape Fear River Estuary $(\mathrm{n}=11)(\mathrm{CFRE})($ Fig. S1). These birds, while unique species with nuanced life histories and food web roles, reflect broadly similar foraging preferences and strategies in coastal and pelagic habitats (Table S2). Literature also implies similar PFAS bioaccumulative capacity between different bird species, enabling a comparison of similar avifauna ${ }^{44-46}$.

The individuals analyzed here span several months in age, ranging from 2-4 week old chicks to $\sim 6$ month old juveniles. Based on the long tissue half-life of PFAS, these young birds predominantly reflect PFAS derived from maternal offloading, and thus the highest internal concentrations experienced by each individual over their lifetime $\mathrm{e}^{46-48}$. More details supporting our use of a multispecies, variable age sample set can be found in the SI. 
The selected habitats represent a continuum of potential PFAS exposure, with the highest likelihood of exposure in the CFRE downstream from a fluoropolymer production facility. Narragansett Bay represents an intermediate potential for PFAS exposure; it is a well-mixed coastal embayment adjacent to a large urban center, but lacks major PFAS production facilities. The lowest likelihood of exposure, based on increased distance from direct human sources of PFAS, is reflected by seabird juveniles collected from Massachusetts Bay. Massachusetts Bay is a productive offshore marine habitat encompassing Stellwagen Bank National Marine Sanctuary, and representative of the offshore pelagic environment of the North Atlantic (Fig. S1).

All individuals were necropsied in a standardized manner ${ }^{49}$. Liver tissue was used for PFAS analysis and stable isotope analysis, while muscle was only used for stable isotope analysis. Additional details about sample condition, sample procurement, and individual sample details can be found in the SI.

Analysis of PFAS

Complete extraction methods are provided in the SI. Briefly, liver samples were lyophilized and solvent-extracted in methanol using sonication, centrifugation, and freezing, paired with graphitized non-porous carbon solid phase extraction. Measurement and quantification of 25 PFAS was achieved using liquid chromatography tandem-mass spectrometry (UPLC-MS/MS) experiments in negative electrospray ionization mode. Further details about quantification and instrumental parameters are provided in the SI. Estimates of PFAS concentrations were obtained by quantification using isotope dilution. Method recovery ranged 
from $14-112 \%$ with a mean recovery of $62 \%$ across all compounds, similar to recoveries reported in other work using avian liver ${ }^{47,50,51}$ (Table S6).

Liver tissue was also assessed via suspect screening using high resolution mass spectrometry (HRMS). Fresh tissue aliquots were extracted for PFEAs of interest by means of protein precipitation and dilution ${ }^{19,52}$. Sample extracts were measured using a ThermoFisher Orbitrap Fusion (Thermo Fisher Scientific, Waltham, MA, USA) operated in heated electrospray ionization in negative mode as previously described ${ }^{19,52}$.

Concentrations of PFEAs were derived from these HRMS experiments. Nafion BP2 and $\mathrm{PFO}_{5}$ DoDA were assessed using native standards and reported here quantitatively while all other PFEAs were assessed based on previously determined accurate mass and spectral information using a semi-quantitative approach (Tables S1, S5) ${ }^{8,53}$. $\mathrm{PFO}_{4} \mathrm{DA}$ was the only PFEA detected in samples that lacked a native standard; $\mathrm{PFO}_{4} \mathrm{DA}$ was reported as raw abundance and excluded from concentration calculations due to the lack of an authentic native standard. Mass-labelled surrogates of similar molecular weight and retention time were used in the absence of matched surrogates for quantitation of Nafion BP2 and $\mathrm{PFO}_{5}$ DoDA (Table S5).

For those compounds analyzed via both UPLC-MS/MS and HRMS, UPLCMS/MS concentrations are reported here due to more rigorous quality assurance and increased sensitivity (Table S1). Further details about sample preparation, 
analysis, and quality assurance for both UPLC-MS/MS and HRMS are provided in the SI.

\section{Stable Isotope and Phospholipid Analysis}

Stable isotope analysis was used to evaluate trophic transfer of PFAS, as well as to ensure trophic comparability of the sample set. $\delta^{15} \mathrm{~N}, \delta^{13} \mathrm{C}$, and $\delta^{34} \mathrm{~S}$ were measured in muscle; $\delta^{15} \mathrm{~N}$ and $\delta^{13} \mathrm{C}$ were also measured in liver to facilitate comparison to a tissue with a faster isotopic turnover rate ${ }^{42}$, given the unknown rate of PFAS turnover in avian tissues.

Total lipid was extracted from liver tissue aliquots using a modified Folch method, and phospholipid content of liver tissue assessed colorimetrically using an EnzyChrom Phospholipid Assay Kit (BioAssay Systems, Hayward, CA) ${ }^{54}$. More details about stable isotope and phospholipid analysis are available in the SI.

\section{Statistical Analysis}

All data manipulation and statistical analyses were performed in $\mathrm{R}$ version 3.6.1 (R Core Team, 2020) ${ }^{55}$. Concentrations were converted to a wet weight basis for comparability to other literature values. Responses not detected or below the linear dynamic range of the curve were labeled as "nd" and assigned a value of zero. Observations below method reporting limits with a detection frequency higher than $50 \%$ were replaced with the half the method reporting limit for statistical analyses, and included as such in calculation of summed concentrations $\left(\sum{ }_{19} \mathrm{PFAS}\right)$ and statistical analysis ${ }^{56}$. Data were checked for normality and homoscedasticity using the Shapiro-Wilk test and Levene's test. Concentration 
data were non-normal despite $\log$ transformation and therefore treated nonparametrically for statistical analyses; habitat groups displayed no significant differences in variance. Differences between habitats were assessed using Kruskal-Wallis tests with post hoc application of Dunn's test with Bonferroni correction for multiple testing, or with the Wilcoxon rank sum test. Relationships between concentrations were assessed using Spearman rank correlation coefficients $\left(\mathrm{R}_{\mathrm{s}}{ }^{2}\right) . \mathrm{R}_{\mathrm{s}}{ }^{2}$ presents the proportion of the rank variance explained by the correlation between variables with test assumptions more suitable for this dataset, providing insight about the relationship similar to the Pearson $\mathrm{R}^{2}$. Liverwater bioaccumulation factors (BAFs) were calculated by dividing geometric mean liver PFAS levels by measured or estimated surface water concentrations adjacent to nesting or collection locations, followed by log transformation; more details about BAF calculations can be found in the SI.

\section{RESULTS AND DISCUSSION}

\section{Observed detection frequencies and patterns by habitat}

Samples were screened for 36 analytes using target and suspect screening; 27 analytes were quantified using native standards. Only one semi-quantitative compound was detected via suspect screening, $\mathrm{PFO}_{4} \mathrm{DA} .19$ of the 27 quantifiable analytes were measured above detection limits in at least one sample, and detection frequencies varied by habitat. PFOS, PFNA, PFDA, and PFUnDA were present in at least $97 \%$ of individuals (Table 1). 
The highest $\sum{ }_{19} \mathrm{PFAS}$ measured was $390 \mathrm{ng} / \mathrm{g}$ w.w. liver comprised of 14 quantifiable analytes, found in a CFRE Royal Tern chick. The lowest $\sum{ }_{19}$ PFAS concentration was observed in a juvenile Great Shearwater from Massachusetts Bay containing $\sum{ }_{19} \mathrm{PFAS}$ of $26 \mathrm{ng} / \mathrm{g}$, comprised of 5 analytes above detection limits (Fig. 1). Chicks from the CFRE system contained significantly greater concentrations and number of PFAS than juveniles from Massachusetts Bay or Narragansett Bay (Dunn's test; $\mathrm{p}<0.001$ ) (Figs. 1a, 3a). There was no significant difference between mean $\sum_{19}$ PFAS levels observed in individuals from Narragansett Bay and Massachusetts Bay (Fig. 3a), though Great Shearwater individuals were older than Narragansett Bay chicks, and may underestimate levels found in chicks of this species. Within each habitat, concentrations were not significantly different between male and female chicks, though the sample size of sexed individuals was small (Table S17).

Multiple PFEAs were not detected, including novel PFECAs PMPA, PEPA, $\mathrm{PFO}_{2} \mathrm{HxA}$, and $\mathrm{PFO}_{3} \mathrm{OA}$, and novel PFESAs NVHOS, Nafion byproduct 4 and 1. No fluorotelomer sulfonates were detected above reporting limits, nor NEtFOSAA or N-MeFOSAA. The lack of bioaccumulation of shorter-chain PFEAs may be analogous to the reduced bioaccumulation potential of short-chain PFCAs in upper trophic level homeotherms ${ }^{57}$. The lack of detection of these compounds may also denote in vivo biotransformation. Research in other biota has shown fluorotelomer sulfonates are precursors to some PFCAs ${ }^{58}$ while N-EtFOSAA and N-MeFOSAA are PFOS precursors ${ }^{7}$. Further work is required to deduce if the non-detects observed in this study are indicative of reduced bioavailability, rapid 
biotransformation, or the true absence of a compound in these environments and food webs. FOSA, a perfluoroalkane sulfonamide, was only detectable in three birds from the CFRE. These detections may be the result of continuous, high levels of FOSA or FOSA-precursors related to production activities or legacy PFAS sources in the region ${ }^{53}$. Such continuous inputs could exceed metabolic capacity and cause tissue residues of FOSA.

Continued dominance of PFOS in juvenile seabirds

PFOS was the most abundant compound in all sampled livers, making up $58 \%$ of total liver concentrations across the sample set. The proportion of PFOS measured in each individual varied by habitat, with the CFRE and Narragansett Bay individuals containing the highest geometric mean of $61 \%$ and $67 \%$ PFOS, respectively (Fig. 2).

These data align well with previous literature that determined a high proportion of PFOS in seabird livers, eggs, and serum in species from multiple ecological provinces ${ }^{20,21,59-63}$. The highest PFOS concentration measured in this study was $280 \mathrm{ng} / \mathrm{g} \mathrm{w.w.} \mathrm{liver} \mathrm{in} \mathrm{a} \mathrm{Sandwich} \mathrm{Tern} \mathrm{chick} \mathrm{from} \mathrm{the} \mathrm{CFRE,} \mathrm{similar}$ or higher than levels measured within the past ten years in other long-lived temperate seabirds ${ }^{20,21,60,61}$. Average avian toxicity reference values (TRVs) for PFOS reported by Newsted et al. of $600 \mathrm{ng}$ PFOS/g liver were approximately 230 times higher than PFOS levels reported in these young birds ${ }^{64}$. Seven of eleven CFRE chicks exceeded the female-specific liver TRV of $140 \mathrm{ng}$ PFOS/g liver; these birds were female or not yet able to be sexed visually ${ }^{64}$. One female 
from Mass. Bay and one unsexed bird from Narra. Bay also exceeded the femalespecific TRV.

Our data indicate the continued occurrence of PFOS in juvenile avifauna at levels of toxicological concern despite a production phase-out of perfluorooctanesulfonyl fluoride (POSF), PFOS, and PFOS precursors in the US in the early $2000 \mathrm{~s}^{1}$. The phase-out in 2000-2002 resulted in decreased PFOS and/or precursor concentrations in select environmental matrices ${ }^{22,65}$. Modeling and empirical results suggest decreased availability of volatile precursor compounds like FOSA, whose environmental occurrence responded quickly to the phase-out, likely caused any decreasing trends of PFOS in wildlife observed after $2002^{22}$. Yet biotic trends in PFOS vary based on spatial habits, proximity to local sources, and with trophic strategy, and there is no consistent global pattern of continually decreasing PFOS across multiple avian matrices $18,60,63,66$. Current PFOS concentrations in wildlife reflect exposure to extant precursor compounds that may transform in situ or in vivo to PFOS, in vivo depuration of PFOS, and sustained transfer of PFOS itself via environmental or trophic interactions ${ }^{65,67,68}$. Our results highlight the continued biological occurrence of PFOS as a function of these exposures.

Variable contribution of PFCAs by Habitat

Concentrations of PFCAs including PFDA, PFNA, and PFUnDA reported in this study were similar to or elevated compared to concentrations previously reported in temperate species, as well as in birds from Great Lakes and Arctic environments ${ }^{4,60-62,66,69}$. In CFRE, mean concentrations of PFCAs increased up to 
PFDA, where PFOA $<$ PFNA $<$ PFDA $>$ PFUnDA, whereas in Massachusetts Bay PFNA $<$ PFDA $<$ PFUnDA and in Narragansett Bay PFNA $<$ PFDA $\approx$ PFUnDA.

The proportion of $\sum$ PFCAs to $\sum$ PFSAs varied between habitats, with individuals from CFRE and Narragansett Bay dominated by PFSAs while individuals from Massachusetts Bay contained a significantly higher proportion of PFCAs (Dunn's test; $\mathrm{p}<0.001$ ) (Fig. 3b). The dominance of PFCAs in offshore Massachusetts Bay individuals was driven partly by significantly higher concentrations of the $\mathrm{C}_{11}$ PFCA, PFUnDA, in offshore individuals (Dunn's test; $p$ $<0.05)$ (Fig. S2). Mean concentrations of other long-chain $\left(\mathrm{C}_{n} \mathrm{~F}_{2 \mathrm{n}-1} \mathrm{COOH}, \mathrm{n} \geq 7\right)$ PFCAs found in both Narragansett Bay and Massachusetts Bay were not significantly different.

The preferential dominance of PFUnDA seen here in seabirds from offshore Massachusetts Bay habitat has been observed in Arctic marine mammals as well as Arctic and temperate seabirds $\mathrm{s}^{4,20,21,70}$. These studies exemplify a broader pattern in which PFCAs with odd chain lengths are more abundant in biota than PFCAs with even chain lengths. This pattern is a result of preferential bioaccumulation of longer-chain homologues, coupled to substantial atmospheric and water-borne transport of PFCAs and PFCA precursors of variable chain length ${ }^{15,23,70-72}$. Notably, our data suggests this pattern only applies to biota exposed to diffuse PFAS sources, as CFRE birds subject to localized point sources of PFAS actually contained greater concentrations of PFDA $\left(\mathrm{C}_{10}\right)$ compared to PFUnDA $\left(\mathrm{C}_{11}\right)$. 
Limited data suggest environmentally relevant exposures of long-chain PFCAs are associated with changes in metabolic rate, oxidative stress, and reproductive behaviors in Arctic black-legged kittiwakes ${ }^{25,73}$. We highlight the need for further research about the formation, long-range transport, occurrence, and effects of PFUnDA and other long-chain PFCAs in marine systems supporting seabirds. Such a comprehensive understanding is necessary given the sustained or increasing occurrence of long-chain PFCAs in remote wildlife $\mathrm{e}^{21,63}$, the substantial suite of stressors currently impacting marine species globally, and the continued importance of marine food resources to human communities ${ }^{74}$.

\section{Potentially Confounding Factors}

The opportunistic sample set of dead chicks and juveniles enabled us to measure PFAS in liver tissue, allowed the acquisition of unique samples from the CFRE before the cessation of certain industrial PFAS discharges, and allowed assessment of PFAS during a critical development window in immature individuals from data-deficient habitats. Yet this opportunistic sampling also introduced potentially confounding factors related to the variable species and ages of collected individuals.

The older Great Shearwater juveniles in this study were self-feeding for approximately two months, while the chicks from other habitats were still being provisioned by their parents. Great Shearwater PFAS profiles may therefore reflect increased input from dietary sources. Immature Shearwaters are thought to eat similar items as adult Great Shearwaters ${ }^{75}$; hence - the PFAS profile should be conserved, allowing their comparison between habitats. More likely, the older 
juveniles reflect growth dilution, and may underestimate PFAS concentrations present in chicks of this species.

Literature to date has yet to identify significant differences between uptake, metabolism, or elimination pathways and rates between similar bird species ${ }^{20,44-}$ 46. bioaccumulation in birds appears driven by habitat and trophic exposures. However, species-specific toxicokinetics have been identified between multiple mammal species ${ }^{76}$. We also have not investigated the possibility of developmental changes in molecular receptors of PFAS in birds or other wildlife, although PFAS have been found at higher levels in juveniles than adults across multiple $\operatorname{taxa}^{48,77,78}$. We suggest additional research on the toxicokinetic behavior of PFAS in different bird species and across developmental stages, given their utility and importance as sentinel predators.

Association with phospholipid content

Lipid moieties play key roles in organismal metabolism, reproduction, migration, and other basic functions key to wildlife health and fitness. Phospholipid levels were significantly $(\mathrm{p}<0.05)$ associated with PFOS in Massachusetts Bay and CFRE individuals $\left(\mathrm{R}_{\mathrm{s}}{ }^{2}=0.52\right.$ and 0.45 , respectively) (Fig. 4a). $\sum{ }_{19}$ PFAS was more weakly associated with measured phospholipids $\left(\mathrm{R}_{\mathrm{s}}{ }^{2}=\right.$ 0.14 and 0.45 for Massachusetts Bay and CFRE individuals, respectively and $\mathrm{R}_{\mathrm{s}}{ }^{2}$ $=0.51$ when assessed as a total sample set, $\mathrm{n}=31$ ). There was also a statistically significant correlation between PFNA and phospholipid in CFRE chicks $\left(\mathrm{R}_{\mathrm{s}}{ }^{2}=\right.$ $0.53, \mathrm{p}<0.05)$ 
The importance of phospholipids in the accumulation of long-chain PFAAs has been supported by both empirical observations from marine mammals as well as modeling results ${ }^{54,79-81}$. Conversely, controlled studies in chickens suggest PFOS may impact lipid metabolism and production via gene suppression, suggesting PFOS may instead indirectly mediate lipid levels $32,33,68,82$. Currently, our understanding of phospholipid-PFAS associations and relationships lacks substantial field-derived data beyond marine mammals.

These data, though derived from a small sample set, suggest an association between environmentally relevant concentrations of PFOS (and possibly PFNA) and decreased liver phospholipid content in wild seabirds, a previously unreported phenomenon (Fig. 4). Lipid levels in seabird livers may be influenced by a variety of physiological and nutritional constraints not measured within the scope of this study; we note the lack of comparative baseline data describing liver phospholipid levels in the species examined within this work as a possible limiting factor. However, lines of evidence from multiple disciplines suggest a high conservation of non-diet lipid composition in a given tissue between similar avian species ${ }^{83-86}$. Controlled animal studies also point to the same relationship between PFOS exposure and changes in phospholipid content, lipid profiles, and lipid metabolism alongside altered expression of genes related to lipid dynamics in the livers of domestic chickens ${ }^{32,33,68,82}$. Most relevant to the results seen here, subcutaneous delivery of $0.02 \mathrm{mg} / \mathrm{ml}$ and $0.1 \mathrm{mg} / \mathrm{ml}$ PFOS resulted in decreased phospholipid content in domestic chicken plasma after 28 days of exposure and 28 days of depuration ${ }^{68}$. 
Laboratory-based studies using model phospholipid bilayers, liposomes, and bacterial membranes exhibit an inverse relationship between PFAS levels and phospholipids, manifested via increased incorporation of PFAS into bilayers and subsequently decreased lipid content. These studies also found increased bilayer disruption by PFAS based on chain-length and functional group ${ }^{87,88}$. The results from these controlled membrane studies are not easily translated to realistic biological conditions, yet in combination with other evidence (our data, previous work in pilot whales ${ }^{54}$, and controlled animal studies referenced above) we highlight a potential and currently undefined relationship between PFOS and phospholipid responses in wild organismal systems at environmentally relevant exposure levels. Further research is warranted to better describe relationships between PFAS-driven lipid responses in combination with the role of lipids in PFAS partitioning.

Relationship to stable isotope data

$\delta^{15} \mathrm{~N}$ is frequently used as a proxy for trophic level across terrestrial and aquatic systems; while system and prey-base specific, enrichment of $\delta^{15} \mathrm{~N}$ typically indicates foraging at a higher trophic level ${ }^{42}$. Legacy persistent organic pollutants (POPs) like polychlorinated biphenyls or DDT frequently increase in concentration with trophic level as approximated by $\delta^{15} \mathrm{~N}$ values, due to bioaccumulation of hydrophobic POPs in lipid-rich consumer matrices ${ }^{89}$. Here, we found $\delta^{15} \mathrm{~N}$ values and calculated trophic level were not significantly associated with concentrations of the majority of individual PFAS in each habitat 
(Fig. S6, Table S23). PFOS and $\sum{ }_{19}$ PFAS were positively associated with $\delta^{15} \mathrm{~N}$ only in Massachusetts Bay individuals (Table S23).

$\delta^{13} \mathrm{C}$ values reflect basal sources of primary production supporting trophic networks ${ }^{42}$ and exhibit significantly less step-wise change with prey-consumer interactions. This allows bulk differentiation between inshore and offshore food chains based on characteristic enrichment or depletion of $\delta^{13} \mathrm{C}$ associated with terrestrial vs marine primary production (Fig. S8) ${ }^{42} . \delta^{13} \mathrm{C}$ ranged from -19.8 to 17.0 in CFRE individuals, and from -22.5 to -19.0 in Narragansett and Massachusetts Bay individuals. In contrast to previous studies, concentrations of $\mathrm{C}_{9}, \mathrm{C}_{10}, \mathrm{C}_{11}$, and $\mathrm{C}_{13}$ PFCAs, PFOS, and $\sum{ }_{19}$ PFAS in CFRE birds were associated with enriched $\delta^{13} \mathrm{C}$ values in seabird muscle and liver, likely reflecting the unique geomorphology of the CFRE system and energy-saving coastal foraging habits of CFRE seabird species (Fig. S7). PFTrDA and PFDA were negatively associated with $\delta^{13} \mathrm{C}$ values in Mass. Bay individuals, which may reflect increased exposure to long-chain PFCAs in offshore environments via long-range transport and transformation of PFCA precursors. More details about relationships between PFAS and stable isotopes are available in the SI.

\section{Unique occurrence of emerging compounds}

HRMS analysis helped us confirm the presence of (previously identified) PFEAs in chick livers for the first time: Nafion BP2 and PFO5DoDA were found in livers of all CFRE individuals $(\mathrm{n}=11)$ while $\mathrm{PFO}_{4} \mathrm{DA}$ was found in 7 of 11 CFRE individuals (Fig. 1b, Table S16). 
The maximum Nafion BP2 concentration was $110 \mathrm{ng} / \mathrm{g}$ w.w. in a Laughing Gull chick and a maximum PFO5DoDA concentration of $30 \mathrm{ng} / \mathrm{g}$ w.w. in a Royal Tern chick (Fig. 1b). Nafion BP2 was also detected in two Great Shearwaters and one Herring Gull from outside the CFRE, or $15 \%$ of the non-CFRE sample set (Figs. 1b, S10).

The detection of these PFEAs in CFRE chicks is due to proximity to an industrial point source and the then-ongoing discharge of PFEAs to surface water. CFRE chicks were hatched in a well-mixed estuary $\sim 145 \mathrm{~km}$ downstream from a fluoropolymer production facility in Fayetteville, NC. "Gen X" or HFPO-DA, along with other PFEAs were detected at high levels in Cape Fear River surface water and Wilmington, $\mathrm{NC}$ drinking water as a result of industrial wastewater discharges into the mainstem Cape Fear River ${ }^{53,90,91}$. Increased research attention following the 2017 termination of the industrial discharge has revealed the presence of multiple PFEAs in downstream environments, fish, and humans ${ }^{52,92}$.

Notably, concentrations of Nafion BP2, PFO5DoDA, and PFO4DA reported here are the highest biotic measurements of these PFEAs at the greatest distance from the production plant recorded to date, similar to measured PFOS concentrations and PFOS bioaccumulation factors in chick livers (Figs. 1, S9). Average PFEA concentrations reported here from seabird chick liver tissue (ppb, $\mathrm{ng} / \mathrm{g}$ ) are approximately 20 times higher than PFEA concentrations found in striped bass serum $(\mathrm{ppb}, \mathrm{ng} / \mathrm{mL})^{52}$.

Observed PFEA concentrations in CFRE chicks are likely not a result of riverine foraging proximate to the plant discharge or incorporation of freshwater 
prey items by seabird parents. The species sampled in this study are strictly marine, and do not inhabit or use freshwater, riverine habitats based on colony observations and their foraging preferences (marine and estuarine prey including forage fish, squid, and crustaceans $)^{93-95}$. PFEAs were previously measured in river water upstream from the breeding colony at very high concentrations while the industrial discharge was ongoing ${ }^{90,96}$. Their detection in seabirds here suggests these emerging PFEAs are environmentally persistent and capable of significant downstream transport and biotic uptake via undefined water-borne, particulate, and/or atmospheric pathways.

The confirmed presence of Nafion BP2 in three individuals outside of the CFRE is the first identification of Nafion BP2 in biota outside of the CFRE region, reiterating the environmental persistence and mobility of Nafion BP2. These detections are difficult to explain because virtually no data exists describing Nafion BP2 environmental occurrence beyond the CFRE region. Nafion BP2 detections in non-CFRE chicks may be due to migratory proximity to the CFRE; we consider this unlikely due to the lack of evidence indicating use or reliance on the CFRE system by non-CFRE populations sampled in this study.

More likely, Great Shearwater juveniles and Herring Gull chicks accumulated Nafion BP2 as a result of exposure to indirect discharges contaminated with Nafion products or related degradation byproducts. Nafion is the brand name of a perfluorinated ionic polymer first discovered in the 1960s via modification of the Teflon polymer. The Fayetteville, NC production facility has 
produced this ionic polymer since the 1980 s. Nafion byproduct- 2 is a side product from the reaction of the polymer precursors. ${ }^{92}$.

Little data exist describing how production, use, or disposal of this perfluorinated polymer may contribute to PFAS to the environment. Evidence from the CFRE suggests Nafion production waste streams may contribute substantial loads of Nafion byproducts to receiving environments ${ }^{52,53,90,92}$. Feng et al. (2015) investigated the thermolysis products of Nafion 117, a typical Nafion membrane and suggested high-temperature uses or disposal of Nafion via incineration may produce multiple perfluorinated structures as a result of incomplete combustion ${ }^{97}$; Nine groups of fluorinated analogues were identified as a result of thermal treatment of Nafion 117 membranes, including breakdown products structurally similar to Nafion $\mathrm{BP} 2^{97}$. Additional research documents chemical and mechanical degradation pathways relevant to Nafion membrane function and efficiency ${ }^{98}$. The (albeit limited) detection of Nafion BP2 beyond CFRE individuals warrants additional screening of Nafion byproducts in diverse environmental matrices, investigation into the life-cycle of Nafion technologies, and potential environmental persistence of PFESAs.

\section{Divergent sources of emerging and legacy PFAS in the CFRE system}

Ratios of PFEAs to PFOS varied between individuals, with PFEA levels on the same order of magnitude as PFOS in select individuals (Fig. 1b inset). PFEA concentrations were not correlated with PFOS or long-chain PFCAs in CFRE chicks, while PFO5DoDA displayed a weak positive correlation with PFDA (Fig. S5). This lack of correlation suggests legacy PFAAs like PFOS were contributed 
to the system via different pathways unassociated with the industrial facility producing PFEAs, in-line with their previous detection in surface waters from the region ${ }^{53}$. Prior research indicates concentrations of PFCAs and PFSAs in surface water are similar above and below the fluoropolymer facility in Fayetteville, NC while concentrations of PFEAs varied starkly upstream and downstream of the facility while active discharges from the facility were ongoing ${ }^{53,90}$. Implications for further research

Our results highlight the potential role of seabirds as key sentinels of marine environments, and confirm the sustained presence of legacy PFAS in marine systems along the US East Coast. We also document PFEAs in seabirds for the first time, reflecting the shifting suite of PFAS in production and in environmental matrices. As our current understanding of PFAS effects in wildlife is limited, future biomonitoring in seabirds and other wildlife should derive responses and effects related to ambient PFAS levels. Understanding PFAS levels and effects in marine food webs and biota ultimately stands to benefit public health and commerce as we continue to rely on marine food webs for economic, nutritional, and aesthetic services.

\section{ASSOCIATED CONTENT}

The following files are available free of charge:

Details about sample preparation, extraction methods, instrumental analysis, quality assurance and quality control, supplementary figures (pdf) 
monitored ions, extraction performance, raw data, summary statistics, data inputs for comparison and baf calculations (excel workbook)

\section{AUTHOR INFORMATION}

Corresponding Author

*Anna Ruth Robuck, annarobuck@gmail.com

Author Contributions

The manuscript was primarily drafted by A. Robuck and finalized through contributions of all authors. Sample analysis and data interpretation were carried out by A. Robuck through contributions of all authors. All authors have given approval to the final version of the manuscript.

\section{Funding Sources}

A. Robuck acknowledges support from the National Oceanic and Atmospheric Administration Dr. Nancy Foster Scholarship program (NOAA Award Number NA17NOS4290028), the Robert and Patricia Switzer Foundation, the STEEP Superfund Research Program (NIEHS Award Number P42ES027706), and the Oak Ridge Institute for Science and Education (ORISE) program.

\section{ACKNOWLEDGMENTS}

The authors are indebted to the Northeast Fisheries Observer Program, Gina Shield, Johanna Pedersen, Michael Moore, Matthew McIver, Marissa Sprouls, Arianna Mouradjian, and the Wildlife Clinic of Rhode Island for assistance 
obtaining and necropsying bird samples used in this study. The authors also acknowledge Christine Gardiner (URI) for laboratory assistance and Michael A. Mallin for context about the Cape Fear River and Estuary. We also acknowledge Melanie Hedgespeth, Andy Lindstrom, and Elsie Sunderland for providing presubmission critical reviews. Although EPA and NOAA employees contributed to this article, this research was not funded by EPA and was conceived, designed, and implemented by URI. The primary author was not an ORISE participant at US EPA when the research was carried out. EPA's role was limited to advising PFAS and stable isotope analysis and therefore not subject to EPA's quality requirements. Consequently, the views, interpretations, and conclusions expressed in the article are solely those of the authors and do not necessarily reflect or represent NOAA or EPA's views or policies.

\section{ABBREVIATIONS}

PFAS: per- and polyfluoroalkyl substances

CFRE: Cape Fear River and Estuary

PFCAs: perfluoroalkyl carboxylate(s)

PFSAs: perfluoroalkyl sulfonates(s)

PFEAs: perfluoroalkyl ether acid(s)

PFECAs: perfluoroalkyl ether carboxylate(s)

PFESAs: perfluoroalkyl ether sulfonate(s)

BCF: bioconcentration factor 
See SI Table S1 for the full names of all individual PFAS discussed in the scope of this analysis

EPA: US Environmental Protection Agency 


\section{LITERATURE CITED}

(1) Buck, R. C.; Franklin, J.; Berger, U.; Conder, J. M.; Cousins, I. T.; Voogt, P. De; Jensen, A. A.; Kannan, K.; Mabury, S. A.; van Leeuwen, S. P. J.

Perfluoroalkyl and Polyfluoroalkyl Substances in the Environment: Terminology, Classification, and Origins. Integr. Environ. Assess. Manag. 2011, 7 (4), 513-541. https://doi.org/10.1002/ieam.258.

(2) Ahrens, L. Polyfluoroalkyl Compounds in the Aquatic Environment: A Review of Their Occurrence and Fate. J. Environ. Monit. 2011, 13 (1), 20-31. https://doi.org/10.1039/c0em00373e.

(3) Giesy, J. P.; Kannan, K. Global Distribution of Perfluorooctane Sulfonate in Wildlife Global Distribution of Perfluorooctane Sulfonate in Wildlife. Environ. Sci. Technol. 2001, 35 (March), 1339-1342. https://doi.org/10.1021/es001834k.

(4) Muir, D.; Bossi, R.; Carlsson, P.; Evans, M.; De Silva, A.; Halsall, C.; Rauert, C.; Herzke, D.; Hung, H.; Letcher, R.; Rigét, F.; Roos, A. Levels and Trends of Poly- and Perfluoroalkyl Substances in the Arctic Environment - An Update. Emerg. Contam. 2019, 5, 240-271.

https://doi.org/10.1016/j.emcon.2019.06.002.

(5) Wang, Z.; Dewitt, J. C.; Higgins, C. P.; Cousins, I. T. A Never-Ending Story of Per- and Polyfluoroalkyl Substances (PFASs)? Environ. Sci. Technol. 2017, 51 (5), 2508-2518. https://doi.org/10.1021/acs.est.6b04806.

(6) Liu, Y.; Agostino, L. A. D.; Qu, G.; Jiang, G.; Martin, J. W. HighResolution Mass Spectrometry (HRMS) Methods for Nontarget Discovery and Characterization of Poly- and Per-Fluoroalkyl Substances (PFASs) in Environmental and Human Samples. Trends Anal. Chem. 2019. https://doi.org/10.1016/j.trac.2019.02.021.

(7) Martin, J. W.; Asher, B. J.; Beesoon, S.; Benskin, J. P.; Ross, M. S. PFOS or PreFOS? Are Perfluorooctane Sulfonate Precursors (PreFOS) Important Determinants of Human and Environmental Perfluorooctane Sulfonate (PFOS) Exposure? J. Environ. Model. 2010, 12, 1979-2004. https://doi.org/10.1039/c0em00295j.

(8) Mccord, J.; Strynar, M. Identification of Per- and Polyfluoroalkyl Substances in the Cape Fear River by High Resolution Mass Spectrometry and Nontargeted Screening. Environ. Sci. Technol. 2019, 53 (9), 4717-4727. https://doi.org/10.1021/acs.est.8b06017.

(9) Zhang, X.; Lohmann, R.; Dassuncao, C.; Hu, X. C.; Weber, A. K.; Vecitis, C. D.; Sunderland, E. M. Source Attribution of Poly- and Perfluoroalkyl Substances (PFASs) in Surface Waters from Rhode Island and the New York 
Metropolitan Area. Environ. Sci. Technol. Lett. 2016, 3 (9), 316-321. https://doi.org/10.1021/acs.estlett.6b00255.

(10) Falk, S.; Stahl, T.; Fliedner, A.; Rüdel, H.; Tarricone, K.; Brunn, H.; Koschorreck, J. Levels, Accumulation Patterns and Retrospective Trends of Perfluoroalkyl Acids (PFAAs) in Terrestrial Ecosystems over the Last Three Decades. Environ. Pollut. 2019, 246, 921-931. https://doi.org/10.1016/j.envpol.2018.12.095.

(11) Yamashita, N.; Taniyasu, S.; Petrick, G.; Wei, S.; Gamo, T.; Lam, P. K. S.; Kannan, K. Perfluorinated Acids as Novel Chemical Tracers of Global Circulation of Ocean Waters. Chemosphere 2008, 70 (7), 1247-1255. https://doi.org/10.1016/j.chemosphere.2007.07.079.

(12) Munoz, G.; Budzinski, H. H.; Labadie, P. Influence of Environmental Factors on the Fate of Legacy and Emerging Per- and Polyfluoroalkyl Substances along the Salinity/Turbidity Gradient of a Macrotidal Estuary. Environ. Sci. Technol. 2017, 51 (21), acs.est.7b03626. https://doi.org/10.1021/acs.est.7b03626.

(13) Eriksson, U.; Roos, A.; Lind, Y.; Hope, K.; Ekblad, A.; Kärrman, A. Comparison of PFASs Contamination in the Freshwater and Terrestrial Environments by Analysis of Eggs from Osprey (Pandion Haliaetus), Tawny Owl (Strix Aluco), and Common Kestrel (Falco Tinnunculus). Environ. Res. 2016, 149, 40-47. https://doi.org/10.1016/j.envres.2016.04.038.

(14) Pickard, H. M.; Criscitiello, A. S.; Spencer, C.; Sharp, M. J.; Muir, D. C. G.; De Silva, A. O.; Young, C. J. Continuous Non-Marine Inputs of per- and Polyfluoroalkyl Substances to the High Arctic: A Multi-Decadal Temporal Record. Atmos. Chem. Phys. 2018, 18 (7), 5045-5058.

https://doi.org/10.5194/acp-18-5045-2018.

(15) Ellis, D. A.; Martin, J. W.; Silva, A. O. D. E.; Hurley, M. D.; De Silva, A. O.; Mabury, S. A.; Hurley, M. D.; Sulbaek Andersen, M. P.; Wallington, T. J. Degradation of Fluorotelomer Alcohols: A Likely Atmospheric Source of Perfluorinated Carboxylic Acids. Environ. Sci. Technol. 2004, 38 (12), 33163321. https://doi.org/10.1021/es049860w.

(16) Langberg, H. A.; Breedveld, G. D.; Grønning, H. M.; Kvennås, M.; Jenssen, B. M.; Hale, S. E. Bioaccumulation of Fluorotelomer Sulfonates and Perfluoroalkyl Acids in Marine Organisms Living in Aqueous Film-Forming Foam Impacted Waters. Environ. Sci. Technol. 2019, 53 (18), 10951-10960. https://doi.org/10.1021/acs.est.9b00927.

(17) White, N. D.; Vena, J. E.; Kannan, K.; Karthikraj, R.; Wolf, B.; Fair, P. A.; Arnott, S. A. Perfluoroalkyl Substances (PFASs) in Edible Fish Species from Charleston Harbor and Tributaries, South Carolina, United States: Exposure and 
Risk Assessment. Environ. Res. 2019, 171 (November 2018), 266-277. https://doi.org/10.1016/j.envres.2019.01.021.

(18) Sun, J.; Bossi, R.; Bustnes, J. O.; Helander, B.; Boertmann, D.; Dietz, R.; Herzke, D.; Jaspers, V. L. B.; Labansen, A. L.; Lepoint, G.; Schulz, R.; Sonne, C.; Thorup, K.; Tøttrup, A. P.; Zubrod, J. P.; Eens, M.; Eulaers, I. White-Tailed Eagle (Haliaeetus Albicilla) Body Feathers Document Spatiotemporal Trends of Perfluoroalkyl Substances in the Northern Environment. Environ. Sci. Technol. 2019. https://doi.org/10.1021/acs.est.9b03514.

(19) Russell, M. C.; Newton, S. R.; McClure, K. M.; Levine, R. S.; Phelps, L. P.; Lindstrom, A. B.; Strynar, M. J. Per- and Polyfluoroalkyl Substances in Two Different Populations of Northern Cardinals. Chemosphere 2019, 222, 295-304. https://doi.org/10.1016/j.chemosphere.2019.01.112.

(20) Roscales, J. L.; Vicente, A.; Ryan, P. G.; González-Solís, J.; Jiménez, B. Spatial and Interspecies Heterogeneity in Concentrations of Perfluoroalkyl Substances (PFASs) in Seabirds of the Southern Ocean. Environ. Sci. Technol. 2019, 53 (16), 9855-9865. https://doi.org/10.1021/acs.est.9b02677.

(21) Miller, A.; Elliott, J. E.; Elliott, K. H.; Lee, S.; Cyr, F. Temporal Trends of Perfluoroalkyl Substances (PFAS) in Eggs of Coastal and Offshore Birds: Increasing PFAS Levels Associated with Offshore Bird Species Breeding on the Pacific Coast of Canada and Wintering near Asia. Environ. Toxicol. Chem. 2015, 34 (8), 1799-1808. https://doi.org/10.1002/etc.2992.

(22) Dassuncao, C.; Hu, X. C.; Zhang, X.; Bossi, R.; Dam, M.; Mikkelsen, B.; Sunderland, E. M. Temporal Shifts in Poly- and Perfluoroalkyl Substances (PFASs) in North Atlantic Pilot Whales Indicate Large Contribution of Atmospheric Precursors. Environ. Sci. Technol. 2017, 51 (8), 4512-4521. https://doi.org/10.1021/acs.est.7b00293.

(23) Ahrens, L.; Bundschuh, M.; Hrens, L. U. T. Z. A.; Undschuh, M. I. B. Fate and Effects of Poly- and Perfluoroalkyl Substances in the Aquatic Environment: A Review. Environ. Toxicol. Chem. 2014, 33 (9), 1921-1929. https://doi.org/10.1002/etc.2663.

(24) Gomis, M. I.; Vestergren, R.; Borg, D.; Cousins, I. T. Comparing the Toxic Potency in Vivo of Long-Chain Perfluoroalkyl Acids and Fluorinated Alternatives. Environ. Int. 2018, 113 (November 2017), 1-9. https://doi.org/10.1016/j.envint.2018.01.011.

(25) Blévin, P.; Tartu, S.; Ellis, H. I.; Chastel, O.; Bustamante, P.; Parenteau, C.; Herzke, D.; Angelier, F.; Gabrielsen, G. W. Contaminants and Energy Expenditure in an Arctic Seabird: Organochlorine Pesticides and Perfluoroalkyl Substances Are Associated with Metabolic Rate in a Contrasted Manner. Environ. 
Res. 2017, 157 (February), 118-126.

https://doi.org/10.1016/j.envres.2017.05.022.

(26) Cassone, C. G.; Vongphachan, V.; Chiu, S.; Williams, K. L.; Letcher, R. J.; Pelletier, E.; Crump, D.; Kennedy, S. W. In Ovo Effects of Perfluorohexane Sulfonate and Perfluorohexanoate on Pipping Success, Development, MRNA Expression, and Thyroid Hormone Levels in Chicken Embryos. Toxicol. Sci. 2012, 127 (1), 216-224. https://doi.org/10.1093/toxsci/kfs072.

(27) Peden-Adams, M. M.; Stuckey, J. E.; Gaworecki, K. M.; Berger-Ritchie, J.; Bryant, K.; Jodice, P. G.; Scott, T. R.; Ferrario, J. B.; Guan, B.; Vigo, C.; Boone, J. S.; McGuinn, W. D.; DeWitt, J. C.; Keil, D. E. Developmental Toxicity in White Leghorn Chickens Following in Ovo Exposure to Perfluorooctane Sulfonate (PFOS). Reprod. Toxicol. 2009, 27 (3-4), 307-318. https://doi.org/10.1016/j.reprotox.2008.10.009.

(28) Costantini, D.; Blévin, P.; Herzke, D.; Moe, B.; Wing, G.; Ove, J.; Chastel, O.; Gabrielsen, G. W.; Bustnes, J. O.; Chastel, O. Higher Plasma Oxidative Damage and Lower Plasma Antioxidant Defences in an Arctic Seabird Exposed to Longer Perfluoroalkyl Acids. Environ. Res. 2019, 168 (October 2018), 278-285. https://doi.org/10.1016/j.envres.2018.10.003.

(29) Custer, C. M.; Custer, T. W.; Dummer, P. M.; Etterson, M. A.; Mckann, P. C. Exposure and Effects of Perfluoroalkyl Substances in Tree Swallows Nesting in Minnesota and Wisconsin, USA. 2014, 2000, 120-138.

https://doi.org/10.1007/s00244-013-9934-0.

(30) Lopez-Antia, A.; Groffen, T.; Lasters, R.; AbdElgawad, H.; Sun, J.; Asard, H.; Bervoets, L.; Eens, M. Perfluoroalkyl Acids (PFAAs) Concentrations and Oxidative Status in Two Generations of Great Tits Inhabiting a Contamination Hotspot. Environ. Sci. Technol. 2019, 53, 1617-1626. https://doi.org/10.1021/acs.est.8b05235.

(31) Nordén, M.; Berger, U.; Engwall, M. Developmental Toxicity of PFOS and PFOA in Great Cormorant ( Phalacrocorax Carbo Sinensis ), Herring Gull ( Larus Argentatus ) and Chicken ( Gallus Gallus Domesticus ). 2016, 1085510862. https://doi.org/10.1007/s11356-016-6285-1.

(32) Geng, D.; Musse, A. A.; Wigh, V.; Carlsson, C.; Engwall, M.; Orešič, M.; Scherbak, N.; Hyötyläinen, T. Effect of Perfluorooctanesulfonic Acid (PFOS) on the Liver Lipid Metabolism of the Developing Chicken Embryo. Ecotoxicol. Environ. Saf. 2019, 170 (October 2018), 691-698. https://doi.org/10.1016/j.ecoenv.2018.12.040.

(33) Jacobsen, A. V.; Nordén, M.; Engwall, M.; Scherbak, N. Effects of Perfluorooctane Sulfonate on Genes Controlling Hepatic Fatty Acid Metabolism 
in Livers of Chicken Embryos. Environ. Sci. Pollut. Res. 2018, 25 (23), 2307423081. https://doi.org/10.1007/s11356-018-2358-7.

(34) Salihovic, S.; Fall, T.; Ganna, A.; Broeckling, C. D.; Prenni, J. E.; Hyötyläinen, T.; Kärrman, A.; Lind, P. M.; Ingelsson, E.; Lind, L. Identification of Metabolic Profiles Associated with Human Exposure to Perfluoroalkyl Substances. J. Expo. Sci. Environ. Epidemiol. 2019, 29 (2), 196-205. https://doi.org/10.1038/s41370-018-0060-y.

(35) Kelly, B. C.; Ikonomou, M. G.; Blair, J. D.; Surridge, B.; Hoover, D.; Grace, R.; Gobas, F. A. P. C. Perfluoroalkyl Contaminants in an Arctic Marine Food Web: Trophic Magnification and Wildlife Exposure. Environ. Sci. Technol. 2009, 43 (11), 4037-4043. https://doi.org/10.1021/es9003894.

(36) Munoz, G.; Budzinski, H. H.; Babut, M.; Drouineau, H.; Lauzent, M.; Menach, K. Le; Lobry, J.; Selleslagh, J.; Simonnet-Laprade, C.; Labadie, P. Evidence for the Trophic Transfer of Perfluoroalkylated Substances in a Temperate Macrotidal Estuary. Environ. Sci. Technol. 2017, 51 (15), 8450-8459. https://doi.org/10.1021/acs.est.7b02399.

(37) Loi, E. I. H.; Yeung, L. W. Y.; Taniyasu, S.; Lam, P. K. S.; Kannan, K.; Yamashita, N. Trophic Magnification of Poly- and Perfluorinated Compounds in a Subtropical Food Web. Environ. Sci. Technol. 2011, 45 (13), 5506-5513. https://doi.org/10.1021/es200432n.

(38) Groffen, T.; Lopez-Antia, A.; Hollander, W. D.; Prinsen, E.; Eens, M. Perfluoroalkylated Acids in the Eggs of Great Tits ( Parus Major ) near a Flurochemical Plant in Flanders, Belgium. Environ. Pollut. 2017, 228, 140-148. https://doi.org/10.1016/j.envpol.2017.05.007.

(39) Piatt, J. F.; Sydeman, W. J.; Wiese, F. Introduction: A Modern Role for Seabirds as Indicators. Mar. Ecol. Prog. Ser. 2007, 352, 199-204. https://doi.org/10.3354/meps07070.

(40) Cairns, D. K. Seabirds as Indicators of Marine Food Supplies. Biol. Oceanogr. 1987, 5 (June), 261-271. https://doi.org/10.1080/01965581.1987.10749517.

(41) Furness, R. W.; Camphuysen, K. Seabirds as Monitors of the Marine Environment. ICES J. Mar. Sci. 1997, 54 (4), 726-737. https://doi.org/10.1006/jmsc.1997.0243.

(42) Hobson, K. A.; Piatt, J. F.; Pitocchelli, J. Using Stable Isotopes to Determine Seabird Trophic Relationships. J. Anim. Ecol. 1994, 63 (4), 786-798. 
(43) Hazen, E. L.; Abrahms, B.; Brodie, S.; Carroll, G.; Jacox, M. G.; Savoca, M. S.; Scales, K. L.; Sydeman, W. J.; Bograd, S. J. Marine Top Predators as Climate and Ecosystem Sentinels. 2019, 1-10. https://doi.org/10.1002/fee.2125.

(44) Lopez-Antia, A.; Dauwe, T.; Meyer, J.; Maes, K.; Bervoets, L.; Eens, M. High Levels of PFOS in Eggs of Three Bird Species in the Neighbourhood of a Fluoro-Chemical Plant. Ecotoxicol. Environ. Saf. 2020, 139 (August 2016), 165171. https://doi.org/10.1016/j.ecoenv.2017.01.040.

(45) Yoo, H.; Kannan, K.; Seong, K. K.; Kyu, T. L.; Newsted, J. L.; Giesy, J. P. Perfluoroalkyl Acids in the Egg Yolk of Birds from Lake Shihwa, Korea.

Environ. Sci. Technol. 2008, 42 (15), 5821-5827. https://doi.org/10.1021/es800447d.

(46) Newsted, J. L.; Coady, K. K.; Beach, S. A.; Butenhoff, J. L.; Gallagher, S.; Giesy, J. P. Effects of Perfluorooctane Sulfonate on Mallard and Northern Bobwhite Quail Exposed Chronically via the Diet. Environ. Toxicol. Pharmacol. 2007, 23 (1), 1-9. https://doi.org/10.1016/j.etap.2006.04.008.

(47) Holmström, K. E.; Berger, U. Tissue Distribution of Perfluorinated Surfactants in Common Guillemot (Uria Aalge) from the Baltic Sea. Environ. Sci. Technol. 2008, 42 (16), 5879-5884. https://doi.org/10.1021/es800529h.

(48) Wang, J.; Zhang, Y.; Zhang, F.; Yeung, L. W. Y.; Taniyasu, S.; Yamazaki, E.; Wang, R.; Lam, P. K. S.; Yamashita, N.; Dai, J. Age- and GenderRelated Accumulation of Perfluoroalkyl Substances in Captive Chinese Alligators (Alligator Sinensis). Environ. Pollut. 2013, 179, 61-67.

https://doi.org/10.1016/j.envpol.2013.04.020.

(49) van Franeker, J. A. Save the North Sea Fulmar-Litter-EcoQO Manual Part 1: Collection and Dissection Procedures. Alterra-rapport 2004, 672, 1-38.

(50) Kannan, K.; Choi, J. W.; Iseki, N.; Senthilkumar, K.; Hoon, D.; Masunaga, S.; Giesy, J. P.; Kim, D. H.; Masunaga, S.; Giesy, J. P. Concentrations of Perfluorinated Acids in Livers of Birds from Japan and Korea. Chemosphere 2002, 49 (3), 225-231. https://doi.org/10.1016/S0045-6535(02)00304-1.

(51) Guruge, K. S.; Yeung, L. W. Y.; Li, P.; Taniyasu, S.; Yamashita, N.; Nakamura, M. Fluorinated Alkyl Compounds Including Long Chain Carboxylic Acids in Wild Bird Livers from Japan. Chemosphere 2011, 83 (3), 379-384. https://doi.org/10.1016/j.chemosphere.2010.12.010.

(52) Guillette, T. C. T.; McCord, J.; Guillette, M.; Polera, M. E.; Rachels, K. T.; Morgeson, C.; Kotlarz, N.; Knappe, D. R. U.; Reading, B. J.; Strynar, M.; Belcher, S. M. Per- and Polyfluoroalkyl Substances Exposure in Cape Fear River Striped Bass (Morone Saxatilis) Is Associated with Biomarkers of Altered 
Immune and Liver Function Research. Environ. Int. 2019, 136 (September), 105358. https://doi.org/10.1016/j.envint.2019.105358.

(53) Strynar, M.; Dagnino, S.; McMahen, R.; Liang, S.; Lindstrom, A.; Andersen, E.; McMillan, L.; Thurman, M.; Ferrer, I.; Ball, C. Identification of Novel Perfluoroalkyl Ether Carboxylic Acids (PFECAs) and Sulfonic Acids (PFESAs) in Natural Waters Using Accurate Mass Time-of-Flight Mass Spectrometry (TOFMS). Environ. Sci. Technol. 2015, 49 (19), 11622-11630. https://doi.org/10.1021/acs.est.5b01215.

(54) Dassuncao, C.; Pickard, H.; Pfohl, M.; Tokranov, A. K.; Li, M.; Mikkelsen, B.; Slitt, A.; Sunderland, E. M. Phospholipid Levels Predict the Tissue Distribution of Poly- and Perfluoroalkyl Substances in a Marine Mammal. Environ. Sci. Technol. Lett. 2019, 6 (3), acs.estlett.9b00031. https://doi.org/10.1021/acs.estlett.9b00031.

(55) R Core Team. R: A Language and Environment for Statistical Computing. R Foundation for Statistical Computing: Vienna, Austria 2020.

(56) Hites, R. A. Correcting for Censored Environmental Measurements. Environ. Sci. Technol. 2019, 53 (19), 11059-11060. https://doi.org/10.1021/acs.est.9b05042.

(57) Conder, J. M.; Hoke, R. A.; Wolf, W.; Russell, M. H.; Buck, R. C. Are PFCAs Bioaccumulative? A Critical Review and Comparison with Regulatory Criteria and Persistent Lipophilic Compounds. Environ. Sci. Technol. 2008, 42, 995-1003.

(58) Liu, J.; Mejia Avendaño, S. Microbial Degradation of Polyfluoroalkyl Chemicals in the Environment: A Review. Environ. Int. 2013, 61, 98-114. https://doi.org/10.1016/j.envint.2013.08.022.

(59) Sedlak, M. D.; Benskin, J. P.; Wong, A.; Grace, R.; Greig, D. J. Per- and Polyfluoroalkyl Substances (PFASs) in San Francisco Bay Wildlife : Temporal Trends , Exposure Pathways, and Notable Presence of Precursor Compounds. Chemosphere 2017, 185, 1217-1226. https://doi.org/10.1016/j.chemosphere.2017.04.096.

(60) Leat, E. H. K.; Bourgeon, S.; Eze, J. I.; Muir, D. C. G.; Williamson, M.; Bustnes, J. O.; Furness, R. W.; Borgå, K. Perfluoroalkyl Substances in Eggs and Plasma of an Avian Top Predator, Great Skua (Stercorarius Skua), in the North Atlantic. Environ. Toxicol. Chem. 2013, 32 (3), 569-576. https://doi.org/10.1002/etc.2101.

(61) Chu, S.; Wang, J.; Leong, G.; Ann, L.; Letcher, R. J.; Li, Q. X.; Woodward, L. A.; Letcher, R. J.; Li, Q. X. Perfluoroalkyl Sulfonates and 
Carboxylic Acids in Liver, Muscle and Adipose Tissues of Black-Footed Albatross (Phoebastria Nigripes) from Midway Island, North Pacific Ocean. Chemosphere 2015, 138 (2015), 60-66.

https://doi.org/10.1016/j.chemosphere.2015.05.043.

(62) Letcher, R. J.; Su, G.; Moore, J. N.; Williams, L. L.; Martin, P. A.; De Solla, S. R.; Bowerman, W. W.; Solla, S. R. De; Bowerman, W. W.

Perfluorinated Sulfonate and Carboxylate Compounds and Precursors in Herring Gull Eggs from across the Laurentian Great Lakes of North America: Temporal and Recent Spatial Comparisons and Exposure Implications. Sci. Total Environ. 2015, 538 (August), 468-477. https://doi.org/10.1016/j.scitotenv.2015.08.083.

(63) Braune, B. M.; Letcher, R. J. Perfluorinated Sulfonate and Carboxylate Compounds in Eggs of Seabirds Breeding in the Canadian Arctic: Temporal Trends (1975-2011) and Interspecies Comparison. Environ. Sci. Technol. 2013, 47 (1), 616-624. https://doi.org/10.1021/es303733d.

(64) Newsted, J. L.; Jones, P. D.; Coady, K.; Giesy, J. P. Avian Toxicity Reference Values for Perfluorooctane Sulfonate. Environ. Sci. Technol. 2005, 39 (23), 9357-9362. https://doi.org/10.1021/es050989v.

(65) Armitage, J. M.; Schenker, U.; Scheringer, M.; Martin, J. W.; Macleod, M.; Cousins, I. T. Modeling the Global Fate and Transport of Perfluorooctane Sulfonate (PFOS) and Precursor Compounds in Relation to Temporal Trends in Wildlife Exposure. Environ. Sci. Technol. 2009, 43 (24), 9274-9280. https://doi.org/10.1021/es901448p.

(66) Gebbink, W. A.; Letcher, R. J.; Hebert, C. E.; Chip Weseloh, D. V. V. Twenty Years of Temporal Change in Perfluoroalkyl Sulfonate and Carboxylate Contaminants in Herring Gull Eggs from the Laurentian Great Lakes. J. Environ. Monit. 2011, 13 (12), 3365-3372. https://doi.org/10.1039/c1em10663e.

(67) Tarazona, J. V.; Rodríguez, C.; Alonso, E.; Sáez, M.; González, F.; San Andrés, M. D.; Jiménez, B.; San Andrés, M. I. Toxicokinetics of Perfluorooctane Sulfonate in Birds under Environmentally Realistic Exposure Conditions and Development of a Kinetic Predictive Model. Toxicol. Lett. 2015, 232 (2), 363368. https://doi.org/10.1016/j.toxlet.2014.11.022.

(68) Yoo, H.; Guruge, K. S.; Yamanaka, N.; Sato, C.; Mikami, O.; Miyazaki, S.; Yamashita, N.; Giesy, J. P. Depuration Kinetics and Tissue Disposition of PFOA and PFOS in White Leghorn Chickens (Gallus Gallus) Administered by Subcutaneous Implantation. Ecotoxicol. Environ. Saf. 2009, 72 (1), 26-36. https://doi.org/10.1016/j.ecoenv.2007.09.007.

(69) Routti, H.; Aars, J.; Fuglei, E.; Hanssen, L.; Lone, K.; Polder, A.; Pedersen, A. Ø.; Tartu, S.; Welker, M.; Yoccoz, N. G. Emission Changes Dwarf 
the In F1 Uence of Feeding Habits on Temporal Trends of Per- and Poly F1 Uoroalkyl Substances in Two Arctic Top Predators. 2017.

https://doi.org/10.1021/acs.est.7b03585.

(70) Martin, J. W.; Smithwick, M. M.; Braune, B. M.; Hoekstra, P. F.; Muir, D. C. G.; Mabury, S. A. Identification of Long-Chain Perfluorinated Acids in Biota from the Canadian Arctic. Environ. Sci. Technol. 2004, 38 (2), 373-380. https://doi.org/10.1021/es034727+.

(71) Armitage, J. M.; MacLeod, M.; Cousins, I. T. Modeling the Global Fate and Transport of Perfluorooctanoic Acid (PFOA) and Perfluorooctanoate (PFO) Emitted from Direct Sources Using a Multispecies Mass Balance Model (Environmental Science and Technology (2009) 43, 1134-1140)). Environ. Sci. Technol. 2009, 43 (16), 6438-6439. https://doi.org/10.1021/es901832b.

(72) Conder, J. M.; Hoke, R. A.; De Wolf, W.; Russell, M. H.; Buck, R. C. Are PFCAs Bioaccumulative? A Critical Review and Comparison with Regulatory Criteria and Persistent Lipophilic Compounds. Environ. Sci. Technol. 2008, 42 (4), 995-1003. https://doi.org/10.1021/es070895g.

(73) Tartu, S.; Gabrielsen, G. W.; Blévin, P.; Ellis, H.; Bustnes, J. O.; Herzke, D.; Chastel, O. Endocrine and Fitness Correlates of Long-Chain Perfluorinated Carboxylates Exposure in Arctic Breeding Black-Legged Kittiwakes. Environ. Sci. Technol. 2014, 48 (22), 13504-13510. https://doi.org/10.1021/es503297n.

(74) Crain, C. M.; Kroeker, K.; Halpern, B. S. Interactive and Cumulative Effects of Multiple Human Stressors in Marine Systems. Ecol. Lett. 2008, 11 (12), 1304-1315. https://doi.org/10.1111/j.1461-0248.2008.01253.x.

(75) Campioni, L.; Granadeiro, J. P.; Catry, P. Niche Segregation between Immature and Adult Seabirds: Does Progressive Maturation Play a Role? Behav. Ecol. 2016, 27 (2), 426-433. https://doi.org/10.1093/beheco/arv167.

(76) Pizzurro, D. M.; Seeley, M.; Kerper, L. E.; Beck, B. D. Interspecies Differences in Perfluoroalkyl Substances (PFAS)Toxicokinetics and Application to Health-Based Criteria. Regul. Toxicol. Pharmacol. 2019, 106 (May), 239-250. https://doi.org/10.1016/j.yrtph.2019.05.008.

(77) Baduel, C.; Lai, F. Y.; Townsend, K.; Mueller, J. F. Size and AgeConcentration Relationships for Perfluoroalkyl Substances in Stingray Livers from Eastern Australia. Sci. Total Environ. 2014, 496, 523-530. https://doi.org/10.1016/j.scitotenv.2014.07.010.

(78) Mondal, D.; Lopez-Espinosa, M. J.; Armstrong, B.; Stein, C. R.; Fletcher, T. Relationships of Perfluorooctanoate and Perfluorooctane Sulfonate Serum Concentrations between Mother-Child Pairs in a Population with 
Perfluorooctanoate Exposure from Drinking Water. Environ. Health Perspect. 2012, 120 (5), 752-757. https://doi.org/10.1289/ehp.1104538.

(79) Ng, C. A.; Hungerbühler, K. Bioaccumulation of Perfluorinated Alkyl Acids: Observations and Models. Environ. Sci. Technol. 2014, 48 (9), 4637-4648. https://doi.org/10.1021/es404008g.

(80) Ng, C. A.; Hungerbühler, K. Bioconcentration of Perfluorinated Alkyl Acids: How Important Is Specific Binding? Environ. Sci. Technol. 2013, 47 (13), 7214-7223. https://doi.org/10.1021/es400981a.

(81) Armitage, J. M.; Arnot, J. A.; Wania, F. Potential Role of Phospholipids in Determining the Internal Tissue Distribution of Perfluoroalkyl Acids in Biota. Environ. Sci. Technol. 2012, 46 (22), 12285-12286. https://doi.org/10.1021/es304430r.

(82) Yeung, L. W. Y.; Guruge, K. S.; Yamanaka, N.; Miyazaki, S.; Lam, P. K. S. Differential Expression of Chicken Hepatic Genes Responsive to PFOA and PFOS. Toxicology 2007, 237 (1-3), 111-125. https://doi.org/10.1016/j.tox.2007.05.004.

(83) Ronconi, R. A.; Koopman, H. N.; McKinstry, C. A. E.; Wong, S. N. P.; Westgate, A. J. Inter-Annual Variability in Diet of Non-Breeding Pelagic Seabirds Puffinus Spp. at Migratory Staging Areas: Evidence from Stable Isotopes and Fatty Acids. Mar. Ecol. Prog. Ser. 2010, 419, 267-282. https://doi.org/10.3354/meps08860.

(84) Calhoon, E. A. Lipid and Phospholipid Species Composition Associated with Life History Variation in North Temperate and Neotropical Birds, Ohio State University, 2016.

(85) Entenman, C. Lipid Content of Chick Tissues. J Biol Chem 1940, No. 65, 231-241.

(86) Blem, C. R. Patterns of Lipid Storage and Utilization in Birds. Integr. Comp. Biol. 1976, 16 (4), 671-684. https://doi.org/10.1093/icb/16.4.671.

(87) Nouhi, S.; Ahrens, L.; Campos Pereira, H.; Hughes, A. V.; Campana, M.; Gutfreund, P.; Palsson, G. K.; Vorobiev, A.; Hellsing, M. S. Interactions of Perfluoroalkyl Substances with a Phospholipid Bilayer Studied by Neutron Reflectometry. J. Colloid Interface Sci. 2018, 511, 474-481. https://doi.org/10.1016/j.jcis.2017.09.102.

(88) Fitzgerald, N. J. M.; Wargenau, A.; Sorenson, C.; Pedersen, J.; Tufenkji, N.; Novak, P. J.; Simcik, M. F. Partitioning and Accumulation of Perfluoroalkyl 
Substances in Model Lipid Bilayers and Bacteria. Environ. Sci. Technol. 2018, 52 (18), 10433-10440. https://doi.org/10.1021/acs.est.8b02912.

(89) Fisk, A. T.; Hobson, K. A.; Norstrom, R. J. Influence of Chemical and Biological Factors on Trophic Transfer of Persistent Organic Pollutants in the Northwater Polynya Marine Food Web. Environ. Sci. Technol. 2001, 35 (4), 732 738. https://doi.org/10.1021/es001459w.

(90) Sun, M.; Arevalo, E.; Strynar, M.; Lindstrom, A.; Richardson, M.; Kearns, B.; Pickett, A.; Smith, C.; Knappe, D. R. U. Legacy and Emerging Perfluoroalkyl Substances Are Important Drinking Water Contaminants in the Cape Fear River Watershed of North Carolina. Environ. Sci. Technol. Lett. 2016, 3 (12), 415-419. https://doi.org/10.1021/acs.estlett.6b00398.

(91) Nakayama, S.; Strynar, M. J.; Helfant, L.; Egeghy, P.; Ye, X.; Lindstrom, A. B. Perfluorinated Compounds in the Cape Fear Drainage Basin in North Carolina. Environ. Sci. Technol. 2007, 41, 5271-5276. https://doi.org/10.1021/es070792y.

(92) Hopkins, Z. R.; Sun, M.; DeWitt, J. C.; Knappe, D. R. U. Recently Detected Drinking Water Contaminants : GenX and Other Per- and Polyfluoroalkyl Ether Acids. J. AWWA 2018, 110 (7), 13-28. https://doi.org/10.1002/awwa.1073.

(93) Sachs, E. Provisioning and Prey Quality in Brown Pelicans (Pelecanus Occidentalis) in Charleston Harbor, South Carolina, Clemson University, 2007.

(94) Baptist, M. J.; Leopold, M. F. Prey Capture Success of Sandwich Terns Sterna Sandvicensis Varies Non-Linearly with Water Transparency. Ibis (Lond. 1859). 2010, 152 (4), 815-825. https://doi.org/10.1111/j.1474919X.2010.01054.X.

(95) Ronconi, R. A.; Steenweg, R. J.; Taylor, P. D.; Mallory, M. L. Gull Diets Reveal Dietary Partitioning, Influences of Isotopic Signatures on Body Condition, and Ecosystem Changes at a Remote Colony. Mar. Ecol. Prog. Ser. 2014, 514 (August 2015), 247-261. https://doi.org/10.3354/meps10980.

(96) Zhang, C.; Hopkins, Z. R.; Mccord, J.; Strynar, M. J.; Detlef, R. U.; Knappe, D. R. U. Fate of Per- and Polyfluoroalkyl Ether Acids in the Total Oxidizable Precursor Assay and Implications for the Analysis of Impacted Water. Environ. Sci. Technol. Lett. 2019. https://doi.org/10.1021/acs.estlett.9b00525.

(97) Feng, M.; Qu, R.; Wei, Z.; Wang, L.; Sun, P.; Wang, Z. Characterization of the Thermolysis Products of Nafion Membrane: A Potential Source of Perfluorinated Compounds in the Environment. 2015, 1-8. https://doi.org/10.1038/srep09859. 
(98) Mauritz, K. A.; Moore, R. B. State of Understanding of Nafion. Chem. Rev. 2004, 104 (10), 4535-4585. https://doi.org/10.1021/cr0207123. 
TABLES AND FIGURES

\begin{tabular}{|c|c|c|c|c|c|c|}
\hline \multicolumn{7}{|c|}{$\%$ Detection by Ecosystem } \\
\hline Compound & Family & $\begin{array}{c}\# \text { Fluorinated } \\
\text { Carbons }\end{array}$ & $\begin{array}{l}\text { Mass. } \\
\text { Bay }\end{array}$ & $\begin{array}{l}\text { Narra. } \\
\text { Bay }\end{array}$ & CFRE & All \\
\hline N-MeFOSAA & FASAA & 8 & 0 & 0 & 0 & 0 \\
\hline $\mathrm{N}$ - EtFOSAA & FASAA & 8 & 0 & 0 & 0 & 0 \\
\hline $\mathrm{FOSA}^{\mathrm{a}}$ & FASA & 8 & 0 & 0 & 27 & 10 \\
\hline $4: 2 \mathrm{FTS}$ & FTS & 4 & 0 & 0 & 0 & 0 \\
\hline 6:2 FTS & FTS & 6 & 0 & 0 & 0 & 0 \\
\hline $8: 2$ FTS & FTS & 8 & 0 & 0 & 0 & 0 \\
\hline PFBA & PFCA & 3 & 100 & 80 & 73 & 84 \\
\hline PFPeA & PFCA & 4 & 0 & 0 & 0 & 0 \\
\hline PFHxA & PFCA & 5 & 0 & 30 & 27 & 19 \\
\hline PFHpA & PFCA & 6 & 0 & 0 & 0 & 0 \\
\hline PFOA & PFCA & 7 & 0 & 0 & 64 & 23 \\
\hline PFNA & PFCA & 8 & 100 & 100 & 100 & 100 \\
\hline PFDA & PFCA & 9 & 90 & 100 & 100 & 97 \\
\hline PFUnDA & PFCA & 10 & 100 & 90 & 100 & 97 \\
\hline PFDoA & PFCA & 11 & 0 & 10 & 27 & 13 \\
\hline PFTrDA & PFCA & 12 & 70 & 20 & 27 & 39 \\
\hline PFTeDA & PFCA & 13 & 0 & 10 & 0 & 3 \\
\hline PMPA & PFECA & 3 & 0 & 0 & 0 & 0 \\
\hline $\mathrm{PFO}_{2} \mathrm{HxA}$ & PFECA & 3 & 0 & 0 & 0 & 0 \\
\hline PEPA & PFECA & 4 & 0 & 0 & 0 & 0 \\
\hline $\mathrm{PFO}_{3} \mathrm{OA}$ & PFECA & 4 & 0 & 0 & 0 & 0 \\
\hline HFPO-DA & PFECA & 5 & 0 & 0 & 9 & 3 \\
\hline $\mathrm{PFO}_{4} \mathrm{DA}^{\mathrm{b}}$ & PFECA & 5 & 0 & 0 & 70 & 23 \\
\hline $\mathrm{PFO}_{5}$ DoDA & PFECA & 6 & 0 & 0 & 100 & 36 \\
\hline Nafion BP4 & PFESA & 6 & 0 & 0 & 0 & 0 \\
\hline Nafion BP2 & PFESA & 7 & 20 & 10 & 100 & 45 \\
\hline Nafion BP1 & PFESA & 7 & 0 & 0 & 0 & 0 \\
\hline NVHOS & PFESA & 4 & 0 & 0 & 0 & 0 \\
\hline PFBS & PFSA & 4 & 0 & 10 & 0 & 3 \\
\hline PFPeS & PFSA & 5 & 20 & 0 & 0 & 7 \\
\hline $\mathrm{PFHxS}$ & PFSA & 6 & 10 & 10 & 55 & 26 \\
\hline PFHpS & PFSA & 7 & 0 & 0 & 55 & 19 \\
\hline PFOS & PFSA & 8 & 100 & 100 & 100 & 100 \\
\hline PFNS & PFSA & 9 & 0 & 0 & 0 & 0 \\
\hline PFDS & PFSA & 10 & 0 & 10 & 9 & 7 \\
\hline PFECHS & $\begin{array}{l}\text { Cyclic } \\
\text { PFSA }\end{array}$ & 8 & 0 & 0 & 0 & 0 \\
\hline
\end{tabular}

Table 2.1. Detection frequency of quantifiable and semi-quantitative analytes in seabird juveniles from each habitat and as a total sample set across all habitats combined. Mass. Bay $=$ massachusetts bay, narra. Bay $=$ narragansett bay, and cfre $=$ cape fear river estuary. Family names are from buck et al. 2011. Compounds highlighted in gray are those compounds detected above reporting levels in at least $97 \%$ of individuals via lc-ms $/ \mathrm{ms}$. ${ }^{\text {a }}$ detection based on raw abundances in comparison to blank raw abundances due to lack of authentic standards. ${ }^{\mathrm{b}}$ low recovery $(14 \%)$ related to sample preparation. 

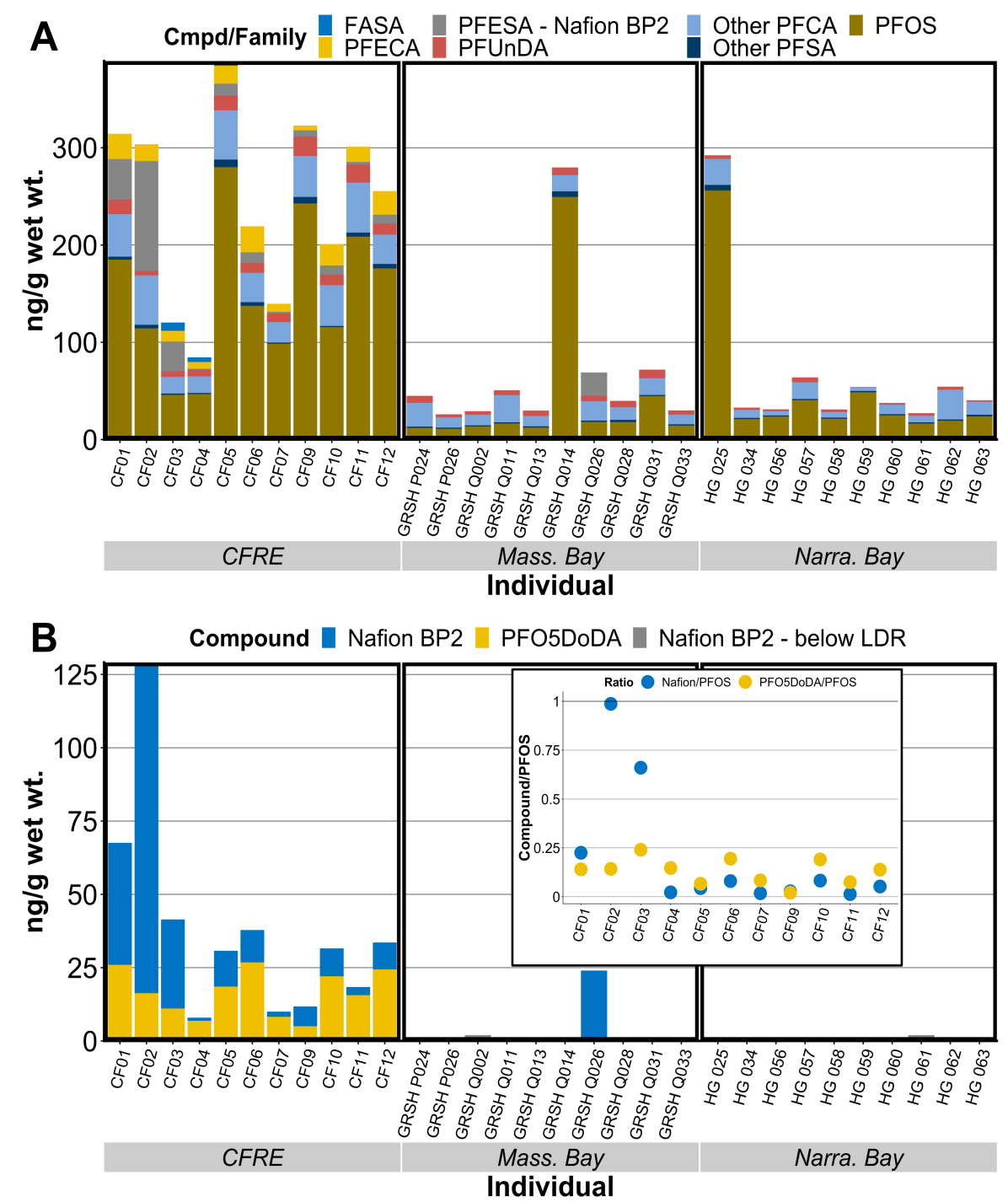

Figure 2.1. Measured concentrations of A) PFAS in juvenile seabird livers measured via LC-MS/MS, B) two emerging PFAS measured via targeted HRMS, alongside B-inset) ratios of emerging PFEAs to PFOS in CFRE chicks. Nafion BP2 concentrations positively identified in non-CFRE chicks but below the linear dynamic curve range are graphed in panel B as half the reporting limit; grey arrows are used to distinguish these data points. 


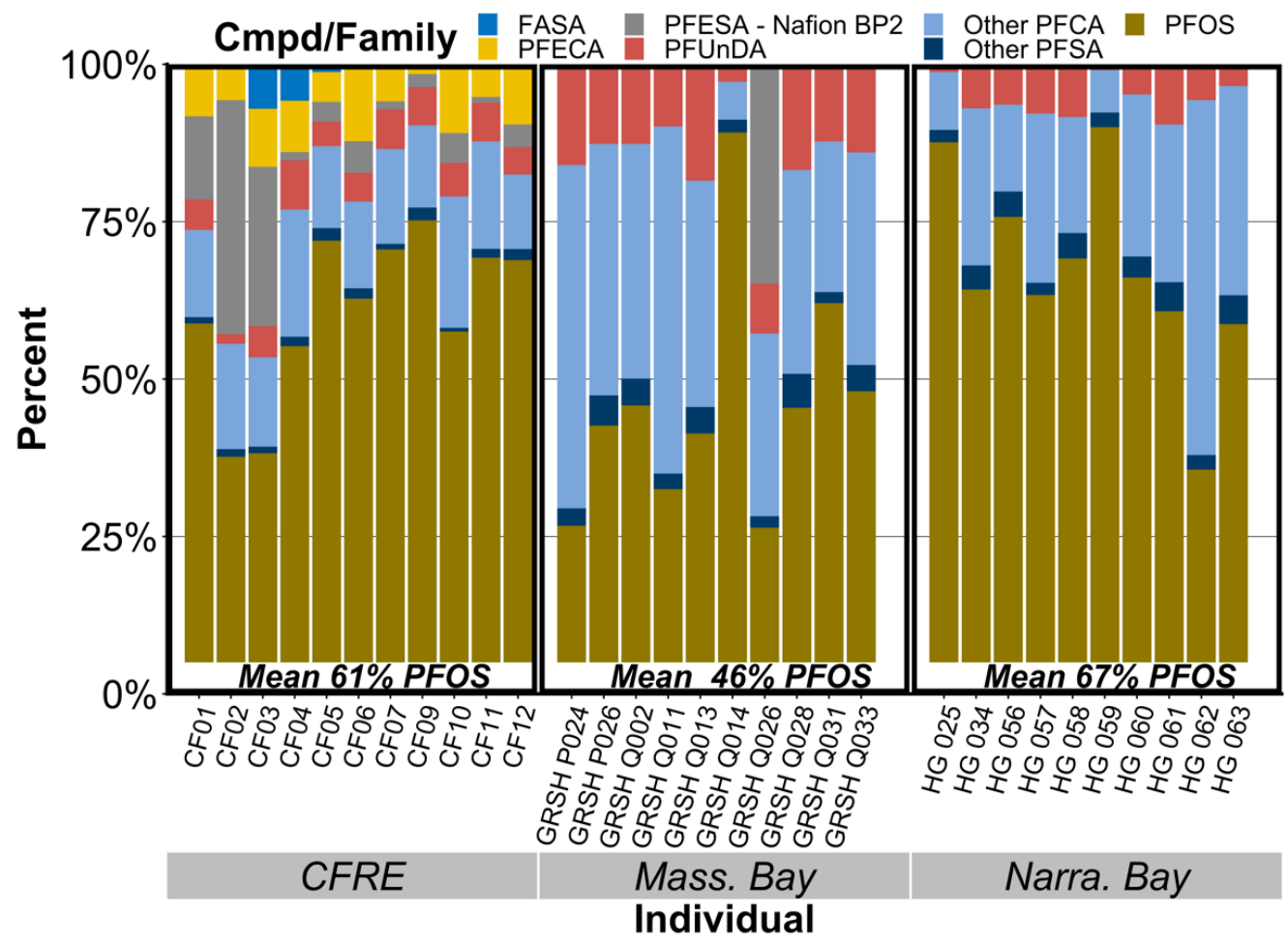

Figure 2.2. The composition of PFAS in liver tissue, presented by individual and grouped by habitat. 

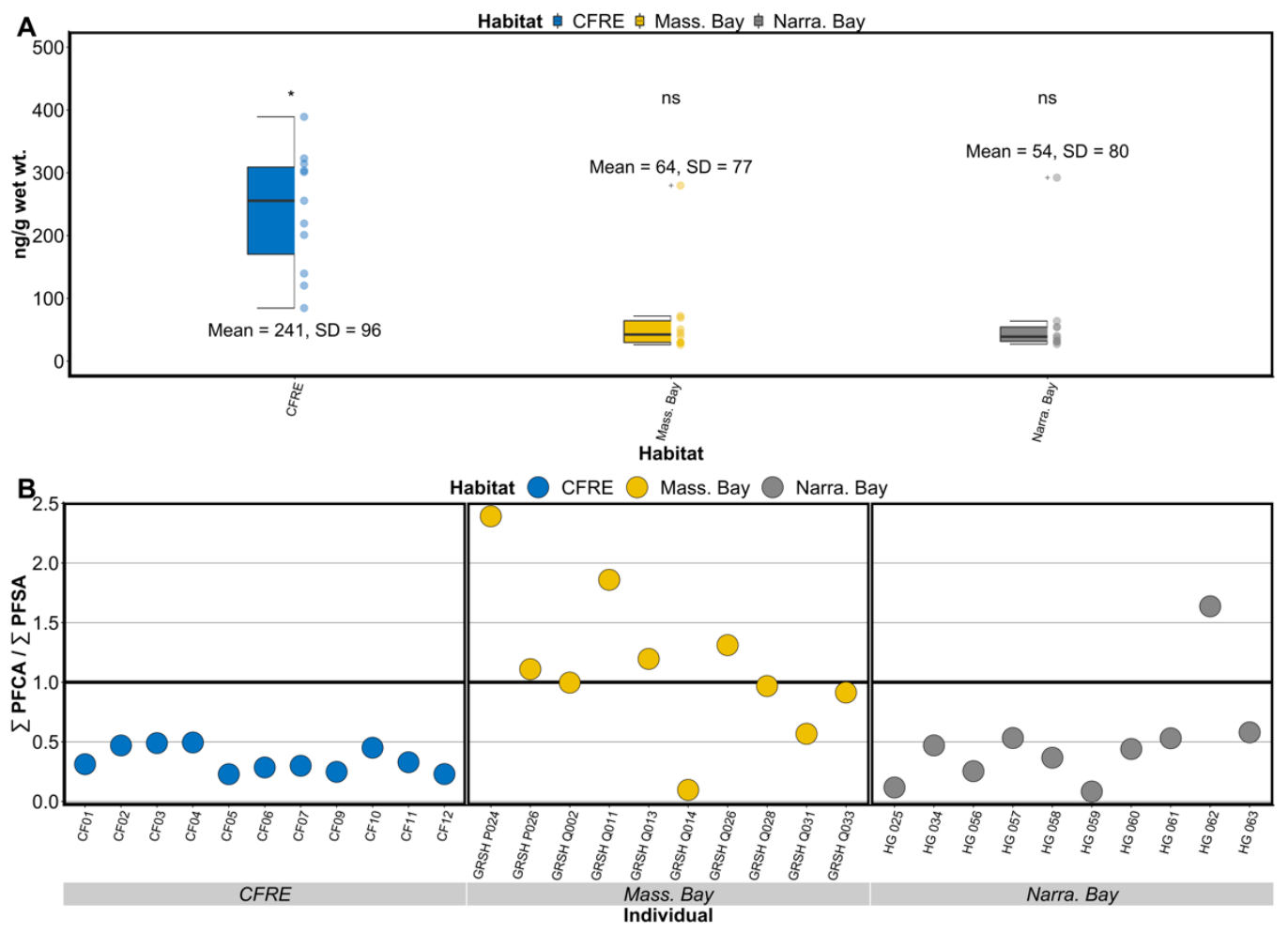

Figure 2.3. $\sum{ }_{19} \mathrm{PFAS}$ presented in A) as a boxplot, with the dark line representing the median, box limits representing the first and third quartiles, whiskers denoting 1.5 times the interquartile range, and crosses denoting outliers. The asterisk indicates a statistically significant difference between habitat mean $\sum{ }_{19}$ PFAS compared via Wilcoxon rank sum test using the CFRE as the reference group, while B) presents ratios of $\sum$ PFCAs to $\sum$ PFSAs in each individual. Ratios above 1 indicate PFCA dominance, while ratios below 1 indicate PFSA dominance. (Small figure, 300 word equivalent) 

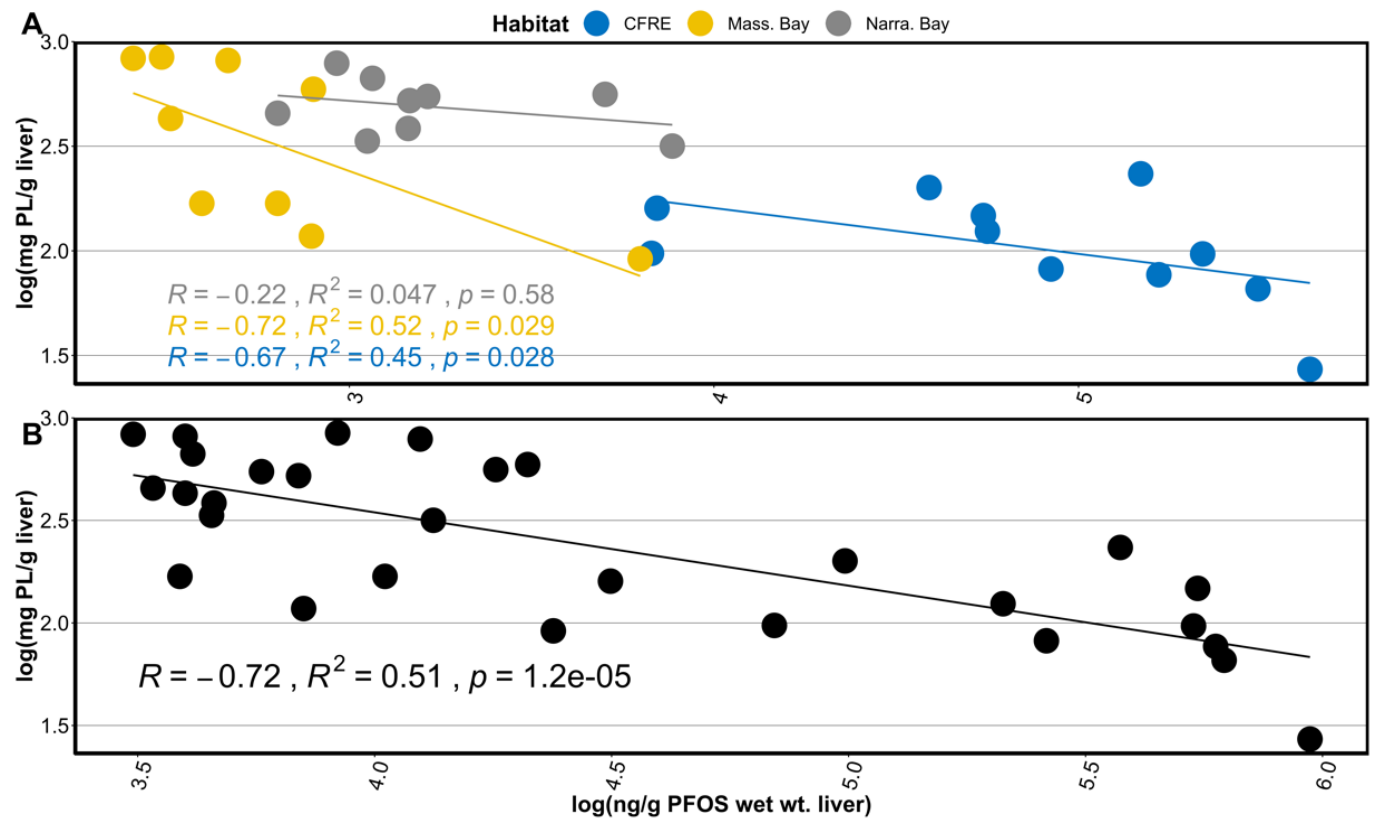

Figure 2.4. A) presents log-transformed concentrations of PFOS vs phospholipid (PL) grouped by habitat, while B) displays log-transformed PFOS and phospholipid (PL), assessed as a total sample set $(n=31)$. Text annotation presents $\mathrm{R}, \mathrm{R}_{\mathrm{s}}{ }^{2}$, and $\mathrm{p}$-value derived from Spearman rank correlation analysis. 


\title{
SUPPORTING INFORMATION
}

\section{LEGACY AND NOVEL PER- AND POLYFLUOROALKYL SUBSTANCES IN JUVENILE SEABIRDS FROM THE U.S. ATLANTIC COAST}

\author{
Anna R. Robuck, Mark G. Cantwell, James McCord, Lindsay M. Addison, Marisa \\ Pfohl, Mark J. Strynar, Richard McKinney, David R. Katz, David N. Wiley, and \\ Rainer Lohmann
}

This document contains 10 figures and 23 tables.

\section{Chemicals, reagents, and materials}

Analytical standards of greater than $99.9 \%$ purity, including mass-labeled surrogates, were purchased from Wellington Laboratories (Guelph, ON, Canada). Native Nafion BP2 and PFO5DoDA were provided by Chemours (Fayetteville, NC) in lieu of a commercial source. HPLC grade methanol was purchased from Fisher Scientific (Waltham, MA, USA). Ultrapure water for equipment cleaning was obtained from a Milli-Q system fit with an HPLC water polisher or via HPLC grade water purchased from Fisher Scientific (Waltham, MA, USA). ENVI Carb $2 \mathrm{~g}$ cartridges were purchased from Sigma-Aldrich (St. Louis, MO, USA). Ammonium acetate was purchased from Fisher Scientific (Waltham, MA, USA)

\section{Sample collection details}


All birds were obtained opportunistically for use in this study, and no birds were killed for the purposes of this study. Birds were collected and stored in accordance with URI Biosafety standards and the Migratory Bird Treaty Act.

Dead Great Shearwater juveniles (Ardenna gravis) were obtained as bycatch from the National Oceanic and Atmospheric Administration (NOAA) Northeast Fisheries Observer Program (Falmouth, MA). Dead Herring Gull (Larus argentatus smithsonianus) chicks were obtained from the Wildlife Clinic of Rhode Island (Narragansett, RI). Dead Royal Tern (Thalasseus maximus), Sandwich Tern (Thalasseus sandvicensis), Laughing Gull (Leucophaeus atricilla), and Brown Pelican (Pelecanus occidentalis) chicks were obtained as part of routine field and nest surveys conducted by North Carolina Audubon (Wilmington, NC).

Great Shearwaters in this study were self-feeding and had completed their first migration from their remote breeding site in the South Atlantic at approximately 5-6 months old, and are therefore considered juveniles. Great Shearwaters were confirmed to be juveniles based on molt status, presence of the bursa of Fabricius, and stage of gonad development. All birds from Rhode Island and North Carolina were pre-fledging birds under 8 weeks of age, still under parental care at time of death. All individuals were hatch-year juveniles, under approximately six months of age. Great Shearwaters were 5-6 years away from reproductive maturity, while gulls, terns, and pelicans, who mature more rapidly, were at least a year away from reproductive maturity (Tables S3-S4).

\section{Support for a multi-species, variable age comparison}


$\underline{\text { Species }}$

The literature does not currently support the hypothesis of significantly different uptake, metabolism, or elimination rates between similar seabird species. The species included in this study are similar in trophic strategy and preferred prey items, facilitating an acceptable comparison.

a) Species-based differences in bioaccumulation capacity

We point to multiple studies across the literature that suggest foraging preferences, migratory strategies, and other life history details that determine exposure potential drive PFAS concentrations rather than innate differences in bioaccumulation capacity between seabird species ${ }^{1,2,11,3-10}$.

The most valuable support of our sample design comes from studies investigating concentration trends in multiple species surrounding concerted PFAS sources. Lopez-Antia et al. (2017) examined PFOS levels in eggs from three species of bird surrounding a fluoropolymer production site in the Netherlands. The study focused on species that were unambiguously different from one another in terms of size and diet, unlike the similar seabirds included in our study. Great tits are a small passerine (songbird) species that primarily feeds on insects; northern lapwings are small-medium marsh wading birds that feed on small aquatic invertebrates or insects. Mediterranean gulls are opportunist seabirds that feed on fish, bivalves, or crustaceans. The study found no statistically significant differences in egg PFOS levels among the three species, suggesting the common exposure source drove PFOS levels in all birds without 
clear species-level differences in adult uptake, metabolism, or elimination via maternal offloading. Similarly, Yoo et al. (2008) examined PFAS in eggs from three bird species nesting on Lake Shiwa in Korea, a large, industrially-influenced artificial seawater lake. The study found no significant differences in PFOS and $\sum_{5}$ PFCA between little egret (a large wading bird), little ringed plover (a small shorebird), and parrot bill (a small songbird-like bird) eggs.

Data from controlled studies indicate approximately equivalent accumulation efficiency in adult quails (a game bird) and mallards (a duck) ${ }^{9}$. Domestically reared adult birds from both species and sexes were exposed to the same PFOS levels via diet. Adult livers and serum were found to contain similar PFOS levels at the completion of exposure; larger differences were apparent between sexes of the same species than between different species. Egg yolk and 14-day old juvenile liver and serum also displayed comparable PFOS levels between both species, without the stark differences between sexes.

Roscales et al. 2019 compared PFAS levels in seabird serum from multiple habitats in or adjacent to the Southern Ocean ${ }^{11}$. Two species were sampled within three colonies across different latitudes and ocean basins. Measurements show larger differences between the same species at different locations, compared to observed differences between different species at the same colony (Roscales et al. 2019, Table 1). This suggests location-based exposure factors, like proximity to PFAS sources or prey PFAS levels, are the primary determinant of PFAS in similar seabird species. 
Beyond avifauna, we note that data from marine mammals suggests taxa with a shared phylogeny conserve cellular and tissue machinery driving internal kinetics of PFAS. For example, evidence in marine mammals demonstrates conservation of metabolic pathways at the family level rather than the species level; data to date suggests all odontocete cetaceans are unable to efficiently metabolize FOSA ${ }^{12-16}$.

Species-specific toxicokinetic processes and rates are certainly possible and have been demonstrated across contrasting mammal species like rats and humans ${ }^{17}$. However, based on the available data showing similar tissue residues in seabirds and other types of birds subject to similar PFAS exposures, we suggest further data is required to rigorously support the claim of significantly different accumulation, metabolism, or elimination pathways or rates at a species level in similar birds. The data we have on hand suggests comparisons across multiple species are acceptable, and any major differences in PFAS levels are due to dietary and habitat factors. We highlight this as an important research gap considering the utility of birds as ecosystem sentinels.

b) Ontogenetic and diet differences that confound comparison between species

As the above evidence suggests, habitat-related exposure and dietary choices likely drive PFAS levels more than major innate differences in toxicokinetics between seabird species. We assert that the birds included in our study are roughly comparable in diet, all feeding primarily on forage fish and invertebrates (Table S2). All species demonstrate some reliance on Atlantic menhaden 
Brevoortia tyrannus, a key forage fish found along the US Atlantic seaboard from Florida to Nova Scotia. All species are plungers and opportunists rather than pursuit divers. This broad similarity in trophic strategy and position allows our study to highlight and compare differences in PFAS levels due to habitat-driven factors like human inputs and proximity to production sources.

Age

Our study focused on how each habitat/lifestyle contributes PFAS to an associated sentinel predator, and by sampling juveniles and chicks we avoid life history complications associated with older birds to focus on this question. While chick and juvenile stages are distinct in many ways, we do not believe the differences in these life stages significantly compromise PFAS measurements in our study due to the influence of maternal offloading, and the long half-life of PFAS.

a) Both chick and juvenile birds primarily reflect maternal offloading PFAS are very persistent in the environment and in biota. PFAS transferred from mother to offspring do not readily dissipate over the first few months of development. The lengthy plasma half-life of PFOS in birds (231 days) means that both chicks $(\sim 14-56$ days old $)$ and juveniles $(\sim 140-170$ days old $)$ within this study retain and reflect the influence of maternal offloading in liver measurements ${ }^{18}$. Short-term exposure trials in juvenile quail and mallard suggest the liver half-life of PFOS is roughly 2.5 times that of the serum half-life. Using the plasma half-life derived in Tarazona et al. 2015, this equates to an estimated liver half-life for PFOS of 578 days in birds, or 1.6 years. Liver elimination of 
PFAS via biliary excretion has been demonstrated to be very slow $(\sim 1 \%$ of supplied dose $)^{19}$, and liver metabolism of most PFAAs is thought to negligible. Therefore we reiterate that all individuals of all ages used in this study primarily reflect the influence of maternal offloading. A longer duration of self-feeding by Great shearwaters is unlikely to offset or surpass the PFAS burden received in ovo; a study examining prey fish concentrations compared to egg and juvenile liver concentrations in guillemots suggested maternal offloading exposure drastically exceeded dietary inputs ${ }^{20}$. Measurement of shearwater prey (sand lance) from Massachusetts Bay points to similar, low levels of PFAS exposure from offshore prey ( $\sum_{12}$ PFAAs: $\sim 1-8 \mathrm{ng} / \mathrm{g}$ whole body, wet weight) (Robuck, unpublished data). We also point to seabird literature that suggests adults of these species provision their young with the same or similar species that they themselves consume, meaning the dietary additions provided to chicks were similar or identical to those prey items that contributed to PFAS burdens in mothers prior to maternal offloading (Table S2).

The duration of dietary exposure was longer for the older juveniles, who had been self-feeding for several months. More importantly, these birds also had a longer growth period, causing growth dilution of maternally offloaded PFAS in liver.

b) There is no evidence that specific cellular and tissue machinery known to drive PFAS accumulation differ with age.

PFAS accumulation is driven by associations with specific proteins in liver and blood, in tandem nonspecific associations with amphiphilic structural lipids ${ }^{21-}$ 
23. We are not aware of any data demonstrating age-based differences in the protein structures or binding efficiency of liver fatty acid binding protein and albumin, the two primary proteins identified to drive PFAS accumulation in liver and blood. Rather, data from in ovo studies demonstrates the ability of developing embryos to readily accumulate PFAS in liver, at levels on par with or exceeding maternal liver concentrations ${ }^{9}$. Data from in utero exposure studies in mice reiterate the same ${ }^{24}$. Current, albeit limited, data suggest no appreciable changes in bulk liver phospholipid levels over chick development ${ }^{25}$, though rapid changes in storage lipid content occur across all tissues during chick development ${ }^{26}$. Our data in Figure 4 reiterates this - chicks from Narragansett Bay had phospholipid levels similar to older Great Shearwater juveniles (possibly due to similar PFOS levels) compared to chicks from the CFRE of the same age and from the same family. Elimination rates have been shown to be sex-dependent, but no data exist describing age-specific elimination differences in birds ${ }^{9,18,27}$. Specific mechanisms of PFAS uptake may change on a bulk level with tissue growth as more tissue is available to interact with PFAS, but we believe this would not be apparent in our measurements normalized to ng PFAS/g tissue basis. More importantly, the maternally offloaded burden of PFAS would be diluted with tissue growth. Overall, more research is needed to explore changes in PFAS uptake with age, but data to date do not indicate substantial changes in tissue components driving bioaccumulation of PFAS.

c) PFAS are not stored in fat 
PFAS are not stored in fat akin to legacy persistent organic pollutants like polychlorinated biphenyls or $\mathrm{DDT}^{28-31}$, meaning the rapid development of adipose fat stores in juveniles that may impact hydrophobic pollutant levels is not likely to impact organ levels of PFAS in chicks or juveniles.

d) PFAS demonstrate an inverse relationship with age

Current literature suggests PFAS do not display the same positive relationships between age like some legacy POPs ${ }^{32}$. Data from several taxa indicate PFAS are significantly higher in juveniles compared to adults ${ }^{33-37}$. Possible explanatory mechanisms for this negative relationship include the influence of maternal offloading combined with the long tissue half-lives of many PFAS, or growth-mediated biodilution. Research examining PFAS in maternal liver, egg, and chick liver have found the highest PFAS levels in egg, with subsequent chick liver containing PFOS on par with or higher than the mother's liver $^{9,20}$. This suggests that like other wildlife taxa, seabirds also display a negative relationship between PFAS and age. By measuring young birds, we are likely measuring the highest concentrations experienced by these individuals throughout their lifetimes, during a critical development window.

e) Juveniles are not impacted by reproductive cycles which confound measurements in adults.

Seabirds have been shown to offload significant concentrations of PFAS in $\operatorname{eggs}^{28,29,38}$.

f) Using juvenile or immature dead seabirds allows measurement of individuals of a known age and sex. 
Most adult seabirds (including all species included in this study) can't be aged or sexed by plumage or morphometric characteristics after their first year of age.

g) Juveniles demonstrate some of the most predictable ties to a given foraging habitat, helping to constrain dietary and habitat influences on PFAS levels.

For chicks, this is caused by parents engaging in central-place foraging, meaning the adults find food for their chicks within a region adjacent to the nesting colony to facilitate continuous provisioning of chicks. For the Great Shearwater juveniles which were self-feeding, multiple years of tracking data suggest hatch year birds forage in a predictable home range across Massachusetts Bay, the Great South Channel off Cape Cod, and Georges Bank while adults may forage more widely across a larger pelagic region ${ }^{39,40}$. 


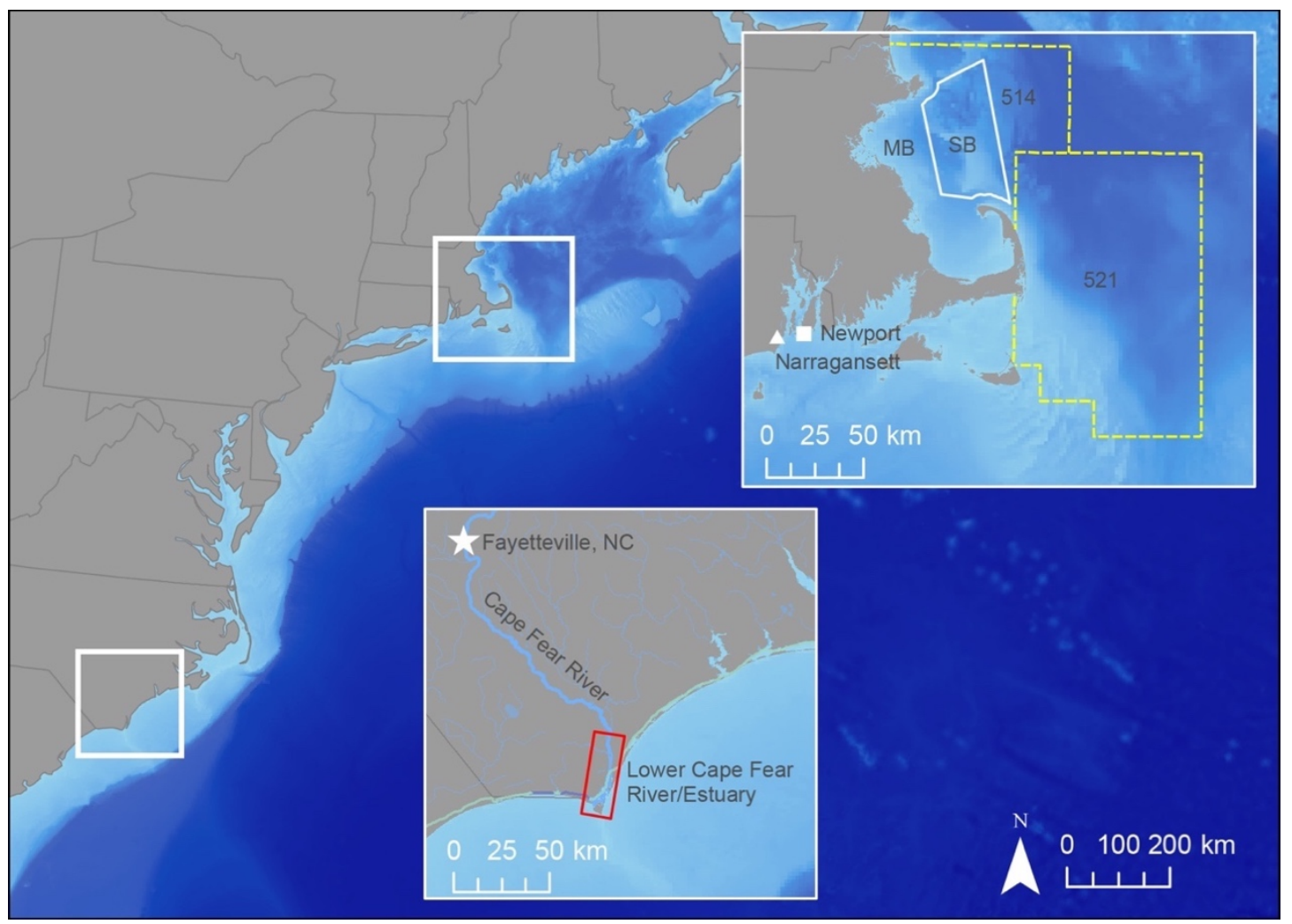

Figure S2.1. Map of the US Atlantic East Coast. Insets provide further detail about collection locations of from each habitat. Great shearwaters were collected as bycatch in Massachusetts Bay and off Cape Cod, designated as NOAA Fisheries Stat Areas 514 and 521. Stellwagen Bank National Marine Sanctuary, a key foraging area for Great Shearwaters, is outlined in white and indicated as "SB" in the top right inset. Herring Gull chicks came from nests located in Narragansett, RI and Newport, RI. The bottom inset shows the Cape Fear River and Estuary system, located in southeastern NC. A Chemours facility is located near Fayetteville, NC, designated on the map as a white star. The red box indicates the lower river and estuary portion of the system; CFRE chicks were hatched on dredge islands near the mouth of the estuary.

\section{Sample condition, cause of death, and necropsy}

Great Shearwaters and Herring Gulls were freshly or very recently dead, and frozen immediately with no decomposition apparent.

The procurement of tissues from juvenile Great Shearwaters caught via fishing bycatch is extremely unlikely to impact liver integrity. Juvenile Great Shearwaters were bycaught via gillnet. Lung hemorrhaging and edema was 
apparent in some bycatch seabirds (indicative of drowning), but there was no evidence of seawater intrusion into the coelomic cavity housing the liver, and no perforations or damage to any other internal organs beyond lung hemorrhaging. As such we consider bycatch birds as excellent sample specimens, in good condition with no apparent liver degradation or trauma.

Tern, Laughing Gull, and Brown Pelican chicks were recently deceased; chicks spent a maximum of 24-48 hours exposed to the elements prior to collection due to the colony monitoring schedule. Some decomposition was apparent externally on a few individuals. This decomposition was entirely external, apparent via reduced eye integrity, mouth mucus membrane color, and skin pallor. No CFRE individuals displayed evidence of scavenging or substantial autolysis internally.

Phosphotidylcholines, the primary type of phospholipid measured with our bulk assay, have demonstrated stability up to 48 hours after death at $24.1 \mathrm{C}, 50 \%$ humidity, and atmospheric pressure, suggesting the lipid measurements from CFRE chicks produced within our study are likely free from majorly compromising impacts related to post-death putrefaction, autolysis, or $\mathrm{pH}$ diminution ${ }^{41,42}$.

Massachusetts and Narragansett Bay birds were otherwise healthy birds that met unfortunate ends. Narragansett Bay birds fell from their nests and broke one or more limbs, while Massachusetts Bay birds drowned or were strangled in fishing nets. There was no indication of other health problems impacting these individuals that could influence contaminant distributions observed. 
Cape Fear birds died due to mostly uncertain causes - predation was not the cause of death because all deceased individuals were collected intact. Colony monitoring data from multiple locations suggests nest abandonment as the most common cause of chick death. Seabirds such as terns and pelicans are very sensitive to disturbance. They will readily abandon a nest due to human, predator, or insect disturbance ${ }^{43-46}$ - the chick would then die of dehydration or cold stress. Chicks may also die from tidal flooding or starvation. Chilling, dehydration, and tidal flooding are unlikely to impact contaminant levels in chick livers. PFAS are minimally stored in fat, therefore any starvation-induced use of fat stores is unlikely to release PFAS to liver and overall circulation, should starvation have been the cause of death for any of these birds. No widespread starvation event was apparent in the CFRE region in 2017, making this final potential cause of death somewhat unlikely.

All birds were frozen following collection and tissues sampled within two months. Species identification was corroborated by both specimen collector and necropsy prosector. Each seabird individual was partially thawed and necropsied according to standard protocol, documenting morphometric features, organ weights, overall body condition, sex, and stomach contents after van Franeker $2004^{47}$. Birds were sexed if gonads were visually identifiable, and aged using morphometric, gonad, and bursa characteristics along with nest observations. Multiple tissues including liver were collected from each bird, and all tissue samples were wrapped in solvent-cleaned aluminum foil, stored in polyethylene baggies, and frozen until analysis at $-15 \mathrm{C}$. More details about sample collection 
locations, bird morphometric data, age, sex, and sample condition can be found in Table S3.

\section{Sample preparation for UPLC-MS/MS}

A modified extraction procedure was developed to maximize sample throughput while minimizing matrix effects and extraction losses, incorporating steps employed in several previously published extraction protocols ${ }^{28,48-50}$. A tissue aliquot was weighed into a polypropylene tube and $4 \mathrm{ml}$ of methanol added, followed by $10 \mathrm{ng}$ of isotopically labeled PFAS surrogate mix (1ng/ $\mu \mathrm{L})$. Samples were vortexed for 30 seconds, and allowed to equilibrate for 30 minutes. Samples were again vortexed for 30 seconds, sonicated for 20 minutes, and centrifuged at 4,000 rpm for ten minutes. The resulting supernatant was decanted into a fresh polypropylene tube, and the extraction procedure repeated with $4 \mathrm{~mL}$ of $2 \mathrm{mM}$ ammonium acetate in methanol. The extract was frozen at $-15 \mathrm{C}$ for at least four hours to encourage precipitation of additional biological material and then centrifuged for three minutes under refrigeration, directly followed by decanting the supernatant for clean-up.

The combined extract was cleaned up using Supelclean ENVI-Carb cartridges (2g, $12 \mathrm{~mL}, 100-400 \mathrm{mesh}$, Supelco, U.S.A.). The cartridges were cleaned with $8 \mathrm{~mL}$ methanol prior to sample introduction. After sample clean-up, each cartridge was rinsed with $2 \mathrm{~mL}$ of methanol. Clean-up was repeated if the final extract contained any hint of color. The extracts were dried to $250 \mathrm{ul}$ at $32^{\circ} \mathrm{C}$ under 5-7 psi $\mathrm{N}_{2}$, and reconstituted to $1 \mathrm{~mL}$ using $2 \mathrm{mM}$ ammonium acetate in water for a final sample makeup ratio of 3 parts aqueous: 1 part organic extract. 
The $1 \mathrm{~mL}$ extracts were centrifuged at $10,000 \mathrm{rpm}$ for fifteen minutes at $5^{\circ} \mathrm{C}$ to remove any remaining tissue residues; the final extract minus any pellet solids was transferred to an autosampler vial in preparation for instrumental analysis.

\section{UPLC-MS/MS analysis}

$40 \mu 1$ of sample extract was injected, and chromatographic separation achieved using a $50 \mathrm{~mm}$ BEH C-18 column interfaced to a Waters Acquity UPLC. The mobile phase was made up of methanol and HPLC-grade water modified with $2 \mathrm{mM}$ ammonium acetate, made fresh before each run. The applied gradient is detailed in Table $\mathrm{S} 10$, the flow rate was set at $0.4 \mathrm{ml} / \mathrm{min}$, and column temperature set at $45^{\circ} \mathrm{C}$.

Detection of PFAS was carried out using UPLC-MS/MS in negative electrospray ionization mode. Multiple reaction monitoring (MRM) was employed, monitoring two transitions for each compound as available, detailed in Table S7 and S9. Desolvation temperature was set at $400^{\circ} \mathrm{C}$, desolvation gas flow at $600 \mathrm{~L} / \mathrm{hr}$, source temperature was set at $150^{\circ} \mathrm{C}$, and cone gas flow set at $30^{\circ} \mathrm{C}$. Optimal transitions and analyte-specific energies were generated using Intellistart software in combination with manually performed direct infusion experiments.

Quantitation was carried out using an isotope-dilution approach; those analytes lacking a matched mass-labelled standard were quantified using a masslabelled surrogate of similar molecular weight and retention time (Table S7).

\section{Sample preparation for HRMS}

A tissue aliquot was diluted with three parts water and homogenized to a uniform slurry. An aliquot of homogenate was diluted $4: 1$ with $0.1 \mathrm{M}$ formic acid 
and vortexed for 30 seconds. The denatured extract was further diluted 5:1 with cold acetonitrile and centrifuged for $5 \mathrm{~min}$ at $10000 \mathrm{rpm}$. An aliquot of the supernatant was removed and combined with dilute ammonium formate buffer $(2.5 \mathrm{mM})$ to obtain a final sample extract ratio of 3 parts aqueous: 1 part organic extract.

\section{HRMS analysis}

$100 \mathrm{ul}$ of sample was injected, and chromatographic separation achieved using a Vanquish UPLC system equipped with an Accucore $100 \mathrm{~mm}$ reversephase $\mathrm{C} 18$ column, at a flow rate of $300 \mathrm{ul} / \mathrm{min}$. Mobile phase constituents included Solvent A (95:5 water: acetonitrile) and Solvent B (95:5 acetonitrile:water). Mobile phase gradient is detailed in Table S11. Nafion byproduct 2 (Nafion BP2, or 2-[1-[difluoro(1,2,2,2-tetrafluoroethoxy)methyl]1,2,2,2-tetrafluoroethoxy]-1,1,2,2-tetrafluoro-ethanesulfonic acid) and $\mathrm{PFO}_{5}$ DoDA $(2,2,4,4,6,6,8,8,10,10,12,12,12$-Tridecafluoro-3,5,7,9,11pentaoxadodecanoic acid) were identified with authentic native standards, while $\mathrm{PFO}_{4} \mathrm{DA}$ (perfluoro-3,5,7,9-tetraoxadecanoic acid), PFMA (2,3,3,3-tetrafluoro-2(trifluoromethoxy)-propanoic acid), $\mathrm{PFO}_{2} \mathrm{HxA}$ (perfluoro-3,5-dioxahexanoic acid), PEPA (Perfluoro-2-ethoxypropanoic acid), PFO3OA (Perfluoro(3,5,7trioxaoctanoic) acid), NVHOS (1,1,2,2-tetrafluoro-2-(1,2,2,2-tetrafluoroethoxy)ethane sulfonic acid), Nafion byproduct 4 (2,2,3,3,4,5,5,5-4-(1,1,2,2tetrafluoro-2- sulfoethoxy) pentanoic acid), Nafion byproduct 1 (2-[1[difluoro[(1,2,2-trifluoroethenyl)oxy]methyl]-1,2,2,2-tetrafluoroethoxy]-1,1,2,2tetrafluoro-ethanesulfonic acid) and PFECHS (perfluoro-4- 
ethylcyclohexanesulfonate) were detected using previous accurate mass assessment information. PFAS were detected using a Thermo Orbitrap Fusion mass spectrometer using heated electrospray ionization in negative mode (Thermo Fisher Scientific, Waltham, MA, USA). Full scan accurate mass spectra were acquired from 70 to $700 \mathrm{Da}$ with a resolving power of $120,000 \mathrm{Rs}$ for MS1 and at 30,000 Rs for MS2, and a mass accuracy of $\pm 5 \mathrm{ppm}$. Data-dependent acquisition was carried out to acquire MS/MS of select features at a resolving power of 30000. The ion transfer tube was set at $250^{\circ} \mathrm{C}$ and vaporizer temp set at $30^{\circ} \mathrm{C}$. Interference from the tissue matrix prevented the use of the Fusion internal lock mass in this experimental method. Data acquisition and analysis was performed using Xcalibur and Compound Discoverer software (Thermo Fisher Scientific, Waltham, MA, USA).

\section{Quality Assurance and Quality Control}

Six-point, processed and matrix-matched calibration curves were prepared, one for each extraction method. Curve preparation entailed taking multiple aliquots of liver tissue (from the same batch of slurried tissue) through each extraction in its entirety. The matrix-matched approach is key to account for the influence or interference of biological co-eluents on PFAS response and derived concentrations $^{50,51}$.

The UPLC-MS/MS curve points were spiked directly before instrumental analysis with appropriate levels of native and mass-labelled standard, ranging from $0.25-100 \mathrm{ng} / \mathrm{ml}$. The HRMS curve points were spiked before extraction, and therefore recovery-corrected all subsequent quantitation; these curve points 
ranged from $0.05-10 \mathrm{ng} / 400 \mu 1$. The curves were used for quantification of samples prepared with the corresponding extraction method. Organic chicken liver, demonstrated to contain low concentrations of targeted PFAS, was used as the curve matrix. PFOS was consistently found in organic chicken liver samples, and thus the curve was corrected for background levels of PFOS by subtracting the average of measured background samples $(n=6)$ from PFOS responses measured in curve point samples. All calibration curves used for quantitation demonstrated an $R^{2} \geq 0.98$, with most demonstrating an $R^{2} \geq 0.99$.

During targeted analysis via UPLC-MS/MS, process blanks were prepared and analyzed with every 10 samples and found to be free of significant contamination; sample concentrations were not blank corrected as a result. Each sample was injected in duplicate, and duplicate injections monitored for stability. Mobile phase blanks (3:1 aqueous:organic) were analyzed between duplicate sample injections to monitor for analyte carryover or contamination.

Five samples consisting of chicken liver spiked with $2 \mathrm{ng}$ of all native compounds were also prepared and used to calculate accuracy and precision metrics for UPLC-MS/MS. Accuracy ranged from 18-154\% with a mean accuracy of $92 \%$. Precision, calculated as percent relative standard deviation ( $\%$ RSD), ranged from $7-150 \%$, with a mean $\%$ RSD of $28 \%$ (Table S13). Precision and accuracy were particularly variable for neutral sulfonamide acetic acids, those compounds lacking an identical mass-labelled surrogate like PFDS, and the $\mathrm{C}_{13}$ and $\mathrm{C}_{14}$ PFCAs. 
Although the matrix-matched curves account for matrix effects in quantitation efforts, matrix effects were calculated for illustrative purposes following methods described by Chambers et al. $2008^{51}$. Matrix calculations indicated variable enhancement or suppression of each analyte, with an average ion suppression of $-20 \%$ and an ionization enhancement of $31 \%$ (Table S14).

Equation 1:

$$
\% \text { Matrix Effects }=\left(\left(\frac{\text { Post-extraction spiked matrix }}{\text { Post-extraction spiked solvent }}\right)-1\right) \times 100
$$

Method detection limits for UPLC-MS/MS ranged from $0.5-4.1 \mathrm{ng} / \mathrm{ml}$ based on spiked replicate samples multiplied by the Student's t-value appropriate for a single-tailed 99th percentile (Table S15). Method recovery ranged from 14 $112 \%$, with a mean recovery of $61 \%$ across all compounds (Table S6).

$$
\text { Equation 2: \% Recovery }=\frac{\text { Pre-extraction spiked sample }}{\text { Post-extraction spiked sample }} \times 100
$$

During HRMS analysis, duplicate process blanks using formic acid and acetonitrile were prepared daily with each sample set, for a total of 8 process blanks. Process blanks were used to identify contamination introduced via sample preparation and instrument background signal. Mobile phase blanks were injected between different types of samples to monitor instrumental background noise and any carryover between samples. No contamination of emerging PFAS was apparent in process blanks; HFPO-DA and several other legacy PFCAs displayed 
high levels of instrumental background noise as displayed in instrumental blanks and process blanks. No significant background noise was apparent for Nafion BP2, PFO5DoDA, or PFO4DA, the three PFEAs of interest reported here using HRMS analysis (Table S12). Method recoveries for HRMS were not tracked; each curve point sample was spiked with appropriate levels of native and internal standard and then taken through the extraction to create a recovery-corrected curve for quantification. Samples were analyzed in four batches, and curve stability monitored between runs. Curve responses at all levels varied less than $4 \%$ across all four runs for the three PFEAs reported using HRMS measurements in this analysis.

Quantification of emerging compounds via HRMS was limited to those samples above the linear range of the calibration curve; the lower detection limit was determined by comparison to blank values plus three times the standard deviation of blank responses (Table S12). Reporting of emerging compounds below the curve range or those without authentic standards is limited to raw abundances in comparison to process and instrumental blank values (Table S16).

PFOS concentrations measured via both HRMS and UPLC-MS/MS were compared for parity, and were generally found to be within $30 \%$ of UPLCMS/MS results and all were within an order of magnitude. UPLC-MS/MS results were considered more precise due to lower levels of instrumental background noise along with higher levels of $\mathrm{QA} / \mathrm{QC}$ and were therefore used for comparison and statistical analyses for all compounds excepting Nafion BP2 and PFO5DoDA. Stable Isotope Analysis Sample Preparation and Analysis 
Liver and muscle of seabird chicks were lyophilized, and ground to fineness using a mortar and pestle. 2-3 mg of tissue was weighed out into tin capsules for carbon and nitrogen stable isotope analysis, while 3-5 mg of muscle tissue were weighed out for sulfur isotope analysis.

Weighed samples were measured via IR-MS analysis, and results interpreted as parts per thousand relative to appropriate references. $\delta^{15} \mathrm{~N}$ and $\delta^{13} \mathrm{C}$ were measured using an Isoprime 100 Isotope Ratio Mass Spectrometer coupled to a Micro Vario Elemental Analyzer (Elementar Americas, Mt.Laurel, NJ). $\delta^{34} \mathrm{~S}$ was measured by UC Davis Stable Isotope Laboratory using an Elementar Vario ISOTOPE cube interfaced to a SerCon 20-22 IRMS (Sercon Ltd., Cheshire, UK).

The nitrogen $\left(\delta^{15} \mathrm{~N}\right)$ isotope composition was expressed as a part per thousand deviation (\%o ) from air. Carbon $\left(\delta^{13} \mathrm{C}\right)$ isotope composition was expressed relative to Vienna Pee Dee Belemnite where $\delta \mathrm{X}=\left[\left(\mathrm{R}_{\text {sample }}-\right.\right.$ $\left.\mathrm{R}_{\text {standard }} / \mathrm{R}_{\text {standard }}\right] \times 10^{3}$, where $\mathrm{X}$ is $\delta^{15} \mathrm{~N}$ or $\delta^{13} \mathrm{C}$, and $\mathrm{R}$ is the ratio of heavy to light isotope (15N: 14N, 13C: 12C). Duplicates were analyzed every 10 samples, and a blue mussel reference material every 15 samples to ensure measurement quality.

\section{Phospholipid analysis}

$50 \mathrm{mg}$ of liver was homogenized at $4^{\circ} \mathrm{C}$ in $1 \mathrm{ml}$ phosphate-buffered saline using a Beadruptor Elite bead mill homogenizer from Omni International. An aliquot of the homogenate was transferred to a $15 \mathrm{ml}$ polypropylene tube, and 3.75 $\mathrm{ml}$ of chloroform:methanol $(2: 1, \mathrm{v} / \mathrm{v})$ was added. The solution was vortexed, and $0.5 \mathrm{ml}$ deionized water added, followed by another $15 \mathrm{sec}$ of vortexing. The 
extract was then centrifuged at $3000 \mathrm{rpm}$ for $5 \mathrm{~min}$ at room temperature, and the organic layer decanted into a pre-weighed glass vial. The organic extract was allowed to evaporate in a fume hood overnight, and the remaining lipid residue was then weighed to ascertain total lipid content. The residue was then resuspended in $200 \mu 1$ of $1 \%$ Triton X-100 in $100 \%$ ethanol and shaken well for at least two hours. $20 \mu \mathrm{l}$ of the extract was then pipetted into a 96-well plate, inoculated with phospholipid kit working reagent, and assessed colorimetrically using a Spectramax M2 Multi-Mode microplate reader for phospholipid content via comparison to a 4-point curve containing $0-200 \mu \mathrm{M}$ phosphatidylcholine ${ }^{52}$.

\section{More detail about stable isotope values and relationships}

There is limited fine-scale comparability between stable isotope measurements between habitats included in this study, due to the species and food web specific nature of isotope fractionation factors, alongside inevitable and stark differences in isotopic composition at the base of each food web. As a result, here we focus on associations between PFAS concentrations and stable isotope measurements within each habitat, rather than considering isotope ratios across the sample set as a whole. Likewise, we apply previously described trophic level calculations to measurements within each habitat for illustrative purposes, noting that bulk trophic level calculations uncoupled from food-web specific data lack fine-scale insight.

Summary statistics associated with stable isotope analysis (SIA) of each tissue are presented in Table S20; a three-dimensional presentation of all SIA data can be found here [http://rpubs.com/Arobuck/555350]. 
Herring Gulls from Narragansett Bay evidenced the widest range of $\delta^{15} \mathrm{~N}$, while Shearwaters from Massachusetts Bay displayed the least variability in $\delta^{15} \mathrm{~N}$ (Table S21). Derived trophic level estimates reflected the same patterns of variability, with all birds evidencing an estimated trophic level between $3.24-$ 4.59 based on liver $\delta^{15} \mathrm{~N}$ (Table S20, S21). Trophic level estimates assumed a calanoid copepod primary consumer, a realistic assumption for all habitats. $\delta^{15} \mathrm{~N}$ values and calculated trophic level were not significantly associated with concentrations of most individual PFAS or $\sum{ }_{19}$ PFAS in each habitat. PFOS and $\sum_{19}$ PFAS were positively associated with $\delta^{15} \mathrm{~N}$ only in Massachusetts Bay individuals (Table S23). The lack of more significant associations with $\delta^{15} \mathrm{~N}$ may be related to similar trophic strategies and prey items utilized by birds included in this study, resulting in a limited range of $\delta^{15} \mathrm{~N}$ (Tables S21, S23). The lack of association likely also relates to the unique partitioning and accumulation pathways governing PFAS distribution in biota compared to legacy POPs ${ }^{11,53}$.

Muscle $\delta^{34} \mathrm{~S}$ was also compared to concentrations of individual PFAS and $\sum$ PFAS. Muscle $\delta^{34} \mathrm{~S}$ was only associated with PFUnDA in Narra. Bay individuals; no other statistically significant or observationally notable associations were present between $\delta^{34} \mathrm{~S}$ and PFAS concentrations. This association may be driven by variable coastal vs inland foraging strategies in herring gulls from Narra. Bay, coupled to the significance of PFUnDA as a PFAS common in marine food webs.

$\delta^{13} \mathrm{C}$ and PFAS concentrations were most notably associated in birds from the CFRE. The association between $\delta^{13} \mathrm{C}$ and PFAS levels in CFRE chicks is 
likely a function of the marine and estuarine foraging habits of species included in this study, coupled to the local geomorphology of the CFRE system. Seabird parents foraging in the CFRE or in the adjacent coastal plume likely obtained resources comparatively enriched in $\delta^{13} \mathrm{C}$ due to the abundance of Sporobolus alterniflora marshes in the CFRE lower estuary. These marshes make up a substantial portion of the undeveloped land area of the lower estuary and serve as habitat and nursery area for ecologically and commercially important fish and invertebrates ${ }^{54,55}$. Sporobolus alterniflora typically reflects an enriched $\delta^{13} \mathrm{C}$ value of $-13.6 \%{ }^{56}$, compared to more depleted $\delta^{13} \mathrm{C}$ ratios observed in offshore food webs based on particulate organic matter and phytoplankton (-20\%o to $26 \%{ }^{57,58}$ ) (Fig. S6). Sporobolus alterniflora marshes inhabit geomorphically protected inshore lagoon and barrier island environments in the CFRE, and were physically closer to upstream PFAS sources. Therefore, they were subject to less open-water dilution, likely increasing PFAS exposure potential for prey and predators reliant on these marsh systems. Surface water studies support this hypothesis, with predictable inverse relationships between salinity and surface water $\sum$ PFAS apparent in estuarine ${ }^{59}$, coastal shelf, and slope ${ }^{60}$ environments. This suggests an increased potential for PFAS exposure in terrestrially-influenced environments like estuaries or brackish marshes. CFRE seabird parents also likely had an incentive to forage as close as possible to estuarine nesting colonies to conserve energy, further encouraging reliance on marsh and estuary resources. Seabird parents utilizing more offshore, marine systems for chick provisioning were likely foraging in environments with increased dilution and mixing, 
obtaining larger prey with decreased PFAS levels and a more depleted $\delta^{13} \mathrm{C}$ signature.

\section{Bioaccumulation factors}

Liver-water bioaccumulation factors (BAFs) were calculated by dividing liver geometric mean PFAS levels by measured or estimated surface water concentrations adjacent to nesting or collection locations, followed by log transformation. Water values for Massachusetts Bay and Narragansett Bay were derived from unpublished data from the Lohmann lab, while surface water estimates for the CFRE were estimated using data inputs from Zhang et al. 2019, assuming a conservation of mass dilution approach and estimating salinity near the collection site at M18 using salinity data from the Lower Cape Fear River Program (Tables S18-S19) ${ }^{61,62}$.

BAFs reported here range from 0.5 - 3.7, with PFOS, PFNA, PFDA, PFO5DoDA, and Nafion BP2 displaying BAFs near or above 2 variably across the three habitats sampled in this study (Fig. S9). BAFs above 3.3 are considered "bioaccumulative" under regulatory protocol designed for persistent organic pollutants ${ }^{63}$. 


\section{Miscellaneous Supporting Figures}

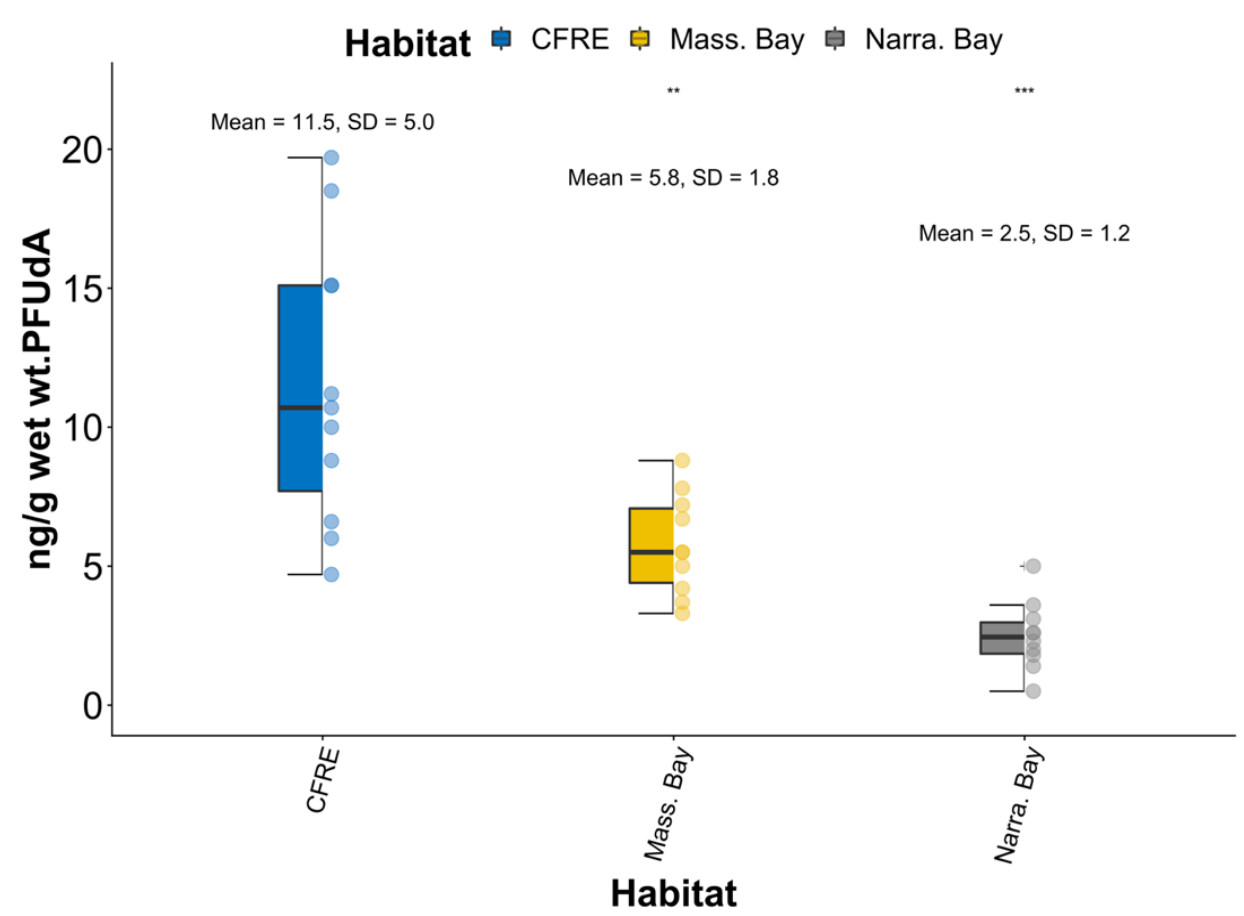

Figure S2.2. Concentrations of PFUnDA by habitat. Habitat mean concentrations of PFUnDA presented as boxplots, with the dark line representing the median, box limits representing the first and third quartiles, whiskers denoting 1.5 times the interquartile range, and crosses denoting outliers. The points reflect measured observations contributing to the summary statistics presented by the boxplot. The asterisks indicate a statistically significant difference between habitat mean PFUnDA compared via the Wilcoxon rank sum test, using the CFRE group as the reference group. PFUnDA concentrations were displayed separately, as concentrations of this compound were significantly different between habitats and this was the second most abundant compound in many individuals. No toxicity reference thresholds exist for this compound in bird to date. 

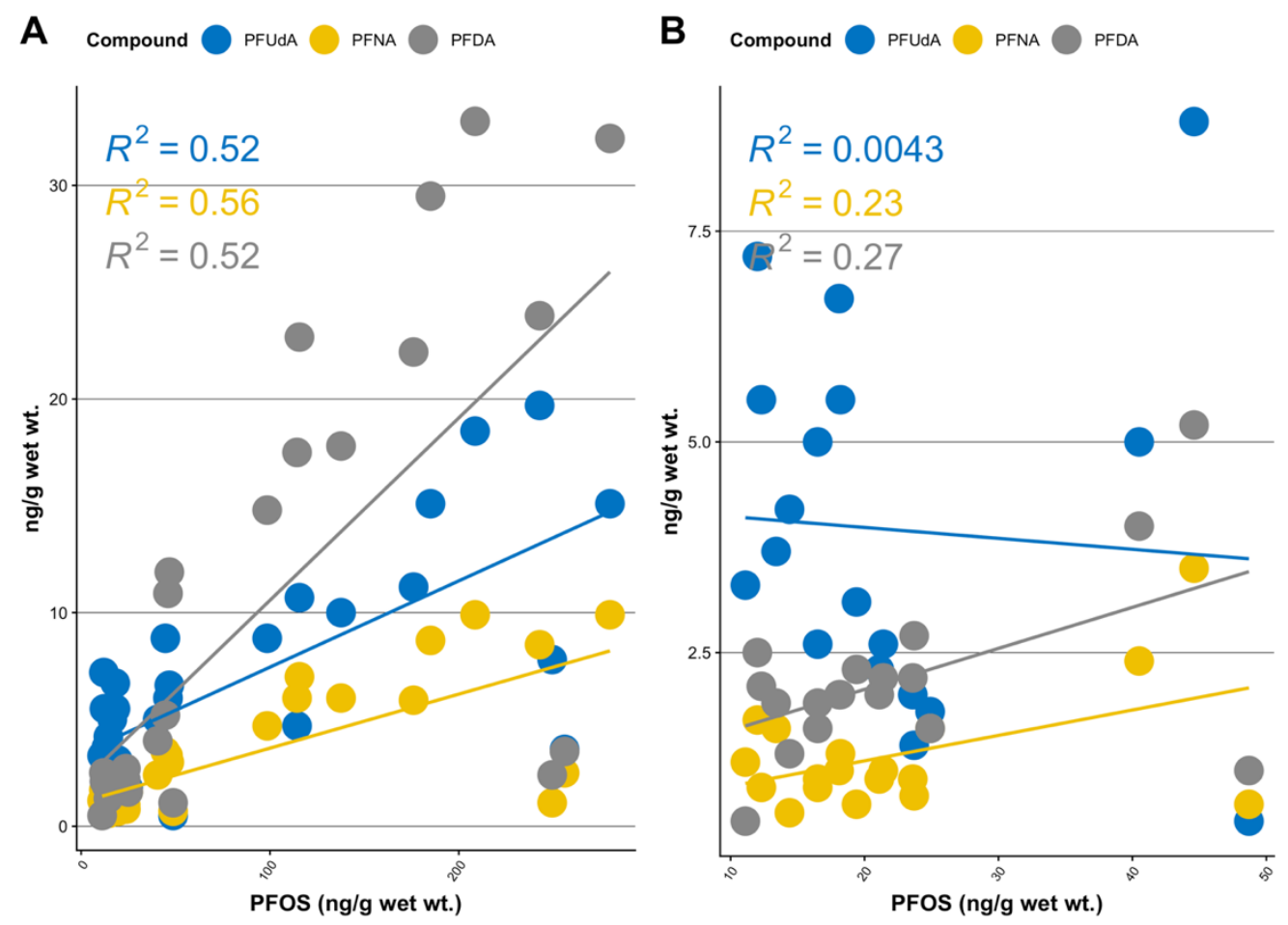

Figure S2.3. Observed concentrations of PFOS versus concentrations of PFNA, PFDA, and PFUnDA in A) all individuals across three habitats, and B) in only individuals from Narragansett Bay and Massachusetts Bay with two outlier individuals removed. All concentrations were measured via UPLC-MS/MS. 

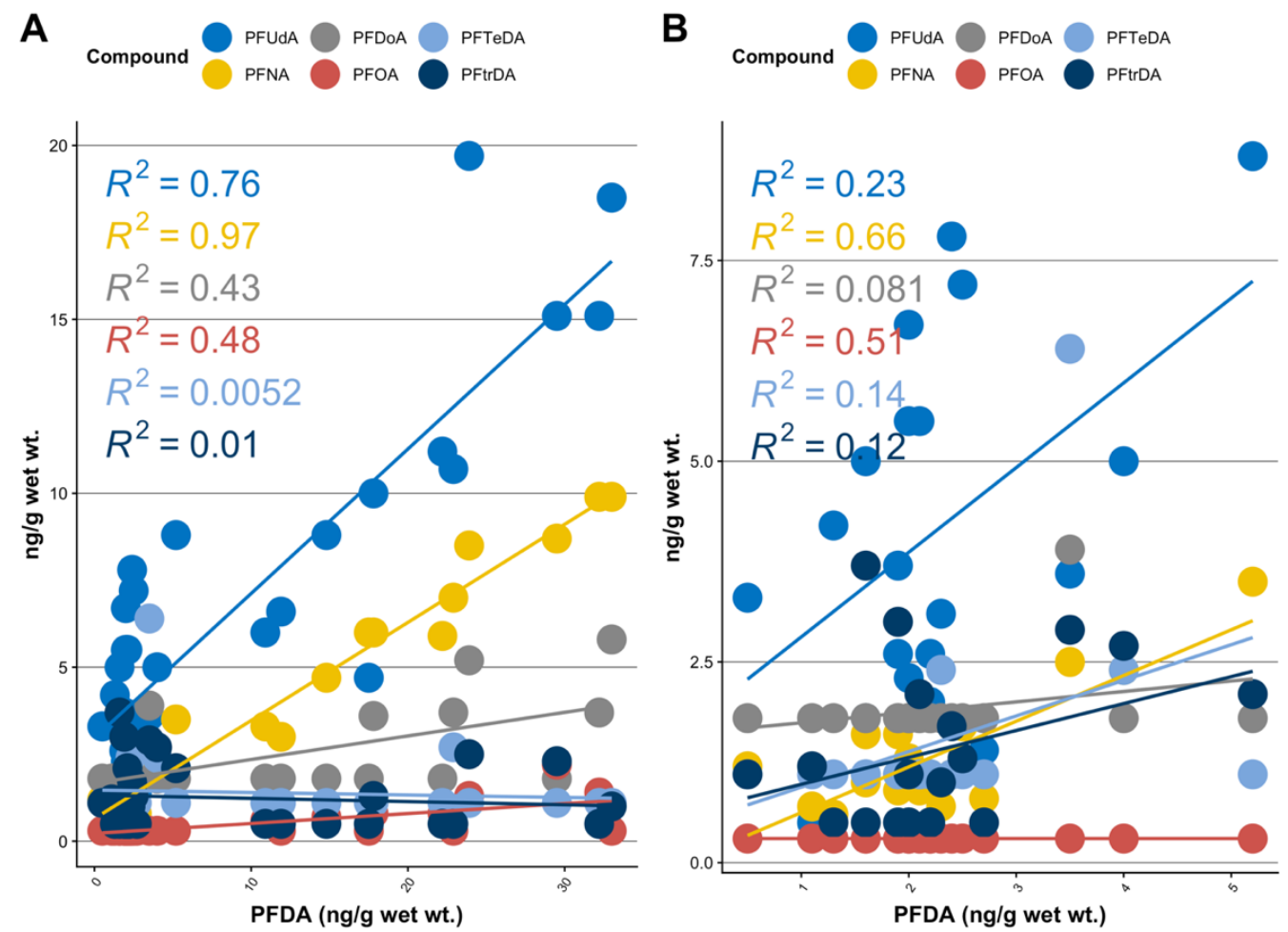

Figure S2.4. Concentrations of $\mathrm{C} 8$ - C14 PFCAs as a function of PFDA measured via LC-MS/MS in A) all individuals across three habitats, and B) in only individuals from Narragansett Bay and Massachusetts Bay. Associations between PFCAs as approximated by linear regression slightly decrease without CFRE data included, likely due to the truncation of the data range as a result of more similar concentrations observed in habitats removed from point sources and inclusion of MDL/2 values. 

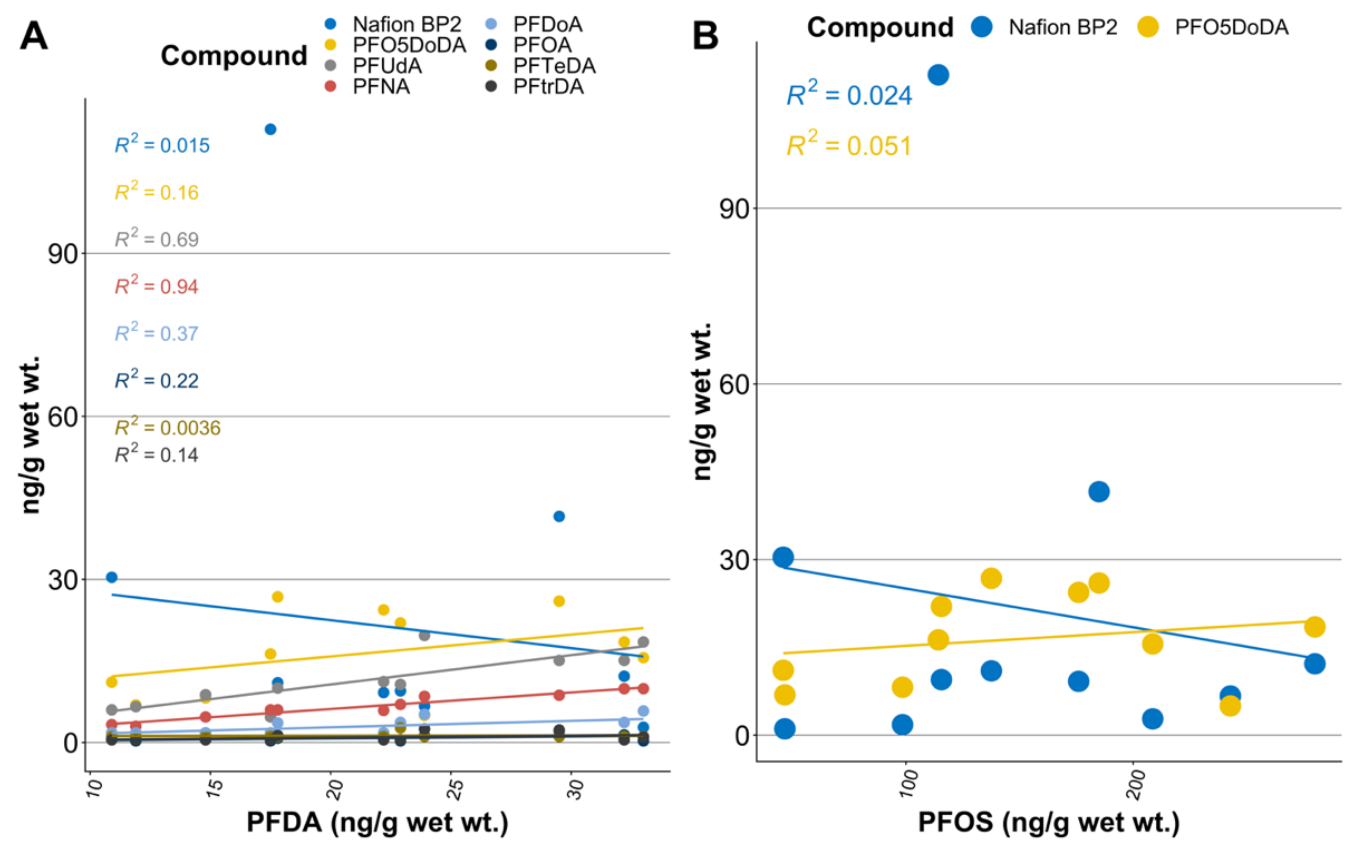

Figure S2.5. Observed concentrations of emerging PFEAs versus legacy PFAS in CFRE chicks with A) displaying concentrations of PFDA vs PFEAs and other long-chain PFCAs, and B) displaying concentrations of PFOS vs PFEAs. PFCA and PFSA concentrations were measured via UPLC-MS/MS while PFEAs were measured via HRMS. 

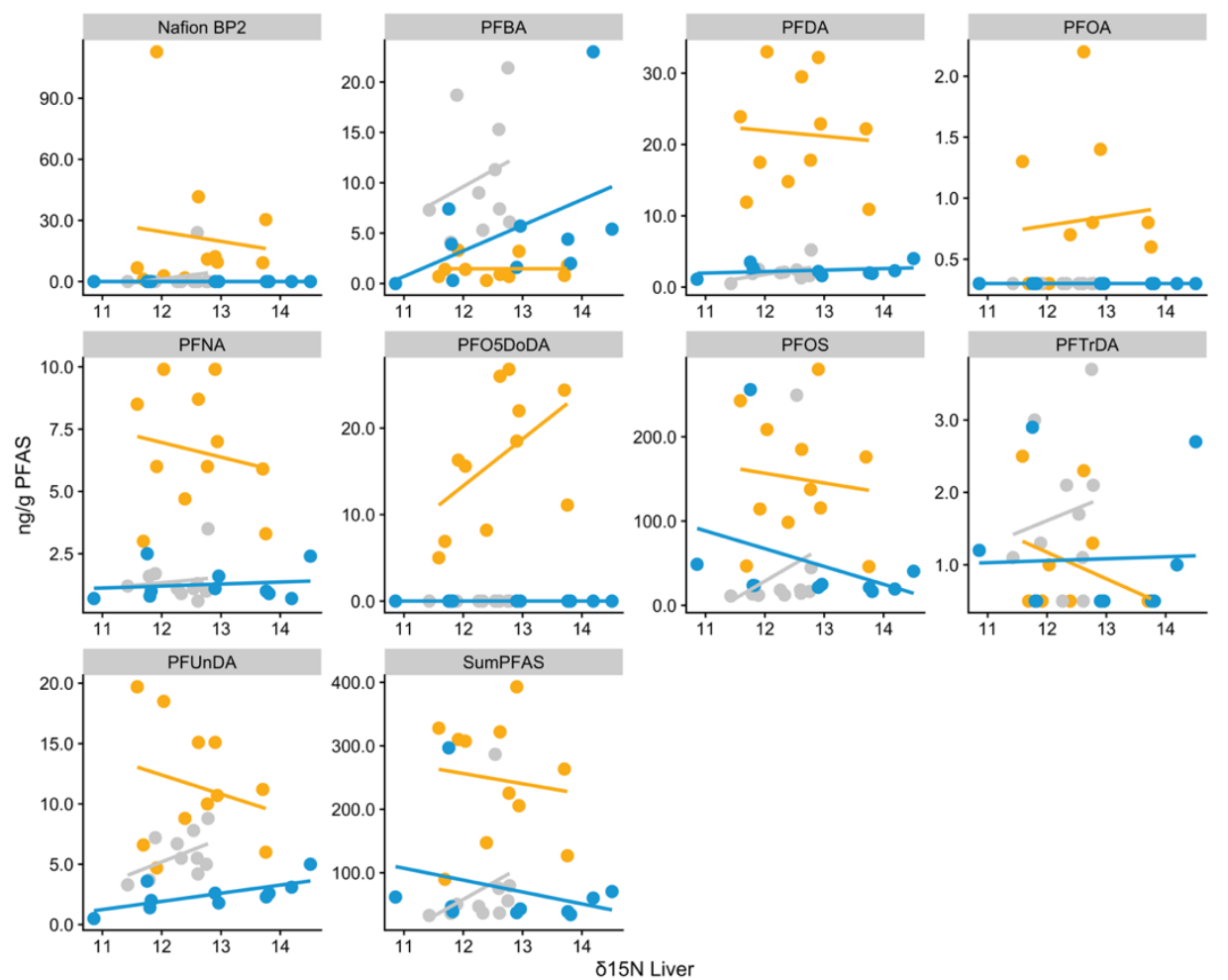

Figure S2.6. $\delta 15 \mathrm{~N}$ vs concentrations of PFAS. Measured $\delta^{15} \mathrm{~N}$ in seabird liver and muscle did not relate to concentrations of individual PFAS, or ${ }_{19} \sum \mathrm{PFAS}$ in seabird liver across any habitat. No correlation was found when the total sample set $(\mathrm{n}=31)$ was assessed in the same way. Muscle $\delta^{15} \mathrm{~N}$ ratios are presented here; facet plots include only those compounds found in $>40 \%$ of the sample set. 

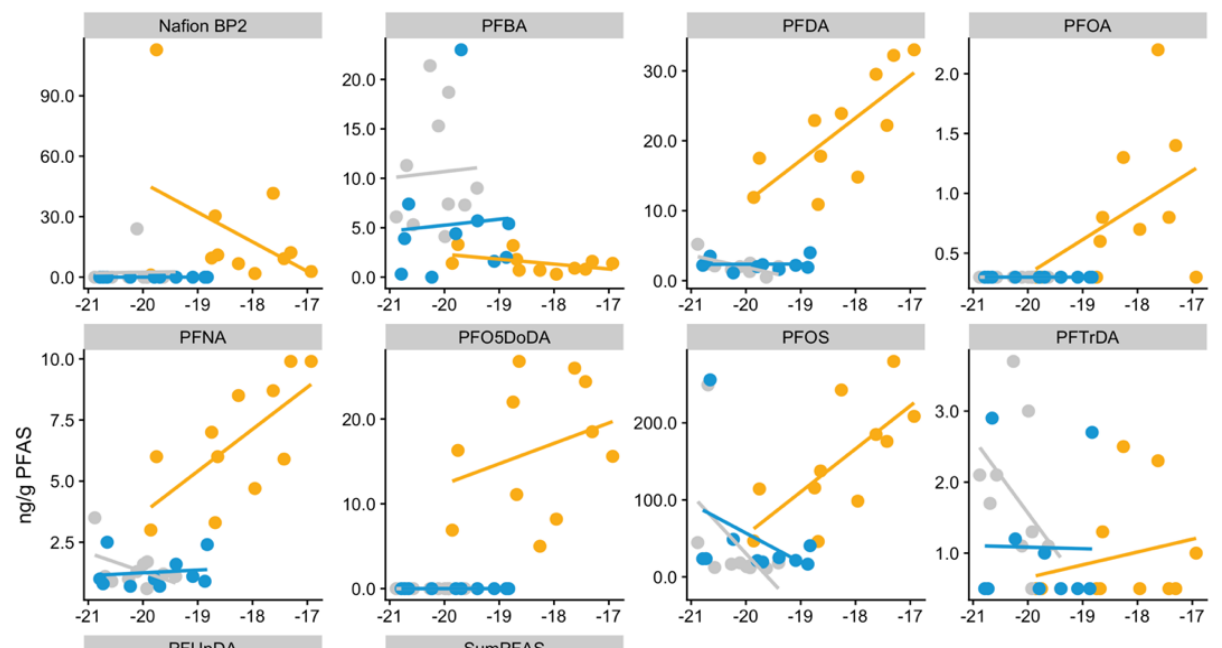

Habitat

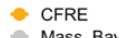

- Mass. Bay
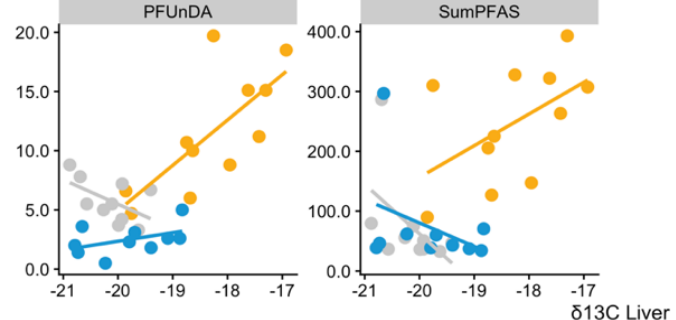

Figure S2.7. $\delta 13 \mathrm{C}$ vs concentrations of PFAS. Measured $\delta^{13} \mathrm{C}$ in seabird liver and muscle did not relate to concentrations of individual PFAS, or $19 \sum$ PFAS in seabird liver from Massachusetts Bay or Narragansett Bay. No correlation was found when the total sample set $(n=31)$ was assessed in the same way. PFDA, PFNA, PFOS, PFUnDA, and 19 2PFAS were moderately associated $(\mathrm{p}<0.05)$ with $\delta^{13} \mathrm{C}$ ratios, only in CFRE chicks. See SI text for further discussion of this limited phenomenon. Muscle $\delta^{13} \mathrm{C}$ ratios are presented here; facet plots include only those compounds found in $>40 \%$ of the sample set. 


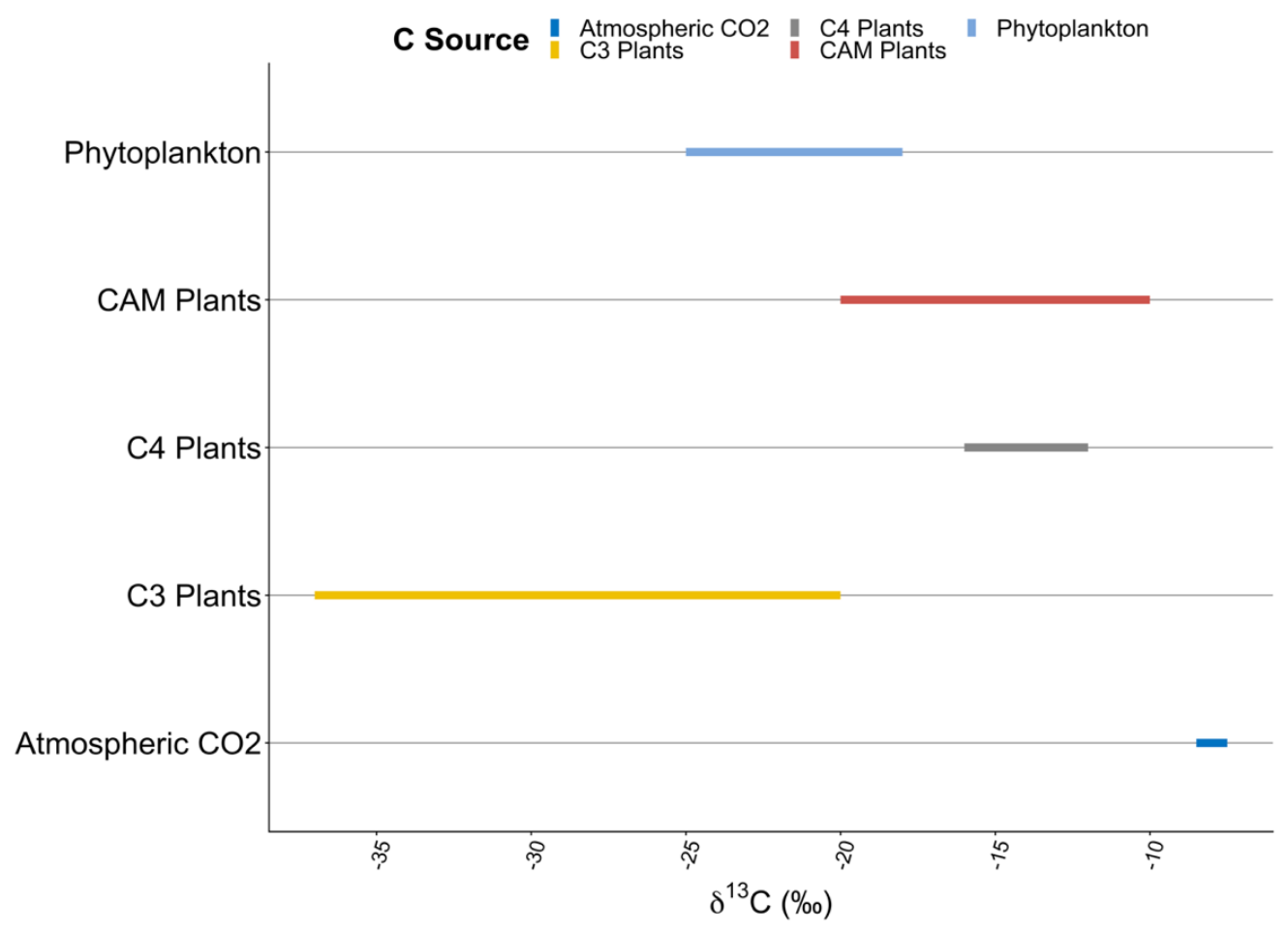

Figure S2.8. $\delta^{13} \mathrm{C}$ in key organic and inorganic matrices. 


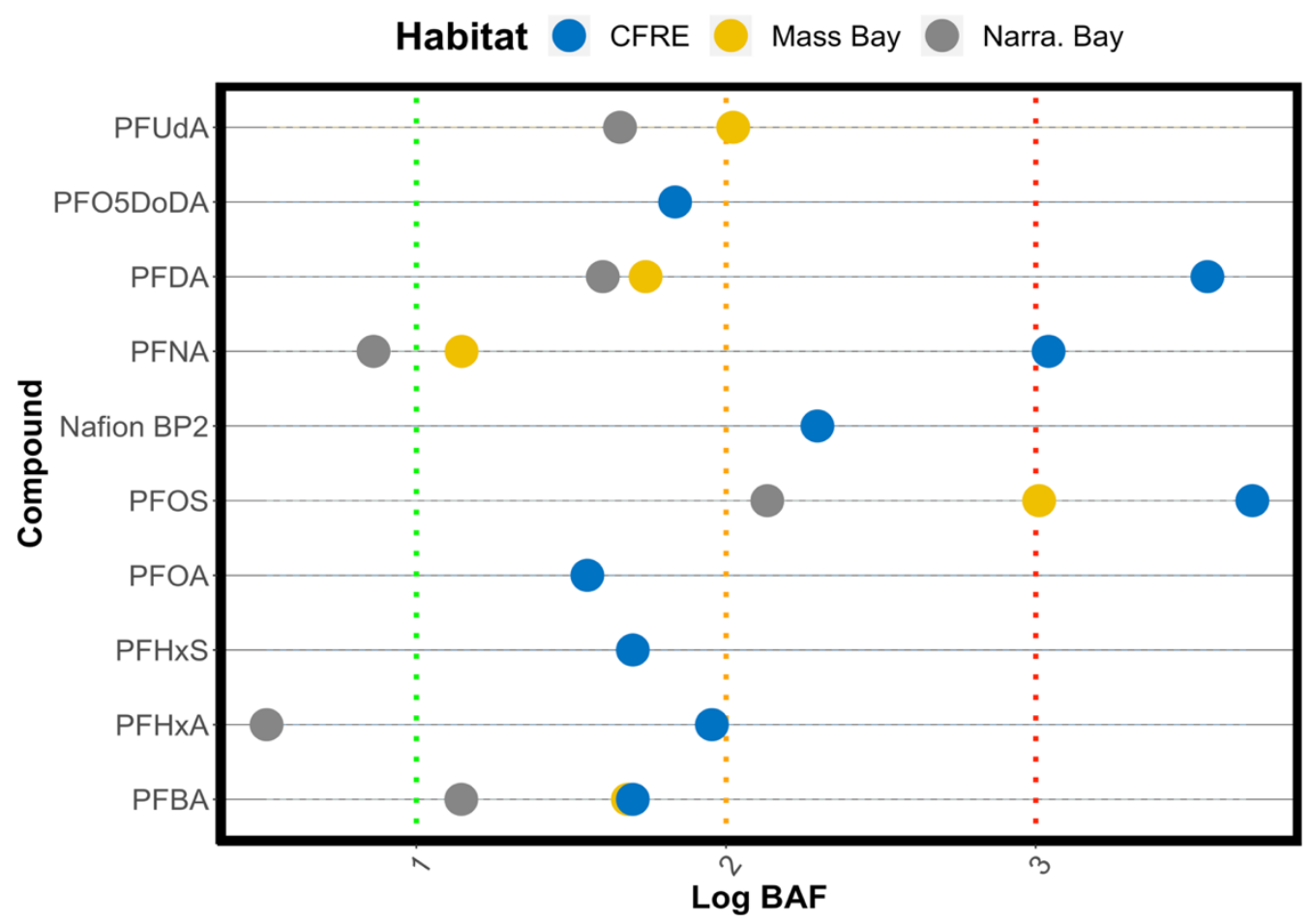

Figure S2.9. Log BAFs for 10 compounds across three habitats. BAFs were calculated by dividing mean liver concentrations observed in each habitat by observed or estimated surface water concentrations. 


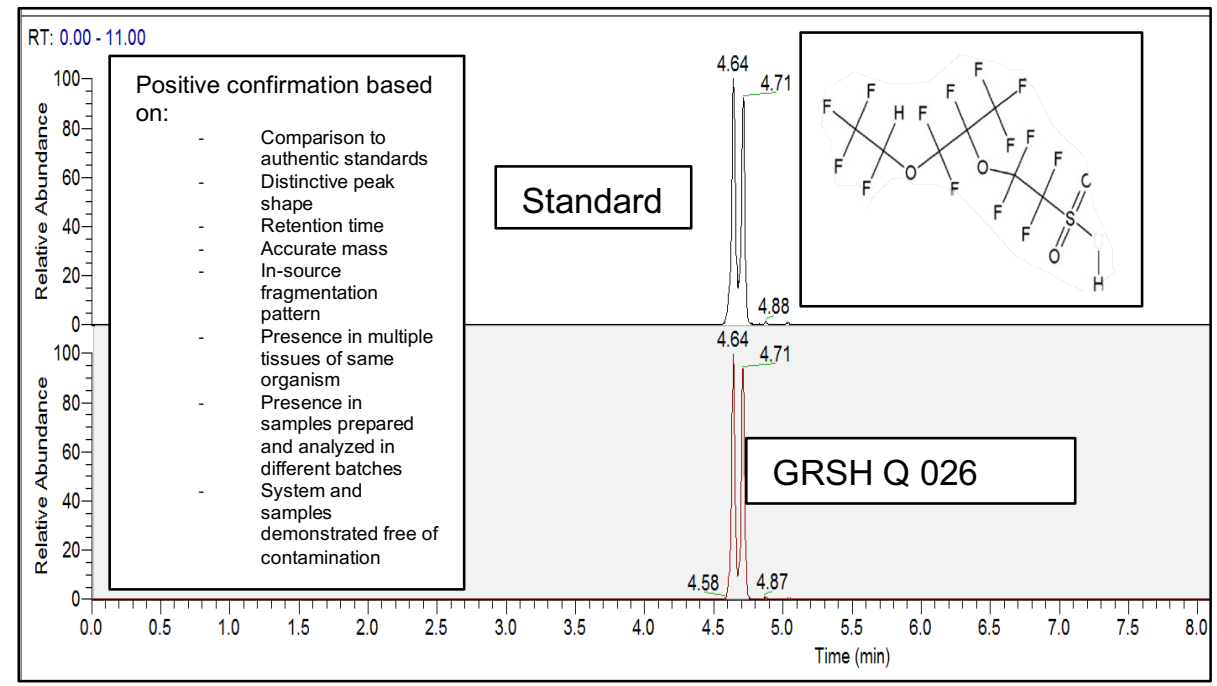

Figure S2.10. Chromatograms of Nafion BP2 in native standard and Great Shearwater liver. Extracted ion chromatogram (EIC) of Nafion BP2, [M-H] ion with $\mathrm{m} / \mathrm{z}=462.9326 \pm 5 \mathrm{ppm}$. The figure shows the authentic standard (top) compared to the EIC observed in a Great Shearwater liver from Massachusetts Bay (bottom). Nafion BP2 was identified in two Great Shearwater juveniles and one Herring Gull chick outside of the CFRE system. Positive identification of Nafion BP2 in these non-CFRE samples was confirmed by comparison to authentic standards, distinctive peak shape, accurate mass, in-source fragmentation patterns, $\mathrm{MS}^{\mathrm{n}}$ data when available, retention time, and sample preparation and analysis information. 


\section{Supporting Tables}

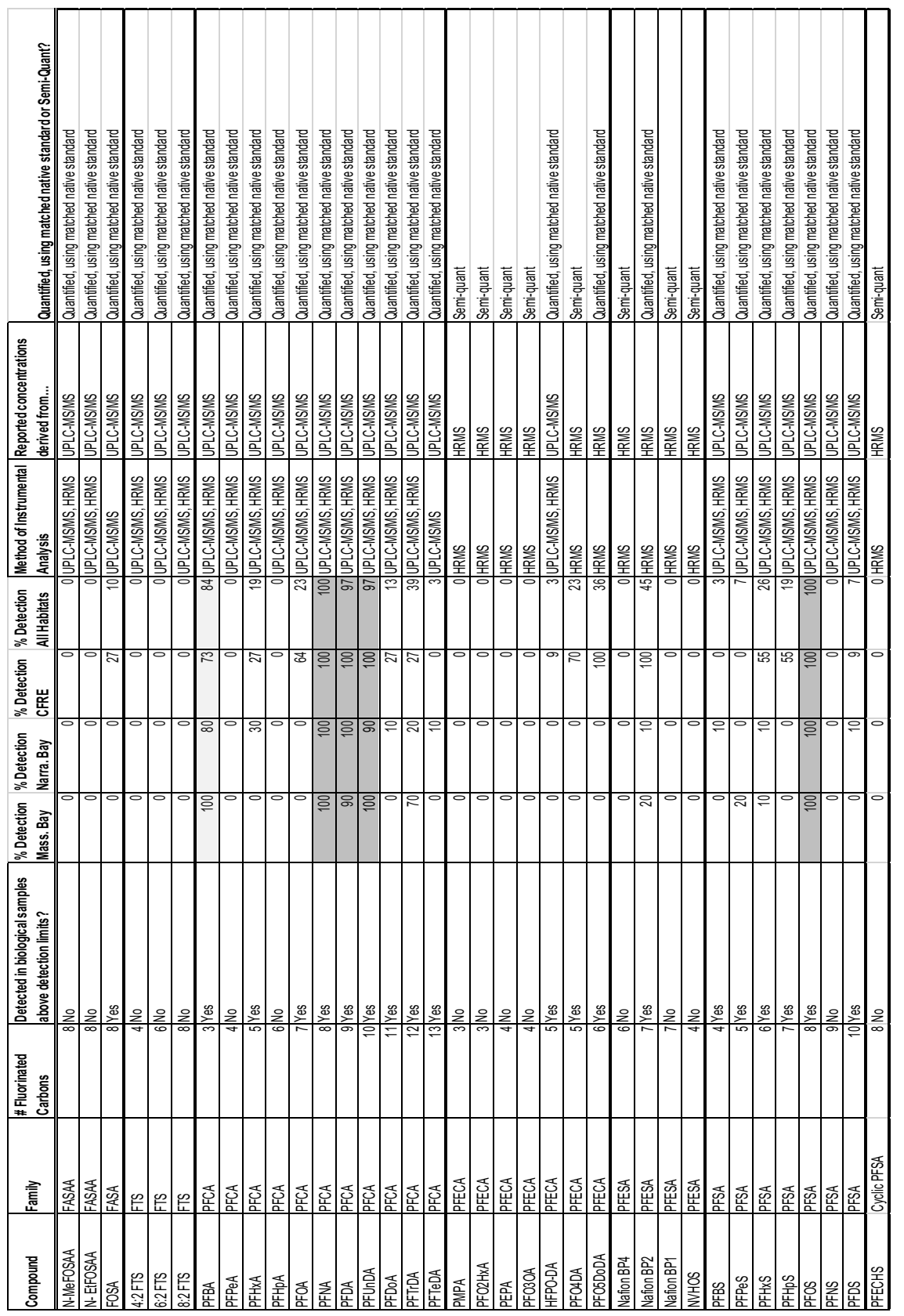

Table S2.1. Table presenting all compounds assessed in this study, method of instrumental analysis applied, method used to report concentrations, quantification approach used based on availability of native standard, and summary of detections in liver extracts. Family designation based on Buck et al. 2011 and Strynar et al. 2015. 


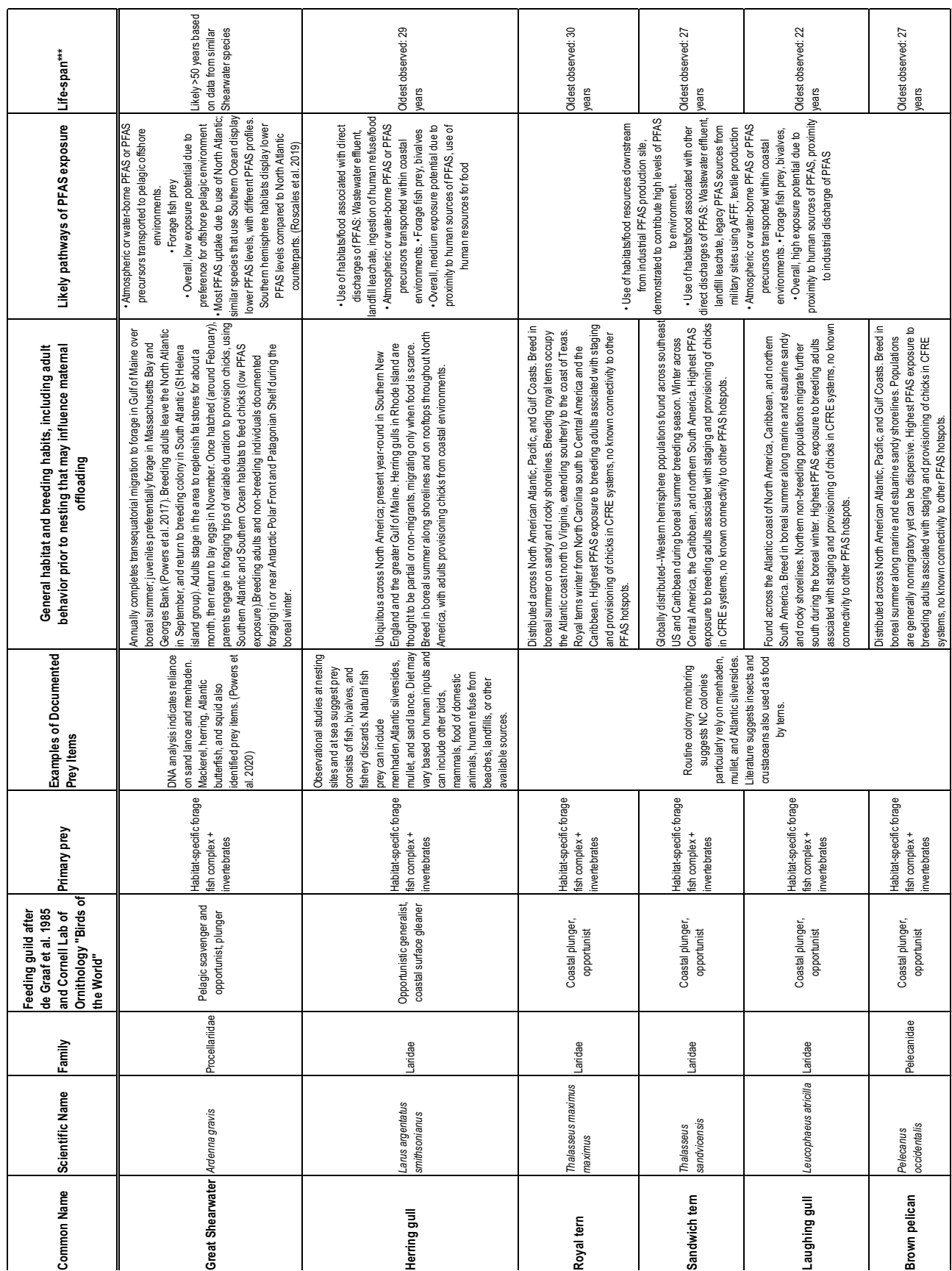

Table S2.2. Table containing relevant life history details of seabird species included in this study. Diet and life history sources include species profiles on the Cornell Lab of Ornithology All About Birds data portal -https://www.allaboutbirds.org/guide/ -- and all references therein -- were referenced for diet information, in addition to personal communications from colony monitoring technicians. Age examples are from https//www.pwrc.usgs.gov/BBL/longevity/longvrec.cfm. 


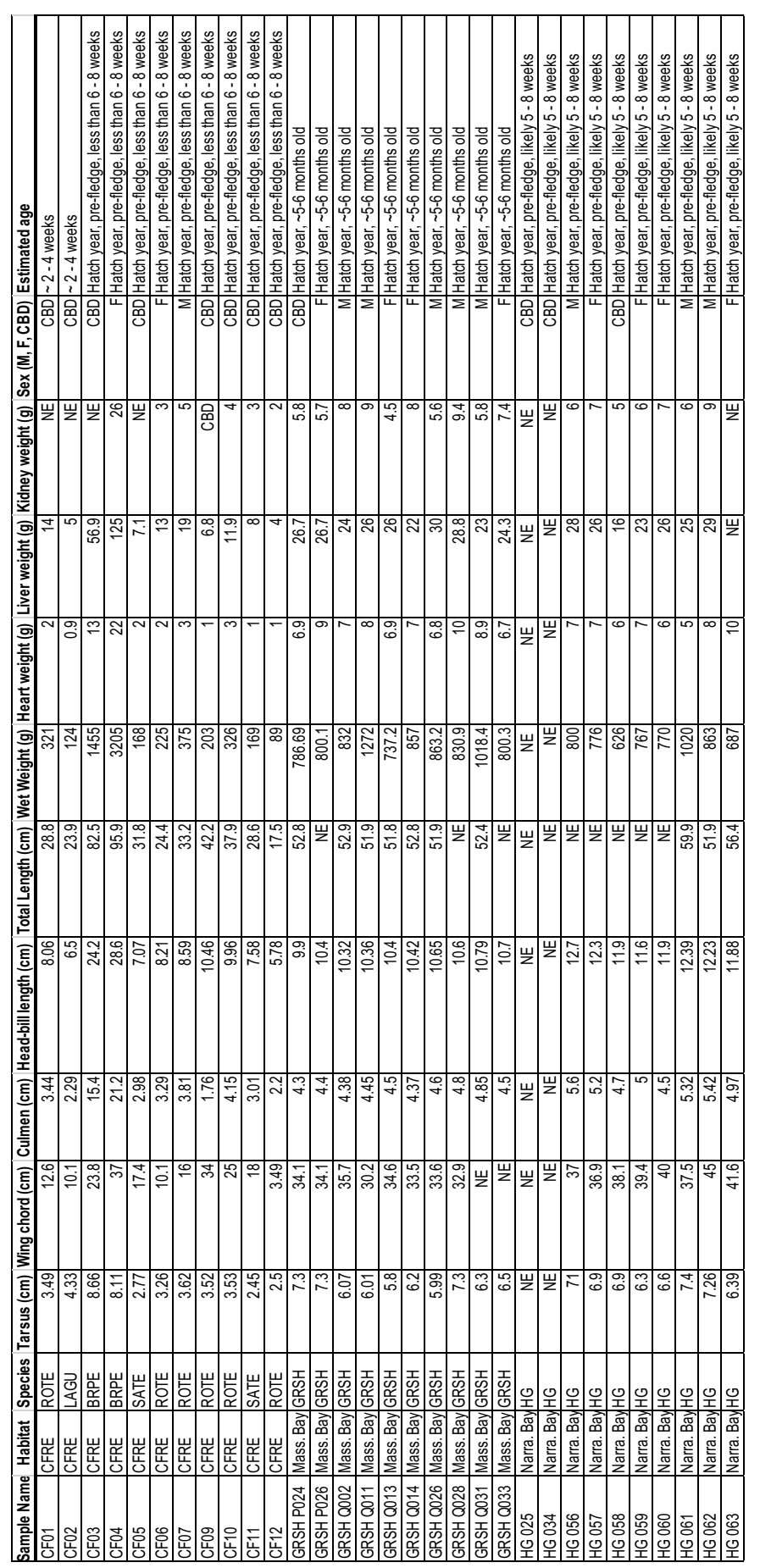

Table S2.3. Table containing relevant morphometric and collection details describing each individual included in this study. "NE" designates "Not Examined", "CBD" designates "Couldn't Be Determined" 


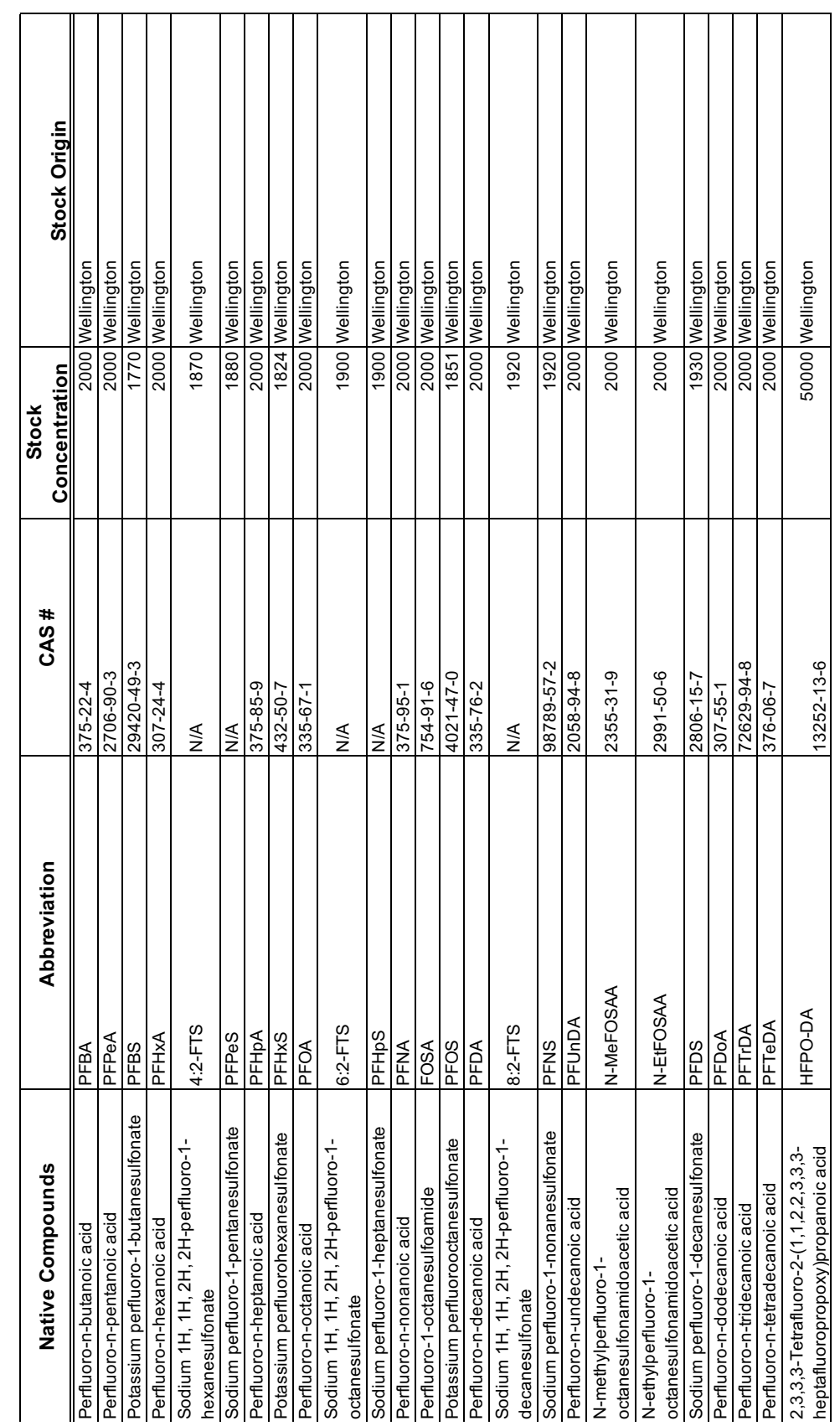

Table S2.4. Table presenting details about all native analytes measured in this study via LC-MS/MS. 


\begin{tabular}{|c|c|c|c|c|c|}
\hline Native Compound & Abbreviation & $\begin{array}{l}\text { Chemical } \\
\text { Formula }\end{array}$ & CAS \# & $\begin{array}{l}\text { Stock } \\
\text { Concentration } \\
(\mathrm{ng} / \mathrm{mL})\end{array}$ & Assigned surrogate \\
\hline $\begin{array}{l}\text { Perfluoro-2-ethoxypropanoic } \\
\text { acid }\end{array}$ & PEPA & C5HF9O3 & $267239-61-2$ & na & MPFBA-13C4 \\
\hline $\begin{array}{c}\text { Perfluoro-2- } \\
\text { methoxypropanoic acid }\end{array}$ & PFMA & $\mathrm{C} 4 \mathrm{HF} 7 \mathrm{O} 3$ & $13140-29-9$ & na & MPFBA-13C4 \\
\hline $\begin{array}{c}\text { Perfluoro-3,5-dioxahexanoic } \\
\text { acid }\end{array}$ & $\mathrm{PFO} 2 \mathrm{H} \times \mathrm{A}$ & $\mathrm{C} 4 \mathrm{HF} 7 \mathrm{O} 4$ & $39492-88-1$ & na & MPFBA-13C4 \\
\hline $\begin{array}{c}\text { Perfluoro(3,5,7- } \\
\text { trioxaoctanoic) acid }\end{array}$ & PFO3OA & C5HF9O5 & $39492-89-2$ & na & M8PFOA-13C8 \\
\hline $\begin{array}{c}\text { Perfluoro-3,5,7,9- } \\
\text { tetraoxadecanoic acid }\end{array}$ & PFO4DA & C6HF1106 & $39492-90-5$ & 100000 & M8PFOA-13C8 \\
\hline $\begin{array}{c}\text { Perfluoro(3,5,7,9,11- } \\
\text { pentaoxadodecanoic) acid }\end{array}$ & PFO5DoDA & $\mathrm{C} 7 \mathrm{HF} 13 \mathrm{O} 7$ & $39492-91-6$ & 100000 & M8PFOA-13C8 \\
\hline $\begin{array}{c}\text { 2-[1-[difluoro[(1,2,2- } \\
\text { trifluoroethenyl)oxy]methyl]- } \\
\text { 1,2,2,2- tetrafluoroethoxy]- } \\
\text { 1,1,2,2-tetrafluoro- } \\
\text { Ethanesulfonic acid, }\end{array}$ & Nafion BP1 & C7HF13O5S & 29311-67-9 & na & M8PFOS-13C8 \\
\hline $\begin{array}{c}\text { 2-[1-[difluoro(1,2,2,2- } \\
\text { tetrafluoroethoxy)methyl]- } \\
\text { 1,2,2,2-tetrafluoroethoxy]- } \\
\text { 1,1,2,2-tetrafluoro- } \\
\text { ethanesulfonic acid }\end{array}$ & Nafion BP2 & $\mathrm{C} 7 \mathrm{H} 2 \mathrm{~F} 14 \mathrm{O} 5 \mathrm{~S}$ & 749836-20-2 & 100000 & M8PFOS-13C8 \\
\hline $\begin{array}{c}2,2,3,3,4,5,5,5-4-(1,1,2,2- \\
\text { tetrafluoro-2- } \\
\text { sulfoethoxy)pentanoic acid }\end{array}$ & Nafion BP4 & $\mathrm{C} 7 \mathrm{H} 2 \mathrm{~F} 12 \mathrm{O} 6 \mathrm{~S}$ & na & na & M8PFOS-13C8 \\
\hline $\begin{array}{c}\text { 1,1,2,2-tetrafluoro-2-(1,2,2,2- } \\
\text { tetrafluoro-ethoxy)ethane } \\
\text { sulfonate }\end{array}$ & NVHOS & $\mathrm{C} 4 \mathrm{H} 2 \mathrm{~F} 8 \mathrm{O} 4 \mathrm{~S}$ & $\begin{array}{c}\text { 801209-99-4 } \\
\text { (free acid) }\end{array}$ & na & M8PFOS-13C8 \\
\hline $\begin{array}{c}\text { 1,2,2,3,3,4,5,5,6,6-decafluoro- } \\
\text { 4-(1,1,2,2,2-pentafluoroethyl)- } \\
\text { cyclohexanesulfonicacid, }\end{array}$ & PFECHS & C8HF15O3S & $646-83-3$ & na & na \\
\hline
\end{tabular}

Table S2.5. Table presenting details about all native analytes measured in this study via LC-HRMS. 


\begin{tabular}{|l|r|}
\hline \multicolumn{2}{|c|}{ Method \% Recovery } \\
\hline \hline \multicolumn{1}{|c|}{ Compound } & \% Recovery \\
\hline 4:2 FTS_IS & 55.5 \\
\hline 6:2 FTS_IS & 64.8 \\
\hline 8:2-FTS_IS & 46.9 \\
\hline EtFOSAA-IS & 52.1 \\
\hline FOSA_IS & 13.8 \\
\hline HFPODA_IS & 78.7 \\
\hline MeFOSAA_IS & 48.5 \\
\hline PFBA_IS & 112.4 \\
\hline PFBS_IS & 72.4 \\
\hline PFDA_IS & 56.4 \\
\hline PFDoA_IS & 31.4 \\
\hline PFHpA_IS & 73.7 \\
\hline PFHxA_IS & 86.0 \\
\hline PFHxS_IS & 69.1 \\
\hline PFNA_IS & 81.1 \\
\hline PFOA_IS & 68.0 \\
\hline PFOS_IS & 59.3 \\
\hline PFPeA_IS & 72.6 \\
\hline PFTeDA_IS & 34.5 \\
\hline PFUnDA_IS & 46.1 \\
\hline Arithmetic Mean \% Recovery & 61.2 \\
\hline Arithmetic Mean \% Recovery & \\
\hline FOSA (outlier) & 63.7 \\
\hline Arithmetic Mean \% Recovery \\
of PFCAs
\end{tabular}

Table S2.6. LC-MS/MS Method recovery as a percentage, following: \% Recovery $=(($ Pre-extraction spiked sample $) /($ Post-extraction spiked sample $)) \times$ 100 


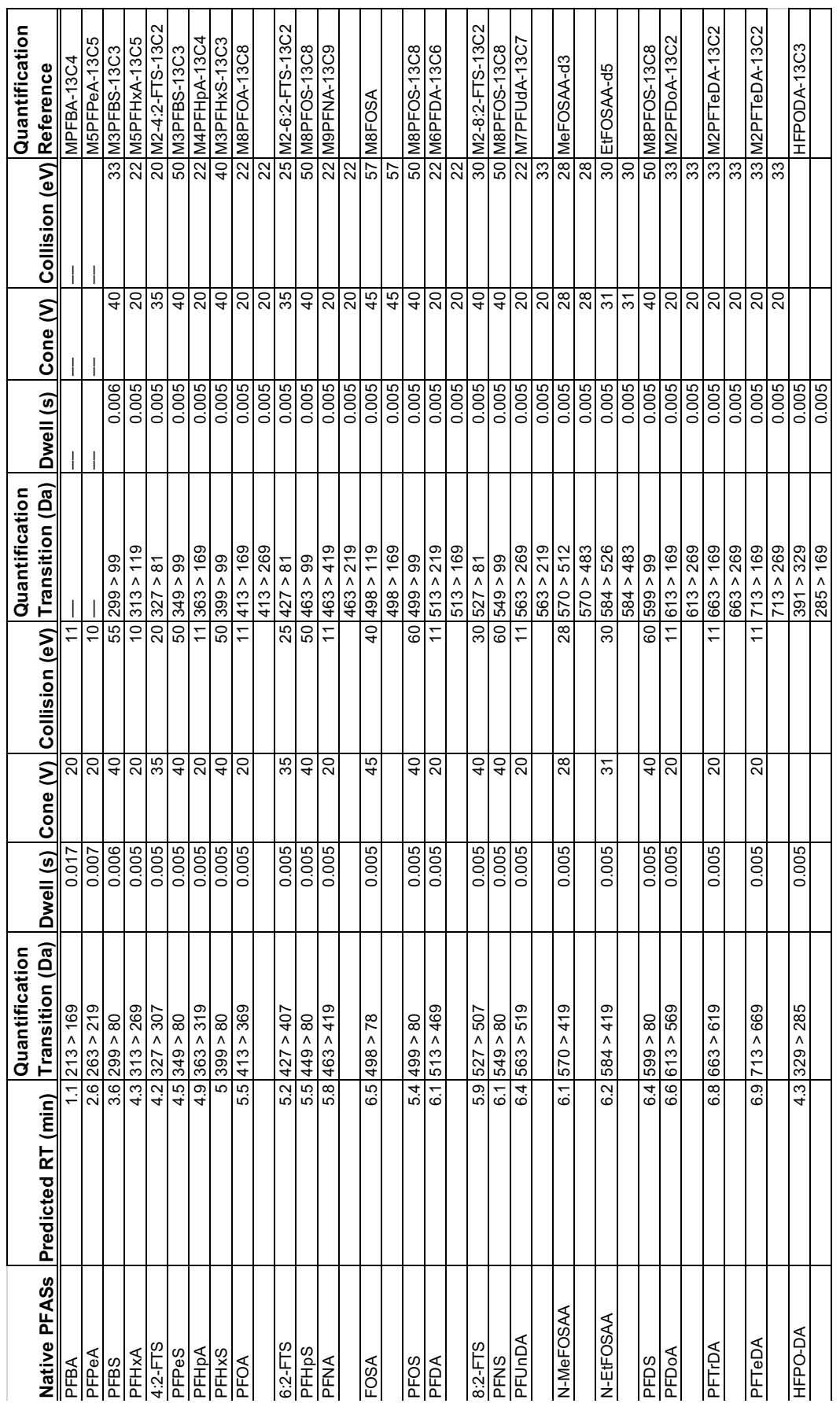

Table S2.7. Table of targeted native analytes, associated instrument parameters, and quantification parameters targeted with LC-MS/MS analysis. 


\begin{tabular}{|c|c|c|}
\hline $\begin{array}{l}\text { Labeled } \\
\text { Compounds }\end{array}$ & Abbreviation & \begin{tabular}{|l|} 
Stock \\
Concentration \\
(ng/mL)
\end{tabular} \\
\hline $\begin{array}{l}\text { Perfluoro-n- }\left[{ }^{13} \mathrm{C} 4\right. \\
\text { ]butanoic acid }\end{array}$ & MPFBA- ${ }^{13} \mathrm{C} 4$ & 1000 \\
\hline $\begin{array}{l}\text { Perfluoro-n- } \\
{\left[{ }^{13} \mathrm{C} 5\right] \text { pentanoic }} \\
\text { acid }\end{array}$ & M5PFPeA- ${ }^{13} \mathrm{C} 5$ & 1000 \\
\hline $\begin{array}{l}\text { Sodium perfluoro- } \\
1-[2,3,4- \\
\left.{ }^{13} \mathrm{C} 3\right] \text { butanesulfon } \\
\text { ate }\end{array}$ & M3PFBS $-{ }^{13} \mathrm{C} 3$ & 929 \\
\hline $\begin{array}{l}\text { Perfluoro-n- } \\
{[1,2,3,4,6-} \\
{ }^{13} \text { C5]hexanoic } \\
\text { acid }\end{array}$ & M5PFHxA- ${ }^{13} \mathrm{C} 5$ & 1000 \\
\hline $\begin{array}{l}\text { Sodium } \\
1 \mathrm{H}, 1 \mathrm{H}, 2 \mathrm{H}, 2 \mathrm{H}- \\
\text { perfluoro-1-[1,2- } \\
{ }^{13} \mathrm{C} 2 \text { ]hexanesulfo } \\
\text { nate }\end{array}$ & M2-4:2-FTS- ${ }^{13} \mathrm{C} 2$ & 935 \\
\hline $\begin{array}{l}\text { Perfluoro-n- } \\
{[1,2,3,4-} \\
\left.{ }^{13} \mathrm{C} 4\right] \text { heptanoic } \\
\text { acid }\end{array}$ & M4PFHpA- $-{ }^{13} \mathrm{C} 4$ & 1000 \\
\hline $\begin{array}{l}\text { Sodium perfluoro- } \\
1-[1,2,3- \\
\left.{ }^{13} \mathrm{C} 3\right] \text { hexanesulfo } \\
\text { nate }\end{array}$ & M3PFHxS $-{ }^{13} \mathrm{C} 3$ & 946 \\
\hline $\begin{array}{l}\text { Perfluoro-n- } \\
\text { [13c8]octanoic } \\
\text { acid }\end{array}$ & M8PFOA-13C8 & 1000 \\
\hline $\begin{array}{l}\text { Sodium } \\
1 \mathrm{H}, 1 \mathrm{H}, 2 \mathrm{H}, 2 \mathrm{H}- \\
\text { perfluoro-1-[1,2- } \\
13 \mathrm{C} 2 \text { ]octanesulfo } \\
\text { nate } \\
\end{array}$ & M2-6:2-FTS-13C2 & 949 \\
\hline \begin{tabular}{l|} 
Perfluoro-n- \\
[13C9]nonanoic \\
acid
\end{tabular} & M9PFNA-13C9 & 1000 \\
\hline $\begin{array}{l}\text { Perfluoro-1- } \\
\text { [13C8]octanesulfo } \\
\text { namide }\end{array}$ & M8FOSA & 1000 \\
\hline $\begin{array}{l}\text { Sodium perfluoro- } \\
1- \\
{[13 \mathrm{C} 8 \text { ]octanesulfo }} \\
\text { nate }\end{array}$ & M8PFOS-13C8 & 957 \\
\hline $\begin{array}{l}\text { Perfluoro-n- } \\
{[1,2,3,4,5,6-} \\
13 C 6] \text { decanoic } \\
\text { acid } \\
\end{array}$ & M6PFDA-13C 6 & 1000 \\
\hline $\begin{array}{l}\text { Sodium } \\
1 \mathrm{H}, 1 \mathrm{H}, 2 \mathrm{H}, 2 \mathrm{H}- \\
\text { perfluoro-1-[1,2- } \\
13 \mathrm{C} 2 \text { decanesulf } \\
\text { onate }\end{array}$ & M2-8:2-FTS-13C2 & 958 \\
\hline $\begin{array}{l}\text { Perfluoro-n- } \\
{[1,2,3,4,5,6,7-} \\
13 C 7] \text { undecanoic } \\
\text { acid }\end{array}$ & M7PFUnDA-13C7 & 1000 \\
\hline $\begin{array}{l}\text { N-methyl-d3- } \\
\text { perfluoro-1- } \\
\text { octanesulfonamid } \\
\text { oacetic acid }\end{array}$ & MeFOSAA-d3 & 1000 \\
\hline $\begin{array}{l}\text { N-ethyl-d5- } \\
\text { perfluoro-1- } \\
\text { octanesulfonamid } \\
\text { oacetic acid }\end{array}$ & EtFOSAA-d5 & 1000 \\
\hline $\begin{array}{l}\text { Perfluoro-n-[1,2- } \\
\text { 13C2]dodecanoic } \\
\text { acid }\end{array}$ & M2PFDoA-13C2 & 1000 \\
\hline $\begin{array}{l}\text { Perfluoro-n-[1,2- } \\
13 C 2] \text { tetradecano } \\
\text { ic acid }\end{array}$ & M2PFTeDA-13C2 & 1000 \\
\hline $\begin{array}{l}2,3,3,3- \\
\text { Tetrafluoro-2- } \\
(1,1,2,2,3,3,3- \\
\text { heptafluoropropo } \\
\text { xy)propanoic } \\
\text { acid }\end{array}$ & HFPODA-13C3 & 50000 \\
\hline
\end{tabular}

Table S2.8. Table of target mass-labeled compounds, associated abbreviations, and concentrations of purchased stock solutions used in both targeted and HRMS analysis. 


\begin{tabular}{|c|c|c|c|c|c|c|c|c|c|}
\hline $\begin{array}{l}\text { Internal } \\
\text { Standards }\end{array}$ & $\begin{array}{l}\text { Predicted } \\
\text { RT (min) }\end{array}$ & $\begin{array}{l}\text { Quantification } \\
\text { Transition } \\
\text { (Da) }\end{array}$ & Dwell (s) & Cone (V) & $\begin{array}{l}\text { Collision } \\
(\mathrm{eV})\end{array}$ & $\begin{array}{l}\text { Quantification } \\
\text { Transition (Da) }\end{array}$ & Dwell (s) & Cone (V) & $\begin{array}{l}\text { Collision } \\
(\mathrm{eV})\end{array}$ \\
\hline MPFBA-13C4 & 1.2 & $217>172$ & \begin{tabular}{|l|}
0.017 \\
\end{tabular} & 20 & 11 & $1--$ & - & 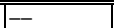 & (-- \\
\hline \begin{tabular}{|l|} 
M5PFPeA-13C5 \\
\end{tabular} & 2.6 & $268>223$ & 0.007 & 20 & 11 & - & - & -- & -- \\
\hline M3PFBS-13C3 & 3.6 & $302>80$ & 0.006 & 40 & 55 & $302>99$ & 0.006 & 40 & 33 \\
\hline M5PFHxA-13C5 & 4.3 & $318>273$ & 0.005 & 20 & 10 & $318>119$ & 0.005 & 20 & 22 \\
\hline M2-4:2-FTS-13C2 & 4.2 & $329>309$ & 0.005 & 35 & 20 & $329>81$ & 0.005 & 35 & 20 \\
\hline M4PFHpA-13C4 & 4.9 & $367>322$ & 0.005 & 20 & 11 & $367>171$ & 0.005 & 20 & 22 \\
\hline M3PFHxS-13C3 & 5 & $402>80$ & 0.005 & 40 & 50 & $402>99$ & 0.005 & 40 & 40 \\
\hline \multirow[t]{2}{*}{\begin{tabular}{|l|} 
M8PFOA-13C8 \\
\end{tabular}} & 5.5 & $421>376$ & 0.005 & 20 & 11 & $421>171$ & 0.005 & 20 & 22 \\
\hline & & & & & & $421>223$ & 0.005 & 20 & 22 \\
\hline M2-6:2-FTS-13C2 & 5.2 & $429>409$ & 0.005 & 35 & 25 & $429>81$ & 0.005 & 35 & 25 \\
\hline \multirow[t]{2}{*}{ M9PFNA-13C9 } & 5.8 & $472>427$ & 0.005 & 20 & 11 & $472>423$ & 0.005 & 20 & 22 \\
\hline & & & & & & $472>171$ & 0.005 & 20 & 22 \\
\hline M8FOSA & 6.5 & $506>78$ & 0.005 & 45 & 40 & $506>172$ & 0.005 & 45 & 57 \\
\hline M8PFOS-13C8 & 5.4 & $507>80$ & 0.005 & 40 & 60 & $507>99$ & 0.005 & 40 & 50 \\
\hline \multirow[t]{2}{*}{\begin{tabular}{|l|} 
M6PFDA-13C6 \\
\end{tabular}} & 6.1 & $519>474$ & 0.005 & 20 & 11 & $519>219$ & 0.005 & 20 & 22 \\
\hline & & & & & & $519>169$ & 0.005 & 20 & 22 \\
\hline M2-8:2-FTS-13C2 & 5.9 & $529>509$ & 0.005 & 40 & 30 & $529>81$ & 0.005 & 40 & 30 \\
\hline \multirow[t]{2}{*}{ M7PFUnDA-13C7 } & 6.4 & $570>525$ & 0.005 & 20 & 11 & $570>269$ & 0.005 & 20 & 22 \\
\hline & & & & & & $570>219$ & 0.005 & 20 & 33 \\
\hline \multirow[t]{2}{*}{ MeFOSAA-d3 } & 6.1 & $573>419$ & 0.005 & 28 & 28 & $573>515$ & 0.005 & 28 & 28 \\
\hline & & & & & & $573>483$ & 0.005 & 28 & 28 \\
\hline \multirow[t]{2}{*}{ EtFOSAA-d5 } & 6.2 & $589>419$ & 0.005 & 31 & 30 & $589>531$ & 0.005 & 31 & 30 \\
\hline & & & & & & $589>483$ & 0.005 & 31 & 30 \\
\hline \multirow[t]{2}{*}{ M2PFDoA-13C2 } & 6.6 & $615>570$ & 0.005 & 20 & 11 & $615>169$ & 0.005 & 20 & 33 \\
\hline & & & & & & $615>269$ & 0.005 & 20 & 33 \\
\hline M2PFTeDA-13C2 & 6.9 & $715>670$ & 0.005 & 20 & 11 & $715>169$ & 0.005 & 20 & 33 \\
\hline
\end{tabular}

Table S2.9. Table of target mass-labeled compounds, associated instrument parameters, and quantification parameters used for targeted LC-MS/MS analysis. 


\begin{tabular}{|r|r|r|r|}
\hline Time & Flow (ml/min) & $\begin{array}{l}\text { \% } \\
\text { aqueous }\end{array}$ & $\begin{array}{l}\text { \% } \\
\text { organic }\end{array}$ \\
\hline \hline 0 & 0.4 & 75 & 25 \\
\hline 0.5 & 0.4 & 75 & 25 \\
\hline 5 & 0.4 & 15 & 85 \\
\hline 5.1 & 0.4 & 5 & 95 \\
\hline 5.6 & 0.4 & 5 & 95 \\
\hline 7 & 0.4 & 5 & 95 \\
\hline 9 & 0.4 & 75 & 25 \\
\hline
\end{tabular}

Table S2.10. Table presenting mobile phase gradient ratios used during sample analysis via LC-MS/MS 


\begin{tabular}{|r|l|l|r|}
\hline \multicolumn{1}{|l|}{ Time } & $\begin{array}{l}\text { Flow } \\
\text { (ml/min) }\end{array}$ & $\begin{array}{l}\text { \% } \\
\text { aqueous }\end{array}$ & $\begin{array}{l}\text { \% } \\
\text { organic }\end{array}$ \\
\hline-3 & 0.3 & 80 & 20 \\
\hline 0 & 0.3 & 80 & 20 \\
\hline 0.5 & 0.3 & 80 & 20 \\
\hline 3 & 0.3 & 50 & 50 \\
\hline 3.5 & 0.3 & 50 & 50 \\
\hline 5.5 & 0.3 & 40 & 60 \\
\hline 6 & 0.3 & 40 & 60 \\
\hline 7 & 0.3 & 0 & 100 \\
\hline 11 & 0.3 & 0 & 100 \\
\hline
\end{tabular}

Table S2.11. Table presenting mobile phase gradient ratios used during sample analysis via HRMS 


\begin{tabular}{|c|c|c|c|c|c|}
\hline Compound & $\begin{array}{c}\text { Mean } \\
\text { Instrumental } \\
\text { Blank }(n= \\
64)\end{array}$ & $\begin{array}{l}\text { Std. Dev. of } \\
\text { Instrumental } \\
\text { Blank }\end{array}$ & $\begin{array}{c}\text { Mean Process } \\
\text { Blank }(n=8)\end{array}$ & $\begin{array}{c}\text { Std. Dev. of } \\
\text { Process Blank }\end{array}$ & $\begin{array}{l}\text { MDL (in raw } \\
\text { abundance) }\end{array}$ \\
\hline HFPO-DA & 6,558 & 7,848 & 50,584 & 12,527 & 88,164 \\
\hline Nafion BP2 & 708 & 127 & 712 & 37 & 822 \\
\hline PFO4DA & 881 & 275 & 803 & $\mathrm{NA}$ & 1,707 \\
\hline PFO5DoDA & 792 & 59 & 814 & 167 & 1,315 \\
\hline
\end{tabular}

Table S2.12. Raw abundances of instrumental and process blanks observed in HRMS analysis. Estimated MDLs are calculated as the mean process blank area plus three times the standard deviation. The estimated MDL for PFO4DA was calculated using instrumental blank values due to limited detection in process blanks, precluding calculation of standard deviation using process blank values. HFPO-DA was analyzed via both HRMS and LC-MS/MS, but high instrumental and process blanks values associated with HRMS prevented quantification of HFPO-DA using this technique. Detections of HFPO-DA reported in this study are derived from LC-MS/MS measurements. Quantification limits for HRMS were constrained by the linear dynamic range of the curve. 


\begin{tabular}{|l|r|r|r|}
\hline \multicolumn{4}{|c|}{ Accuracy and Precision } \\
\hline Compound & $\begin{array}{r}\text { \% } \\
\text { Accuracy }\end{array}$ & $\begin{array}{r}\text { SD } \% \\
\text { Accuracy }\end{array}$ & \% RSD \\
\hline 4:2 FTS & 118.0 & 9.2 & 7.8 \\
\hline 6:2 FTS & 114.3 & 16.0 & 14.0 \\
\hline 8:2-FTS & 111.6 & 28.5 & 25.6 \\
\hline EtFOSAA & 101.3 & 26.4 & 26.1 \\
\hline FOSA & 92.2 & 56.4 & 61.1 \\
\hline HFPODA & 102.2 & 12.7 & 12.4 \\
\hline N- & 95.2 & 27.1 & 28.5 \\
MeFOSAA & & & \\
\hline PFBA & 154.0 & 10.8 & 7.0 \\
\hline PFBS & 84.2 & 6.2 & 7.3 \\
\hline PFDA & 101.8 & 16.2 & 15.9 \\
\hline PFDoA & 89.1 & 28.8 & 32.3 \\
\hline PFDS & 17.9 & 26.8 & 149.6 \\
\hline PFHpA & 103.6 & 10.3 & 9.9 \\
\hline PFHpS & 110.3 & 25.4 & 23.0 \\
\hline PFHxA & 124.6 & 8.6 & 6.9 \\
\hline PFHxS & 83.0 & 12.7 & 15.3 \\
\hline PFNA & 93.1 & 6.4 & 6.9 \\
\hline PFNS & 41.1 & 28.1 & 68.4 \\
\hline PFOA & 99.3 & 7.7 & 7.8 \\
\hline PFOS & 76.5 & 8.3 & 10.8 \\
\hline PFPeA & 101.3 & 8.4 & 8.3 \\
\hline PFPeS & 114.2 & 10.2 & 8.9 \\
\hline PFTeDA & 67.4 & 20.3 & 30.1 \\
\hline PFTrDA & 19.6 & 16.7 & 85.5 \\
\hline PFUnDA & 86.9 & 17.6 & 20.3 \\
\hline MEN & & & \\
\hline
\end{tabular}

Table S2.13. Method accuracy and precision as relative standard deviation (RSD); both metrics were calculated using five spiked replicate samples consisting of organic chicken liver spiked with 2 ng native stock and $10 \mathrm{ng}$ of isotopically labeled surrogate stock. 


\begin{tabular}{|l|r|}
\hline \multicolumn{2}{|c|}{ \% Matrix Effects } \\
compared to spiked \\
solvent \\
\hline \hline Compound & \multicolumn{1}{|c|}{$\%$ Effect } \\
\hline 4:2 FTS_IS & 39.019 \\
\hline 6:2 FTS_IS & 68.15 \\
\hline 8:2-FTS_IS & 44.619 \\
\hline EtFOSAA-IS & -39.162 \\
\hline FOSA_IS & -37.001 \\
\hline HFPODA_IS & -5.387 \\
\hline MeFOSAA_IS & -20.812 \\
\hline PFBA_IS & -40.869 \\
\hline PFBS_IS & -5.92 \\
\hline PFDA_IS & -2.305 \\
\hline PFDoA_IS & -42.043 \\
\hline PFHpA_IS & -1.74 \\
\hline PFHXA_IS & -20.683 \\
\hline PFHXS_IS & -4.617 \\
\hline PFNA_IS & 2.815 \\
\hline PFOA_IS & -3.067 \\
\hline PFOS_IS & 1.688 \\
\hline PFPeA_IS & -10.996 \\
\hline PFTeDA_IS & -55.018 \\
\hline PFUnDA_IS & -16.539 \\
\hline
\end{tabular}

Table S2.14. Table presenting the degree of ion enhancement or suppression associated with matrix interference calculated using: \% Matrix Effects $=(($ Post Extraction Spike in Chicken Matrix)/(Post Extraction Spike in Solvent)-1) $\times 100$ 


\begin{tabular}{|l|r|}
\hline \multicolumn{2}{|c|}{$\begin{array}{c}\text { Method Detection } \\
\text { Limits, } \mathbf{n g} \text { /ml }\end{array}$} \\
\hline \hline Compound & \multicolumn{1}{c|}{ MDL } \\
\hline 4:2 FTS & \multicolumn{1}{c|}{0.548} \\
\hline 6:2 FTS & 1.24 \\
\hline 8:2-FTS & 2.379 \\
\hline EtFOSAA & 4.139 \\
\hline FOSA & 3.674 \\
\hline HFPODA & 0.895 \\
\hline N-MeFOSAA & 2.185 \\
\hline PFBA & 0.69 \\
\hline PFBS & 0.502 \\
\hline PFDA & 0.983 \\
\hline PFDS & 1.541 \\
\hline PFDoA & 3.552 \\
\hline PFHpA & 0.625 \\
\hline PFHpS & 1.686 \\
\hline PFHxA & 0.781 \\
\hline PFHxS & 0.845 \\
\hline PFNA & 0.459 \\
\hline PFNS & 1.729 \\
\hline PFOA & 0.539 \\
\hline PFOS & 0.559 \\
\hline PFPeA & 0.569 \\
\hline PFPeS & 0.875 \\
\hline PFTeDA & 2.197 \\
\hline PFUnDA & 1.089 \\
\hline PFTrDA & 0.957 \\
\hline
\end{tabular}

Table S2.15. Method reporting limits for LC-MS/MS analysis calculated using the standard deviation of spiked replicate samples multiplied by the Student's tvalue appropriate for a single-tailed 99th percentile, according to: 『MDL】 s $\mathrm{s}=\mathrm{t} \_((\mathrm{n}-1, \quad 1-\alpha=0.99)) \times \mathrm{S} \_\mathrm{s}$ 


\begin{tabular}{|c|c|c|}
\hline Sample ID & Compound & $\begin{array}{c}\text { Detect vs } \\
\text { non- } \\
\text { detect }\end{array}$ \\
\hline CF10_G & Nafion BP2 & Detect \\
\hline CF01_G & Nafion BP2 & Detect \\
\hline CF02_G & Nafion BP2 & Detect \\
\hline CF03_G & \begin{tabular}{|l|} 
Nafion BP2 \\
\end{tabular} & Detect \\
\hline CF04_G & Nafion BP2 & Detect \\
\hline CF05_G & \begin{tabular}{|l|} 
Nafion BP2 \\
\end{tabular} & Detect \\
\hline CF06_G & Nafion BP2 & Detect \\
\hline CF07_G & Nafion BP2 & Detect \\
\hline CF08_G & Nafion BP2 & Detect \\
\hline CF09_G & Nafion BP2 & Detect \\
\hline CF11_G & Nafion BP2 & Detect \\
\hline CF12_G & Nafion BP2 & Detect \\
\hline GRSH_Q002_G & \begin{tabular}{|l|} 
Nafion BP2 \\
\end{tabular} & Detect \\
\hline GRSH_Q026_G & Nafion BP2 & Detect \\
\hline HG_061_G & Nafion BP2 & Detect \\
\hline HG_062_G & Nafion BP2 & Nondetect \\
\hline P88026_G & \begin{tabular}{|l|} 
Nafion BP2 \\
\end{tabular} & Nondetect \\
\hline CF010_G & PFO4DA & Nondetect \\
\hline CF01_G & PFO4DA & Detect \\
\hline CF05_G & PFO4DA & Detect \\
\hline CF06_G & PFO4DA & Detect \\
\hline CF07_G & PFO4DA & Detect \\
\hline CF08_G & PFO4DA & Detect \\
\hline CF11_G & PFO4DA & Detect \\
\hline CF12_G & PFO4DA & Detect \\
\hline CF010_G & PFO5DoDA & Detect \\
\hline CF01_G & PFO5DoDA & Detect \\
\hline CF02_G & PFO5DoDA & Detect \\
\hline CF03_G & PFO5DoDA & Detect \\
\hline CF04_G & PFO5DoDA & Detect \\
\hline CF05_G & PFO5DoDA & Detect \\
\hline CF06_G & PFO5DoDA & Detect \\
\hline CF07_G & PFO5DoDA & Detect \\
\hline CF08_G & PFO5DoDA & Detect \\
\hline CF09_G & PFO5DoDA & Detect \\
\hline CF11_G & PFO5DoDA & Detect \\
\hline CF12_G & PFO5DoDA & Detect \\
\hline HG_062_G & PFO5DoDA & Nondetect \\
\hline
\end{tabular}

Table S2.16. Detection of PFEAs, based on compariston to estimated method detection limit (mean process blank abundance $+3 *$ std. dev.) or the lowest curve point. 


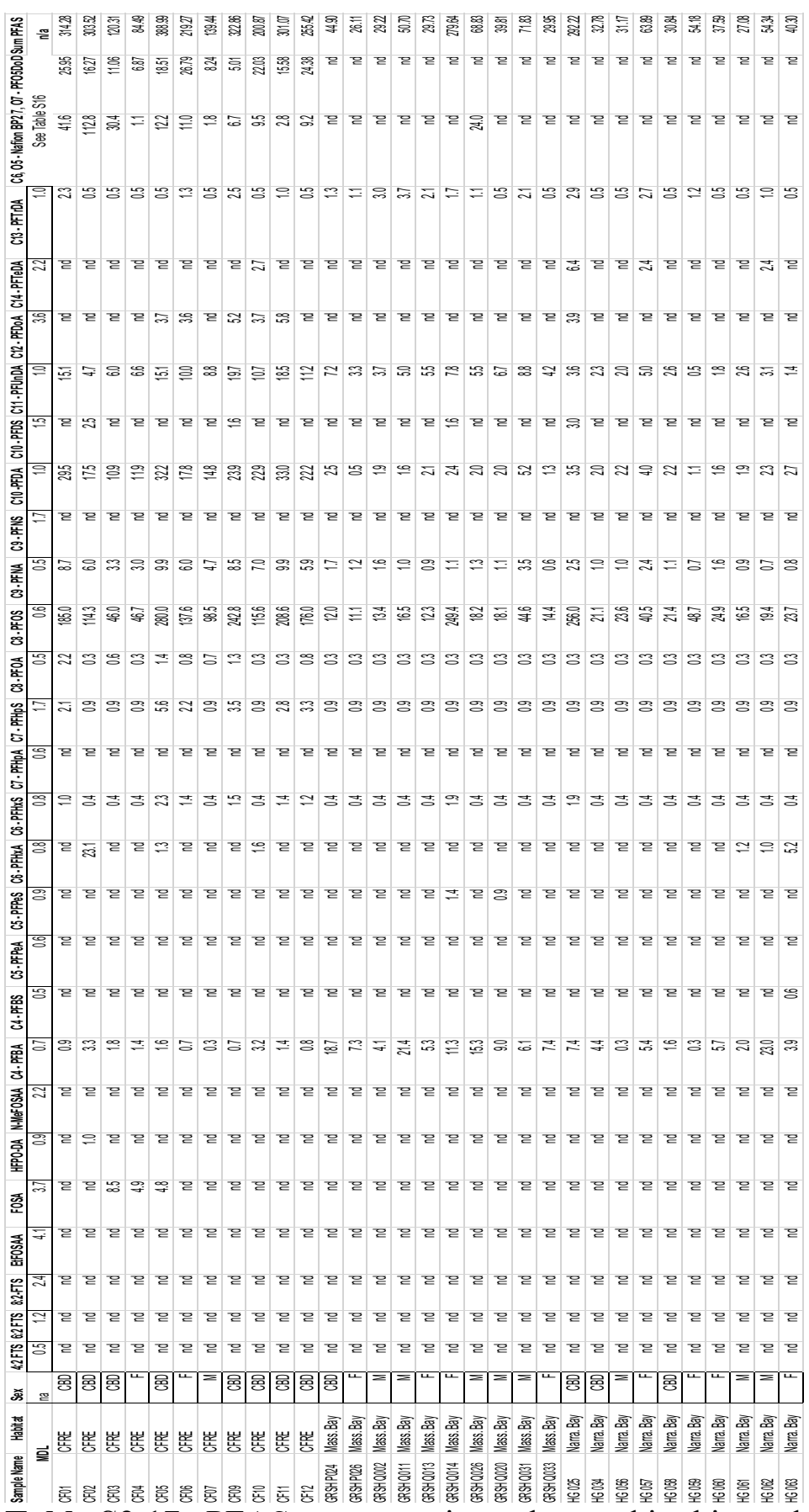

Table S2.17. PFAS concentrations observed in this study. Responses not detected or outside the linear dynamic range of the curve were labeled as "nd" and assigned a value of zero. Observations below method detection limits but with clear signal, clear qualifier ions, and detected in at least $50 \%$ of samples in one habitat were replaced with the half the method detection limit for statistical analyses, and included as such in calculation of summed concentrations. 


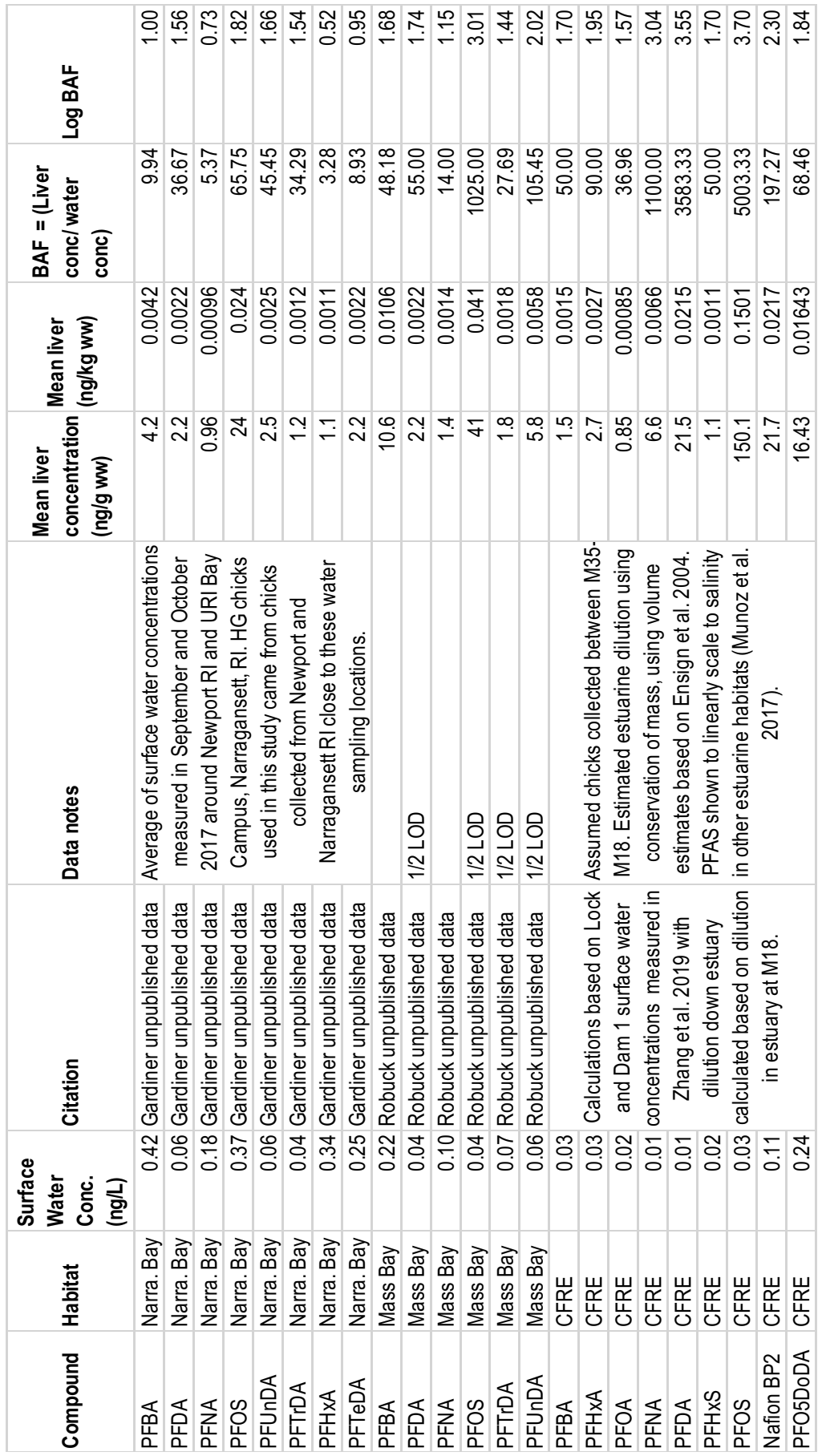

Table S2.18. Table providing surface water concentrations, mean liver concentrations, and calculation of bioconcentration factors (BCFs). 


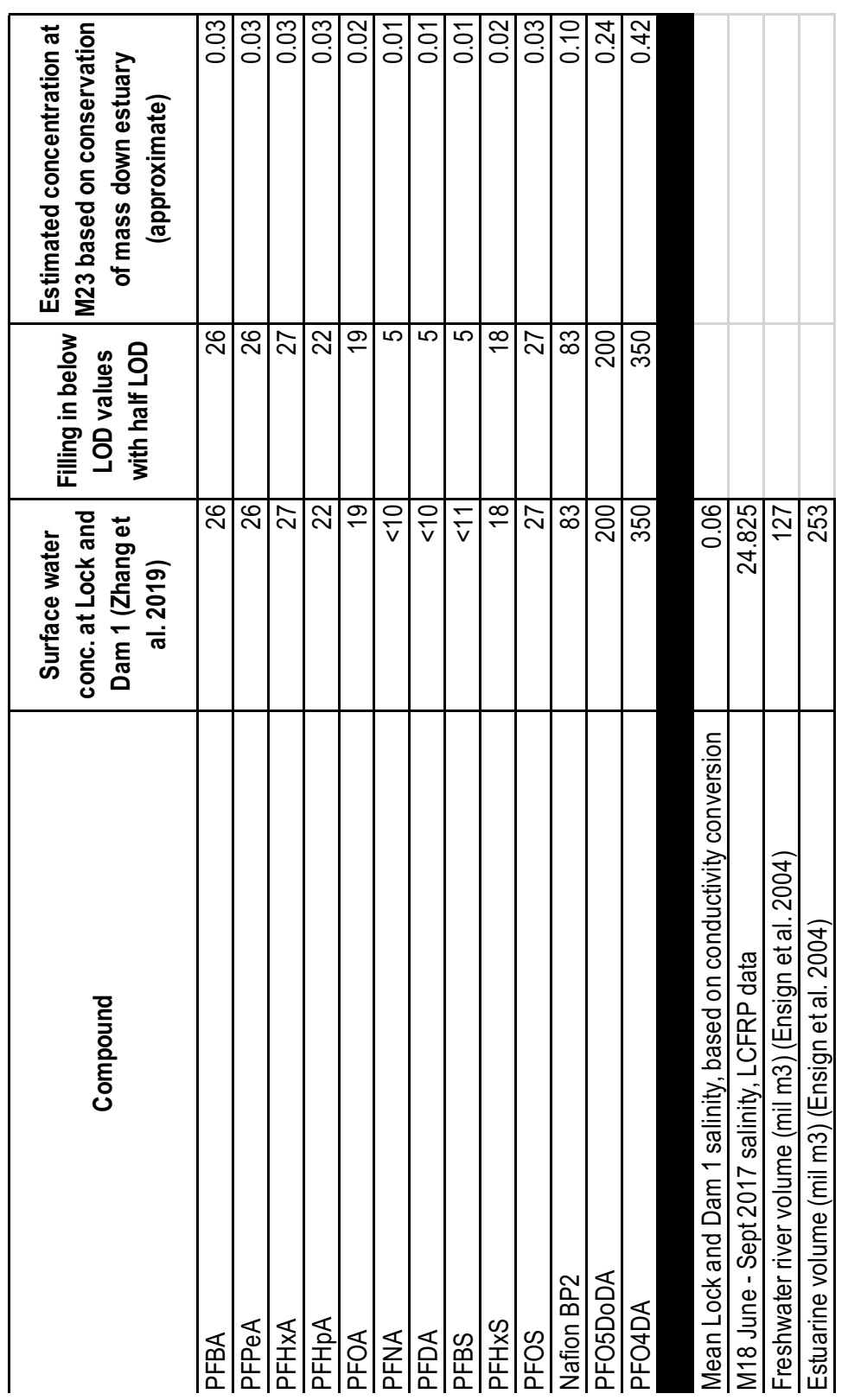

Table S2.19. Worksheet containing surface water measurements from Lock and Dam 1 in the CFRE derived from Zhang et al. 2019, and subsequent estimation of estuarine water concentrations based on conservation of mass and salinity data. Water data from 10.1016/j.cageo.2004.03.015 and https://doi.org/10.1021/acs.estlett.9b00525. 


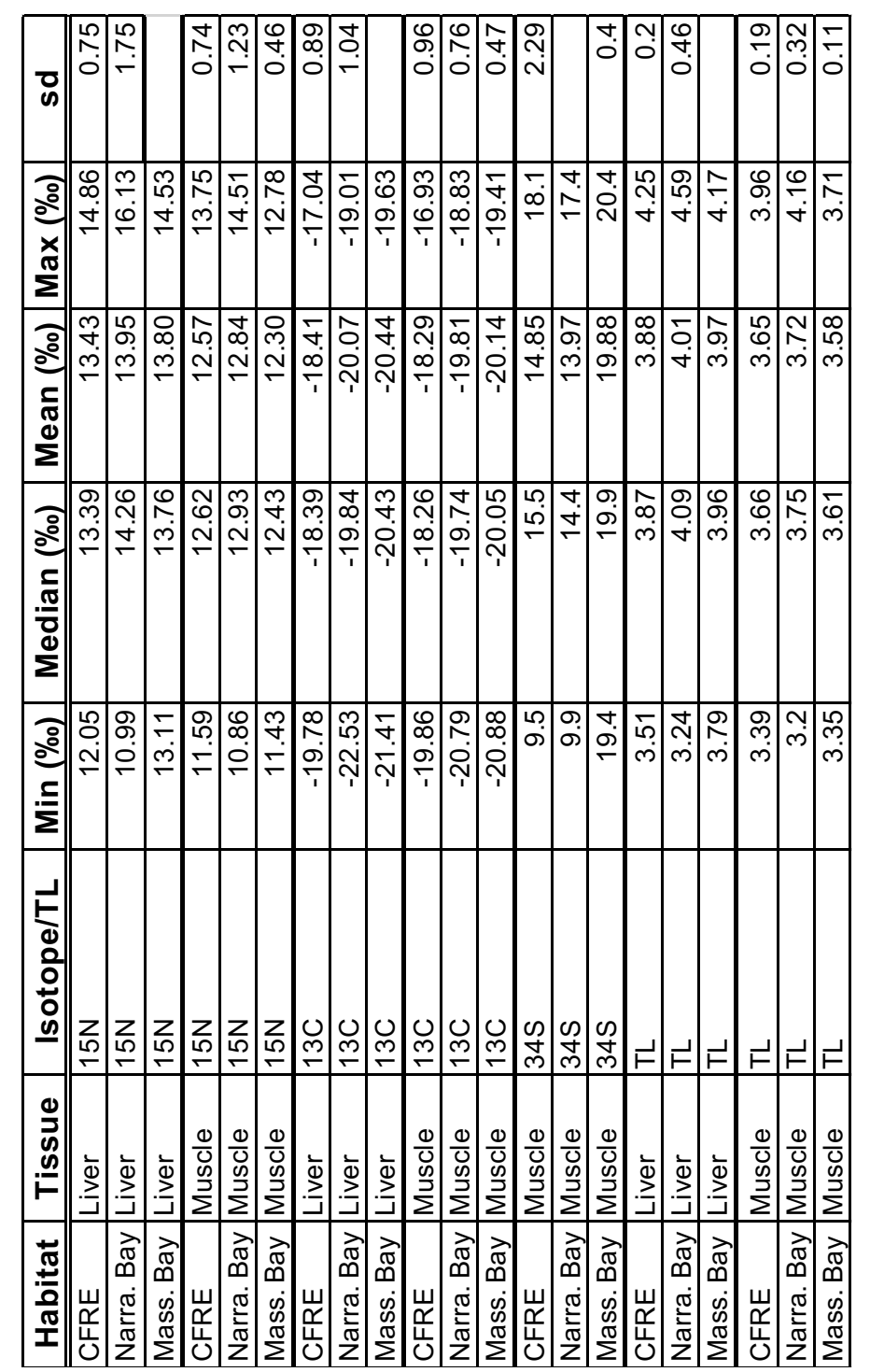

Table S2.20. Summary statistics describing stable isotope measurements of bird liver and muscle. Trophic levels were calculated following methods of Fisk et al. 2001 and Hobson et al. 1994. 


\begin{tabular}{|l|l|r|r|r|r|}
\hline Sample & Habitat & N15 Liver (\%) & Liver TL & N15 Muscle (\%) & Muscle TL \\
\hline \hline CF01 & CFRE & 13.31 & 3.84 & 12.62 & 3.66 \\
\hline CF02 & CFRE & 12.05 & 3.51 & 11.92 & 3.48 \\
\hline CF03 & CFRE & 14.86 & 4.25 & 13.75 & 3.96 \\
\hline CF04 & CFRE & 12.68 & 3.68 & 11.69 & 3.42 \\
\hline CF05 & CFRE & 13.82 & 3.98 & 12.90 & 3.74 \\
\hline CF06 & CFRE & 13.53 & 3.90 & 12.77 & 3.70 \\
\hline CF07 & CFRE & 13.39 & 3.87 & 12.39 & 3.60 \\
\hline CF09 & CFRE & 13.28 & 3.84 & 11.59 & 3.39 \\
\hline CF10 & CFRE & 14.21 & 4.08 & 12.94 & 3.75 \\
\hline CF11 & CFRE & 12.93 & 3.74 & 12.03 & 3.51 \\
\hline CF12 & CFRE & 13.72 & 3.95 & 13.70 & 3.95 \\
\hline GRSH P024 & Mass. Bay & 13.71 & 3.95 & 11.89 & 3.47 \\
\hline GRSH P026 & Mass. Bay & 13.11 & 3.79 & 11.43 & 3.35 \\
\hline GRSH Q002 & Mass. Bay & 14.01 & 4.03 & 11.79 & 3.44 \\
\hline GRSH Q011 & Mass. Bay & NA & & 12.75 & 3.70 \\
\hline GRSH Q013 & Mass. Bay & 13.34 & 3.85 & 12.33 & 3.59 \\
\hline GRSH Q014 & Mass. Bay & 13.76 & 3.96 & 12.54 & 3.64 \\
\hline GRSH Q026 & Mass. Bay & 14.53 & 4.17 & 12.60 & 3.66 \\
\hline GRSH Q028 & Mass. Bay & 14.05 & 4.04 & 12.26 & 3.57 \\
\hline GRSH Q031 & Mass. Bay & 14.23 & 4.09 & 12.78 & 3.71 \\
\hline GRSH Q033 & Mass. Bay & 13.42 & 3.87 & 12.61 & 3.66 \\
\hline HG 025 & Narra. Bay & 12.93 & 3.74 & 11.75 & 3.44 \\
\hline HG 034 & Narra. Bay & 14.32 & 4.11 & 13.76 & 3.96 \\
\hline HG 056 & Narra. Bay & 12.44 & 3.62 & 11.82 & 3.45 \\
\hline HG 057 & Narra. Bay & 16.10 & 4.58 & 14.51 & 4.16 \\
\hline HG 058 & Narra. Bay & 12.24 & 3.56 & 12.90 & 3.74 \\
\hline HG 059 & Narra. Bay & 10.99 & 3.24 & 10.86 & 3.20 \\
\hline HG 060 & Narra. Bay & 14.20 & 4.08 & 12.96 & 3.75 \\
\hline HG 061 & Narra. Bay & 15.51 & 4.42 & 13.81 & 3.98 \\
\hline HG 062 & Narra. Bay & 16.13 & 4.59 & 14.19 & 4.08 \\
\hline HG 063 & Narra. Bay & 14.67 & 4.20 & 11.80 & 3.45 \\
\hline & Mean & 13.72 & 3.95 & 12.57 & 3.65 \\
\hline & GeoMean & 13.67 & 3.94 & 12.54 & 3.64 \\
\cline { 2 - 6 } & SD & 1.12 & 0.29 & 10.86 & 0.23 \\
\hline & Min & 10.99 & 3.24 & 14.51 & 4.20 \\
\hline Max & 16.13 & 4.59 & 4.16 \\
\hline
\end{tabular}

Table S2.21. Measured 15N ratios alongside estimates of trophic level (TL), assuming a copepod primary consumer after Fisk et al. 2001. 


\begin{tabular}{|r|l|l|}
\hline \multicolumn{2}{|c|}{ Correlation Coefficient } & Dancey \& Reidy (2007) \\
\hline 1 & -1 & Perfect \\
\hline 0.9 & -0.9 & Strong \\
\hline 0.8 & -0.8 & Strong \\
\hline 0.7 & -0.7 & Strong \\
\hline 0.6 & -0.6 & Moderate \\
\hline 0.5 & -0.5 & Moderate \\
\hline 0.4 & -0.4 & Moderate \\
\hline 0.3 & -0.3 & Weak \\
\hline 0.2 & -0.2 & Weak \\
\hline 0.1 & -0.1 & Weak \\
\hline 0 & & Zero \\
\hline
\end{tabular}

Table S2.22. Definition of correlation coefficient significance and descriptors, from https://dx.doi.org/10.1016\%2Fj.tjem.2018.08.001. 


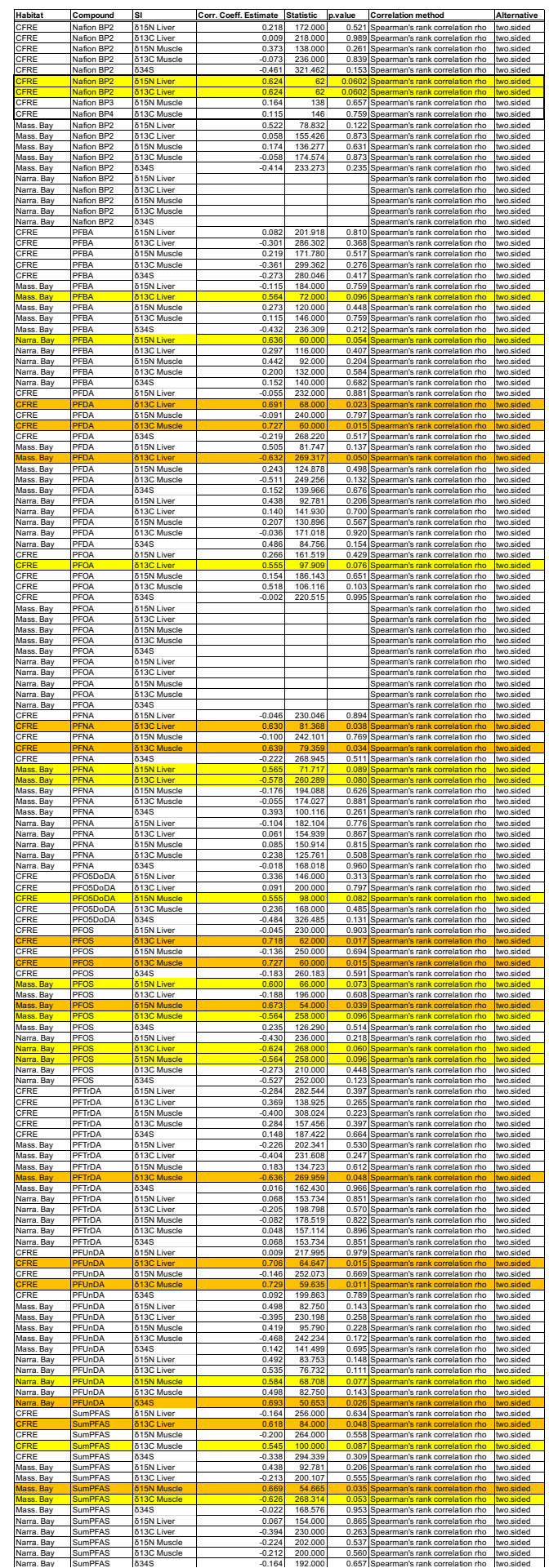

Table S2.23. Results of Spearman correlation analysis assessing relationships between each PFAS and stable isotope ratios. Statistically significant ( $p 0.05$ ) relationships are highlighted in orange. Non-statistically significant relationships with $0.05<$ p.value $<0.1$ are highlighted in yellow for illustrative purposes. 


\section{LITERATURE CITED}

(1) Giesy, J. P.; Kannan, K. Global Distribution of Perfluorooctane Sulfonate in Wildlife Global Distribution of Perfluorooctane Sulfonate in Wildlife. Environ. Sci. Technol. 2001, 35 (March), 1339-1342. https://doi.org/10.1021/es001834k.

(2) Kannan, K.; Franson, J. C.; Bowerman, W. W.; Hansen, K. J.; Jones, P. D.; Giesy, J. P. Perfluorooctane Sulfonate in Fish-Eating Water Birds Including Bald Eagles and Albatrosses. Environ. Sci. Technol. 2001, 35 (15), 3065-3070. https://doi.org/10.1021/es001935i.

(3) Braune, B. M.; Letcher, R. J. Perfluorinated Sulfonate and Carboxylate Compounds in Eggs of Seabirds Breeding in the Canadian Arctic: Temporal Trends (1975-2011) and Interspecies Comparison. Environ. Sci. Technol. 2013, 47 (1), 616-624. https://doi.org/10.1021/es303733d.

(4) Letcher, R. J.; Su, G.; Moore, J. N.; Williams, L. L.; Martin, P. A.; De Solla, S. R.; Bowerman, W. W.; Solla, S. R. De; Bowerman, W. W.

Perfluorinated Sulfonate and Carboxylate Compounds and Precursors in Herring Gull Eggs from across the Laurentian Great Lakes of North America: Temporal and Recent Spatial Comparisons and Exposure Implications. Sci. Total Environ. 2015, 538 (August), 468-477. https://doi.org/10.1016/j.scitotenv.2015.08.083.

(5) Miller, A.; Elliott, J. E.; Elliott, K. H.; Lee, S.; Cyr, F. Temporal Trends of Perfluoroalkyl Substances (PFAS) in Eggs of Coastal and Offshore Birds:

Increasing PFAS Levels Associated with Offshore Bird Species Breeding on the Pacific Coast of Canada and Wintering near Asia. Environ. Toxicol. Chem. 2015, 34 (8), 1799-1808. https://doi.org/10.1002/etc.2992.

(6) Miller, A.; Elliott, J. E.; Wilson, L. K.; Elliott, K. H.; Drouillard, K. G.; Verreault, J.; Lee, S.; Idrissi, A. Influence of Overwinter Distribution on Exposure to Persistent Organic Pollutants (POPs) in Seabirds, Ancient Murrelets (Synthliboramphus Antiquus), Breeding on the Pacific Coast of Canada. Environ. Pollut. 2019, 113842. https://doi.org/10.1016/j.envpol.2019.113842.

(7) Kannan, K.; Choi, J. W.; Iseki, N.; Senthilkumar, K.; Hoon, D.; Masunaga, S.; Giesy, J. P.; Kim, D. H.; Masunaga, S.; Giesy, J. P. Concentrations of Perfluorinated Acids in Livers of Birds from Japan and Korea. Chemosphere 2002, 49 (3), 225-231. https://doi.org/10.1016/S0045-6535(02)00304-1.

(8) Lopez-Antia, A.; Dauwe, T.; Meyer, J.; Maes, K.; Bervoets, L.; Eens, M. High Levels of PFOS in Eggs of Three Bird Species in the Neighbourhood of a Fluoro-Chemical Plant. Ecotoxicol. Environ. Saf. 2020, 139 (August 2016), 165171. https://doi.org/10.1016/j.ecoenv.2017.01.040. 
(9) Newsted, J. L.; Coady, K. K.; Beach, S. A.; Butenhoff, J. L.; Gallagher, S.; Giesy, J. P. Effects of Perfluorooctane Sulfonate on Mallard and Northern Bobwhite Quail Exposed Chronically via the Diet. Environ. Toxicol. Pharmacol. 2007, 23 (1), 1-9. https://doi.org/10.1016/j.etap.2006.04.008.

(10) Newsted, J. L.; Beach, S. A.; Gallagher, S. P.; Giesy, J. P. Acute and Chronic Effects of Perfluorobutane Sulfonate (PFBS) on the Mallard and Northern Bobwhite Quail. Arch. Environ. Contam. Toxicol. 2008, 54 (3), 535545. https://doi.org/10.1007/s00244-007-9039-8.

(11) Roscales, J. L.; Vicente, A.; Ryan, P. G.; González-Solís, J.; Jiménez, B. Spatial and Interspecies Heterogeneity in Concentrations of Perfluoroalkyl Substances (PFASs) in Seabirds of the Southern Ocean. Environ. Sci. Technol. 2019, 53 (16), 9855-9865. https://doi.org/10.1021/acs.est.9b02677.

(12) Galatius, A.; Bossi, R.; Sonne, C.; Rigét, F. F.; Kinze, C. C.; Lockyer, C.; Teilmann, J.; Dietz, R. PFAS Profiles in Three North Sea Top Predators: Metabolic Differences among Species? Environ. Sci. Pollut. Res. 2013, 20 (11), 8013-8020. https://doi.org/10.1007/s11356-013-1633-x.

(13) Letcher, R. J.; Chu, S.; McKinney, M. A.; Tomy, G. T.; Sonne, C.; Dietz, R. Comparative Hepatic in Vitro Depletion and Metabolite Formation of Major Perfluorooctane Sulfonate Precursors in Arctic Polar Bear, Beluga Whale, and Ringed Seal. Chemosphere 2014, 112, 225-231.

https://doi.org/10.1016/j.chemosphere.2014.04.022.

(14) Dassuncao, C.; Hu, X. C.; Zhang, X.; Bossi, R.; Dam, M.; Mikkelsen, B.; Sunderland, E. M. Temporal Shifts in Poly- and Perfluoroalkyl Substances (PFASs) in North Atlantic Pilot Whales Indicate Large Contribution of Atmospheric Precursors. Environ. Sci. Technol. 2017, 51 (8), 4512-4521. https://doi.org/10.1021/acs.est.7b00293.

(15) Lynch, K. M.; Fair, P. A.; Houde, M.; Muir, D. C. G.; Kannan, K.; Bossart, G. D.; Bartell, S. M.; Gribble, M. O. Temporal Trends in Per- and Polyfluoroalkyl Substances in Bottlenose Dolphins (Tursiops Truncatus) of Indian River Lagoon, Florida and Charleston, South Carolina. Environ. Sci. Technol. 2019, 53 (24), 14194-14203. https://doi.org/10.1021/acs.est.9b04585.

(16) Houde, M.; Wells, R. S.; Fair, P. A.; Bossart, G. D.; Hohn, A. A.; Rowles, T. K.; Sweeney, J. C.; Solomon, K. R.; Muir, D. C. G. Polyfluoroalkyl Compounds in Free-Ranging Bottlenose Dolphins (Tursiops Truncatus) from the Gulf of Mexico and the Atlantic Ocean. Environ. Sci. Technol. 2005, 39 (17), 6591-6598. https://doi.org/10.1021/es0506556.

(17) Pizzurro, D. M.; Seeley, M.; Kerper, L. E.; Beck, B. D. Interspecies Differences in Perfluoroalkyl Substances (PFAS)Toxicokinetics and Application 
to Health-Based Criteria. Regul. Toxicol. Pharmacol. 2019, 106 (May), 239-250. https://doi.org/10.1016/j.yrtph.2019.05.008.

(18) Tarazona, J. V.; Rodríguez, C.; Alonso, E.; Sáez, M.; González, F.; San Andrés, M. D.; Jiménez, B.; San Andrés, M. I. Toxicokinetics of Perfluorooctane Sulfonate in Birds under Environmentally Realistic Exposure Conditions and Development of a Kinetic Predictive Model. Toxicol. Lett. 2015, 232 (2), 363368. https://doi.org/10.1016/j.toxlet.2014.11.022.

(19) Heuvel, J. P. V.; Kuslikis, B. I.; Van Rafelghem, M. J.; Peterson, R. E. Tissue Distribution, Metabolism, and Elimination of Perfluorooctanoic Acid in Male and Female Rats. J. Biochem. Toxicol. 1991, 6 (2), 83-92. https://doi.org/10.1002/jbt.2570060202.

(20) Holmström, K. E.; Berger, U. Tissue Distribution of Perfluorinated Surfactants in Common Guillemot (Uria Aalge) from the Baltic Sea. Environ. Sci. Technol. 2008, 42 (16), 5879-5884. https://doi.org/10.1021/es800529h.

(21) Armitage, J. M.; Arnot, J. A.; Wania, F. Potential Role of Phospholipids in Determining the Internal Tissue Distribution of Perfluoroalkyl Acids in Biota.

Environ. Sci. Technol. 2012, 46 (22), 12285-12286. https://doi.org/10.1021/es304430r.

(22) Ng, C. A.; Hungerbühler, K. Bioaccumulation of Perfluorinated Alkyl Acids: Observations and Models. Environ. Sci. Technol. 2014, 48 (9), 4637-4648. https://doi.org/10.1021/es404008g.

(23) Ng, C. A.; Hungerbühler, K. Bioconcentration of Perfluorinated Alkyl Acids: How Important Is Specific Binding? Environ. Sci. Technol. 2013, 47 (13), 7214-7223. https://doi.org/10.1021/es400981a.

(24) Fenton, S. E.; Reiner, J. L.; Nakayama, S. F.; Delinsky, A. D.; Stanko, J. P.; Hines, E. P.; White, S. S.; Lindstrom, A. B.; Strynar, M. J.; Petropoulou, S. E. Analysis of PFOA in Dosed CD-1 Mice. Part 2: Disposition of PFOA in Tissues and Fluids from Pregnant and Lactating Mice and Their Pups. Reprod. Toxicol. 2009, 27 (3-4), 365-372. https://doi.org/10.1161/CIRCULATIONAHA.110.956839.

(25) Entenman, C. Lipid Content of Chick Tissues. J Biol Chem 1940, No. 65, 231-241.

(26) Cuthbert, R. J. Breeding Biology, Chick Growth and Provisioning of Great Shearwaters (Puffinus Gravis) at Gough Island, South Atlantic Ocean. Ети 2005, 105 (4), 305-310. https://doi.org/10.1071/MU05036. 
(27) Yoo, H.; Guruge, K. S.; Yamanaka, N.; Sato, C.; Mikami, O.; Miyazaki, S.; Yamashita, N.; Giesy, J. P. Depuration Kinetics and Tissue Disposition of PFOA and PFOS in White Leghorn Chickens (Gallus Gallus) Administered by Subcutaneous Implantation. Ecotoxicol. Environ. Saf. 2009, 72 (1), 26-36. https://doi.org/10.1016/j.ecoenv.2007.09.007.

(28) Verreault, J.; Houde, M.; Gabrielsen, G. W.; Berger, U.; Haukås, M.; Letcher, R. J.; Muir, D. C. G. Perfluorinated Alkyl Substances in Plasma, Liver, Brain, and Eggs of Glaucous Gulls (Larus Hyperboreus) from the Norwegian Arctic. Environ. Sci. Technol. 2005, 39 (19), 7439-7445. https://doi.org/10.1021/es051097y.

(29) Gebbink, W. A.; Letcher, R. J. Comparative Tissue and Body Compartment Accumulation and Maternal Transfer to Eggs of Perfluoroalkyl Sulfonates and Carboxylates in Great Lakes Herring Gulls. Environ. Pollut. 2012, 162, 40-47. https://doi.org/10.1016/j.envpol.2011.10.011.

(30) Ahrens, L.; Siebert, U.; Ebinghaus, R. Total Body Burden and Tissue Distribution of Polyfluorinated Compounds in Harbor Seals (Phoca Vitulina) from the German Bight. Mar. Pollut. Bull. 2009, 58 (4), 520-525. https://doi.org/10.1016/j.marpolbul.2008.11.030.

(31) Borg, D.; Bogdanska, J.; Sundström, M.; Nobel, S.; Håkansson, H.; Bergman, A.; DePierre, J. W.; Halldin, K.; Bergström, U. Tissue Distribution of 35S-Labelled Perfluorooctane Sulfonate (PFOS) in C57Bl/6 Mice Following Late Gestational Exposure. Reprod. Toxicol. 2010, 30 (4), 558-565. https://doi.org/10.1016/j.reprotox.2010.07.004.

(32) Wang, J.; Caccamise, S. A. L.; Woodward, L. A.; Li, Q. X. L. Polychlorinated Biphenyls in the Plasma and Preen Oil of Black-Footed Albatross (Diomedea Nigripes) Chicks and Adults on Midway Atoll, North Pacific Ocean. PLoS One 2015, 10 (4), 1-12. https://doi.org/10.1371/journal.pone.0123041.

(33) Mondal, D.; Lopez-Espinosa, M. J.; Armstrong, B.; Stein, C. R.; Fletcher, T. Relationships of Perfluorooctanoate and Perfluorooctane Sulfonate Serum Concentrations between Mother-Child Pairs in a Population with Perfluorooctanoate Exposure from Drinking Water. Environ. Health Perspect. 2012, 120 (5), 752-757. https://doi.org/10.1289/ehp.1104538.

(34) Houde, M.; Balmer, B. C.; Brandsma, S.; Wells, R. S.; Rowles, T. K.; Solomon, K. R.; Muir, D. C. G. Perfluoroalkyl Compounds in Relation to LifeHistory and Reproductive Parameters in Bottlenose Dolphins (Tursiops Truncatus) from Sarasota Bay, Florida, USA. Environ. Toxicol. Chem. 2006, 25 (9), 2405-2412. https://doi.org/10.1897/05-499R.1. 
(35) Baduel, C.; Lai, F. Y.; Townsend, K.; Mueller, J. F. Size and AgeConcentration Relationships for Perfluoroalkyl Substances in Stingray Livers from Eastern Australia. Sci. Total Environ. 2014, 496, 523-530. https://doi.org/10.1016/j.scitotenv.2014.07.010.

(36) Wang, J.; Zhang, Y.; Zhang, F.; Yeung, L. W. Y.; Taniyasu, S.; Yamazaki, E.; Wang, R.; Lam, P. K. S.; Yamashita, N.; Dai, J. Age- and GenderRelated Accumulation of Perfluoroalkyl Substances in Captive Chinese Alligators (Alligator Sinensis). Environ. Pollut. 2013, 179, 61-67. https://doi.org/10.1016/j.envpol.2013.04.020.

(37) Cui, Q.; Pan, Y.; Zhang, H.; Sheng, N.; Wang, J.; Guo, Y.; Dai, J. Occurrence and Tissue Distribution of Novel Perfluoroether Carboxylic and Sulfonic Acids and Legacy Per/Polyfluoroalkyl Substances in Black-Spotted Frog (Pelophylax Nigromaculatus). Environ. Sci. Technol. 2018, 52 (3), 982-990. https://doi.org/10.1021/acs.est.7b03662.

(38) Lasters, R.; Groffen, T.; Lopez-Antia, A.; Bervoets, L.; Eens, M. Variation in PFAA Concentrations and Egg Parameters throughout the Egg-Laying Sequence in a Free-Living Songbird (the Great Tit, Parus Major): Implications for Biomonitoring Studies. Environ. Pollut. 2019, 246 (2019), 237-248. https://doi.org/10.1016/j.envpol.2018.12.014.

(39) Powers, K. D.; Wiley, D. N.; Allyn, A. J.; Welch, L.; Ronconi, R. A. Movements and Foraging Areas of Great Shearwaters in the Gulf of Maine. Mar. Ecol. Prog. Ser. 2017, 574, 1-57.

(40) Ronconi, R. A.; Koopman, H. N.; McKinstry, C. A. E.; Wong, S. N. P.; Westgate, A. J. Inter-Annual Variability in Diet of Non-Breeding Pelagic Seabirds Puffinus Spp. at Migratory Staging Areas: Evidence from Stable Isotopes and Fatty Acids. Mar. Ecol. Prog. Ser. 2010, 419, 267-282. https://doi.org/10.3354/meps08860.

(41) Lorente, J. A.; Lorente, M.; Villanueva, E. Postmortem Stability of Lung Surfactant Phospholipids. J. Forensic Sci. 1992, 37 (5), 13322J. https://doi.org/10.1520/jfs13322j.

(42) Noble, R. C.; Cocchi, M. Lipid Metabolism and the Neonatal Chicken. Prog. Lipid Res 1990, 29, 107-140.

(43) Schreiber, R. Nesting Chronology of the Eastern Brown Pelican. Auk 1980, 97 (3), 491-508. https://doi.org/10.1093/auk/97.3.491.

(44) Condor, S. T.; Winter, N.; Gensch, R. H.; Brown, C. P. Ticks as a Factor in Nest Desertion of California Brown Pelicans Author ( $\mathrm{s}$ ): Kirke A . King, James O . Keith, Christine A. Mitchell and James E . Keirans Published by: 
Oxford University Press Stable URL : Https://Www.Jstor.Org/Stable/1367739

REFEREN. 2020, 79 (4), 507-509.

(45) Brooke, M. de L.; Bonnaud, E.; Dilley, B. J.; Flint, E. N.; Holmes, N. D.; Jones, H. P.; Provost, P.; Rocamora, G.; Ryan, P. G.; Surman, C.; Buxton, R. T. Seabird Population Changes Following Mammal Eradications on Islands. Anim. Conserv. 2018, 21 (1), 3-12. https://doi.org/10.1111/acv.12344.

(46) Nisbet, I. C. T.; Welton, M. J. Seasonal Variations in Breeding Success of Common Terns: Consequences of Predation. Condor 1984, 86 (1), 53. https://doi.org/10.2307/1367345.

(47) van Franeker, J. A. Save the North Sea Fulmar-Litter-EcoQO Manual Part 1: Collection and Dissection Procedures. Alterra-rapport 2004, 672, 1-38.

(48) Malinsky, M. D.; Jacoby, C. B.; Reagen, W. K. Determination of Perfluorinated Compounds in Fish Fillet Homogenates: Method Validation and Application to Fillet Homogenates from the Mississippi River. Anal. Chim. Acta 2011, 683 (2), 248-257. https://doi.org/10.1016/j.aca.2010.10.028.

(49) Powley, C. R.; George, S. W.; Ryan, T. W.; Buck, R. C. Matrix EffectFree Analytical Methods for Determination of Perfluorinated Carboxylic Acids in Environmental Matrixes. Anal. Chem. 2005, 77 (19), 6353-6358. https://doi.org/10.1021/ac0508090.

(50) Berger, U.; Haukås, M.; Hauk, M. Validation of a Screening Method Based on Liquid Chromatography Coupled to High-Resolution Mass Spectrometry for Analysis of Perfluoroalkylated Substances in Biota. $J$. Chromatogr. A 2005, 1081 (2), 210-217. https://doi.org/10.1016/j.chroma.2005.05.064.

(51) Chambers, E.; Wagrowski-Diehl, D. M.; Lu, Z.; Mazzeo, J. R. Systematic and Comprehensive Strategy for Reducing Matrix Effects in LC/MS/MS Analyses. J. Chromatogr. B Anal. Technol. Biomed. Life Sci. 2007, 852 (1-2), 22-34. https://doi.org/10.1016/j.jchromb.2006.12.030.

(52) Dassuncao, C.; Pickard, H.; Pfohl, M.; Tokranov, A. K.; Li, M.; Mikkelsen, B.; Slitt, A.; Sunderland, E. M. Phospholipid Levels Predict the Tissue Distribution of Poly- and Perfluoroalkyl Substances in a Marine Mammal.

Environ. Sci. Technol. Lett. 2019, 6 (3), acs.estlett.9b00031. https://doi.org/10.1021/acs.estlett.9b00031.

(53) Leat, E. H. K.; Bourgeon, S.; Eze, J. I.; Muir, D. C. G.; Williamson, M.; Bustnes, J. O.; Furness, R. W.; Borgå, K. Perfluoroalkyl Substances in Eggs and Plasma of an Avian Top Predator, Great Skua (Stercorarius Skua), in the North 
Atlantic. Environ. Toxicol. Chem. 2013, 32 (3), 569-576.

https://doi.org/10.1002/etc.2101.

(54) Mallin, M. A.; Lewitus, A. J. The Importance of Tidal Creek Ecosystems. J. Exp. Mar. Bio. Ecol. 2004, 298 (2), 145-149. https://doi.org/10.1016/S00220981(03)00356-3.

(55) NOAA Office for Coastal Management. C-CAP Land Cover Atlas https://coast.noaa.gov/digitalcoast/tools/lca.html.

(56) Haines, E. B. Stable Carbon Isotope Ratios in the Biota, Soils and Tidal Water of a Georgia Salt Marsh. Estuar. Coast. Mar. Sci. 1976, 4 (6), 609-616. https://doi.org/10.1016/0302-3524(76)90069-4.

(57) Druffel, E. R. M.; Williams, P. M.; Bauer, J. E.; Ertel, J. R. Cycling of Dissolved and Particulate Organic Matter in the Open Ocean. J. Geophys. Res. 1992, 97 (C10). https://doi.org/10.1029/92jc01511.

(58) Hobson, K. A.; Piatt, J. F.; Pitocchelli, J. Using Stable Isotopes to Determine Seabird Trophic Relationships. J. Anim. Ecol. 1994, 63 (4), 786-798.

(59) Munoz, G.; Budzinski, H. H.; Labadie, P.; Budzinski, H. H.; Labadie, P.; Budzinski, H. H.; Labadie, P. Influence of Environmental Factors on the Fate of Legacy and Emerging Per- and Polyfluoroalkyl Substances along the Salinity/Turbidity Gradient of a Macrotidal Estuary. Environ. Sci. Technol. 2017, 51 (21), acs.est.7b03626. https://doi.org/10.1021/acs.est.7b03626.

(60) Zhang, X.; Lohmann, R.; Sunderland, E. M. Poly- and Perfluoroalkyl Substances (PFAS) in Seawater and Plankton from the Northwestern Atlantic Margin. Environ. Sci. Technol. 2019, 53 (21), 12348-12356. https://doi.org/10.1021/acs.est.9b03230.

(61) Zhang, C.; Hopkins, Z. R.; Mccord, J.; Strynar, M. J.; Detlef, R. U.; Knappe, D. R. U. Fate of Per- and Polyfluoroalkyl Ether Acids in the Total Oxidizable Precursor Assay and Implications for the Analysis of Impacted Water. Environ. Sci. Technol. Lett. 2019. https://doi.org/10.1021/acs.estlett.9b00525.

(62) Ensign, S. H.; Halls, J. N.; Mallin, M. A. Application of Digital Bathymetry Data in an Analysis of Flushing Times of Two Large Estuaries. Comput. Geosci. 2004, 30 (5), 501-511. https://doi.org/10.1016/j.cageo.2004.03.015.

(63) Arnot, J. A.; Gobas, F. A. P. C. A Review of Bioconcentration Factor (BCF) and Bioaccumulation Factor (BAF) Assessments for Organic Chemicals in Aquatic Organisms. Environ. Rev. 2006, 14 (4), 257-297. https://doi.org/10.1139/A06-005. 


\section{CHAPTER 3}

TIME TRENDS OF PER- AND POLYFLUOROALKYL SUBSTANCES FROM 2010 - 2019 IN A TRANS-EQUATORIAL MIGRATORY SEABIRD

This manuscript is formatted for submission to the journal Environmental Science and Technology

Anna R. Robuck ${ }^{*}$, Mark G. Cantwell ${ }^{2}$, David N. Wiley ${ }^{3}$, Mengyang Liu ${ }^{4}$, Lindsay Agvent ${ }^{1}$, Kevin D. Powers ${ }^{3}$, Richard McKinney ${ }^{2}$, Elsie M. Sunderland ${ }^{5,6}$, and Rainer Lohmann ${ }^{1}$

${ }^{1}$ University of Rhode Island Graduate School of Oceanography, Narragansett, RI 02882

${ }^{2}$ Atlantic Coastal Environmental Sciences Division, Center for Environmental Measurement and Modeling, US Environmental Protection Agency, Narragansett, RI 02882

${ }^{3}$ Stellwagen Bank National Marine Sanctuary, National Oceanic and Atmospheric Administration, Scituate, MA 02066

${ }^{4}$ College of Ocean \& Earth Sciences, Xiamen University, China

${ }^{5}$ Harvard T. H. Chan School of Public Health, Boston, Massachusetts 02215, United States

${ }^{6}$ Harvard John A. Paulson School of Engineering and Applied Sciences, Cambridge, Massachusetts 02138, United States 
*Corresponding author, present address:

Anna Robuck, 215 S Ferry Rd Narragansett RI 02882, annarobuck@gmail.com

KEYWORDS: PFOS, PFAS, seabirds

ABSTRACT

Per- and polyfluoroalkyl substances, or PFAS, are industrial chemicals used widely in a range of consumer, industrial, and military applications. Increased regulatory scrutiny and research attention have brought about changes in the production and use of PFAS, affecting PFAS trends within environmental and biological matrices over the last two decades. Here we measured 27 PFAS in 116 Great Shearwaters collected in Massachusetts Bay, USA from 2010 - 2019. We found limited differences between sexes and no significant differences between age groups. Concentrations of PFOS and FOSA declined over the ten-year time series, while C8 - C11 PFCAs showed no significant trends over time. The lack of trends in long-chain PFCAs over time may be indicative of an ongoing change point, as long-chain PFCAs may no longer be increasing in the environment. Two less studied PFAS, Nafion BP2 and 7:3 FTCA, were detected in 36\% and 64\% of the sample set over time, respectively. Multiple PFAS were significantly associated with reduced liver and kidney weights and depth of body fat, while PFHxS and PFNA were negatively associated with mass and count of ingested plastics. Continued evaluation of long-chain PFCAs and emerging compounds is required to discern any changing trends and better describe the impacts of these compounds in biota subject to sustained concentrations. 


\section{INTRODUCTION}

Per- and polyfluoroalkyl compounds, or PFAS, are a large family of anthropogenic chemicals first created in the $1940 \mathrm{~s}^{1}$. PFAS are highly persistent under a range of environmental and applied conditions ${ }^{1}$. Many PFAS are also amphiphilic in nature which allows them to repel both oil and water due to the inclusion of hydrophobic carbon backbones coupled to a hydrophilic functional groups like $-\mathrm{SO}_{4}$ or $-\mathrm{COOH}$. These unique and attractive qualities have resulted in the incorporation of PFAS into a range of industrial, military, and consumer applications; a recent meta-analysis found over 200 distinct use categories involving over 1400 different $\mathrm{PFAS}^{2}$.

Their prolific use within the built environment has resulted in their ubiquity in natural environments, including remote polar regions ${ }^{3}$ as well as environments closer to direct human sources ${ }^{4,5}$. The spatial distributions of PFAS in the global environment are generally a function of their unique physico-chemical properties that facilitate long-range transport as well as their emissions during manufacturing and use. For example, emissions of perfluorooctane sulfonic acid (PFOS) are dominated by release from use of consumer and industrial products that previously incorporated PFOS directly or currently incorporate precursor compounds that transform into PFOS in vivo or in situ (like Me-FOSAA used in foot packaging products $)^{4,6}$. Local and regional "hot spots" of specific compounds or classes of PFAS may also be linked to manufacturing sites $^{7}$ or surrounding locations where extensive product use or disposal has occurred (e.g., military 
bases, wastewater treatment plants $)^{8,9}$. Population density can also serve as a proxy for relative exposure in fresh and coastal waters where emissions are more diffuse and driven predominantly by use of consumer products ${ }^{4,6}$.

Regardless of specific emission source or compound, PFAS ultimately pose the most significant risk to marine environments, as the ocean is thought to be the ultimate reservoir for perfluorooctanoic acid (PFOA), PFOS, and other PFAS. PFAS have been identified in the surface ocean and at depth ${ }^{10}$, as well as in marine mammals ${ }^{11}$, seabirds ${ }^{12}$, fish ${ }^{13}$, crustacea and invertebrates ${ }^{14,15}$. PFAS with more than 7 carbons have been documented to strongly bioaccumulate and biomagnify in marine food webs, particularly those supporting air-breathing organisms like seabirds and marine mammals ${ }^{15,16}$. However, PFAS preferentially bind to protein ${ }^{17}$ or amphiphilic fatty acids ${ }^{18}$, unlike the preference for hydrophobic fatty matrices demonstrated by well-studied hydrophobic organic contaminants such as polychlorinated biphenyls. Therefore their occurrence and accumulation within different organisms, life stages, sexes, or food web scenarios is still under investigation.

Temporal trends of PFAS in marine environments are likewise of interest, as PFAS production and use has undergone significant changes with increased evidence of adverse effects within humans and wildlife ${ }^{19}$. For example, perfluoroalkyl acids (PFAAs) like PFOA and PFOS were phased out in the early 2000 s, with increased use of replacements with fewer than seven fluorinated carbons, polyfluorinated structures, and/or structurally modified iterations of legacy PFAS, such as perfluoroalkyl ether acids (PFEAs) incorporating different 
numbers of ether linkages ${ }^{20,21}$. Changes to PFAS production and use patterns have previously been demonstrated to alter environmental concentrations of PFAS over time, as PFOS and FOSA rapidly decreased in wildlife and environmental matrices following the phase out of these compounds in the early $2000 \mathrm{~s}^{11}$. It is therefore expected that continued interventions aimed at curtailing the use of PFAS will result in further changes in the occurrence of PFAS over time and space.

In the present study we measured PFAS over a ten-year time series in a longlived Atlantic seabird. In summary, the goals of the study were to (i) ascertain any temporal trends of PFAS over the last decade; (ii) assess the presence of novel and understudied PFAS in these birds, and (iii) explore PFAS dynamics between sexes, age groups, and in association with other biological and pollutant covariates.

\section{MATERIALS AND METHODS}

\section{Study species}

The Great Shearwater (Ardenna gravis) is a long-lived, trans-equatorial migratory seabird that inhabits the pelagic Gulf of Maine in the NW Atlantic during its non-breeding period over the boreal summer. Satellite tracking data and necropsy data suggest age-specific segregation of their non-breeding range, with preferential use of the SW Gulf of Maine and Massachusetts Bay by juveniles ${ }^{22}$. This species breeds during the boreal winter in the South Atlantic, on Gough Island, Inaccessible Island, and Nightingale Island in the St. Helena Island Group 
and Kidney Island in the Falkland Islands ${ }^{23}$. Contaminant levels measured in this species are primarily thought to reflect the influence of foraging in the Northern Hemisphere, due to the much lower levels of PFAS measured in other petrels at similar or higher trophic levels that solely rely on the Southern Atlantic Ocean ${ }^{24}$. Specimen Collection, Necropsy, and Sample Condition

Dead Great Shearwater juveniles and adults were obtained as bycatch from the National Oceanic and Atmospheric Administration (NOAA) Northeast Fisheries Observer Program (Falmouth, MA) from Massachusetts Bay. Bycatch collections spanned $2010-2019$ and the majority of samples were collected in the months of August - November each year, after approximately 2 or more months of foraging in the NW Atlantic. Each individual was frozen individually as a whole specimen upon collection and stored at $-20{ }^{\circ} \mathrm{C}$ until necropsy. All individuals were necropsied in a standardized manner ${ }^{25}$, including measurements of morphometric characteristics, body condition, and organ weights. Age group was determined following the age characterization of Great Shearwaters as defined by Powers et al. (2020) ${ }^{22}$ using gonad size and the presence of the bursa of Fabricius, and sex based upon gonad presentation.

\section{Chemicals, reagents, and materials}

Analytical standards of greater than $99.9 \%$ purity, including mass-labeled surrogates, were purchased from Wellington Laboratories (Guelph, ON, Canada). Native Nafion BP2 was provided by Chemours (Fayetteville, NC) in lieu of a commercial source. HPLC grade methanol and acetonitrile were purchased from Fisher Scientific (Waltham, MA, USA). Ultrapure water for equipment cleaning 
was obtained from a Milli-Q system fit with an HPLC water polisher or via HPLC grade water purchased from Fisher Scientific (Waltham, MA, USA). ENVI Carb bulk powder was purchased from Sigma-Aldrich (St. Louis, MO, USA). Ammonium acetate was purchased from Fisher Scientific (Waltham, MA, USA) Extraction and analysis of PFAS

A total of 116 Great Shearwaters (Ardenna gravis) were analyzed for PFAS. 95 were juveniles and 21 were mature birds. The sample set was evenly split between males and females, with 55 females and 54 males examined. 7 birds could not be accurately sexed.

Aliquots of liver from each individual were homogenized and solventextracted in acetonitrile following addition of isotope-labelled surrogates, as previously described ${ }^{26}$. Extracts were cleaned up using high-speed centrifugation paired with $25 \mathrm{mg}$ of graphitized non-porous carbon and glacial acetic acid. Measurement and quantification of 27 PFAS was achieved using liquid chromatography tandem-mass spectrometry (UPLC-MS/MS) experiments in negative electrospray ionization mode. Estimates of PFAS concentrations were obtained by quantification using isotope dilution.

Matrix spikes and procedural blanks were included with the sample set, to monitor matrix effects, process recovery, and background contamination. Matrix effects were addressed using a 7-point matrix-matched curve, made up of chicken liver homogenate extracted in an identical fashion to GRSH liver samples, and spiked with native and isotope-labelled standards directly prior to analysis. The chicken liver matrix used for the curve contained trace levels of PFAS (namely L 
- PFOS), and was corrected for background PFAS using the average of triplicate samples taken through the extraction. Data reported in this study were not blank corrected, due to low levels of process contamination identified in procedural blanks. PFBA and PFBS were not quantitatively reported here due to poor chromatography. Method detection limits were defined as procedural blank levels of a given analyte plus 3 times the standard deviation; in the absence of quantifiable blank concentrations, the lowest curve point $(0.025 \mathrm{ng} / \mathrm{mL})$ was deemed the method detection limit. Values below MDLs were considered zero for summation purposes. Method recovery ranged from $11-130 \%$ with a mean recovery of $76 \%$ across all compounds, similar to recoveries reported in other work using avian liver ${ }^{27-29}$. FOSA, PFTeDA, and PFTrDA evidenced the lowest recoveries due to predictable loss of these analytes during sample preparation. Analysis for total mercury

We used body feathers for analysis of total mercury (THg). The samples were washed with Milli-Q water and acetone in an ultrasonic bath respectively, and then rinsed with Milli-Q water 3 times. After freeze drying overnight, each sample was cut up and homogenized, and about $15 \mathrm{mg}$ weighed into a nickel boat for analysis. All samples were analyzed using a direct thermal decomposition mercury analyzer (MA-3000, Nippon Instruments Corporation). Quality control methods included blanks, analytical duplicates and the certified reference materials (TORT-3 Lobster hepatopancreas and ERM-DB001 Human hairs, National Research Council of Canada) analyzed every 10 samples. Average 
recoveries for the certified reference materials were $93.1 \pm 5.8 \%$ (TORT-3) and $95.9 \pm 5.0 \%$.

\section{Stable isotope analysis}

Body feathers were analyzed for $\delta^{15} \mathrm{~N}$ and $\delta^{13} \mathrm{C}$ to deduce any influence of trophic trends on results. Feathers were cleaned with acetone, and ground to fineness using a mortar and pestle. $\sim 1 \mathrm{mg}$ of feather was weighed out into tin capsules for carbon and nitrogen stable isotope analysis. Weighed samples were measured via IR-MS, and results interpreted as parts per thousand relative to appropriate references. $\delta^{15} \mathrm{~N}$ and $\delta^{13} \mathrm{C}$ were measured using an Isoprime 100 Isotope Ratio Mass Spectrometer coupled to a Micro Vario Elemental Analyzer (Elementar Americas, Mt.Laurel, NJ).

The nitrogen $\left(\delta^{15} \mathrm{~N}\right)$ isotope composition was expressed as a part per thousand deviation (\%o ) from air. Carbon $\left(\delta^{13} \mathrm{C}\right)$ isotope composition was expressed relative to Vienna Pee Dee Belemnite where $\delta \mathrm{X}=\left[\left(\mathrm{R}_{\text {sample }}-\right.\right.$ $\left.\left.\mathrm{R}_{\text {standard }}\right) / \mathrm{R}_{\text {standard }}\right] \times 10^{3}$, where $\mathrm{X}$ is $\delta^{15} \mathrm{~N}$ or $\delta^{13} \mathrm{C}$, and $\mathrm{R}$ is the ratio of heavy to light isotope (15N: 14N, 13C: 12C). Duplicates were analyzed every 10 samples, and a blue mussel reference material every 15 samples to ensure measurement quality.

\section{Statistical treatment}

All data manipulation and statistical analyses were performed in $\mathrm{R}$ version 3.6.1 (R Core Team, 2020) ${ }^{30}$. Quantitative data were checked for normality and homoscedasticity using the Shapiro-Wilk test and Levene's test. Concentrations were non-normal despite log transformation and therefore treated non- 
parametrically as log-transformed variables for statistical analyses; year groups displayed no significant differences in variance. Summary statistics were derived using censored and uncensored data using the cenfit functions in the R package, NADA, version 1.6 - 1.1 (Lee 2020) to better account for left-censored data (Helsel 2012). Differences between years or groups were assessed using KruskalWallis tests with post hoc application of Dunn's test with Bonferroni correction for multiple testing. Temporal trends were evaluated for compounds found in $>80 \%$ of samples using generalized linear models (GLMs) with year as the sole predictor as a numeric variable to deduce any sustained temporal trends.

Temporal trend assessment included all individuals with concentrations above detection limits given the lack of significant differences between mature and juvenile birds. 7:3 FTCA was also assessed for temporal trends despite its lower detection frequency (64\%) using a generalized linear model, but these results were not reported quantitatively. Relationships between continuous, logtransformed numeric variables were assessed using Spearman rank correlation coefficients $\left(\mathrm{R}_{\mathrm{s}}{ }^{2}\right)$. Effects observed within all statistical tests were considered significant when $\mathrm{p}<0.05$.

\section{RESULTS AND DISCUSSION}

\section{Observed detection frequencies and concentrations}

Samples were screened for 27 analytes, and 17/27 compounds were above detection limits in at least one sample. FOSA, PFOA, PFNA, PFDA, PFUnDA, PFDoDA, and PFOS were detected in at least $80 \%$ of the sample set (Table 1). 
PFOS was the most abundant compound in each individual and within each year, making up $49 \%$ of $\sum_{17} \mathrm{PFAS}$ concentrations across all years with a mean concentration of $41 \mathrm{ng} / \mathrm{g}$ across all years and a maximum of $111 \mathrm{ng} / \mathrm{g}$ in a 2010 individual. After PFOS, PFUnDA or 7:3 FTCA generally alternated as the second most abundant compound in each sample. Concentrations of PFOS and PFUnDA were similar to concentrations previously observed in this species in 2017, as well as similar to concentrations observed in other temperate petrels ${ }^{12,31-33}$.

The highest $\sum_{17}$ PFAS measured was 234 ng/g w.w. liver comprised of 15 quantifiable analytes, found in a 2010 juvenile GRSH. The lowest $\sum_{17} \mathrm{PFAS}$ concentration was observed in a 2019 juvenile Great Shearwater from Massachusetts Bay containing $\sum{ }_{17} P F A S$ of $9 \mathrm{ng} / \mathrm{g}$, comprised of 6 analytes above detection limits.

Similarities and differences between age groups and sexes

Younger birds generally contained higher concentrations of individual and $\sum_{17}$ PFAS, but these results were not statistically significant in any given year or overall, possibly related to the low number of adults included in the sample set.

Considering solely mature individuals across all years $(n=21)$, mature females possessed lower concentrations of PFOA and PFHxS compared to mature males (Kruskal-Wallis, $\mathrm{p}<0.05$ ). Controlled studies in chickens and other animal models indicate differences in PFAS levels between sexes are related to differences in elimination efficiency mediated by sex hormone levels. For example, in a controlled study using adult mice, Kudo et al. (2002) experimentally castrated male rats which subsequently exhibited increased renal 
clearance of PFOA approaching rates observed in females; reduced renal clearance rates were again apparent in the castrated males following testosterone treatment ${ }^{34}$. We believe a similar sexually-mediated mechanism drives differences in PFOA and PFHxS levels in mature birds within our sample set, as higher concentrations in male individuals have likewise been identified in controlled studies involving mallards and quail ${ }^{35,36}$.

However, concentration differences between sexes were not observed for any compound in juvenile birds within each year or overall. The lack of differences between sexes in young birds has been previously reported ${ }^{37}$, and may be due to slow sexual maturation in long-lived seabirds in combination with the strong influence of maternal off-loading. The juveniles assessed within our study were primarily hatch year individuals less than 6 months in age, and therefore may be too young to exhibit the hormonally-mediated elimination differences apparent in older individuals due to their limited expression of sexual hormones. Like other Procellariiform seabirds, Great Shearwaters require an extended period of development (6-9 years) to reach sexual maturity. Changes in hormonal characteristics over this maturation period have been identified in other Procellariiforms (e.g. Wandering Albatross, Diomedea exulans) with very young males possessing significantly less testosterone compared to older males approaching or at sexual maturity ${ }^{38}$; Great Shearwaters have not been specifically tested for such hormonal differences over maturation.

Additionally, young individuals likely reflect a disproportionate amount of PFAS derived from maternal offloading, which may obfuscate sex-based 
concentration differences in the short-term. The tremendous persistence of PFAS in biological matrices means that PFAS transferred from mother to offspring are not likely to dissipate over the first months of development. Tarazona et al. (2015) established a plasma half-life estimate for PFOS in birds (231 days) using environmentally relevant doses of PFOS in chickens ${ }^{39}$. Short-term exposure trials in juvenile quail and mallard suggest the liver half-life of PFOS is roughly 2.5 times that of the serum half-life ${ }^{36}$. Using the plasma half-life derived in Tarazona et al. 2015 scaled using the exposure data relating plasma half-life and liver halflife, this equates to an estimated liver half-life for PFOS of 578 days in birds, or 1.6 years. Liver elimination of PFAS via biliary excretion has been demonstrated to be very slow ( $\sim 1 \%$ of supplied dose $)^{40}$, and biological metabolism of most PFAAs is thought to negligible. Therefore, the young birds (under about 6 months of age) assessed in this study likely reflect maternally-derived PFAS rather than unique PFAS profiles driven by hormonally-mediated processes. Few data sets exploring PFAS levels in birds over time or space report differences between sexes or lack thereof, and we encourage increased assessment of sex-based differences in other long-lived bird taxa to ascertain if juveniles of other species reflect the same apparent lack of sex-based dissimilarities early in development. Detection of novel PFAS over time

Here we provide the first estimates describing the occurrence of two emerging PFAS within a sample set collected across a decade (Fig. 1). Nafion byproduct 2, or Nafion BP2, and 7:3 FTCA were detected in 36\% and $64 \%$ of our 
sample set, respectively. Little or no data to date reports on the occurrence of these compounds in wildlife.

Nafion BP2 is an ether-based PFAS derived from the production of ionic polymers that are used across diverse industrial applications. Nafion BP2 has been previously reported in $\operatorname{birds}^{37}$, fish ${ }^{41}$, and humans ${ }^{42}$ from the Cape Fear River watershed in southeast North Carolina, due to ongoing industrial activity in the region $^{43}$. Prior work from our group likewise identified this compound in a limited number of individuals from Massachusetts Bay and Narragansett Bay in $2017^{37}$. Here we found Nafion BP2 in 36\% (42/116) of Great Shearwaters dating back to 2010, with a maximum concentration of $0.230 \mathrm{ng} / \mathrm{g}$ in liver from a juvenile Great Shearwater bycaught in 2015. Temporal trends were not statistically assessed due to the limited detection frequency of this analyte. However, despite this limitation, our data is the first to measure this compound in biota before or after 2017-2018.

Additionally, we believe this is the first study to identify this compound over multiple years in a wildlife population with no significant connection to known industrial sources. Great Shearwaters have no migratory connectivity to the Cape Fear region, as only $15 \%$ of individuals use the offshore US East Coast for transequatorial migration, while $85 \%$ use the margins of the eastern Atlantic adjacent to the coasts of Africa and Europe. Those individuals that use the western Atlantic migration corridor stay well offshore, and have not been documented to use the riverine or estuarine habitats of the Cape Fear River region. This suggests the occurrence of Nafion BP2 in our sample set is a result of environmental 
persistence facilitating long-range transport of this compound to pelagic environments; this work is some of the first to establish the long-range transport of Nafion BP2. Alternatively, our results also point to multiple additional source(s) beyond the Cape Fear region, which in turn suggests we may be underestimating exposure to this compound by assuming it may only be derived from one highly visible source. We recommend more comprehensive assessment of this ether-based sulfonic acid across a wider suite of environmental matrices, considering its demonstrated yet underexplored capacity for environmental occurrence

7:3 FTCA is a stable fluorotelomer carboxylic acid (FTCA), previously reported in a handful of studies examining marine mammals ${ }^{26}$, waterbirds ${ }^{29,44}$, birds of prey ${ }^{45}$, Flesh-Footed and Wedge-tailed shearwaters from the Southern Hemisphere ${ }^{46}$, as well as humans. Concentrations up to $1131 \mathrm{ng} / \mathrm{g}$ in a polar bear adult and $192 \mathrm{ng} / \mathrm{g}$ in an adult harbor seal were recently reported in marine mammals from the NW Atlantic ${ }^{26} .7: 3$ FTCA was detected in $64 \%$ of our sample set at concentrations up to $62 \mathrm{ng} / \mathrm{g}$. This compound alternated with PFUnDA as the second most abundant compound, after PFOS, within each given individual. To the best of our knowledge, this marks the first time this compound has been measured over time in a pelagic seabird. No significant differences in concentration were observed between sexes or ages considering the total sample set as a whole, and no temporal trends were apparent over time.

The lack of any temporal trend here is likely due to continued, widespread use and emissions of precursors that transform into FTCAs and PFCAs. 7:3 
FTCA is derived from aerobic or anaerobic degradation of atmospherically-labile fluorotelomer alcohols, primarily 8:2 FTOH; degradation of FTOHs also results in formation of PFCAs at low yields ${ }^{47}$. 7:3 FTCA may also be formed from in vivo degradation of polyfluoroalkyl phosphate surfactants (PAPs), nonpolymeric PFAS used in food contact paper products ${ }^{48}$.

The documented source pathways of this compound suggest respiratory uptake from atmospheric reservoirs is likely driving 7:3 FTCA concentrations observed in seabirds within this study. Limited evidence from food web investigations underscores the assumed importance of atmospheric exposure; 27 forage fish from Massachusetts Bay were measured for 7:3 FTCA (Robuck et al. 2021, in prep), with no detections of 7:3 FTCA above limits of detection in fish. Loi et al. (2011) also did not find 7:3 FTCA in a range of marine fish and crustaceans ${ }^{44}$. Any role of trophic transfer in 7:3 FTCA accumulation is made additionally unclear given its weak - moderate negative association with $\delta^{15} \mathrm{~N}$, suggesting birds feeding higher in the food web with higher ratios of $\delta^{15} \mathrm{~N}$ contain less 7:3 FTCA (Table 2), unlike the typical positive relationship between $\delta^{15} \mathrm{~N}$ and traditionally biomagnified pollutants ${ }^{49}$. This negative relationship may instead suggest biotic metabolism of 7:3 FTCA into PFOA, previously documented in a range of biological systems ${ }^{47}$. Biological metabolism may therefore limit the transfer of this compound between trophic levels, leaving respiratory uptake from atmospheric reservoirs as the primary route of exposure.

Neutral, volatile FTCA precursors such as 8:2 FTOH continue to enter the environment via use and disposal of consumer products ${ }^{50}$, industrial activities, the 
built environment ${ }^{51}$, and waste infrastructure ${ }^{52}$. As a result, 8:2 FTOH remains the dominant compound found in air at remote environments ${ }^{53}$ and adjacent to urban centers $^{54}$, suggesting this precursor will continue to impart sustained biotic occurrence of downstream products like 7:3 FTCA. More research is warranted into local and global occurrence of 7:3 FTCA and other FTCAs, given limited but concerning toxicity data suggesting FTCAs may be significantly more toxic than $\mathrm{PFCAs}^{55}$.

\section{Trends over time}

Temporal trends were assessed for FOSA, PFOS, PFOA, PFNA, PFDA, and PFUnDA using generalized linear models. FOSA and PFOS significantly decreased over $2010-2019(\mathrm{p}<0.05)$. This aligns with trends observed in gannets from the United Kingdom over 1977 - 2014, white-tailed eagles from northern Europe measured over 2008 - 2017, several species of pelagic seabird from the Pacific coast of Canada from 1990 - 2011 ${ }^{31}$, as well as multiple taxa from the Arctic across varying time scales ${ }^{3}$. Declining concentrations of these compounds here and across other environmental matrices are likely driven by the voluntary phase out of PFOS and its precursors in the early 2000s in the US and Europe.

Concentrations of PFCAs in Great Shearwaters did not significantly change over 2010 - 2019 within our study, although PFUnDA increased marginally but insignificantly $(\mathrm{p}=0.067)$. This is in contrast to other datasets, as most temporal analyses of long-chain PFCAs in other biotic matrices have found increased concentrations of PFCAs over time. However, few currently published data sets 
include data from the most recent decade. Assessing PFAS specifically over this period of time is crucial because of policy and industrial interventions that have altered PFAS use and management over the course of 2000 - 2020, many of which stand to alter ambient PFAS levels in the environment. For example, Prevedouros et al. (2006) suggested that long-chain PFCAs primarily enter the environment due to their use as processing aids in creation of fluoropolymers ${ }^{56}$, or via in situ degradation of volatile precursors ${ }^{57}$. Fluoropolymer production has instead increasingly shifted to rely upon ether-based PFEAs ${ }^{7,58}$ rather than the C8 or C9 PFCA formulations originally used; PFEAs are terminal endpoints themselves, and are not thought to further degrade to PFCAs ${ }^{59}$.

We believe the sustained, rather than increasing, concentrations of PFCAs observed in our study may be suggestive of an ongoing change point where $\mathrm{C} 8$ C11 PFCAs cease to increase in the environment and marine biota, due to shifts in production away from structures that degrade into long-chain PFCAs or process streams that produce long-chain PFCAs as impurities. A similar change point was observed for PFOS and FOSA following cessation of its use in the early 2000s ${ }^{11}$. Limited data from other more recent datasets tenuously suggests the same for long-chain PFCAs. Jouanneau et al. assessed PFAS in plasma of white-tailed eagle nestlings from 2008 - 2017, and likewise found no significant trends in PFOA or PFNA, while PFUnDA and PFTrDA ceased to increase after approximately $2012^{60}$. In the US, PFOA and PFUnDA levels in bald eagle nestlings' plasma was found to decline from 2006 to $2011^{61}$. 
Further study is required to describe developing trends of PFCAs in different biological receptors, to truly discern any change in environmental and biological abundance; the lack of clear trends may also merely indicate sustained but level emissions or formation of PFCAs or PFCA precursors in recent years.

Additionally, continued investigation is warranted to describe potential adverse effects associated with long-chain PFCAs, given the paucity of data exploring PFCA levels in relation to acute or chronic impacts in wildlife populations and their sustained concentrations in the environment.

\section{Correlation to morphometric, stable isotope, and mercury data}

Morphometric measurements were collected for each individual as part of necropsy, in tandem with stable isotope ratios, plastic ingestion, and total mercury levels, which were collected in support of other datasets derived from this bird time series. Correlation analyses suggested a range of weak to moderate relationships between these covariates and specific PFAS concentrations (Table 2). Associations were considered weak with $\rho=|0.2-0.3|$, weak-moderate with $\rho$ $=|0.3-0.4|$, and moderate with $\rho=|0.4-0.5|$.

Plastic mass and count were weakly to moderately negatively associated with PFHxS and PFNA levels. This is the first documented significant association between plastic ingestion and PFAS in seabirds, and suggests plastic pieces are not a significant vector of PFAS to wildlife that ingest plastic. This intuitively makes sense given the amphiphilic and hydrophilic chemical properties of most PFAS that reduce their proclivity for sorption to non-polar matrices. This relationship also reflects the divergent exposure pathways for these two pollutant 
groups, as seabirds are primarily exposed to PFAS via diet as opposed to direct ingestion of plastics via discovery in the environment. While plastic ingestion is a growing and popular research topic, we emphasize the lack of relationship and inverse relationships between plastics and PFAS identified here, and caution that further research investigating PFAS and plastics should beware of spurious positive significant relationships in juxtaposition to the physicochemical tendencies of PFAS.

$\delta^{13} \mathrm{C}$ ratios measured in body feathers ranged from $-19.6 \%$ to $-16.6 \%$; no consistent trends in $\delta^{13} \mathrm{C}$ were apparent over time. However, PFOS and FOSA were positively associated with $\delta^{13} \mathrm{C}$. This may suggest birds utilizing prey sources more reliant on terrestrial environments with higher (less negative) $\delta^{13} \mathrm{C}$ influenced by $\mathrm{C} 4$ primary production are more exposed to PFOS and FOSA compared to individuals reliant on more pelagic environments and forage items reflective of a more negative, marine $\delta^{13} \mathrm{C}$ signature ${ }^{62}$. These two compounds were likewise positively associated with total mercury, possibly indicative of similar biomagnification capacity among these highly bioaccumulative pollutants ${ }^{63}$.

7:3 FTCA, PFDA, PFDoA, PFHxS, PFNA, PFOA, PFOS, PFTrDA, and PFUnDA were negatively associated with kidney and liver weights. Though a mechanism driving these associations is unclear, organ weight changes have been previously demonstrated as sensitive indicators of chemical influences. Decreased organ weights may impact bird health through reduced volume capacity to perform physiological functions. PFHpS was positively associated with wing 
chord, along with PFTrDA although this association was only marginally significant $(p=0.066)$. These morphometric measurements were not significantly different between mature and juvenile individuals. Positive significant associations between wing chord and PFDA and PFTrDA have previously been reported in two species of Pacific shearwaters ${ }^{46}$. PFDoA, PFOS, PFTrDA, and PFUndA were also positively associated with body condition. The body condition index is a unitless measurement of bird condition based on the visual robustness of pectoral muscle, intestinal fat appearance, adipose fat layers, and prominence of the keel documented upon initiation of necropsy. Associations between morphometric characteristics and PFAS levels may be related to the demonstrated capacity of PFAS to impact hormonal dynamics that influence growth and development in birds and other taxa $a^{64-66}$.

Additionally, PFDoA, PFHxS, PFTrDA, and PFUnDA were negatively associated with fat depth, measured adjacent to pectoral muscle. Unlike other hydrophobic pollutants, PFAS do not preferentially partition into adipose fat stores, therefore we believe this association reflects as-of-yet undetermined physiological or ecological dynamics rather than starvation-induced release of pollutants from fat stores. PFAS, specifically PFOS and PFNA, have been previously associated with changes in lipid dynamics in this species ${ }^{12}$.

Observations from other field studies and controlled research likewise demonstrate associations between PFAS and lipid dynamics in other taxa and molecular models ${ }^{18,67,68}$. Reduced fat stores may impact organismal function and fitness, as seabirds rely on finely tuned fat reserves to successfully carry out 
migration and reproduction. Given the importance of body condition and fat stores for individual and population health, we argue for more comprehensive evaluation of relationships between PFAS and sublethal endpoints like lipid and hormonal expression in seabirds and other wildlife, as detailed mechanistic or empirical explanations of the associations found in this study are lacking.

\section{Implications}

Our results confirm sustained concentrations of PFAS in multiple life stages of a pelagic seabird over a ten-year time series. PFOS and FOSA were found to decrease over time, while PFOA, PFNA, PFDA, and PFUnDA show no clear trends over time, indicating sustained concentrations. These results, in tandem with other emerging datasets, suggest a change point may be underway whereby PFCAs stop increasing in the environment and biota. Continued assessment of temporal trends is vital to ascertain the veracity of any such shifts, as well as to define environmental trends of understudied compounds like Nafion BP2 and 7:3 FTCA, two compounds that were identified in a significant portion of samples within this study. More focus should likewise be paid to deducing sublethal impacts of PFAS on wildlife, given the suite of relationships identified here between concentrations of specific PFAS and morphometric measurements. Continued attention to PFAS in wildlife over time ultimately stands to benefit humans and our own understanding of PFAS trends, as we continue to use air, water, and food resources subject to the same ambient environmental exposures as wildlife. 
ASSOCIATED CONTENT

NA

AUTHOR INFORMATION

Corresponding Author

*Anna Ruth Robuck, annarobuck@gmail.com

Author Contributions

The manuscript was primarily drafted by A. Robuck and finalized through contributions of all authors. Sample analysis and data interpretation were carried out by A. Robuck, alongside contributions of all authors. All authors have given approval to the final version of the manuscript.

\section{Funding Sources}

A. Robuck acknowledges support from the National Oceanic and Atmospheric Administration Dr. Nancy Foster Scholarship program (NOAA Award Number NA17NOS4290028), the Robert and Patricia Switzer Foundation, the STEEP Superfund Research Program (NIEHS Award Number P42ES027706), and the Oak Ridge Institute for Science and Education (ORISE) program.

Competing Interests

The authors have no competing interests to declare.

\section{ACKNOWLEDGMENTS}

The authors are indebted to the Northeast Fisheries Observer Program, Gina Shield, and Stephanie DePasquale for their assistance obtaining samples used in this study. Although NOAA and EPA employees contributed to this article, this 
research was conceived, designed, and implemented by URI. Consequently, the views, interpretations, and conclusions expressed in the article are solely those of the authors and do not necessarily reflect or represent NOAA views or policies. 


\section{LITERATURE CITED}

(1) Buck, R. C.; Franklin, J.; Berger, U.; Conder, J. M.; Cousins, I. T.; Voogt, P. De; Jensen, A. A.; Kannan, K.; Mabury, S. A.; van Leeuwen, S. P. J.

Perfluoroalkyl and Polyfluoroalkyl Substances in the Environment: Terminology, Classification, and Origins. Integr. Environ. Assess. Manag. 2011, 7 (4), 513-541. https://doi.org/10.1002/ieam.258.

(2) Glüge, J.; Scheringer, M.; Cousins, I. T.; DeWitt, J. C.; Goldenman, G.; Herzke, D.; Lohmann, R.; Ng, C. A.; Trier, X.; Wang, Z. An Overview of the Uses of Per- and Polyfluoroalkyl Substances (PFAS). Environ. Sci. Process.

Impacts 2020. https://doi.org/10.1039/d0em00291g.

(3) Muir, D.; Bossi, R.; Carlsson, P.; Evans, M.; De Silva, A.; Halsall, C.; Rauert, C.; Herzke, D.; Hung, H.; Letcher, R.; Rigét, F.; Roos, A. Levels and Trends of Poly- and Perfluoroalkyl Substances in the Arctic Environment - An Update. Emerg. Contam. 2019, 5, 240-271. https://doi.org/10.1016/j.emcon.2019.06.002.

(4) Zhang, X.; Lohmann, R.; Dassuncao, C.; Hu, X. C.; Weber, A. K.; Vecitis, C. D.; Sunderland, E. M. Source Attribution of Poly- and Perfluoroalkyl Substances (PFASs) in Surface Waters from Rhode Island and the New York Metropolitan Area. Environ. Sci. Technol. Lett. 2016, 3 (9), 316-321. https://doi.org/10.1021/acs.estlett.6b00255.

(5) Zhang, X.; Lohmann, R.; Sunderland, E. M. Poly- and Perfluoroalkyl Substances (PFAS) in Seawater and Plankton from the Northwestern Atlantic Margin. Environ. Sci. Technol. 2019, 53 (21), 12348-12356. https://doi.org/10.1021/acs.est.9b03230.

(6) Hu, X. C.; Andrews, D. Q.; Lindstrom, A. B.; Bruton, T. A.; Schaider, L. A.; Grandjean, P.; Lohmann, R.; Carignan, C. C.; Blum, A.; Balan, S. A.; Higgins, C. P.; Sunderland, E. M. Detection of Poly- and Per Fl Uoroalkyl Substances (PFASs) in U.S. Drinking Water Linked to Industrial Sites, Military Fire Training Areas, and Wastewater Treatment Plants. 2016. https://doi.org/10.1021/acs.estlett.6b00260.

(7) Strynar, M.; Dagnino, S.; McMahen, R.; Liang, S.; Lindstrom, A.; Andersen, E.; McMillan, L.; Thurman, M.; Ferrer, I.; Ball, C. Identification of Novel Perfluoroalkyl Ether Carboxylic Acids (PFECAs) and Sulfonic Acids (PFESAs) in Natural Waters Using Accurate Mass Time-of-Flight Mass Spectrometry (TOFMS). Environ. Sci. Technol. 2015, 49 (19), 11622-11630. https://doi.org/10.1021/acs.est.5b01215.

(8) Houtz, E. F.; Sutton, R.; Park, J.; Sedlak, M. Poly- and per Fl Uoroalkyl Substances in Wastewater : Signi Fi Cance of Unknown Precursors, 
Manufacturing Shifts, and Likely AFFF Impacts. Water Res. 2016, 95, 142-149. https://doi.org/10.1016/j.watres.2016.02.055.

(9) Lanza, H. A.; Cochran, R. S.; Mudge, J. F.; Olson, A. D.; Blackwell, B. R.; Maul, J. D.; Salice, C. J.; Anderson, T. A. Temporal Monitoring of Perfluorooctane Sulfonate Accumulation in Aquatic Biota Downstream of Historical Aqueous Film Forming Foam Use Areas. Environ. Toxicol. Chem. 2017, 36 (8), 2022-2029. https://doi.org/10.1002/etc.3726.

(10) Yamashita, N.; Taniyasu, S.; Petrick, G.; Wei, S.; Gamo, T.; Lam, P. K. S.; Kannan, K. Perfluorinated Acids as Novel Chemical Tracers of Global Circulation of Ocean Waters. Chemosphere 2008, 70 (7), 1247-1255. https://doi.org/10.1016/j.chemosphere.2007.07.079.

(11) Dassuncao, C.; Hu, X. C.; Zhang, X.; Bossi, R.; Dam, M.; Mikkelsen, B.; Sunderland, E. M. Temporal Shifts in Poly- and Perfluoroalkyl Substances (PFASs) in North Atlantic Pilot Whales Indicate Large Contribution of Atmospheric Precursors. Environ. Sci. Technol. 2017, 51 (8), 4512-4521. https://doi.org/10.1021/acs.est.7b00293.

(12) Robuck, A.; Cantwell, M. G.; McCord, J. P.; Addison, L. M.; Pfohl, M.; Strynar, M. J.; McKinney, R. A.; Katz, D. R.; Wiley, D. N.; Lohmann, R. Legacy and Novel Per- and Polyfluoroalkyl Substances (PFAS) in Juvenile Seabirds from the US Atlantic Coast. Environ. Sci. Technol. 2020. https://doi.org/10.1021/acs.est.0c01951.

(13) White, N. D.; Vena, J. E.; Kannan, K.; Karthikraj, R.; Wolf, B.; Fair, P. A.; Arnott, S. A. Perfluoroalkyl Substances (PFASs) in Edible Fish Species from Charleston Harbor and Tributaries, South Carolina, United States: Exposure and Risk Assessment. Environ. Res. 2019, 171 (November 2018), 266-277. https://doi.org/10.1016/j.envres.2019.01.021.

(14) Loi, E. I. H.; Yeung, L. W. Y.; Taniyasu, S.; Lam, P. K. S.; Kannan, K.; Yamashita, N. Trophic Magnification of Poly- and Perfluorinated Compounds in a Subtropical Food Web. Environ. Sci. Technol. 2011, 45 (13), 5506-5513. https://doi.org/10.1021/es200432n.

(15) Tomy, G. T.; Pleskach, K.; Ferguson, S. H.; Hare, J.; Stern, G.; Macinnis, G.; Marvin, C. H.; Loseto, L. Trophodynamics of Some PFCs and BFRs in a Western Canadian Arctic Marine Food Web. Environ. Sci. Technol. 2009, 43 (11), 4076-4081. https://doi.org/10.1021/es900162n.

(16) Kelly, B. C.; Ikonomou, M. G.; Blair, J. D.; Surridge, B.; Hoover, D.; Grace, R.; Gobas, F. A. P. . Perfluoroalkyl Contaminants in an Arctic Marine Food Web: Trophic Magnification and Wildlife Exposure. Environ. Sci. Technol. 2009, 43 (15), 6112. https://doi.org/10.1021/es9013079. 
(17) Ng, C. A.; Hungerbühler, K. Bioaccumulation of Perfluorinated Alkyl Acids: Observations and Models. Environ. Sci. Technol. 2014, 48 (9), 4637-4648. https://doi.org/10.1021/es404008g.

(18) Dassuncao, C.; Pickard, H.; Pfohl, M.; Tokranov, A. K.; Li, M.; Mikkelsen, B.; Slitt, A.; Sunderland, E. M. Phospholipid Levels Predict the Tissue Distribution of Poly- and Perfluoroalkyl Substances in a Marine Mammal.

Environ. Sci. Technol. Lett. 2019, 6(3), 119-125.

https://doi.org/10.1021/acs.estlett.9b00031.

(19) Reiner, J. J. L.; Place, B. L. Perfluorinated Alkyl Acids in Wildlife. In Toxicological Effects of Perfluoroalkyl and Polyfluoralkyl Substances; DeWitt, J. C., Ed.; Humana Press, 2015; pp 127-150. https://doi.org/https://doi.org/10.1007/978-3-319-15518-0_5.

(20) Wang, Z.; Dewitt, J. C.; Higgins, C. P.; Cousins, I. T. A Never-Ending Story of Per- and Polyfluoroalkyl Substances (PFASs)? Environ. Sci. Technol. 2017, 51 (5), 2508-2518. https://doi.org/10.1021/acs.est.6b04806.

(21) Liu, Y.; Agostino, L. A. D.; Qu, G.; Jiang, G.; Martin, J. W. HighResolution Mass Spectrometry (HRMS) Methods for Nontarget Discovery and Characterization of Poly- and Per-Fluoroalkyl Substances (PFASs) in Environmental and Human Samples. Trends Anal. Chem. 2019. https://doi.org/10.1016/j.trac.2019.02.021.

(22) Powers, K. D.; Wiley, D. N.; Robuck, A. R.; Olson, Z. H.; Welch, L. J.; Thompson, M. A.; Kaufman, L. Spatiotemporal Characterization of NonBreeding Great Shearwaters Ardenna Gravis within Their Wintering Range. Mar. Ornithol. 2020, 48, 215-229.

(23) Cuthbert, R. J. Breeding Biology, Chick Growth and Provisioning of Great Shearwaters (Puffinus Gravis) at Gough Island, South Atlantic Ocean. Етu 2005, 105 (4), 305-310. https://doi.org/10.1071/MU05036.

(24) Roscales, J. L.; Vicente, A.; Ryan, P. G.; González-Solís, J.; Jiménez, B. Spatial and Interspecies Heterogeneity in Concentrations of Perfluoroalkyl Substances (PFASs) in Seabirds of the Southern Ocean. Environ. Sci. Technol. 2019, 53 (16), 9855-9865. https://doi.org/10.1021/acs.est.9b02677.

(25) van Franeker, J. A. Save the North Sea Fulmar-Litter-EcoQO Manual Part 1: Collection and Dissection Procedures. Alterra-rapport 2004, 672, 1-38.

(26) Spaan, K. M.; Van Noordenburg, C.; Plassmann, M. M.; Schultes, L.; Shaw, S.; Berger, M.; Heide-Jørgensen, M. P.; Rosing-Asvid, A.; Granquist, S. M.; Dietz, R.; Sonne, C.; Rigét, F.; Roos, A.; Benskin, J. P. Fluorine Mass Balance and Suspect Screening in Marine Mammals from the Northern 
Hemisphere. Environ. Sci. Technol. 2020, 54 (7), 4046-4058.

https://doi.org/10.1021/acs.est.9b06773.

(27) Kannan, K.; Choi, J. W.; Iseki, N.; Senthilkumar, K.; Hoon, D.;

Masunaga, S.; Giesy, J. P.; Kim, D. H.; Masunaga, S.; Giesy, J. P. Concentrations of Perfluorinated Acids in Livers of Birds from Japan and Korea. Chemosphere 2002, 49 (3), 225-231. https://doi.org/10.1016/S0045-6535(02)00304-1.

(28) Holmström, K. E.; Berger, U. Tissue Distribution of Perfluorinated Surfactants in Common Guillemot (Uria Aalge) from the Baltic Sea. Environ. Sci. Technol. 2008, 42 (16), 5879-5884. https://doi.org/10.1021/es800529h.

(29) Guruge, K. S.; Yeung, L. W. Y.; Li, P.; Taniyasu, S.; Yamashita, N.; Nakamura, M. Fluorinated Alkyl Compounds Including Long Chain Carboxylic Acids in Wild Bird Livers from Japan. Chemosphere 2011, 83 (3), 379-384. https://doi.org/10.1016/j.chemosphere.2010.12.010.

(30) R Core Team. R: A Language and Environment for Statistical Computing. R Foundation for Statistical Computing: Vienna, Austria 2020.

(31) Miller, A.; Elliott, J. E.; Elliott, K. H.; Lee, S.; Cyr, F. Temporal Trends of Perfluoroalkyl Substances (PFAS) in Eggs of Coastal and Offshore Birds: Increasing PFAS Levels Associated with Offshore Bird Species Breeding on the Pacific Coast of Canada and Wintering near Asia. Environ. Toxicol. Chem. 2015, 34 (8), 1799-1808. https://doi.org/10.1002/etc.2992.

(32) Leat, E. H. K.; Bourgeon, S.; Eze, J. I.; Muir, D. C. G.; Williamson, M.; Bustnes, J. O.; Furness, R. W.; Borgå, K. Perfluoroalkyl Substances in Eggs and Plasma of an Avian Top Predator, Great Skua (Stercorarius Skua), in the North Atlantic. Environ. Toxicol. Chem. 2013, 32 (3), 569-576.

https://doi.org/10.1002/etc.2101.

(33) Chu, S.; Wang, J.; Leong, G.; Woodward, L. A.; Letcher, R. J.; Li, Q. X. Perfluoroalkyl Sulfonates and Carboxylic Acids in Liver, Muscle and Adipose Tissues of Black-Footed Albatross (Phoebastria Nigripes) from Midway Island, North Pacific Ocean. Chemosphere 2015, 138, 60-66. https://doi.org/10.1016/j.chemosphere.2015.05.043.

(34) Kudo, N.; Katakura, M.; Sato, Y.; Kawashima, Y. Sex HormoneRegulated Renal Transport of Perfluorooctanoic Acid. Chem. Biol. Interact. 2002, 139 (3), 301-316. https://doi.org/10.1016/S0009-2797(02)00006-6.

(35) Newsted, J. L.; Coady, K. K.; Beach, S. A.; Butenhoff, J. L.; Gallagher, S.; Giesy, J. P. Effects of Perfluorooctane Sulfonate on Mallard and Northern Bobwhite Quail Exposed Chronically via the Diet. Environ. Toxicol. Pharmacol. 2007, 23 (1), 1-9. https://doi.org/10.1016/j.etap.2006.04.008. 
(36) Newsted, J. L.; Beach, S. A.; Gallagher, S. P.; Giesy, J. P.

Pharmacokinetics and Acute Lethality of Perfluorooctanesulfonate (PFOS) to Juvenile Mallard and Northern Bobwhite. Arch. Environ. Contam. Toxicol. 2006, 50 (3), 411-420. https://doi.org/10.1007/s00244-005-1137-x.

(37) Robuck, A. R.; Cantwell, M. G.; McCord, J. P.; Addison, L. M.; Pfohl, M.; Strynar, M. J.; McKinney, R.; Katz, D. R.; Wiley, D. N.; Lohmann, R. Legacy and Novel Per- and Polyfluoroalkyl Substances in Juvenile Seabirds from the U.S. Atlantic Coast. Environ. Sci. \&amp; Technol. 2020, 54 (20). https://doi.org/10.1021/acs.est.0c01951.

(38) Hector, J. A. L.; Pickering, S. P. C.; Croxall, J. P.; Follett, B. K. The Endocrine Basis of Deferred Sexual Maturity in the Wandering Albatross, Diomedea Exulans. L. Funct. Ecol. 1990, 4 (1), 59. https://doi.org/10.2307/2389653.

(39) Tarazona, J. V.; Rodríguez, C.; Alonso, E.; Sáez, M.; González, F.; San Andrés, M. D.; Jiménez, B.; San Andrés, M. I. Toxicokinetics of Perfluorooctane Sulfonate in Birds under Environmentally Realistic Exposure Conditions and Development of a Kinetic Predictive Model. Toxicol. Lett. 2015, 232 (2), 363368. https://doi.org/10.1016/j.toxlet.2014.11.022.

(40) Heuvel, J. P. V.; Kuslikis, B. I.; Van Rafelghem, M. J.; Peterson, R. E. Tissue Distribution, Metabolism, and Elimination of Perfluorooctanoic Acid in Male and Female Rats. J. Biochem. Toxicol. 1991, 6 (2), 83-92. https://doi.org/10.1002/jbt.2570060202.

(41) Guillette, T. C.; McCord, J.; Guillette, M.; Polera, M. E.; Rachels, K. T.; Morgeson, C.; Kotlarz, N.; Knappe, D. R. U.; Reading, B. J.; Strynar, M.; Belcher, S. M. Per- and Polyfluoroalkyl Substances Exposure in Cape Fear River Striped Bass (Morone Saxatilis) Is Associated with Biomarkers of Altered Immune and Liver Function Research. Environ. Int. 2019, 2 (1), 105358. https://doi.org/10.1016/j.envint.2019.105358.

(42) Kotlarz, N.; McCord, J.; Collier, D.; Lea, C.; Strynar, M.; Lindstron, A.; Wilkie, A.; Islam, J.; Matney, K.; Tarte, P.; Polera, M.; Burdette, K.; DeWitt, J.; May, K.; Smart, R.; Knappe, D.; Hoppin, J. A. Measurement of Novel, Drinking Water-Associated PFAS in Blood from Adults and Children in Wilmington, North Carolina. Env. Heal. Perspect 2020, 128 (July), 1-12.

(43) Hopkins, Z. R.; Sun, M.; DeWitt, J. C.; Knappe, D. R. U. Recently Detected Drinking Water Contaminants : GenX and Other Per- and Polyfluoroalkyl Ether Acids. J. AWWA 2018, 110 (7), 13-28. https://doi.org/10.1002/awwa.1073. 
(44) Loi, E. I. H.; Yeung, L. W. Y.; Taniyasu, S.; Lam, P. K. S.; Kannan, K.; Yamashita, N. Trophic Magnification of Poly- and Perfluorinated Compounds in a Subtropical Food Web. Environ. Sci. Technol. 2011, 45 (13), 5506-5513. https://doi.org/10.1021/es200432n.

(45) Eriksson, U.; Roos, A.; Lind, Y.; Hope, K.; Ekblad, A.; Kärrman, A. Comparison of PFASs Contamination in the Freshwater and Terrestrial Environments by Analysis of Eggs from Osprey (Pandion Haliaetus), Tawny Owl (Strix Aluco), and Common Kestrel (Falco Tinnunculus). Environ. Res. 2016, 149, 40-47. https://doi.org/10.1016/j.envres.2016.04.038.

(46) Szabo, D.; Lavers, J. L.; Shimeta, J.; Green, M. P.; Mulder, R. A.; Clarke, B. Correlations between Per- and Polyfluoroalkyl Substances (PFASs) and Body Morphometrics in Fledgling Shearwaters Impacted by Plastic Consumption from a Remote Pacific Island. Environ. Toxicol. Chem. 2021, Accepted. https://doi.org/10.1016/s0740-5472(96)90021-5.

(47) Butt, C. M.; Muir, D. C. G.; Mabury, S. A. Biotransformation Pathways of Fluorotelomer-Based Polyfluoroalkyl Substances: A Review. Environ. Toxicol. Chem. 2014, 33 (2), 243-267. https://doi.org/10.1002/etc.2407.

(48) D'Eon, J. C.; Mabury, S. A. Production of Perfluorinated Carboxylic Acids (PFCAs) from the Biotransformation of Polyfluoroalkyl Phosphate Surfactants (PAPS): Exploring Routes of Human Contamination. Environ. Sci. Technol. 2007, 41 (13), 4799-4805. https://doi.org/10.1021/es070126x.

(49) Fisk, A. T.; Hobson, K. A.; Norstrom, R. J. Influence of Chemical and Biological Factors on Trophic Transfer of Persistent Organic Pollutants in the Northwater Polynya Marine Food Web. Environ. Sci. Technol. 2001, 35 (4), 732 738. https://doi.org/10.1021/es001459w.

(50) Kotthoff, M.; Müller, J.; Jürling, H.; Schlummer, M.; Fiedler, D. Perfluoroalkyl and Polyfluoroalkyl Substances in Consumer Products. Environ. Sci. Pollut. Res. 2015, 22 (19), 14546-14559. https://doi.org/10.1007/s11356015-4202-7.

(51) Wang, Z.; Scheringer, M.; MacLeod, M.; Bogdal, C.; Müller, C. E.; Gerecke, A. C.; Hungerbühler, K. Atmospheric Fate of Poly- and Perfluorinated Alkyl Substances (PFASs): II. Emission Source Strength in Summer in Zurich, Switzerland. Environ. Pollut. 2012, 169, 204-209. https://doi.org/10.1016/j.envpol.2012.03.037.

(52) Chen, C.; Wang, J.; Li, L.; Xu, W.; Liu, J. Comparison of Fluorotelomer Alcohol Emissions from Wastewater Treatment Plants into Atmospheric and Aquatic Environments. Environ. Int. 2020, 139 (March). https://doi.org/10.1016/j.envint.2020.105718. 
(53) Wong, F.; Shoeib, M.; Katsoyiannis, A.; Eckhardt, S.; Stohl, A.; BohlinNizzetto, P.; Li, H.; Fellin, P.; Su, Y.; Hung, H. Assessing Temporal Trends and Source Regions of Per- and Polyfluoroalkyl Substances (PFASs) in Air under the Arctic Monitoring and Assessment Programme (AMAP). Atmos. Environ. 2018, 172 (June 2017), 65-73. https://doi.org/10.1016/j.atmosenv.2017.10.028.

(54) Gawor, A.; Shunthirasingham, C.; Hayward, S. J.; Lei, Y. D.; Gouin, T.; Mmereki, B. T.; Masamba, W.; Ruepert, C.; Castillo, L. E.; Shoeib, M.; Lee, S. C.; Harner, T.; Wania, F. Neutral Polyfluoroalkyl Substances in the Global Atmosphere. Environ. Sci. Process. Impacts 2014, 16 (3), 404-413. https://doi.org/10.1039/c3em00499f.

(55) Phillips, M. M.; Dinglasan-Panlilio, M. J. A.; Mabury, S. A.; Solomon, K. R.; Sibley, P. K. Fluorotelomer Acids Are More Toxic than Perfluorinated Acids. Environ. Sci. Technol. 2007, 41 (20), 7159-7163. https://doi.org/10.1021/es070734c.

(56) Prevedouros, K.; Cousins, I. T.; Buck, R. C.; Korzeniowski, S. H. Sources, Fate and Transport of Perfluorocarboxylates. Environ. Sci. Technol. 2006, 40 (1), 32-44. https://doi.org/10.1021/es0512475.

(57) Ellis, D. A.; Martin, J. W.; Silva, A. O. D. E.; Hurley, M. D.; De Silva, A. O.; Mabury, S. A.; Hurley, M. D.; Sulbaek Andersen, M. P.; Wallington, T. J. Degradation of Fluorotelomer Alcohols: A Likely Atmospheric Source of Perfluorinated Carboxylic Acids. Environ. Sci. Technol. 2004, 38 (12), 33163321. https://doi.org/10.1021/es049860w.

(58) McCord, J. P.; Strynar, M. J.; Washington, J. W.; Bergman, E. L.; Goodrow, S. M. Emerging Chlorinated Polyfluorinated Polyether Compounds Impacting the Waters of Southwestern New Jersey Identified by Use of Nontargeted Analysis. Environ. Sci. Technol. Lett. 2020. https://doi.org/10.1021/acs.estlett.0c00640.

(59) Zhang, C.; Hopkins, Z. R.; Mccord, J.; Strynar, M. J.; Detlef, R. U.; Knappe, D. R. U. Fate of Per- and Polyfluoroalkyl Ether Acids in the Total Oxidizable Precursor Assay and Implications for the Analysis of Impacted Water. Environ. Sci. Technol. Lett. 2019. https://doi.org/10.1021/acs.estlett.9b00525.

(60) Jouanneau, W.; Bårdsen, B.-J.; Herzke, D.; Johnsen, T. V.; Eulaers, I.; Bustnes, J. O. Spatiotemporal Analysis of Perfluoroalkyl Substances in WhiteTailed Eagle (Haliaeetus Albicilla) Nestlings from Northern Norway-A TenYear Study. Environ. Sci. Technol. 2020, 54 (8), 5011-5020. https://doi.org/10.1021/acs.est.9b06818.

(61) Route, W. T.; Key, R. L.; Russell, R. E.; Lindstrom, A. B.; Strynar, M. J. Spatial and Temporal Patterns in Concentrations of Perfluorinated Compounds in 
Bald Eagle Nestlings in the Upper Midwestern United States. Environ. Sci. Technol. 2014, 48 (12), 6653-6660. https://doi.org/10.1021/es501055d.

(62) Hobson, K. A.; Piatt, J. F.; Pitocchelli, J. Using Stable Isotopes to Determine Seabird Trophic Relationships. J. Anim. Ecol. 1994, 63 (4), 786-798.

(63) Ackerman, J. T.; Eagles-Smith, C. A.; Herzog, M. P.; Hartman, C. A.; Peterson, S. H.; Evers, D. C.; Jackson, A. K.; Elliott, J. E.; Vander Pol, S. S.; Bryan, C. E. Avian Mercury Exposure and Toxicological Risk across Western North America: A Synthesis. Sci. Total Environ. 2016, 568, 749-769. https://doi.org/10.1016/j.scitotenv.2016.03.071.

(64) Tartu, S.; Gabrielsen, G. W.; Blévin, P.; Ellis, H.; Bustnes, J. O.; Herzke, D.; Chastel, O. Endocrine and Fitness Correlates of Long-Chain Perfluorinated Carboxylates Exposure in Arctic Breeding Black-Legged Kittiwakes. Environ. Sci. Technol. 2014, 48 (22), 13504-13510. https://doi.org/10.1021/es503297n.

(65) Hull, K. L.; Cockrem, J. F.; Bridges, J. P.; Candy, E. J.; Davidson, C. M. Effects of Corticosterone Treatment on Growth, Development, and the Corticosterone Response to Handling in Young Japanese Quail (Coturnix Coturnix Japonica). Comp. Biochem. Physiol. - A Mol. Integr. Physiol. 2007, 148 (3), 531-543. https://doi.org/10.1016/j.cbpa.2007.06.423.

(66) Costantini, D.; Blévin, P.; Herzke, D.; Moe, B.; Wing, G.; Ove, J.; Chastel, O.; Gabrielsen, G. W.; Bustnes, J. O.; Chastel, O. Higher Plasma Oxidative Damage and Lower Plasma Antioxidant Defences in an Arctic Seabird Exposed to Longer Perfluoroalkyl Acids. Environ. Res. 2019, 168 (October 2018), 278-285. https://doi.org/10.1016/j.envres.2018.10.003.

(67) Geng, D.; Musse, A. A.; Wigh, V.; Carlsson, C.; Engwall, M.; Orešič, M.; Scherbak, N.; Hyötyläinen, T. Effect of Perfluorooctanesulfonic Acid (PFOS) on the Liver Lipid Metabolism of the Developing Chicken Embryo. Ecotoxicol. Environ. Saf. 2019, 170 (October 2018), 691-698.

https://doi.org/10.1016/j.ecoenv.2018.12.040.

(68) Fitzgerald, N. J. M.; Wargenau, A.; Sorenson, C.; Pedersen, J.; Tufenkji, N.; Novak, P. J.; Simcik, M. F. Partitioning and Accumulation of Perfluoroalkyl Substances in Model Lipid Bilayers and Bacteria. Environ. Sci. Technol. 2018, 52 (18), 10433-10440. https://doi.org/10.1021/acs.est.8b02912. 


\section{TABLES AND FIGURES}

\begin{tabular}{|c|c|c|c|c|c|}
\hline Compound & Family & $\begin{array}{c}\text { \# Fluorinated } \\
\text { Carbons }\end{array}$ & $\begin{array}{c}\% \\
\text { Detection } \\
\end{array}$ & Mean \pm SE & Range \\
\hline N-MeFOSAA & FASAA & 8 & 0 & - & - \\
\hline $\mathrm{N}$ - EtFOSAA & FASAA & 8 & 0 & - & - \\
\hline FOSA & FASA & 8 & 83 & $0.96 \pm 0.08$ & $0-3.7$ \\
\hline 4:2 FTS & FTS & 4 & 0 & - & - \\
\hline 6:2 FTS & FTS & 6 & 0 & - & - \\
\hline 8:2 FTS & FTS & 8 & 0 & - & - \\
\hline PFBA & PFCA & 3 & $\mathrm{nr}$ & - & - \\
\hline PFPeA & PFCA & 4 & 0 & - & - \\
\hline PFHxA & PFCA & 5 & 9 & $0.24 \pm 0.03$ & $0-1.3$ \\
\hline PFHpA & PFCA & 6 & 60 & $0.28 \pm 0.04$ & $0-2.2$ \\
\hline PFOA & PFCA & 7 & 95 & $0.30 \pm 0.04$ & $0-2.6$ \\
\hline PFNA & PFCA & 8 & 99 & $2.2 \pm 0.13$ & $0-6.8$ \\
\hline PFDA & PFCA & 9 & 99 & $2.4 \pm 0.11$ & $0-6.6$ \\
\hline PFUnDA & PFCA & 10 & 99 & $7.45 \pm 0.38$ & $0-24$ \\
\hline PFDoA & PFCA & 11 & 99 & $3.1 \pm 0.18$ & $0-10$ \\
\hline PFTrDA & PFCA & 12 & 75 & $2.62 \pm 0.17$ & $0-8$ \\
\hline PFTeDA & PFCA & 13 & 60 & $1.73 \pm 0.13$ & $0-5$ \\
\hline 7:3 FTCA & FTCA & 7 & 64 & $6.54 \pm 1.01$ & $0-62$ \\
\hline HFPO-DA & PFECA & 5 & 0 & - & - \\
\hline Nafion BP2 & PFESA & 7 & 36 & $0.04 \pm 0.008$ & $0-0.2$ \\
\hline PFBS & PFSA & 4 & $\mathrm{nr}$ & - & - \\
\hline PFPeS & PFSA & 5 & 20 & - & - \\
\hline PFHxS & PFSA & 6 & 73 & $1.32 \pm 0.10$ & $0-5$ \\
\hline PFHpS & PFSA & 7 & 43 & $0.52 \pm 0.05$ & $0-2$ \\
\hline PFOS & PFSA & 8 & 99 & $38 \pm 2.6$ & $0-111$ \\
\hline PFNS & PFSA & 9 & 57 & $0.18 \pm 0.02$ & $0-0.9$ \\
\hline PFDS & PFSA & 10 & 41 & $0.09 \pm 0.03$ & $0-0.3$ \\
\hline
\end{tabular}

Table 3.1. Detection frequency, arithmetic mean, standard error, and range of concentrations included in this study. PFBA and PFBS were not reported, or "nr" due to chromatographic imprecision. Family designations are after Buck et al. 2011. 

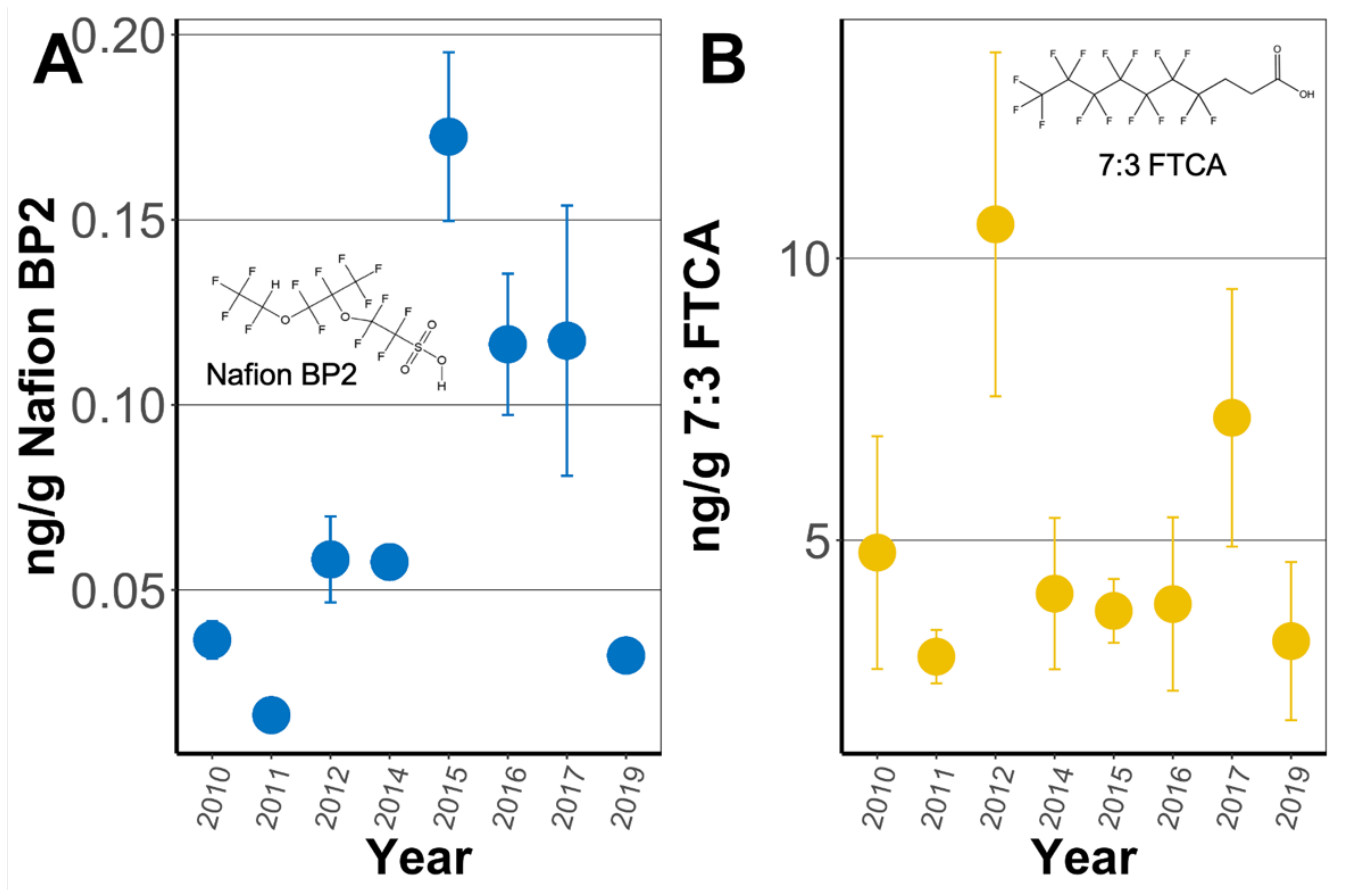

Figure 3.1. Mean concentrations of A) Nafion BP2 and B) 7:3 FTCA in ng/g wet weight over time, with bars representing standard error of the mean. 


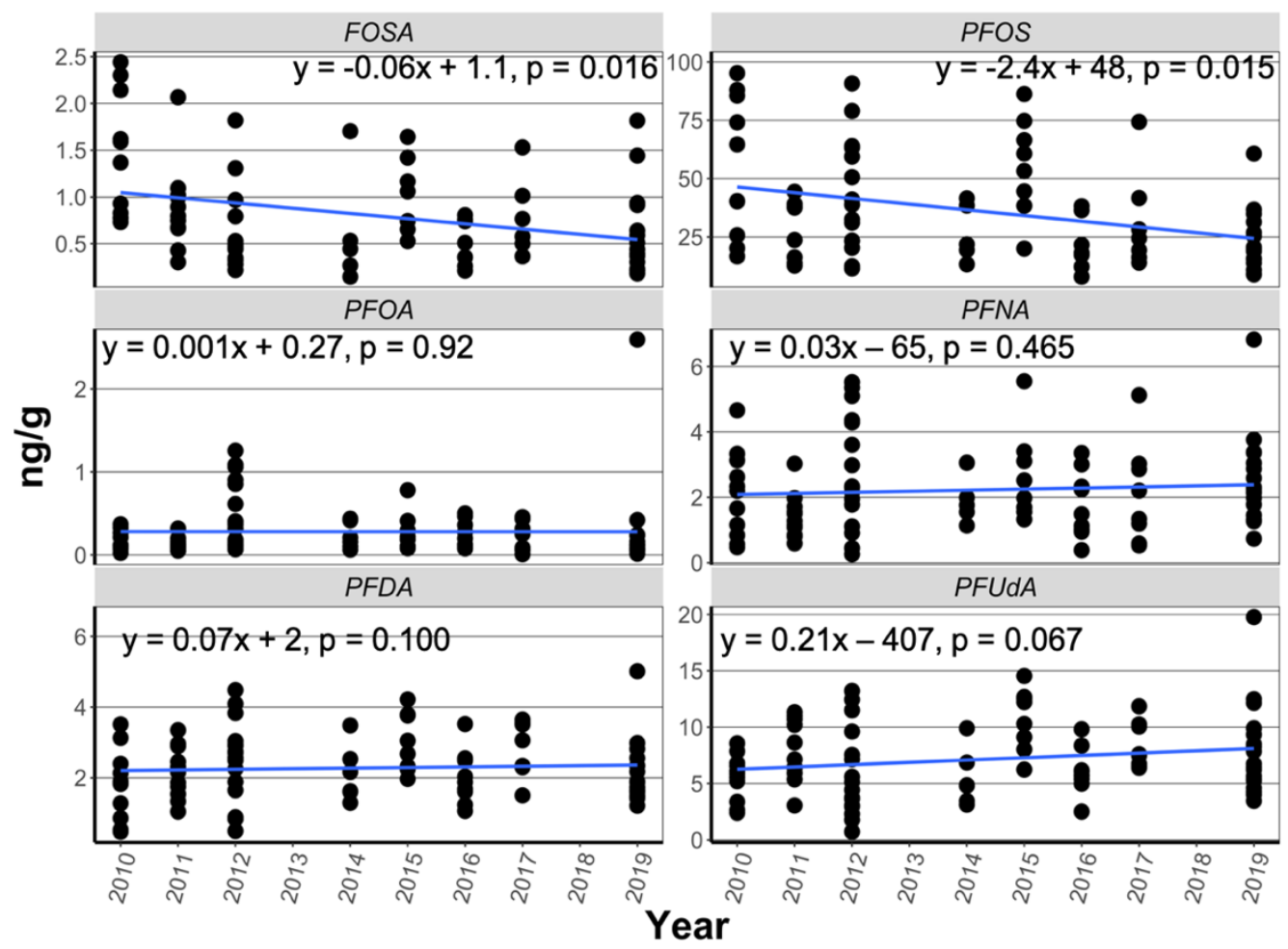

Figure 3.2. Concentrations of six PFAS over 2010 - 2019; the blue line represents a linear model using year as the sole predictor to deduce any time trends. Only compounds with a detection frequency above $80 \%$ were meaningfully assessed for temporal trends. 


\begin{tabular}{|c|c|c|c|c|}
\hline $\begin{array}{c}\text { Biological/chemical } \\
\text { parameter }\end{array}$ & $\begin{array}{l}\text { Associated } \\
\text { compound(s) }\end{array}$ & $\begin{array}{c}\text { Rho } \\
\text { estimate }\end{array}$ & $p$-value & $\begin{array}{l}\text { Strength of } \\
\text { association }\end{array}$ \\
\hline \multirow{2}{*}{$\mathrm{d} 15 \mathrm{~N}$} & 7:3 FTCA & -0.332 & 0.037 & Weak - Moderate \\
\hline & PFHxS & -0.244 & 0.050 & Weak \\
\hline \multirow{2}{*}{ Wet Weight (g) } & 7:3 FTCA & -0.309 & 0.018 & Weak - Moderate \\
\hline & FOSA & 0.260 & 0.018 & Weak \\
\hline \multirow{7}{*}{ Liver Weight (g) } & 7:3 FTCA & -0.409 & 0.001 & Moderate \\
\hline & PFDA & -0.230 & 0.016 & Weak \\
\hline & PFDoA & -0.275 & 0.004 & Weak \\
\hline & PFHxS & -0.309 & 0.006 & Weak - Moderate \\
\hline & PFOS & -0.276 & 0.012 & Weak \\
\hline & PFtrDA & -0.263 & 0.077 & Weak \\
\hline & PFUdA & -0.274 & 0.004 & Weak \\
\hline \multirow{6}{*}{ Kidney Weight (g) } & 7:3 FTCA & -0.364 & 0.005 & Weak - Moderate \\
\hline & PFDA & -0.247 & 0.011 & Weak \\
\hline & PFDoA & -0.286 & 0.003 & Weak \\
\hline & PFNA & -0.210 & 0.033 & Weak \\
\hline & PFOA & -0.217 & 0.030 & Weak \\
\hline & PFUdA & -0.304 & 0.002 & Weak - Moderate \\
\hline \multirow{2}{*}{ d13C } & FOSA & 0.248 & 0.045 & Weak \\
\hline & PFOS & 0.285 & 0.021 & Weak \\
\hline \multirow{2}{*}{$\mathrm{Hg}$} & FOSA & 0.339 & 0.014 & Weak - Moderate \\
\hline & PFOS & 0.239 & 0.095 & Weak \\
\hline \multirow{5}{*}{ Tarsus } & PFDoA & -0.318 & 0.001 & Weak - Moderate \\
\hline & PFHxS & -0.218 & 0.054 & Weak \\
\hline & PFOS & -0.266 & 0.016 & Weak \\
\hline & PFtrDA & -0.400 & 0.006 & Moderate \\
\hline & PFUdA & -0.294 & 0.002 & Weak \\
\hline \multirow{4}{*}{ Body Condition Index } & PFDoA & 0.210 & 0.030 & Weak \\
\hline & PFOS & 0.255 & 0.022 & Weak \\
\hline & PFtrDA & 0.418 & 0.004 & Moderate \\
\hline & PFUdA & 0.260 & 0.007 & Weak \\
\hline \multirow{4}{*}{ Fat Depth (mm) } & PFDoA & -0.301 & 0.003 & Weak - Moderate \\
\hline & PFHxS & -0.263 & 0.023 & Weak \\
\hline & PFtrDA & -0.397 & 0.008 & Weak - Moderate \\
\hline & PFUdA & -0.284 & 0.005 & Weak \\
\hline \multirow{2}{*}{ Wing Chord (cm) } & PFHpS & 0.292 & 0.079 & Weak \\
\hline & PFtrDA & 0.279 & 0.066 & Weak \\
\hline Plastic Count & PFHxS & -0.376 & 0.001 & Weak - Moderate \\
\hline \multirow{2}{*}{ Mass of Plastic (mg) } & PFHxS & -0.351 & 0.002 & Weak - Moderate \\
\hline & PFNA & -0.224 & 0.026 & Weak \\
\hline Head-Bill Length (cm) & PFHxS & -0.217 & 0.054 & Weak \\
\hline
\end{tabular}

Table 3.2. Table presenting significant associations identified via Spearman rho correlation analysis between continuous biological covariates and PFAS concentrations. Associations above the pre-determined significance level ( $p=$ 0.05 ) but below $p=0.1$ are highlighted in orange. 


\section{CHAPTER 4}

\section{TISSUE-SPECIFIC DISTRIBUTION OF LEGACY AND NOVEL PER- AND POLYFLUOROALKYL SUBSTANCES IN JUVENILE SEABIRDS}

This manuscript is in review at Environmental Science and Technology

Letters

Anna R. Robuck ${ }^{\dagger^{*}}$, James P. McCord ${ }^{\bigcirc}$, Mark J. Strynar ${ }^{\bigcirc}$, Mark G. Cantwell ${ }^{\ddagger}$, David N. Wiley", and Rainer Lohmann ${ }^{\dagger}$

† University of Rhode Island Graduate School of Oceanography, Narragansett, RI 02882

O US Environmental Protection Agency, Center for Environmental Measurement and Modeling, Durham, NC 27709

tUS Environmental Protection Agency, Center for Environmental Measurement and Modeling, Atlantic Coastal Environmental Sciences Division, Narragansett, RI 02882

' National Oceanic and Atmospheric Administration Stellwagen Bank National Marine Sanctuary, Scituate, MA 02066

KEYWORDS: PFOS, PFAS, seabirds, bioaccumulation, partitioning 


\section{ABSTRACT}

Of the thousands of per- and polyfluoroalkyl substances (PFAS) potentially in the environment, only few have been investigated in detail. Little is also known about their partitioning among multiple wildlife tissues and organs, which is needed to better predict the ecological risk PFAS may pose. In this study, we analyzed 36 legacy and emerging PFAS in up to 8 juvenile seabird tissues collected from three habitats along the US Eastern Seaboard (Massachusetts Bay, Narragansett Bay and Cape Fear River Estuary). PFOS was the dominant compound across multiple tissues, while long-chain PFCAs dominated in brain (mean $=44 \%$ of total concentrations). Emerging perfluoroalkyl ether acids (PFEAs) Nafion byproduct2 and PFO5DoDA were detected in greater than $90 \%$ of tissues from juveniles obtained from the Cape Fear River Estuary, a nesting region downstream from a major fluorochemical production site. This marks the first environmental detection of these compounds in multiple vertebrate organs, including the brain. PFEAs displayed a preference for partitioning into blood compared to liver, possibly due to the increased flexibility of albumin to accommodate multi-ether PFAS, while PFO5DoDA showed a reduced preference for brain. These results suggest future monitoring efforts and toxicological studies should focus on impacts of novel PFAS and long-chain PFCAs in multiple tissues beyond liver and blood, while exploring the unique binding mechanisms driving uptake of multi-ether PFEAs. 


\section{INTRODUCTION}

Global biomonitoring studies document the pervasive accumulation of perand polyfluoroalkyl substances (PFAS) in wildlife adjacent to human sources of PFAS $^{1-4}$ but also in animals from remote regions. The majority of these studies measured PFAS in select matrices only, such as whole body homogenate, serum, liver, feather, or egg, to evaluate hypotheses focused on spatial and temporal dynamics $^{5-7}$. The most commonly measured matrices often encompass tissues collected using non-invasive methods. A smaller subset of research has investigated the distributions of PFAS within multiple tissues of wild organisms, including tissues such as brain, lungs, heart, muscle, kidney, gonads, and adipose tissue (fat) ${ }^{8-14}$. In this study, we analyzed PFAS in 8 different seabird tissues. Knowing the abundance of PFAS in multiple tissues and organs is key to better understand PFAS internal partitioning, identify tissue reservoirs, and predict PFAS physiological and ecological risks.

Existing tissue-specific work demonstrates the unique PFAS partitioning behavior compared to legacy persistent organic pollutants such as polychlorinated biphenyls (PCBs). Verreault et al. (2005) examined glaucous gull plasma, liver, egg, and brain and determined perfluorooctanesulfonic acid (PFOS) concentrations were greatest in plasma, followed by approximately equal concentrations in liver and egg, with the lowest concentrations in the brain ${ }^{9}$. Investigations in herring gull and guillemots subsequently found liver to contain higher concentrations of PFOS compared to plasma or muscle ${ }^{10,15}$. Results from multiple taxa beyond avifauna demonstrate that blood, liver, and kidney contain 
the highest concentrations of multiple perfluoroalkyl acids (PFAAs) normalized by tissue weight, with some indication of preferential uptake of long-chain perfluoroalkyl carboxylic acids (PFCAs, $\mathrm{C}_{n} \mathrm{~F}_{2 n+1} \mathrm{COOH}, \mathrm{n} \geq 7$ ) in the brains of marine mammals ${ }^{8,14,16}$. This is in stark contrast to the behavior of hydrophobic organic chemicals like PCBs, which show a distinct preference for fatty tissue storage.

Beyond contrasting to legacy pollutants, tissue-specific measurements help constrain drivers of PFAS bioaccumulation. Empirical data from tissue-specific studies, modeling efforts, and data from controlled laboratory studies indicate interactions with specific proteins like albumin and liver fatty acid binding protein (L-FABP) determine the accumulation of various PFAS in liver, kidney, and blood matrices ${ }^{17,18}$. For instance, the L-FABP interaction with perfluoroalkyl sulfonic acids (PFSAs, $\mathrm{C}_{\mathrm{n}} \mathrm{F}_{2 \mathrm{n}+1} \mathrm{SO}_{3} \mathrm{H}, \mathrm{n} \geq 6$ ) and PFCAs is driven by hydrophobic and hydrogen-bonding in the protein, mediated by the size and rigidity of the PFAS molecule ${ }^{19}$. PFCAs with an alkyl chain consisting of $4-11$ carbons and PFSAs with 6-10 carbons favorably bind to L-FABP, with a decrease in binding observed beyond 11 carbons. Non-specific phospholipid-mediated pathways have also been hypothesized to drive PFAA uptake in brain and liver ${ }^{18,20,21}$. Sizable data gaps exist describing these mechanisms empirically and mechanistically across different taxa and trophic web levels.

Notably, little information exists evaluating bioaccumulation mechanisms or tissue-specific behavior of novel and replacement PFAS ${ }^{11,12}$, despite shifts towards production and use of PFAS with fewer than seven fluorinated carbons, 
polyfluorinated structures, and/or structurally modified iterations of PFAAs, such as such as per- and polyfluoroalkyl ether acids (PFEAs) incorporating ether linkages $^{22}$.

To address this knowledge gap, we present tissue-specific distributions of legacy and emerging PFAS using high resolution mass spectrometry (HRMS) in seabirds from the U.S. Atlantic Coast. We screened for the most widely assessed PFCAs and PFSAs along with 11 PFEAs. In summary, the aims of this study were to (i) derive the tissue distributions of 36 legacy and emerging PFAS in birds from three habitats and (ii) explore possible factors driving any differences in observed bioaccumulation between novel and legacy PFAS.

\section{MATERIALS AND METHODS}

\section{Sample Collection}

Deceased juvenile seabirds were obtained from three habitats reflecting different PFAS exposure potential. Habitats included the Cape Fear River Estuary (CFRE) downstream from a major fluoropolymer manufacturing site, Narragansett Bay adjacent to a major metropolitan center, and Massachusetts Bay, an offshore region removed from direct contact with PFAS point sources. The rationale supporting the evaluation of PFAS in similar seabird species from multiple habitats has been described previously ${ }^{23}$. All individuals were necropsied according to standard protocol, and intact tissues wrapped in methanol-cleaned foil and stored in polyethylene bags or vials. Tissues collected included heart, brain, kidney, lungs, uropygial gland, adipose fat, liver, whole blood, pectoral 
muscle, spleen, and feces; tissues were collected on an as-available basis, as some individuals were too small for accurate collection of all tissues. Tissues were frozen at $-15^{\circ} \mathrm{C}$ until chemical analysis.

PFAS analysis via HRMS

A total of 36 PFAS were assessed in juvenile seabird livers via targeted and suspect screening analysis. A complete analyte list is available in Table S4. All tissues were extracted by means of protein precipitation in acetonitrile and dilution as previously described ${ }^{24}$. PFAS were detected using with a Thermo Orbitrap Fusion mass spectrometer (ThermoFisher Scientific, Waltham, MA) with a heated electrospray ionization (HESI) source operated in negative mode, following a previously described HRMS workflow ${ }^{25-27}$. Further details about sample preparation, analysis, quantification, and quality control are available in the Supplementary Information (SI).

\section{RESULTS AND DISCUSSION}

Detection of PFAS by tissue and region

17 of 36 analytes were detected in at least one tissue from juvenile seabirds using HRMS (Fig. 1). Nine PFAS (Nafion BP2, PFO5DoDA, PFO4DA, PFOS, PFNA, PFDA, PFUnDA, PFDoA, and PFTrDA) were found $\geq 50 \%$ of at least two tissues from at least one habitat. The dominant five compounds (PFOS, PFDA, PFUnDA, PFDoA, and PFTrDA) were detected in at least two tissues in all three habitats. PFNA was only detected in livers from Massachusetts Bay and Narragansett Bay birds, but was detected across six tissues in CFRE individuals. 
Nafion BP2 was detected in muscle, blood, and liver of birds outside of the CFRE, but levels in Narragansett Bay birds were below the linear dynamic range of the curve $(<0.5 \mathrm{ng} / \mathrm{mL})$ and were not quantifiable. The largest number of analytes and the highest detection frequencies across each tissue type were observed in individuals from the Cape Fear sample set (Fig. 1).

Multiple short-chain PFCAs and PFEAs, fluorotelomer sulfonates, and acetic acid precursors like Et-FOSAA were not detected in any tissue (Table S5), suggesting reduced bioaccumulation potential compared to the detected longchain structures, in vivo transformation of these compounds to other terminal endpoints, or simply levels below the detection limits applicable in this study. PFOS and PFCA concentrations across multiple tissues

Concentrations of each analyte varied by tissue and habitat. PFOS was found at the highest mean concentration in most tissues where it was detected. It also made up the largest single percentage of the total sum concentration in most tissues (Fig. 2). The abundance of PFOS is noted across multiple wildlife taxa due to its highly persistence and bioaccumulative nature. Considering birds from all habitats, the highest mean concentrations of PFOS were observed in liver, kidney, lungs, and blood across all habitats (Fig. 3). High PFOS abundances in these relative to other tissues has been reported in wildlife from regions unimpacted by PFOS point sources ${ }^{9,10,15}$. PFOS was found in all CFRE tissues examined, and at the highest concentrations (up to $480 \mathrm{ng} / \mathrm{g}$ in blood) (Table S2). When grouped by tissue type solely considering CFRE birds, PFOS decreased in concentration according to: Kidney $=$ Liver $>$ Blood $=$ Lungs $>$ Brain $>$ Heart $>$ 
Muscle $>$ Fat. The abundance of PFOS in CFRE samples is likely related to high ambient levels of PFOS across the wider Cape Fear region, and has likewise been noted in fish and human samples from the region ${ }^{27-29}$.

A total of 5 CFRE, 1 Massachusetts Bay, and 3 Narragansett Bay bird contained liver concentrations above the $140 \mathrm{ng} / \mathrm{g}$ female-specific avian toxicity references value $(\mathrm{TRV})^{30}$, though it is unclear if reproductive impacts are occurring in these seabird populations due to data defiencies and potential differences in sensitivity to PFAS between avian species. No birds contained tissue residues above the overall avian TRV of $600 \mathrm{ng} / \mathrm{g}$ liver. TRVs are not available for specific avian tissues beyond liver, serum, and egg; this deficit stands as a large data gap as our dataset further demonstrates kidney, lungs, and brain act as reservoirs containing the highest mean concentrations of PFOS or other PFAS across multiple taxa (Fig. 3) ${ }^{17,31}$.

CFRE birds displayed the largest number of PFCA detections across multiple tissues (Fig. S2). PFUnDA was the most abundant PFCA found in liver, with a mean concentration of 10, 10, and $5 \mathrm{ng} / \mathrm{g}$ in CFRE, Massachusetts Bay, and Narraganset Bay livers, respectively (Table S2). PFTrDA was the most abundant PFCA detected in the lungs and brain of Massachusetts Bay individuals, at a mean concentration of $19 \mathrm{ng} / \mathrm{g}$ in both tissues. Elevated levels of this $\mathrm{C}_{13}$ PFCA solely in Massachusetts Bay individuals likely relates to their pelagic habits that entail disproportionate exposure to atmospherically transported precursor compounds that may oxidize in situ or in vivo to PFCAs ${ }^{32}$. Relatively high abundances of 
long-chain PFCAs have been noted in other work investigating PFAS in remote wildlife ${ }^{3,33-35}$, as well as in other tissue-specific research ${ }^{8,13,15}$.

PFEA concentrations across multiple tissues of CFRE birds

PFEAs made up the largest single proportion of total PFAS in fat (54\% in CFRE birds); PFEA levels in fat were dominated by Nafion BP2, but concentrations were overall low in fat compared to levels in other tissues (Fig. 3, Table S2). The highest PFEA levels were found in brain of CFRE individuals, with a maximum of $360 \mathrm{ng} / \mathrm{g}$ Nafion BP2 (Table S2). Mean concentrations of Nafion BP2 in blood from CFRE individuals (mean $=21 \mathrm{ng} / \mathrm{ml}$ ) were approximately 70 times higher than Nafion BP2 levels observed in striped bass from the Cape Fear River $(\text { mean }=0.3 \mathrm{ng} / \mathrm{ml})^{27}$, and about 7 times higher than Nafion BP2 levels observed in human serum from Wilmington, NC (median $=2.7$ $\mathrm{ng} / \mathrm{ml}$ ), a community where upstream industrial PFAS discharges in the Cape Fear River are known to impact finished drinking water ${ }^{36}$. These concentrations in CFRE bird blood are also approximately two orders of magnitude higher than concentrations observed in humans surrounding a fluoropolymer production site in China (median $=0.097 \mathrm{ng} / \mathrm{ml}$ ), which were found to be associated with altered glucose, cholesterol, and other enzymes and proteins in human serum ${ }^{37}$.

Mean PFO5DoDA concentrations in blood of CFRE chicks ( mean $=27$ $\mathrm{ng} / \mathrm{ml}$ ) were about 90 times higher than concentrations measured in human serum from Wilmington residents (median $=0.3 \mathrm{ng} / \mathrm{ml}$ ). Human populations in Cape Fear communities are exposed via air, drinking water, and any fish consumed from recreational fishing, while regional seabirds are wholly reliant on the 
ecosystem for both food and water throughout the duration of their occupancy. Beyond differential reliance on the natural system, the higher observed concentrations of PFEAs in birds may be related to dose as a function of body weight and maternal offloading, with the smaller birds evidencing higher tissue concentrations when subjected to the same air/water dose.

Relative body burdens by tissue and body weight

We derived relative body burdens (RBBs) as the product of PFAS concentrations, organ weights, and total body weight (Eq. S1). The highest RBB of PFOS was found in liver, followed by blood and muscle (Fig. S2). In muscle, the high RBB of PFOS is driven by the large mass fraction of this component ( $\sim 15-20 \%$ of total body weight) whereas in blood ( $\sim 10 \%$ of total body weight) and liver ( $4-6 \%$ of total body weight) the large RBB is the product of the relatively large mass fraction of these compartments in tandem with the demonstrated affinity of PFOS for specific uptake mechanisms in these tissues including albumin, L-FABP, and membrane phospholipids ${ }^{17,19,38}$.

RBBs observed in CFRE chicks suggest a chain-length dependence of PFCA accumulation in liver and brain (Fig. S2). $\mathrm{C}_{9}$ PFNA had the highest relative mass in liver, with decreasing relative mass with increasing chain length (Fig. S2). $\mathrm{C}_{12}$ PFDoDA and $\mathrm{C}_{13}$ PFTrDA showed significantly decreased relative masses in liver and increased mass in brain compared to $\mathrm{C}_{9}-\mathrm{C}_{11}$ PFCAs. Binding affinity of PFCAs with specific proteins in brain and blood-brain membrane transport pathways likely drives these trends. Binding displacement assays conducted using human liver fatty acid binding protein (L-FABP) suggest L-FABP interacts most 
optimally with PFCAs that possess less than eleven carbon constituents, due to the rigidity of the fluorocarbon tail and the size of the L-FABP active site pocket ${ }^{19}$, while associations with phospholipid or pathways that act upon phospholipids have been shown to disproportionately prefer long-chain PFAAs, leading to increased concentrations of these in brain ${ }^{14,17}$

The largest RBBs of Nafion BP2, PFO4DA, PFO5DoDA, and HFPO-DA were found in blood, driven by the large mass fraction of this compartment coupled to relatively high concentrations of PFEAs in whole blood (Fig. S2).

\section{Legacy and novel PFAS in brain}

10 compounds were detected in at least one brain sample and 8 detected in at least $50 \%$ of brain samples, demonstrating that multiple PFAS can migrate across the blood-brain barrier. PFOS displayed the highest mean concentration in brain, followed by Nafion BP2 $>>$ PFUnDA $>$ PFDA $>$ PFTrDA $=$ PFO5DoDA $>$ PFDoA $>$ PFO4DA (Table S2). PFCAs made up the largest fraction observed in brain ( $44 \%$ of total concentration across all birds), with PFUnDA found at the highest mean concentration, with mean concentrations of 13,9 , and $3 \mathrm{ng} / \mathrm{g}$ in brains from CFRE, Massachusetts Bay, and Narragansett Bay individuals, respectively. The dominance of PFCAs was particularly notable in bird brains from Massachusetts Bay and Narragansett Bay individuals in which [PFOS] was lower, with PFCAs constituting a mean of $57 \%$ of total concentrations observed in brains $(n=6)($ Table S5). This implies PFOS uptake into brain tissue is less consistent compared to PFCA uptake into brain tissue, which showed consistent brain tissue uptake across variable and distinct habitat exposure scenarios. 
Pairwise concentration comparison suggests PFTrDA is most preferentially taken up into the brain compared to structurally analogous $\mathrm{C}_{9}-\mathrm{C}_{12}$ PFCAs (Table S3). These observations are in agreement with results from marine mammals demonstrating the preference of long-chain PFCAs in brain ${ }^{14}$, hypothesized to be driven by phospholipid-mediated uptake.

Nafion byproduct-2, PFO4DA, and PFO5DoDA were all detected in brain tissue of individuals from the CFRE, with detection frequencies of $80 \%, 60 \%$, and $100 \%$ of brain samples, respectively. Migration across the blood-brain barrier (BBB) is highly restricted for ionic species like PFAAs and PFEAs, and is thought to occur via active transport pathways ${ }^{39}$. OATP1A2 is a key BBB influx transporter in mammals, and has been previously demonstrated to reject PFOA as a substrate ${ }^{40}$. Instead, PFOA, PFNA, and PFDA inhibit uptake of the native substrate E3S by OATP1A2 when subjected to a mixture of $\mathrm{C}_{4}-\mathrm{C}_{12}$ PFCAs at a concentration of $100 \mathrm{uM}^{40}$. However, empirical observations in marine mammals and birds demonstrate the presence of long-chain PFCAs, PFOS, and PFDS in brain tissue, suggesting BBB transport of PFCAs with $\mathrm{C}>9$ or PFSAs with $\mathrm{C} \geq 8$ is possible, whether by OATP1A2 or another unspecified transport pathway related to or mediated by phospholipid interactions ${ }^{9,13,14}$. Our data highlight that ether-based PFAS chemistries are also transported through this highly selective membrane.

Our results represent the first evidence that PFEAs are able to migrate across the blood-brain barrier in vertebrate wild animals subject to environmentally relevant PFEA exposures. We note that shifts in production towards ether-based 
PFAS may not translate to reduced tissue accumulation in vertebrate wildlife receptors like seabirds, nor a reduction in any associated adverse effects of PFAS given ether-based chemicals are readily transported to multiple body compartments and sensitive tissues such as the brain. Data from polar bears ${ }^{16}$, frogs $^{41}$, and mice ${ }^{42}$ suggest some legacy PFAS (PFOS, PFCAs) are associated with alterations of neurochemical signaling and proteins critical for brain development, with uncertain long-term implications. Modeling suggests etherbased PFAS have similar or higher toxic potency compared to PFAAs ${ }^{43}$. Further research is required to examine PFEA impacts in the brain, given their proven ability to migrate into this tissue compartment.

\section{Decreased preference of PFO5DoDA for brain}

Concentration ratios facilitate the evaluation of compound partitioning between different tissues. While tissue concentrations are determined by exposure levels, distribution among organs probably reflects multiple pathways of varying importance. This leads to similar ratios between tissues subject to similar exposure and distribution pathways and divergent ratios between tissues subject to dissimilar PFAS exposure. Using ratios, we note that PFO5DoDA, a PFOA substitute with a backbone incorporating 6 carbons and 5 oxygens in ether linkages, partitions differently in brain than in other tissues when contrasted with PFUnDA, PFTrDA, PFOS, and Nafion BP2 (Fig. 4, Table S3).

This suggests a combination of structural features, such as the inclusion of multiple ether linkages $(>2)$ that increase backbone flexibility, the molecular size of PFO5DoDA (11-member backbone), and/or the - $\mathrm{COOH}$ head group, reduce 
transport across the BBB compared to other examined PFAAs and the PFESA Nafion BP2. We consider the ether-linkages, molecular size, and/or increased backbone flexibility of PFO5DoDA to be the most important features for its accumulation in brain, given that the $-\mathrm{COOH}$ functional group is also present in long-chain PFCAs that preferentially partition to brain. The ether-based chemicals distribute across most other tissues in a similar manner as long-chain PFAA analogues, with a preference for blood and kidney apparent in some comparisons (Table S3).

Relative sorption strength of PFEAs to proteins

The high PFAS concentrations observed in blood suggests albumin may offer increased binding opportunity for PFEAs via its multiple binding sites with distinct binding affinities that favor a range of PFAS chain lengths and functional groups $^{44,45}$. The flexible binding opportunities afforded by albumin is in contrast to the chain-length and charge-mediated interactions between PFAAs and the primary binding site of L-FABP ${ }^{19}$. Binding of PFCAs and PFSAs within the primary L-FABP cavity has been demonstrated to heavily rely on hydrophobic interactions, which may be altered with PFEAs that incorporate multiple ether linkages associated with alterations in dipole distribution ${ }^{45}$.

Quantum chemical calculations performed via COSMOconf/TURBOMOLE confirm differences in surface charge density between the lowest energy PFEA conformations compared to their respective PFAA homologues (Table S7). Nafion BP2 possessed increased probabilities of positive surface charge density compared to PFOS, and reduced probabilities of neutral surface charge; given the 
sensitivity of binding cavity interactions to charge, we hypothesize that reduced ability to engage in hydrophobic interactions may help explain the reduced presence of Nafion BP2 in liver compared to blood. However, PFO5DoDA possessed an increased probability of neutral surface charge compared to PFOA, but was found at higher concentrations in blood compared to liver. Steric effects may drive differences in binding of PFO5DoDA to L-FABP compared to C8 C10 PFAAs, which were found at higher concentrations in liver compared to blood in CFRE individuals. Sheng et al. (2018) found that several ether- and chlorine-containing emerging PFAS were structurally distorted during the binding process in orientations distinct from the "head-out" binding observed for PFAAs; they also interacted with a different suite of amino acid residues ${ }^{46}$.

Based on both calculated and observational results, we suggest multi-ether PFEAs engage with the L-FABP active site in a way distinct from the structural deformation and binding interactions observed between L-FABP and long-chain PFAAs. These differences may reduce the favorability of interaction in the primary L-FABP cavity, leading to reduced liver concentrations compared to blood (Figs. S2, S3). Alternatively, phospholipid binding is an additionally important mechanism driving PFAS accumulation in liver, in tandem with LFABP interactions ${ }^{18}$. The multi-ether PFAS, with altered electron densities or conformational behavior compared to PFAAs, may be less likely to engage with uptake pathways that favor phospholipid-like species with a distinctly hydrophobic tail and a hydrophilic head group. Little research to date has examined the binding behavior of multi-ether PFEAs, which is needed 
considering their burgeoning environmental relevance and continued incorporation in industrial processes.

\section{Implications}

This study documents the presence of PFAAs and PFEAs in multiple tissues and organs of chick and juvenile seabirds, including the first documentation of PFEAs in brain. Multi-ether PFEAs were most abundant in blood rather than liver, suggesting compounds containing multiple ether linkages may find more flexible binding sites within serum albumin compared to the primary active site of L-FABP. These important findings contribute to the prediction and testing of potential adverse effects and mechanisms related to PFAS in avian kidney, lungs, and brain. This work also provides the first estimates of PFEA internal distribution behavior in vertebrate wildlife with implications for other systems and receptors subject to similar environmental PFEA exposures due to ongoing production and use of these compounds. Overall, we caution that an industrial switch to PFEAs is unlikely to lead to reduced bioaccumulation in vertebrate wildlife (or humans for that matter), and is cause for concern and continued investigation. Our results reinforce the benefits of using birds as sentinels of bioaccumulation and their utility to better understand internal distributions of emerging and legacy PFAS, and identify target tissues of concern.

\section{ASSOCIATED CONTENT}

Supporting Information. 
The following files are available free of charge.

Additional summary data, analyte list (Excel workbook)

Additional text describing methods, quality assurance, and providing additional figures in support of the text (PDF)

AUTHOR INFORMATION

Corresponding Author

*Anna Ruth Robuck, annarobuck@gmail.com

Author Contributions

The manuscript was primarily drafted by A. Robuck and finalized through contributions of all authors. Sample analysis and data interpretation were carried out through contributions of all authors. All authors have given approval to the final version of the manuscript.

Funding Sources

A. Robuck acknowledges support from the National Oceanic and Atmospheric Administration Dr. Nancy Foster Scholarship program, the Robert and Patricia Switzer Foundation, the STEEP Superfund Research Program (NIEHS Award Number P42ES027706), and the Oak Ridge Institute for Science and Education (ORISE) program.

\section{ACKNOWLEDGMENTS}

The authors are indebted to the Northeast Fisheries Observer Program, Gina Shield, Johanna Pedersen, Michael Moore, Lindsay Addison, Matthew McIver, the Wildlife Clinic of Rhode Island, and Kevin Powers for assistance obtaining and necropsying bird samples used in this study. The authors also thank Detlef 
Knappe for connecting members of this team to bring the study to fruition. The authors also acknowledge Michaela Cashman, Diane Nacci, and Steve Rego for critical feedback prior to peer review. The TOC art was created with BioRender.com, with the tern photo provided courtesy of Lindsay Addison. The authors also thank Andrea Ebert and Kai Uwe Goss for assistance with COSMOconf analysis and interpretation. Although EPA employees contributed to this article, the research presented was not funded by EPA and was conceived, designed, and implemented by URI. EPA's role was limited to advising PFAS analysis and therefore not subject to EPA's quality system requirements.

Consequently, the views, interpretations, and conclusions expressed in the article are solely those of the authors and do not necessarily reflect or represent EPA's views or policies.
ABBREVIATIONS
PFOS - Perfluorooctanesulfonic acid
PFAAs - Perfluoroalkyl acids
PFEAs - Per- and polyfluoroalkyl ether acids 


\section{LITERATURE CITED}

(1) Giesy, J. P.; Kannan, K. Global Distribution of Perfluorooctane Sulfonate in Wildlife Global Distribution of Perfluorooctane Sulfonate in Wildlife. Environ. Sci. Technol. 2001, 35 (March), 1339-1342. https://doi.org/10.1021/es001834k.

(2) Gebbink, W. A.; Letcher, R. J.; Hebert, C. E.; Chip Weseloh, D. V. V. Twenty Years of Temporal Change in Perfluoroalkyl Sulfonate and Carboxylate Contaminants in Herring Gull Eggs from the Laurentian Great Lakes. J. Environ. Monit. 2011, 13 (12), 3365-3372. https://doi.org/10.1039/c1em10663e.

(3) Braune, B. M.; Letcher, R. J. Perfluorinated Sulfonate and Carboxylate Compounds in Eggs of Seabirds Breeding in the Canadian Arctic: Temporal Trends (1975-2011) and Interspecies Comparison. Environ. Sci. Technol. 2013, 47 (1), 616-624. https://doi.org/10.1021/es303733d.

(4) Ahrens, L. Polyfluoroalkyl Compounds in the Aquatic Environment: A Review of Their Occurrence and Fate. J. Environ. Monit. 2011, 13 (1), 20-31. https://doi.org/10.1039/c0em00373e.

(5) Jahnke, A.; Berger, U. Trace Analysis of Per- and Polyfluorinated Alkyl Substances in Various Matrices-How Do Current Methods Perform? J. Chromatogr. A 2009, 1216 (3), 410-421. https://doi.org/10.1016/j.chroma.2008.08.098.

(6) Nakayama, S. F.; Yoshikane, M.; Onoda, Y.; Nishihama, Y.; IwaiShimada, M.; Takagi, M.; Kobayashi, Y.; Isobe, T. Worldwide Trends in Tracing Poly- and Perfluoroalkyl Substances (PFAS) in the Environment. TrAC - Trends Anal. Chem. 2019, No. xxxx. https://doi.org/10.1016/j.trac.2019.02.011.

(7) Valsecchi, S.; Rusconi, M.; Polesello, S. Determination of Perfluorinated Compounds in Aquatic Organisms: A Review. Anal. Bioanal. Chem. 2013, 405 (1), 143-157. https://doi.org/10.1007/s00216-012-6492-7.

(8) Greaves, A. K.; Letcher, R. J.; Sonne, C.; Dietz, R.; Born, E. W. TissueSpecific Concentrations and Patterns of Perfluoroalkyl Carboxylates and Sulfonates in East Greenland Polar Bears. Environ. Sci. Technol. 2012, 46 (21), 11575-11583. https://doi.org/10.1021/es303400f.

(9) Verreault, J.; Houde, M.; Gabrielsen, G. W.; Berger, U.; Haukås, M.; Letcher, R. J.; Muir, D. C. G. Perfluorinated Alkyl Substances in Plasma, Liver, Brain, and Eggs of Glaucous Gulls (Larus Hyperboreus) from the Norwegian Arctic. Environ. Sci. Technol. 2005, 39 (19), 7439-7445. https://doi.org/10.1021/es051097y. 
(10) Gebbink, W. A.; Letcher, R. J. Comparative Tissue and Body Compartment Accumulation and Maternal Transfer to Eggs of Perfluoroalkyl Sulfonates and Carboxylates in Great Lakes Herring Gulls. Environ. Pollut. 2012, 162, 40-47. https://doi.org/10.1016/j.envpol.2011.10.011.

(11) Shi, Y.; Vestergren, R.; Nost, T. H.; Zhou, Z.; Cai, Y. Probing the Differential Tissue Distribution and Bioaccumulation Behavior of Per- and Polyfluoroalkyl Substances of Varying Chain-Lengths, Isomeric Structures and Functional Groups in Crucian Carp. Environ. Sci. Technol. 2018, 52 (8), 45924600. https://doi.org/10.1021/acs.est.7b06128.

(12) Shi, Y.; Vestergren, R.; Zhou, Z.; Song, X.; Xu, L.; Liang, Y.; Cai, Y. Tissue Distribution and Whole Body Burden of the Chlorinated Polyfluoroalkyl Ether Sulfonic Acid F-53B in Crucian Carp (Carassius Carassius): Evidence for a Highly Bioaccumulative Contaminant of Emerging Concern. Environ. Sci. Technol. 2015, 49 (24), 14156-14165. https://doi.org/10.1021/acs.est.5b04299.

(13) Ahrens, L.; Siebert, U.; Ebinghaus, R. Total Body Burden and Tissue Distribution of Polyfluorinated Compounds in Harbor Seals (Phoca Vitulina) from the German Bight. Mar. Pollut. Bull. 2009, 58 (4), 520-525. https://doi.org/10.1016/j.marpolbul.2008.11.030.

(14) Dassuncao, C.; Pickard, H.; Pfohl, M.; Tokranov, A. K.; Li, M.; Mikkelsen, B.; Slitt, A.; Sunderland, E. M. Phospholipid Levels Predict the Tissue Distribution of Poly- and Perfluoroalkyl Substances in a Marine Mammal. Environ. Sci. Technol. Lett. 2019, 6 (3), 119-125. https://doi.org/10.1021/acs.estlett.9b00031.

(15) Holmström, K. E.; Berger, U. Tissue Distribution of Perfluorinated Surfactants in Common Guillemot (Uria Aalge) from the Baltic Sea. Environ. Sci. Technol. 2008, 42 (16), 5879-5884. https://doi.org/10.1021/es800529h.

(16) Eggers Pedersen, K.; Basu, N.; Letcher, R.; Greaves, A. K.; Sonne, C.; Dietz, R.; Styrishave, B. Brain Region-Specific Perfluoroalkylated Sulfonate (PFSA) and Carboxylic Acid (PFCA) Accumulation and Neurochemical Biomarker Responses in East Greenland Polar Bears (Ursus Maritimus). Environ. Res. 2015, 138, 22-31. https://doi.org/10.1016/j.envres.2015.01.015.

(17) Ng, C. A.; Hungerbühler, K. Bioconcentration of Perfluorinated Alkyl Acids: How Important Is Specific Binding? Environ. Sci. Technol. 2013, 47 (13), 7214-7223. https://doi.org/10.1021/es400981a.

(18) Ng, C. A.; Hungerbühler, K. Bioaccumulation of Perfluorinated Alkyl Acids: Observations and Models. Environ. Sci. Technol. 2014, 48 (9), 4637-4648. https://doi.org/10.1021/es404008g. 
(19) Zhang, L.; Ren, X. M.; Guo, L. H. Structure-Based Investigation on the Interaction of Perfluorinated Compounds with Human Liver Fatty Acid Binding Protein. Environ. Sci. Technol. 2013, 47 (19), 11293-11301. https://doi.org/10.1021/es4026722.

(20) Armitage, J. M.; Arnot, J. A.; Wania, F. Potential Role of Phospholipids in Determining the Internal Tissue Distribution of Perfluoroalkyl Acids in Biota. Environ. Sci. Technol. 2012, 46 (22), 12285-12286. https://doi.org/10.1021/es304430r.

(21) Armitage, J. M.; Arnot, J. A.; Wania, F.; Mackay, D. Development and Evaluation of a Mechanistic Bioconcentration Model for Ionogenic Organic Chemicals in Fish. Environ. Toxicol. Chem. 2013, 32 (1), 115-128. https://doi.org/10.1002/etc.2020.

(22) Zhao, H. X.; Jiang, J. Q.; Wang, Y. L.; Xie, Q.; Qu, B. C. Phototransformation of 2,4,6-Tribromophenol in Aqueous Solution: Kinetics and Photolysis Products. J. Environ. Sci. Heal. Part A 2017, 52 (1), 45-54. https://doi.org/10.1080/10934529.2016.1229926.

(23) Robuck, A.; Cantwell, M. G.; McCord, J. P.; Addison, L. M.; Pfohl, M.; Strynar, M. J.; McKinney, R. A.; Katz, D. R.; Wiley, D. N.; Lohmann, R. Legacy and Novel Per- and Polyfluoroalkyl Substances (PFAS) in Juvenile Seabirds from the US Atlantic Coast. Environ. Sci. Technol. 2020. https://doi.org/10.1021/acs.est.0c01951.

(24) Russell, M. C.; Newton, S. R.; McClure, K. M.; Levine, R. S.; Phelps, L. P.; Lindstrom, A. B.; Strynar, M. J. Per- and Polyfluoroalkyl Substances in Two Different Populations of Northern Cardinals. Chemosphere 2019, 222, 295-304. https://doi.org/10.1016/j.chemosphere.2019.01.112.

(25) Mccord, J.; Strynar, M. Identifying Per- and Polyfluorinated Chemical Species with a Combined Targeted and Non-Targeted-Screening High-Resolution Mass Spectrometry Workflow. J. Vis. Exp. 2019, 146 (e59142), 1-15. https://doi.org/10.3791/59142.

(26) Strynar, M.; Dagnino, S.; McMahen, R.; Liang, S.; Lindstrom, A.; Andersen, E.; McMillan, L.; Thurman, M.; Ferrer, I.; Ball, C. Identification of Novel Perfluoroalkyl Ether Carboxylic Acids (PFECAs) and Sulfonic Acids (PFESAs) in Natural Waters Using Accurate Mass Time-of-Flight Mass Spectrometry (TOFMS). Environ. Sci. Technol. 2015, 49 (19), 11622-11630. https://doi.org/10.1021/acs.est.5b01215.

(27) Guillette, T. C.; McCord, J.; Guillette, M.; Polera, M. E.; Rachels, K. T.; Morgeson, C.; Kotlarz, N.; Knappe, D. R. U.; Reading, B. J.; Strynar, M.; Belcher, S. M. Per- and Polyfluoroalkyl Substances Exposure in Cape Fear River 
Striped Bass (Morone Saxatilis) Is Associated with Biomarkers of Altered Immune and Liver Function Research. Environ. Int. 2019, 2 (1), 105358. https://doi.org/10.1016/j.envint.2019.105358.

(28) Kotlarz, N.; McCord, J.; Collier, D.; Lea, C.; Strynar, M.; Lindstron, A.; Wilkie, A.; Islam, J.; Matney, K.; Tarte, P.; Polera, M.; Burdette, K.; DeWitt, J.; May, K.; Smart, R.; Knappe, D.; Hoppin, J. A. Measurement of Novel, Drinking Water-Associated PFAS in Blood from Adults and Children in Wilmington, North Carolina. Env. Heal. Perspect 2020, 128 (July), 1-12.

(29) Nakayama, S.; Strynar, M. J.; Helfant, L.; Egeghy, P.; Ye, X.; Lindstrom, A. B. Perfluorinated Compounds in the Cape Fear Drainage Basin in North Carolina. Environ. Sci. Technol. 2007, 41, 5271-5276. https://doi.org/10.1021/es070792y.

(30) Newsted, J. L.; Jones, P. D.; Coady, K.; Giesy, J. P. Avian Toxicity Reference Values for Perfluorooctane Sulfonate. Environ. Sci. Technol. 2005, 39 (23), 9357-9362. https://doi.org/10.1021/es050989v.

(31) Martin, J. W.; Whittle, D. M.; Muir, D. C. G.; Mabury, S. A. Perfluoroalkyl Contaminants in a Food Web from Lake Ontario. Environ. Sci. Technol. 2004, 38 (20), 5379-5385. https://doi.org/10.1021/es049331s.

(32) Ellis, D. A.; Martin, J. W.; Silva, A. O. D. E.; Hurley, M. D.; De Silva, A. O.; Mabury, S. A.; Hurley, M. D.; Sulbaek Andersen, M. P.; Wallington, T. J. Degradation of Fluorotelomer Alcohols: A Likely Atmospheric Source of Perfluorinated Carboxylic Acids. Environ. Sci. Technol. 2004, 38 (12), 33163321. https://doi.org/10.1021/es049860w.

(33) Mallory, M. L.; Braune, B. M. Tracking Contaminants in Seabirds of Arctic Canada: Temporal and Spatial Insights. Mar. Pollut. Bull. 2012, 64 (7), 1475-1484. https://doi.org/10.1016/j.marpolbul.2012.05.012.

(34) Costantini, D.; Blévin, P.; Herzke, D.; Moe, B.; Wing, G.; Ove, J.; Chastel, O.; Gabrielsen, G. W.; Bustnes, J. O.; Chastel, O. Higher Plasma Oxidative Damage and Lower Plasma Antioxidant Defences in an Arctic Seabird Exposed to Longer Perfluoroalkyl Acids. Environ. Res. 2019, 168 (October 2018), 278-285. https://doi.org/10.1016/j.envres.2018.10.003.

(35) Boisvert, G.; Sonne, C.; Rigét, F. F.; Dietz, R.; Letcher, R. J. Bioaccumulation and Biomagnification of Perfluoroalkyl Acids and Precursors in East Greenland Polar Bears and Their Ringed Seal Prey. Environ. Pollut. 2019, 252, 1335-1343. https://doi.org/10.1016/j.envpol.2019.06.035.

(36) Hopkins, Z. R.; Sun, M.; DeWitt, J. C.; Knappe, D. R. U. Recently Detected Drinking Water Contaminants : GenX and Other Per- and 
Polyfluoroalkyl Ether Acids. J. AWWA 2018, 110 (7), 13-28.

https://doi.org/10.1002/awwa.1073.

(37) Yao, J.; Pan, Y.; Sheng, N.; Su, Z.; Guo, Y.; Wang, J.; Dai, J. Novel Perfluoroalkyl Ether Carboxylic Acids (PFECAs) and Sulfonic Acids (PFESAs): Occurrence and Association with Serum Biochemical Parameters in Residents Living Near a Fluorochemical Plant in China. Environ. Sci. Technol. 2020. https://doi.org/10.1021/acs.est.0c02888.

(38) Jones, P. D.; Hu, W. Y.; De, C.; Newsted, J. L.; Giesy, J. P. Binding of Perfluorinated Fatty Acids to Serum Proteins. Environ. Toxicol. Chem. 2003, 22, 2639-2649.

(39) Barar, J.; Rafi, M. A.; Pourseif, M. M.; Omidi, Y. Blood-Brain Barrier Transport Machineries and Targeted Therapy of Brain Diseases. BioImpacts 2016, 6 (4), 225-248. https://doi.org/10.15171/bi.2016.30.

(40) Yang, C. H.; Glover, K. P.; Han, X. Characterization of Cellular Uptake of Perfluorooctanoate via Organic Anion-Transporting Polypeptide 1A2, Organic Anion Transporter 4, and Urate Transporter 1 for Their Potential Roles in Mediating Human Renal Reabsorption of Perfluorocarboxylates. Toxicol. Sci. 2010, 117 (2), 294-302. https://doi.org/10.1093/toxsci/kfq219.

(41) Foguth, R. M.; Hoskins, T. D.; Clark, G. C.; Nelson, M.; Flynn, R. W.; de Perre, C.; Hoverman, J. T.; Lee, L. S.; Sepúlveda, M. S.; Cannon, J. R. Single and Mixture Per- and Polyfluoroalkyl Substances Accumulate in Developing Northern Leopard Frog Brains and Produce Complex Neurotransmission Alterations. Neurotoxicol. Teratol. 2020, 81 (June), 106907. https://doi.org/10.1016/j.ntt.2020.106907.

(42) Johansson, N.; Eriksson, P.; Viberg, H. Neonatal Exposure to PFOS and PFOA in Mice Results in Changes in Proteins Which Are Important for Neuronal Growth and Synaptogenesis in the Developing Brain. Toxicol. Sci. 2009, 108 (2), 412-418. https://doi.org/10.1093/toxsci/kfp029.

(43) Gomis, M. I.; Vestergren, R.; Borg, D.; Cousins, I. T. Comparing the Toxic Potency in Vivo of Long-Chain Perfluoroalkyl Acids and Fluorinated Alternatives. Environ. Int. 2018, 113 (November 2017), 1-9.

https://doi.org/10.1016/j.envint.2018.01.011.

(44) Chen, Y. M.; Guo, L. M. Fluorescence Study on Site-Specific Binding of Perfluoroalkyl Acids to Human Serum Albumin. Arch. Toxicol. 2009, 83, 255261. https://doi.org/10.1007/s00204-008-0359-x.

(45) Allendorf, F.; Berger, U.; Goss, K.-U. U.; Ulrich, N. Partition Coefficients of Four Perfluoroalkyl Acid Alternatives between Bovine Serum Albumin (BSA) 
and Water in Comparison to Ten Classical Perfluoroalkyl Acids. Environ. Sci. Process. Impacts 2019, 21 (11), 1852-1863. https://doi.org/10.1039/c9em00290a.

(46) Sheng, N.; Cui, R.; Wang, J.; Guo, Y.; Wang, J.; Dai, J. Cytotoxicity of Novel Fluorinated Alternatives to Long-Chain Perfluoroalkyl Substances to Human Liver Cell Line and Their Binding Capacity to Human Liver Fatty Acid Binding Protein. Arch. Toxicol. 2018, 92 (1), 359-369.

https://doi.org/10.1007/s00204-017-2055-1. 
TABLES AND FIGURES

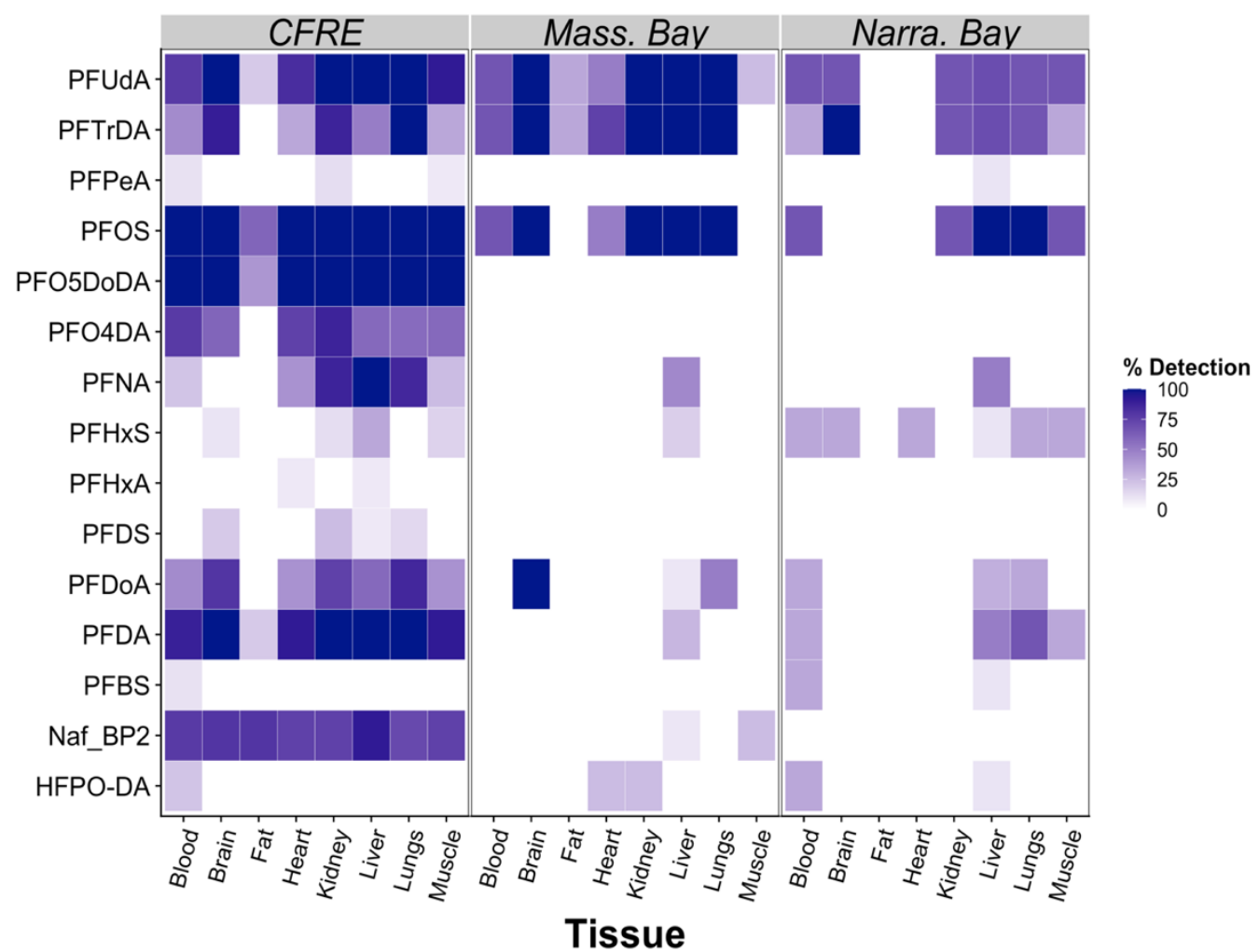

Figure 4.1. Detection frequencies by habitat and tissue type, including 15 compounds that were found at least two samples. See SI for compound abbreviation definitions. 


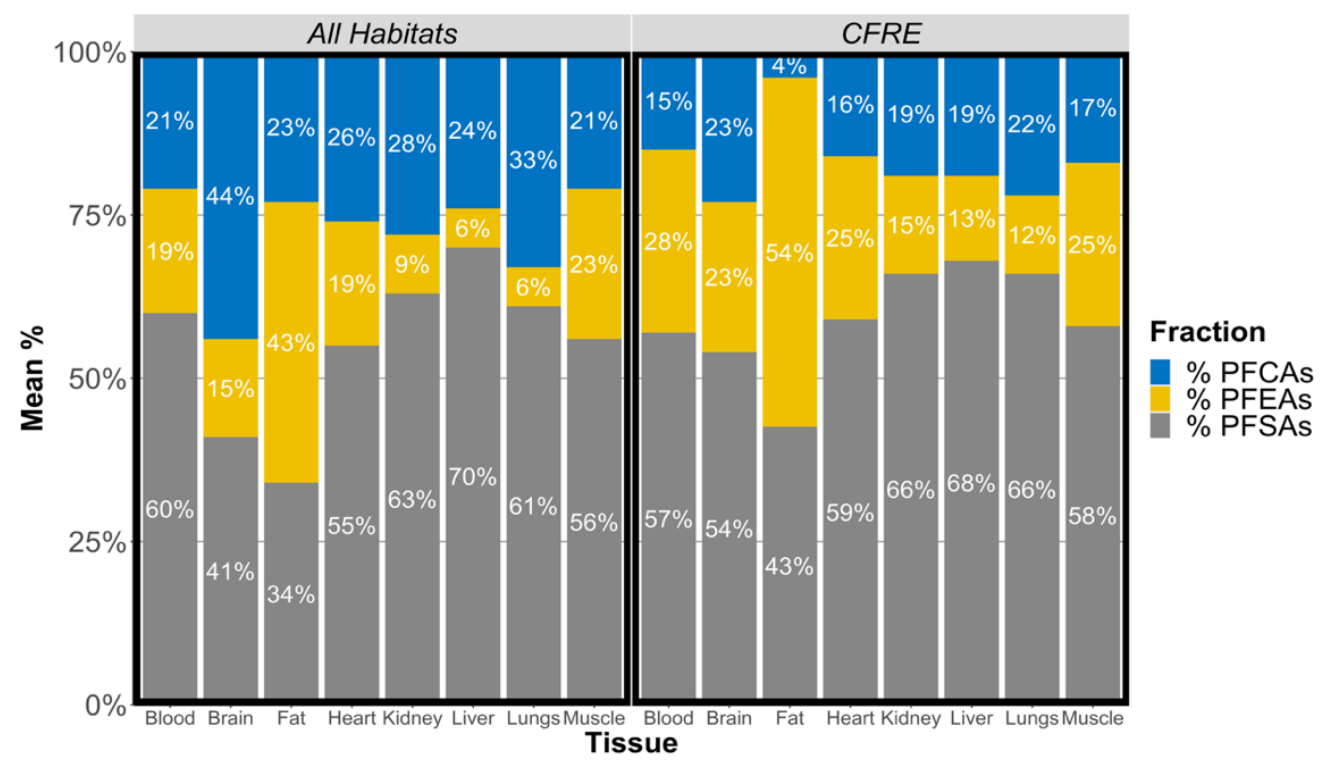

Figure 4.2. Mean percentages of three subclasses of PFAS observed in eight tissues, averaged across all habitats (left) and solely considering CFRE individuals (right). Percentages reported reflect the concentration of the given PFAS group, divided by the total concentration (in $\mathrm{ng} / \mathrm{g}$ ) observed in a given tissue. The proportion of PFSA in each tissue is majorly dominated by PFOS. PFEA proportions are driven by Nafion BP2. Proportions stratified by habitat and compound are presented in the SI, along with relative mass weighted proportions. 


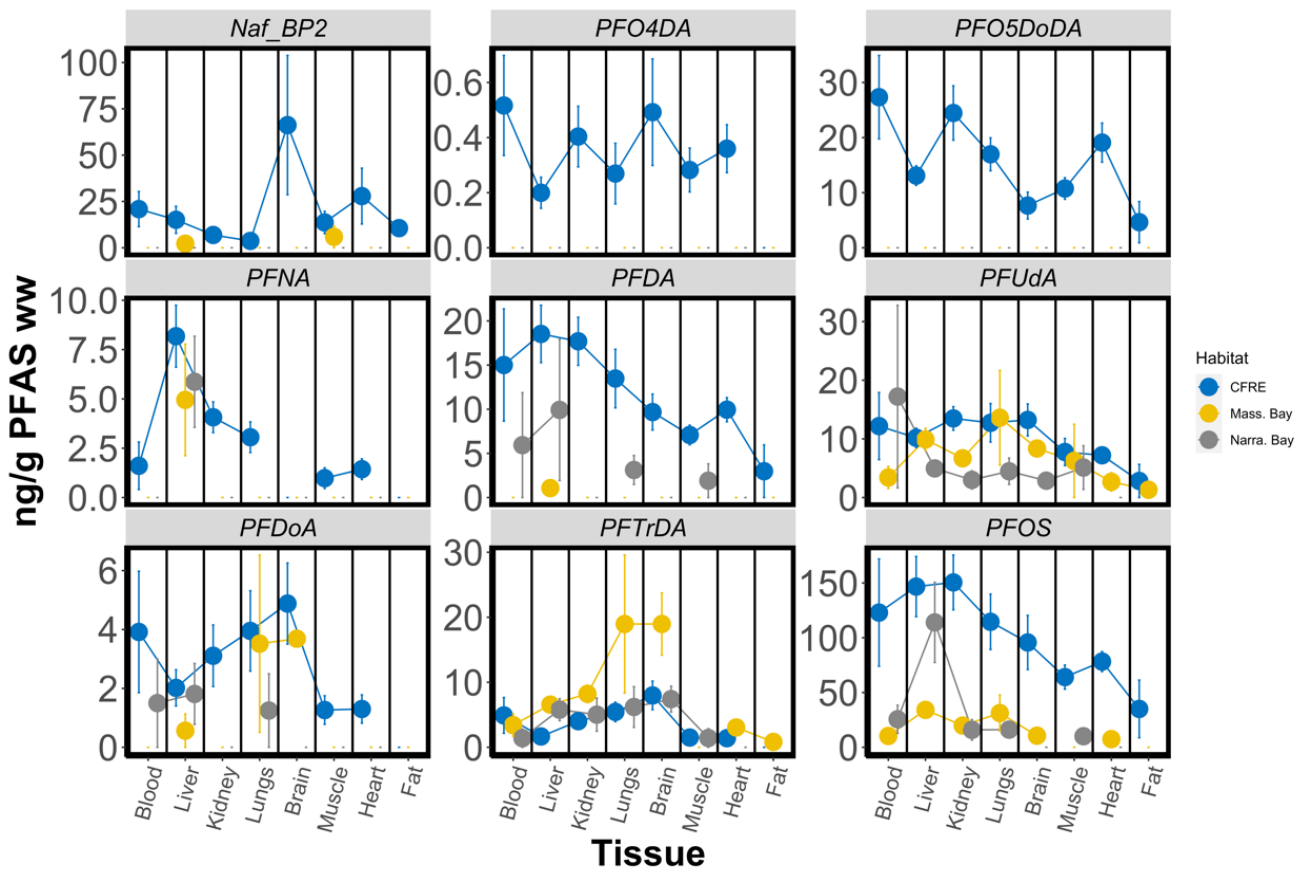

Figure 4.3. Mean concentrations and standard errors of each compound, observed in eight tissues, stratified by habitat as indicated by point and line color. 


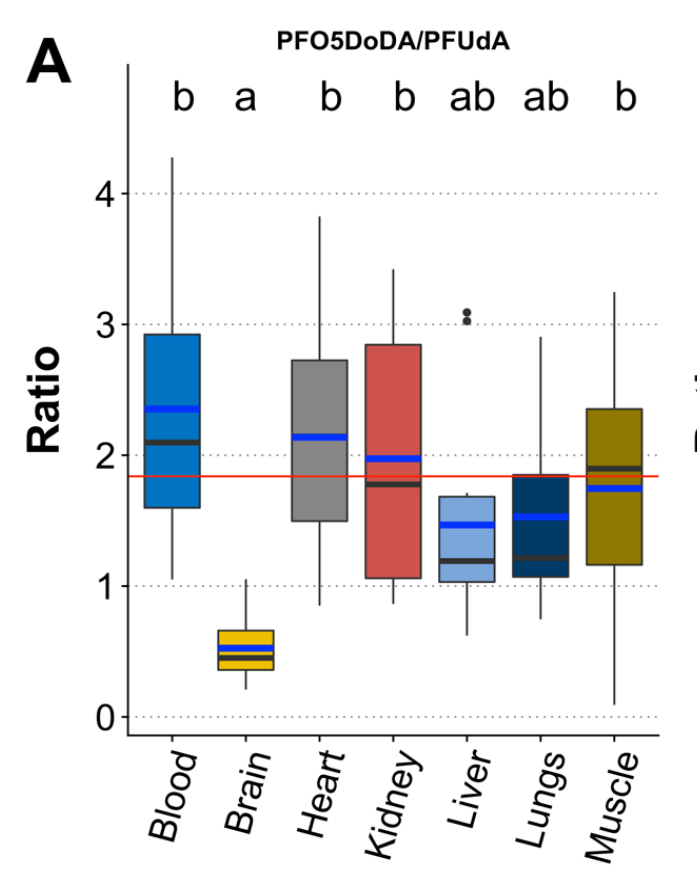

Tissue

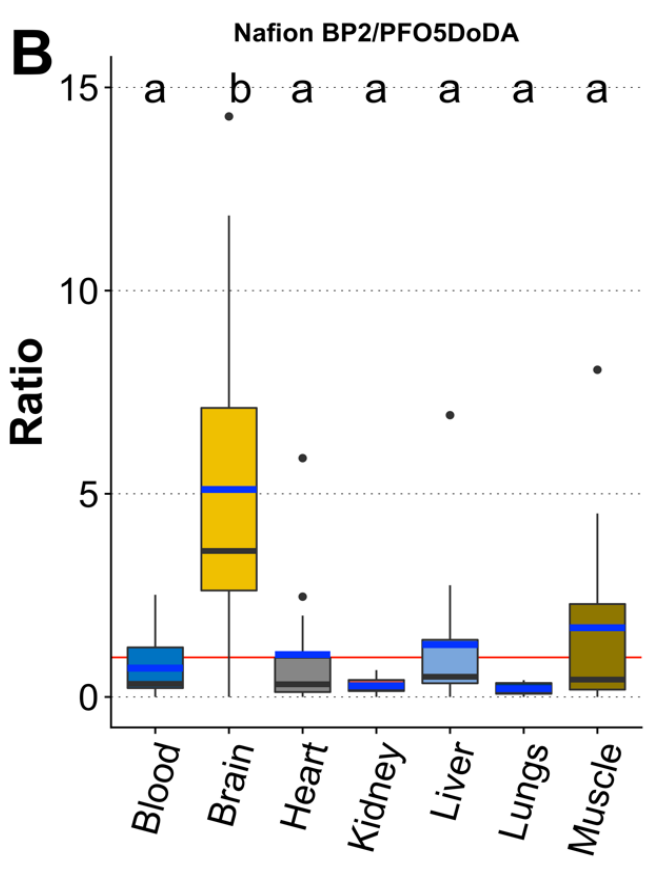

Tissue

Figure 4.4. Ratios of PFAS concentrations in six tissues from CFRE individuals, with A) presenting concentrations of PFO5DoDA divided by PFUnDA and B) presenting concentrations of Nafion BP2 divided by concentrations of PFO5DoDA. The blue line within each box plot indicates the arithmetic mean while the black line indicates the median. The box hinges represent the first and third quartiles, and the whiskers indicate 1.5 times the interquartile range. Outliers are indicated by black dots. The red line indicates the mean of each concentration ratio including all tissues except brain. Common letters above each boxplot indicate tissues that share statistically indistinguishable concentration ratios as determined using Tukey's post-hoc test. 


\title{
SUPPORTING INFORMATION
}

\section{TISSUE-SPECIFIC DISTRIBUTION OF LEGACY AND NOVEL PER- AND POLYFLUOROALKYL SUBSTANCES IN JUVENILE SEABIRDS}

\author{
Anna R. Robuck, James McCord, Mark J. Strynar, Mark G. Cantwell, David N. \\ Wiley", and Rainer Lohmann ${ }^{\dagger}$
}

This document contains 3 figures and 7 tables

Chemicals, reagents, and materials. Analytical standards of greater than $99.9 \%$ purity, including mass-labeled surrogates, were purchased from Wellington Laboratories (Guelph, ON, Canada). Native Nafion BP2 and PFO5DoDA were provided by Chemours (Fayetteville, NC) in lieu of a commercial source. HPLC grade methanol, acetonitrile, and formic acid were purchased from Fisher Scientific (Waltham, MA, USA). Ultrapure water for equipment cleaning was obtained from a Milli-Q system fit with an HPLC water polisher or via HPLC grade water purchased from Fisher Scientific (Waltham, MA, USA). Ammonium formate was purchased from Fisher Scientific (Waltham, MA, USA).

Sample collection details. All birds were obtained opportunistically for use in this study, and no birds were killed for the purposes of this study. Birds were collected and stored in accordance with URI Biosafety standards and the Migratory Bird Treaty Act. 
Dead Great Shearwater juveniles (Ardenna gravis) were obtained as bycatch from the National Oceanic and Atmospheric Administration (NOAA) Northeast Fisheries Observer Program (Falmouth, MA). Dead Herring Gull (Larus argentatus smithsonianus) chicks were obtained from the Wildlife Clinic of Rhode Island (Narragansett, RI). Dead Royal Tern (Thalasseus maximus), Sandwich Tern (Thalasseus sandvicensis), Laughing Gull (Leucophaeus atricilla), and Brown Pelican (Pelecanus occidentalis) chicks were obtained as part of routine field and nest surveys conducted by North Carolina Audubon (Wilmington, NC).

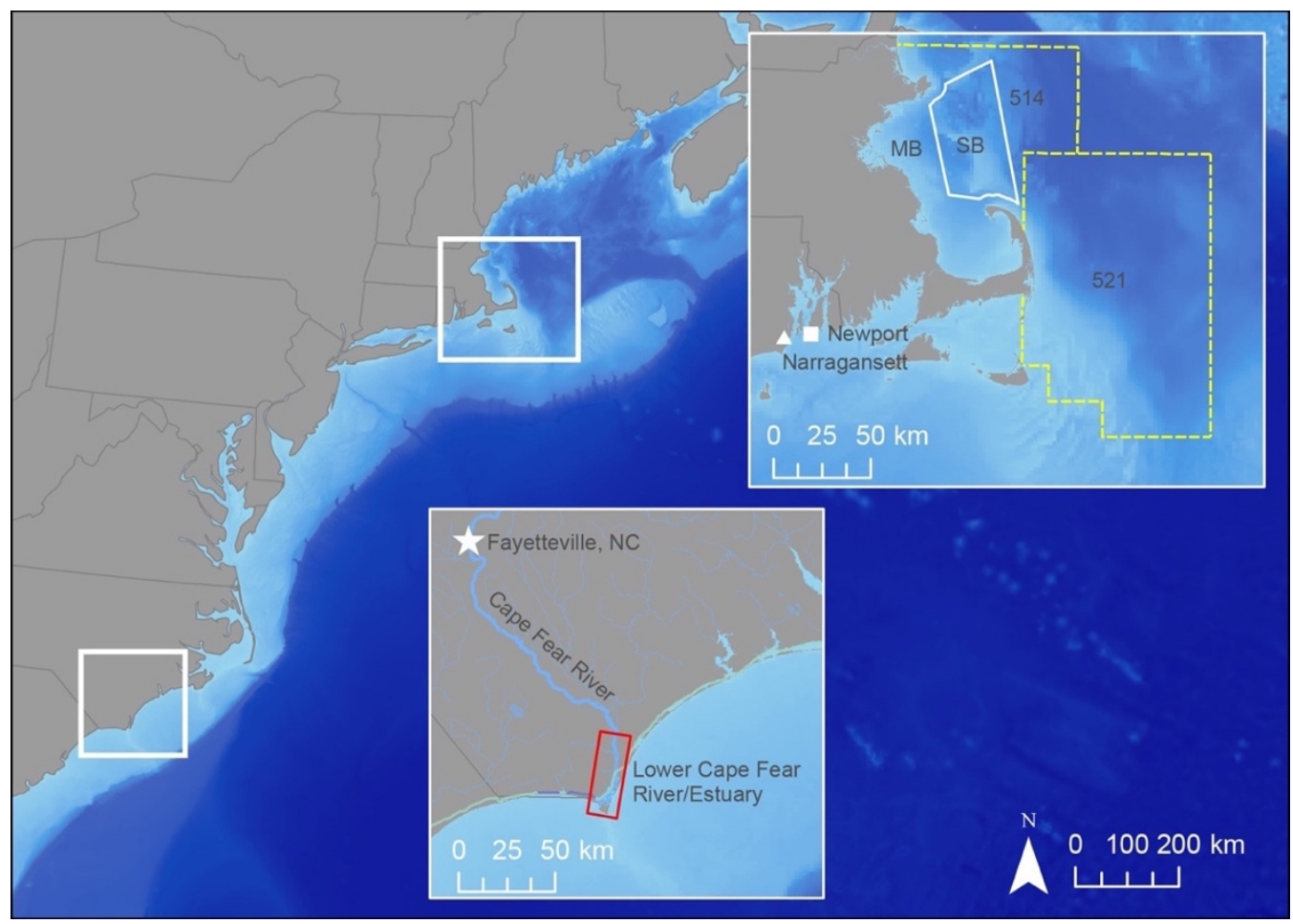

Figure S4.1. Map of the US Atlantic East Coast. Insets provide further detail about collection locations of from each habitat. Great shearwaters were collected as bycatch in Massachusetts Bay and off Cape Cod, designated as NOAA Fisheries Stat Areas 514 and 521. Stellwagen Bank National Marine Sanctuary, a key foraging area for Great shearwaters, is outlined in white and indicated as "SB" in the top right inset. Herring gull chicks came from nests located in Narragansett, RI and Newport, RI. The bottom inset shows the Cape Fear River and Estuary system, located in southeastern NC. A Chemours facility is located 
near Fayetteville, NC, designated on the map as a white star. The red box indicates the lower river and estuary portion of the system; CFRE chicks were hatched on dredge islands near the mouth of the estuary.

The rationale supporting the use of similar seabird species to discuss bioaccumulation was presented elsewhere ${ }^{1}$.

Sample preparation for HRMS. A tissue aliquot was diluted with three parts water and homogenized to a uniform slurry. An aliquot of homogenate was diluted 4:1 with $0.1 \mathrm{M}$ formic acid and vortexed for 30 seconds. The denatured extract was further diluted 5:1 with cold acetonitrile and centrifuged for $5 \mathrm{~min}$ at $10000 \mathrm{rpm}$. An aliquot of the supernatant was removed and combined with dilute ammonium formate buffer $(2.5 \mathrm{mM})$ to obtain a final sample extract ratio of 3 parts aqueous: 1 part organic extract.

HRMS analysis. $100 \mathrm{ul}$ of sample was injected, and chromatographic separation achieved using a Vanquish UPLC system equipped with an Accucore $100 \mathrm{~mm}$ reverse-phase $\mathrm{C} 18$ column, at a flow rate of $300 \mathrm{ul} / \mathrm{min}$. Mobile phase constituents included Solvent A (95:5 water: acetonitrile) and Solvent B (95:5 acetonitrile: water). Mobile phase gradient is detailed in Table S11. Nafion byproduct 2 (Nafion BP2, or 2-[1-[difluoro(1,2,2,2-tetrafluoroethoxy)methyl]1,2,2,2-tetrafluoroethoxy]-1,1,2,2-tetrafluoro-ethanesulfonic acid) and $\mathrm{PFO}_{5}$ DoDA $(2,2,4,4,6,6,8,8,10,10,12,12,12$-Tridecafluoro-3,5,7,9,11pentaoxadodecanoic acid) were identified with authentic native standards, while $\mathrm{PFO}_{4} \mathrm{DA}$ (perfluoro-3,5,7,9-tetraoxadecanoic acid), PFMA (2,3,3,3-tetrafluoro-2(trifluoromethoxy)-propanoic acid), $\mathrm{PFO}_{2} \mathrm{HxA}$ (perfluoro-3,5-dioxahexanoic acid), PEPA (Perfluoro-2-ethoxypropanoic acid), PFO3OA (Perfluoro(3,5,7- 
trioxaoctanoic) acid), NVHOS (1,1,2,2-tetrafluoro-2-(1,2,2,2-tetrafluoroethoxy)ethane sulfonic acid), Nafion byproduct 4 (2,2,3,3,4,5,5,5-4-(1,1,2,2tetrafluoro-2- sulfoethoxy) pentanoic acid), Nafion byproduct 1 (2-[1[difluoro[(1,2,2-trifluoroethenyl)oxy]methyl]-1,2,2,2-tetrafluoroethoxy]-1,1,2,2tetrafluoro-ethanesulfonic acid) and PFECHS (perfluoro-4ethylcyclohexanesulfonate) were detected using previous accurate mass assessment information ${ }^{1}$. PFAS were detected using a Thermo Orbitrap Fusion mass spectrometer using heated electrospray ionization in negative mode (Thermo Fisher Scientific, Waltham, MA, USA). Full scan accurate mass spectra were acquired from 70 to $700 \mathrm{Da}$ with a resolving power of 120,000 Rs for MS1 and at $30,000 \mathrm{Rs}$ for MS2, and a mass accuracy of $\pm 5 \mathrm{ppm}$. Data-dependent acquisition was carried out to acquire MS/MS of select features at a resolving power of 30000. The ion transfer tube was set at $250^{\circ} \mathrm{C}$ and vaporizer temp set at $30^{\circ} \mathrm{C}$. Interference from the tissue matrix prevented the use of the Fusion internal lock mass in this experimental method. Data acquisition and analysis was performed using Xcalibur and Compound Discoverer software (Thermo Fisher Scientific, Waltham, MA, USA).

\section{Quality Assurance and Quality Control}

Six-point, processed and matrix-matched calibration curves were prepared. Curve preparation entailed taking multiple aliquots of "blank" liver tissue (from the same batch of slurried tissue confirmed to be mostly clean of PFAS by HRMS analysis) through each extraction in its entirety. The matrix-matched approach or another approach to account for matrix effects is vital to account for the influence 
or interference of biological co-eluents on PFAS response and derived concentrations $s^{2,3}$.

HRMS curve points were spiked before extraction, and therefore recoverycorrected all subsequent quantitation; these curve points ranged from $0.05-10$ $\mathrm{ng} / \mathrm{ml}$. PFOS was consistently found in organic chicken liver samples, and thus the curve was corrected for background levels of PFOS by subtracting the average of measured background samples $(n=6)$ from PFOS responses measured in curve point samples. All calibration curves used for quantitation demonstrated an $\mathrm{R}^{2} \geq 0.98$, with most demonstrating an $\mathrm{R}^{2} \geq 0.99$.

During HRMS analysis, duplicate process blanks using formic acid and acetonitrile were prepared daily with each sample set, for a total of 8 process blanks. Process blanks were used to identify contamination introduced via sample preparation and instrument background signal. Mobile phase blanks were injected between different types of samples and different tissue types to monitor instrumental background noise and any carryover between samples. No contamination of emerging PFAS was apparent in process blanks; HFPO-DA and several other legacy PFCAs displayed high levels of instrumental background noise as displayed in instrumental blanks and process blanks. No significant background noise was apparent for Nafion $\mathrm{BP} 2, \mathrm{PFO}_{5} \mathrm{DoDA}$, or $\mathrm{PFO}_{4} \mathrm{DA}$, the three PFEAs of interest reported here using HRMS analysis. Samples were analyzed in four batches, and curve stability monitored between runs. Curve responses at all levels varied less than $4 \%$ across all four runs for the three PFEAs reported using HRMS measurements in this analysis. 
Quantification of emerging compounds via HRMS was limited to those samples above the linear range of the calibration curve; the method reporting limit was determined by comparison to blank values plus three times the standard deviation of blank responses or the lowest calibration curve point. Samples below method detection limits were assumed to be zero for summation purposes.

PFOS concentrations were previously measured in liver via both HRMS and LC-MS/MS for parity ${ }^{1}$, and HRMS results were generally found to be within 30\% of LC-MS/MS results and all were within an order of magnitude. Duplicate samples were processed for each tissue matrix, and were found to be within $40 \%$ of each other, with most samples within $15 \%$ of each other.

\section{Relative body burdens}

Relative body burdens (RBBs) were calculated, based on an equation modified from Shi et al. following Equation $1^{4}$ :

$$
\text { Equation 1: } R B B=\frac{C_{\text {tissue }} \times m_{\text {tissue }}}{\text { Total body weight }} \times 100
$$

Tissue weights and body weights were measured for each organ in each individual. Organ weights that could not be completely measured (blood, fat, muscle) were approximated based on literature values or estimates for seabirds or other avifauna. 


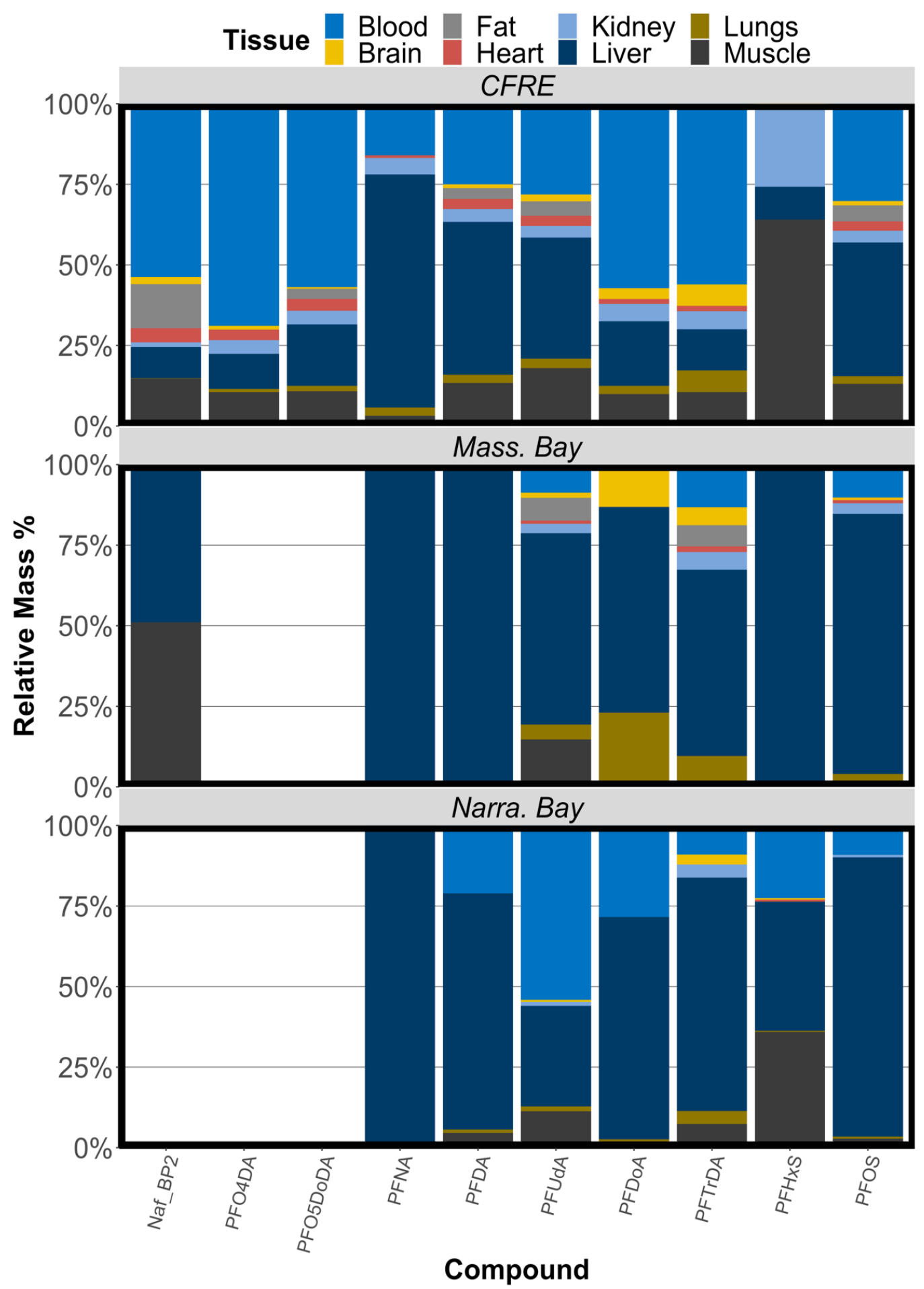

Figure S4.2. RBBs by habitat. Relative body burdens (RBBs) of each PFAS by tissue compartment, stratified by habitat. 


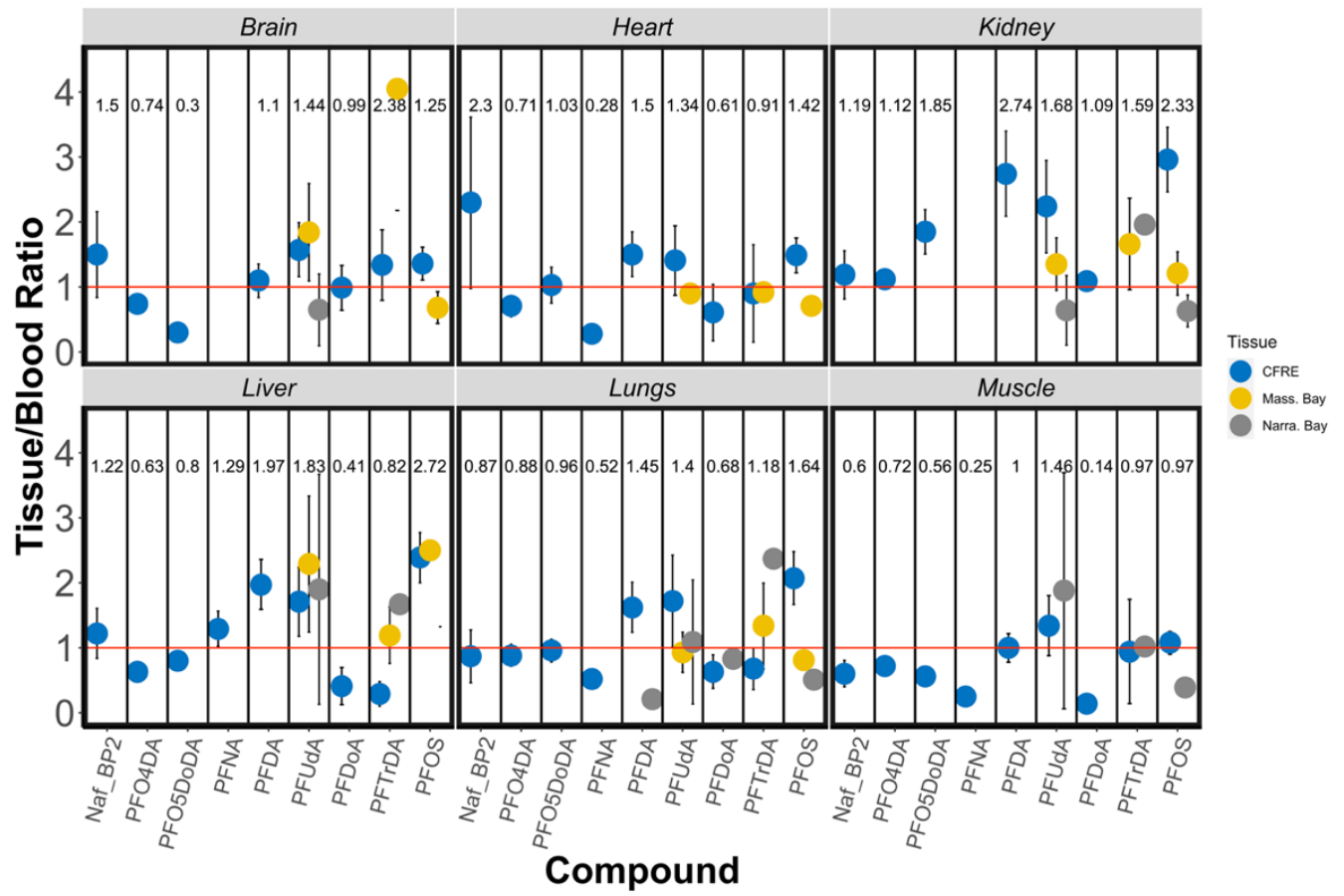

Figure S4.3. TBRs by Habitat. Tissue-blood ratios of each PFAS by tissue compartment; point color denotes habitat, while the black bars delineate the standard error of the mean. 


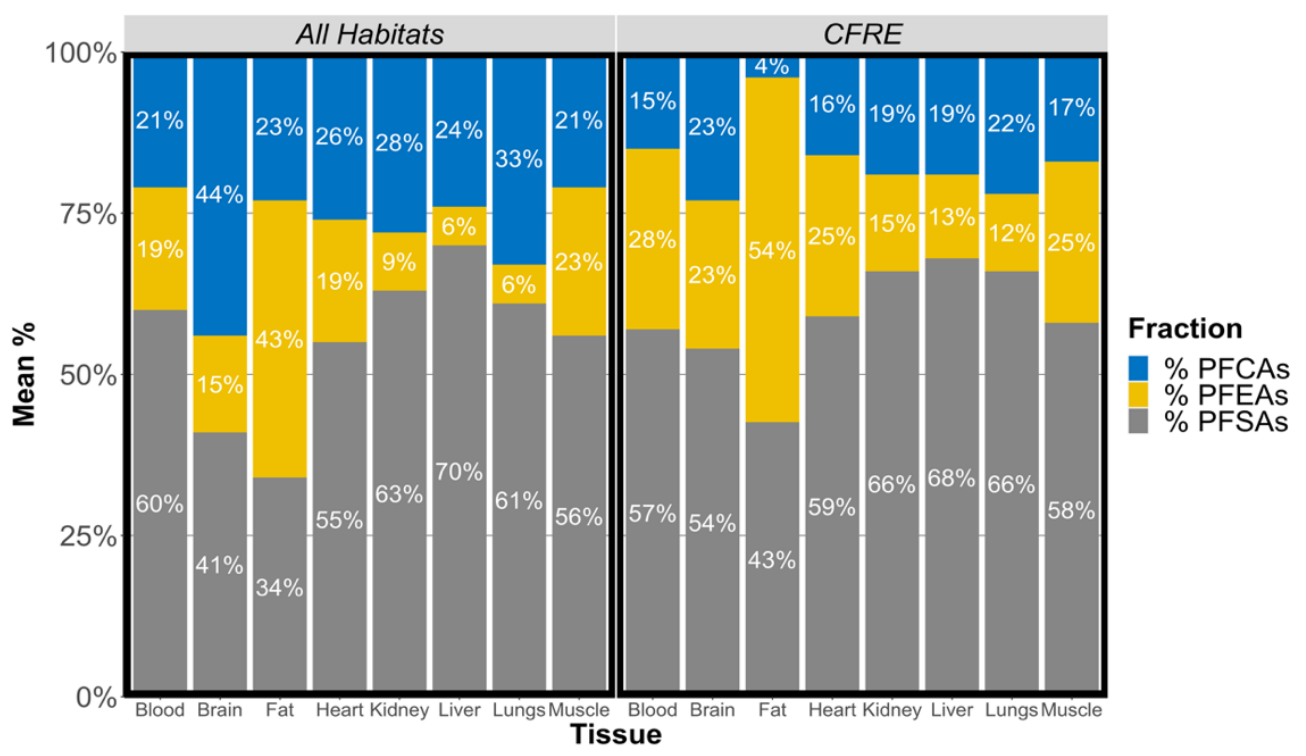

Figure S4.4. Proportions by Tissue. Mean percentages of three subclasses of PFAS observed in eight tissues, averaged across all habitats (left) and solely considering CFRE individuals (right). Percentages reported reflect the concentration of the given PFAS group, divided by the total concentration (in $\mathrm{ng} / \mathrm{g}$ ) observed in a given tissue. The proportion of PFSA in each tissue is majorly dominated by PFOS. PFEA proportions are driven by Nafion BP2. Proportions stratified by habitat and compound are presented in the SI, along with relative mass weighted proportions. 


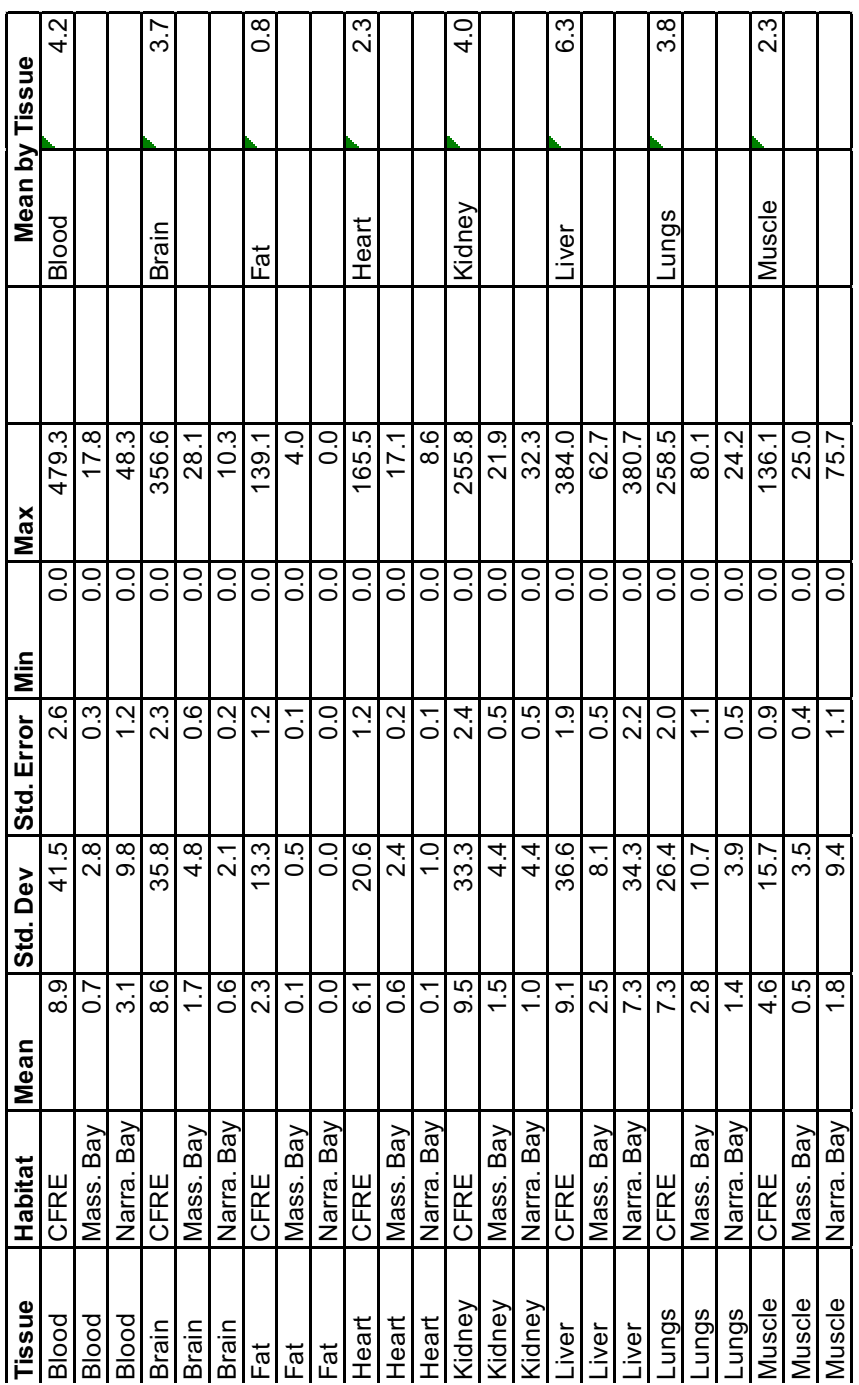

Table S.4.1. Table presenting summary statistics of the sum PFAS in each tissue, stratified by habitat. 


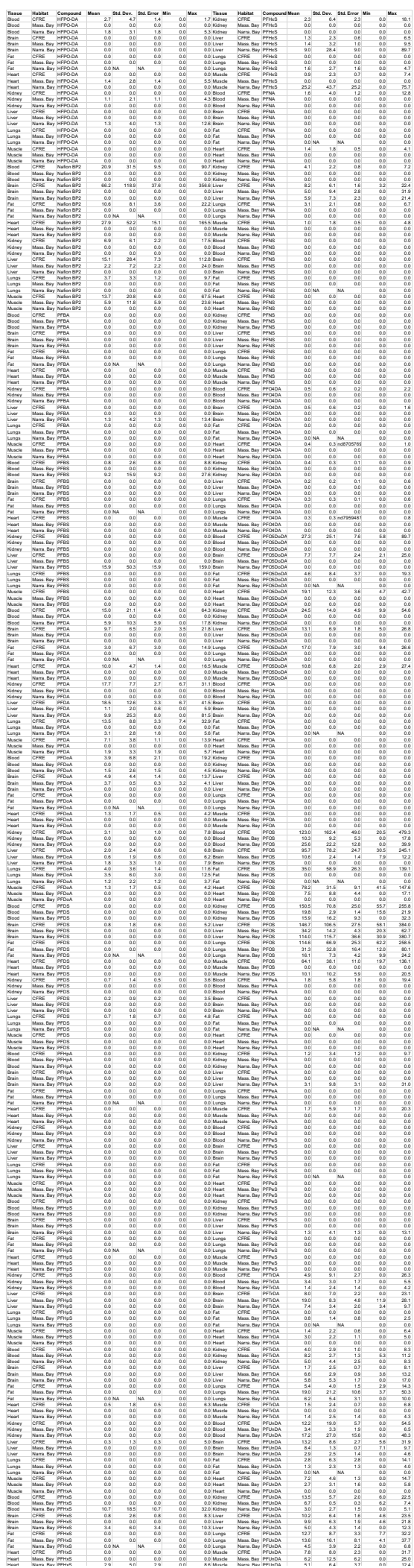

Table S4.2. Table presenting summary statistics of the each PFAS identified in at least one tissue in eight different tissues, stratified by habitat. 


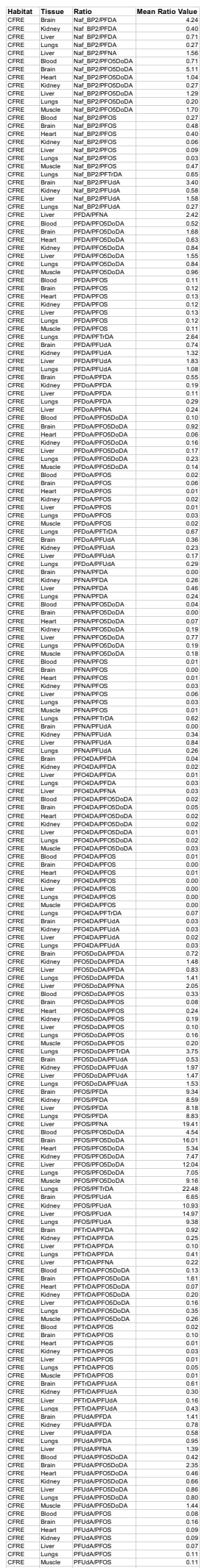

Table S4.3. Table presenting non-zero and non-infinite pairwise ratios in each tissue, stratified by habitat. 


\begin{tabular}{|c|c|c|c|c|c|}
\hline Compound & CAS \# & Family & $\begin{array}{l}\text { \# Fluorinated } \\
\text { Carbons }\end{array}$ & $\begin{array}{c}\text { Detected in at least } \\
\text { one biological sample } \\
\text { above detection } \\
\text { limits? } \\
\end{array}$ & Quantified, using matched native standard or Semi-Quant? \\
\hline N-MeFOSAA & $2355-31-9$ & FASAA & 8 & No & Quantified, using matched native standard \\
\hline N-EtFOSAA & $2991-50-6$ & FASAA & 81 & No & Quantified, using matched native standard \\
\hline FOSA & $754-91-6$ & FASA & 8 & No & Quantified, using matched native standard \\
\hline $4: 2$ FTS & & FTS & 41 & No & Quantified, using matched native standard \\
\hline 6:2 FTS & & FTS & 6 & No & Quantified, using matched native standard \\
\hline $8: 2$ FTS & & FTS & $8 \mid$ & No & Quantified, using matched native standard \\
\hline PFBA & $375-22-4$ & PFCA & 3 & Yes & Quantified, using matched native standard \\
\hline PFPeA & $2706-90-3$ & PFCA & 4 & Yes & Quantified, using matched native standard \\
\hline PFHxA & $307-24-4$ & PFCA & 5 & Yes & Quantified, using matched native standard \\
\hline PFHpA & $375-85-9$ & PFCA & 6 & No & Quantified, using matched native standard \\
\hline PFOA & $335-67-1$ & PFCA & 7 & Yes & Quantified, using matched native standard \\
\hline PFNA & $375-95-1$ & PFCA & 8 & Yes & Quantified, using matched native standard \\
\hline PFDA & $335-76-2$ & PFCA & 9 & Yes & Quantified, using matched native standard \\
\hline PFUnDA & $2058-94-8$ & PFCA & 10 & Yes & Quantified, using matched native standard \\
\hline PFDoA & $307-55-1$ & PFCA & 11 & Yes & Quantified, using matched native standard \\
\hline PFTrDA & $72629-94-8$ & PFCA & 12 & Yes & Quantified, using matched native standard \\
\hline PFTeDA & $376-06-7$ & PFCA & 13 & No & Quantified, using matched native standard \\
\hline PMPA & $13140-29-9$ & PFECA & 31 & No & Semi-quant \\
\hline PFO2HxA & $39492-88-1$ & PFECA & 31 & No & Semi-quant \\
\hline PEPA & 267239-61-2 & PFECA & 4 & No & Semi-quant \\
\hline PFO3OA & $39492-89-2$ & PFECA & 4 & No & Semi-quant \\
\hline HFPO-DA & 13252-13-6 & PFECA & 5 & Yes & Quantified, using matched native standard \\
\hline PFO4DA & $39492-90-5$ & PFECA & 5 & Yes & Semi-quant \\
\hline PFO5DoDA & $39492-91-6$ & PFECA & 6 & Yes & Quantified, using matched native standard \\
\hline Nafion BP4 & na & PFESA & 6 & No & Semi-quant \\
\hline Nafion BP2 & $749836-20-2$ & PFESA & 7 & Yes & Quantified, using matched native standard \\
\hline Nafion BP1 & $29311-67-9$ & PFESA & 7 & No & Semi-quant \\
\hline NVHOS & $\begin{array}{l}801209-99-4 \\
\text { (free acid) }\end{array}$ & PFESA & 4 & No & Semi-quant \\
\hline PFBS & $29420-49-3$ & PFSA & 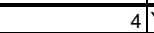 & Yes & Quantified, using matched native standard \\
\hline PFPeS & & PFSA & 5 & Yes & Quantified, using matched native standard \\
\hline PFHxS & $432-50-7$ & PFSA & 6 & Yes & Quantified, using matched native standard \\
\hline PFHpS & & PFSA & 7 & No & Quantified, using matched native standard \\
\hline PFOS & $4021-47-0$ & PFSA & 8 & Yes & Quantified, using matched native standard \\
\hline PFNS & 98789-57-2 & PFSA & 9 & No & Quantified, using matched native standard \\
\hline PFDS & $2806-15-7$ & PFSA & 10 & Yes & Quantified, using matched native standard \\
\hline PFECHS & $646-83-3$ & Cyclic PFSA & 81 & No & Semi-quant \\
\hline
\end{tabular}

Table S4.4. Table presenting all analytes included in this study. Semi-quantitative analytes lacked a matched native and internal standard. Quantitative analytes were measured using at least one exact matched standard. 


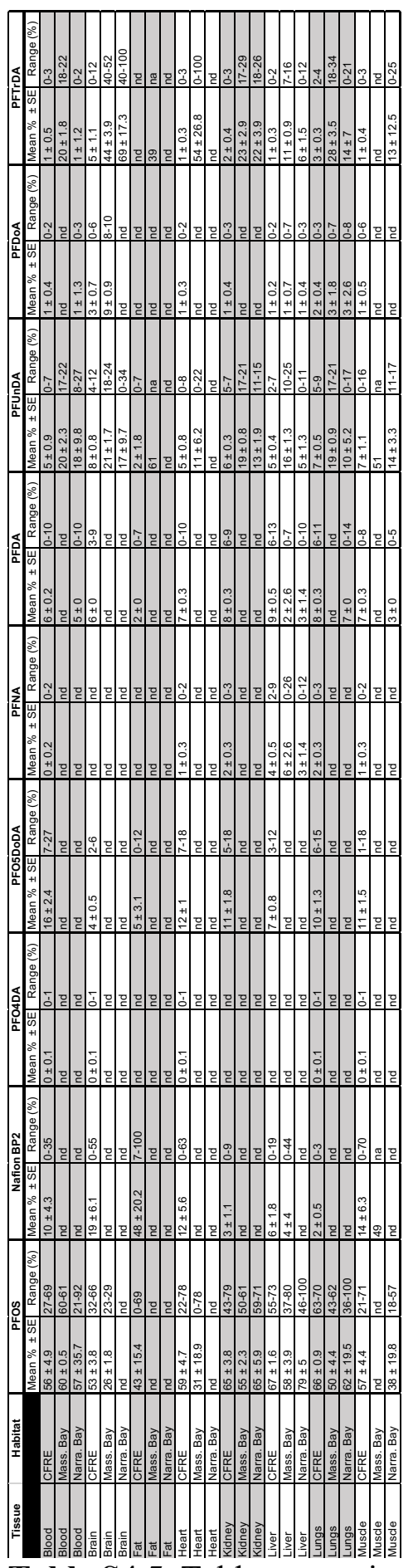

Table S4.5. Table presenting proportions of each PFAS in each tissue, stratified by habitat. 


\begin{tabular}{|c|c|c|c|c|c|c|c|}
\hline Tissue & Fraction & Mean & Std. Dev. (\%) & Median (\%) & Range (\%) & $\mathbf{n}$ & Habitat \\
\hline Blood & $\%$ PFCAs & 10 & 8 & 16 & $0-27$ & 9 & CFRE \\
\hline Brain & $\%$ PFCAs & 23 & 8 & 24 & $9-36$ & 10 & CFRE \\
\hline Fat & $\%$ PFCAs & 4 & 7 & 0 & $0-14$ & 5 & CFRE \\
\hline Heart & $\%$ PFCAs & 16 & 5 & 16 & $4-22$ & 12 & CFRE \\
\hline Kidney & $\%$ PFCAs & 19 & 3 & 21 & $14-23$ & 8 & CFRE \\
\hline \begin{tabular}{|l|} 
Liver \\
\end{tabular} & $\%$ PFCAs & 19 & 4 & 19 & $13-28$ & 12 & CFRE \\
\hline Lungs & $\%$ PFCAs & 22 & 3 & 24 & $17-25$ & 7 & CFRE \\
\hline Muscle & $\%$ PFCAs & 17 & 8 & 16 & $0-28$ & 12 & CFRE \\
\hline Blood & $\%$ PFEAs & 28 & 20 & 21 & $10-73$ & 9 & CFRE \\
\hline Brain & $\%$ PFEAs & 23 & 20 & 17 & $5-60$ & 10 & CFRE \\
\hline Fat & $\%$ PFEAs & 54 & 35 & 49 & $16-100$ & 5 & CFRE \\
\hline Heart & $\%$ PFEAs & 25 & 20 & 18 & $7-74$ & 12 & CFRE \\
\hline Kidney & $\%$ PFEAs & 15 & 7 & 13 & $7-24$ & 8 & CFRE \\
\hline Liver & $\%$ PFEAs & 13 & 6 & 11 & $7-24$ & 12 & CFRE \\
\hline Lungs & $\%$ PFEAs & 12 & 4 & 10 & $9-18$ & 7 & CFRE \\
\hline Muscle & $\%$ PFEAs & 25 & 21 & 22 & $4-79$ & 12 & CFRE \\
\hline Blood & $\%$ PFSAs & 57 & 15 & 61 & $27-73$ & 9 & CFRE \\
\hline Brain & $\%$ PFSAs & 54 & 13 & 58 & $32-70$ & 10 & CFRE \\
\hline Fat & $\%$ PFSAs & 43 & 31 & 51 & $0-69$ & 5 & CFRE \\
\hline Heart & $\%$ PFSAs & 59 & 16 & 66 & $22-78$ & 12 & CFRE \\
\hline Kidney & $\%$ PFSAs & 66 & 9 & 65 & 53-79 & 8 & CFRE \\
\hline \begin{tabular}{|l} 
Liver \\
\end{tabular} & $\%$ PFSAs & 68 & 5 & 69 & $55-73$ & 12 & CFRE \\
\hline Lungs & $\%$ PFSAs & 66 & 2 & 66 & $63-70$ & 7 & CFRE \\
\hline Muscle & $\%$ PFSAs & 58 & 15 & 63 & 21-71 & 12 & CFRE \\
\hline Blood & $\%$ PFCAs & 21 & 13 & 18 & $0-42$ & 15 & All Habitats \\
\hline Brain & $\%$ PFCAs & 44 & 31 & 29 & $9-100$ & 16 & All Habitats \\
\hline Fat & $\%$ PFCAs & 23 & 44 & 0 & $0-100$ & 9 & All Habitats \\
\hline Heart & $\%$ PFCAs & 26 & 29 & 17 & $0-100$ & 19 & All Habitats \\
\hline Kidney & $\%$ PFCAs & 28 & 11 & 23 & $14-47$ & 15 & All Habitats \\
\hline \begin{tabular}{|l|} 
Liver \\
\end{tabular} & $\%$ PFCAs & 24 & 12 & 21 & $0-55$ & 33 & All Habitats \\
\hline Lungs & $\%$ PFCAs & 33 & 18 & 25 & $0-57$ & 14 & All Habitats \\
\hline Muscle & $\%$ PFCAs & 21 & 13 & 17 & $0-51$ & 19 & All Habitats \\
\hline Blood & $\%$ PFEAs & 19 & 21 & 16 & $0-73$ & 15 & All Habitats \\
\hline Brain & $\%$ PFEAs & 15 & 19 & 7 & $0-60$ & 16 & All Habitats \\
\hline Fat & $\%$ PFEAs & 43 & 39 & 40 & $0-100$ & 9 & All Habitats \\
\hline Heart & $\%$ PFEAs & 19 & 20 & 16 & $0-74$ & 19 & All Habitats \\
\hline Kidney & $\%$ PFEAs & 9 & 9 & 8 & $0-24$ & 15 & All Habitats \\
\hline Liver & $\%$ PFEAs & 6 & 10 & 0 & $0-44$ & 33 & All Habitats \\
\hline Lungs & $\%$ PFEAs & 6 & 7 & 4 & $0-18$ & 14 & All Habitats \\
\hline Muscle & $\%$ PFEAs & 23 & 22 & 22 & $0-79$ & 19 & All Habitats \\
\hline Blood & $\%$ PFSAs & 60 & 16 & 61 & $27-92$ & 15 & All Habitats \\
\hline Brain & $\%$ PFSAs & 41 & 22 & 45 & $0-70$ & 16 & All Habitats \\
\hline Fat & $\%$ PFSAs & 34 & 33 & 42 & $0-69$ & 9 & All Habitats \\
\hline Heart & $\%$ PFSAs & 55 & 27 & 65 & $0-100$ & 19 & All Habitats \\
\hline Kidney & $\%$ PFSAs & 63 & 9 & 61 & $50-79$ & 15 & All Habitats \\
\hline \begin{tabular}{|l|} 
Liver \\
\end{tabular} & $\%$ PFSAs & 70 & 13 & 70 & $37-100$ & 33 & All Habitats \\
\hline Lungs & $\%$ PFSAs & 61 & 14 & 63 & $43-100$ & 14 & All Habitats \\
\hline Muscle & $\%$ PFSAs & 56 & 22 & 62 & $0-84$ & 19 & All Habitats \\
\hline
\end{tabular}

Table S4.6. Table presenting proportions of each PFAS family in each tissue, stratified by CFRE and all habitats combined. 


\begin{tabular}{|l|r|r|r|}
\hline \multicolumn{5}{|c|}{$\%$ Difference } \\
\hline Sigma (Range) & PFOS - Nafion BP2 & PFOA - PFO5DoDA & PFOA - PFO4DA \\
\hline \hline$-0.03--0.021$ & 0 & 0 & 0 \\
\hline$-0.011--0.02$ & 6.96 & 0 & 0 \\
\hline$-0.01--0.006$ & 4.174 & 4.43 & 3.607 \\
\hline $0.001--0.005$ & -17.175 & 16.392 & 2.437 \\
\hline 0 & -22.757 & 1.888 & -7.988 \\
\hline $0.001-0.005$ & 29.071 & 20.393 & 5.465 \\
\hline $0.006-0.01$ & 0.306 & 1.062 & 1.111 \\
\hline $0.011-0.02$ & 0.298 & 1.157 & -0.348 \\
\hline $0.021-0.03$ & 0 & -0.219 & -0.155 \\
\hline
\end{tabular}

Table S4.7. Percent difference of sigma probabilities between the lowest energy conformer of each PFEA anion, compared to an appropriate low energy PFAA anion analogue. Negative sigma values correspond to a positive surface charge density, while positive values correspond to a negative surface charge density 


\section{LITERATURE CITED}

(1) Robuck, A.; Cantwell, M. G.; McCord, J. P.; Addison, L. M.; Pfohl, M.; Strynar, M. J.; McKinney, R. A.; Katz, D. R.; Wiley, D. N.; Lohmann, R. Legacy and Novel Per- and Polyfluoroalkyl Substances (PFAS) in Juvenile Seabirds from the US Atlantic Coast. Environ. Sci. Technol. 2020.

https://doi.org/10.1021/acs.est.0c01951.

(2) Berger, U.; Haukås, M.; Hauk, M. Validation of a Screening Method Based on Liquid Chromatography Coupled to High-Resolution Mass Spectrometry for Analysis of Perfluoroalkylated Substances in Biota. $J$. Chromatogr. A 2005, 1081 (2), 210-217. https://doi.org/10.1016/j.chroma.2005.05.064.

(3) Chambers, E.; Wagrowski-Diehl, D. M.; Lu, Z.; Mazzeo, J. R. Systematic and Comprehensive Strategy for Reducing Matrix Effects in LC/MS/MS Analyses. J. Chromatogr. B Anal. Technol. Biomed. Life Sci. 2007, 852 (1-2), 22-34. https://doi.org/10.1016/j.jchromb.2006.12.030.

(4) Shi, Y.; Vestergren, R.; Nost, T. H.; Zhou, Z.; Cai, Y. Probing the Differential Tissue Distribution and Bioaccumulation Behavior of Per- and Polyfluoroalkyl Substances of Varying Chain-Lengths, Isomeric Structures and Functional Groups in Crucian Carp. Environ. Sci. Technol. 2018, 52 (8), 45924600. https://doi.org/10.1021/acs.est.7b06128. 


\section{CHAPTER 5}

PLASTIC INGESTION IN THE GREAT SHEARWATER, AN ENDEMIC ATLANTIC SEABIRD

This manuscript has been formatted for Frontiers in Marine Science, and the abstract has been accepted to a special topic issue on plastic ingestion (March 2021)

Anna R. Robuck ${ }^{*}$, David N. Wiley ${ }^{2}$, Christine A. Hudak ${ }^{3}$, Lindsay Agvent ${ }^{1}$, Gwenyth Emery ${ }^{1}$, Peter G. Ryan ${ }^{4}$, Vonica A. Perold ${ }^{4}$, Kevin D. Powers ${ }^{2}$, Johanna Pedersen, Michael A. Thompson², Michael J. Moore ${ }^{5}$, Craig Harms ${ }^{6}$, Richard McKinney $^{7}$, and Rainer Lohmann ${ }^{1}$

${ }^{1}$ University of Rhode Island Graduate School of Oceanography, Narragansett, RI 02882

${ }^{2}$ Stellwagen Bank National Marine Sanctuary, National Oceanic and Atmospheric Administration, Scituate, MA 02066

${ }^{3}$ Center for Coastal Studies, Provincetown, MA 02657

${ }^{4}$ Percy FitzPatrick Institute of African Ornithology, DST/NRF Centre of Excellence, University of Cape Town, Rondebosch 7701, South Africa

${ }^{5}$ Woods Hole Oceanographic Institute, Woods Hole, MA 02540

${ }^{6}$ Center for Marine Sciences and Technology, North Carolina State University, Morehead City, NC 28557 
${ }^{7}$ Atlantic Coastal Environmental Sciences Division, Center for Environmental Measurement and Modeling, US Environmental Protection Agency, Narragansett, RI 02882

*Corresponding author, present address:

Anna Robuck,

215 S Ferry Rd Narragansett RI 02882, annarobuck@gmail.com

KEYWORDS: plastic ingestion, seabirds

ABSTRACT

Limited work to date has examined plastic ingestion in temperate, endemic Atlantic seabirds like Great Shearwaters (Ardenna gravis), although this species is particularly prone to plastic ingestion as wide-ranging petrels. We examined 175 Great Shearwaters obtained from 2010 - 2019 in the Northwest and South Atlantic to assess accumulation of ingested plastic as well as trends over time and between locations. Over 1500 plastic fragments were documented in the proventriculus portion of the gastrointestinal tract, with an average of 8 plastic fragments per bird. Juveniles accumulated plastic more frequently than adults, with an ingestion frequency of $98 \%$ compared to $58 \%$ in breeding adults. The frequency of plastic occurrence (FO) observed in juveniles was higher than in previous studies focused on this species in prior decades. Each fragment was assessed using 
Fourier-transform ion spectroscopy to discern polymer identity. We found lowand high-density polyethylene fragments dominated within all years and locations. Breeding adults contained a higher proportion of larger polypropylene fragments compared to juveniles and non-breeding adults, likely indicative of an increased use of remote, pelagic habitats subject to reduced inputs of smaller, land-derived plastics. Different signatures of polymer type and size between breeding and non-breeding mature birds likewise suggests rapid turnover of ingested plastics commensurate with migratory stage and location. No fragments of the same size (4-10 $\mathrm{mm}$ in largest dimension) were found in the primary forage fish prey (sand lance, $n=202$ ) that supports Great Shearwaters in Massachusetts Bay, suggesting this species directly ingest the bulk of their plastic loads rather than accumulating via trophic transfer. This work is the first to comprehensively measure the accumulation of ingested plastics by Great Shearwaters over the last decade and across multiple locations spanning their yearly migration cycle, and emphasizes their utility as sentinels of plastic pollution in Atlantic systems.

\section{INTRODUCTION}

Mismanaged plastic waste has emerged as a globally significant pollutant in aquatic ecosystems. Current estimates suggest $4.8-12.7$ million metric tons of mismanaged plastic waste enter the global ocean each year as debris ${ }^{1}$, with modeling efforts suggesting the amount of plastic debris entering marine environments will increase by an order of magnitude by 2025 without significant 
policy and consumer interventions. The current amount of ocean plastic and projected additional releases are problematic due to potential for plastic debris to degrade habitat integrity, entangle marine life, act as a vector of invasive species transport, or be ingested by marine fauna ${ }^{2}$. These impacts are predicted in marine life at every level of the food chain due to the wide spectrum of sizes and materials constituting ocean plastic loads ${ }^{2,3}$.

Marine plastic debris originates from a variety of land-based human uses and activities, as well as from maritime sources such as shipping and fishing activities, leading to inputs of variable types and sizes of plastic materials to the marine environment ${ }^{4}$. Yet a key unifying characteristic of marine plastic debris is its inevitable degradation into progressively smaller pieces over time, leading to proliferation of meso-, micro- and nanoplastics in all compartments of the marine environment, even within the remotest marine habitats ${ }^{5,6}$.

A significant portion of microplastics $(<5 \mathrm{~mm})$ entering the ocean are thought to become fouled ${ }^{7}$ and settle out of the surface water compartment into intermediate depths or ocean sediments ${ }^{8}$, while plastic items of variable size and composition remain buoyant. An estimated 5 - 125 trillion pieces of buoyant plastic $>100$ um reside within the ocean surface layer, engaging in biophysical interactions and subject to oceanic scale circulation ${ }^{9-11}$. Plastic pieces within coastal and pelagic surface environments are predominantly composed of polyethylene and polypropylene ${ }^{12}$, two of the most highly produced olefin plastics used in an array of single-use consumer and industrial products. 
Abundance of small plastic pieces within ocean surface environments results in an increased probability of encounter between euphotic marine fauna and plastic items, as well as increased opportunity for ingestion of plastic items. Ingestion of plastic may occur via direct ingestion of plastic pieces ${ }^{13}$, or ingestion of prey items containing plastic ${ }^{14}$. In vertebrate species, ingested plastics can result in blockages or tears within the digestive tract ${ }^{15}$, reduced feeding due to false satiation ${ }^{16}$, as well as potential uptake of adsorbed chemical pollutants or innate chemical additives ${ }^{17}$.

The accumulation of ingested plastics in seabirds has been documented since the 1960s; Wilcox et al. (2015) found that 59\% of seabird species examined between 1962 and 2012 had ingested plastic, and, within those studies, on average $29 \%$ of individuals had plastic in their gastrointestinal tract ${ }^{4}$. Seabirds in the order Procellariiformes (e.g. albatrosses, shearwaters, petrels, and storm petrels) are thought to be most vulnerable to plastic ingestion ${ }^{18,19}$ related to their wide-ranging pelagic habits ${ }^{20}$ and heavy use of olfactory cues ${ }^{21}$, yet the frequency of plastic ingestion varies widely between members of this order. Some albatross species have been shown to infrequently ingest plastics, while other tube-nosed seabirds such as short-tailed shearwaters ${ }^{22}$, Northern fulmars ${ }^{23}$, and great shearwaters ${ }^{24}$ have been found to ingest plastic at high rates ( $\sim 80-100 \%$ frequency of occurrence). Modeling and review efforts suggest the incidence of plastic ingestion in seabirds increases with abundance of plastics in the environment ${ }^{25}$. For example, Northern fulmars in the North Sea have been monitored for plastic ingestion at their breeding colonies since the 1970s; the ingestion of industrial 
pellets, or nurdles, within this monitored population has been found to roughly track the measured abundance of industrial pellets in ocean surface water ${ }^{25}$. Efforts to curb mismanagement and loss of industrial pellets in the 1980s and 1990s resulted in reduced concentrations of nurdles within the North Atlantic subtropical gyre, and declines in the geometric mean of industrial pellets in fulmar GITs. Seabird plastic ingestion also increases with use of plastic geographical hotspots ${ }^{4,26}$; subtropical gyres and the southern boundary of the Atlantic, Pacific, and Indian Oceans are considered hotspots of plastic in the surface layer. Incidence of plastic ingestion also varies according to foraging strategy, with trophic generalists and surface seizing birds reliant on crustacean diets the most vulnerable ${ }^{27}$.

However, data describing the occurrence of ingested plastic as well as trends over time and space is irregular. Existing studies often focus on a narrow subset of accessible species during the breeding season when chicks are immobile and adults are subject to area-restricted foraging behaviors, limiting understanding of plastic ingestion over the complete annual cycle and overall life cycle of a diversity of species. Likewise, few studies couple measurement of plastic ingestion with comprehensive necropsy data, description of plastic piece morphology, and identification of polymer type. Assessment of plastic polymer identity is particularly valuable, as it aids in identification of potential sources or source products. Those studies that incorporate polymer analysis indicate that polyethylene and polypropylene are typically the dominant polymers ingested in seabirds, and item composition is strongly correlated to item morphology ${ }^{28}$. The 
polymer composition of ingested plastics is related to spatial patterns of plastic abundance, rather than a preference for ingestion of any one polymer type.

We set out to investigate the accumulation of ingested plastics in Great Shearwaters (Ardenna gravis), an endemic Atlantic Procellariiform seabird. The US East Coast and the Gulf of Maine are more poorly described in terms of plastic ingestion in marine biota compared to other regions, despite the high density of human populations and potential plastic sources along this coastal margin. Great Shearwaters frequent the Gulf of Maine and Massachusetts Bay along the US East Coast, and have been previously assessed for plastic ingestion. However, no comprehensive efforts have harmonized methodology and pieced together trends describing occurrence over space and time in this species. Monitoring of plastic in this species is valuable due to its abundance ${ }^{29}$, welldescribed habitat use $\mathrm{e}^{30,31}$, its colocation with key marine megafauna $\mathrm{a}^{32}$, and its compact representation of divergent Atlantic habits, spanning developed coastal margins within the Gulf of Maine as well as vast pelagic regions of the South Atlantic.

Here we comprehensively describe plastic ingestion in this species, using bycatch and beach-cast samples obtained over 2010 - 2019 from multiple habitats included in the annual cycle of this trans-equatorial migrant. The goal of this study included a) evaluation of ingested plastics over time, to deduce any temporal trends, b) comparison of plastic categories and polymer types found between different locations and time periods, and c) assess the trophic or environmental origin of ingested plastics. 


\section{MATERIALS AND METHODS}

\section{Study species}

The Great Shearwater (Ardenna gravis) is a long-lived, pelagic seabird in the Procellariiformes order of seabirds. Generally between $600-1100 \mathrm{~g}$, this medium-sized "tube nose" is a trans-equatorial migrant that inhabits the Gulf of Maine in the NW Atlantic during its non-breeding period over the boreal summer. Tracking and necropsy data suggest age-specific segregation of their nonbreeding range, with preferential use of the SW Gulf of Maine and Massachusetts Bay by juveniles ${ }^{31}$. This species breeds during the boreal winter in the South Atlantic, on Gough Island, Inaccessible Island, and Nightingale Island in the St. Helena Island Group and Kidney Island in the Falkland Islands ${ }^{29}$. Great Shearwaters feed on squid, crustacea, and small forage fish including sand lance (Ammodytes spp. $)^{31}$, and have regularly been observed attending fishing vessels seeking fisheries offal. Most foraging activities occur within the top $10 \mathrm{~m}$ of the water column via surface plunging and seizing behaviors, with most dives less than $2 \mathrm{~m}$ in depth ${ }^{33}$.

The accumulation of ingested plastics has been previously assessed in this seabird within its breeding and non-breeding range over the past sixty years via studies covering a single year, or a span up to 14 years, including variable numbers of individuals $(n=1-84)$. The most recent published work examined 84 individuals collected between 2000 - 2011 from Sable Island, Nova Scotia, Canada in the NW Atlantic. These efforts have generally possessed low yearly 
sample sizes from one geographic region, and have been unable to assess any temporal or spatial trends. Specimen Collection, Necropsy, and Sample Condition

A total of 175 Great Shearwaters were analyzed for plastics, from three habitats (Fig. 1). 135 dead Great Shearwater juveniles and adults were obtained as bycatch from the National Oceanic and Atmospheric Administration (NOAA) Northeast Fisheries Observer Program (Falmouth, MA) from Massachusetts Bay. Bycatch collections spanned $2010-2019$ and the majority of samples were collected in the months of August - November each year, after approximately 2 or more months of foraging in the NW Atlantic. 15 Great Shearwaters were obtained in 2017 from the southeastern coast of North Carolina, USA, after having washed up dead on shorelines along the Outer Banks and the Crystal Coast region of North Carolina. 25 Great Shearwaters were obtained from long-lining activities around Gough Island in 2017 - 2018, in the St Helena Island group in the south Atlantic. Each individual was frozen individually as a whole specimen upon collection and stored at -20C until necropsy.

All individuals were necropsied in a standardized manner ${ }^{34}$, including measurements of morphometric characteristics, body condition, and organ weights. Age was determined following the age characterization of Great Shearwaters as defined by Powers et al. $(2020)^{31}$, and sex based upon gonad presentation. Multiple tissues, including the entire gastrointestinal tract (GIT), were collected and frozen at $-15^{\circ} \mathrm{C}$ until analysis. Lung hemorrhaging and edema was apparent in some bycatch seabirds (indicative of drowning), but there was no 
evidence of seawater intrusion into the coelomic cavity, and no perforations or damage to other internal organs excluding lung hemorrhaging.

Analysis of accumulated, ingested plastics

Food debris was commonly found in the esophagus and mouth of sampled bycatch individuals, likely due to regurgitation during entanglement and drowning. Given the abundance of bycatch samples used in this study, the esophagus and proventriculus were not assessed for plastic contents, assuming any assessment of plastic occurrence in these compartments may be biased by regurgitation. Plastics were instead assessed in the ventriculus, or "gizzard", a muscular organ used to grind up squid beaks and other indigestible, hard diet materials. In Procellariiform seabirds, the isthmus connecting the proventriculus and ventriculus is angled and narrow, unlike the more linear junctions seen in other seabirds. This unique configuration is thought to restrict the passage of materials from the ventriculus back into the proventriculus, insulating evaluated organ contents from bias related to regurgitation. A small test $(\mathrm{n}=5)$ assessing plastic in both the proventriculus and ventriculus demonstrated this approach was appropriate, as $\sim 90 \%$ of accumulated plastics occurred in the ventriculus; this is further supported in the literature ${ }^{35}$.

Plastics were obtained from the ventriculus as previously described ${ }^{25,34,36}$. Briefly, the organ was cut open and flushed thoroughly using deionized water over a sieve with a mesh size of $1 \mathrm{~mm}^{2}$. While plastics smaller than $1 \mathrm{~mm}^{2}$ may be present within the samples, we did not screen for this size fraction due to facility and equipment constraints. Plastic items were visually separated from natural 
items with the aid of a dissection microscope, magnifying lenses, or density separation techniques as necessary. Natural and plastic materials were combined in plastic or glass petri dishes (respectively), and dried in a fume food for $\sim 2$ days. Plastic material was further sorted into established qualitative categories outlined by van Franeker et al. (2015) and Provencher et al. (2017) ${ }^{36,37}$. Each plastic item was first classified as an industrial (nurdle or pellet) or user plastic (non-industrial items likely sourced from consumer use) and further differentiated into five subcategories including sheet-like plastics (e.g., bag and film), threadlike plastics (e.g., microfibers, rope), foam (e.g., polystyrene), fragments (unidentifiable hard plastics), and other (e.g., balloon, rubber, melted

plastic). Each piece of plastic was weighed to the nearest $0.0001 \mathrm{~g}$ using an analytical balance, and length, width, and thickness measured to the nearest 0.01 $\mathrm{mm}$ using a calibrated electronic caliper. Soft plastics were weighed but not measured for morphology. Plastic pieces were also classified into a plastic size category (e.g. micro-, meso-, and macro-) after Barnes et al. ${ }^{5}$

\section{Polymer analysis}

1000 plastic pieces were analyzed for polymer identity using Fourier Transform Infrared (FTIR) spectroscopy. Prior to analysis, each individual fragment was cleaned with deionized water to remove biological debris and filed with a metal file to obtain a flat surface or further remove biological debris for the analysis. The fragments were scanned through a Thermo Scientific Nicolet TM iS5 FTIR spectrometer with an ATR accessory, using the Thermo Scientific Omnic software (Thermo Fisher Scientific, Waltham, MA). A diamond crystal 
ATR plate was used for most analyses, however for analysis of black plastic a germanium crystal ATR plate was used due to the crystal's high refractive index and its capacity to accurately scan high absorbing substances such as carbonblack colored objects.

A spectrum was obtained for each plastic fragment and run through the spectral library search. The principal spectral libraries used were the Aldrich Polymers library, the Hummel Polymer and Additives library, and the Center for Coastal Studies (Provincetown, MA) private library. Each result from the spectral library was verified by identifying the spectrum's wavelength peaks and matching wavelength numbers to the known wavelength ranges of the corresponding polymer. Only fragments with greater than a $70 \%$ confidence of assignment were reported $^{38}$.

Each identified plastic fragment was categorized under one of 17 polymer groups, while the non-plastic fragments were categorized under four groups (biological, vegetation, rock, or natural fiber blend). Polyethylene was further divided into low density polyethylene (LDPE), high density polyethylene (HDPE), and polyethylene (inconclusive on the type of polyethylene). Additives ranged from poly(ethylene:propylene:diene) (EPDM rubber) to oil based additives. Mixed polymers were labeled under the primary polymer.

\section{Statistical Analysis}

All data manipulation and statistical analyses were performed in $\mathrm{R}$ version 3.6.1 (R Core Team, 2020) ${ }^{39}$. Quantitative data were checked for normality and homoscedasticity using the Shapiro-Wilk test and Levene's test. Plastic count and 
mass data were non-normal despite log transformation and therefore treated nonparametrically for statistical analyses; year groups displayed no significant differences in variance. Differences between years or groups were assessed using Kruskal-Wallis tests with post hoc application of Dunn's test with Bonferroni correction for multiple testing. Relationships between continuous variables were assessed using Spearman rank correlation coefficients $\left(\mathrm{R}_{\mathrm{s}}{ }^{2}\right)$. Potential predictors of plastic ingestion mass or count were evaluated using generalized linear models (GLMs) considering year, age, sex, and morphometric measurements as potential predictors. Model evaluation was performed stepwise manually starting with the saturated model. Model significance and performance was further evaluated if needed using Tukey's Honest Significant Differences post hoc test for multiple comparisons and Akaike's Information Criterion (AIC) to rank models, with a lower AIC assumed to indicate the better model. Effects observed within all statistical tests were considered significant when $\mathrm{p}<0.05$.

\section{RESULTS AND DISCUSSION}

Accumulation and frequency of ingested plastics

The mass and number of plastics varied by location and year. Considering all years of data, Massachusetts Bay birds contained an average of 8.5 plastic items and $151 \mathrm{mg}$ plastic, Gough Island individuals contained an average of 9.6 plastic items and $236 \mathrm{mg}$ plastic, and individuals found off the coast of North Carolina contained an average of 12 items and $181 \mathrm{mg}$ plastic (Table 1). Breeding phase adults in the South Atlantic contained a significantly higher mass of plastic 
compared to their North Atlantic counterparts (Kruskal-Wallis test, $\mathrm{p}<0.01$ ), but not a higher count of plastics. Count and mass data were highly correlated across all locations $\left(\left(\mathrm{R}_{\mathrm{s}}{ }^{2}=0.88, \mathrm{p}<0.001\right)\right.$. Wet weight, tarsus length, culmen length, wing chord, d13C, d15N, body condition index, liver weight, heart weight, kidney weight, and adipose fat depth were not significantly related to the count or mass of plastic individually or interactively, suggesting a lack of relationship between individual health and plastic occurrence/accumulation.

No statistically significant trends in count or mass were apparent over 2010 2019 considering all Massachusetts Bay individuals, likely related to low sample size and the resulting variability of plastic metrics apparent in some years (e.g. 2014, 2016, 2018). Only considering those years with sufficient sample size (n > 15 birds) from Massachusetts Bay, plastic mass increased over time between 2010 and $2019\left(\mathrm{R}_{\mathrm{s}}^{2}=0.87, \mathrm{p}=0.044\right)$.

Count and mass averages found in birds from each location are similar to previously published estimates (mean number of plastics: $9.5-22.0$, mean mass plastics: $0.17-2.52 \mathrm{~g})^{24}$, but results are not directly comparable. Previous studies report mass and number of plastics in the total gastrointestinal tract rather than in specific compartments (i.e. the ventriculus) as we report here.

Frequency of occurrence, or FO, describes the number of individuals containing plastic as a function of the total sample set. FO varied by location, year, and age in this study. Young and hatch year birds possessed the highest FO, with bycatch individuals from Massachusetts Bay demonstrating an FO of $98 \%$ (n $=103$ ) and young beach-cast birds from North Carolina evidencing an FO of 
$100 \%(n=15)$. Mature birds from Massachusetts Bay possessed an FO of $87 \%(n$ $=23$ ) while mature breeding birds from Gough Island possessed an FO of 58\% (n $=58)$ (Table 2). Differential plastic ingestion between juvenile and adult birds has been previously found in short-tailed shearwaters from eastern Australia, where juveniles were found to ingest plastic more frequently than adults ( $85 \%$ vs. $62.7 \%$, respectively), as well as a larger number of plastics compared to adults.

Our data adds nuance to age-specific plastic ingestion by suggesting adults during or directly after the chick-rearing phase have much lower rates of plastic ingestion compared to juveniles assessed during the non-breeding phase, while adults during the non-breeding phase have lower but more comparable rates to non-breeding juveniles. The substantially lower frequency of plastics in breeding adults may be due to offloading of plastic pieces to chicks prior to fragments traveling to the ventriculus and lower GIT beyond the influence of regurgitation, or this may be due to reduced plastic occurrence in habitats supporting adults during the chick-rearing phase. We consider the latter unlikely, as the South Atlantic subtropical gyre is thought to be a growing hotspot of plastic occurrence and seabird plastic ingestion ${ }^{9,10,26}$. The lower rates of plastic ingestion in nonbreeding adults may be due to use of more remote habitats within the NW Atlantic ${ }^{31}$, or increased foraging experience compared to naïve young birds ${ }^{40}$.

The FO observed in juveniles is higher than previous literature estimates from studies of comparable sample size conducted within the same species. Moser and Lee (1992) estimated an FO of 64\% based on assessment of 55 individuals from the NW Atlantic collected between $1975-1989^{18}$. More recently, Bond et 
al. (2018) measured an FO of $88 \%$ based upon assessment of 84 Great

Shearwaters beach-cast on Sable Island, Nova Scotia, Canada in the NW Atlantic from $2000-2011^{24}$.

The increased FO in this study may suggest an increased prevalence of plastic ingestion in juveniles of this species compared to previous points in time, but more likely reflects artifacts of study design confounding direct comparison of reported results between studies ${ }^{37}$. Prior work does not report FO as a function of age, and therefore previous literature estimates may be lower due to an increased proportion of mature birds with lower plastic ingestion rates. Additionally, prior work investigating plastics in Great Shearwaters incorporates a variety of sampling and reporting approaches that preclude direct comparability of reported metrics.

Plastic mass and count data were likewise not associated with any significant changes in morphological or body condition variables, similar to results observed in other work ${ }^{24,28}$. We suggest future work evaluates the association between plastic ingestion and other biological endpoints related to nutritional condition or short-term biological responses like blood chemistry, considering plastic's potential interference with effective ingestion of natural food items and the uncertain but likely limited retention time of plastics in seabirds ${ }^{35,46}$.

\section{Plastic item morphology}

Industrial plastics are defined as the raw plastic materials, often in the form of pellets or beads, that are melted down to create consumer plastic products; they are often called "nurdles". These made up a small proportion of observed plastics 
from each year and location, with no significant changes in the percent of industrial plastics over time or between locations. The percentage of industrial plastics within this sample set $($ mean $=8.2 \%)$ was equal to or lower than percent industrial metrics reported in previous studies from the North Atlantic ${ }^{23,25,28}$. The mean number of industrial pellets reported here is also consistent with measurements conducted between 1999 - 2006 using Great Shearwaters collected from the South Atlantic and western Indian Oceans, suggesting the ingestion of pellets from industrial sources remains consistent and lower than observed in prior decades ( $\underline{\text { Ryan, 2008) }}$.

Each plastic item was classified into one of five morphological categories, including fragments, thread-like plastics, sheet-like plastics, foams, or "other". Proportions of each plastic morphological category were assessed as a percentage of the total count of plastic items measured within a given year and location. Category ratios differed between years in Massachusetts Bay (Fisher's exact test, two-sided, $\mathrm{p}<0.001$ ), but were consistently dominated by fragments (range: $70-$ 93\% fragments) (Fig. 2).

Category ratios differed between habitats (Fisher's exact test, two-sided, $\mathrm{p}=$ 0.004) when considering all individuals from all habitats. However, the Gough Island sample set contained an apparent outlier, one individual with a count and mass of plastics much higher than all other sampled individuals. When this outlier was excluded, differences between the occurrence of plastic categories between habitats disappeared and all habitats were dominated by fragments (mean $=88 \%)$ (Fisher's exact test, two-sided, $\mathrm{p}=0.669$ ). Excluding fragments from the analysis, 
no differences existed between ratios of other morphologies between habitats ((Fisher's exact test, two-sided, $\mathrm{p}=0.6202)$ (Fig. 2).

There was no difference between ratios of plastic categories between Massachusetts Bay and Gough Island mature birds, despite a slightly higher but statistically insignificant proportion of thread-like pieces in Massachusetts Bay mature birds. Likewise, there was no difference between category ratios observed in young and mature Massachusetts Bay birds.

We explored the occurrence of plastics found in surface waters and forage fish used by this species in the Gulf of Maine to deduce if birds inherited their consistent category signature from their environment or diet. Limited data details the occurrence of different plastic categories in surface water within the Gulf of Maine and South Atlantic habitats specifically used by Great Shearwaters, as most large-scale datasets present abundance by size rather than plastic piece category ${ }^{10}$. However, 2013 surveys conducted near Isles of Shoals in the SW Gulf of Maine found microfibers dominated $(84 \%)$ in the surface layer $(\sim 1 \mathrm{~m})$ compared to fragments $(14 \%)^{11}$, suggesting Great Shearwaters in the Gulf of Maine may seek out plastic fragments as opposed to accumulating plastics based on likelihood of encounter. Additionally, stomach content analyses were performed on 202 Ammodytes spp. collected in 2019, the primary prey species of Great Shearwaters while in the Gulf of Maine. We found no plastic fragments of the same size or morphology as those found in Great Shearwater stomachs (Suca et al. 2020, in review). Only one thread-like item was found in one fish, suggesting Great Shearwaters in Massachusetts Bay are not deriving their accumulated plastic loads 
from trophic transfer. This corroborates evidence from other seabirds suggesting the transfer of plastics between fish prey and seabird predators may be limited ${ }^{41}$.

The size of plastic items varied widely, yet the largest item measured from each location measured approximately $13 \mathrm{~mm}$ in its widest dimension, suggesting a consistent limit on the size of plastic items based on bird gape size.

We compared the size of fragments between habitats and found mature birds from Gough Island contained the largest fragments compared to items found in individuals from Massachusetts Bay or SE North Carolina (Dunn's test, $\mathrm{p}<$ 0.001) (Fig. 3). This difference was likewise apparent when the comparison was restricted to mature birds from Massachusetts Bay and mature birds from Gough Island (Kruskal-Wallis test, $\mathrm{p}=0.0238$ ).

The abundance of larger plastic fragments in Gough Island adults is likely related to size-mediated distribution of plastic items in the surface ocean ${ }^{42,43}$ and maritime sources of intact plastic items ${ }^{44}$, coupled to the highly pelagic foraging range of Great Shearwaters during their breeding and chick rearing phases. Smaller plastic items with higher surface area:volume ratios are thought to settle out of the water column rapidly moving away from coastal regions due to biofouling and other biophysical processes ${ }^{42}$, with larger, more buoyant pieces preferentially remaining in the surface ocean to circulate to more remote regions ${ }^{43}$. During their breeding phase Great Shearwaters forage across highly pelagic regions along the boundary of the South Atlantic, South Pacific, and SW Indian Oceans ${ }^{45}$, and therefore likely encounter a higher proportion of larger plastic items that have been retained through size-mediated dispersal processes to 
date. Additionally, major ship-borne inputs of whole consumer items may likewise contribute to the abundance of larger plastic items in Great Shearwaters from Gough Island. The incidence of plastic debris derived from shipping and fishing activities is rapidly increasing in the South Atlantic around the breeding islands used by Great Shearwaters, providing inputs of intact consumer items to an otherwise remote region ${ }^{44}$. These consumer products (e.g. beverage bottles) are often made of buoyant plastics, and would first fragment into larger pieces. An interplay of these two drivers is likely driving the size trends we see here.

Regardless of specific cause, the increased abundance of larger plastic items in breeding birds is problematic, as larger plastic items are likely retained in the GIT longer and pose an increased risk of physical impairment or injury ${ }^{46}$.

\section{Polymer patterns and differences}

1000 plastic pieces were assessed for polymer type using FTIR, and these results indicated the type of polymer ingested varied by age and habitat. Mature individuals from Gough Island contained higher proportions of polypropylene mixes (PP mix) compared to other locations and ages. Considering only fragments, mature birds from Massachusetts Bay possessed a significantly different polymer composition compared to their South Atlantic counterparts (Fisher's exact test, two-sided, $\mathrm{p}=0.002$ ), with North Atlantic birds possessing a lower proportion of PP mix, a higher proportion of polyethylene, and increased incidence of "Other" items comprised mostly of balloons (Fig. 3).

The different polymer signatures may indicate turnover of plastic pieces over time, allowing Great Shearwaters to reflect variable signatures of plastic 
associated with divergent habitats. The retention time of plastic items in seabirds is generally unclear, but is thought to vary between different taxa based on other diet items present in the GIT as well as the size threshold required to pass through the lower GIT. Retention time is also based on initial item size, item composition, and rate of wear within the GIT ${ }^{35}$. Fragments, the dominant morphology found in individuals within this study, may also be prone to reduced retention times due to increased exposure to UV and other environmental stressors that caused and continued their fragmentation from intact items ${ }^{35}$.

van Franeker and Law (2015) suggested Northern fulmars excrete approximately $75 \%$ of their plastic load within one month based on assessment of plastic loads in fulmars between their breeding and non-breeding locations. However, their inference did not account for possible transfer from adults to chicks during chick-rearing, which may reduce the incidence of plastics in adults via offloading to chicks. Alternatively, direct studies of plastic pellet breakdown in seabirds suggest slow breakdown of plastics in vivo, as industrial pellets fed to white-chinned petrel fledgings lost only $\sim 1 \%$ of their mass over 12 days within the GIT, making excretion within a month improbable (Ryan and Jackson 1987).

Our data strongly suggest that plastic fragments within the size ranges observed here are not be retained indefinitely by Great Shearwaters, but rather are replaced in less than 5-6 months to reflect changes associated with migrationrelated shifts in ambient environment. However, we acknowledge there is likely a lag of uncertain duration as birds excrete plastic from previous environments and assimilate new plastic items from current environments. We do not believe 
changes in polymer signature between locations are due to dilution of signature by accumulation of more plastic items (e.g. apparent lower proportion of PP mixes in North Atlantic birds due to accumulation of more plastics overall), because birds from all locations contained similar counts and mass of plastic items (Table 1). We also note that our inference is based on comparisons more removed from the influence of intergenerational transfer, as samples reflecting breeding phase adults were collected at the end of chick-rearing in March/April 2018, while adults and juveniles from Massachusetts Bay were collected in August - November after use of NW Atlantic regions for $>2$ months. Recommendations for future monitoring

Over $50 \%$ of Great Shearwaters sampled in this study surpass the EcoQ standard for plastic ingestion in seabirds, which suggests no more than $10 \%$ of a given seabird population should contain a mass of plastic greater than $0.1 \mathrm{~g}^{24,25,34}$.

We recommend increased standardization of approaches within studies focusing on plastic ingestion in Great Shearwaters to more fully leverage the utility of this species as an indicator of the subtropical and temperate Atlantic regions upon which it relies. Mass, count, and FO should be presented for each compartment of the GIT as a function of month, age, and sex ${ }^{37}$, given the highly variable mass, count, and FO reported between different ages and locations across time. We particularly highlight the need for reporting plastic ingestion within each GIT compartment separately to facilitate increased use of bycatch samples within monitoring studies. Bycatch samples are prone to regurgitation impacting plastic loads in the proventriculus, but are more consistently available as Great Shearwaters are the most abundantly bycaught species in the Gulf of Maine ${ }^{48,49}$ 
and are also bycaught in the South Atlantic along the Patagonian Shelf ${ }^{50}$. Bycatch samples are also considered healthy prior to death and lack bias potentially associated with beach-cast samples which may have been sickly or compromised leading to death ${ }^{51,52}$.

The subtropical North and South Atlantic stand to experience continued accumulation of plastic pieces in the coming decades ${ }^{47}$; with increased parity of approach, Great Shearwaters offer an abundant, compact method to monitor future plastic dynamics in these regions, and assess the efficacy of any future interventions to address plastic pollution. We encourage transboundary cooperation to more fully develop monitoring of this species, using the body of work surrounding the Northern fulmar in the North Atlantic as a model.

\section{Conclusions}

Our results confirm sustained plastic ingestion in multiple life stages of a Procellariiform seabird reliant on both the North and South Atlantic. Juveniles and non-breeding adults accumulated plastics more frequently than mature adults in their breeding phase. Fragments were the dominant plastic item ingested across all life stages and locations, although breeding birds in the South Atlantic were found to ingest larger plastic fragments compared to North Atlantic individuals. Significantly different polymer types between North Atlantic and South Atlantic birds suggests plastics turnover within the GIT commensurate with migratory phase, allowing Great Shearwaters to reflect their ambient environment after an assumed acclimation period. Crucially, this suggests mitigation of plastic sources within both the North and South Atlantic are key to reduce the incidence of plastic 
ingestion in this species, which occurs at levels well above EcoQ thresholds considered acceptable for ecological health. Understanding plastic ingestion levels and effects in marine food webs and biota such as seabirds remains an important priority, as ocean plastic pollution is predicted to exponentially increase over the coming decades 


\section{ASSOCIATED CONTENT}

NA

AUTHOR INFORMATION

Corresponding Author

*Anna Ruth Robuck, annarobuck@gmail.com

Author Contributions

The manuscript was primarily drafted by A. Robuck and finalized through contributions of all authors. Sample analysis and data interpretation were carried out by A. Robuck, C. Hudak, G. Emery, L. Agvent, and D. Wiley, alongside contributions of all authors. All authors have given approval to the final version of the manuscript. Funding Sources

A. Robuck acknowledges support from the National Oceanic and Atmospheric Administration Dr. Nancy Foster Scholarship program (NOAA Award Number NA17NOS4290028), the Robert and Patricia Switzer Foundation, the STEEP Superfund Research Program (NIEHS Award Number P42ES027706), and the Oak Ridge Institute for Science and Education (ORISE) program.

Competing Interests

The authors have no competing interests to declare.

\section{ACKNOWLEDGMENTS}

The authors are indebted to the Northeast Fisheries Observer Program, Gina Shield, and Stephanie DePasquale for their assistance obtaining samples used in this study. Although NOAA employees contributed to this article, this research was conceived, designed, and implemented by URI. Consequently, the views, 
interpretations, and conclusions expressed in the article are solely those of the authors and do not necessarily reflect or represent NOAA views or policies.

\author{
ABBREVIATIONS \\ GIT - gastrointestinal tract \\ GRSH - Great Shearwater \\ FO - Frequency of occurrence
}

\title{
LITERATURE CITED
}

(1) Jambeck, J. R.; Geyer, R.; Wilcox, C.; Siegler, T. R.; Perryman, M.; Andrady, A.; Narayan, R.; Law, K. L. Plastic Waste Inputs from Land into the Ocean. Science (80-. ). 2015, 347 (6223).

(2) Gall, S. C.; Thompson, R. C. The Impact of Debris on Marine Life. Mar. Pollut. Bull. 2015, 92 (1-2), 170-179. https://doi.org/10.1016/j.marpolbul.2014.12.041.

(3) Law, K. L. Plastics in the Marine Environment. 2017, 205-232. https://doi.org/10.1146/annurev-marine-010816-060409.

(4) Ryan, P. G.; Moore, C. J.; van Franeker, J. A.; Moloney, C. L. Monitoring the Abundance of Plastic Debris in the Marine Environment. Philos. Trans. R. Soc. B Biol. Sci. 2009, 364 (1526), 1999-2012. https://doi.org/10.1098/rstb.2008.0207.

(5) Barnes DK, Galgani F, Thompson RC, B. M. Accumulation and Fragmentation of Plastic Debris in Global Environments. Philos. Trans. R. Soc. Lond. B. Biol. Sci. 2009, 364 (1526), 1985-1998.

(6) Horton, A. A.; Barnes, D. K. A. Microplastic Pollution in a Rapidly Changing World: Implications for Remote and Vulnerable Marine Ecosystems. Sci. Total Environ. 2020, 738, 140349. https://doi.org/10.1016/j.scitotenv.2020.140349.

(7) Kooi, M.; Van Nes, E. H.; Scheffer, M.; Koelmans, A. A. Ups and Downs in the Ocean: Effects of Biofouling on Vertical Transport of Microplastics. Environ. Sci. Technol. 2017, 51 (14), 7963-7971. https://doi.org/10.1021/acs.est.6b04702. 
(8) Kane, I. A.; Clare, M. A. Dispersion, Accumulation, and the Ultimate Fate of Microplastics in Deep-Marine Environments: A Review and Future Directions. Front. Earth Sci. 2019, 7 (April). https://doi.org/10.3389/feart.2019.00080.

(9) Sherman, P.; Sebille, E. Van; Koelmans, A. A.; Kooi, M.; Law, L.; Kaiser, D.; Kowalski, N.; Sebille, E. Van; Wilcox, C.; Lebreton, L.; Maximenko, N.; Hardesty, B. D. A Global Inventory of Small Floating Plastic Debris. 2015.

(10) Eriksen, M.; Lebreton, L. C. M.; Carson, H. S.; Thiel, M.; Moore, C. J.; Borerro, J. C.; Galgani, F.; Ryan, P. G.; Reisser, J. Plastic Pollution in the World's Oceans: More than 5 Trillion Plastic Pieces Weighing over 250,000 Tons Afloat at Sea. PLoS One 2014, 9 (12), 1-15. https://doi.org/10.1371/journal.pone.0111913.

(11) Lindeque, P. K.; Cole, M.; Coppock, R. L.; Lewis, C. N.; Miller, R. Z.; Watts, A. J. R.; Wilson-McNeal, A.; Wright, S. L.; Galloway, T. S. Are We Underestimating Microplastic Abundance in the Marine Environment? A Comparison of Microplastic Capture with Nets of Different Mesh-Size. Environ. Pollut. 2020, 265, 114721. https://doi.org/10.1016/j.envpol.2020.114721.

(12) Erni-Cassola, G.; Zadjelovic, V.; Gibson, M. I.; Christie-Oleza, J. A. Distribution of Plastic Polymer Types in the Marine Environment; A Meta-Analysis. J. Hazard. Mater. 2019, 369 (November 2018), 691-698. https://doi.org/10.1016/j.jhazmat.2019.02.067.

(13) Savoca, M. S.; Wohlfeil, M. E.; Ebeler, S. E.; Nevitt, G. A. Marine Plastic Debris Emits a Keystone Infochemical for Olfactory Foraging Seabirds. Sci. Adv. 2016, 2 (11), 1-9. https://doi.org/10.1126/sciadv.1600395.

(14) Nelms, S. E.; Galloway, T. S.; Godley, B. J.; Jarvis, D. S.; Lindeque, P. K. Investigating Microplastic Trophic Transfer in Marine Top Predators. Environ. Pollut. 2018, 238, 999-1007. https://doi.org/10.1016/j.envpol.2018.02.016.

(15) Pierce, K. E.; Harris, R. J.; Larned, L. S.; Pokras, M. A. Obstruction and Starvation Associated with Plastic Ingestion in a Northern Gannet Morus Bassanus and a Greater Shearwater Puffinus Gravis. Mar. Ornithol. 2004, 32 (2), 187-189.

(16) Santos, R. G.; Andrades, R.; Demetrio, G. R.; Kuwai, G. M.; Sobral, M. F.; Vieira, J. de S.; Machovsky-Capuska, G. E. Exploring Plastic-Induced Satiety in Foraging Green Turtles. Environ. Pollut. 2020, 265. https://doi.org/10.1016/j.envpol.2020.114918.

(17) Koelmans, A. A.; Bakir, A.; Burton, G. A.; Janssen, C. R. Microplastic as a Vector for Chemicals in the Aquatic Environment: Critical Review and ModelSupported Reinterpretation of Empirical Studies. Environ. Sci. Technol. 2016, 50 (7), 3315-3326. https://doi.org/10.1021/acs.est.5b06069. 
(18) Moser, Mary L., Lee, D. S. A Fourteen-Year Survey of Plastic Ingestion by Western North Atlantic Seabirds Author ( s ): Mary L . Moser and David S . Lee Published by: Waterbird Society Stable URL : Http://Www.Jstor.Org/Stable/1521357 REFERENCES Linked References Are Available on J. 2016, 15 (1), 83-94.

(19) Provencher, J. F.; Bond, A. L.; Hedd, A.; Montevecchi, W. A.; Bin, S.; Courchesne, S. J.; Gilchrist, H. G.; Jamieson, S. E.; Merkel, F. R.; Falk, K.; Durinck, J.; Mallory, M. L. Prevalence of Marine Debris in Marine Birds from the North Atlantic. Mar. Pollut. Bull. 2014, 84 (1-2), 411-417. https://doi.org/10.1016/j.marpolbul.2014.04.044.

(20) Davies, R. G.; Irlich, U. M.; Chown, S. L.; Gaston, K. J. Ambient, Productive and Wind Energy, and Ocean Extent Predict Global Species Richness of Procellariiform Seabirds. Glob. Ecol. Biogeogr. 2010, 19 (1), 98-110. https://doi.org/10.1111/j.1466-8238.2009.00498.x.

(21) Nevitt, G. A. Sensory Ecology on the High Seas: The Odor World of the Procellariiform Seabirds. J. Exp. Biol. 2008, 211 (11), 1706-1713. https://doi.org/10.1242/jeb.015412.

(22) Acampora, H.; Schuyler, Q. A.; Townsend, K. A.; Hardesty, B. D. Comparing Plastic Ingestion in Juvenile and Adult Stranded Short-Tailed Shearwaters (Puffinus Tenuirostris) in Eastern Australia. Mar. Pollut. Bull. 2014, 78 (1-2), 63-68. https://doi.org/10.1016/j.marpolbul.2013.11.009.

(23) Avery-Gomm, S.; Provencher, J. F.; Liboiron, M.; Poon, F. E.; Smith, P. A. Plastic Pollution in the Labrador Sea: An Assessment Using the Seabird Northern Fulmar Fulmarus Glacialis as a Biological Monitoring Species. Mar. Pollut. Bull. 2017, No. October, 0-1. https://doi.org/10.1016/j.marpolbul.2017.10.001.

(24) Bond, A. L.; Provencher, J. F.; Daoust, P. Y.; Lucas, Z. N. Plastic Ingestion by Fulmars and Shearwaters at Sable Island, Nova Scotia, Canada. Mar. Pollut. Bull. 2014, 87 (1), 68-75. https://doi.org/10.1016/j.marpolbul.2014.08.010.

(25) Van Franeker, J. A.; Law, K. L.; Franeker, J. A. Van; Lavender, K. Seabirds, Gyres and Global Trends in Plastic Pollution. Environ. Pollut. 2015, 203, 89-96. https://doi.org/10.1016/j.envpol.2015.02.034.

(26) Wilcox, C.; Van Sebille, E.; Hardesty, B. D.; Sebille, E. Van; Denise, B. Threat of Plastic Pollution to Seabirds Is Global, Pervasive, and Increasing. Proc. Natl. Acad. Sci. 2015, 112 (38), 1-6. https://doi.org/10.1073/pnas.1502108112.

(27) Roman, L.; Bell, E.; Wilcox, C.; Hardesty, B. D.; Hindell, M. Ecological Drivers of Marine Debris Ingestion in Procellariiform Seabirds. Sci. Rep. 2019, 9 (1), 1-8. https://doi.org/10.1038/s41598-018-37324-w. 
(28) Kühn, S.; van Oyen, A.; Bravo Rebolledo, E. L.; Ask, A. V.; van Franeker, J. A. Polymer Types Ingested by Northern Fulmars (Fulmarus Glacialis) and Southern Hemisphere Relatives. Environ. Sci. Pollut. Res. 2020.

https://doi.org/10.1007/s11356-020-10540-6.

(29) Cuthbert, R. J. Breeding Biology, Chick Growth and Provisioning of Great Shearwaters (Puffinus Gravis) at Gough Island, South Atlantic Ocean. Emu 2005, 105 (4), 305-310. https://doi.org/10.1071/MU05036.

(30) Powers, K. D.; Wiley, D. N.; Allyn, A. J.; Welch, L.; Ronconi, R. A. Movements and Foraging Areas of Great Shearwaters in the Gulf of Maine. Mar. Ecol. Prog. Ser. 2017, 574, 1-57.

(31) Powers, K. D.; Wiley, D. N.; Robuck, A. R.; Olson, Z. H.; Welch, L. J.; Thompson, M. A.; Kaufman, L. Spatiotemporal Characterization of Non-Breeding Great Shearwaters Ardenna Gravis within Their Wintering Range. Mar. Ornithol. 2020, 48, 215-229.

(32) Silva, T. L.; Wiley, D. N.; Thompson, M. A.; Hong, P.; Kaufman, L.; Suca, J. J.; Llopiz, J. K.; Baumann, H.; Fay, G. High Collocation of Sand Lance and Protected Top Predators: Implications for Conservation and Management. Conserv. Sci. Pract. 2020, No. July, 1-8. https://doi.org/10.1111/csp2.274.

(33) Ronconi, R. A.; Ryan, P. G.; Ropert-Coudert, Y. Diving of Great Shearwaters (Puffinus Gravis) in Cold and Warm Water Regions of the South Atlantic Ocean. PLoS One 2010, 5 (11), 1-7. https://doi.org/10.1371/journal.pone.0015508.

(34) van Franeker, J. A. Save the North Sea Fulmar-Litter-EcoQO Manual - Part 1: Collection and Dissection Procedures. Alterra-rapport 2004, 672, 1-38.

(35) Ryan, P. G. How Quickly Do Albatrosses and Petrels Digest Plastic Particles? Environ. Pollut. 2015, 207, 438-440. https://doi.org/10.1016/j.envpol.2015.08.005.

(36) Van Franeker, J. a; Meijboom, A. LITTER NSV, Marine Litter Monitoring by Northern Fulmars: A Pilot Study; 2002.

(37) Provencher, J. F.; Borrelle, S. B.; Bond, A. L.; Lavers, J. L.; Van, J. A.; Kühn, S.; Hammer, S.; Avery-gomm, S.; Mallory, M. L. Recommended Best Practices for Plastic and Litter Ingestion Studies in Marine Birds : Collection, Processing, and Reporting. 2019, 111-130. https://doi.org/10.1139/facets-2018-0043.

(38) Lusher, A. L.; McHugh, M.; Thompson, R. C. Occurrence of Microplastics in the Gastrointestinal Tract of Pelagic and Demersal Fish from the English Channel. Mar. Pollut. Bull. 2013, 67 (1-2), 94-99. https://doi.org/10.1016/j.marpolbul.2012.11.028. 
(39) R Core Team. R: A Language and Environment for Statistical Computing. R Foundation for Statistical Computing: Vienna, Austria 2020.

(40) Campioni, L.; Granadeiro, J. P.; Catry, P. Niche Segregation between Immature and Adult Seabirds: Does Progressive Maturation Play a Role? Behav. Ecol. 2016, 27 (2), 426-433. https://doi.org/10.1093/beheco/arv167.

(41) Provencher, J. F.; Ammendolia, J.; Rochman, C. M.; Mallory, M. L. Assessing Plastic Debris in Aquatic Food Webs: What We Know and Don't Know about Uptake and Trophic Transfer. Environ. Rev. 2019, 27 (3), 304-317. https://doi.org/10.1139/er2018-0079.

(42) Van Sebille, E.; Aliani, S.; Law, K. L.; Maximenko, N.; Alsina, J. M.; Bagaev, A.; Bergmann, M.; Chapron, B.; Chubarenko, I.; Cózar, A.; Delandmeter, P.; Egger, M.; Fox-Kemper, B.; Garaba, S. P.; Goddijn-Murphy, L.; Hardesty, B. D.; Hoffman, M. J.; Isobe, A.; Jongedijk, C. E.; Kaandorp, M. L. A.; Khatmullina, L.; Koelmans, A. A.; Kukulka, T.; Laufkötter, C.; Lebreton, L.; Lobelle, D.; Maes, C.; MartinezVicente, V.; Morales Maqueda, M. A.; Poulain-Zarcos, M.; Rodríguez, E.; Ryan, P. G.; Shanks, A. L.; Shim, W. J.; Suaria, G.; Thiel, M.; Van Den Bremer, T. S.; Wichmann, D. The Physical Oceanography of the Transport of Floating Marine Debris. Environ. Res. Lett. 2020, 15 (2), 23003. https://doi.org/10.1088/17489326/ab6d7d.

(43) Ryan, P. G. Does Size and Buoyancy Affect the Long-Distance Transport of Floating Debris? Environ. Res. Lett. 2015, 10 (8), 84019. https://doi.org/10.1088/1748-9326/10/8/084019.

(44) Ryan, P. G.; Dilley, B. J.; Ronconi, R. A.; Connan, M. Rapid Increase in Asian Bottles in the South Atlantic Ocean Indicates Major Debris Inputs from Ships. 2019, 1-6. https://doi.org/10.1073/pnas.1909816116.

(45) Ronconi, R. A.; Schoombie, S.; Westgate, A. J.; Wong, S. N. P.; Koopman, H. N.; Ryan, P. G. Effects of Age, Sex, Colony and Breeding Phase on Marine Space Use by Great Shearwaters Ardenna Gravis in the South Atlantic. Mar. Biol. 2018, 165 (3). https://doi.org/10.1007/s00227-018-3299-x.

(46) Roman, L.; Hardesty, B. D.; Hindell, M. A.; Wilcox, C. A Quantitative Analysis Linking Seabird Mortality and Marine Debris Ingestion. Sci. Rep. 2019, 9 (1), 1-7. https://doi.org/10.1038/s41598-018-36585-9.

(47) Wilcox, C.; Hardesty, B. D.; Law, K. L. Abundance of Floating Plastic Particles Is Increasing in the Western North Atlantic Ocean. Environ. Sci. Technol. 2020, 54 (2), 790-796. https://doi.org/10.1021/acs.est.9b04812. 
(48) Hatch, J. M.; Wiley, D.; Murray, K. T.; Welch, L. Integrating Satellite-Tagged Seabird and Fishery-Dependent Data: A Case Study of Great Shearwaters (Puffinus Gravis) and the U.S. New England Sink Gillnet Fishery. Conserv. Lett. 2016, 9 (1), 43-50. https://doi.org/10.1111/conl.12178.

(49) Hatch, J. M. Comprehensive Estimates of Seabird-Fishery Interactions for the US Northeast and Mid-Atlantic. Aquat. Conserv. Mar. Freshw. Ecosyst. 2018, 28 (1), 182-193. https://doi.org/10.1002/aqc.2812.

(50) Bugoni, L.; Mancini, P. L.; Monteiro, D. S.; Nascimento, L.; Neves, T. S. Seabird Bycatch in the Brazilian Pelagic Longline Fishery and a Review of Capture Rates in the Southwestern Atlantic Ocean. Endanger. Species Res. 2008, 5 (2-3), 137147. https://doi.org/10.3354/esr00115.

(51) Colabuono, F. I.; Barquete, V.; Domingues, B. S.; Montone, R. C. Plastic Ingestion by Procellariiformes in Southern Brazil. Mar. Pollut. Bull. 2009, 58 (1), $93-$ 96. https://doi.org/10.1016/j.marpolbul.2008.08.020.

(52) Rodríguez, A.; Ramírez, F.; Carrasco, M. N.; Chiaradia, A. Seabird Plastic Ingestion Differs among Collection Methods: Examples from the Short-Tailed Shearwater. Environ. Pollut. 2018, 243, 1750-1757. https://doi.org/10.1016/j.envpol.2018.09.007. 


\section{TABLES AND FIGURES}

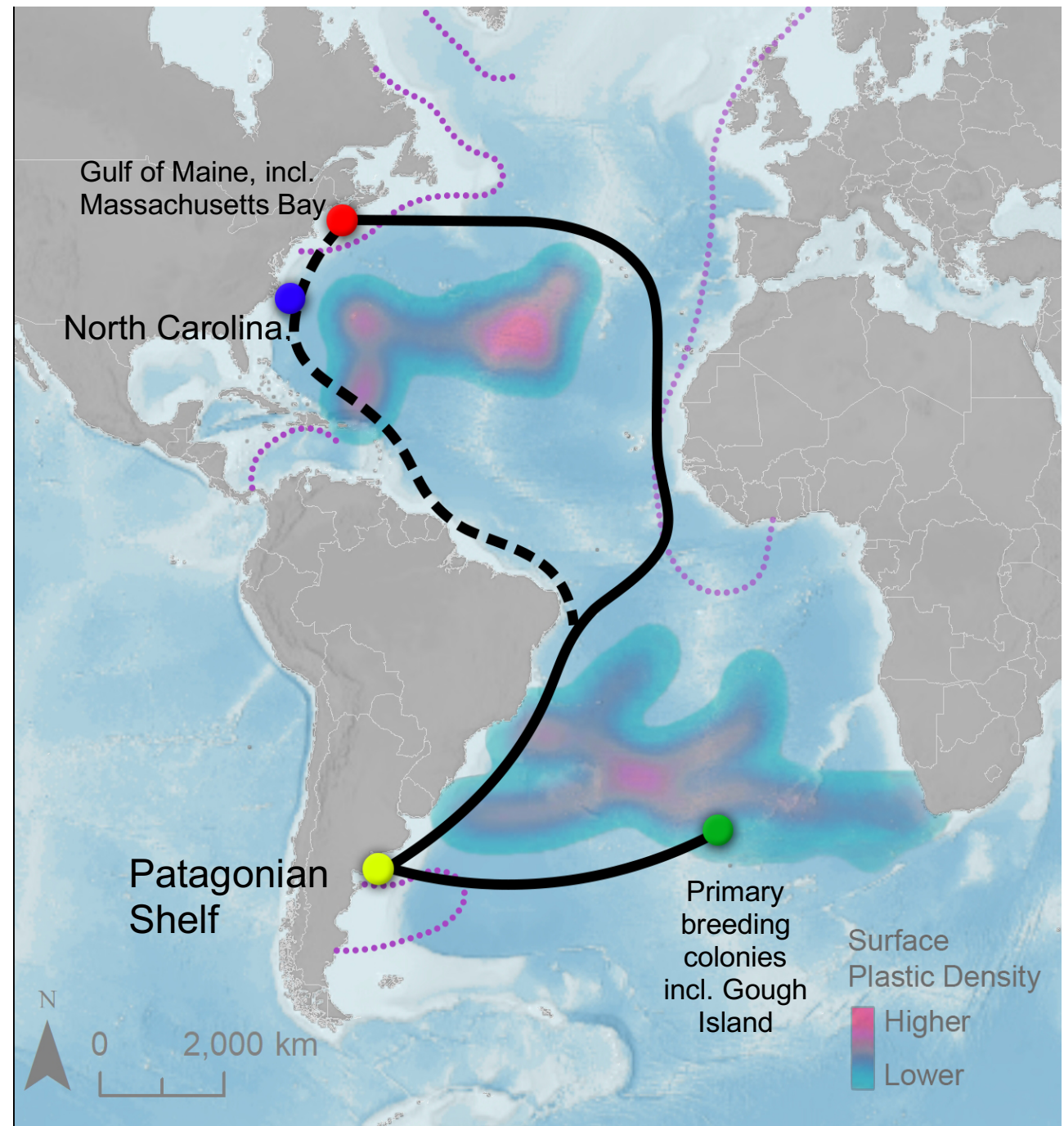

Figure 5.1. Map of Great Shearwater migratory routes (black lines), and sample collection locations (marked by colored points). Surface plastic density is indicated by heatmap gradients in pink and blue. No samples were collected off the Patagonian Shelf (marked by the yellow dot), but this location is heavily used by both adult and juvenile Great Shearwaters as a staging location before and after trans-equatorial migration. 


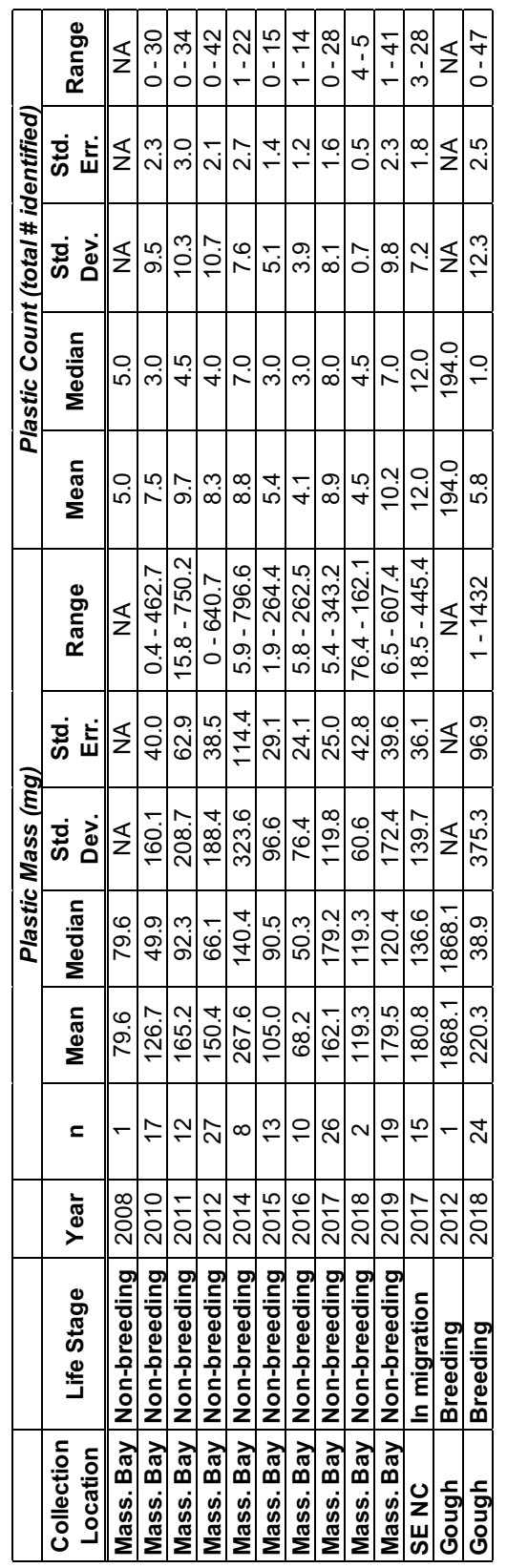

Table 5.1. Summary statistics describing accumulated ingested plastics by mass and count, in different regions and years. 


\begin{tabular}{|c|l|c|c|c|c|c|}
\hline Habitat & Life Stage & Year & Age & HYIAHY & $\mathbf{n}$ & FO (\%) \\
\hline \hline Mass Bay & Non-breeding & All & Mature & na & 23 & 87 \\
\hline Mass Bay & Non-breeding & All & Young & na & 103 & 98 \\
\hline Mass Bay & Non-breeding & All & Young & HY & 53 & 98 \\
\hline Mass Bay & Non-breeding & All & Young & AHY & 16 & 94 \\
\hline Mass Bay & Non-breeding & 2008 & All & na & 1 & 100 \\
\hline Mass Bay & Non-breeding & 2010 & All & na & 17 & 94 \\
\hline Mass Bay & Non-breeding & 2011 & All & na & 12 & 92 \\
\hline Mass Bay & Non-breeding & 2012 & All & na & 27 & 89 \\
\hline Mass Bay & Non-breeding & 2014 & All & na & 8 & 100 \\
\hline Mass Bay & Non-breeding & 2015 & All & na & 13 & 85 \\
\hline Mass Bay & Non-breeding & 2016 & All & na & 10 & 100 \\
\hline Mass Bay & Non-breeding & 2017 & All & na & 26 & 92 \\
\hline Mass Bay & Non-breeding & 2018 & All & na & 2 & 100 \\
\hline Mass Bay & Non-breeding & 2019 & All & na & 19 & 100 \\
\hline SE NC & In migration & 2017 & Young & na & 15 & 100 \\
\hline Gough & Breeding & 2012 & Mature & na & 1 & 100 \\
\hline Gough & Breeding & $2017-2018$ & Mature & na & 24 & 58
\end{tabular}

Table 5.2. Frequency of occurrence (FO) of ingested plastic by location, year, and age. 


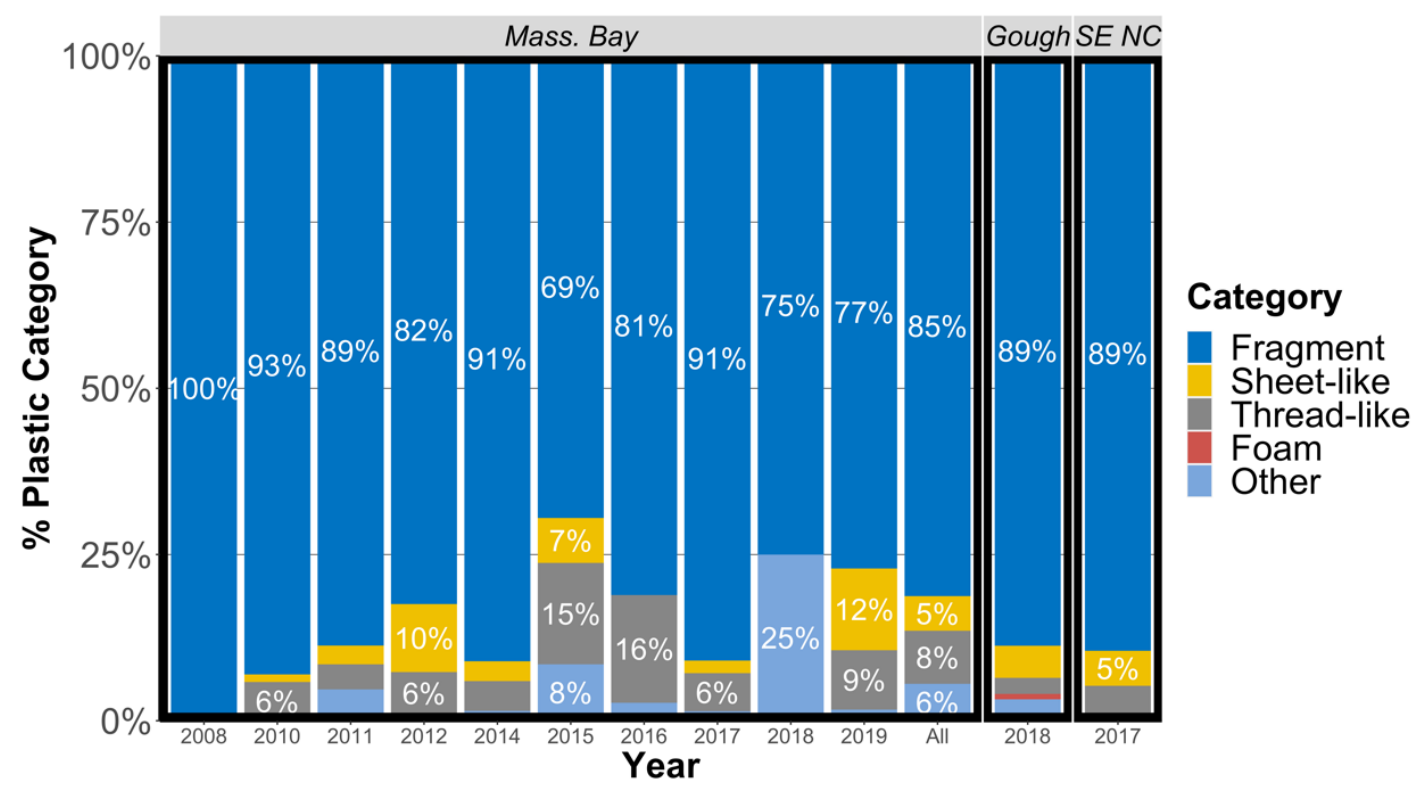

Figure 5.2. Proportions of each plastic morphology, as a percentage of the total count of plastic items measured within a given year and location. The summary of the Gough Island dataset presented herein omits one clear outlier that possessed 194 plastic pieces of greater category diversity than observed within other mature birds from Gough Island. 


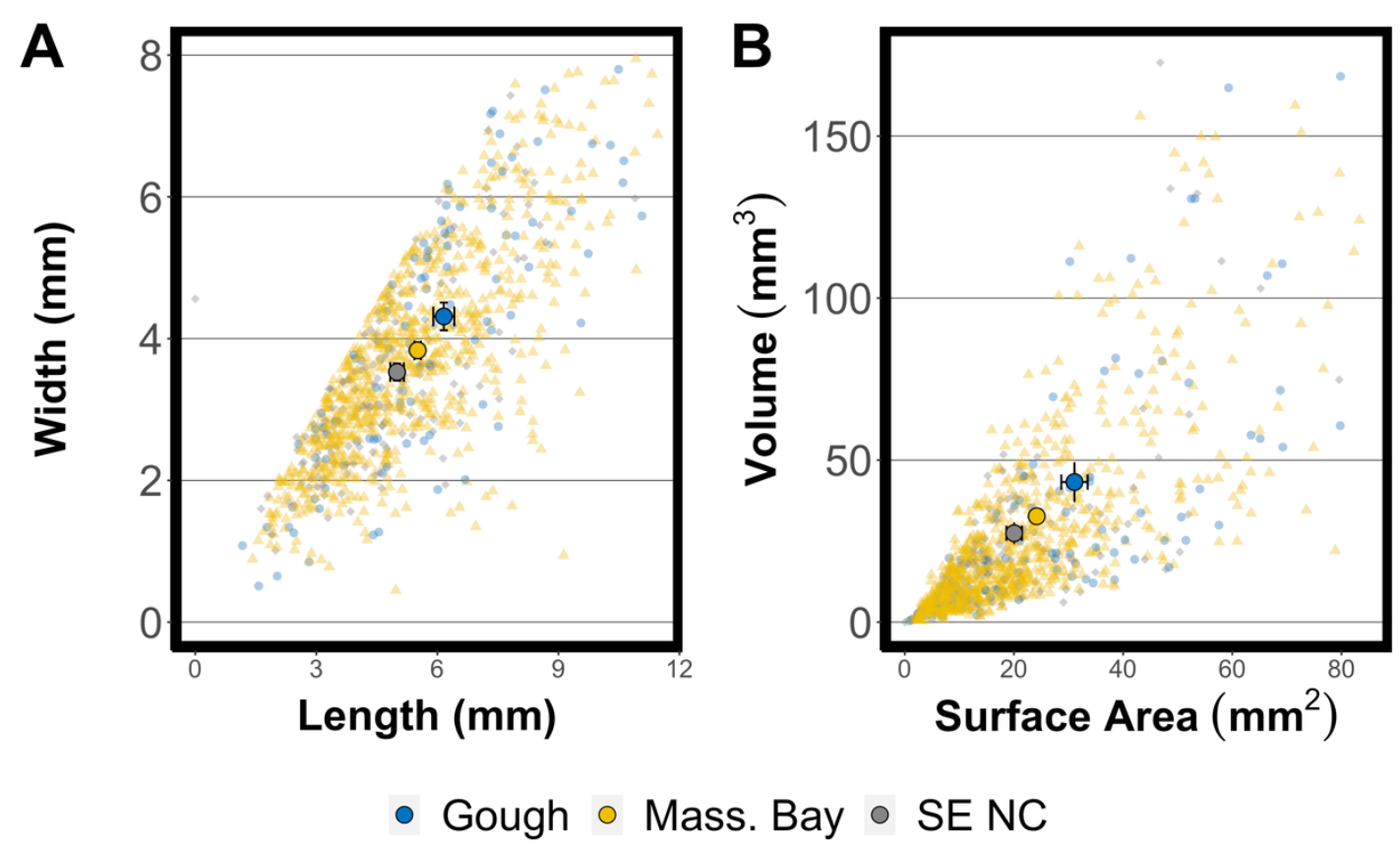

Figure 5.3. A) Length and width and B) surface area and volume of plastic fragments, with colors indicating collection region. $95 \%$ of the data is displayed, with the upper $5 \%$ removed. Error bars present the standard error of the mean. 

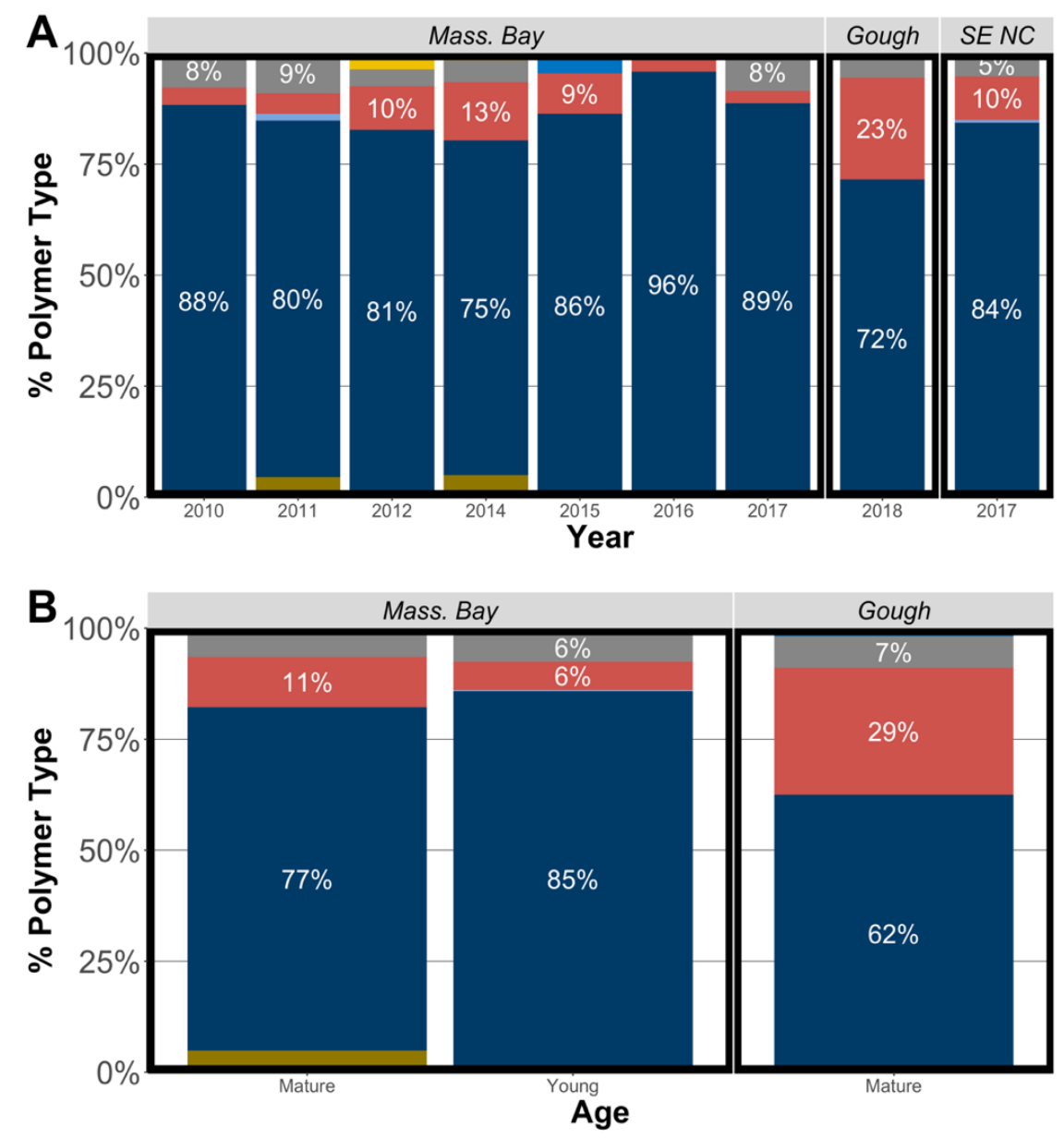

Category

Polystyrene

Resin (syntheti

Polypropylene

PP mix

PE mix

Polyethylene

Other

Figure 5.4. Polymer composition by A) year and B) age presented by location. 


\title{
CHAPTER 6
}

\section{NAPE COLORATION IS NOT A RELIABLE MARKER OF AGE IN GREAT SHEARWATERS ARDENNA GRAVIS}

This manuscript is formatted for submission to the journal Avian Biology

\author{
Anna R. Robuck ${ }^{1}$, Ewan D. Wakefield ${ }^{2}$, Kevin D. Powers ${ }^{3}$, Robert A. Ronconi ${ }^{4}$, Peter \\ G. Ryan ${ }^{5}$, David N. Wiley ${ }^{3}$
}

${ }^{1}$ University of Rhode Island Graduate School of Oceanography, Narragansett, RI, 02882, USA

${ }^{2}$ University of Glasgow, Institute of Biodiversity, Animal Health and Comparative Medicine, Graham Kerr Building, Glasgow, G12 8QQ, UK.

${ }^{3}$ Stellwagen Bank National Marine Sanctuary, National Oceanic and Atmospheric Administration, Scituate, MA 02066

${ }^{4}$ Canadian Wildlife Service, Environment and Climate Change Canada, Dartmouth, NS B2Y 2N6, Canada

${ }^{5}$ Percy FitzPatrick Institute of African Ornithology, DST/NRF Centre of Excellence, University of Cape Town, Rondebosch 7701, South Africa 


\section{ABSTRACT}

Interpreting observations of petrels at sea is generally limited by a lack of differentiating plumage characteristics indicating individual sex or age. Exceptions exist, including great shearwaters (Ardenna gravis), which have been previously proposed to display age-based nape coloration. Pyle (2008) suggested after hatch year and after second year individuals possess a more complete white collar compared to hatch year or second year individuals. Here we combine observations of known age great shearwaters across their breeding and non-breeding range to evaluate nape as an honest indicator of age. Data from necropsies, at-sea photos, and in-colony photos $(\mathrm{n}=$ 333) were scored for nape aspect and compared to true age as ascertained via presentation of gonads and bursa of Fabricius or known breeding status. Pooling all data, nape presentation was not significantly different between age classes, and the accuracy of the nape ageing method was only $19 \%$. Nape was significantly different between sexes in non-breeding individuals, with a higher proportion of white, "C" napes in females observed or necropsied in Massachusetts Bay. There was no evidence of the same sexual dimorphism in breeding individuals bycaught or observed at South Atlantic breeding colonies. We suggest nape is not a reliable indicator of age in this petrel species and suggest further evaluation of sex or trophic drivers as causes of plumage polymorphism in great shearwaters.

\section{INTRODUCTION}

The order Procellariiformes (hereafter petrels) includes many of the world's most abundant pelagic seabird species, as well as many of its most threatened (Brooke 
2004, Croxall et al. 2012). Petrels are long-lived compared to most other birds, with maturation taking from 3 to 12 years (Warham 1990). Recent interest in the early life history of petrels has been stimulated by rapid advances in bird-borne tracking technologies, which have revealed sometimes marked differences in both distribution and behavior between adults and immatures (Riotte-Lambert and Weimerskirch 2013, Campioni et al. 2020). However, notwithstanding technological advances, it remains challenging to track petrels during immaturity because most species are very wideranging and return rarely to land during this life history stage (Rodríguez et al. 2019). Direct observational study of seabirds at sea could provide additional data on agerelated distribution, habitat use, and behavior. However, plumage coloration is largely uniform across age classes in most petrels (Onley and Scofield 2007), making it impractical to differentiate between adult and immatures observed visually at sea.

Some exceptions to this rule do occur. For example, plumage coloration changes systematically with age in albatrosses (especially Diomedea spp.) and giant petrels (Macronectes spp.) (Hyrenbach 2002, Carlos and Voisin 2008, Prince et al. 1997, Weimerskirch et al. 1989). However, plumage coloration can also vary due to sex, breeding status, diet, predation pressure, molt, and feather wear (Arkos 2007, Le Corre 1999, Meunier et al. 2011, Leal et al. 2019, Soravia et al. 2020). In some cases, these factors can confound attempts to age birds based on plumage alone. For example, among Diomedea spp. there is considerable overlap in the plumage aspect of immatures and females (Onley and Scofield 2007).

Great shearwaters (Ardenna gravis) are abundant (world population approx.15 million individuals (Brooke 2004)) medium-sized petrels that breed in the Tristan da 
Cunha group of islands, South Atlantic. In the austral summer adults forage in temperate to polar waters, including the Patagonian shelf, Benguela upwelling and intervening oceanic areas (Ronconi et al. 2018). In the boreal summer, great shearwaters migrate into the Northwest Atlantic, with high concentrations in coastal and pelagic waters of the Gulf of Maine, before continuing clockwise through the North Atlantic and back to breeding colonies in the fall (Voous and J. 1963, Powers et al. 2020). Despite recent at-sea study and tracking (Bugoni et al. 2015, Powers et al. 2017, Ronconi et al. 2018, Hong et al. 2019), there remains considerable uncertainty about the movements of immature great shearwaters, so the ability to discriminate between adults and immatures at sea would be very useful ag-related differences in life histories (Powers et al. 2020).

Great shearwaters have a dark brown cap and mantle, separated by a partial or complete collar of cream-colored feathers (Fig. 1). Pyle (2008) suggested that juvenile to hatching year/second (HY/SY) great shearwaters have a 'Nape with [an] indistinct to incomplete white collar', whilst after hatching year/after second year (AHY/ASY) they have a 'Nape with [an] indistinct to distinct white collar'. Pyle's Figure 201 illustrates three distinct nape classes, A, B or C forming a spectrum from dark, through partial, to light coloration (inset, Fig. 1). The caption adds that 'it is possible that AHY/ASYs resembling C can reliably be aged to ASY/ATY [after second/third year] or older' but notes that more study is required to confirm these patterns.

Here we assess nape plumage scores of known-status great shearwaters sampled in their colonies and bycaught in the South and North Atlantic in order to test whether 
nape coloration is a reliable marker of age class. In addition, we test for variation in nape coloration by sex, region, and over time.

\section{METHODS}

Bycaught birds

From 2010 - 2017 the National Oceanic and Atmospheric Administration Northeast Fisheries Observer Program (Falmouth, MA) supplied us with dead great shearwaters bycaught in fisheries in Massachusetts Bay, USA $(n=174)$. Bycatch birds were obtained between May and December, with most birds collected in August, September, and November across all years. Additional dead great shearwaters were obtained in March and April 2018 during the chick-rearing phase adjacent to the breeding colony at Gough Island in the South Atlantic as longline bycatch in collaboration with Tristan Conservation $(n=25)$. Each individual was partially thawed and necropsied following van Franeker (2004) in order to obtain the following data: plumage characteristics, external morphometrics, organ weights, body condition, sex and stomach contents. Individuals were aged based on molt status, presence of the bursa of Fabricius, and stage of gonad development as previously described (Table 1) (Powers et al. 2020, Broughton 1994). Napes were classified following Pyle (2008) (Fig. 1) as A, B or C by ARR and/or KDP prior to necropsy.

\section{Birds in the colony}

The napes of adult and fledgling great shearwaters were photographed on Gough Island during the breeding season. Adults were photographed between colony arrival and early chick-rearing (September 22, 2017 to January 16, 2018) and fledglings just 
prior to leaving the colony (May 3 to 8, 2018). Birds were removed by hand from burrows during the daytime, photographed and then returned to their burrows. Adults were either known breeders, previously marked as part of a long-term demographic and tracking studies, or unmarked non-breeders. Fledglings were taken from active breeding burrows. At the time, they had attained their first plumage, but some retained traces of down.

Napes of adults and unknown age great shearwaters were also photographed on Gough Island and Inaccessible Island during the 2009 breeding season. On Gough, birds were photographed during initial colony arrival and courtship period (September 22 to October 02), including individuals $(\mathrm{n}=2)$ and pairs outside of burrows at night ( $\mathrm{n}=5$ pairs, 10 individuals), individuals in flight at dusk $(\mathrm{n}=9)$, and individuals handled in tagging study which were removed from burrows during the daytime ( $\mathrm{n}=$ 2). Though these birds were attending nests and some pairs were observed copulating, age was not known and some may not have been mature, but all were likely after second year. On Inaccessible Island, during early incubation (November 10 to December 01) birds were photographed during tagging studies $(\mathrm{n}=4$ males and $\mathrm{n}=5$ females; Ronconi et al. 2018) or opportunistically at burrow entrances $(n=3)$. Due to opportunistic nature of photographs, nape scoring was not possible for all images.

Napes were scored A, B, or C from these photographs independently by the two coauthors who classified the necropsied birds. To ensure that this was done doubleblind, EDW supplied ARR and KDP with a pdf file of the photographs, which ARR and KDP had not seen previously. The pdf was generated using an R script which arranged the images in a random order. The script also generated a file containing the 
true ages of the birds in each image. This file was not consulted until after the napes had been classified. Nape classifications were highly consistent between both observers, with little or no disagreement between nape designations. Nape designations where disagreement occurred were removed from the sample set.

\section{Statistical analyses}

Birds assessed from the South Atlantic were designated as within the breeding/chick-rearing phase while birds from Massachusetts Bay were considered non-breeding. Chi-squared Tests of Independence or Fisher's exact tests were used to compare ratios of nape classes between age groups, sexes, locations and sampling years. Confusion matrices using Pyle's age classes (HY/SY, AHY/ASY) were used to assess classification accuracy of known-age individuals (Fielding and Bell 1997). Necropsied birds classified as "AHY/Not Mature" were omitted from this part of the analysis, as this category could include both HY/SY and AHY/ASY classes. All analyses were performed in R statistical software, version 1.3.959 (R Core Team, 2020)

\section{RESULTS}

We assessed the nape plumage class of 333 birds, comprising 135 photographed in colonies and 198 necropsied birds. Pooling all data, the ratio of nape classes (A:B:C) did not differ significantly between age classes, defined following either Pyle $(2008)\left(X^{2} 4,333=3.43, p=0.489\right)$, or Powers et al. $(2020),\left(X^{2} 4,333=4.01, p=0.405\right)$ (Fig. 2). 
In our dataset, $218 / 333$ individuals were assigned a B nape. This means that $65 \%$ of the individuals we observed could not be reliably aged using Pyle's suggested nape plumage method alone (according to the latter, B napes could be any age from HY to ASY). Based on breeding/chick-rearing status observed in the colony or on criteria assessed during necropsies, we were able to unambiguously classify 254 birds as HY or mature. As in the wider dataset, the majority of individuals (66\%) in this subset had type B nape, and therefore could not be assigned to either HY/SY or AHY/ASY according to the Pyle method. Of the remaining 86 birds designated as A or C, $55 \%$ of HY birds were appropriately assigned as HY/SY based on nape aspect, while $43 \%$ of mature birds were correctly assigned to AHY/ASY. The resulting overall accuracy of the nape method, with consideration of the B napes, was therefore only 19\% (Table 2).

Considering only birds bycaught in Massachusetts Bay from 2010 - 2017 (Fig. 3 ), the ratio of nape classes (A:B:C) differed significantly among some years (Fisher's exact test, two-sided, $\mathrm{p}<0.001)$.

Nape class ratios also varied with location and sex. At all locations, some mature birds had type A napes, while some immatures from both Gough and Massachusetts Bay possessed type C napes. Nape presentation differed in Massachusetts Bay HY and mature birds compared to individuals from the same life history stages in the South Atlantic, with an increased frequency of Type $\mathrm{C}$ napes in Massachusetts Bay $\left(X_{2,41}^{2}=\right.$ $74.95, p<0.001)$. Nape presentation was not different between HY and Mature birds at Gough Island $\left(X_{2,147}^{2}=4.04, \mathrm{p}=0.133\right)$, nor between birds at Gough and Inaccessible (Fisher's exact test, two-sided, $\mathrm{p}=0.112$ ), but nape presentation was 
different between age classes in Massachusetts Bay $\left(X^{2}{ }_{2,174}=10.88, \mathrm{p}=0.028\right.$ ) (Fig. 4).

Further stratification by sex reveals that the relatively high proportion of type $\mathrm{C}$ napes in Mass. Bay is driven by females, which have a higher proportion of type $\mathrm{C}$ napes than males in that location $\left(X^{2}{ }_{2,174}=12.16, \mathrm{p}=0.002\right)($ Fig. 5). This variable presentation of nape aspect and the increased proportion of $\mathrm{C}$ napes in Massachusetts Bay females occurs in each age class observed in this area $\left(\mathrm{HY}: X^{2}{ }_{2,70}=6.63, \mathrm{p}=\right.$ 0.036, AHY/Not Mature: $X^{2}{ }_{2,74}=8.93, \mathrm{p}=0.011$, Mature: $\left.X^{2}{ }_{2,30}=46.42, \mathrm{p}<0.001\right)$.

However, this is not apparent among birds necropsied from bycatch in the South Atlantic adjacent to Gough Island where mature males have patterns of nape presentation statistically indistinguishable from mature females $\left(X^{2}{ }_{2,28}=4.26, \mathrm{p}=\right.$ 0.119). Though limited in size, lack of $\mathrm{C}$ nape scored in mature males $(\mathrm{n}=$ ?) and females $(n=?)$ from Inaccessible further suggests lack of sexual differences during the breeding/chick-rearing period. We could not ascertain if this also applies in immature birds from the breeding colony because the sexes of these birds were unknown.

\section{DISCUSSION}

Our results show that while a slightly higher proportion of fledgling and immature great shearwaters have all-dark napes compared to adults, this effect is small and not a reliable way of discriminating between HY/SY and AHY/ASY or older birds as hypothesized by Pyle (2008). The occurrence of known hatch-year great shearwaters with type C (light collared) napes and known mature, breeding individuals with type A (all dark) napes reinforces this point. Indeed, most great shearwaters 
(65\%), regardless of age, have nape coloration intermediate between all dark and a complete light collar. Thus, we suggest there is no evidence to suggest a lifetime progression from dark to light collars as suggested by Pyle (2008).

Given the lack of systematic variation in nape aspect with age, remaining causes of the differences that we observed potentially include environmental, sexual, or genetic factors. Specifically, our data suggests that nape plumage varies subtly but systematically with sex and location, as females in the NW Atlantic displayed a higher proportion of type $\mathrm{C}$ napes, with most samples informing this observation collected from August - November directly preceding or overlapping the timing of courtship, burrow staging, or breeding activities (September - January). Moreover, among females in the NW Atlantic, mature birds had a higher a proportion of type C napes than either HY and young birds. However, during the breeding/chick-rearing period at the colony, mature males and females displaying a statistically similar proportion of $\mathrm{C}$ napes ( $17 \%$ and $10 \%$, respectively). The fact that this sex-specific shift of $\mathrm{C}$ nape presentation between locations occurs among mature birds suggests that nape plumage may have a sexual display function. Mature birds included in this study were not aged specifically using banding or colony monitoring, and therefore we are unable to comment on how nape characteristics may change over the long reproductive period of mature birds spanning decades of breeding seasons, as seen in other petrels (Hyrenbach 2002).

Evidence from seabirds and other bird taxa provides context regarding potential sexual drivers of nape characteristics in great shearwaters. Allopreening, especially of the head and neck, is a common pair formation/maintenance behavior among birds 
(Kenny et al. 2017) and in some species more robust neck biting occurs. In Northern gannets, both male and females start the breeding season with yellow heads, yet the female head and nape become increasingly speckled with white plumage over the course of the breeding season (Redman et al. 2002). Males of this species engage in neck biting, which may damage feathers on the females' neck and prompt their replacement with white feathers. However, Nelson (2001) suggested the occurrence of white speckling on female birds cannot be related to courtship or sexual behaviors, due to the presence of white speckling in immature females and some males (Nelson 2001). Great shearwaters have also been observed to engage in allopreening during courtship, but it is unlikely that any white feathers on female napes lost during these behaviors would be replaced with energetically more costly brown plumage. . Additionally, this dataset lacks data detailing sex of immature birds in the South Atlantic to ascertain any transition (or lack thereof) to darker napes in non-breeding females not subject to courtship behaviors.

Nelson (2001) also suggested that gannet chicks have dark plumage because white plumage elicits an aggressive reaction from adults. In the case of great shearwaters, a white nape may be preferred for females only during pre-breeding and courtship stages, thereafter the white collar is replaced with darker nape plumage to avoid aggression from males. This may also explain the slightly higher incidence of dark, type A napes in immature individuals.

Additionally, great shearwaters are unusual among the small-medium petrels in that they engage in courtship display in the daylight, usually in the evening. They are also more patterned than other shearwaters and have less pronounced sexual 
differences in vocalizations (Brooke 1988). These lines of evidence suggest this species may rely more on visual cues about conspecifics and potential mates. A higher proportion of white napes in mature females may therefore play a role in visual courtship displays and mate identification. In multiple taxa including and beyond petrels, plumage coloration and brightness indicate nutritional condition and quality of the individual with implications for reproductive behaviors (Hill 1991, McGraw et al. 2002, Meunier et al. 2011, Soravia et al. 2020). For example, nocturnal Mediterranean Storm Petrels (Hydrobates pelagicus melitensis) show condition-dependent presentation of chest and tail plumage in breeding males based on UV spectrometry measurements (Soravia et al. 2020). Differences in presentation were solely apparent in colonies subject to unfavorable environmental conditions. Soravia et al. hypothesized this sexual dimorphism in achromatic plumage may function as a signal of male quality during mate selection by females, or as an indicator of body condition to rival males during defense of burrow territories. Within our study species, disparity in plumage presentation between sexes during the pre-breeding period in Massachusetts Bay may likewise function in mate selection or other sexually-mediated roles not explored within the scope of this dataset.

In summary, our findings show that nape color is not a reliable signal of age as previously suggested and that underlaying causes and patterns of sex-specific nape polymorphs in great shearwaters warrant further investigation across their breeding and non-breeding range. Despite the long-standing challenges associated with sexing and aging petrels at sea, the development of methods based on plumage and other external characteristics remains a very worthwhile goal. This is not least because this 
group includes some of the world's most threatened birds (Croxall et al. 2012) and understanding and mitigating threats such as bycatch requires an understanding of if and how they affect different demographic groups asymmetrically at sea (Dias et al. 2019). Beak scaling, hormonal differences, telomere length, and pre-maxillary gape width have proven useful in age determination in other seabird taxa, and merit further exploration as ageing tools in great shearwaters and other petrels lacking age-based plumage characteristics.

\section{ACKNOWLEDGEMENTS}

We are grateful to Michael J. Moore for necropsy facility use and to Vonica A. Perold and Maëlle Connan for necropsy assistance in South Africa; to Johanna Pedersen for necropsy and data entry assistance in the US; to Fabrice le Bouard, Jaimie Cleeland and Kate Lawrence for collecting data on Gough and to the Royal Society for the Protection of Birds for facilitating fieldwork there and to the Tristan da Cunha Government for permitting this work. Funding was provided by the UK Natural Environmental Research Council (grant NE/M017990/1, awarded to EW). A. Robuck acknowledges support from the National Oceanic and Atmospheric Administration Dr. Nancy Foster Scholarship program, the Robert and Patricia Switzer Foundation, the STEEP Superfund Research Program (NIEHS Award Number P42ES027706), and the Oak Ridge Institute for Science and Education (ORISE) program. During the 2009 work on Gough and Inaccessible Islands, R. Ronconi was supported by a scholarship

from the Natural Sciences and Engineering Research Council of Canada (NSERC) and the Killam Trusts, Dalhousie University. Funding for Massachusetts Bay work was 
provided by the Volgenau Foundation and Stellwagen Bank National Marine Sanctuary

\section{LITERATURE CITED}

1) Arcos JM. 2007. Frequency-dependent morph differences in kleptoparasitic chase rate in the polymorphic Arctic skua Stercorarius parasiticus. Journal of Ornithology, 148:167-171.

2) Bolton, M. and Thomas, R. 2001. Moult and ageing of storm petrels hydrobates pelagicus. Ringing \& Migration, 20: 193-201.

3) Bradley, J.S., Gunn, B.M., Skira, I.J., Meathrel, C.E. And Wooller, R.D. 1999. Age- dependent prospecting and recruitment to a breeding colony of Shorttailed Shearwaters Puffinus tenuirostris. Ibis, 141(2): 277-285.

4) Brooke, M. 2004. The Petrels, Oxford, Oxford University Press.

5) Broughton, J.M. 1994. Size of the bursa of Fabricius in relation to gonad size and age in Laysan and Black-footed Albatrosses. The Condor, 96: 203207.

6) Bugoni, L., Naves, L. C. And Furness, R. W. 2015. Moult of three Tristan da Cunha seabird species sampled at sea. Antarctic Science, 27: 239-250.

7) Campioni, L., Dias, M. P., Granadeiro, J. P. And Catry, P. 2020. An ontogenetic perspective on migratory strategy of a long-lived pelagic seabird: Timings and destinations change progressively during maturation. Journal of Animal Ecology, 89: 29-43.

8) Carlos, C. J. and Voisin, J.F. 2008. Identifying giant petrels, Macronectes giganteus and M. halli, in the field and in the hand. Seabird, 21: 1-15.

9) Croxall, J. P., Butchart, S. H. M., Lascelles, B., Stattersfield, A. J., Sullivan, B. J., Symes, A. and Taylor, P. 2012. Seabird conservation status, threats and priority actions: a global assessment. Bird Conservation International, 22: 134.

10) M.P. Dias, R. Martin, E.J. Pearmain, I.J. Burfield, C. Small, R.A. Phillips, O. Yatesd, B. Lascelles, P.G. Borboroglue, J.P. Croxall. 2019. Threats to seabirds: a global assessment. Biol. Conserv., 237: 525-537 
11) Fielding A. H. and Bell J. F. 1997. A review of methods for the assessment of prediction errors in conservation presence/absence models. Environ. Conserv. 24: 38-49.

12) Flood, B. and A., F. 2020. Multimedia Identification Guide to North Atlantic Seabirds: Shearwaters: Jouanin's \& White-Chinned Petrels, Pelagic Birds \& Birding Multimedia Identification Guides.

13) Hill, G. 1991. Plumage coloration is a sexually selected indicator of male quality. Nature 350, 337-339. DOI: https://doi.org/10.1038/350337a0

14) Hong, P., D. N. Wiley, K. D. Powers, R. H. Michener, L. Kaufman, and K. A. Hatch. 2019. Stable Isotope Analyses of Multiple Tissues of Great Shearwaters (Ardenna Gravis) Reveals Long-Term Dietary Stability, Short-Term Changes in Diet, and Can be Used as a Tool to Monitor Food Webs. Diversity, 11:163.

15) Hyrenbach, K. D. 2002. Plumage-Based Ageing Criteria for the Black Footed Albatross Phoebastria Nigripes. Marine Ornithology, 30: 85 - 93.

16) Keijl, G. 2011. Sooty Shearwaters Puffinus griseus in the North Atlantic Moult Studies Using Digital Cameras. Marine Ornithology, 39: 141-142.

17) Kenny, E., T. R. Birkhead, and J. P. Green. 2017. Allopreening in birds is associated with parental cooperation over offspring care and stable pair bonds across years. Behavioral Ecology, 28:1142-1148.

18) Lack, D. 1968. Ecological adaptations for breeding in birds, London, Methuen.

19) Leal, G.R., Nunes, G.T., Oliveira, G., Bugoni, L. 2019. Assortative mating, sexual size dimorphism and sex determination in a seabird with plumage polymorphism. Marine Biology Research, 15(1): $74-83$.

20) Le Corre M. 1999. Plumage polymorphism of red-footed boobies (Sula sula) in the western Indian Ocean: an indicator of biogeographic isolation. Journal of Zoology, 249:411-415.

21) Lee, D.S. 2009. Mass die-offs of Greater Shearwaters in the western North Atlantic: $\quad$ Effects of weather patterns on mortality of a trans-equatorial migrant. Chat, 37- 47.

22) McGraw KJ, Mackillop EA, Dale J, and Hauber ME. 2002. Different colors reveal different information: how nutritional stress affects the expression of melanin- and structurally based ornamental plumage. J Exp Biol, 205(Pt 23):3747-55. 
23) Meier, R. E., Votier, S. C., Wynn, R. B., Guilford, T., Mcminn Grivé, M., Rodríguez, A., Newton, J., Maurice, L., Chouvelon, T., Dessier, A. and Trueman, C. N. 2017. Tracking, feather moult and stable isotopes reveal foraging behaviour of a critically endangered seabird during the non-breeding season. Diversity and Distributions, 23: 130-145.

24) Meunier, J., Figueiredo Pinto, S., Burri, R. and Roulin, A. 2011. Eumelaninbased coloration and fitness parameters in birds: a meta-analysis. Behav Ecol Sociobiol, 65, 559-567. DOI: Https://doi.org/10.1007/s00265-010-1092-z

25) Onley, D. and Scofield, P. 2007. Albatrosses, petrels and shearwaters of the world, London, Christopher Helm.

26) Powers, K. D., D. N. Wiley, A. J. Allyn, L. J. Welch, and R. A. Ronconi. 2017. Movements and foraging habitats of great shearwaters Puffinus gravis in the Gulf of Maine. Marine Ecology Progress Series, 574:211-226.

27) Powers, K. D., D. N. Wiley, A. R. Robuck, Z. H. Olson, L. J. Welch, M. A. Thompson, and L. Kaufman. 2020. Spatiotemporal characterization of nonbreeding great shearwaters Ardenna gravis within their wintering range. Marine Ornithology, 48: 215-229.

28) Prince, P. A., Weimerskirch, H., Huin, N. and Rodwell, S. 1997. Molt, Maturation of Plumage and Ageing in the Wandering Albatross. The Condor, 99: $58-72$.

29) Pyle, P. 2008. Identification Guide to North American Birds, Part 2: Anatidae to Alcidae, Slate Creek Press.

30) Redman, K., Lewis, S., Griffiths, R., Wanless, S., \& Hamer, K. 2002. Sexing Northern Gannets from DNA, Morphology and Behavior. Waterbirds, 25(2), 230-234.

31) Riotte-Lambert, L. and Weimerskirch, H. 2013. Do naive juvenile seabirds forage differently from adults? Proceedings. Biological sciences / The Royal Society, 280: 20131434.

32) Rodríguez, A., Arcos, J. M., Bretagnolle, V., Dias, M. P., Holmes, N. D., Louzao, M., Provencher, J., Raine, A. F., Ramírez, F., Rodríguez, B., Ronconi, R. A., Taylor, R. S., Bonnaud, E., Borrelle, S. B., Cortés, V., Descamps, S., Friesen, V. L., Genovart, M., Hedd, A., Hodum, P., Humphries, G. R. W., Le Corre, M., Lebarbenchon, C., Martin, R., Melvin, E. F., Montevecchi, W. A., Pinet, P., Pollet, I. L., Ramos, R., Russell, J. C., Ryan, P. G., Sanz-Aguilar, A., Spatz, D. R., Travers, M., Votier, S. C., Wanless, R. M., Woehler, E. and Chiaradia, A. 2019. Future Directions in Conservation Research on Petrels and Shearwaters. Frontiers in Marine Science, 6. 
33) Ronconi, R. A., S. Schoombie, A. J. Westgate, S. N. P. Wong, H. N. Koopman, and P. G. Ryan. 2018. Effects of age, sex, colony and breeding phase on marine space use by Great Shearwaters Ardenna gravis in the South Atlantic. Marine Biology, 165(58).

34) Voous, K. H., and W. J. 1963. Distribution and migration of the Greater Shearwater. Ardea 51:143-157.

35) Van Franeker, J. A. Save the North Sea Fulmar-Litter-ecoqo Manual - Part 1: Collection and Dissection Procedures. Alterra-rapport 2004, 672, 1-38.

36) Warham, J. 1990. The petrels: their ecology and breeding systems, San Diego, Academic Press.

37) Weimerskirch, H., Lequette, B. and Jouventin, P. 1989. Development and maturation of plumage in the wandering albatross Diomedea exulans. Journal of Zoology, 219: 411-421. 

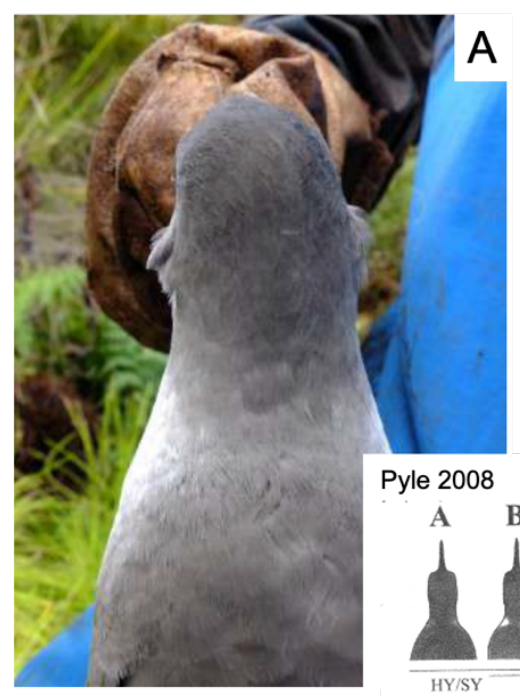

Pyle 2008
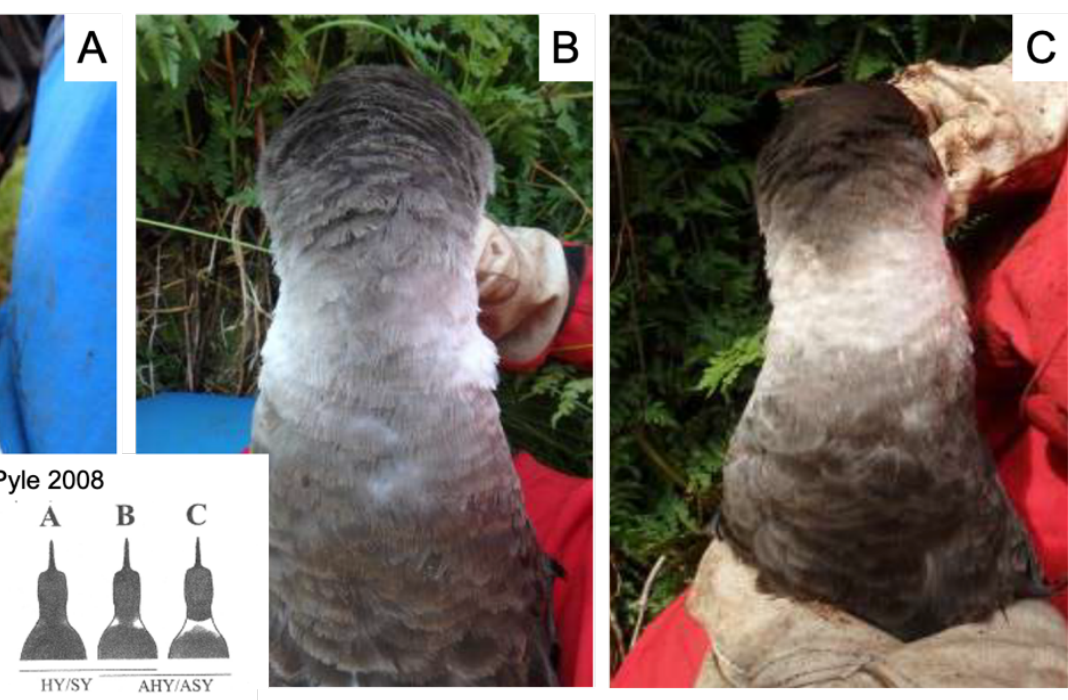

Figure 6.1: Examples of each nape class as observed in live birds. The diagram inset is reproduced from Pyle 2008. 


\begin{tabular}{|c|c|c|c|c|c|c|c|}
\hline $\begin{array}{l}\text { Classification } \\
\text { scheme }\end{array}$ & Age Class & $\begin{array}{c}\text { Bursa } \\
\text { presen } \\
\mathbf{t}\end{array}$ & $\begin{array}{c}\text { Molt } \\
\text { score = } \\
50^{\mathrm{a}}\end{array}$ & $\begin{array}{c}\text { Molt score } \\
<50^{\mathrm{a}}\end{array}$ & $\begin{array}{c}\text { Gonad } \\
\text { class = } 1 \\
\text { or } 2\end{array}$ & $\begin{array}{c}\text { Gonad } \\
\text { class }=3 \\
\text { or } 4\end{array}$ & $\begin{array}{l}\text { Gonad } \\
\text { size } \\
\text { overlap } \\
\text { with } \\
\text { known } \\
\text { breeder }\end{array}$ \\
\hline Pyle & HYISY & $\mathrm{X}$ & $X$ & $X$ & $\mathrm{X}$ & & \\
\hline Pyle & AHYIASY & & & $\mathrm{X}$ & $\mathrm{X}$ & $\mathrm{X}$ & $\mathrm{X}$ \\
\hline Powers et al. & $\mathrm{HY}$ & $\mathrm{X}$ & $x$ & & $\mathrm{X}$ & & \\
\hline Powers et al. & $\begin{array}{l}\text { AHY/Not } \\
\text { Mature }^{b}\end{array}$ & & $\mathrm{X}$ & $\mathrm{X}$ & $x$ & & \\
\hline Powers et al. & Mature & & $\mathrm{X}$ & $\mathrm{X}$ & & $\mathrm{X}$ & $\mathrm{X}$ \\
\hline
\end{tabular}

Table 6.1: Age classes and associated criteria from Pyle (2008) and Powers et al. (2020). 

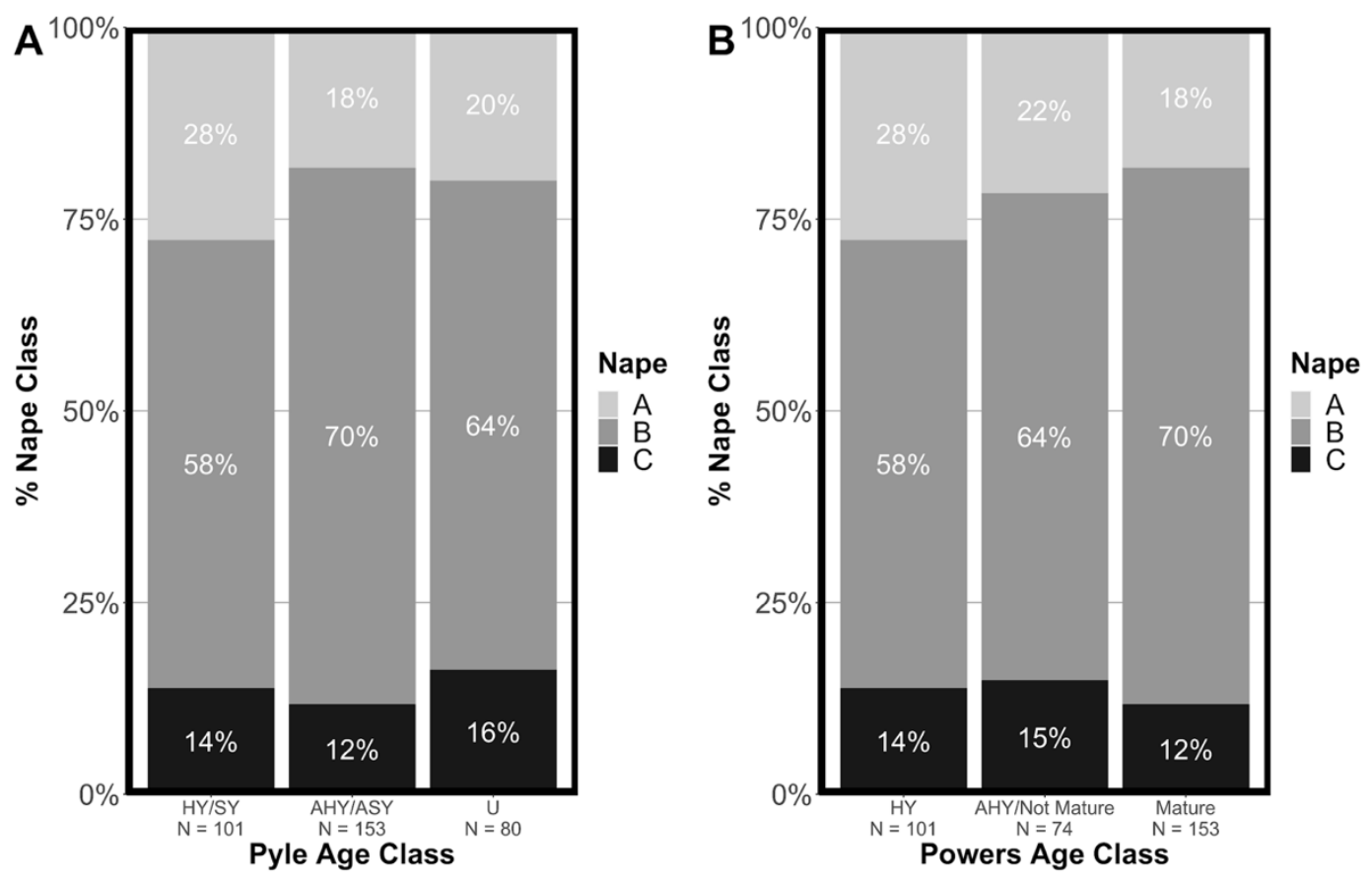

Figure 6.2. Variation in nape class (as defined in Fig. 1) by age class defined following either (A) Pyle (2008) or (B)Powers et al. (2020). Both panels include observations from all locations. 


\begin{tabular}{|c|r|r|r|}
\hline & \multicolumn{3}{|c|}{ Inferred from nape } \\
\hline True & AHYIASY & HY/SY & \multicolumn{1}{c|}{ Unk } \\
\hline \hline AHY/ASY & 20 & 26 & 107 \\
\hline HY/SY & 12 & 28 & 59 \\
\hline
\end{tabular}

Table 6.2. Confusion matrix presenting counts of known age (HY or Mature) birds, compared to the age class as predicted according to Pyle's nape classification system, under the "Inferred from nape" heading. 


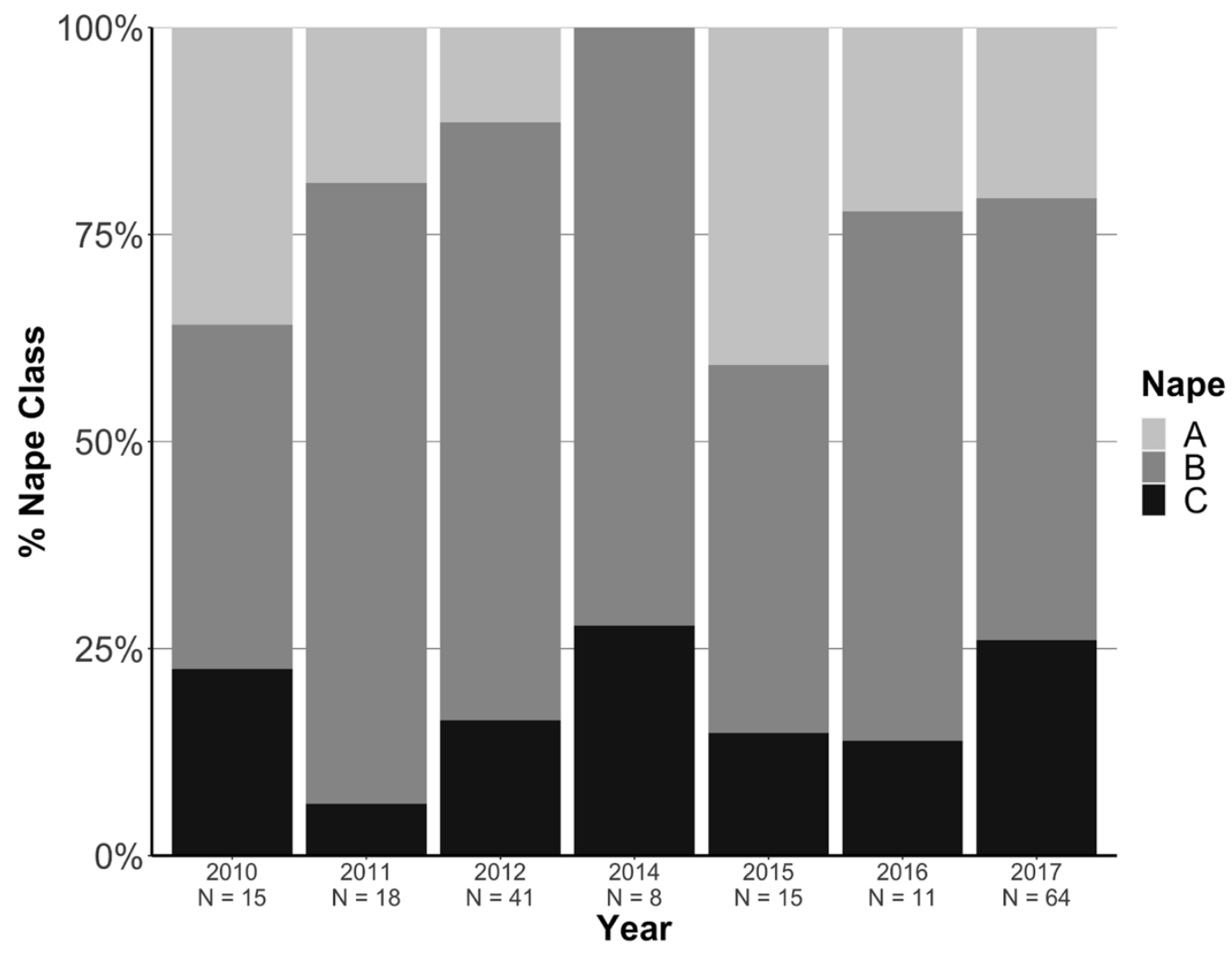

Figure 6.3. Nape classification of Massachusetts Bay bycatch individuals by year 


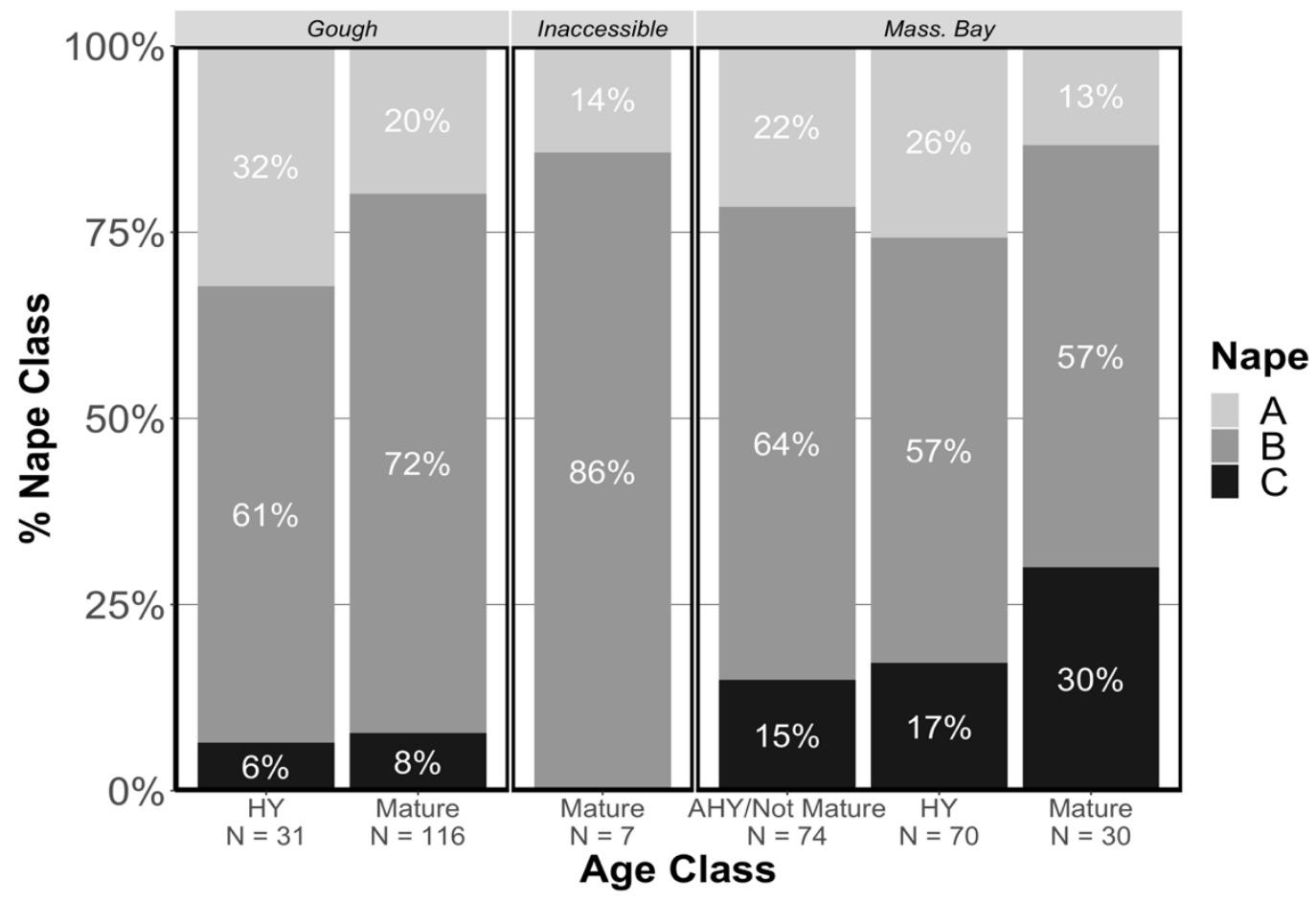

Figure 6.4. Nape classification by age and location. 


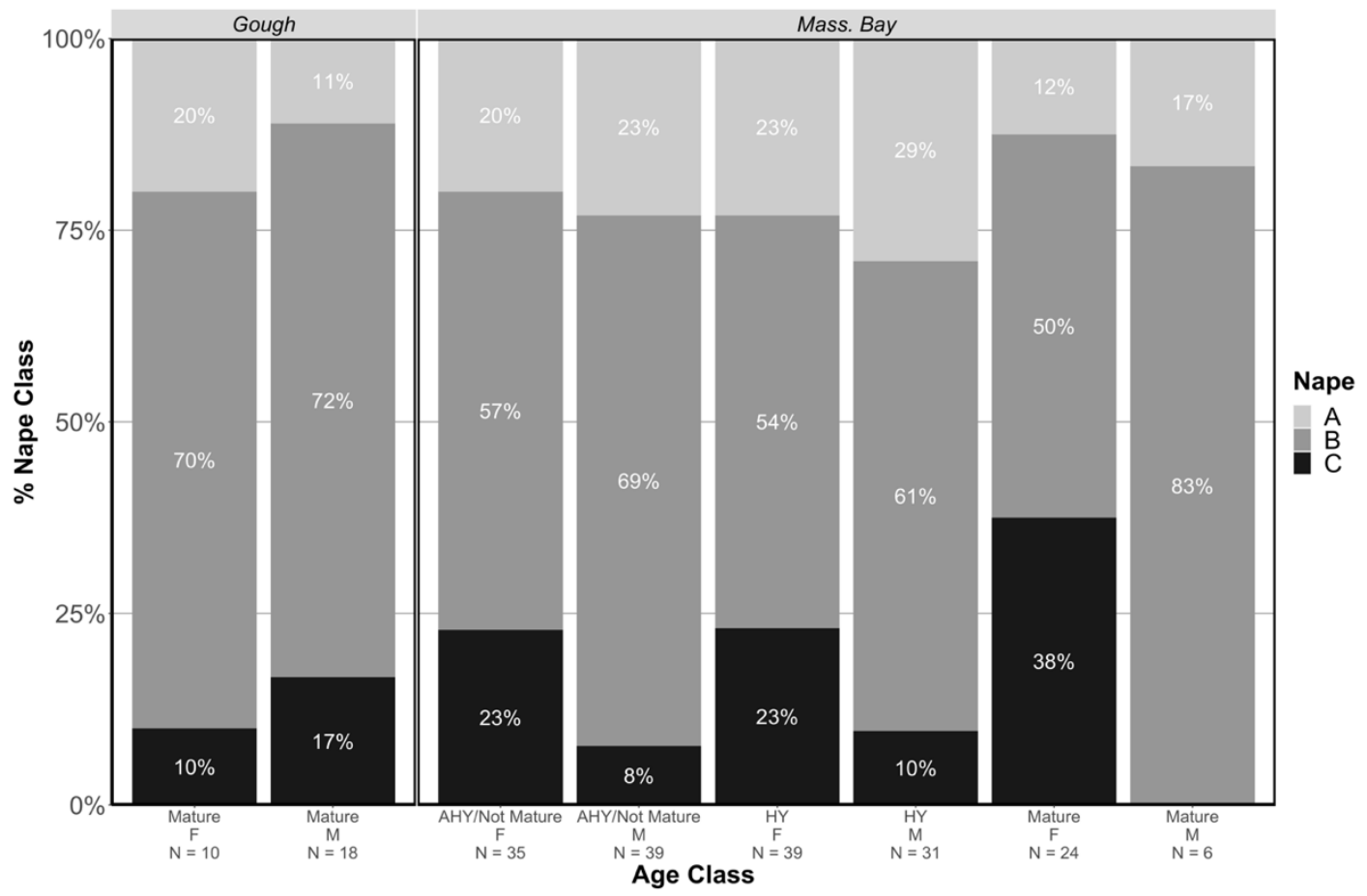

Figure 6.5. Nape classification by sex, age, and location. 


\section{CHAPTER 7}

\section{CONCLUSION}

\section{Improved characterization of Great Shearwater demographics and contributions to}

Stellwagen Bank National Marine Sanctuary research priorities. Execution of the contaminant studies that were the primary focus of this dissertation required a largescale necropsy program that entailed collection of comprehensive demographic and morphometric data. To date, 366 Great Shearwaters and 22 individuals from other species collected from 2009 - 2019 were necropsied in support of various studies

presented herein. Biological data from this necropsy work was used to collaboratively answer several questions about Great Shearwaters, a key sentinel species in Massachusetts Bay ${ }^{1}$. Primarily, demographic data paired with plumage observations were used to correct guidance from previous work suggesting plumage is an indicator of age in Great Shearwaters (Chapter 5). Pyle (2008) previously suggested that nape coloration is an indicator of maturity, with completely dark napes associated with hatch year or second year birds while completely white napes were associated with older individuals. Here we found nape is not an honest signal of age in juvenile or adult Great Shearwaters, at any point in their life cycle. This finding enables future atsea observational studies to avoid misleading age classifications based on plumage coloration. However, we noted a higher abundance of complete white napes in females, suggesting nape coloration may potentially act in mate selection or other reproductive processes. Further work is required to determine any significance of nape coloration in reproductive behaviors for this species; observations of nape aspect over 
the duration of the breeding phase may be helpful to parse out sexual drivers of nape plumage.

Great Shearwaters have been previously documented to be the most commonly bycaught species in Massachusetts Bay ${ }^{2,3}$; necropsy data obtained via this dissertation also revealed that $\sim 90 \%$ of bycatch Great Shearwaters from Massachusetts Bay are juveniles, and males and females are equally represented in the bycatch sample set. This is the first quantitative description of Great Shearwater bycatch demography and leaves outstanding questions regarding how the preferential loss of pre-reproductive individuals may impact overall population dynamics, as bycatch of Great Shearwaters can remove thousands of individuals per year, though Great Shearwaters as a species are not considered threatened due to a previous population estimate around 15 million individuals in the early 2000s. A follow-up project exploring this question is underway using the demographic data collected within this dissertation and demographic modeling tools previously applied in other seabird-fishery interaction scenarios ${ }^{4}$.

Morphometric and demographic data combined with satellite tracking data of live birds also suggests that Massachusetts Bay and the SW Gulf of Maine are preferentially used by juvenile individuals as a "nursery" area upon their arrival to the Gulf of Maine, likely due to consistent supplies of sand lance (Ammodytes spp.), a key forage fish that favors sandy bottom substrates and shallow environments (co-author work, Powers et al. 2020) $)^{5}$. This work has implications for protected area management and resource conservation, due to potential sand mining and wind lease exploration within the SW Gulf of Maine. Additionally, time series data of bulk $\delta^{15} \mathrm{~N}$ and $\delta^{13} \mathrm{C}$ stable isotope ratios were collected to ascertain if pollutant dynamics were related to 
shifts in trophic level or primary productivity (Chapter 2). These data suggest slight shifts in Great Shearwater trophic level based on variable $\delta^{15} \mathrm{~N}$ ratios over $2010-$ 2019. While the changes over time are slight and are not thought to meaningfully impact pollutant dynamics seen here, they may indicate potential fluctuations in prey use, such as variable reliance on sand lance, menhaden, or squid each given year. Temperatures in the Gulf of Maine and Massachusetts Bay are rapidly changing due to climate change, with commensurate changes in regional fish assemblages that may be playing a role in the shifts we see here ${ }^{6,7}$. Further work will continue analyzing stable isotope ratios in Great Shearwaters over time to better quantify any long-term shifts in trophic level, and incorporate baseline measurements from a wider suite of forage fish.

Additionally, involvement in Stellwagen Bank National Marine Sanctuary's Great Shearwater research team facilitated collaboration with sand lance research ongoing in the Sanctuary. In collaboration with another PhD candidate, bulk lipid measurements were carried out in 202 sand lance collected over the 2019 growing season, and results indicated that fish lipid content varied by up to $30 \%$ over growth and reproductive phases spanning February to November. Sand lance from Stellwagen Bank National Marine Sanctuary reached their peak lipid content commensurate with the arrival of Great Shearwaters in June - August. This work also predicted tremendous changes to sand lance abundance due to climate-related drivers (Co-author work, Suca et al., in review), and further research is required to understand how this may impact seabirds heavily reliant on the abundance of this lipid-rich species at a specific time and location. Ongoing work is also exploring PFAS and hydrophobic contaminants in the same sand lance previously measured for lipid content. 
Distributions of PFAS in seabirds across space and time. PFAS were last assessed in seabirds from the US East Coast in the late 1990s and early 2000s. This dissertation provides an updated description of PFAS in select US East Coast seabirds by measuring legacy and novel PFAS in livers from several species of seabird from habitats representative of low, medium, and high PFAS exposure potential. We also measured PFAS in Great Shearwaters from Massachusetts Bay, an offshore environment associated with low PFAS exposure potential, from 2010 - 2019 (Chapters $1 \& 2$ ).

We found seabirds reared downstream from a major fluoropolymer production site in SE North Carolina contained elevated concentrations of PFAS, including novel PFEAs Nafion BP2, PFO5DoDA, and PFO4DA. Nafion BP2 was also detected in seabirds with no known connection to the region downstream from the industrial region, dating back to 2010 in Great Shearwaters. Our measurements represent the first identification of these PFEAs in seabirds, and at the furthest distances from the known production site to date. Our results also indicate the capacity for long-range transport of Nafion BP2, and raise the possibility of as-of-yet unidentified additional sources of PFEAs to the environment. Future work should prioritize measurements of PFEAs, specifically Nafion BP2, in multiple environmental and biological receptors to better constrain sources, distributions, and bioaccumulation potential of these emerging compounds.

We also found significant concentrations of legacy PFAAs including PFOS and C8-C13 PFCAs in seabirds from all three habitats, and in Great Shearwaters over 2010 
- 2019. Individuals reared downstream from the industrial site in North Carolina possessed the highest total concentration of legacy PFAAs, while $\sum{ }_{19}$ PFAS was statistically similar between Massachusetts Bay and Narragansett Bay birds despite their differential proximity to point sources of PFAS.

Despite being phased out in the early 2000s, PFOS was the most abundant compound identified in most individuals, at levels associated with toxicological significance (> $140 \mathrm{ng} / \mathrm{g}$ wet wt. in liver) in other bird taxa ${ }^{8}$. Measurements in Great Shearwater livers from 2010 - 2019 suggest PFOS and FOSA levels are slowly decreasing over time in this species due to the voluntary phase-out of PFOS and its salts in the early 2000s; this decline is in line with observations from other taxa and locations ${ }^{9,10}$.

C8 - C13 PFCAs were also found in most individuals measured in this work; the C11 PFUnDA was generally the most abundant PFCA identified. PFCAs made up a larger proportion of $\sum{ }_{19} \mathrm{PFAS}$ in Massachusetts Bay birds compared to Narragansett Bay or Cape Fear individuals, and no consistent trends in PFCAs were identified in Massachusetts Bay Great Shearwaters over time. The lack of a clear temporal trend is in contrast to prior work that has identified increasing PFCA levels over time in birds and other taxa ${ }^{11,12}$, and may indicate an ongoing change point whereby long-chain PFCAs are ceasing to increase in the environment ${ }^{13}$.

This work also described concentrations of 7:3 FTCA, a fluorotelomer carboxylic acid formed through degradation of volatile PFAS, in Great Shearwaters from Massachusetts Bay from 2010 - 2019. 7:3 FTCA likewise demonstrated no clear linear trend over time, suggesting sustained emissions over the duration of our time series, 
but alternated with PFUnDA as the second most abundant compound found in each individual. This compound has been previously identified in marine mammals from the NW Atlantic, and our work marks the first identification of this compound in a seabird from the Atlantic Basin. This compound has demonstrated toxicity thresholds up to 10,000 times lower than PFCAs, and requires further research to constrain possible impacts or trends.

These results lead to further questions regarding current PFAS levels in other marine fauna. Further work should prioritize monitoring of PFAS in US East Coast environments and biota, considering the high population density along the US Eastern Seaboard, the abundance of PFAS sources present within this region ${ }^{14}$, and the importance of commercial fisheries to regional economies. Future efforts would benefit from inclusion of a broad compound list to evaluate trends in emerging PFAS like PFEAs and 7:3 FTCA, given the noted paucity of data describing their environmental and biological occurrence.

In particular, PFAS and other emerging contaminants should remain a monitoring focus in Massachusetts Bay, given the demonstrated ubiquity of these compounds in seabirds from the region, sustained pollutant inputs from regional urban sources, associations between PFAS and bird individual condition (see below), and the importance of this productive offshore region for commercial fisheries and critically endangered megafauna like the North Atlantic Right Whale.

Follow-up work based on the findings presented within this dissertation will measure novel and emerging PFAS in baleen, liver, fat, and skin from several species of whale that inhabit the Gulf of Maine and Massachusetts Bay, to provide first-of-its- 
kind information and improve our understanding of PFAS levels in apex marine biota from this region. Additionally, a food web study incorporating sediment, water, plankton, fish, and Great Shearwaters from Massachusetts Bay is nearing completion (delayed due to COVID-19) and will further develop understanding of PFAS in the region.

Further work should also continue to monitor trends in PFAS levels over time in wildlife populations across the broader US Atlantic Coast, in tandem with characterization of PFAS levels in ambient natural waters using water grab sampling or passive sampling techniques. We expect changes in emissions or production of PFAS to be rapidly reflected in US East Coast biota and environments, due to the region's embedded population centers and PFAS sources. An increased body of time series data from the US East Coast would therefore allow evaluation of fluctuations or trend responses that may indicate the efficacy (or lack thereof) of future policy and production interventions; such data would also provide an indication of potential trend changes expected in other, more remote regions around the globe (i.e. the Arctic).

Associations between PFAS and bird condition. This work assessed relationships between PFAS levels measured in seabird livers and a suite of biological and morphometric variables measured in each bird, using Spearman correlation analyses. We found negative relationships between liver phospholipid levels and PFOS and PFNA concentrations in birds from Massachusetts Bay and the Cape Fear River Estuary, as well as negative relationships between fat depth and several PFAS (Chapters 1, 2). Negative associations between PFAS levels and lipid expression have been previously identified in controlled studies ${ }^{15}$, yet this marks the first 
documentation of this phenomena in a wildlife population. The range of exposure associated with lipid changes in the offshore birds $(11-44 \mathrm{ng} / \mathrm{g})$ was much lower than thresholds identified by controlled studies and field-based studies (140 ng/g). Further work evaluating associations between PFAS and lipids in wildlife is crucial, given the importance of finely-tuned lipid dynamics for growth, reproduction, migration, and other key developmental milestones, and the potential for individual adverse effects to scale to population-level impacts. To date, population level impacts of PFAS in birds or other wildlife is completely unexplored. This finding has also led to a follow-up study involving measurement of 1,300 lipid moieties in wild birds and hepatocyte cultures of bird cells, to specifically identify the types of phospholipid most related to PFAS levels. Further exploration of this phenomena could be conducted using serum samples collected in live birds with commensurate measurement of blood chemical parameters using inexpensive veterinary testing kits; this would allow measurement of PFAS levels and serum lipids alongside other biomarkers indicative of physiological changes.

We also found varied associations between kidney weight, liver weight, wing chord, body condition of Great Shearwaters, and various PFAS levels (Chapter 2). Some of the same relationships between morphometric measurements and PFAS levels were recently identified in two species of Pacific shearwater ${ }^{16}$. These results are challenging to frame, because scant data exists describing the mechanism through which PFAS may be associated with or driving these relationships, but these changes may be related to PFAS-driven hormonal disruptions that impact structural growth. Additionally, it is unclear how synergistic or additive effects from unidentified co- 
contaminants or trophic factors may be influencing these results. More controlled and field study investigating sublethal outcomes in avifauna exposed to environmentally relevant levels of PFAS and other pollutants would be helpful to better clarify if or how PFAS may be related to these morphometric outcomes. Again, it is unclear what, if any, population level impacts may result from the associations between PFAS and bird condition and size observed within this study.

Internal behavior of PFAS. PFAS were measured in eight tissues of seabirds from Massachusetts Bay, Narragansett Bay, and the Cape Fear River Estuary (Chapter 3). These tissue-specific measurements were particularly valuable, because they facilitated exploration of mechanisms driving partitioning of legacy and novel PFAS through comparative means.

This study found PFAAs and PFEAs throughout multiple tissues of birds from each habitat (Chapter 3). This is the first work to document PFO4DA, PFO5DoDA, and Nafion BP2 in multiples tissues of environmentally exposed vertebrates. Blood, liver, kidney, lungs, and brain were found to possess the highest PFAS concentrations. PFOS and PFNA were most abundant in liver, while PFEAs were more abundant in blood. Quantum chemistry calculations suggest the preferred partitioning of PFEAs into blood may be due to different surface charge potential and/or folding conformations that reduce the ability of PFEAs to bind to liver proteins in the same way as PFAAs. Long-chain PFCAs preferentially partitioned into brain tissue; PFEAs were also identified in brain, marking the first time these emerging compounds have been documented to cross the highly selective blood-brain barrier. 
Further work should examine the tissue-specific distributions of PFEAs in other biota, given the increasing use of these emerging PFAS in fluoropolymer production and the lack of research parameterizing internal distributions of these ether-based compounds. Additionally, more research is required to determine levels of toxicological concern associated with potential impacts in multiple tissues. Existing thresholds of concern focus on liver, egg, or blood, yet this data and a limited number of other studies ${ }^{17-19}$ investigating PFAAs in a tissue-specific manner confirm that kidney, lungs, and brain are also important PFAS reservoirs.

The totality of PFAS research and associated expertise documented within this dissertation likewise contributed to development of a large critical review, as part of a wider team effort seeking to review human and wildlife exposure to PFAS (Co-author work, De Silva et al. 2021, Accepted).

Accumulation of ingested plastics in seabirds. The accumulation of ingested plastics was assessed in 175 adult and juvenile Great Shearwaters necropsied as part of this dissertation (Chapter 4). An average of 8 plastic pieces were found in each individual. Juveniles accumulated plastic more frequently than adults, with an ingestion frequency of $98 \%$ compared to $58 \%$ in breeding adults. The frequency of plastic occurrence (FO) observed in juveniles was higher than in previous studies focused on this species in prior decades ${ }^{20,21}$. The physical dimensions, morphological category, and polymer makeup of each plastic piece were assessed, and revealed that breeding phase adults from Gough Island in the South Atlantic ingested larger plastic fragments compared to mature and juvenile Great Shearwaters in the NW Atlantic, likely due to size-mediated transport of larger plastic pieces to more remote pelagic environments ${ }^{22}$. While 
polyethylene was the primary polymer ingested across all habitats, Gough Island adults ingested a higher proportion of polypropylene mixes compared to a higher incidence of polyethylene and "other" type plastics, composed primarily of balloons. These results suggest Great Shearwaters turn over plastics rapidly enough to reflect different plastic signatures associated with contrasting ambient environments. This contribution adds evidence to the otherwise still-uncertain retention time of ingested plastics in seabirds ${ }^{23}$. Future work monitoring plastics in Great Shearwaters should prioritize use of standard approaches ${ }^{24}$, while incorporating measurements from multiple age groups and locations to further refine the retention of plastic pieces in this sentinel species.

Overall Policy and Management Implications. The seabird demographic data produced within this work provide the first quantitative description of Great Shearwaters using the Massachusetts Bay region, and may be useful for future resource management. By demonstrating the importance of Massachusetts Bay for juvenile Great Shearwaters, this finding suggesting the need to balance the timing or location of compatible uses with use of the region by young, naïve seabird individuals reliant on consistent food resources and environmental conditions. These results also highlight the importance of bycatch practices that reduce potential for seabird-fishery interactions, considering bycatch is preferentially removing potentially thousands of juveniles per year with uncertain implications for the population.

The descriptions of PFAS occurrence and possible impacts described in this work may also be useful to inform policy and management initiatives. Primarily, this work documented widespread occurrence of PFOS, C9-C13 PFCAs, Nafion BP2, and 7:3 
FTCA in seabirds from several habitats. Only PFOS has been vetted for effects in controlled and field settings, highlighting the need for science and policy to expand to understand and potentially mitigate the proliferation of other PFAS beyond PFOS in biota.

The decreasing trends of PFOS and FOSA identified in this dissertation and elsewhere indicate that phase-out or regulation of specific, well-studied PFAS leads to decreases in environmental matrices on observable time scales. As more PFAS are deemed harmful, policies mandating cessation of use are essential to protect human and ecological health. More logically, this work suggests PFAS and other humancreated chemicals should be vetted for the totality of environmental behaviors before production and introduction to the environment to prevent regrettable, unintended, and irreversible environmental outcomes. For example, long-chain PFCAs, rarely produced intentionally, along with a degradation compound 7:3 FTCA, were some of the most abundant compounds found in seabirds from multiple habitats. The downstream bioaccumulation of these inadvertently created compounds underscores the importance of understanding and assessing the risk of the total potential life-cycle of a given PFAS prior to use and release to prevent the environmental formation of bioaccumulative and ubiquitous terminal end products like long-chain PFCAs or 7:3 FTCA.

Given this proactive approach is not currently formalized in policy or practice within the US, results obtained within this dissertation indicate the need to avoid policy or scientific assumptions that assume novel PFAS are innocuous or environmentally irrelevant. For example, the use of novel PFEAs should better 
account for the newly discovered bioaccumulation potential and environmental mobility of these novel PFAS. PFEAs are currently in use as replacements for some legacy PFAS, and these results demonstrate these new compounds spread in the environment and accumulate across a range of tissues akin to the compounds PFEAs are intended to safely replace, and therefore should receive the same scientific and regulatory scrutiny as their legacy counterparts. The preference of PFEAs to partition to blood compared to other tissues suggests blood monitoring may be an important tool to understand PFEA distributions in other species or locations, yet further research is required to parametrize accumulation and potential impacts of PFEAs in multiple tissues considering the abundant concentrations of these compounds found across eight tissues included in this study.

The sustained occurrence of both legacy and novel PFAS in seabirds and their association with a range of body condition changes at environmentally relevant concentrations demonstrated herein suggests sublethal impacts may be ongoing, yet little work meaningfully explores the implications of PFAS in marine food webs despite the importance of these environments for seafood production and human economies. Funding mechanisms should prioritize exploration of PFAS in marine food webs to better parameterize the underexplored range of individual and population-scale impacts of PFAS in marine biota at ambient exposure levels. Chemical production and clean-up policies should then follow suit to ensure marine food webs and seafood resources are safe for human and wildlife consumption.

This work additionally underscores the importance of policies and practices that support waste management and effective recycling, considering upwards of $90 \%$ of 
plastic items found within seabirds were recyclable polymers that likely ended up in the environment due to waste mismanagement. 


\section{LITERATURE CITED}

(1) Silva, T. L.; Wiley, D. N.; Thompson, M. A.; Hong, P.; Kaufman, L.; Suca, J. J.; Llopiz, J. K.; Baumann, H.; Fay, G. High Collocation of Sand Lance and Protected Top Predators: Implications for Conservation and Management. Conserv. Sci. Pract. 2020, No. July, 1-8. https://doi.org/10.1111/csp2.274.

(2) Hatch, J. M.; Wiley, D.; Murray, K. T.; Welch, L. Integrating Satellite-Tagged Seabird and Fishery-Dependent Data: A Case Study of Great Shearwaters (Puffinus Gravis) and the U.S. New England Sink Gillnet Fishery. Conserv. Lett. 2016, 9 (1), 43-50. https://doi.org/10.1111/conl.12178.

(3) Hatch, J. M. Comprehensive Estimates of Seabird-Fishery Interactions for the US Northeast and Mid-Atlantic. Aquat. Conserv. Mar. Freshw. Ecosyst. 2018, 28 (1), 182-193. https://doi.org/10.1002/aqc.2812.

(4) Anderson, C. M.; Iverson, S. A.; Black, A.; Mallory, M. L.; Hedd, A.; Merkel, F.; Provencher, J. F. Modelling Demographic Impacts of a Growing Arctic Fishery on a Seabird Population in Canada and Greenland. Mar. Environ. Res. 2018, 142 (September), 80-90. https://doi.org/10.1016/j.marenvres.2018.09.021.

(5) Powers, K. D.; Wiley, D. N.; Robuck, A. R.; Olson, Z. H.; Welch, L. J.; Thompson, M. A.; Kaufman, L. Spatiotemporal Characterization of Non-Breeding Great Shearwaters Ardenna Gravis within Their Wintering Range. Mar. Ornithol. 2020, 48, 215-229.

(6) Pershing, A. J.; Alexander, M. A.; Hernandez, C. M.; Kerr, L. A.; Le Bris, A.; Mills, K. E.; Nye, J. A.; Record, N. R.; Scannell, H. A.; Scott, J. D.; Sherwood, G. D.; Thomas, A. C. Slow Adaptation in the Face of Rapid Warming Leads to Collapse of the Gulf of Maine Cod Fishery. Science (80-. ). 2015, 350 (6262), 809-812. https://doi.org/10.1126/science.aac9819.

(7) Nye, J. A.; Baker, M. R.; Bell, R.; Kenny, A.; Kilbourne, K. H.; Friedland, K. D.; Martino, E.; Stachura, M. M.; Van Houtan, K. S.; Wood, R. Ecosystem Effects of the Atlantic Multidecadal Oscillation. J. Mar. Syst. 2014, 133, 103-116.

https://doi.org/10.1016/j.jmarsys.2013.02.006.

(8) Newsted, J. L.; Coady, K. K.; Beach, S. A.; Butenhoff, J. L.; Gallagher, S.; Giesy, J. P. Effects of Perfluorooctane Sulfonate on Mallard and Northern Bobwhite Quail Exposed Chronically via the Diet. Environ. Toxicol. Pharmacol. 2007, 23 (1), 1-9. https://doi.org/10.1016/j.etap.2006.04.008.

(9) Dassuncao, C.; Hu, X. C.; Zhang, X.; Bossi, R.; Dam, M.; Mikkelsen, B.; Sunderland, E. M. Temporal Shifts in Poly- and Perfluoroalkyl Substances (PFASs) in North Atlantic Pilot Whales Indicate Large Contribution of Atmospheric Precursors. Environ. Sci. Technol. 2017, 51 (8), 4512-4521. https://doi.org/10.1021/acs.est.7b00293. 
(10) Sun, J.; Bossi, R.; Bustnes, J. O.; Helander, B.; Boertmann, D.; Dietz, R.; Herzke, D.; Jaspers, V. L. B.; Labansen, A. L.; Lepoint, G.; Schulz, R.; Sonne, C.; Thorup, K.; Tøttrup, A. P.; Zubrod, J. P.; Eens, M.; Eulaers, I. White-Tailed Eagle (Haliaeetus Albicilla) Body Feathers Document Spatiotemporal Trends of Perfluoroalkyl Substances in the Northern Environment. Environ. Sci. Technol. 2019. https://doi.org/10.1021/acs.est.9b03514.

(11) Muir, D.; Bossi, R.; Carlsson, P.; Evans, M.; De Silva, A.; Halsall, C.; Rauert, C.; Herzke, D.; Hung, H.; Letcher, R.; Rigét, F.; Roos, A. Levels and Trends of Polyand Perfluoroalkyl Substances in the Arctic Environment - An Update. Emerg.

Contam. 2019, 5, 240-271. https://doi.org/10.1016/j.emcon.2019.06.002.

(12) Miller, A.; Elliott, J. E.; Elliott, K. H.; Lee, S.; Cyr, F. Temporal Trends of Perfluoroalkyl Substances (PFAS) in Eggs of Coastal and Offshore Birds: Increasing PFAS Levels Associated with Offshore Bird Species Breeding on the Pacific Coast of Canada and Wintering near Asia. Environ. Toxicol. Chem. 2015, 34 (8), 1799-1808. https://doi.org/10.1002/etc.2992.

(13) Jouanneau, W.; Bårdsen, B.-J.; Herzke, D.; Johnsen, T. V.; Eulaers, I.; Bustnes, J. O. Spatiotemporal Analysis of Perfluoroalkyl Substances in White-Tailed Eagle (Haliaeetus Albicilla) Nestlings from Northern Norway-A Ten-Year Study. Environ. Sci. Technol. 2020, 54 (8), 5011-5020. https://doi.org/10.1021/acs.est.9b06818.

(14) Hu, X. C.; Andrews, D. Q.; Lindstrom, A. B.; Bruton, T. A.; Schaider, L. A.; Grandjean, P.; Lohmann, R.; Carignan, C. C.; Blum, A.; Balan, S. A.; Higgins, C. P.; Sunderland, E. M. Detection of Poly- and Perfluoroalkyl Substances (PFASs) in U.S. Drinking Water Linked to Industrial Sites, Military Fire Training Areas, and Wastewater Treatment Plants. Environ. Sci. Technol. Lett. 2016, 3 (10), 344-350. https://doi.org/10.1021/acs.estlett.6b00260.

(15) Geng, D.; Musse, A. A.; Wigh, V.; Carlsson, C.; Engwall, M.; Orešič, M.; Scherbak, N.; Hyötyläinen, T. Effect of Perfluorooctanesulfonic Acid (PFOS) on the Liver Lipid Metabolism of the Developing Chicken Embryo. Ecotoxicol. Environ. Saf. 2019, 170 (December 2018), 691-698. https://doi.org/10.1016/j.ecoenv.2018.12.040.

(16) Szabo, D.; Lavers, J. L.; Shimeta, J.; Green, M. P.; Mulder, R. A.; Clarke, B. Correlations between Per- and Polyfluoroalkyl Substances (PFASs) and Body Morphometrics in Fledgling Shearwaters Impacted by Plastic Consumption from a Remote Pacific Island. Environ. Toxicol. Chem. 2021, Accepted. https://doi.org/10.1016/s0740-5472(96)90021-5.

(17) Verreault, J.; Houde, M.; Gabrielsen, G. W.; Berger, U.; Haukås, M.; Letcher, R. J.; Muir, D. C. G. Perfluorinated Alkyl Substances in Plasma, Liver, Brain, and 
Eggs of Glaucous Gulls (Larus Hyperboreus) from the Norwegian Arctic. Environ. Sci. Technol. 2005, 39 (19), 7439-7445. https://doi.org/10.1021/es051097y.

(18) Chu, S.; Wang, J.; Leong, G.; Woodward, L. A.; Letcher, R. J.; Li, Q. X. Perfluoroalkyl Sulfonates and Carboxylic Acids in Liver, Muscle and Adipose Tissues of Black-Footed Albatross (Phoebastria Nigripes) from Midway Island, North Pacific Ocean. Chemosphere 2015, 138, 60-66.

https://doi.org/10.1016/j.chemosphere.2015.05.043.

(19) Gebbink, W. A.; Letcher, R. J. Comparative Tissue and Body Compartment Accumulation and Maternal Transfer to Eggs of Perfluoroalkyl Sulfonates and Carboxylates in Great Lakes Herring Gulls. Environ. Pollut. 2012, 162, 40-47. https://doi.org/10.1016/j.envpol.2011.10.011.

(20) Bond, A. L.; Provencher, J. F.; Daoust, P. Y.; Lucas, Z. N. Plastic Ingestion by Fulmars and Shearwaters at Sable Island, Nova Scotia, Canada. Mar. Pollut. Bull. 2014, 87 (1), 68-75. https://doi.org/10.1016/j.marpolbul.2014.08.010.

(21) Moser, Mary L., Lee, D. S. A Fourteen-Year Survey of Plastic Ingestion by Western North Atlantic Seabirds Author ( s ): Mary L . Moser and David S . Lee Published by : Waterbird Society Stable URL : Http://Www.Jstor.Org/Stable/1521357 REFERENCES Linked References Are Available on J. 2016, 15 (1), 83-94.

(22) Ryan, P. G. Does Size and Buoyancy Affect the Long-Distance Transport of Floating Debris? Environ. Res. Lett. 2015, 10 (8), 84019.

https://doi.org/10.1088/1748-9326/10/8/084019.

(23) Ryan, P. G. How Quickly Do Albatrosses and Petrels Digest Plastic Particles? Environ. Pollut. 2015, 207, 438-440. https://doi.org/10.1016/j.envpol.2015.08.005.

(24) Provencher, J. F.; Borrelle, S. B.; Bond, A. L.; Lavers, J. L.; Van, J. A.; Kühn, S.; Hammer, S.; Avery-gomm, S.; Mallory, M. L. Recommended Best Practices for Plastic and Litter Ingestion Studies in Marine Birds : Collection, Processing, and Reporting. 2019, 111-130. https://doi.org/10.1139/facets-2018-0043. 


\section{APPENDIX A}

\section{COLLABORATIVE CONTRIBUTIONS}

This appendix contains co-author and collaborative works undertaken during the PhD

Candidacy, beyond the primary research aims.

Contribution 1: Authorship of Stellwagen Bank National Marine Sanctuary's Condition Report sections on water quality and contaminant monitoring (pages 323 348)

Contribution 2: Co-author in Markham et al. 2018; contributed analysis of PBDE-209 in seal milk samples via GC/ECNI-MS (pages 349 - 358)

Contribution 3: Co-author in Powers et al. 2020; contributions including participation in field work, provision of all necropsy data, drafting and editing of text detailing necropsy/ageing methods and significance, and active role in manuscript editing and revision (pages $359-373$ ).

Contribution 4: Co-author in Suca et al. 2020, in review; contributions included extraction and analysis of 202 fish for lipid content with primary author, active role in manuscript revisions related to lipid content hypotheses (pages $374-418$ ).

Contribution 5: Co-author in De Silva et al. 2021, accepted; contributions included authorship of wildlife exposure and bioaccumulation section in collaboration with two co-authors (pages $419-476$ ) 


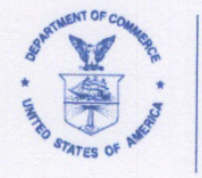

UNITED STATES DEPARTMENT OF COMMERCE

National Oceanic and Atmospheric Administration Office of National Marine Sanctuaries

Stellwagen Bank National Marine Sanctuary

175 Edward Foster Rd Scituate, Massachusetts 02066

August $28^{\text {th }}, 2020$

Dear Dr Lohmann and the Dissertation Committee of Anna Robuck,

I am writing to attest to the phenomenal work PhD candidate Anna Robuck contributed to the development of the Stellwagen Bank National Marine Sanctuary (SBNMS) 2020 Condition Report. The selected portions contained here reflect text and figures Anna contributed to this effort.

The Condition Report is a way for the sanctuary to evaluate and document the changing conditions of the resources protected and managed by the Sanctuary. It is a precursor to the planned revision to the SBNMS management plan, and as such provides data and information which will guide and inform any revisions that may be needed to more effectively manage these resources. To prepare the Condition Report, SBNMS staff (including Anna, as our Nancy Foster Scholar) developed background papers describing the current status of resources, and then presented their data and preliminary status report to an external expert panel, who made recommendations on the conditions and trends of these resources. Topic authors were further involved in multiple rounds of review and copyediting to arrive at the final resource management tool.

Anna was asked to provide write-ups on the following topics:

- What is the eutrophic condition of sanctuary waters and how is it changing?

- Do sanctuary waters pose risks to human health and how are they changing?

- Have recent changes in climate altered water conditions and how are they changing?

- Are other stressors, individually or in combination, affecting water quality, and how are they changing?

- What are the levels of human activities that may adversely influence water quality and how are they changing?

- What are contaminant concentrations in sanctuary habitats and how are they changing?

Anna's efforts in this regard vastly exceeded the contributions of a typical Nancy Foster scholar and fully attest to her consummate professionalism. She did an extraordinary job preparing her write-ups, demonstrating thorough research and comprehensive understanding of each topic. Her initial literature review provided to our Expert Panel included 28 pages of literature review and figures. At our Expert Workshop in February 2019, Anna presented her sections, and meaningfully discussed her assessment of the literature with experts who were advocating 
contrary viewpoints, and overall defended her work with strong and well-supported arguments. She further worked with expert reviewers and NOAA staff to narrow the literature review down to the sections in print within the final 2020 Condition Report. Anna's contributions to this effort were significant and illustrate her unlimited potential as a research scientist should she decide to pursue this career.

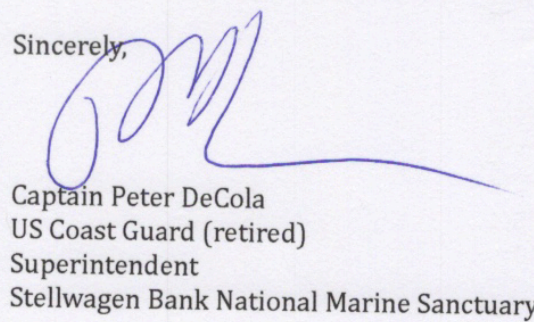

Enclosures: As stated. 


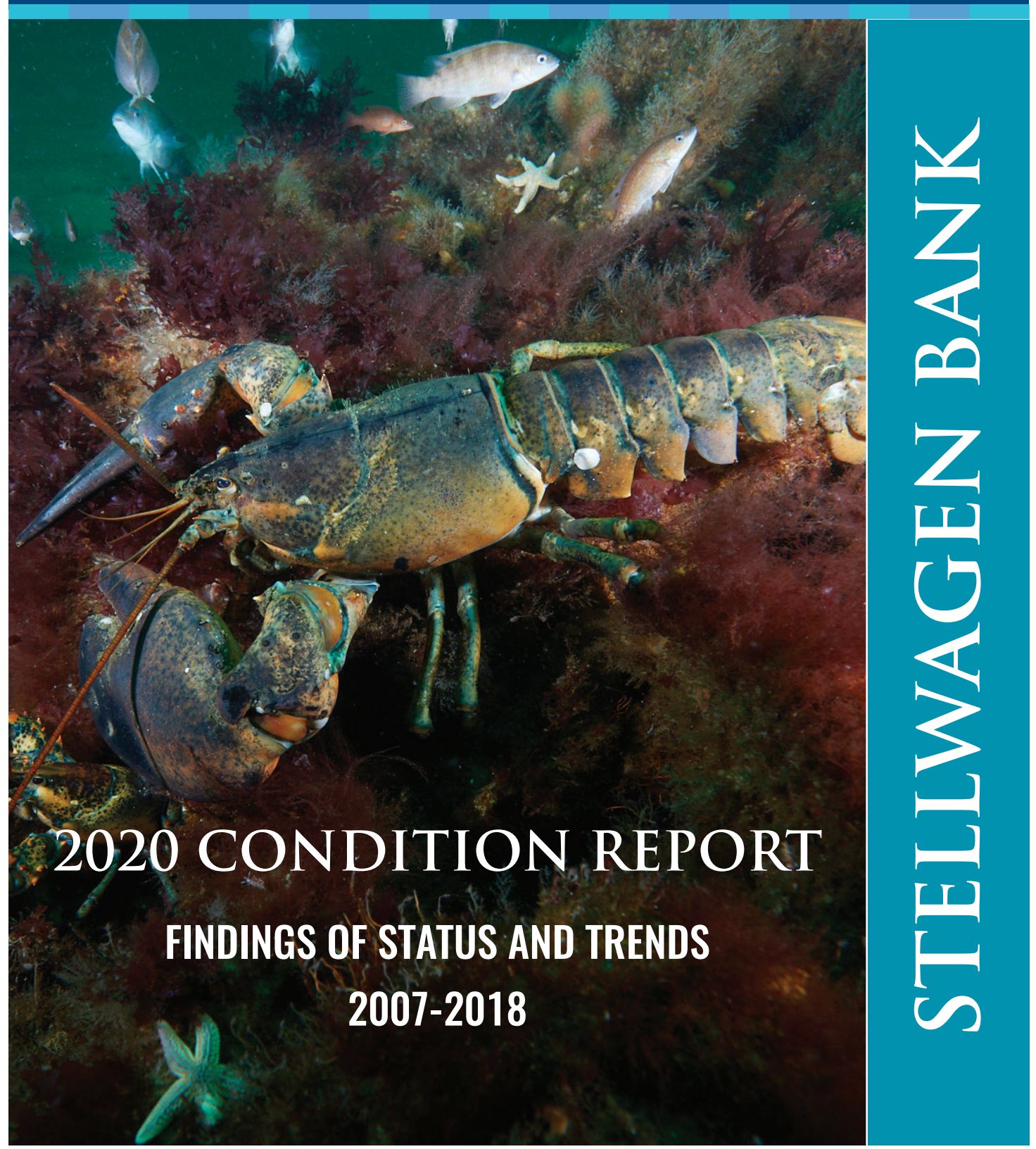


U.S. Secretary of Commerce

Wilbur Ross

Under Secretary of National Oceanic and Atmospheric Administration (NOAA)

Dr. Neil Jacobs (acting)

Assistant Administrator for National Ocean Service

Nicole LeBoeuf (acting)

Director, Office of National Marine Sanctuaries

John Armor
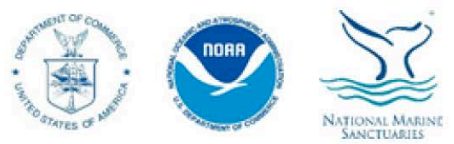

Cover photo: Two American lobsters (Homarus americanus) and a school of cunner (Tautogolabrus adspersus) use a historic shipwreck as home and habitat. The underwater photographer, Keith Ellenbogen, is the recipient of a Ernest F. Hollings Ocean Awareness Award to use the art of underwater photography to raise awareness about Stellwagen Bank National Marine Sanctuary. Photo: Keith Ellenbogen

Suggested Citation: Office of National Marine Sanctuaries. 2020. 2020 Condition Report: Findings of Status and Trends for 2007-2018. U.S. Department of Commerce, National Oceanic and Atmospheric Administration, Office of National Marine Sanctuaries, Silver Spring, MD. 263 pp.

Stellwagen Bank $\mid$ Condition Report 2020 


\section{AUTHORS}

Stellwagen Bank National Marine Sanctuary

Ben Haskell

Leila Hatch, Ph.D.

Matthew Lawrence

Anne-Marie Runfola

Tammy Silva, Ph.D. ${ }^{1}$

Anne Smrcina

Alice Stratton

Michael Thompson

David Wiley, Ph.D.

NOAA Office of National Marine Sanctuaries

Brad Barr, Ph.D.

Kathy Broughton

Stephen R. Gittings, Ph.D.

Vernon R. Leeworthy, Ph.D.

Danielle Schwarzmann, Ph.D.

Mystic Aquarium

Peter J. Auster, Ph.D.

Rochelle Kurth

University of Connecticut

Christian W. Conroy, Ph.D.

University of Rhode Island Graduate School of Oceanography

Anna Robuck ${ }^{3}$

${ }^{1}$ Tammy Silva was supported by the NOAA Dr. Nancy Foster Scholar ship to conduct dissertation research on toothed whale occurrence in SBNMS relevant to this report.

${ }^{2}$ Peter Auster was supported by a grant from the National Marine Sanctuary Foundation to conduct analyses and synthesize information related to habitat, living marine resources, and biodiversity presented in this report.

${ }^{3}$ Anna Robuck is supported by the NOAA Dr. Nancy Foster Scholarship in pursuit of Ph.D. research relevant to SBNMS water and habitat quality. 


\section{Water Quality}

Despite several potential stressors, sanctuary water quality is good/fair and does not appear to be adversely impacted by human activities. The largest effluent contributor in the region and the primary potential human source of nutrients is the Massachusetts Water Resources Authority (MWRA) outfall, which is located approximately 12 nautical miles from the western boundary of SBNMS. Ongoing monitoring suggests that the MWRA outfall is currently not adversely influencing monitored water quality parameters in SBNMS, and no evidence suggests that eutrophication is occurring. The Massachusetts Bay Disposal Site (MBDS) is directly adjacent to the sanctuary's western boundary and receives dredged material deemed suitable for open water disposal. This site incorporates the areas of two historic disposal sites containing toxic materials, though deposited toxic materials show limited mobility, and assessments have not shown any associated contamination of SBNMS. Maintenance dredging and expansion of Boston Harbor will generate 12 million cubic yards of dredged material for disposal in MBDS, but this is not expected to impact sanctuary water quality. Limited data exist to thoroughly evaluate potential impacts to water quality from vessel discharge and sediment perturbation by mobile fishing gear.

Monitoring suggests that concentrations of bacteria and toxigenic phytoplankton species rarely reach or exceed levels of concern in SBNMS and likely pose low risk to human health. However, the presence of toxigenic phytoplankton species indicates the potential for harmful algal blooms (HABs). HAB dynamics in the Gulf of Maine may be related to climate change, but current data show no explicit association between HAB occurrence and increasing temperatures in SBNMS.

Climate change impacts in SBNMS are measurable, and the threat of climate change to ecological integrity is increasing. The Gulf of Maine is warming faster than $99 \%$ of the global ocean; increases in both surface and bottom temperatures in SBNMS reflect these trends. Recent work suggests changes in seasonal temperature dynamics, longer summer seasons, and changes to primary production in and around SBNMS. Climate change is causing shifts in phenology and distributions of plankton, fish, whales, and other organisms in the Gulf of Maine. Impacts of climate change on important prey (foundation) species like sand lance and the copepod Calanus finmarchicus are particularly concerning, as these changes have the potential to drive cascading ecosystem effects and impact abundance, distribution, and health of top predators. In addition, climate change is causing impacts on commercial and recreational fisheries, local businesses, and communities. More robust monitoring of climate change effects and ocean acidification conditions in SBNMS is necessary to understand trends, seasonal fluctuations, and the possible ramifications for water quality, shell-forming invertebrates, and the larger ecosystem.

\section{Habitat}

The sanctuary's diverse underwater landscape is a patchwork of habitats composed of both geologic and biogenic components. Benthic substrate types are generally correlated with seafloor communities and constitute important geologic habitat components. Data suggest measurable degradation of habitat quality

10

Stellwagen Bank | Condition Report 2020 
over the past ten years, primarily due to direct impacts of bottom-contact gear used in commercial fishing, which occurs extensively throughout SBNMS. Mobile, bottom-tending fishing gear can alter or remove important structural characteristics and/or biological components of the seabed, which is a concern for maintaining habitat integrity. Lower levels of direct impact are evident in the Western Gulf of Maine Closure Area, and the closure led to recovery in some areas. Significant increases in scallop dredging may have impacted the northwest corner of Stellwagen Bank in 2017; this effort has been reduced, but is expected to continue. Overall fishing effort has declined by around $55 \%$ since 2009; however, it is unclear whether reductions in effort have resulted in improved sanctuary benthic habitat integrity. Fixed gear has less impact on the seabed, but poses an entanglement risk for protected species. Fishing effort reductions and gear modifications have been implemented to reduce bycatch of small marine mammals and seabirds and to attempt to reduce serious injury and mortality of large whales.

Localized disturbance of benthic habitats was observed after the installation of the sanctuary's only submerged cable, the Hibernia Atlantic cable, in the year 2000. Post-installation monitoring showed that impacts to benthic communities were not significantly different than those caused by commercial fishing. Other potential localized seabed impacts, including anchoring of recreational fishing vessels, should be evaluated.

Legacy contaminants and metals in benthic habitats have been reported; however, they do not appear to remobilize beyond sites where they have been identified, and no population effects have been documented. Limited data suggest emerging contaminants are present within Massachusetts Bay at low concentrations, with uncertain biological ramifications, and may be shifting or increasing with the continued introduction of new chemicals. No data exist regarding microplastics in SBNMS, though surface water concentrations of microplastics in the Gulf of Maine are far lower than in the North Atlantic. Abundance of marine debris in SBNMS depends on natural forces and human drivers, which may increase with increasing population growth and coastal development. Additional work to characterize and quantify emerging contaminants and microplastics in SBNMS habitats is needed.

SBNMS is an urban sanctuary, and impacts to its acoustic environment are a concern. Designated shipping lanes such as the Boston Traffic Separation Scheme (TSS) cut through the sanctuary and are used by most commercial vessels transiting to the port of Boston. Commercial fishing vessels use the sanctuary year-round. The whale watching industry has expanded, and the numbers of recreational boaters and whale watchers are rising. Human-generated underwater noise from vessels, particularly from large commercial ships, can degrade habitat quality and interrupt behavior and communication of many marine species. Increasing noise levels and impacts of noise to some marine mammal and fish species have been documented in the sanctuary and are expected to continue. SBNMS has been at the forefront of raising awareness of the potential threat of noise to organisms and has pioneered the use of several advanced passive acoustic monitoring methods and technologies to further the study of ocean noise and its impacts. 


\section{STATE OF SANCTUARY RESOURCES}

\section{Water Quality (Questions 6-9)}

The following information provides an assessment of the status and trends of key water quality indicators in SBNMS for the period 2007-2018. Eutrophic conditions and their influence on primary production in sanctuary waters is the focus of Question 6. Eutrophication is the accelerated production of organic matter, particularly algae, usually caused by an increase in the amount of nutrients (primarily nitrogen and phosphorus) in surface waters. Eutrophication can impact the condition of sanctuary resources, for example, by promoting nuisance and toxic algal blooms or impacting dissolved oxygen levels.

Question 7 focuses on parameters affecting public health. Human health concerns can arise from water, beach, and/or seafood contamination (bacteria or chemical). Indications of health impacts may include fishery closures and seafood consumption advisories.

Question 8 focuses on shifts in water quality due to climate drivers. Climate indicators include indices of large-scale climate patterns, water temperature, acidity, upwelling intensity and timing, and dissolved oxygen. Shifts in water temperature can affect species growth rates, phenology, distribution, and susceptibility to disease. Acidification can affect organism survival, growth, and reproduction. Upwelling influences oxygen content and nutrient cycling.

Question 9 assesses other biotic and abiotic stressors, individually or in combination, that may influence sanctuary water quality, but were not addressed in other questions. Examples include non-point source contaminants, and hard-to-quantify stressors that influence the condition of habitats and living resources. Such inputs may include industry discharges and emissions, fertilizers, pesticides, heavy metals, and sewage. 
6. What is the eutrophic condition of sanctuary waters and how is it changing? Status Description: Eutrophication has not been
documented, or does not appear to have the potential to negatively affect ecological integrity.

Rationale: MWRA hydrographic modelling (Zhao et al. 2017a) suggests that eutrophication is not occurring. Dissolved oxygen has not approached hypoxic or anoxic conditions over time. Background nitrogen may be decreasing regionally, which would decrease the probability of eutrophication.

The 2007 rating for this question was "good" (very high confidence) and "not changing" (very high confidence). Since 2007 , regional monitoring suggests no sustained increases of key nutrient concentrations or nutrient ratios. Observed nutrient dynamics within the sanctuary and greater Massachusetts Bay are primarily driven by regional circulation and hydrodynamies of the Gulf of Maine (Townsend et al. 2015, 2010, McManus et al. 2014, Libby et al. 2017). Thus, the rating remains unchanged.

SBNMS receives nutrient and pollutant inputs from coastal point and non-point sources and via atmospheric deposition from proximate and distant inland regions. Anthropogenic nutrient sources of primary importance include wastewater treatment plants discharging into rivers or directly into Massachusetts Bay. Notably, the MWRA Deer Island Wastewater Treatment Plant sewage outfall discharges approximately 350 million gallons of effluent daily into the offshore Massachusetts Bay environment, roughly 12 nautical miles west of the SBNMS boundary (Werme et al. 2017).

MWRA conducts ongoing water quality monitoring across Boston Harbor and Massachusetts Bay to identify impacts of the Deer Island Wastewater Treatment Plant outfall within the coastal and offshore environment. The monitoring program assesses an extensive suite of physical and chemical parameters, such as temperature, salinity, dissolved oxygen, and nutrient concentrations, in addition to biological parameters, such as chlorophyll concentrations and phytoplankton community composition. Monitoring has been ongoing since 1992, with increased monitoring in SBNMS directly following outfall relocation in 2000 until 2010, when it was determined that a smaller subset of offshore stations adequately represented offshore conditions. MWRA currently maintains one monitoring station in the southwest corner of SBNMS (F29), one station slightly outside the northwest corner of SBNMS (F22), and two stations within Cape Cod Bay ( F01 and F02). These stations are monitored nine months of the year. The Northeastern Regional Association of Coastal Ocean Observing Systems also maintains a monitoring buoy along the northwest boundary of the sanctuary, collecting data on water temperature, salinity, turbidity, chlorophyll concentration, density, dissolved oxygen, wind, waves, and currents on an hourly basis. These current and historical data sources provide an excellent platform from which to identify and infer long-term water quality trends within and around SBNMS.

The 2007 condition report and 2010 management plan both suggested that eutrophication was not a pressing problem in SBNMS waters. Nitrogen and phosphorus (in elevated concentrations) are the primary nutrients that may contribute to eutrophication. Ammonium and nitrate are two forms of nitrogen that may particularly contribute. Nutrient dynamics in the region are dominated by large-scale circulation of the Gulf of Maine and influx of Gulf of Maine water into Massachusetts Bay (Figure S.WQ.6.1,

89

Stellwagen Bank | Condition Report 2020 
HydroQual 2000). Nutrient availability in surface and bottom waters is influenced by stratification and circulation patterns that are regulated by long-term climate dynamics and seasonal forcing. Surface water nutrient concentrations have remained roughly constant or declined over the course of MWRA monitoring (Figure S.WQ.6.3). Bottom water nutrient trends roughly approximate surface water results, yet bottom water reservoirs display predictably higher nutrient concentrations compared to surface waters (Figure S.WQ.6.4). Specifically, total nitrogen and total phosphorus have decreased in concentration since the early $2000 \mathrm{~s}$, possibly related to circulation shifts driven by the North Atlantic Oscillation (Figure S.WQ.6.4). SBNMS waters contain sustained concentrations of ammonium and nitrate, consistent with seasonal nutrient cycles observed across the region (McManus et al. 2014, Costa et al. 2017, Libby et al. 2017). Concentrations of orthophosphate and silicate, two other types of nutrients important for primary productivity, have decreased in the offshore environment since roughly 2010-2011. These nutrients typically come from terrestrial environments, so changes may reflect larger-scale shifts in circulation or precipitation altering riverine inputs or connectivity to offshore habitats (Figure S. WQ.6.3, Townsend et a1. 2010, 2015, Berton et al. 2017). Overall, nutrient data from 1994-2016 suggest no evidence of increased eutrophication in sanctuary waters (Townsend et al. 2010, 2015, Costa et al. 2017, Libby et al. 2017). Modeling results support a lack of eutrophication potential in surface and bottom waters of Massachusetts Bay and SBNMS due to effluent inputs (Zhao et al. 2016, 2017a). Bottom water dissolved oxygen concentrations also do not indicate hypoxic or anoxic conditions indicative of eutrophication (Libby et al. 2017).

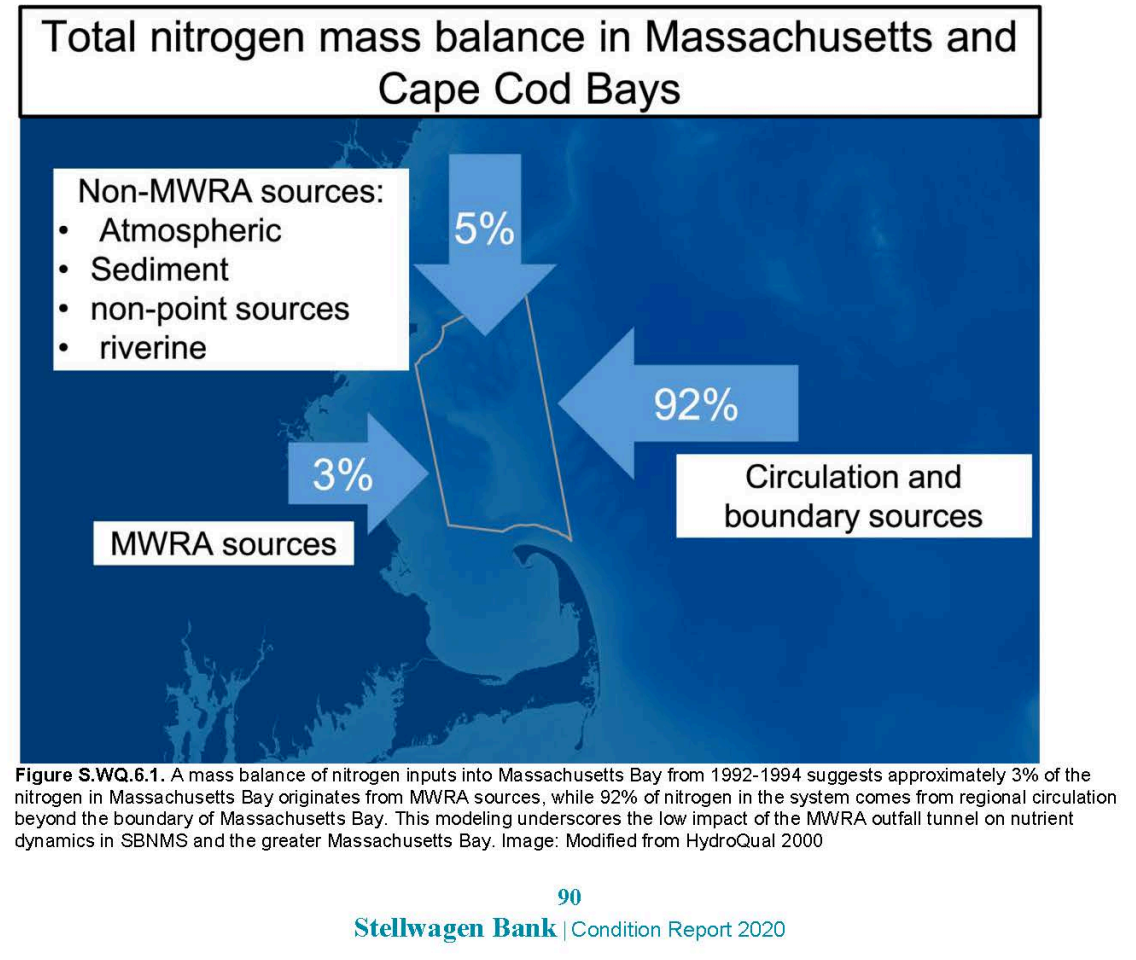




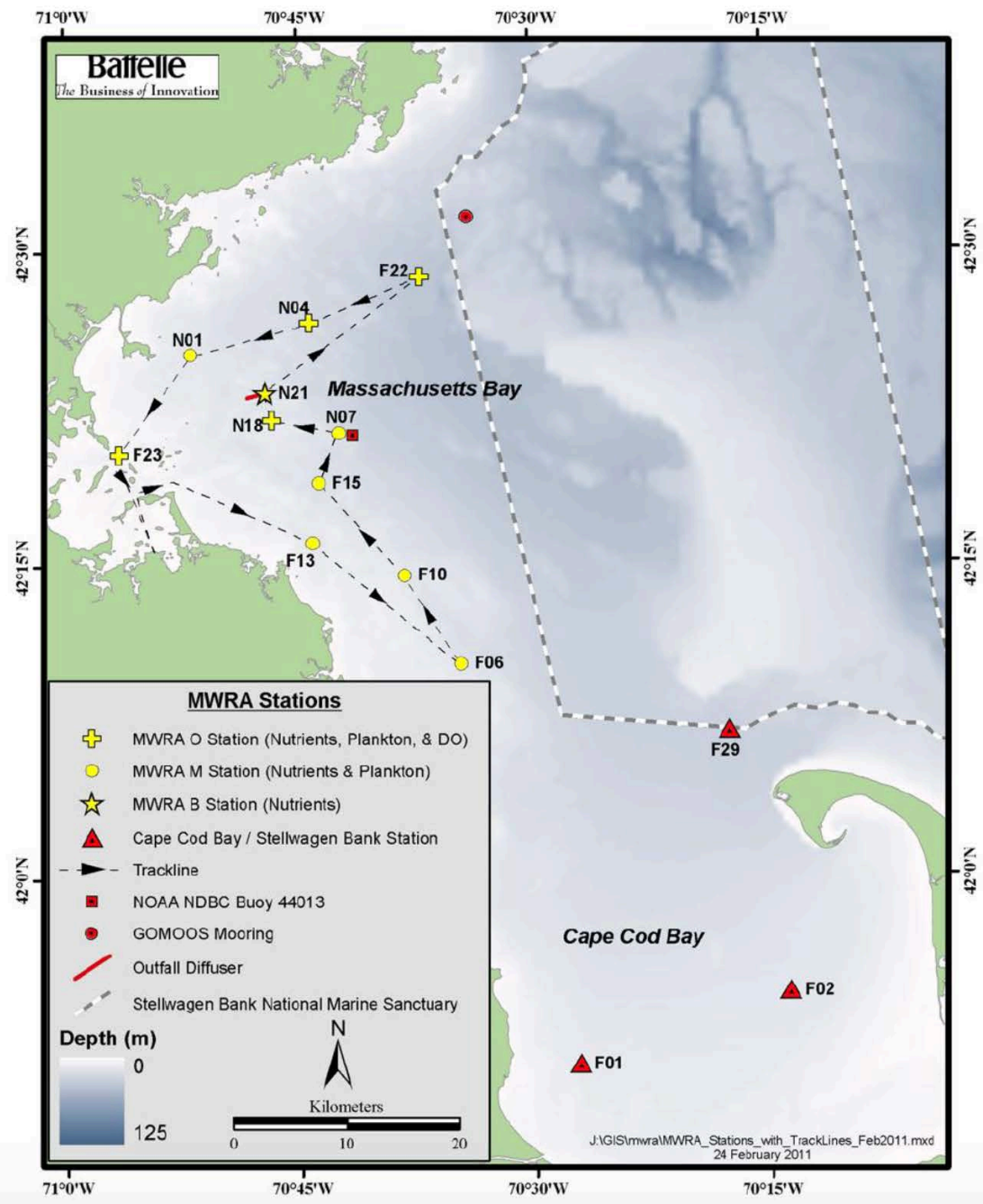

S.WQ.6.2. Location of MWRA monitoring sites across Massachusetts Bay Station F29 is at the southwest corner of the sanctuary, while F22 is slightly outside sanctuary boundaries to the west. These two stations were used as representations of sanctuary water quality as captured within this monitoring program. Image: Libby et al. 2017

91

Stellwagen Bank | Condition Report 2020 
State of Sanctuary Resources
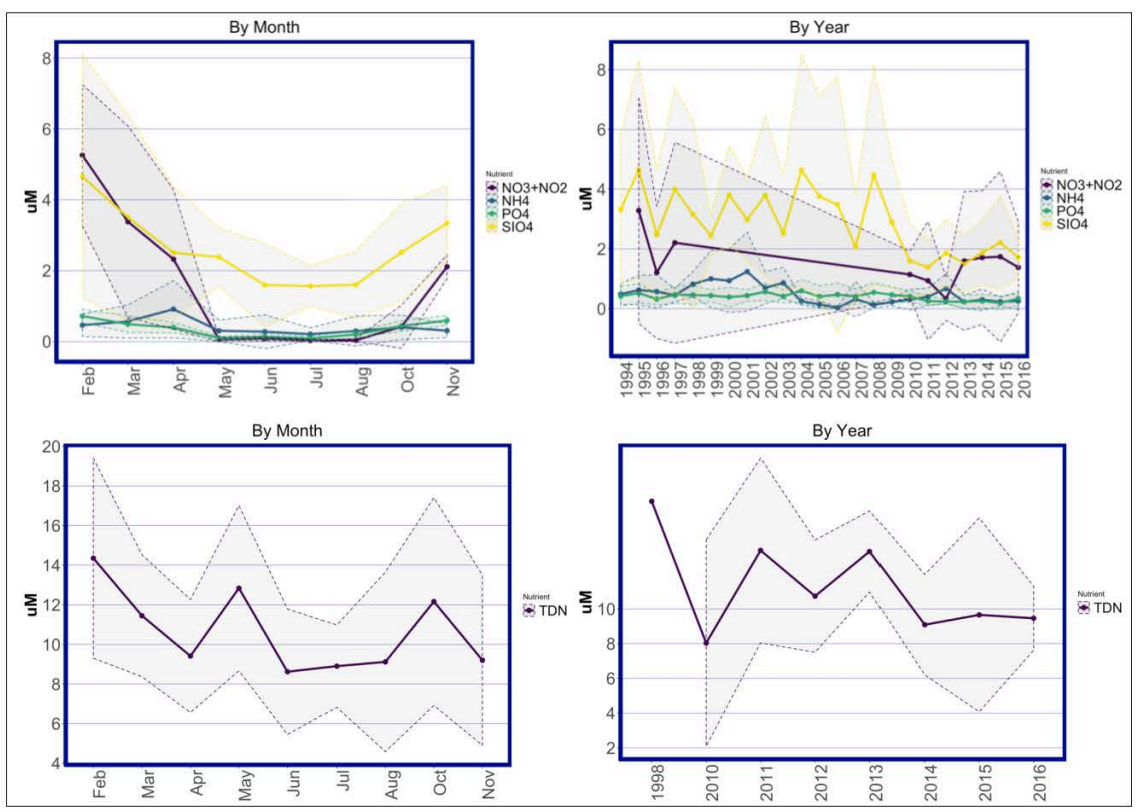

Figure S.WQ.6.3. Intra- and interannual nutrient data from F29, an MWRA monitoring station within SBNMS. Figures present monthly and yearly average concentrations of nitrate + nitrite, ammonium, orthophosphate, and silicate (top row) along with total dissolved nitrogen (TDN, bottom row). These data suggest an apparent lack of eutrophication as indicated by concentrations of key nutrients. Quasi-predictable seasonal fluctuations coupled with regional biogeochemical and hydrological dynamics drive observed nutrient concentrations and subsequent primary productivity in SBNMS and Massachusetts Bay. Gaps in nitrogen data reflect gaps in measurement of these parameters at station F29. Image: MNRA yearly average nutrient data, stations F02, F06, F29, F22, and N20. MWRA data courtesy of Kenneth Keay and Douglas Hersh

92

Stellwagen Bank|Condition Report 2020 

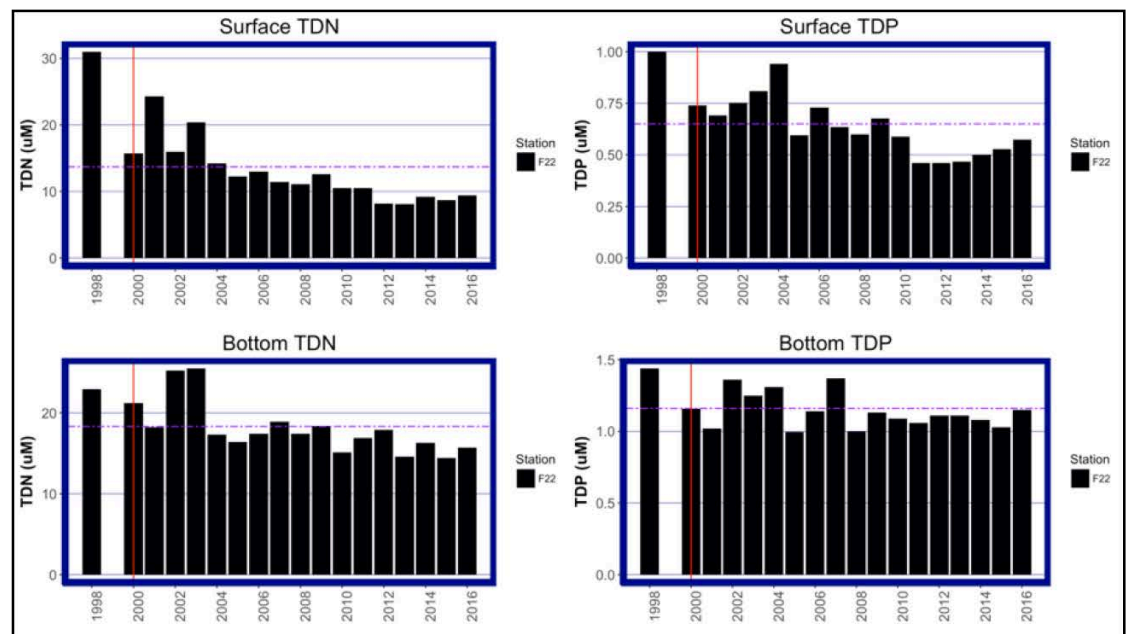

Figure S.WQ.6.4. Data from F22, an MWRA monitoring station directly adjacent to SBNMS boundaries, also suggest that eutrophication is not a pressing problem in SBNMS surface or bottom waters, or the surrounding far-field environment. Dashed purple lines indicate mean parameter concentration across time series, while red vertical lines demarcate MWRA outfall introduction identical data at F29. Image: MWRA yearly average nutrient data, stations F02, F06, F29, F22, and N20. MWRA data courtesy of Kenneth Keay and Douglas Hersh

Further evidence against eutrophication includes constant or declining regional primary production (Figure S.WQ.6.5, NEFSC 2017b, Libby et al. 2017, Oviatt et al. 2007). Notably, phytoplankton and zooplankton abundance and community makeup show significant seasonal and annual variation since MWRA monitoring began in 1992; however, these changes are likely driven by regional, decadal scale processes in the Gulf of Maine rather than eutrophication-related drivers (Oviatt et al. 2007, McManus et a1. 2014, Costa et al. 2017, Libby et al. 2017).

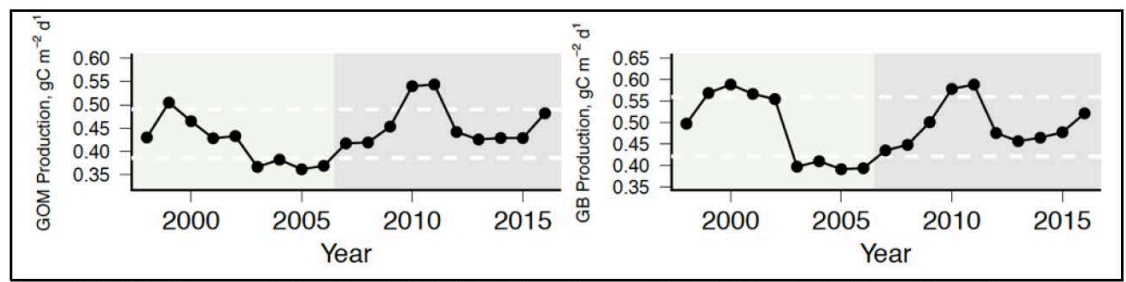

Figure S.WQ.6.5. Primary production in the Gulf of Maine, left, and Georges Bank, right, has fluctuated over time, with current Figure S.WQ.6.5. Primary production in the Gulf of Maine, left, and Georges Bank, right, has fluctuated over time, with current
conditions suggesting stable and average overall system production. Dashed lines indicate $+/-1$ standard deviation. Image: NEFSC $2017 \mathrm{~b}$

93

Stellwagen Bank | Condition Report 2020 


\section{Do sanctuary waters pose risks to human health and how are they changing?}

Status Description: One or more water quality indicators 001 Good/Fair -000 suggest the potential for human health impacts, but human health impacts have not been reported.

Rationale: Toxigenic algae are present but not observed to cause demonstrable threats to human health. Observed water quality changes over the last 12 years may be related to changes in the North Atlantic Oscillation.

In the 2007 condition report, this question was rated "good" and "not changing." The status has now been downgraded to "good/fair." Ongoing bacterial monitoring suggests bacteria are rarely detectable. The downgraded rating stems from the documented occurrence of several toxigenic phytoplankton species with varying degrees of predictability; this suggests the potential for human health impacts, though these impacts have not been reported. Harmful algal bloom dynamies in SBNMS and the wider Gulf of Maine are thought to be mediated by large-scale circulation and climatic dynamics.

At present, SBNMS waters likely pose low risk to human health. Bacterial monitoring has been ongoing across the region by MWRA related to the Deer Island Wastewater Treatment Plant outfall, and indicates that water column bacterial counts consistently remain below detection limits, with a very low likelihood of exceeding thresholds protective of public health and resource use (Codiga et al. 2016).

The most likely source of possible human health effects in the region stems from the presence of toxigenic phytoplankton species (Figure S.WQ.7.1). Identified species of note include Pseudo-nitzschia spp., Alexandrium spp. (Pittman 2019), and Dinophysis spp., which have all been documented in SBNMS, Massachusetts Bay, and the greater Gulf of Maine. Pseudo-nitzschia spp. are classified as diatoms, while the latter two species are dinoflagellates; all produce toxins that may bioaccumulate, particularly in shellfish, and can cause widespread economic loss or health effects in humans (Anderson et al. 2000, Etheridge 2010, Lefebvre and Robertson 2010).

There is no definitive understanding regarding the risk of harmful algal blooms (HABs) to humans or wildlife that utilize the sanctuary, although risk from these or other toxigenic algae may be changing due to evolving $\mathrm{HAB}$ dynamics in the greater Gulf of Maine. Alexandrium spp. and Dinophysis spp. have been irregularly identified at low numbers within SBNMS and surrounding waters as measured via MWRA whole water phytoplankton assessments and regional research (Figure S.WQ.7.1, Anderson et al. 2014a, Gobler et al. 2017, Libby et a1. 2017). Alexandrium spp. blooms are frequently a health concern in waters further north of the sanctuary (Anderson et al. 2014a, 2014b). Literature review and regional monitoring suggest Alexandrium spp. concentrations in excess of 1000 cells $/ \mathrm{L}$ require increased monitoring for shellfish toxicity; MWRA maintains a caution threshold of 100 cells $/ \mathrm{L}$ for the same taxa. Concentrations of Alexandrium spp. have infrequently surpassed either threshold, with notable occurrence of Alexandrium in the sanctuary only in 2005 according to MWRA cell count data.

Over 14 Pseudo-nitzschia species have been identified in the Gulf of Maine, seven of which are known to produce domoic acid, a neurotoxin. Pseudo-nitzschia spp. has been regularly documented in SBNMS according to MWRA survey data, with notable occurrences in sanctuary waters in the autumns of 2012, 2013, and 2016 (Figure S.WQ.7.1). Literature review, regional monitoring, and safety thresholds established by states in the region suggest $P$ seudo-nitzschia cell counts in excess of 20,000 cells $/ \mathrm{L}$ merit

94 
increased monitoring for potential adverse effects in shellfish. Data exist regarding the relationship between cell count and domoic acid production or bioaccumulation specific to the offshore environment (Fernandes et al. 2014), which makes understanding risks associated with cell count thresholds challenging. It is also unclear if or to what extent the presence of this toxigenic diatom may translate to domoic acid exposure in the sanctuary food web. Blooms of toxigenic Pseudo-nitzchia spp. have been implicated in marine mammal and bird deaths around the globe, yet no SBNMS-specific mortality events have been documented (Lefebvre and Robertson 2010, D'Agostino et al. 2017).

Climate change likely plays a pivotal role in local and regional $\mathrm{HAB}$ dynamics. Recent research examining the relationship between sea surface temperature and $\mathrm{HAB}$ dynamics across the greater Northwest Atlantic from 1982-2016 found that bloom season and growth rate of Alexandrium spp. and Dinophysis spp. were positively correlated with increasing sea surface temperature, suggesting that climate change may have already exacerbated these or other $\mathrm{HAB}$ species dynamics in the region ( $\mathrm{O}$ 'Neil et al. 2012, Gobler et al. 2017). Modeling work considering the greater Gulf of Maine also suggests that climatically-mediated variables, such as river discharge, wind patterns, and cross-shore transport, are specifically associated with paralytic shellfish poisoning toxicity events caused by Alexandrium fundyense in a given year; it is uncertain if or how these variables may be shifting within SBNMS and how this may translate to increased risk for toxic $\mathrm{HAB}$ events (Nair et al. 2013). Phytoplankton cell counts obtained via MWRA monthly monitoring data from F22 and F29 do not indicate an explicit association between $\mathrm{HAB}$ species occurrence and warming SBNMS surface water temperatures or repositioning of the outfall. Although this may be a function of sampling frequency or a similar artifact, substantial regional evidence of decadal variation modulating $\mathrm{HAB}$ dynamics suggests the same factors are moderating HAB occurrence locally in SBNMS (Anderson et al. 2014a, 2014b, Libby et al. 2017,

Nair et al. 2013). Note that MWRA cell count and monitoring data, taken monthly from February until December, can only be used to moderately comment on the occurrence or risk of quickly evolving HAB blooms, as blooms may develop and dissipate over the course of a few days or weeks. Further study of phytoplankton communities and toxigenic algae dynamics in the face of changing regional dynamics is warranted. 


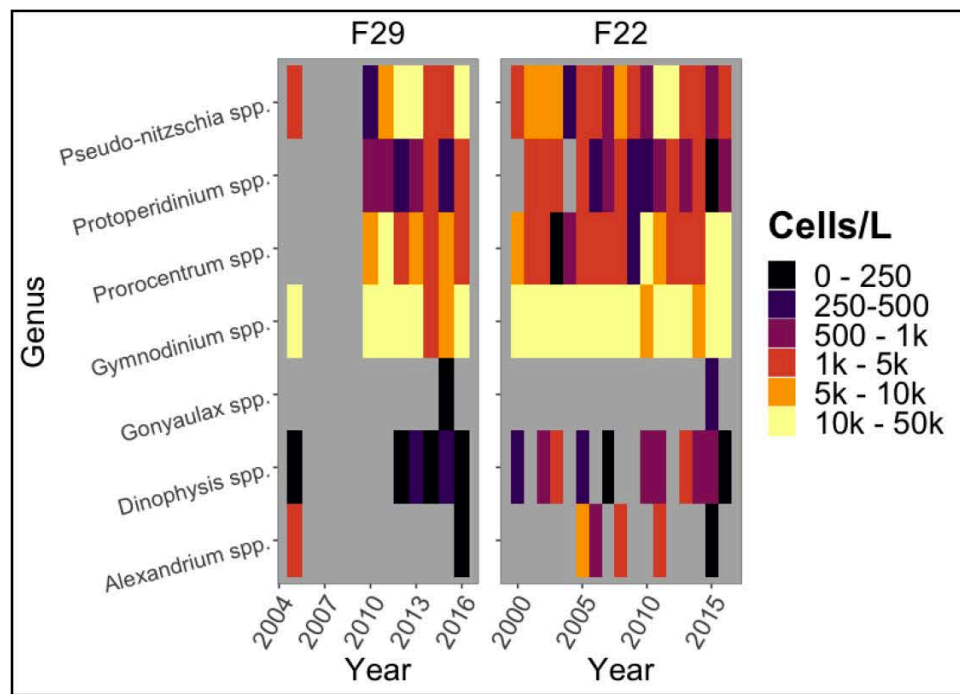

Figure S.WQ.7.1. Yearly average cell counts of toxigenic algae genera at two MWRA ambient monitoring stations. Grey area indicates the species was not observed. While there is no clear trend associated with cell counts of toxigenic algae species over time, several types of toxigenic algae occur regularly or semi-regularly in cell count surveys conducted at MWRA stations F29 and F22, in or directly adjacent to SBNMS waters. Image: MWRA monthly surface phytoplankt on count data, stations F29 and F22
MWRA data courtesy of Kenneth Keay and Douglas Hersh

8. Have recent changes in climate altered water conditions and how are they changing?

Status Description: Climate-related changes have caused measurable, but not severe, degradation in some attributes of ecological integrity.

Rationale: Climate change is influencing the primary production cycle in the region, and has the demonstrated capacity to produce cascading effects within the ecosystem. Additional changes in water temperature, dissolved oxygen, stratification, sea level, precipitation, and storm activity have been documented or modeled, with some suggestion of changes in $\mathrm{pH}$, though more monitoring is needed to more robustly identify acidification trends and effects.

This question is new and was not assessed in the 2007 condition report. SBNMS, as part of the Gulf of Maine, is one of the fastest warming regions around the globe. The current rating is based on changes in the phenology and distribution of fish, plankton, and other organisms that are being observed and attributed to climate change (Staudinger et al. 2019, Pershing et al. 2015, Nye et al. 2009), all of which are affecting production and food web dynamics within the ecosystem.

Climate change impacts have manifested regionally in remarkable and dramatic ways. Overall, the Gulf of Maine is warming $99 \%$ faster than the global ocean. This $1.5^{\circ} \mathrm{C} /$ decade temperature increase is likely due to the influence of decadal-scale cycles and the northward shift of the Gulf Stream (Pershing et al.

96

Stellwagen Bank |Condition Report 2020 
2015, Thomas et al. 2017). The Gulf of Maine is experiencing areas of increasingly warmer surface water and decreases in areas of cold water since 1980 (Figure S.WQ.8.1, NEFSC 2017a). Using monitoring data from the MWRA monitoring site in SBNMS, linear regression of yearly average water temperature data suggests increases in both surface water and bottom water commensurate with regional trends (Figure S.WQ.8.2). Seasonal water temperature changes have also been documented in the Gulf of Maine, with recent research suggesting an earlier summer start date, a later summer end date, and longer summer length based on data from a 33-year period (Thomas et al. 2017). Recent national predictions also suggest average annual air temperatures in the northeast may increase between 3.98 and $5.09^{\circ} \mathrm{F}$ as early as 2036 (Wuebbles et al. 2017).

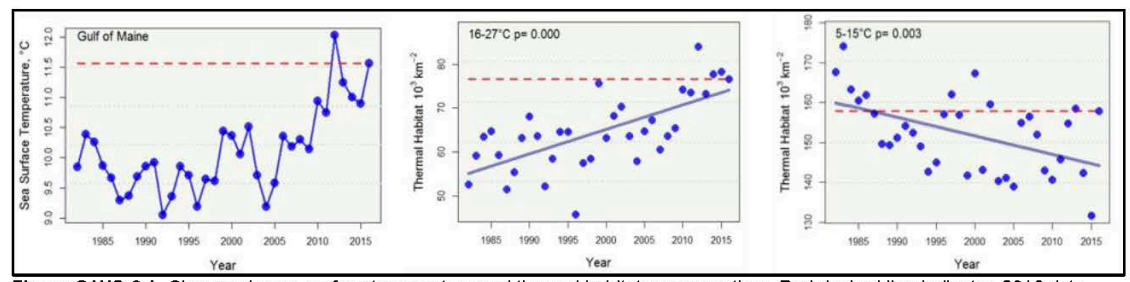

Figure S.WQ.8.1. Changes in sea surface temperature and thermal habitat area over time. Red dashed line indicates 2016 data Image: NEFSC 2017b

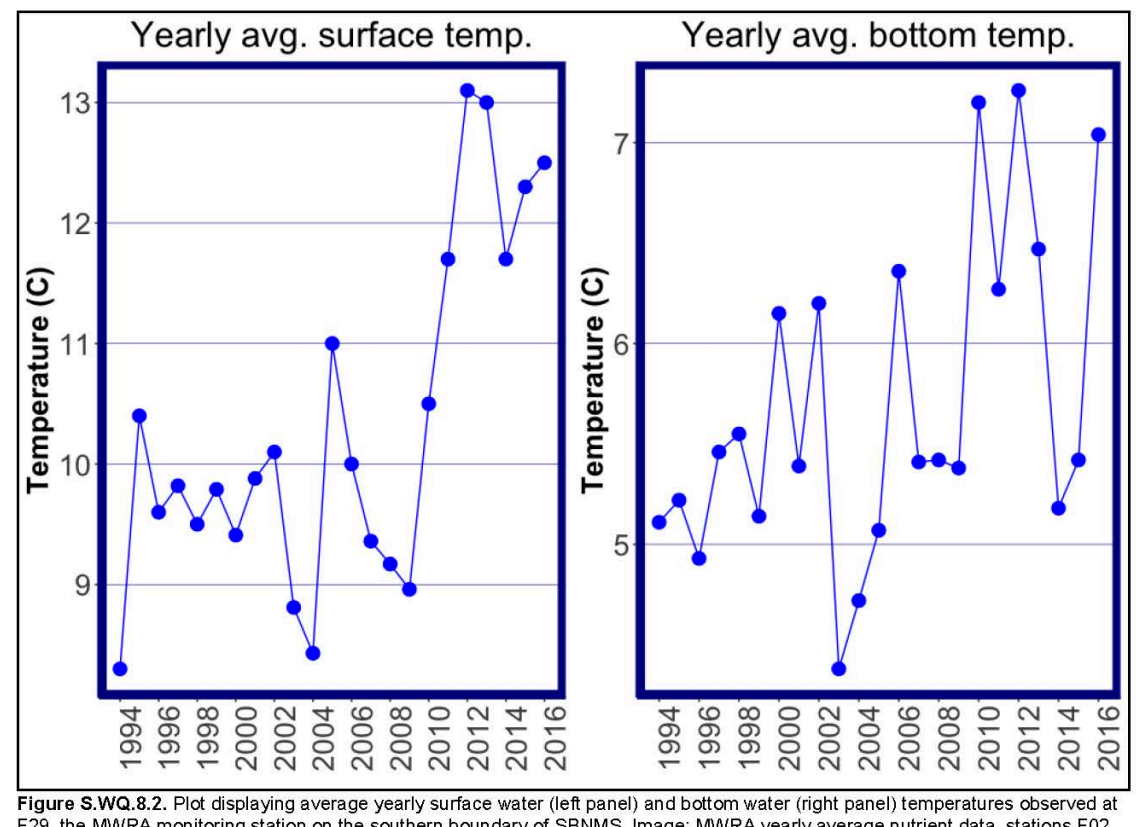

F29, the MWRA monitoring station on the southern boundary of SBNMS. Image: MWRA yearly average nutrient data, stations F02 F29, the MWRA monitoring station on the southern boundary of SBNMS. Image: MWRA
F06, F29, F22, and N20. MWRA data courtesy of Kenneth Keay and Douglas Hersh

97

Stellwagen Bank | Condition Report 2020 
Dissolved oxygen concentrations have decreased over time in SBNMS and Massachusetts Bay according to MWRA monitoring data. This may be due to sampling artifacts or related to the reduced capacity of warmer water for oxygen. Note that decreased oxygen concentrations are likely not yet ecologically relevant, as bottom water consistently maintains saturation levels above $80 \%$ based on MWRA monitoring data (Libby et al. 2017).

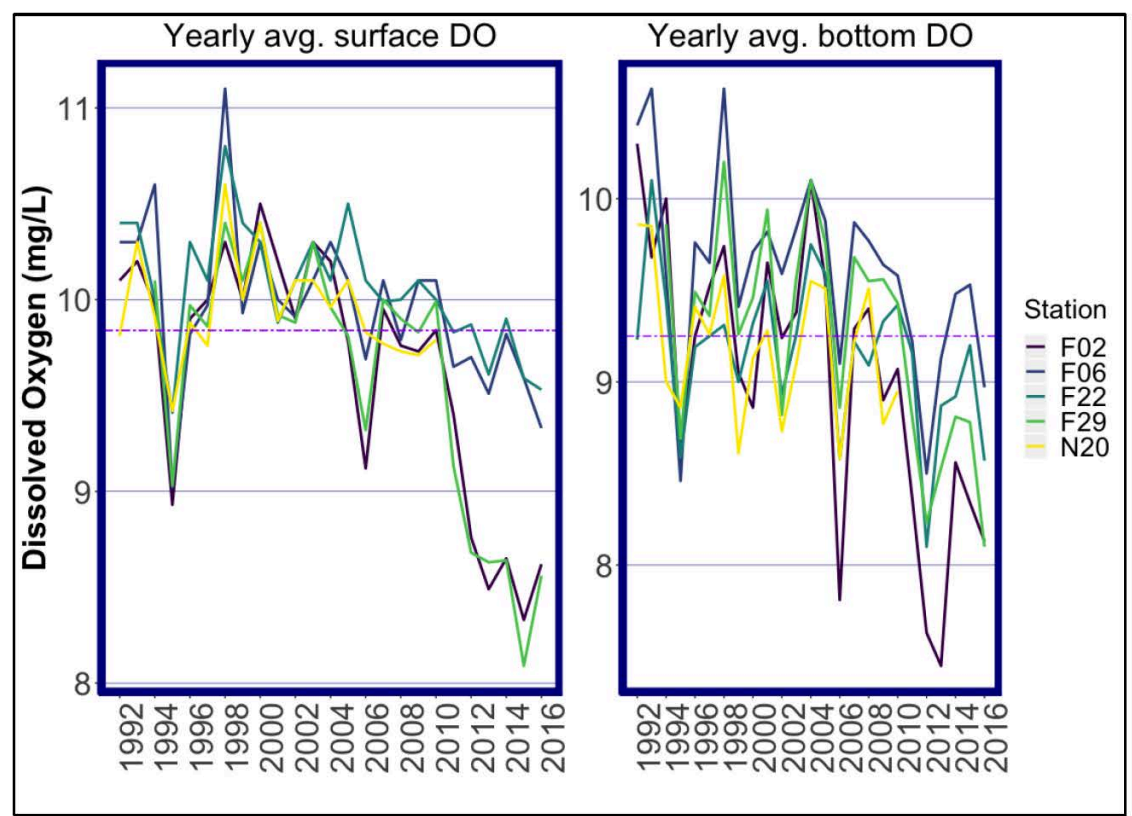

Figure S.WQ.8.3. Plot displaying average annual surface (left panel) and bottom water dissolved oxygen (DO) concentrations (right panel) observed at five MWRA monitoring stations in Massachusetts Bay, with the purple line depicting the mean dissolved oxygen level observed over the time series. Image: MWRA yearly average nutrient data, stations F02, F06, F29, F22, and N20. MWRA data courtesy of Kenneth Keay and Douglas Hersh

Climate change is also likely affecting primary production within SBNMS and the surrounding offshore environment, with uncertain cascading effects within the regional food web (McManus et al. 2014, Costa et al. 2017, Libby et al. 2017). Discharge from the Merrimack River has been demonstrated to strongly correlate with conditions associated with key climate indices, the Atlantic Multidecadal Oscillation, and the North Atlantic Oscillation, with greater than average river discharge anticipated in future (Berton et al. 2017). Climatically-mediated river discharge from the Merrimack and other coastal rivers, Western Maine Coastal Current intrusion, wind stress, and inflow contributions to the greater region from lowsalinity Scotian Shelf waters act to freshen the surface layer and increase stratification, producing variable and dynamic levels of primary production and nutrients (McManus et al. 2014, Townsend et al. 2015).

The Northeast is expected to experience higher rates of sea level rise by 2100 compared to the global average (NEFSC 2017b, Wuebbles et al. 2017). Massachusetts coastlines are projected to experience an average sea level rise of $2.83 \mathrm{~mm} / \mathrm{year}$. Cape Cod and outlying islands are particularly at risk in terms of

98 
sea level rise (NOAA 2019b). The offshore location of SBNMS insulates it from direct effects of sea level rise. It is unclear how inundation of coastal areas and changing sediment sources/transport may impact offshore environments in Massachusetts Bay.

The Gulf of Maine region is particularly susceptible to ocean acidification, due to poor buffering capacity and existing low calcium carbonate concentrations as a result of substantial freshwater and low-salinity inputs from coastal rivers and Scotian Shelf flow (Gledhill et al. 2015, Townsend et al. 2015, Wang et al. 2017). However, recent increases in the temperature and salinity of the Gulf of Maine as a result of the northward shift in the Gulf Stream appear to be buffering against the impacts of ocean acidification on $\mathrm{pH}$ and aragonite saturation state (Salisbury and Jönsson 2018). Regional work has demonstrated that inorganic carbon concentrations are determined by seasonal cycles driven by primary productionrespiration dynamics, with apparent $\mathrm{CaCO}_{3}$ dissolution in bottom water during fall and winter (Wang et al. 2017). A pilot study in SBNMS from December 2011-June 2012 indicated decoupling of surface and bottom water $\mathrm{pCO}_{2}$ in response to the spring bloom, with corresponding decreases in bottom water $\mathrm{pH}$ and calcium carbonate saturation state, a derived index of carbonate ion availability (SBNMS 2013). Sustained ocean acidification may result in sustained subsurface aragonite undersaturation across the region in 30-40 years, with variable and uncertain consequences for water quality or shell-forming invertebrates in SBNMS and the greater Gulf of Maine (Gledhill et al. 2015, Ekstrom et al. 2015, Wang et a1. 2017). More robust monitoring incorporating both surface and bottom water measurements is necessary across SBNMS and the wider region to understand acidification trends, seasonal fluctuations, and possible ramifications for shellfish and the larger ecosystem.

A warming atmosphere and ocean also translates to increased water vapor in the atmosphere, impacting precipitation and tropical cyclone activity. In the northeast, the annual maximum daily precipitation was 17\% higher between 1981 and 2015 than it was in the years between 1901 and 1960, while extreme precipitation from 1996-2014 was 53\% higher than 1901-1995 (Figure S. WQ.8.5, Huang et al. 2017, Wuebbles et al. 2017). Though numbers of tropical cyclones are predicted to remain the same or even decrease globally, tropical cyclone intensity has increased over the past 40 years as illustrated by the doubling of the number of category 4 and 5 tropical cyclones since the 1970s. Moreover, more storms are predicted to track poleward under current emission and temperature change scenarios, likely translating to increased tropical activity reaching SBNMS and the greater North Atlantic (Tamarin-Brodsky and Kaspi 2017, Wuebbles et al. 2017). It is uncertain how increased hurricane and tropical storm exposure may impact local resources and processes. 


9: Are other stressors, individually or in combination, affecting water quality, and how
are they changing?
Status Description: Undetermined
Rationale: Ongoing contaminant monitoring has focused on a handful of legacy contaminants,
leaving the majority of emerging organic contaminants unmeasured. No data exists to determine
changes over time, which is the primary factor driving the rating and trend. More monitoring is
needed in this area.

In the 2007 condition report, this question was rated "good/fair" and "not changing." Since that report, limited data has emerged to document other water quality stressors. Sediment monitoring has indicated concentrations of man-made organic contaminants and metals have decreased or remained steady in the SBNMS region. Both modeling and observational work indicate that dredge material and any associated contaminants remain confined within disposal sites, and are not likely to impact water quality. However, ongoing contaminant monitoring has focused on only a handful of legacy contaminants, leaving the majority of emerging organic contaminants unmeasured, with no indication of how they may be changing in the system, which is the primary uncertainty driving the "undetermined" rating for status and trend for this question.

As an offshore region adjacent to urbanized margins, SBNMS is subjected to a variety of stressors with variable influence on observed water quality. Anthropogenic contaminants, wastewater discharges, and vessel discharges are stressors of particular relevance that may impact water quality within the sanctuary.

Current organic pollutant monitoring by MWRA within the region entails sediment sampling and measuring legacy hydrophobic chemicals that readily adsorb to particles and prefer to remain particlebound (versus dissolved). Few data exist on concentrations of dissolved or suspended, particulateassociated hydrophobic pollutants within SBNMS or greater Massachusetts Bay (Dahlen et al. 2006, Nestler et al. 2017). Based on existing sediment monitoring and well-established partitioning behavior of these hydrophobic chemicals, dissolved concentrations of legacy organic contaminants are likely very low and not a significant stressor of water quality within SBNMS (Nestler et al. 2015).

However, there are no data describing dissolved concentrations of emerging contaminants with waterloving or hydrophilic chemistries, such as water repellents (PFASs) or personal care products (Wang et al. 2011, 2017), whose partitioning behavior is poorly understood. Microplastics and nanomaterials are also poorly described in the region. These emerging pollutants have been identified in water samples and biotic tissues regionally and are likely present in SBNMS due to its proximity to urban source areas (Shaw et al. 2009b, Costa 2012, Cantwell et al. 2016a, 2016b). Dissolved concentrations of emerging contaminants are likely below levels associated with acute impacts due to vigorous flushing mechanisms within Massachusetts Bay or possible degradation, but this has yet to be demonstrated empirically (Jones et al. 2005, Corcoran et al. 2010, Zhao et al. 2016). Testing for emerging contaminants by the EPA and the University of Rhode Island will occur in 2019. There are also no data informing how dissolved emerging organic contaminants may be changing over time.

SBNMS receives continued inputs of dissolved trace metals via offshore circulation, the MWRA outfall and other wastewater streams, and seasonally-mediated resuspension from local sediments (Kalnejais et al. 2015). While sediment metal concentrations are characterized within Massachusetts Bay via ongoing

100

Stellwagen Bank $\mid$ Condition Report 2020 
MWRA monitoring, dissolved concentrations are less clearly documented. Modeling work suggests differential distributions of dissolved metals between surface and bottom water, while existing sediment monitoring implies that dissolved concentrations are likely below biologically meaningful thresholds due to generally low sediment concentrations ( $\mathrm{Li}$ et al. 2010, Nestler et a1. 2017, Werme et al. 2015).

However, it is unclear how seasonal and climatic cycles impacting relevant physicochemical water column parameters may change the availability of dissolved metals over time (Atkinson et al. 2007).

Effluent volume from the MWRA Deer Island Wastewater Treatment Plant is not significantly increasing over time. Discharge volume mirrors regional rainfall trends, with an increasing percentage of effluent receiving full secondary treatment (Figure S.WQ.9.1, Werme et al. 2017). Additionally, the Merrimack River brings significant and rain-dependent flow to the northern area of the sanctuary; its discharge has the capacity to carry point and non-point source pollution from the large Merrimack watershed, which could threaten SBNMS water quality.

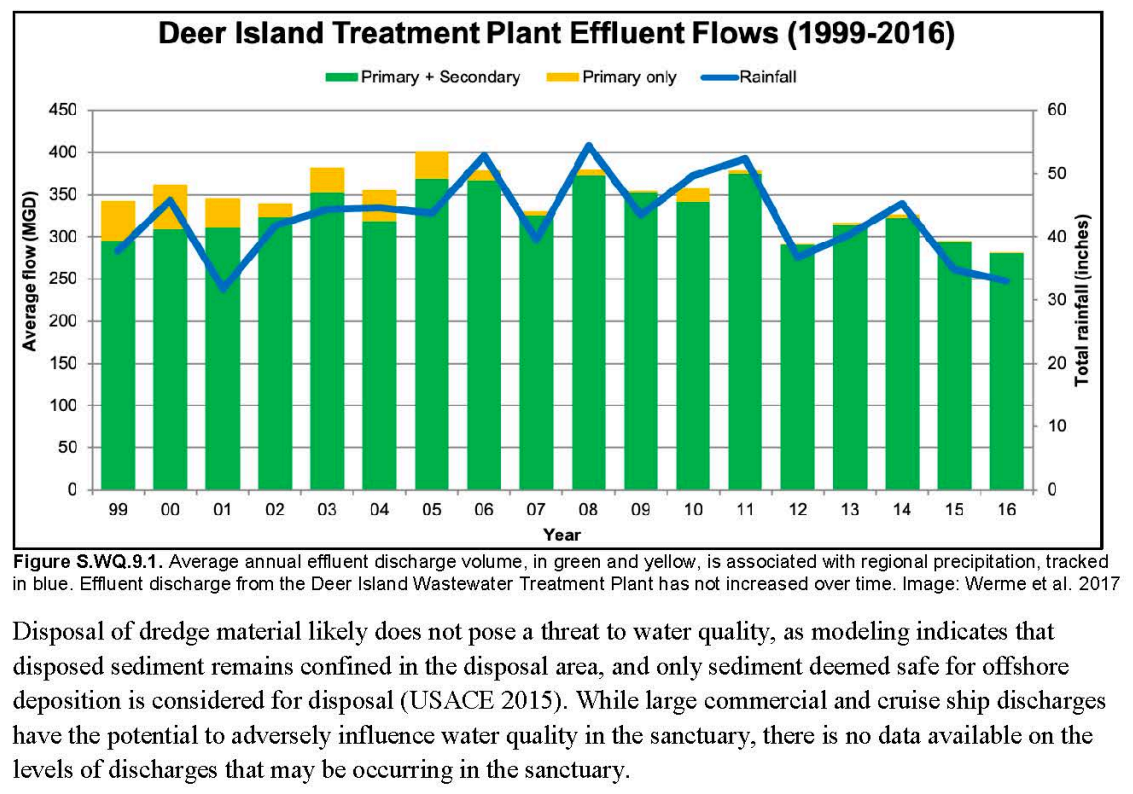

101

Stellwagen Bank |Condition Report 2020 


\section{Habitat (Questions 10-11)}

The following information provides an assessment of the status and trends of key habitat indicators in SBNMS for the period 2007-2018. Question 10 focuses on the integrity of major habitats within the sanctuary, including biologically (biogenic) and abiotically (physical) structured habitats. Physical habitats are abiotic structures, such as sand waves, mud-draped gravel, and piled boulders. Biogenic habitats are structure-forming species, which create habitat structures used by other living marine resources, including hydroids, anemones, and sponges. Biogenic habitats are layered on top of, and are often determined by, the coincident physical habitat. Changes to both biotic and abiotic habitat can significantly alter the diversity of living marine resources and ecosystem services. Question 11 examines concentrations and variability of contaminants in major sanctuary habitats.

\section{What is the integrity of major habitat types and how is it changing?}

Status Description: Selected habitat loss or alteration has Fair - 000 caused measurable but not severe degradation in some attributes of ecological integrity.

Rationale: Data suggest measurable changes in habitat quality, likely due to the use of bottomcontact commercial fishing gear. Some habitat attributes show degradation, while others show improvement. Significant habitat degradation is observed in isolated areas due to chronic disturbance. Use of bottom-contact gear is intensive in SBNMS, but diminishing due to regulatory controls and fleet consolidation. An increase in scallop dredging started in 2017 and will continue. Seabed disturbance from anchoring and other activities might also be locally important and should be evaluated.

102 
11. What are contaminant concentrations in sanctuary habitats and how are they changing?

\section{Undetermined ? S10 Status Description: Undetermined}

Rationale: Legacy contaminants have been reported in benthic habitats. However, they infrequently exceed thresholds of concern, do not appear to remobilize beyond sites where they have been identified (e.g., MBDS), and no indications of acute life history or population effects have been observed. Compounds of emerging concern are present, but poorly documented or monitored; thus, their status and trends could not be assessed. More monitoring is needed in this area.

In the 2007 condition report, this question was rated "good/fair" and "not changing". The current undetermined ratings primarily stem from the abundance of unknowns surrounding contaminant occurrence, trends, and biological effects. MWRA and other regional monitoring has demonstrated the continued, low-level presence of legacy contaminants in benthic habitats, occasionally at concentrations above established regulatory thresholds of low concern. Legacy contaminants in sediments do not appear to be subject to remobilization or transport beyond sites where they have been identified (e.g., MBDS), and there are currently no indications of acute life history or population effects related to legacy contaminant exposures despite demonstrated bioaccumulation in regional fauna. However, biological impacts of contaminants are particularly difficult to parse out in wildlife and marine systems given the abundance of other abiotic and biotic stressors, compounding uncertainty related to assessing contaminant degradation in the sanctuary habitat. Compounds of emerging concern are also present in sanctuary habitats and biota, but are not well-documented or effectively monitored; limited information suggests

107

Stellwagen Bank $\mid$ Condition Report 2020 
that emerging contaminant concentrations may be shifting or increasing with continued introduction of new chemicals to replace regulated compounds. Therefore, status and trends of these emerging compounds could not be assessed with the limited information available.

Contaminant concentrations in SBNMS sedimentary habitats are dynamic. Observed concentrations reflect the influence of local, regional, and global processes that distribute pollutants via atmospheric, particulate, or water-based transport at different time scales. Compound-specific partitioning and degradation pathways also determine environmental distribution and possible biological activity.

MWRA monitors locations across the nearshore-offshore continuum for sediment condition and infauna community structure annually, and sediment contaminant levels are determined every three years (Rutecki et al. 2017). This ongoing program, in combination with additional regional monitoring of sediments and biota, provides a relevant dataset describing concentrations of metals and selected organic contaminants. Regional monitoring of sediments indicates low yet persistent concentrations of metals, such as mercury, and legacy persistent organic pollutants (POPs), such as PAHs, PCBs, and organochlorine pesticides (OCPs) (Figures S.H.11.1 - S.H.11.2, Nestler et al. 2015, Sunderland et al 2012, Kimbrough et al. 2009). Most metals and legacy POPs continue to decrease or remain steady within SBNMS, as documented at one current MWRA monitoring site within sanctuary boundaries. These local trends are commensurate with declines seen regionally and worldwide, as stringent regulation translates to decreased or negligible environmental inputs of many metals and legacy POPs (Zhao et al. 2017b, Werme et al. 2015, Nestler et al. 2015).

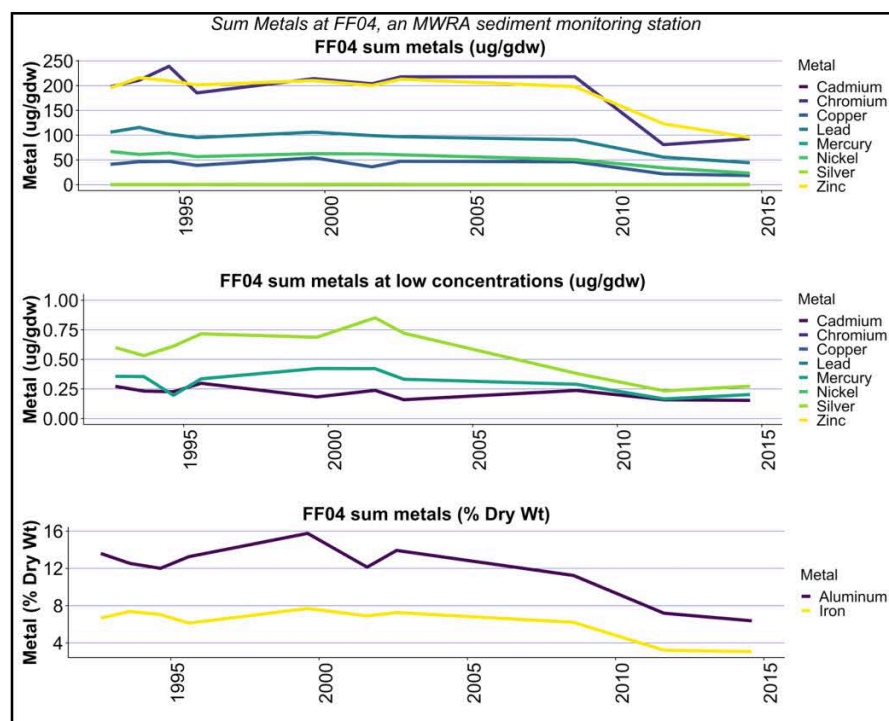

Figure S.H.11.1. Sum metal concentrations have decreased over time at the MWRA monitoring station in SBNMS (FF04), according to MWRA monitoring data. Images: MWRA sediment chemistry data, stations FF04, FF09, FF01A, NF12. MWRA data courtesy of Kenneth Keay and Douglas Hersh

108

Stellwagen Bank |Condition Report 2020 


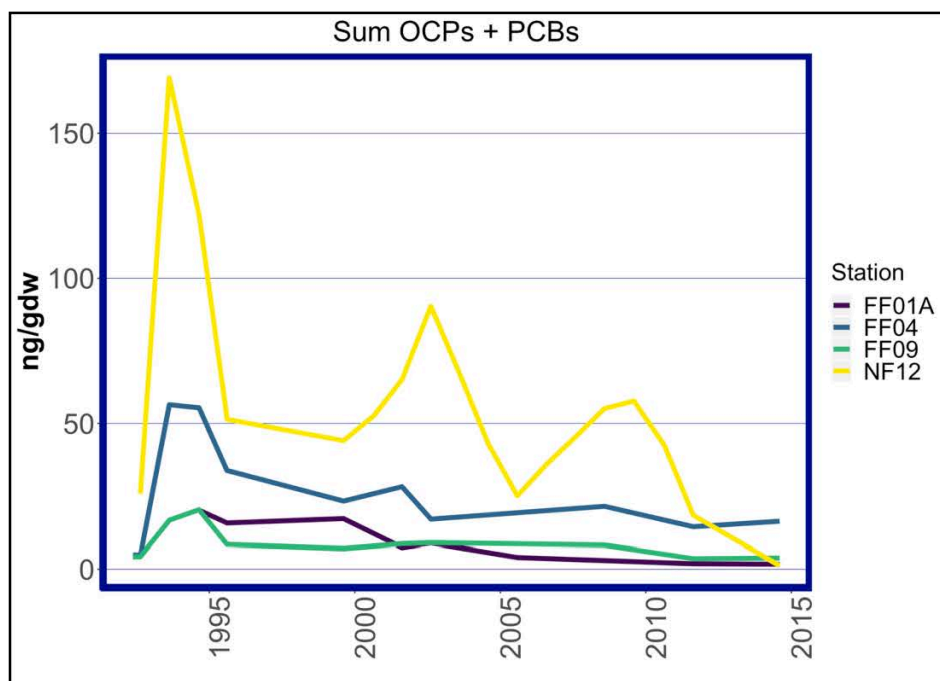

Figure S.H.11.2. Sum concentrations of all chlorinated persistent organic pollutants (POPs), including select organochlorine pesticides (OCPS) and polychlorinated biphenyls (PCBs), in sediment at four MWRA benthic monitoring stations across Massachusetts Bay; FF04 is the station within SBNMS. Image: MWRA sediment chemistry data, stations FF04, FF09, FF01A NF12. MWRA data courtesy of Kenneth Keay and Douglas Hersh

Biotic monitoring also indicates sustained concentrations of metals and legacy POPs. Some regional studies of mussels, fish, pinnipeds, and cetaceans indicate significant tissue burdens of metals and/or legacy POPs, suggesting these compounds remain relevant in the offshore food web, are capable of bioaccumulation, and, in some cases, exceed estimated immunotoxicity thresholds (Nestler et al. 2016, Shaw et al. 2014, 2009b, 2005, Sunderland et al. 2012, Elfes et al. 2010, Kimbrough et al. 2009).

Additionally, a plethora of emerging contaminants remain uncharacterized within the Massachusetts Bay environment and food web, including per- and polyfluorinated substances, novel flame retardants, plasticizers, pharmaceuticals and personal care products, endocrine disrupting compounds, currently used biocides, and more. Few studies have documented various compounds regionally in water, sediment, or biota, and there is no substantial understanding regarding their presence, risk, or trends in SBNMS (Cantwell et al. 2016a, Costa 2012, Kimbrough et al. 2009, Shaw et al. 2009a, 2009b, Kolpin et al. 2002). Limited existing data suggest emerging contaminants are probably present within the overall Massachusetts Bay habitat, likely at low concentrations, with uncertain biological ramifications.

No data were found detailing concentrations of microplastic particles or fibers in SBNMS sediments, or how plastic levels may be changing over time; this is a major data gap, considering marine sediments are a major sink for microplastic pieces (Woodall et al. 2014). Surface water concentrations confirm the presence of microplastic contamination in the region. Surface tows between 1986-2008 suggest Gulf of Maine surface water concentrations of $1534+/-200$ plastic pieces per square kilometer, an order of magnitude lower than concentrations observed in the North Atlantic subtropical gyre (Law et al. 2010, Eriksen et al. 2014) 
Concentrations of Clostridium perfringens, an anaerobic bacterium that acts as a tracer of wastewater effluent influence in sediments, have remained low or declined within SBNMS and at other offshore locations since outfall diversion. Observed concentrations are comparable to baseline levels at MWRA monitoring sites in and around SBNMS following the relocation of the outfall in 2000, suggesting that discharge from the outfall has minimal impact on bacterial loads in SBNMS sediments (Nestler et al. 2015, 2017). 


\section{Time Trends of Polybrominated Diphenyl Ethers (PBDEs) in Antarctic Biota}

Erin Markham, ${ }^{\dagger}$ Emily K. Brault, ${ }^{\ddagger}, \#$ Mohammed Khairy, ${ }^{\dagger, \nabla}$ Anna R. Robuck, ${ }^{\dagger}$ Michael E. Goebel, ${ }^{\S}$ Mark G. Cantwell, ${ }^{\|}$Rebecca M. Dickhut, ${ }^{\ddagger, \perp}$ and Rainer Lohmann*,†

${ }^{\dagger}$ Graduate School of Oceanography, University of Rhode Island, 215 South Ferry Road, Narragansett, Rhode Island 02882, United States

${ }^{\ddagger}$ Virginia Institute of Marine Science, 1208 Greate Road, Gloucester Point, Virginia 23062, United States

${ }^{\S}$ Antarctic Ecosystem Research Division, NOAA Fisheries, Southwest Fisheries Science Center, 8901 La Jolla Shores Drive, La Jolla, California 92037, United States

"Atlantic Ecology Division, U.S. Environmental Protection Agency, Narragansett, Rhode Island 02882, United States

S Supporting Information

ABSTRACT: Polybrominated diphenyl ethers (PBDEs) are "emerged" contaminants that were produced and used as flame retardants in numerou consumer and industrial applications for decades until banned. They remain ubiquitously present in the environment today. Here, a unique set of $>200$ biotic samples from the Antarctic was analyzed for PBDEs, including phytoplankton, krill, fish, and fur seal milk, spanning several sampling seasons phytoplankton, krill, fish, and fur seal milk, spanning several sampling seasons
over 14 years. PBDE-47 and -99 were the dominant congeners determined in all samples, constituting $>60 \%$ of total PBDEs. A temporal trend was observed for $\sum_{7} \mathrm{PBDE}$ concentrations in fur seal milk, where concentrations significantly increased $\left(R^{2}=0.57, p<0.05\right)$ over time $(2000-2014)$. Results for krill and phytoplankton also suggested increasing PBDE concentrations over time. Trends of PBDEs in fur seal milk of individual seals sampled 1 or more years apart showed no clear temporal trends. Overall, there was no indication of PBDEs decreasing in Antarctic biota yet, whereas numerous

studies have reported decreasing trends in the northern hemisphere. Similar PBDE concentrations in perinatal versus nonperinatal milk implied the importance of local PBDE sources for bioaccumulation. These results indicate the need for continued assessment of contaminant trends, such as PBDEs, and their replacements, in Antarctica.

\section{INTRODUCTION}

Antarctica is one of the most pristine places on the planet. However, even in this remote region, anthropogenic effects are measurable. Scientific exploration in Antarctica has occurred for decades and in the summer season, the continent hosts over 100 active facilities operated by 30 different nations. ${ }^{1}$ Although pollution in Antarctica has typically been orders of magnitude lower than concentrations reported elsewhere around the globe, organic contaminants, particularly (semi-) volatile ones, have reached the region via long-range environmental transport by processes of global fractionation and cold condensation. ${ }^{2}$ Legacy contaminants, such as polychlorinated biphenyls (PCBs) and organochlorine pesticides (OCPs), have been reported along with more recent contaminants, such as polybrominated diphenyl ethers (PBDEs) and perfluoroalkyl substances, in numerous environmental matrices from the region. ${ }^{3-9}$ Some of this contamination has also been found to originate from research stations themselves. ${ }^{10,11}$

Polybrominated diphenyl ethers are "emerged" contaminants that have been used as additive flame retardants for decades in a wide range of consumer and industrial applications (e.g.

ACS Publications upholstery, electronics) and are easily leached from these manufactured goods into the environment, food webs, and ultimately reaching humans. ${ }^{12}$ PBDEs, like many legacy persistent organic pollutants (POPs) (e.g., PCBs), are hydrophobic and lipophilic. The height of PBDE production was dominated by three different commercial mixtures (penta-, octa-, and deca-BDE). ${ }^{13}$ In 2009, penta- and octa-PBDE mixtures were listed by the Stockholm Convention. ${ }^{13}$ However, production of the deca-BDE has persisted in many countries and a massive reserve of products that contain PBDEs exist around the globe and will continue to leach them into the environment.

Several studies have reported PBDE concentrations starting to decrease over the last 5-10 years. For example, time trends of PBDEs in samples collected from Swedish mothers indicate a decreasing trend for most PBDEs, except for BDEs 153 and 209, from 1996 to 2010. ${ }^{17}$ PBDEs also decreased in Baltic

Received: April 6, 2018 Accepted: June 1, 2018 Published: June 19, 2018 


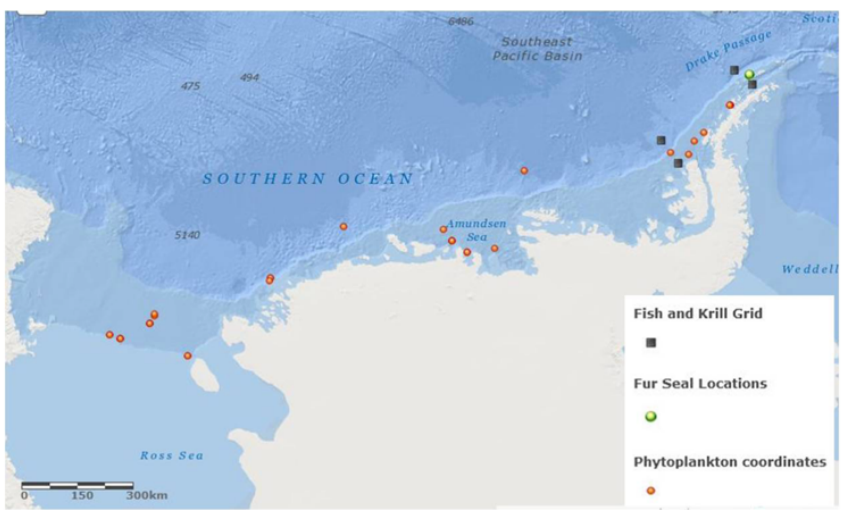

Figure 1. Map of sampling locations. Black squares denote the boundaries of the Long Term Ecological Research (LTER) grid. Created with ArcGIS Explorer.

herring over the last decade. ${ }^{18}$ Across the Great Lakes in North America, PBDEs in fish started to decline in $1999-2000 .^{19}$ So far, no consistent set of Antarctic samples has been available to document time trends of PBDEs in Antarctic marine biota, although several previous studies have detected PBDEs in the

Antarctic environment. ${ }^{3,4,9,10,20}$

Kelly et al. in 2008 presented evidence from a Canadian Arctic marine food web in which many PBDEs appeared to exhibit negligible biomagnification, with the exception of BDE47, which did demonstrate food web biomagnification, albeit at a much lower level than PCBs. ${ }^{21}$ Yet in the same study, PBDEs in macroalgae were excluded from the trophic magnification factor calculation as their concentrations exceeded those from other trophic levels by $5-10$ times.

We obtained a unique set of biotic samples from West Antarctica (Figure 1), including phytoplankton, krill, fish, and fur seal milk, spanning several years (2000-2014). A previous paper reported generally declining trends of several but not all PCBs and legacy OCPs in these fur seal milk samples; PBDE concentrations were not measured at the time. ${ }^{6}$ We used these samples to assess the presence and trophic transfer of PBDEs in the West Antarctic food web and to identify the PBDE temporal trends in this region either from yearly averages or in the case of repeatedly sampled fur seals, from individual trends over time. The food web structure was assessed using stable isotopes of carbon $\left(\delta^{13} \mathrm{C}\right)$ and nitrogen $\left(\delta^{15} \mathrm{~N}\right)$

Specific goals in this research were to (i) determine which PBDEs are being detected in different Antarctic biota; (ii) establish temporal trends of common PBDE congeners over a time period where global regulations and restrictions on production had been implemented (i.e., 2000s); (iii) contrast trends of PBDEs in Antarctic fur seal milk to those from the Arctic; and (iv) assess the difference of milk sampled before and after fur seals begin foraging locally in waters off the Antarctic Peninsula.

\section{- MATERIALS AND METHODS}

Sample Collection. Milk samples were collected from Antarctic fur seals (Arctocephalus gazella) approximately 100 $\mathrm{km}$ off the Antarctic Peninsula at Cape Shirreff, Livingston Island $\left(62.47^{\circ} \mathrm{S}, 60.77^{\circ} \mathrm{W}\right)$ over the austral summers of $2000 /$ 2001, 2001/2002, 2004/2005, 2009/2010, 2010/2011, $2011 /$ 2012, 2012/2013, and 2013/2014 (Figure 1 and Tables S1S8). Most seals were multiparous females in their perinatal stage (i.e., the seals had all bred prior to the year of sample collection and milk was collected during the perinatal period, 1-2 days postpartum, prior to initiation of offshore foraging trips), except for the 2011/2012, 2012/2013, and 2013/2014 samples, which consisted of both perinatal and nonperinatal (i.e., after initiation of foraging cycles) milk samples (Tables S6-S8). Seals were assumed to have had at least one pup prior to the breeding season sampled as all seals were age 5 or older, with the majority being over the age of 7 . Seal capture was performed following methods described in Polito and Goebel and as reported in Brault et al. ${ }^{6,22}$ In brief, seals were captured with hoop nets, sedated with $5 \mathrm{mg}$ of midazolam, and anesthetized with isoflurane. Milk was collected after an intramuscular injection of oxytocin $(0.25 \mathrm{~mL}, 10 \mathrm{UI} / \mathrm{mL})$ in precleaned vials and stored at $-20{ }^{\circ} \mathrm{C}$ until analysis. ${ }^{6,22}$ Temperature loggers sampling every $10 \mathrm{~min}$ were kept with samples to confirm storage temperature.

Phytoplankton samples were collected in a region of Antarctica that spans from the West Antarctic Peninsula to the Ross Sea $\left(64.78^{\circ} \mathrm{S}, 64.07^{\circ} \mathrm{W}\right.$ to $78.64^{\circ} \mathrm{S}, 164.30^{\circ} \mathrm{W}$, Figure 1) over the austral summers of $2007 / 2008,2009 / 2010$, and 2010/2011 using ring net tows (Table S9). Phytoplankton samples consisted largely of diatoms (Antarctic Peninsula) and Phaeocystis sp. (Amundsen and Ross Seas). Further specifics on sample collection can be referenced in Brault et al. ${ }^{6,2}$

All krill and fish samples were collected from within the Palmer Long Term Ecological Research (LTER) grid survey region (approx. $66.99^{\circ} \mathrm{S}, 69.28^{\circ} \mathrm{W}$ to $61.94^{\circ} \mathrm{S}, 73.78^{\circ} \mathrm{W}$, Figure 1) via $700 \mu \mathrm{m}$ ring net tows (taken at oblique angles, Table S10). Krill samples consisted predominantly of Euphausia superba and were collected during the austral summers of 2007/ 2008 and 2010/2011 and split into three size classes (juveniles, adults (including mature females), and gravid females). Fish samples consisted of either silverfish (Pleuragramma antarcti- 
Table 1. Comparison of Average PBDE Concentrations in Antarctic Biota (ng/g lipid) \pm 1 Standard Deviation from This and Previous Studies

\begin{tabular}{|c|c|c|c|c|c|}
\hline & $\sum$ PBDEs' & BDE-28 & $\mathrm{BDE}-47$ & BDE-99 & BDE-100 \\
\hline plankton $^{a}$ & & & $23 \pm 3.5$ & $22 \pm 3.4$ & $4.5 \pm 0.7$ \\
\hline phytoplankton ${ }^{b}$ & $53 \pm 76$ & $1.9 \pm 2.7$ & $20 \pm 27$ & $19 \pm 32$ & $4.8 \pm 8.1$ \\
\hline juvenile krill ${ }^{a}$ & & & $570 \pm 210$ & $620 \pm 250$ & $130 \pm 51$ \\
\hline juvenile kritit ${ }^{b}$ & $0.65 \pm 0.27$ & $0.07 \pm 0.11$ & $0.49 \pm 0.20$ & $0.05 \pm 0.05$ & 0.04 \\
\hline adult krill ${ }^{a}$ & & & $2.0 \pm 0.5$ & $2.5 \pm 0.6$ & $0.5 \pm 0.1$ \\
\hline adult krill ${ }^{b}$ & $0.51 \pm 0.78$ & $0.04 \pm 0.05$ & $0.28 \pm 0.23$ & $0.13 \pm 0.43$ & 0.01 \\
\hline gravid krill ${ }^{b}$ & $0.35 \pm 0.19$ & $0.06 \pm 0.05$ & $0.18 \pm 0.12$ & $0.04 \pm 0.06$ & 0.02 \\
\hline "krill" & $5.6 \pm 1.1$ & & & & \\
\hline${\text { krit } 1^{f}}^{f}$ & 0.095 & 0.001 & 0.011 & 0.009 & 0.002 \\
\hline krit $\left.\right|^{g}$ & 0.027 & & & & \\
\hline adult krill ${ }^{d}$ & 0.94 & 0.03 & 0.17 & 0.2 & 0.05 \\
\hline rockcod muscle $e^{c}$ & $5.8 \pm 2.3$ & & & & \\
\hline rockcod muscle $e^{e}$ & 7.5 & & & & \\
\hline Antarctic silverfish $^{b}$ & $<\mathrm{LOD}$ & & $<1.5$ & $<1.5$ & \\
\hline myctophid ${ }^{b}$ & $\angle L D$ & & $<0.20$ & $<0.20$ & \\
\hline Weddell seal ${ }^{h}$ & 2.0 & $<L O D$ & $<\mathrm{LOD}$ & 2.0 & $<\mathrm{LOD}$ \\
\hline Weddell seal ${ }^{i}$ & 1.5 & $\angle L O D$ & 1.5 & $<\mathrm{LOD}$ & $\angle L O D$ \\
\hline fur seal milk ${ }^{b}$ & $2.3 \pm 1.9$ & $0.07 \pm 0.09$ & $1.3 \pm 1.4$ & $0.36=0.36$ & $0.14 \pm 0.15$ \\
\hline
\end{tabular}

${ }^{a}$ Chiuchiolo et al., ${ }^{5} 64.7^{\circ} \mathrm{S}, 64.0^{\circ} \mathrm{W}$. ${ }^{b} \mathrm{This}$ study, $\sum_{7} \mathrm{BDEs}$, Ross Sea to Antarctic Peninsula; results were averaged over all sampling seasons. ${ }^{c}$ Corsolini et al., ${ }^{4}$ Ross Sea, approx $74^{\circ} 04^{\prime} \mathrm{S}, 179^{\circ} 06^{\prime} \mathrm{E}$. ${ }^{d}$ Bengtson Nash et al., ${ }^{25}$ arithmetic means, $\sim 63-69^{\circ} \mathrm{S}, 30-80^{\circ} \mathrm{E}$. ${ }^{\mathrm{C}} \mathrm{Cincinelli}$ et al., ${ }^{\circ}$ assuming

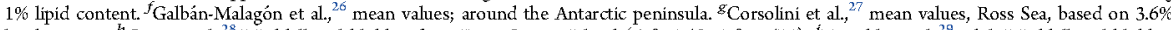
lipid content ${ }^{h} \mathrm{C}$ ipro et al ${ }^{28}$ Weddell seal blubber from King George Island ( $62^{\circ} 050^{\prime} \mathrm{S}, 58^{\circ} 230^{\prime} \mathrm{W}$ ). 'Trumble et al ${ }^{29}$ adult We ddell seal blubbe near McMurdo Station, Antarctica $\left(77^{\circ} 55^{\prime} \mathrm{S}, 166^{\circ} 39^{\prime} \mathrm{E}\right)$. Note that the number of BDE congeners included in the $\sum$ PBDEs varies between studies.

cum) or myctophids (Electrona antarctica) and were collected in the same manner as krill (Table \$11).

Sample Extraction. Fur seal milk extraction was conducted in two batches. The first batch $(n=59)$, which consists of samples from the five austral summers spanning from $2000 /$ 2001 to $2010 / 2011$, was extracted at the Virginia Institute of Marine Science (VIMS) following previously established POP procedures as reported in Geisz et al. ${ }^{24}$ In short, fur seal milk was freeze-dried, homogenized, subsampled ( $1 \mathrm{~g}$ of dry weight), solvent-extracted (65:35 dichloromethane/acetone), and analyzed for several POPs (e.g., 1,1,1-trichloro-2,2-bis ( $p$ chlorophenyl)ethane (DDT), PCBs, and chlordane) as well as lipid content. ${ }^{6}$ Sample extracts were shipped to the University of Rhode Island's Graduate School of Oceanography (URI-GSO) for PBDE analysis.

The second batch of fur seal milk samples (samples from $2011 / 2012,2012 / 2013$, and $2013 / 2014, n=71$ ) was extracted at URI-GSO, as detailed in the Supporting information (SI). Briefly, $2 \mathrm{~mL}$ of fur seal milk was spiked with PBDE surrogates, extracted three times with $20 \mathrm{~mL}$ each of $n$-hexane/acetone (2:1), treated with concentrated sulfuric acid to denature lipids, and cleaned on solid-phase extraction cartridges. Percent lipid was measured separately.

Phytoplankton, fish, and krill samples were also extracted at VIMS. Samples were manually homogenized with a Virtis " 45 " tissue homogenizer (Virtis Co. Inc.), freeze-dried at $-80^{\circ} \mathrm{C}$ for approximately $72 \mathrm{~h}$, and solvent-extracted. Further details on sample preparation can be gathered from Brault. ${ }^{23}$ Following analysis at VIMS for several legacy POPs ${ }^{6}$ phytoplankton, fish, and krill, sample extracts were shipped to URI-GSO to be analyzed for PBDEs.

PBDE Analysis. All samples were analyzed for monothrough hepta-brominated congeners (BDE-2, $-8,-15,-30,-28$, $-49,-47,-100,-99,-154,-153$, and -183 ) via gas chromatography (GC) tandem mass spectrometry (MS) on an Agilent $6890 \mathrm{~N}$ GC coupled to a Waters Quattro Micro MS/ MS under electron ionization/MS/MS in multiple reaction monitoring mode using a DB-5MS column (Agilent J\&W GC Columns, 122-5532, length $30 \mathrm{~m}$, i.d. $0.250 \mathrm{~mm}$, film $0.25 \mu \mathrm{m}$ ) and splitless injection (for more details, see SI). Analysis of BDE-209 was conducted separately, and procedures are detailed in the SI.

Sample extracts were spiked with $10 \mu \mathrm{L}$ of a $5 \mathrm{ng} / \mu \mathrm{L}^{13} \mathrm{C}_{12}$ labeled PBDE surrogate $\left({ }^{13} \mathrm{C}_{12}\right.$ BDEs-28, 47, 99, 153, and 183 , Cambridge Isotope Laboratories) for a total concentration of $50 \mathrm{ng}$ and $5.0 \mu \mathrm{L}$ of a $5.0 \mathrm{ng} / \mu \mathrm{L}$ injection standard (pterphenyl- $d_{14}$, AccuStandard) for a total concentration of $25 \mathrm{ng}$. To the samples originally extracted at VIMS, the surrogate was added postextraction. These samples were corrected for the average recoveries of previously analyzed POPs (e.g., DDT, PCBs, and chlordane), which were $79 \pm 3.7 \%$ for phytoplankton, $69 \pm 1.8 \%$ for krill, and $78 \pm 1.8 \%$ for fur seal milk. The second batch of fur seal milk samples (2011) $2012,2012 / 2013$, and 2013/2014) was spiked prior to extraction at URI-GSO directly with $20 \mu \mathrm{L}$ of a $2.0 \mathrm{ng} / \mu \mathrm{L}$ PBDE surrogate standard in nonane. Results presented below are only for compounds that were detected $>30 \%$ of the time.

Quality Control. Laboratory blanks of a hydromatrix material were initially extracted alongside real samples, and any blanks included in the vial files for shipment from VIMS to URI-GSO were analyzed for PBDEs. All samples were blankcorrected; main detected congeners were BDE 47 and -99 with concentrations averaging $0.18 \pm 0.20 \mathrm{ng}$ (standard deviation (SD)) and $0.18 \pm 0.23 \mathrm{ng}$, respectively (Table S12, SI). For BDE-209, amounts in blanks were similar to fur seal milk results, so results are not reported here.

At URI-GSO, laboratory blanks, matrix spikes $(n=8)$, blank spikes $(n=4)$, and duplicates of fur seal milk $(n=10)$ were included. Limit of detection (LOD) was calculated as the average detected blank concentrations +3 times the standard 

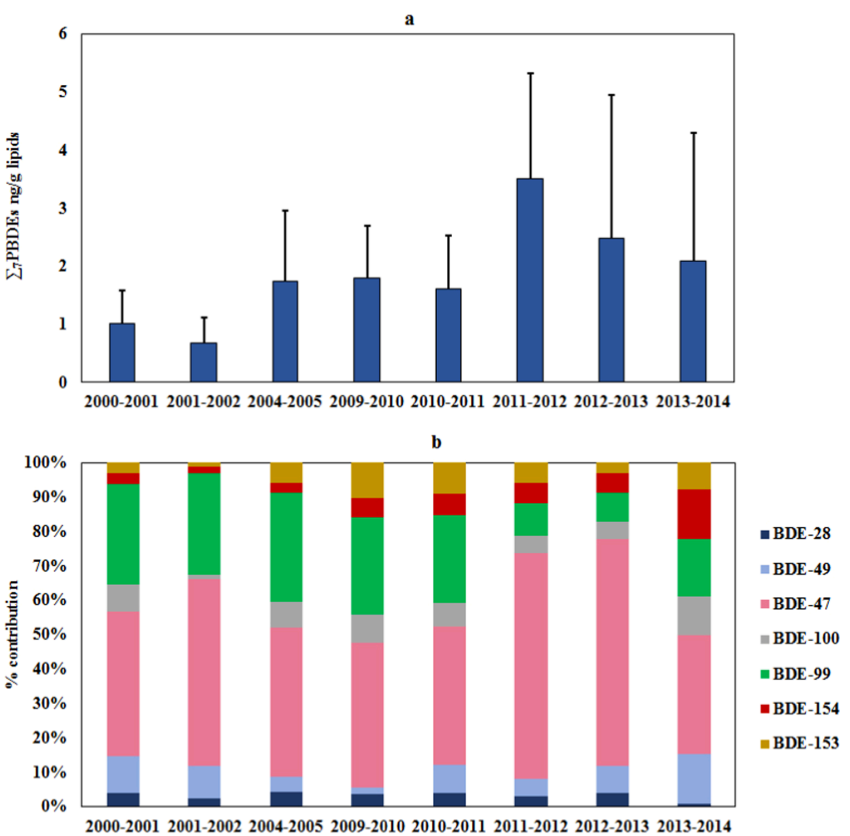

Figure 2. (a) Average sum of PBDEs per breeding season for the eight nonconsecutive austral summers sampled. Bars represent standard deviation The first five austral summers (2000/2001-2010/2011) have had a recovery correction of $77.86 \%$ applied. (b) Average percent composition of PBDEs in fur seal milk from $2000 / 2001$ to $2013 / 2014$.

deviation (Table S13). For congeners that were not detected, the noise was used to derive LODs (Table S14); LODs ranged from $0.011 \mathrm{ng} / \mathrm{g}$ lipid (BDE-2, 15) to $0.16 \mathrm{ng} / \mathrm{g}$ lipid (BDE99). Recoveries of the surrogate standards ranged from $83 \pm$ $2.4 \%$ (BDE-183) to $94+3.1 \%$ (BDE-47). Recoveries of the congeners in the matrix and blank spikes generally ranged from $92 \%$ (BDE-2) to $102 \%$ (BDE-47), as shown in Table S15. Relative percent difference for the duplicates ranged from $6.2 \%$ (BDE-100) to $21 \%$ (BDE-2).

$\delta^{13} \mathrm{C}$ and $\delta^{15} \mathrm{~N}$ Analysis. $\delta^{13} \mathrm{C}$ and $\delta^{15} \mathrm{~N}$ stable isotopes for the majority of samples were determined via an elemental analyzer isotopic ratio mass spectrometer at VIMS, as described elsewhere (Figure S1). ${ }^{6}$ Some plankton samples were analyzed at the University of California, Santa Cruz (UCSC) on a Carlo Erba EA 1108 elemental analyzer coupled to a Finnegan DeltaPlus isotopic ratio mass spectrometer. Average values were -29 $\pm 0.40 \%$ for $\delta^{13} \mathrm{C}$ and $1.6 \pm 0.50 \%$ for $\delta^{15} \mathrm{~N}$.

Statistical Analysis. Data were tested for normality using the Shapiro-Wilks test in RStudio, IBM SPSS Statistics 22, and SigmaPlot 12 software packages. Concentrations were natural $\log$ transformed to make data have a normal or near-normal distribution. Linear regressions were performed for each congener with $>30 \%$ detection against fur seal age, breeding season, $\delta^{13} \mathrm{C}$, and $\delta^{15} \mathrm{~N}$. Any difference between PBDE concentrations in perinatal and nonperinatal milk was tested using a student's two-sample $t$ test assuming unequal variances.
A student's $t$ test was used to compare between detected PBDE concentrations in the krill samples of 2007 and 2011. Similarly, the one-way repeated analysis of variance (ANOVA) test was used to compare between detected PBDE concentrations in (i) the different krill samples (juveniles, adults, and gravid); (ii) phytoplankton samples collected in 2007-2011; and (iii) between the calculated trophic levels for the different seal samples collected in 2000,2001, 2004, 2009, and 2010.

\section{RESULTS AND DISCUSSION}

PBDE-47 and -99 were the dominant congeners determined in all samples, generally constituting $>60 \%$ of total PBDEs. Phytoplankton samples displayed the highest overall concentrations, followed by fur seal milk, krill, and lastly fish, in which no PBDEs were detected (Table 1). The lower brominated congeners (BDE-2, -8, -15, and -30) and BDE-183 were not regularly detected until 2011/2012. These trends could, at least partially, be explained by the switch in extraction procedures between VIMS (2000/2001-2010/2011) and URI-GSO. To ensure consistency across the various samples, we focus on the seven most routinely detected congeners (BDE-28, -49, -47 , $-100,-99,-154$, and -153), which were also summed ( $\left.\sum_{7} \mathrm{BDEs}\right)$

Fur Seal Milk. Lipids in fur seal milk were high with an average value of $64 \pm 9 \%$ (SD; range $14-84 \%$ ). PBDEs were detected in all fur seal milk samples $(n=130$; Tables S15- 


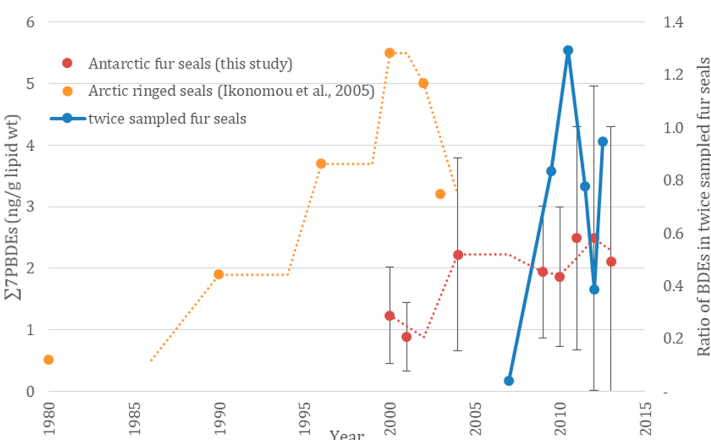

Figure 3. $\sum_{7} \mathrm{PBDE}$ trends in Arctic fur seals (red dotted line), reported BDE concentrations in Arctic ringed seals (orange dashed line, both left axis), and ratios of BDE concentrations in fur seal milk sampled twice over several years (blue solid line).

S22). The $\sum_{7}$ PBDEs in all fur seal milk samples ranged from 0.14 to $17 \mathrm{ng} / \mathrm{g}$ lipid with a mean $\pm \mathrm{SD}$ of $2.1 \pm 1.9 \mathrm{ng} / \mathrm{g}$ lipid (median of $1.7 \mathrm{ng} / \mathrm{g}$ lipid). BDE-47 was the most dominant congener with a range of $0.14-12 \mathrm{ng} / \mathrm{g}$ lipid and a mean of 1.3 $\pm 1.4 \mathrm{ng} / \mathrm{g}$ lipid (median of $0.95 \mathrm{ng} / \mathrm{g}$ lipid). BDE-99 was the second most dominant congener but showed less variability with a range from $<$ LOD to $2.7 \mathrm{ng} / \mathrm{g}$ lipid, mean of $0.36 \pm 0.36$ $\mathrm{ng} / \mathrm{g}$ lipid (median of $0.27 \mathrm{ng} / \mathrm{g}$ lipid). Similar PBDE concentrations of around $1.5-2.0 \mathrm{ng} / \mathrm{g}$ lipid (Table 1) have been reported for Antarctic Weddell seal blubber. ${ }^{28,29}$ For comparison, ringed seal blubber in the Canadian Arctic contained more elevated $\sum_{15}$ PBDEs at $11-14 \mathrm{ng} / \mathrm{g}$ lipid. ${ }^{30}$

Stable isotope analysis was only available for fur seal milk collected during the first five austral summers $(2000 / 2001-$ $2010 / 2011, n=59$ ). Both $\delta^{13} \mathrm{C}$ and $\delta^{15} \mathrm{~N}$ demonstrated variability: $\delta^{13} \mathrm{C}$ ranged from -26 to $-20 \%$ with a mean \pm SD of $-23 \pm 1.4 \%$; $; \delta^{15} \mathrm{~N}$ ranged from 8.0 to $14 \%$, with a mean \pm SD of $10 \pm 1.2 \%$.

Fur Seal Milk Trends. In fur seal milk, the dominant congeners BDE-47 and -99 showed no significant correlation with age, similar to results for legacy POPs, implying that older animals did not display greater concentrations. ${ }^{6}$ No significant relationships were found between fur seal milk PBDE concentration and $\delta^{13} \mathrm{C}$ value from regression analysis. Few significant trends were observed between PBDE concentration and $\delta^{15} \mathrm{~N}$ value; most notably in 2000/2001, BDE-47 and $\sum_{7} \mathrm{PBDEs}$ versus $\delta^{15} \mathrm{~N}$ both demonstrate significantly negative trends (i.e., decreasing concentration with increasing $\delta^{15} \mathrm{~N}$ value or trophic level). We have no explanation for this trend, but note that it disappears when looking at all fur seal milk results from $2000 / 2001$ to $2010 / 2011$ combined.

$\sum_{7}$ PBDE concentrations for the 2011/2012 breeding season were significantly greater than for the other breeding seasons, except for 2009/2010 and 2013/2014 (one-way repeated ANOVA, $p<0.001$ ). Additionally, a temporal trend was observed for $\sum_{7} \mathrm{PBDE}$ concentrations (Figure 2), where concentrations significantly increased $\left(R^{2}=0.57, p=0.03\right)$ over time (from 2000 to 2014). We note that the (increasing) slope of PBDE concentrations over time was not significantly different when considering either all 8 sampling years or just the first 5 years (those extracted at the VIMS).
Fur Seal Milk Trends in the Same Individuals. The previous discussion was based on average values from randomly sampled female fur seals over time. Milk samples from 18 of these individuals were collected twice with at least 1 year between sampling times. From 11 seals, only perinatal milk was obtained, from 3 seals only nonperinatal milk was obtained, and from four seals both perinatal and nonperinatal milk was obtained. Ratios for individual BDE congeners were calculated as the more recent concentration divided by the previous concentrations (Figure S3). No clear trends of changes in PBDE concentrations over time were discernible across all paired milk samples. These results contrast with time trends observed for PBDEs in the northern hemisphere.

In the Great Lakes, PBDE concentrations in fish peaked mostly from 1999 to $2000 .{ }^{19}$ Further away from source regions, PBDE concentrations in ringed seals in East Greenland started to decrease in the early $2000 \mathrm{~s},{ }^{30}$ a trend reported for most Arctic biota. ${ }^{31}$ PBDEs also decreased in Canadian seabirds with a significant and rapid decline after 2003.32 An analysis of PBDE mass flows in the U.S. and Canada predicted penta and octa-BDEs stocks to peak in their use around 2004, whereas BDE-209 stock peaked in 2008. ${ }^{15}$ These diverging trends between the northern hemisphere and our results for the Western Antarctic suggest that transport of PBDEs to the remote Southern Hemisphere has been delayed by a decade or so relative to the Arctic region, which is closer to the primary source region (Figure 3). The vast majority of PBDEs were produced and used in the northern hemisphere; ${ }^{33}$ while they are certainly present in the southern hemisphere, it is unclear whether PBDEs in Antarctica are predominantly reflecting southern or northern hemisphere sources.

Perinatal vs Nonperinatal Milk. After breeding, female fur seals from Cape Shirreff spend up to 8 months away at one of three foraging grounds (off the Chilean coast, Patagonian shelf break, or around South Georgia) during the austral winter. ${ }^{34}$ Perinatal milk thus represents PBDEs accumulated during the winter migration. In contrast, nonperinatal milk reflects PBDEs accumulated while foraging offshore Cape Shirreff's breeding beaches and any mobilization of PBDEs from lipid reserves. Across all fur seal milk samples, there were few significant differences in PBDE concentrations between perinatal and nonperinatal milk. The exception was for the 2012/2013 6599

DOI: 10.1021/acsomega.8b00440
ACS Omega 2018, 3. 6595-6604 


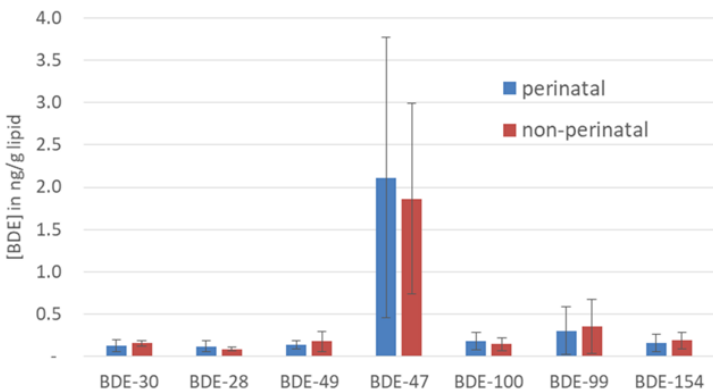

Figure 4. Comparison of $\mathrm{BDE}$ concentrations in perinatal and nonperinatal milk sampled from fur seals sampled twice during the same season $(n=$ 10). Error bars represent one standard deviation.

(a)

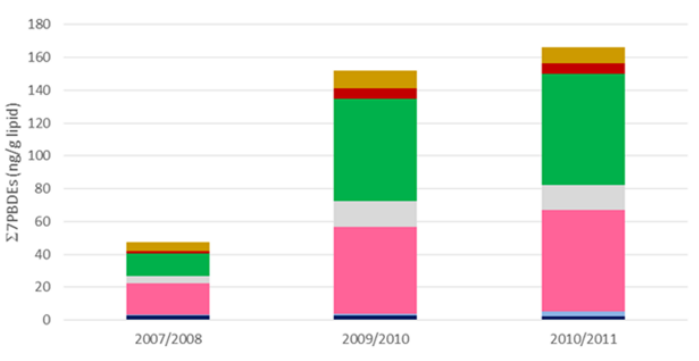

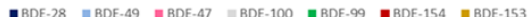

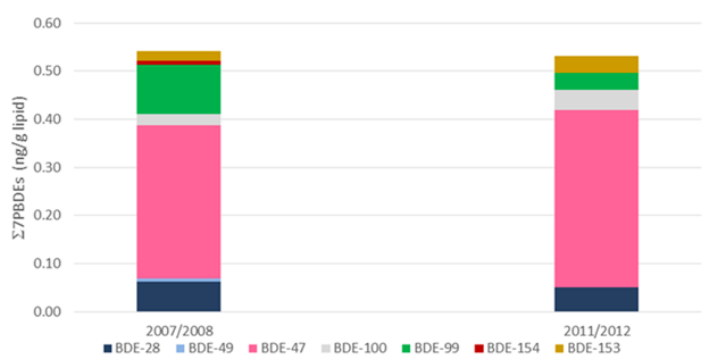

Figure 5. Average $\sum$ PBDEs (ng/g lipid) in (a) phytoplankton and (b) krill per sampling season. Note the uneven interval between years.

sampling period, in which mean \pm SD concentrations of BDE$49(0.33 \pm 0.34 \mathrm{ng} / \mathrm{g}$ lipid $)$ and BDE-99 $(0.28 \pm 0.19 \mathrm{ng} / \mathrm{g}$ lipid) in nonperinatal milk were significantly higher than those in perinatal milk $(0.19 \pm 0.15$ and $0.15 \pm 0.10 \mathrm{ng} / \mathrm{g}$ lipid, respectively; Mann-Whitney rank sum test $p<0.05$ ). This is consistent with expectations based on the maternal body burden of lipid-bound pollutants being passed onto the pup during lactation, particularly in the case of the first pup. ${ }^{35}$
The previous discussion was based on average values from randomly milked fur seals both perinatally and nonperinatally. Again, milk from several fur seals was sampled twice during the same season ( $n=10$ : nine from 2011/2012, one from 2012/ 2013). We thus compared PBDE concentrations in perinatal and nonperinatal milk collected from the same individuals, typically 6 weeks apart. There was no significant difference in individual PBDE congener concentrations between perinatal and nonperinatal milk (Figure 4). On average, fur seals 
accumulated similar BDE concentrations during their winter migration and feeding away from Cape Shirreff as they did while foraging offshore Cape Shirreff's breeding beaches. This implies that within their breeding area and foraging region PBDEs are present in similar concentrations and/or the importance of mobilizing PBDEs from their lipid reserves while at Cape Shirreff.

The average values mask a wide range of individual changes in PBDE concentrations (note large standard deviations in Figure 4). For two fur seals, PBDEs decreased about 2-fold over the course of their foraging trips around Cape Shirreff, whereas it increased for two other seals about 2-fold over this period. Median ratios of nonperinatal divided by perinatal milk were greatest for BDE-183 and BDE-99 implying preferential accumulation of these congeners off Cape Shirreff and/or their less-efficient biodilution within that time period. Overall, profiles of PBDEs in perinatal and nonperinatal milk were very similar (Figure 4), suggesting similar uptake/metabolism of these chemicals in fur seals.

In summary, there is a wide range of trends of $\mathrm{BDE}$ concentrations in individual fur seal milk sampled approximately 9 weeks apart. On the contrary to expectations, there were no significant trends of decreasing concentrations once fur seals started feeding off Cape Shirreff (Figure 4). This might indicate that fur seals, in some years, forage locally prior to coming ashore to give birth.

Phytoplankton. Lipid values in phytoplankton were very low ranging from 0.1 to $6.7 \%$; only samples with lipid values $>0.5 \%$ are presented here to avoid biasing PBDE concen$>0.5 \%$ are prese
trations high.

Phytoplankton $\sum_{P}$ PBDEs ranged from 3.8 to $320 \mathrm{ng} / \mathrm{g}$ lipid with a mean $\pm S D$ of $53 \pm 76 \mathrm{ng} / \mathrm{g}$ lipid (median of $26 \mathrm{ng} / \mathrm{g}$ lipid, Table S23). BDE-47 and -99 were the two most prevalent congeners (Figure 5), with detection 97 and $91 \%$ of the time, respectively. Previously reported PBDE concentrations in plankton in 2005 were 2-3 times lower (Table 1). Macroalgae in the Canadian Arctic similarly contained $\sum_{15}$ PBDEs at 320 $\mathrm{ng} / \mathrm{g}$ lipid. $^{11}$

Stable isotope analysis of $\delta^{15} \mathrm{~N}$ on phytoplankton samples resulted in a wide range of values from -1.1 to $6.1 \%$. We note that although the dominant composition of the plankton samples were identified as phytoplankton (i.e., diatoms or Phaeocystis sp.), samples may have some microzooplankton present despite efforts to remove any nonphytoplankton species. The $\delta^{13} \mathrm{C}$ values of phytoplankton also had a large range, spanning from -33 to $-19 \%$, with a mean of $-29 \pm$ $0.61 \% 0$ (median of $-31 \%$ ).

Phytoplankton Trends. Few significant correlations were detected between PBDE phytoplankton concentrations and $\delta^{15} \mathrm{~N}$ values except for the $2010 / 2011$ season, where almost all congeners detected showed a negative trend of decreasing concentration with increasing $\delta^{15} \mathrm{~N}$ value. BDE-47, -100, -99, $-154,-153$, and $\sum_{7}$ PBDEs versus $\delta^{15} \mathrm{~N}$ all had significantly negative trends $(p<0.05)$

Correlations of phytoplankton PBDE concentrations versus sampling time were only significant for the 2010/2011 sampling season, in which all congeners with $>30 \%$ detection (BDE-28, -49, -47, -100, -99, -154, -153, and $\sum_{7}$ PBDEs) have significantly negative trends; i.e., PBDE concentrations decreased toward the end of austral summer $(p<0.05)$. The austral summer 2010/2011 sampling season, spanning the period from December to March, was longer than the 2007/ 2008 or $2009 / 2010$ seasons. The temporal trend in the $2010 /$
2011 austral summer may reflect a spike in concentrations picked up from the snow/ice melt early in the austral summer, with either a fading signal or dilution occurring as the season progressed. Legacy organic contaminants (e.g., PCBs, DDT, polycyclic aromatic hydrocarbons) have been detected in snow packs and glacial ice from both Arctic and Antarctic environments, and it has been proposed that in colder regions, where the timing of the melt may be more concentrated as compared to a temperate environment, there is a stronger pulse of organic contaminants released to the surrounding water column ${ }^{36}$ Chiuchiolo et al. in 2004 detected various OCPs and BDEs $(-47,-99$, and -100$)$ in phytoplankton and suggested that phytoplankton incorporated POPs from snow and ice melt. Furthermore, POPs may be removed from the water column via sedimentation and organic carbon particle export, which occurs in a relatively short time following phytoplankton blooms in this region (i.e., December and January) ${ }^{5}$ Additionally, Geisz et al. in 2008 present further evidence of glacial meltwater acting as a source of, at least, DDT to the Antarctic marine food web. ${ }^{24}$

There was a significant difference $(p<0.05$, one-tailed twosample $t$ test assuming unequal variances) between diatoms and Pharocystis for BDE-28, -47, -100, -153, and $\sum_{7}$ PBDEs, with Phaeocystis sp. having greater mean concentrations than diatoms. Similarly, the mean PBDE concentrations in phytoplankton $\left(\delta^{15} \mathrm{~N}\right.$ values $<2 \%$ ) were greater than the mean of phytoplankton with possibly greater microzooplankton contamination $\left(\delta^{15} \mathrm{~N}\right.$ values $\left.>2 \% 0\right)$ for BDE-153 $(p=0.036)$ and with lower significance for $\sum_{7}$ PBDEs $(p=0.059)$.

Krill. Average lipid \% in krill ranged from 14 to $33 \%$, much greater than for phytoplankton. PBDEs were detected in all krill samples, with lower concentrations and less variation in contaminant concentrations than was observed for phytoplankton. The average $\sum_{7} \mathrm{PBDEs}$ ranged from 0.14 to $3.5 \mathrm{ng} / \mathrm{g}$ lipid with a mean $\pm \mathrm{SD}$ of $0.61 \pm 0.57 \mathrm{ng} / \mathrm{g}$ lipid (median of 0.49 $\mathrm{ng} / \mathrm{g}$ lipid). BDE-47 was the dominant congener present in all size classes of krill, averaging around $70 \%$ of the total composition (Table S24, SI), followed by BDE-28 and -99 ,

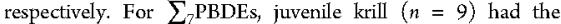
highest concentrations among $E$. superba age classes with a mean of $0.65 \pm 0.27 \mathrm{ng} / \mathrm{g}$ lipid, followed by adult krill $(n=18)$ with a mean of $0.51 \pm 0.78 \mathrm{ng} / \mathrm{g}$ lipid, and gravid krill $(n=7)$ with a mean of $0.35 \pm 0.19 \mathrm{ng} / \mathrm{g}$ lipid (Figure S2). The two Thysanoessa sp. krill samples displayed fairly high concentrations at $\sum$ PBDEs of 1.2 and $0.66 \mathrm{ng} / \mathrm{g}$ lipid. Previously reported PBDE concentrations in krill in 2005 were at least 10 times greater than what we measured, possibly indicating contamination (Table 1$)^{5}$

Stable isotope analysis was performed on a subset of each size class of krill, with the exception of Thysanoessa sp. The $\delta^{15} \mathrm{~N}$ values (mean $+\mathrm{SD}$ ) were similar among the different size classes: $4.0 \pm 0.58 \%$ for juvenile krill, $4.2 \pm 0.19 \%$ for adult krill, and $4.1 \pm 0.43 \% \circ$ for gravid krill. There was slightly more variability for $\delta^{13} \mathrm{C}$ values among the krill age classes; $\delta^{13} \mathrm{C}$ values (mean \pm SD) were: $-24 \pm 1.41,-25 \pm 0.47$, and $-23 \pm$ $0.68 \%$ for juvenile, adult, and gravid krill, respectively.

Trends of Krill. No significant relationships were found between krill concentration and $\delta^{13} \mathrm{C}$ or $\delta^{15} \mathrm{~N}$ value. No significant differences $(p<0.05)$ were found between krill concentration means from the two sampling years of 2007 2008 and 2010/2011 (Figure 5). When comparing different size classes of krill (i.e., juveniles vs adults, adults vs gravid, gravid vs Thysanoessa sp.), BDE-47 and $\sum_{7}$ PBDEs were both

DOl: 10, 1021/7acsomega.8b00440
AC5 Omega 2018, $3,6595-6504$ 
found to be significantly higher in juveniles than adults $(p<$ 0.05 ).

Fish. Lipid values in fish ranged from 22 to $52 \%$. PBDEs were not detected in any of the five fish samples. Low masses of BDE-47 and $-99(0.1-0.2 \mathrm{ng} / \mathrm{sample})$ were initially determined, however, after blank corrections, all PBDEs in fish samples were $<$ LOD (Table 1). Previous studies have detected PBDEs in Antarctic fish samples, though they have generally been able to extract larger amounts of tissue. $3,4,9,10,37,38$ The total ranges of $\delta^{15} \mathrm{~N}$ and $\delta^{13} \mathrm{C}$ values for fish samples were 9.2 to 11 and -24 to $-21 \% 0$, correspondingly. For the myctophid fish species, the mean \pm SD of $\delta^{15} \mathrm{~N}$ values was $9.5 \pm 0.42$ and -24 $+0.24 \%$ for $\delta^{13} \mathrm{C}$. In comparison, the mean $+\mathrm{SD}$ of Antarctic silverish $\delta^{15} \mathrm{~N}$ and $\delta^{13} \mathrm{C}$ values were both higher $(11 \pm 0.63$ and $-21 \pm 1.1 \%$, respectively) than those of the myctophid fish species.

\section{IMPLICATIONS}

Biota from other parts of the world, primarily regions closer to industrialized areas, have started to see a reduction in PBDE concentrations as a reflection of their phase-out. ${ }^{17,18,30,32}$ Time trends of PBDEs in Antarctic biota strongly demonstrate that a decrease of PBDE concentrations in Antarctic biota over the last decade has not (yet) occurred. These surprising results indicate the need for further research to see if and when PBDE concentrations in the Antarctic will start declining as reported in the Arctic. Similarly, the phase-out of PBDEs has led to various novel flame retardants being detected in the environment. $^{39,40}$ As of yet, it is unclear whether these new flame retardants have been transported to Antarctica and have been accumulating in Antarctic biota. This will be an important research area in the near future.

Comparing trends of PBDEs in milk from randomly selected fur seals over several years were by and large similar to trends of the same fur seals milked years apart. Results show that there are large variations in PBDE trends in individuals over time, highlighting the need for large sample sets to determine representative trends. A better understanding of time trends in individual seals is warranted but would require a concerted effort to resample the same individuals over several years, ideally collecting both perinatal and nonperinatal milk.

The high concentrations of PBDEs in phytoplankton compared to the upper trophic level Antarctic fur seal were unexpected but not unprecedented and illustrate the complexity of the Antarctic food web and sampling under challenging circumstances (Table S25). For the phytoplankton sample collections on board a ship, we cannot rule out the possibility of contamination by PBDEs during sampling. ${ }^{41,42}$ Trends of PBDEs in biota are further complicated by the presence of point sources, such as snow and ice melt, which can release pollutant pulses in the austral summer. Finally, the differences in geographic sampling of biota (which were logistically constrained) and the migratory nature and diverse diet of Antarctic fur seals further add complexity to understanding PBDE trends in biota. Monitoring emerging pollutants in remote regions, such as Antarctica, can highlight important global trends of contaminants of concern and further our understanding of long-range transport and global response times to pollutant dynamics.

\section{ASSOCIATED CONTENT}

S Supporting Information

The Supporting Information is available free of charge on the ACS Publications website at DOI: 10.1021 /acsomega.8b00440. Additional details related to sample IDs, chemical analysis, and concentrations of PBDEs (PDF)

\section{- AUTHOR INFORMATION}

Corresponding Author

*E-mail: rlohmann@uri.edu. Phone: 401-874-6612. Fax: 401874-6811.

ORCID

Rainer Lohmann: 0000-0001-8796-3229

\section{Present Addresses}

${ }^{\nabla}$ Department of Environmental Sciences, Faculty of Science, Alexandria University, 21511 Moharam Bek, Alexandria, Egypt (M.K.).

"University of California Santa Cruz, 1156 High Street, Santa Cruz, California 95064, United States (E.K.B.).

Notes

The authors declare no competing financial interest.

${ }^{\perp}$ Author is deceased.

\section{- ACKNOWLEDGMENTS}

R.L. acknowledges funding from the National Science Foundation (ANT 1332492) to understand emerging pollutant dynamics in the Antarctic. We acknowledge Hugh Ducklow (LDEO) and the National Science Foundation Palmer LTER (PLR 1440435) for intellectual and logistical support and thank Rebecca Dickhut (Virginia Institute of Marine Science) for her prior leadership on this grant. We thank Dr. Deborah Steinberg, Kate Ruck (Virginia Institute of Marine Science), and Dr. Heid Geisz (Florida State University) for sample collection. We are grateful to the many field assistants that helped in capture and sample collection of fur seal milk, especially G.I. McDonald, Y. Tremblay, J. Lyons, R. Burner, R. Buchheit, N. Pussini, C. Bonin, and K. Pietrzak. We are also grateful for the support of Drs. Rennie S. Holt and George Watters, directors of the USAMLR program, and to Dr. D.P. Costa, UCSC for thei support of Pinniped research at Cape Shirreff. All Antarctic fur seal research and sample collection were conducted under Marine Mammal Protection Act Permit Nos. 1024, 774-1649, 774-1847, and 16472-01 granted by the Office of Protected Resources, National Marine Fisheries Service.

\section{REFERENCES}

(1) Council of Managers of National Antarctic Programs (COMNAP). Antarctic Facilities List https://www.comnapaq/ Members/SiteAssets/SitePages/Home/COMNAP Antarctic Facilities List 31 March 2017.xlsx.

(2) Wania, F.; MacKay, D. Tracking the distribution of persistent organic pollutants. Emviron. Sci. Technol. 1996, 30, 390A-396A

(3) Borghesi, N.; Corsolini, S.; Focardi, S. Levels of polybrominated diphenyl ethers (PBDEs) and organochlorine pollutants in two species of Antarctic fish (Chionodraco hamatus and Trematomus bernacchit). Chemosphere 2008, 73, 155-160.

(4) Corsolini, S.; Covaci, A; Ademollo, N.; Focardi, S.; Schepens, P. (4) Corsolini, S.; Covac1, A; Ademollo, N.; Focardi, S.; Schepens, P. Occurrence of organochlorine pesticides (OCPs) and their enantiomeric signatures, and concentrations of polybrominated dipheny Pollut. 2006, 140, 371-382. 
(5) Chiuchiolo, A. L.; Dickhut, R. M.; Cochran, M. A; Ducklow, H. W. Persistent organic pollutants at the base of the Antarctic marine food web. Environ. Sci. Technol. 2004, 38, 3551-3557.

(6) Bravlt, E. K. Goebel, M. E. Geisz, H. N. Canuel, E. A.; Dickhut, R. M. Inter-Annual Variation of Persistent Organic Pollutants (POPS) in an Antarctic Top Predator Arctocephalus gazella. Environ. Sci Technol. 2013, 47, 12744-12752.

(7) Khairy, M. A; Luek, J. L.; Dickhut, R; Lohmann, R. Levels, sources and chemical fate of persistent organic pollutants in the atmosphere and snow along the western Antarctic Peninsula. Environ Pollut. 2016, 216, 304-313.

(8) Vecchiato, M.; Argiriadis, E.; Zambon, S.; Barbante, C.; Toscano, G.; Gambaro, A; Piazza, R. Persistent Organic Pollutants (POPs) in Antarctica: Occurrence in continental and coastal surface snow. Microchem. J. 2015, 119, 75-82.

(9) Cincinelli, A; Martellini, T.; Pozo, K; Kukucka, P.; Sáňka, O. Corsolini, S. Trematomus bernacchii as an indicator of POP temporal Corsolini, S. Trematomus bernacchii as an indicator of POP temporal
trend in the Antarctic seawaters. Environ. Poilut. 2016, 217, 19-25.

(10) Hale, R. C.; Kim, S. L.; Harvey, E.; La Guardia, M. J.; Mainor, T. M.; Bush, E. O.; Jacobs, E. M. Antarctic research bases: local sources of polybrominated diphenyl ether (PBDE) flame retardants. Environ. Sci. Technol. 2008, 42, 1452-1457.

(11) Wild, S.; McLagan, D.; Schlabach, M.; Bossi, R; Hawker, D.; Cropp, R; King, C. K.; Stark, J. S.; Mondon, J.; Nash, S. B. An Antarctic research station as a source of brominated and perfluorinated persistent organic pollutants to the local environment. Environ. Sci. Technol. 2015, 49, 103-112.

(12) Hale, R C.; Alaee, M.; Manchester-Neesvig, J. B.; Stapleton, H M.; Ikonomou, M. G. Polybrominated diphenyl ether flame retardants M.; lkonomou, M. G. Polybrominated diphenyl ether flame retardants in the North American environment. Enwiron. Int. 2003, 29,771-779.
(13) United Nations Environment Programme. The New POPs, (13) United Nations Environment Programme. The New POPs,
2018. http:/ wwwwipo int/edocs/lexdocs/treaties/en/unep-pop/trt 2018. http://www.wipo int/edocs/lexdocs/

unep_pop_2.pdf (accessed May 2, 2018).
(14) Hites, R. A. Critical Review Polybrominated Diphenyl Ethers in (14) Hites, R. A. Critical Review Polybrominated Diphenyl Ethers in
the Environment and in People: A Meta-Analysis of Concentrations. Enwiron. Sci. Technol. 2004, 38, 945-956.

(15) Abbasi, G.; Buser, A. M.; Soehl, A; Murray, M. W.; Diamond, M. L. Stocks and Flows of PBDEs in Products from Use to Waste in the U.S. and Canada from 1970 to 2020. Environ. Sci. Technol. 2015, 49, 1521-1528.

(16) Sacks, V. P.; Lohmann, R. Freely dissolved PBDEs in water and porewater of an urban estuary. Environ. Pollut. 2012, 162, 287-293.

(17) Darnerud, P.; Lignell, S.; Aune, M.; Isaksson, M.; Cantillana, T; Redeby, J.; Glynn, A. Time trends of polybrominated diphenylether (PBDE) congeners in serum of Swedish mothers and comparisons to breast milk data. Emivirn. Res. 2015, 138, 352-360.

(18) Airaksinen, R; Hallikainen, A; Rantakokko, P.; Ruokojarvi, P.; Vuorinen, P. J.; Parmanne, R.; Verta, M.; Mannio, J.; Kiviranta, H. Time trends and congener profiles of $\mathrm{PCDD} / \mathrm{Fs}, \mathrm{PCBs}$, and PBDEs in Baltic herring off the coast of Finland during 1978-2009. Chemosphere $2014,114,165-171$

(19) Crimmins, B. S.; Pagano, J. J.; Xia, X; Hopke, P. K; Milligan, M. S.; Holsen, T. M. Polybrominated Diphenyl Ethers (PBDEs): Turning the Corner in Great Lakes Trout 1980-2009. Environ. Sci. Technol. 2012, 46, 9890-9897.

(20) Corsolini, S.; Borghesi, N.; Schiamone, A; Focardi, S. (20) Corsolini, S.; Borghesi, N.; Schiamone, A; Focardi, S.
Polybrominated diphenyl ethers, polychlorinated dibenzo-dioxins,
-furans, and -biphenyls in three species of Antarctic penguins. Environ. -furans, and -biphenyls in three species
Sci. Pollut Res. 2007, 14, 421-429.

Sci. Pollut. Res. 2007, 14, 421-429.
(21) Kelly, B. C.; Ikonomou, M. G.; Blair, J. D.; Gobas, F. A. P. C. Bioaccumulation behaviour of polybrominated diphenyl ethers (PBDEs) in a Canadian Arctic marine food web. Sci. Total Environ 2008, 401, 60-72.

(22) Polito, M. J.; Goebel, M. E. Investigating the use of stable isotope analysis of milk to infer seasonal trends in the diets and foraging habitats of female Antarctic fur seals. J. Exp. Mar. Biol Ecol $2010,395,1-9$.

(23) Brault, E. Evaluating Persistent Organic Pollutants (POPs) and Mercury in the West Antarctic Peninsula (WAP) Food Web, with a
Focus on Antarctic Fur Seals (Arctocephalus gazella). M.S. Thesis, Virginia Institute of Marine Sciences, Gloucester Point, VA, 2012. (24) Geisz, H. N.; Dickhut, R. M.; Cochran, M. A; Fraser, W. R. Ducklow, H. W. Melting glaciers: A probably source of DDT to the Ducklow, H. W. Melting glaciers: A probably source of DDT to the 3962.

(25) Bengtson Nash, S. M.; Poulsen, A. H.; Kawaguchi, S.; Vetter, W; Schlabach, M. Persistent organohalogen contaminant burdens in Antarctic krill (Euphausia superba) from the eastern Antarctic sector: A baseline study. Sci. Total Environ. 2008, 407, 304-314.

(26) Galbán-Malagón, C. J.; Hernán, G.; Abad, E.; Dachs, J. Persistent organic pollutants in krill from the Bellingshausen, South Scotia, and Weddell Seas. Sci. Total Environ. 2018, 610-611, 14871495

(27) Corsolini, S; Ademollo, N.; Martellini, T.; Randazzo, D Vacchi, M.; Cincinelli, A. Legacy persistent organic pollutants including PBDEs in the trophic web of the Ross Sea (Antarctica). Chemosphere 2017, 185, 699-708

(28) Cipro, C. V. Z.; Bustamante, P.; Taniguchi, S.; Montone, R. C Persistent organic pollutants and stable isotopes in pinnipeds from King George Island, Antarctica. Mar. Pollut. Bull. 2012, 64, $2650-$ 2655 .

(29) Trumble, S. J.; Robinson, E. M.; Noren, S. R.; Usenko, S.; Davis, J.; Kanatous, S. B. Assessment of legacy and emerging persistent organic pollutants in Weddell seal tissue (Leptonychotes weddellii) near McMurdo Sound, Antarctica. Sci. Total Environ 2012, 439, 275-283. (30) Vorkamp, K; Riget, F.; Bossi, R; Dietz, R. Temporal Trends of Hexabromocyclododecane, Polybrominated Diphenyl Ethers and Polychlorinated Biphenyls in Ringed Seals from East Greenland. Environ. Sci. Technol 2011, 45, 1243-1249.

(31) de Wit, C. A.; Herzke, D.; Vorkamp, K. Brominated flame retardants in the Arctic environment - trends and new candidates. $S_{c i}$ Total Environ. 2010, 408, 2885-2918.

(32) Braune, B. M.; Letcher, R. J.; Gaston, A. J.; Mallory, M. L. Trends of polybrominated diphenyl ethers and hexabromocyclododecane in eggs of Canadian Arctic seabirds reflect changing use patterns. Environ. Res. 2015, 142, 651-661.

(33) Alaee, M.; Arias, P.; Sjodin, A; Bergman, $\AA$ An overview of commercially used brominated flame retardants, their applications, their use patterns in different countries/regions and possible modes of release. Environ. Int. 2003, 29, 683-689.

(34) Arthur, B.; Hindell, M.; Bester, M.; De Bruyn, P. J. N.; Trathan, P.; Goebel, M.; Lea, M.A. Winter habitat predictions of a key Southern Ocean predator, the Antarctic fur seal (Arctocephalus gazella). Deep Sea Res, Part II 2017, 140, 171-181.

(35) Beckman, K. B.; Ylitalo, G. M.; Towell, R. G.; Krahn, M. M. O'Hara, T. M.; Blake, J. E. Factors affecting organochlorine contaminant concentrations in milk and blood of northern fur sea (Callorhinus ursinus) dams and pups from St. George Island, Alaska. Sci. Total Environ. 1999, 231, 183-200.

(36) Wania, F.; Ho, J. T.; Jia, C. Q.; Mackay, D. The effects of snow and ice on the environmental behaviour of hydrophobic organic chemicals. Environ. Pollut. 1998, 102, 25-41.

(37) Lana, N. B.; Berton, P.; Covaci, A.; Ciocco, N. F. N. F.; BarreraOro, E.; Atencio, A. A; Altamirano, J. C. Fingerprint of persistent organic pollutants in tissues of Antarctic notothenioid fish. Sci. Total Environ. 2014, 499, 89-98.

(38) Borghesi, N.; Corsolini, S.; Leonards, P.; Brandsma, S.; de Boer, J.; Focardi, S. Polybrominated diphenyl ether contamination levels in fish from the Antarctic and the Mediterranean Sea. Chemosphere 2009 $77,693-698$.

(39) Hoh, E.; Hites, R A Brominated flame retardants in the atmosphere of the east-central United States. Environ. Sci. Technol 2005, 39, 7794-7802.

(40) Ma, Y.; Salamova, A; Venier, M.; Hites, R. A. Has the PhaseOut of PBDEs Affected Their Atmospheric Levels? Trends of PBDE and Their Replacements in the Great Lakes Atmosphere. Enwiron. Sci. Technol. 2013, 47, 11457-11464. 
(41) Lohmann, R; Klanova, J.; Kukucka, P.; Yonis, S.; Bollinger, K Concentrations, fluxes, and residence time of PBDEs across the tropical Atlantic Ocean. Environ. Sci. Technol. 2013, 47, 13967-13975. (42) Lohmann, R.; Jaward, F. M.; Durham, L; Barber, J. L; Ockenden, W.; Jones, K C.; Bruhn, R; Lakaschus, S.; Dachs, J.; Booij, Ockenden, W.; Jones, K C.; Bruhn, R; Lakaschus, S.; Dachs, J.; Booij,
K Potential contamination of shipboard air samples by diffusive $\mathrm{K}$ Potential contamination of shipboard air samples by diffusive
emissions of PCBs and other organic pollutants: Implications and solutions. Environ. Sci. Technol. 2004, 38, 3965-3970. 


\title{
SPATIOTEMPORAL CHARACTERIZATION OF NON-BREEDING GREAT SHEARWATERS ARDENNA GRAVIS WITHIN THEIR WINTERING RANGE
}

\author{
KEVIN D. POWERS ${ }^{1 *}$, DAVID N. WILEY 1 ANNA R. ROBUCK 2 ZACHARY H. OLSON ${ }^{3}$, \\ LINDA J. WELCH ${ }^{4}$, MICHAEL A. THOMPSON ${ }^{1}$ \& LES KAUFMAN ${ }^{5}$
IStelwagen Bank National Marne Sanchuary. NOAA National Ocean Service. Scituate, MA 0206o. USA *kapowers24@gmailcom! "University of Rhode lsiand, Graduate School of Oceanography, Namagansett, RI 02832, USA

Received 22 Janiwary 2020 , accepted 17 June 2020

ABSTRACT

POWERS, K.D., WILEY, D.N., ROBUCK, A.R., OLSON, Z.H, WELCH, L.J, THOMPSON, M.A \& KAUFMAN, L, 2020. Spatioteinporal characterization of non-breeding Great Shearwaters Ardenna gravis withio their wintering range. Marne Omitholagy 48: 215-229.

Movements of Great Shearwaters Ardenna gravis wintering in the Northwest Alantic showed age-based spatial and temporal flexibility, with fotaging tactics linked to a defined physical preference of their primary prey. From 2013 to 2018, we tracked 58 Great Shearwaters duting their wintering season using platform teminal transmittess deployed in the same area of the southwest Gulf of Maine. Utilization distributions (UDs) for individual birds were created from convex lulls, which were then combined for spatial and einporal analyses. of the $95 \%$ kenel UDs, $55^{\circ}$ were contained within the Gulf of Maine and the remainder extended to the Scotian Shelf off Nova Scotia and the Grand Banks off New foundland. Analysis of fecal DNA from tagged bits and others captured with them indicated that Nothern sand lance Ammodytes ditbius were the prinary prey while in the Gulf of Maine. This relationship was suppoted by the overlap of UDs and sand lanee habitat. The spatial ceurrence of sand lance from botomfish trawl survey data demonstrated that these fish preferred shallow water $(<100 \mathrm{~m}$ deep) with subsirates consisting of high sand content (> 50\%) and grain sizes ranging from $0.35-2.00 \mathrm{~mm}$ in diameter. These same properties were associaled and spatially aligned with the collective $25 \%$ kemel UD of Great Shearwaters. Necropsy of bycaught Great Shear waters from an atea that overlapped in space and time with tagged individuals and sand lanee habitat demonstrated that most biris $(89 \%)$ were young (0-2 years), based on gonadal development, molt score, andor bursa of Fabricius. Couphing demographic infornation from necropsies with spatial habis and movement timing of tagged birds suggests this region serves as a winter "nursery" for Creat Shearwaters.

Key words: Great Shearwaters, utilization distribution, wintering range, satellite tracking, fecal DNA analysis, sandlance, bycatch tiecropsies

\section{INTRODUCTION}

The distribution of marine vertebrates is the prodnct of a herarchat structure of processes driven at multiple spatiotemporal scales (Mannoci ef al. 2017). Many of these driving processes are in flux due to human-induced or climatically mediated stress, resuting in changes in faunal distribution and abundance (Nye et al. 2009 Moore et al. 2018). In seabirds, shifting enviromnental conditions ate implicated in precipitous dedines actoss taxa and ecoregion (Croxall et al. 2012, Palecany of al. 2015, Dias et al 2019). Characterizing corrent distribution patterns of seabirds is critical to identifying and managing seabird and ecosystem tesponses to cument and predicted environmental changes (González-Solis \& Slaffer 2009, Faleczny el al. 2015), and there is a paucity of dat for juyenile seabirds in particular (Hazen er al. 2012). However, in order to be rigorous tools for ecosystem. management, descriptions of seabird distributions nust be augmented with explanations of the physical and biological processes that contribute to the dynamic nature of such patens (Fauchald of al. 2000. Fauchald 2009, Tremblay et al. 2009 ).

Seabirds may forage across vast coastal and pelagic areas during breeding phases (Weimerskitch et al. 1993, Weimerskitch et at 1994, Jouventin et al. 1994), and many undertake trans-equatorial migrations beween non-breeding and breeding locations (Schaffer et al 2006, González-Solís ef al. 2007, Hedd et al. 2012). Great Shearwaters Ardenna gravis breed on islands in the Sonth Allantic and spend their wintering season in the North Alantic arriving in the western half in May. They retum to the Sonthem Hemisphere passing down the east-central Aflantic starting in September (Browt1 1986). Juverniles and sub-adnits arrive in the western North Alantic later (Inly) and are thought to return south in November, after breeding adults (Brown 1986, Powers et al. 2017). The boreal summer distribution of Great Shearwaters off the notheastem United States and Allantic Canada has teen examined in parl by a series of loosely connected and spatially overlapping studies using shipboatd and aerial obsetvations (Powers 1983, Brown 1986, Winship et al. 2018. Wong et al. 2018, Carvalho \& Davoren 2019). These studies indicate foraging flexibility of wintering shearwaters based on biophysical drivers. For example, Great Shearwaters exhibited inter-annual variability and dietary partitioning with Sooty Shearwaters A. grisec as prey availability shifted within the Bay of Fundy (Ronconi ef al. 2010), Great Shearwater movements within the Gulf of Maine, more tecently characterized with satellite tracking data (Powers et al 2017), showed that bitds preferted to forage in shallower waters $(<100 \mathrm{~m})$, primarily over sloped tims fringing deeper central basins. However, this analysis of foraging, babitat showed inconsistent telationships in terms 
of depth, chlorophyll $a$, and sea surface temperature between Georges Bank/the southern Gulf of Maine and the Bay of Fundy (Powers et al. 2017). The breeding biology of two closely related shearwaters, Short-tailed Shearwater A tenuirostris and Cory's Shearwater Calonectris borealis (Bradley et al. 1999, Campioni $e$ al. 2020), indicates that age-specific demographics may also play an important role in Great Shearwater movement ecology in the Gulf of Maine, which is postulated as a wintering area for mostly nonbreeding birds (Huettmann \& Diamond 2000, Powers et al. 2017, Ronconi et al. 2018)

Platform terminal transmitters (PTTs) provide geographical information that is tracked by Argos, an international satellite system. Using this information, researchers can describe foraging and wintering ranges, migration corridors, and seasonal movements of seabirds (e.g., Pinet et al. 2011, Jodice et al. 2015). Such information allows mapping of the broad-scale distribution of a population, which is often related to oceanographic variables. Spatial data can also provide insight into fine-scale distribution which is mostly influenced by prey distribution (Schneider \& Piatt 1986, Fauchald et al. 2000, Davoren et al. 2003, Suryan et al. 2016). However, exploration into the role of prey in seabird distributions requires further examination of seabird diet in tandem with tracking data.
Here, we report results of Argos data from PTTs placed on 58 Great Shearwaters that were captured in the southwestern Gulf of Maine over six years (2013-2018). We used these results to characterize the spatial and temporal extent of a defined portion of their wintering range in the Northwest Atlantic over a timeframe that can accommodate cycles of seasonal and inter-annual variability. We explored differences in utilization distributions (UDs), estimating range sizes and interactions with other individuals on an annual basis. We derived behavioral traits from the aggregated spatial data to assess revisitation and flight pattems between areas of differing foraging intensity, in order to corroborate if foraging behaviors align with changes in bird density. Temporal shifts in overall distribution over the Northwest Atlantic were examined on a monthly basis to determine the timing of bird movement through the region and the extent of movement between and within areas in the study region. Characteristics of key foraging areas and the distributions of preferred prey were compared with information on prey selection, which was determined by fecal DNA analysis. Age demographics were determined from primary molt scores and the timing of departure, in comparison with a gonad and bursa analysis of fishery-related bycaught birds. We suggest that primarily young non-breeding Great Shearwaters utilize the southern Gulf of Maine and Georges Bank during their wintering period. This pattern of habitat $\mathrm{u}$ tilization is associated with the distribution of a key prey

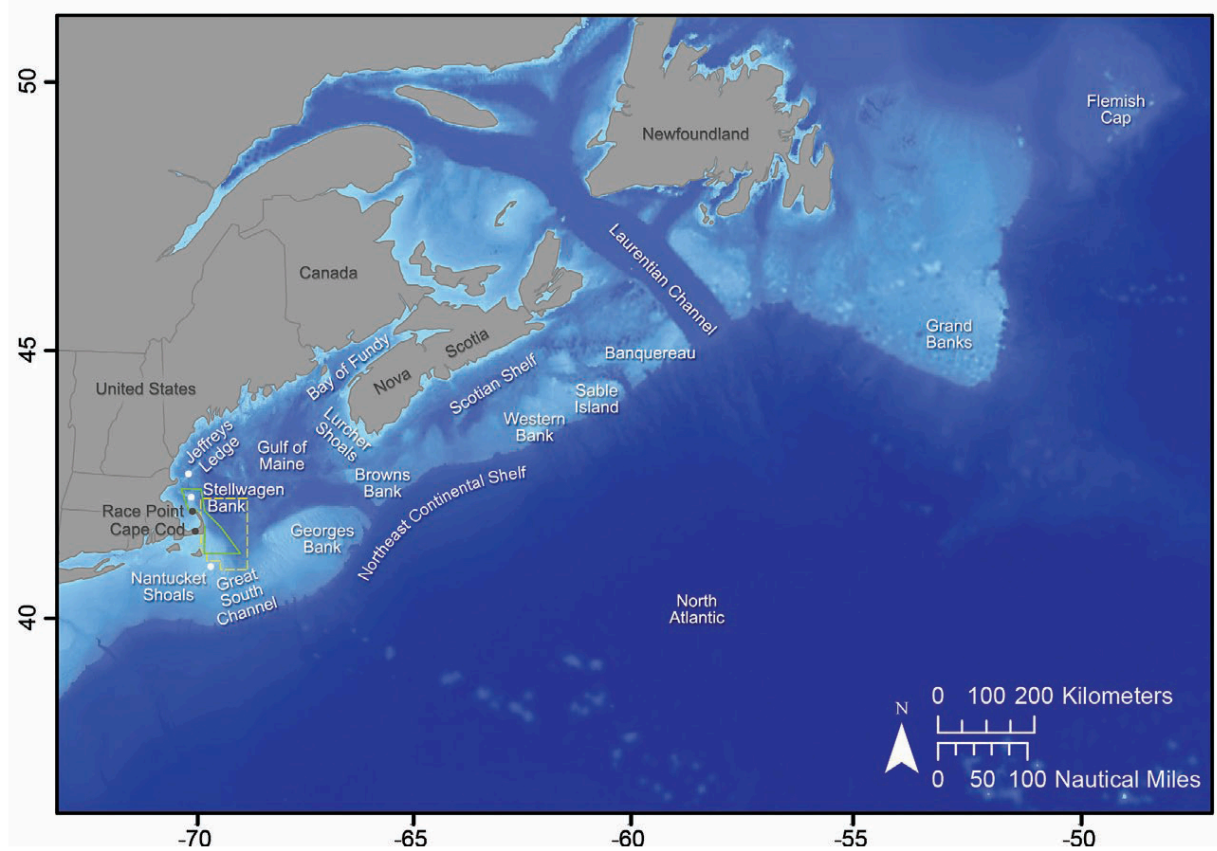

Fig. 1. Map of study area in the northwestern Atlantic Ocean. The shearwater capture area is noted by the green polygon in the sou thwestern Gulf of Maine. Bycatch specimens were collected in NOAA/NEFOP Statistical Area 521, which is noted by white dashed line east of Cape Cod, Massachusetts, USA. 
species that las distinet biological, physical, and geographical habitat preferences

\section{STUDY AREA AVD METHODS}

Data collection

We tagged Great Shearwaters from 2013 to 2018 off the coast of Massachusetts in the sonthwestern Gulf of Maine, from the Stellwagen Bank National Marine Sanchary to the coastal waters east of Cape Cod $41.3^{\circ}$ to $43.0^{\circ}$ latiude, $-70.5^{\circ}$ to $-69.5^{\circ}$ longitude; Fig. 1). We used small vessels to access birds at known foraging areas and attracted birds to the vessel using cut fish and squid. Once they were close enough to the vessel, birds were caught with handled landing nets.

Over six consecutive years, we placed PTTs on 58 shearwaters $(c a$ $10 \mathrm{yt}^{-1}$; Table 1). Solar PTT-100 ags (15 g, Microwave Telemetry, 8835 Columbia 100 Parkway, Suites K \& L. Columbia, MD 21045 , USA) wete used with duty cycles of $2 A$ hours on (i.e., continuous). Tags were attached dorsally, central to the body mass of the bird below the nape and between the wings, using four subcutareous sutures (Prolene suture 4.0 Ethicon) as per MacLeod et al. (2008). All tags eventually stopped transmitting, and we expected that they fell off when the Prolene sutures degraded. Tags represented less than $3 \%$ of body mass for all tagged birds to minimize effect on flight efficieticy (see Ptillips et al. 2003). Tags were typically deployed in Iuly, except for 2017, when poor weather condition delayed tagging until early Angust, and a single bird in Septembe 2016. Body weight and prinary nolt were scored using the BTO method (Ginn \& Melville 1983) for each tagged bird. Sex was identified genetically from blood of feather samples provided (see Acknowledgements)

Data analysis

Range estimation using kemel density

We used the Time Loeal Convex Hu1l (T-LoCoH, http//tlocohtforge.r-projectorg) method (Lyons et al. 2013) in R version 3.4 .0 (R Development Core Team 2018) to analyze our Argos location dataset. T-LoColl generalizes the non-parametric UD construction method $\mathrm{LCCH}$ (Getz et al 2007 ) and integrates time with space in the construction of local hulls through a scaling that relates distance and time to the individual's oharacteristic velocity (Lyons of al. 2013). A hull is defined as a minimum convex polygon constructed around each Atgos location point using a at of "neatest neighbots' (i.e., other Argos location points). Hulls tepresent space-use; if constructed with time, they are localized in space and time oyons et al. 2013). The resulting hulls also enable estimation of behavioral metrics describing both 1) movement phase and 2) multiple dimensions of time use, including revisitation and duration (Lyons et al. 2013). Space-state models (Jonsen et al. 2005, Jonsen et al. 2007 ) provide temporal nomality and flight behavior to asynchronons satellite telemetry data, but the incorporation of time into range construction using the T-LoCoH approach expands the concept of UDs beyond a traditional densify gradient facilitated via state-space models to spatial models of movement and time (Lyons et al. 2013). Location error is problematic with Argos locations using either method with respect to partitions of space or edges of utilization. But, compated to location etror introduced via either modeling approach, gaps in temporal resolntion have larger consequences on space and time use as well as belavioral characterizations (Powers et al. 2017 supplement). PTTs in this study transmitted continuously, and temporal resolution was limited only by satellite availability

Atgos locations wete converted to Universal Thansverse Mercator (UTM) projections usiog. Easting (X) and Northing $(Y)$ coordinates based on UTM Zone 19 for this analysis. This Merator projection preserves angles and ditection but distorts distance over multiple zones. So, we confined the analysis to an area bounded by $38^{\circ}$ to $50^{\circ}$ latitude and $-50^{\circ}$ to $-73^{\circ}$ longitude (Fig. 1). Argos location classes in the set $(3,2,1,0, A, B)$ were used for the observed data. T-LoCoH's ' $g$ ' parameter controls the degree to which the time difference between two locations is factoted into the calculation of their physical 'distance", which is used to identify 'nearest neighibors' (Lyons 2014). Nearest-neighbor selection is based on a distance metric (time-scaled distance, TSD) (Lyons et al. 2013), and we selected ' $g$ ' to scale hull creation into 24-hour intervals per Argos location. The effect of time-distance is to separate points that are far away in time epen though they may be cloce in twodimensional space (Lyons et af. 2013). Hulls were created using the adaptive a-method, which selects all points whose cumulatiwe distance to a parent point, ordered smallest to largest, is less than or equal to $a$. The aninethod (vs, r- fixed radius nethod and $k$-nearest neighbor method) produces more robust UDs because this method is the least sensitive to subopimal choices for its kemel paraneter a (Getz et al. 2007); thus, it provides good results in both dense and sparse ateas of use. In this study, UDs wete created from density isopleths of hull patent points (i.e, centroid locations) for $95 \%, 50 \%$, and $25 \%$ kemel derisites, with the $25 \%$ kernel UD representing areas of greatest bird concentration and $95 \%$ kemel UD ateas of the largest spatial extent. We then determined the amosnt of oserlap or individual interactions within these UDs, both anong individuals in the sane year and among all individuals during the entire study. Physical covatiates (depth and substrate properties) were assigned according to the parent point of each hull (centroid) using available data layers. Substrate properties included sediment sand content $(\%)$ and mean grain size ( $\mathrm{mm}$ ) for the United States northeast continental she1f (NOAANCCOS unpubl. data)

Correlation of individual range size and PTT duration was determined statistically using Pearson's product moment test. If a contration was found, we then exannined whether assumptions of homogeneity of variance differed in individual tag duration by year and number of tag days by year using Barlett's test before proceding 10 ANOVA. ANOVA (F-statistic) was used to determine whether UD groups (by year) or behavioral groups were different, using Tukey"s test in post-hoc anal yses. We also used the Kruskal-Wallis test to detemine whether characieristics (e.g, tag days, range size, body weight, moli scores, sex ratiof of population distributions were identical when we conld not assume a normal distribution.

The-ise and beharoral characteristes

Time-use metrics were used as another line of evidence to examine spatial use (Lyons ef al. 2013). We used an inter-yisit gap (IVG) petiod of $24 \mathrm{~h}$ to understand daily movements. TVG is a unit of time that must pass before another occurrence in a hull is considered a separate visit. So, for daily foraging behavior, we did not want two locations that were a day apart to be considered nearest neighbors, even if they were close in space. To explote the relationships among the distribution of hulls in time-use space, we examined dirtation 
(i.e, the number of locations in a hull within the IVG) and equated that to an amonnt of time a bitd spent in a hull on each wisit. Wo expected that birds should spend more of their time each day in areas of higher foraging intensity. We also expected that revisitation, or the number of times a bird revisited the same lull outside of the IVG, would reflect longer petiods of daily usage of an ared.

Estimation of directional movement of shearwaters was based on a behavioral metric eccentricity (Lyons $e$ al. 2013). Eccentricity is measured on the bounding ellipsoid of a hull. The eccentricity of an ellipse vaties from 0 for a perfect circle to 1 for a line. Hull with low eccentricity represent areas of non-directional movement, such as area-restricted searching or foraging, whereas higber values indicate areas where an animal is moving directionally, such as migrating or traversing habitat of low resource value (Lyons et al 2013). The a-mehod reduces the number of neighbors per hull in outlying areas, where hulls become more eccentric (more directionat travel , compared to lulls constricted in atesis of mote equidistan neighbors, which are less eccentric (more area-restricted searching) We also examined ternporal patterns of spatial use (latitude and longitude, by montb) witbin the $95 \%-50 \%, 50 \%-25 \%$, and $<25 \%$ bands of kernel density by using the aggregate datase

\section{Fecal DNA andysis}

For food habits analysis, birds were captured in 20172018 and temporarily placed into individual pet carriers on mesh netting suspended ower an aluminum foil coveted floor. Teeal material captured on the foil was collected for each individual and frozen either dry or in ethanol. Fecal samples were transported to genetics lab and stored at $-20^{\circ} \mathrm{C}$ until processing. Frozen samples were seraped into $2.0 \mathrm{~mL}$. tubes using flame-sterilized forceps and frequent giove changes. Genomic DNA was extracted from each sample using a Qiagen (Valencia, Califonia, USA) stool mini-kit following manufacturer protocols, with the following modifications: samples were incubated at $60 \mathrm{C}$ overnight in the first buffer and we added $1 \mu \mathrm{L}$ of carrier RNA after the second incubation. We employed polymerase chain reaction (PCR) to target a fragment of the mitochondrial 165 gene that is about $110-130 \mathrm{bp}$ long. We used the Chord 165 F Tag A (forward) and Chord 16S_R Shor (reverse) primers from Deagle et al. (2009) standard PCR conditions, and a blocking primer (GtSh-block1 5'-TGTGGAACTTAAAAATCAACGA-3') that we destgned fo this study to terminate in a $\mathrm{C} 3$ spacer, which wonld limit predato reads. Previous work demonstrated that this primer set (forward, reverse, and blocking) conld reconstruct vertebrate prey item from seal fecal samples collected from the Gulf of Maine, with species-level taxonomic tesolution for most ítems (K. Flanders pers comm.). Pre-PCR and PCR workflows took place in dedicated lab in separate buildings to minimize the potential for contamination.

Amplicons were cleaned and sent to a getomics facility, where they were indexed and sequenced on $1 / 8$ th of a flow cell on an Illumina (San Diego, Califomia, USA) MiSeq using $2 \times 300$ bp cbenistry (ca. two million reads). Forward and reverse reads were assembled in PANDAseq (Masella et al. 2012) using the default primer-match threshold After trimming adaptets and primess, de nowo operational taxonomic units (OTUS) were picked nsing a threshold of $97 \%$ sequence similarity using QIME2 (Caporaso et al. 2010).

OTUs with $<2$ teads were trimmed using the "phyloseq" package (McMurdie \& Holmes 2013) in R yersion 3.2.5 (R Develommen
Core Tean 2018). Renaining OTUs above the $1 \%$ threshold of Deagle et al. (2009) were identified to the lowest possible taxonomic resolution with NCBI Blast+ in the Cloud softwate accessed through Amazon Web Services Markerpiace (Seatile, Washington, USA), which queried OTUs against a server copy of the nucleotide database. Taxonony was assigned to each oTU based on the lowest expect value (e-value), with a maximum threshold of $1 \mathrm{e}-20$ (see Thomas et al. 2014). If mnltiple database matches shared the same lowest e-value, we selected the match whose spectes is known to occlu in the study area. If the oTU matched best (i.e., lowest e-value) to more than one geographically appropriate species, the oTU was assigned to the lowest taxonomy shared between the matches (e.g., family or genus instead of species. OTUs with identical taxonony wete combined using the "pbyloseq" package, and those identified as obviously not prey leg. human, shearwater) were removed as contaminants.

\section{Distributions of potential prey}

We examined the National Oceanic and Atmospheric Administration's (NOAA) bottomfish population assessmen database for comparisons of potential prey distribution to Great Sherwater distribution (Politis $e t a l$. 2014). NOAA trawl lors included location (latitude and longitude), depth, and number of fish canght, but we independently assigned substrate properties (sand content and grain size) based on location using our snbstrate data layers (NOA.NACOS unpubl. data). Based on insight gleaned from the fecal analysis, we investigated distributions of Nontem sand lance Ammodyles dubits, Alantic miackerel Scomber scombrus, and Allantic herring Chapea harengus. Mackerel feed throughout the water column, so bottomfish surveys may be inconclusive in mackerel stock assessments. Alantic menthaden Brevoortia fyronns is a mid-water fish, so it was not represented in these botom traw surveys. While sand lance is not specitically targeted in trawl surveys, it is caught as byeatch and is included in trawl survey datasets. We examined spring (April May) and fall (October-November) logs of trawls from 2013 to 2017 covering the northeastem US continental shelf (east of -73 ) including Georges Banls. These surveys did not indude the Scotian Shelf in Canada. We identifed all traw stations as spatial points to define the scope of bottom tram sampling, and we charactetized bathymetry, substrate, slitface temperatures, and botton temperatures at locations where targeted fish were caught. Sampling of fish was unifom across the northeastern US continental shelf for each spring and fall survey (Politis et al. 2014), so we summed the locations of fish calught over the entire period.

\section{Age analysis using bycatch birds}

Data from bycaught individuals was used to examine age and sex demographics in the southwesien Guif of Maine. Bycaugh shearwaters were obtained from the US NOAA Northeast Fisheries Observer Progtam (NEFOP) in the Gulf of Maine Northeast Statistical Area 521 in Appendix A OF NEFSC FSB 2016) and from scientific longline surveys around Gough Island in the Tristan da Cunha archinelago in the South Aflantic. Individuals were frozen upon collection and necropsied as part of a larger study following the methods of vant Franeker (2004). Morphometric data were collected on live bitds captured in the southwestern Gulf of Matine (see Fig. 1) in July 2019 for comparison to bycanght specimens. 
Within this study, we relied upon gonad development, molt score, and the presencelsize of the bursa of Fabricius to gauge sex and likely age. Based on visual assessment of gonad color and quantitative measurement of gonad size, gonad development was scored as one four classes: hatch year, immature, sub-adulthonbreeder, or breeding bird. As noted in perious studies incorporating necropsy data, the sub-adult category (Class 3) includes both immature birds close to maturity and non-breding adnlts, since gonadal development of these two stages is very similar (Cortes $e t$ al. 2018, Hector et al. 1986\%. Mot score was examined following BTO methods, and each specimen was assigned a score from 0 to 50. Bursa presence and size was also noted when possible tecause these can be used as non-plumage-based evidence to estimate the age of procellar form seabirds (Broughton 1994) instead of the nape plumage characteristics noted by Pyle (2008). The bursa begins to enlarge shorly afier hatching and reaches is maximum size in fou to six months (Broughton 1994). Involution is typically complete by the end of a bird's first year of life, and the burs is absent in adult birds (Glick 1983, Mase \& Oishi 1991). Vestigial bursas have internal walls that cannot be visibly differentiated from the doacal wall (Broughton 1994).

Gonad development, molt score, and bursa presence/size were assessed together for each bitd to assign a likely age class: young or mature. Collected measurements were compared between Cape Cod (Northwest Alantic as assuned non-breeders) and Gough Island (South Alantic as suspeced or confimned breeders). The

Wilcoxon test was used to compare patameter means as we could not assume that the measurements wete notmally distributed

\section{RESULTS}

PTTs provided an average of 19.1 Aroos locations per day per tag. These were used 10 create TSD hulls for individual Great Shearwaters with an IVG of 24 hours. A total of 73302 convex hulls were determined for 4477,5 days (Table 1 ).

Antual ranges, expressed in TSD units, were calculated per bird Table 1). The $50 \%$ kernel UD in any year typically represented $21.8 \%$ of an individual's $95 \%$ kernel UD. The number of tange intersections among individual birds in any year increased proportionately with range size: $32.3 \%$ in the $25 \%$ kernel UD and $623 \%$ in the $50 \%$ kernel UD, relative to total intersections within the $95 \%$ kernel UD. But, the area of intersection was disproportionately larger in the $95 \%$ kemel UD, and $83.6 \%$ of the operlap occurred outside of the $50 \%$ kenel UD (Table 1 ).

Compating range size by year, we found a positive cortelation between tag days and the estimated $95 \%$ kernel UD $P=3.578 \mathrm{e}-06$. $R^{2}=0.552$ ). As a tesult we examined, but found to difference in, both the length of individual tag duration (variance) by year (Bartlett's $K^{2}=2.1842$, of $=5, P=0.8231$ ) and the number of lag days (samplitig effort) by year $(F=1.0712$, $d f=5$ and 52 . $P=0.3871$ ). Howeyet, the $95 \%$ kennel UD size by year was significantly different $(f=3.162, d f=5$ and $52, P=0.0144)$ between 2013 and 2015 (Tukey's lest, $P=0.028$ ) and between 2015 and 2018 (Tukey's test, $P=0.035$; Tabie 1). Ploting 95\% kemel UDs by yeat (Fis. 2) showed that most individuals in 2013 and 2018 stayed within the Gulf of Maine, as opposed to 2015, when

TABLE 1

Annual breakdown of platiorm terminal transmitter (PTT) metrics related to sampling effort, kernel density range estimates as sealed by lime-sealed distance (TSD), individnal range intersections, and morphological measurements for Great Shearwaters $(n=58)$ tagged in 2013-2018

\begin{tabular}{|c|c|c|c|c|c|c|c|}
\hline Statistic & Densily & 2013 & 2014 & 2015 & 2016 & 2017 & 2018 \\
\hline Total tags (PTT) & & 10 & 11 & 10 & 10 & 9 & 8 \\
\hline Mean tag duration (day) & & 59.2 & 85.1 & 96.2 & 69.3 & 79.7 & 72.1 \\
\hline Total tag days & & 591.8 & 936.4 & 962.0 & 693,3 & 717.6 & 576.4 \\
\hline Total convex hulls & & 10878 & 14037 & 12981 & 13079 & 11889 & 10438 \\
\hline \multirow{3}{*}{ Mean range by density (TSD*1e-6) } & 0.25 & 2319 & 6160 & 8655 & 2806 & 1251 & 2212 \\
\hline & 0.50 & 9144 & 11256 & 21399 & 13222 & 11777 & 6879 \\
\hline & 0.95 & 33457 & 74914 & 98053 & 48388 & 49160 & 31434 \\
\hline \multirow{3}{*}{ Mean tag intersections by density } & 0.25 & 13 & 25 & 17 & 23 & 19 & 7 \\
\hline & 0,50 & 31 & 35 & 37 & 32 & 27 & 15 \\
\hline & 0.95 & 45 & 55 & 45 & 44 & 36 & 28 \\
\hline \multirow{3}{*}{ Mean intersection area by tensity (TSD*1e-6) } & 0.25 & 325 & 982 & 1275 & 950 & 774 & 617 \\
\hline & 0.50 & 594 & 2990 & 2845 & 1842 & 1545 & 886 \\
\hline & 0.05 & 3742 & 17685 & 21035 & 8417 & 8863 & 4939 \\
\hline Mean body weight pet bitd (g) & & 754 & 940 & 874 & 859 & 716 & 734 \\
\hline Mean primary moll score $(0-50)$ & & & 45.6 & 35.8 & 48.9 & 48.9 & 47.6 \\
\hline Range primary molt score & & & $41-50$ & $6-50$ & $45-50$ & 4450 & $41-30$ \\
\hline Gender (M/F) & & & & $5 / 4$ & 46 & $2 / 6$ & $5 / 3$ \\
\hline Tagging date (dd-mmm) & & $12-J_{\mathrm{ul}}$ & 20 . In & 09-Jal & $12-\mathrm{I}$ Iul & O4-Aug & $9-\mathrm{Jul}$ \\
\hline
\end{tabular}

Marine Omithology 48: 215 229(2020) 
the UDs of half of the individuals extended east to the Scotian Shelf and north to the Grand Banks. The breadth of the 95\% kernel UD in 2018 was also underrepresented, as two individuals (D numbers: 112710 and 112717), within three weeks of capture, moved east of the study area $\left(<-50^{\circ}\right.$ longitude): one to the Flemish Cap $\left(47^{\circ}\right.$ $\left.-44^{\circ}\right)$ and the other to the mid-Atlantic Ridge $\left(41^{\circ},-30.5^{\circ}\right)$. Thus the overall wintering range of Great Shearwaters extends east of Atlantic Canada $\left(-50^{\circ}\right.$ longitude) and beyond the longitudinal boundary used in this study.

In the context of UD size, we rearranged metrics by individual to derive a table showing birds that primarily used the Gulf of Maine compared to those that also extended to the Scotian Shelf or the Grand Banks (Table 2). In this configuration, there was a significant difference in tag days per range group (Kruskal-Wallis $\chi^{2}=7.3174$ $d f=2, P=0.02577$ ), so we could not statistically compare UD sizes. Birds restricted to the Gulf of Maine had the smallest $95 \%$ kemel UD compared to those that also used the Scotian Shelf (median
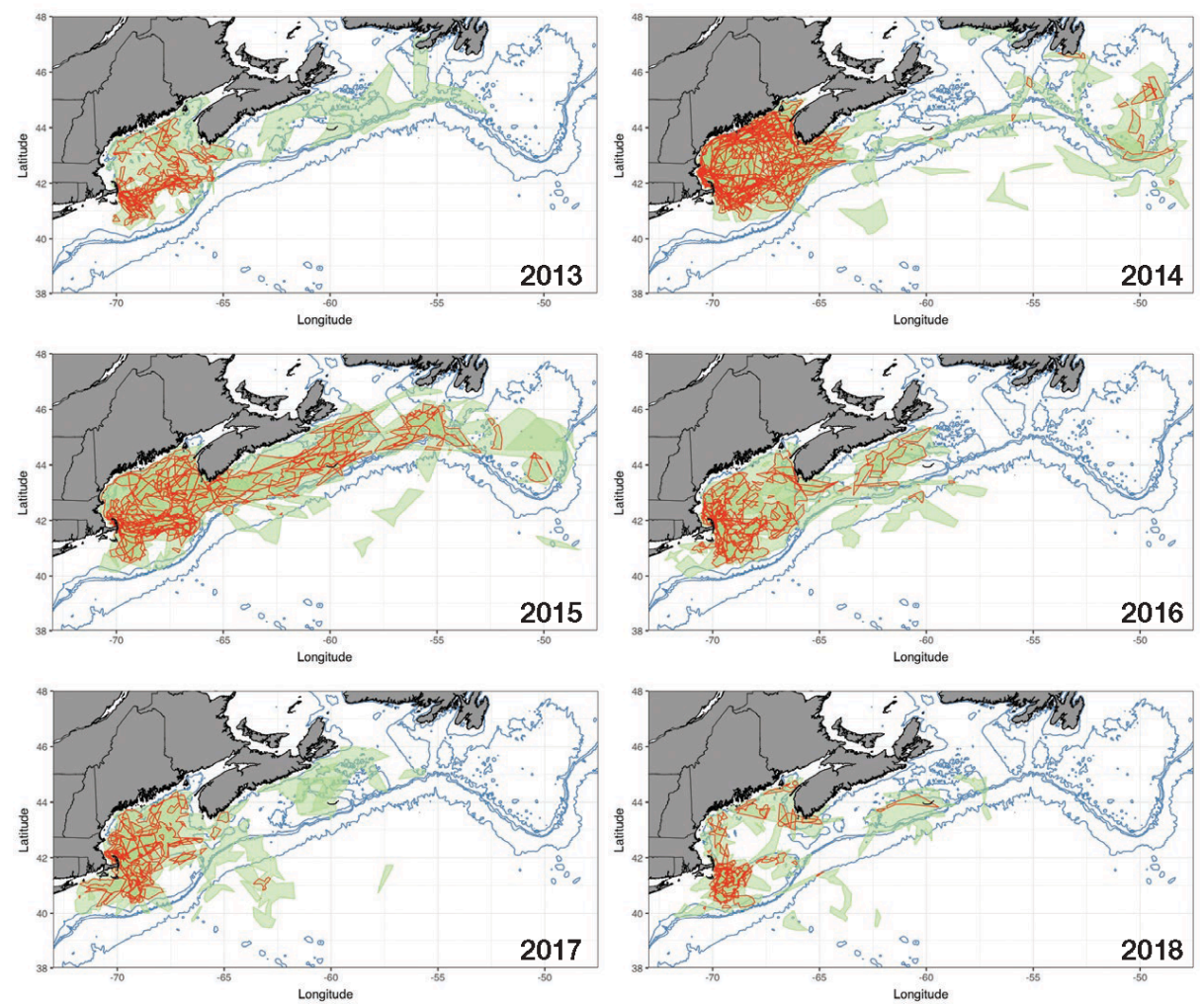

Fig. 2. Plots of Great Shearwater $95 \%$ kernel utilization distributions (UDs) derived from convex hulls of individual birds tagged per year (2013-2018). Individual UDs are filled in green. Red borders denote intersections of individuals within that year. In 2018, two UDs extended east of $-45^{\circ}$ longitude outside of the study area.

Marine Omithology 48: 215-229 (2020) 
the southern New England shelf west of Nantucket Shoals in 2017. Thus, the majority of $50 \%$ kernel UDs were in tangential areas across the southwestern Gulf of Maine, but the 25\% kernel UDs shifted from year to year.

The UDs of all individuals tagged in 2013-2018 were collectively plotted to show individual boundaries with aggregated $95 \%$, $50 \%$, and $25 \%$ kernel density isopleths (Fig. 3). Isolated from an annual perspective, $25 \%$ kernel UDs became contiguous within the southwestern Gulf of Maine, along with separate isolated pockets in the northern Gulf of Maine (including Bay of Fundy and the northeastern corner of the Scotian Shelf (Banquereau)). The centralized 25\% kemel UDs within the Gulf of Maine were connected by the $50 \%$ kernel UDs, which also encapsulated those 25\% kernel UDs on the Scotian Shelf (Fig. 3)

In the aggregate view, more hulls from between the annual $25 \%$ $50 \%$ kemel density isopleths collapsed into a single $25 \%$ kernel UD, and the overall number of hulls between the agrregate $50 \%$ $95 \%$ isopleths were disproportionately reduced. A comparison of behavioral characteristics by density groups (Table 3 ) showed significant differences in revisitation $(F=3347, d f=2$ and 71623 , $P<2.2 \mathrm{e}-16$, duration $(F=622$, $d f=2$ and $71623, P<2.2 \mathrm{e}-16)$, and eccentricity $(F=497, d f=2$ and $71623, P<2.2 \mathrm{e}-16)$ (Table 3 ).

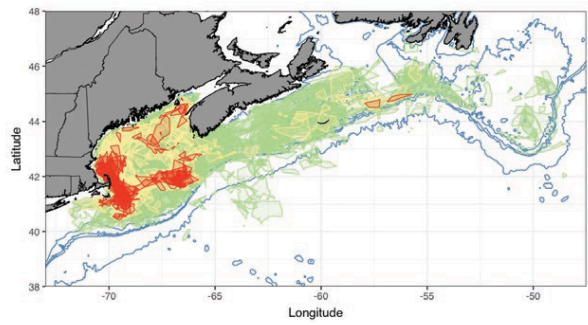

Fig. 3. Aggregate plot of all individual utilization distributions of Great Shearwaters $(n=58)$ from 2013 to 2018 with kernel density groupings based on entire convex hull dataset: $<25 \%$ (red), $25 \%-50 \%$ (yellow), and $50 \%-95 \%$ (green). Shading is caused by overlapping ranges of differing density.

TABLE 2

Platform terminal transmitter metrics related to sampling effort, area of $95 \%$ kernel utilization distribution (UD) as scaled by time-scaled distance (TSD), and morphological measurements by inclusive regions of the continental shelf in the Northwest Atlantic for Great Shearwaters $(n=58)$ tagged in 2013-2018 (median, 1st-3rd quartiles)

\begin{tabular}{|c|c|c|c|}
\hline Measurement & Gulf of Maine & Scotian Shelf & Grand Banks \\
\hline Total individuals & 32 & 14 & 12 \\
\hline Tag days & $59.2(29.9-97.2)$ & $85.1(98.0-119.7)$ & $96.2(46.8-188.3)$ \\
\hline Area of $95 \%$ kernel UD (TSD* $1 \mathrm{e}-6$ ) & $20250(6395-38150)$ & $87000(60525-112250)$ & $115500(61075-138500)$ \\
\hline Body weight (g) & $758(699-853)$ & $858(793-885)$ & $898(826-943)$ \\
\hline Primary molt score & $50(45-50)$ & $49(46-50)$ & $46(43-48)$ \\
\hline Total male & 10 & 4 & 2 \\
\hline Total female & 9 & 6 & 4 \\
\hline
\end{tabular}

TABLE 3

Physical properties and behavioral characteristics of convex hull locations for Great Shearwaters $(n=58)$ tracked in 2013-2018, as summarized by three density groupings with median, 1st-3rd quartiles of each group. Substrate properties represent locations within only the Gulf of Maine and on the western Scotian Shelf.

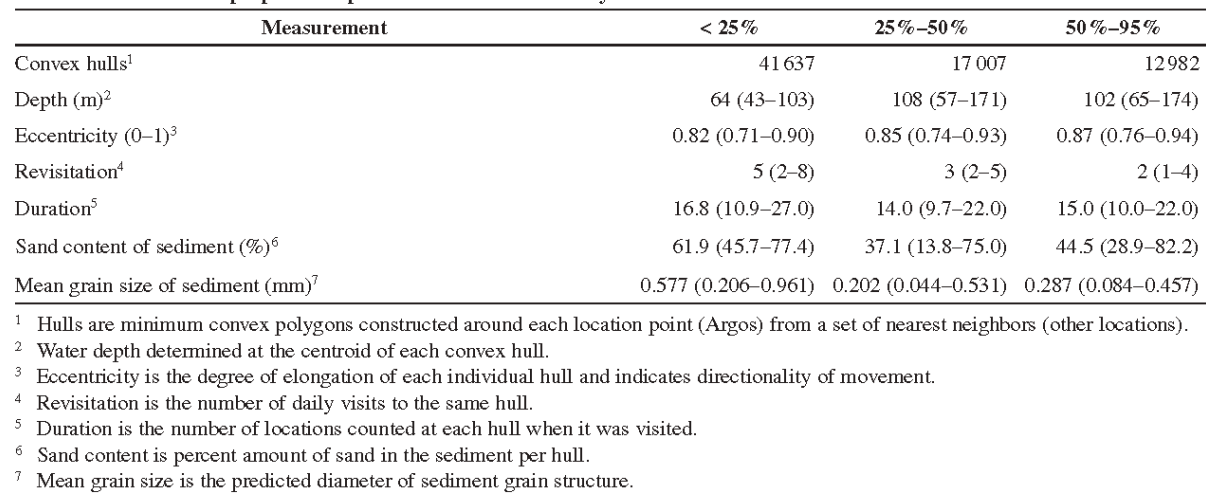

Marine Omithology 48: 215-229 (2020) 
Revisitation significantly increased from 2 to 5 visits per hull as kernel density decreased (i.e., $95 \%-25 \%$ density), indicating that birds went back to the same hulls on more different days in the $<25 \%$ kernel band. Regarding revisitation, each of the density bands was statistically different (Tukey's test, $P=0.00$ ). A expected, duration was significantly higher in the collective $<25 \%$ kernel density band, indicating that birds stayed in those hull for longer periods during each daily visit: 16.8 locations hu11vs. 14-15 locations hull ${ }^{-1}$ when kernel density was $25 \%-95 \%$ (Table 3). Outside of the $<25 \%$ kemel density band, there was no significant difference in duration between the $25 \%-50 \%$ or the $50 \%-95 \%$ kernel density groups (Tukey's test, $P=0.775$ ). Once outside of the primary foraging areas, birds did not linger but instead moved to other areas. Regarding eccentricity, the $<25 \%$
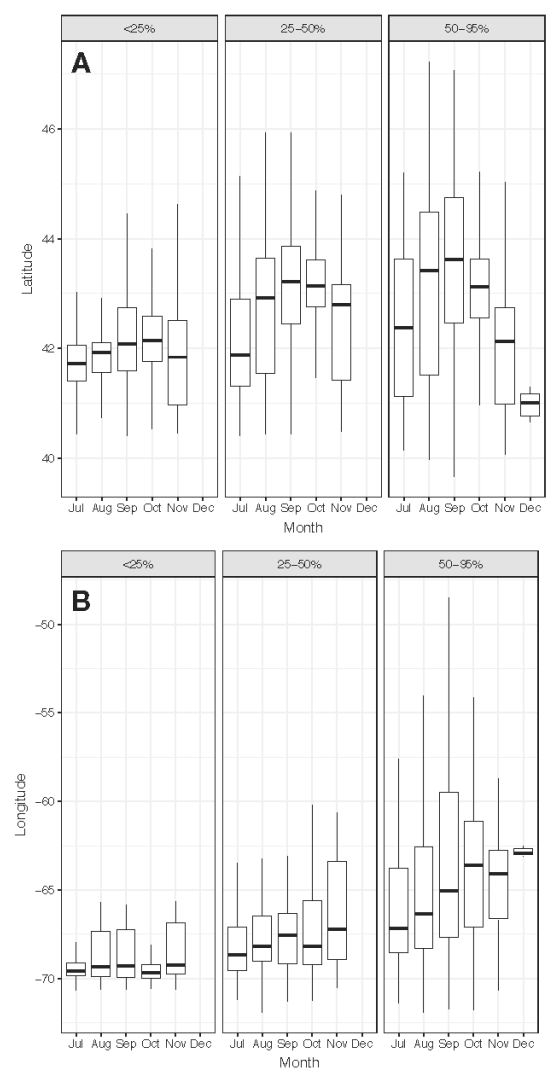

Fig. 4. Histograms of monthly (Jul-Nov) average latitude (A) an longitude (B) by kernel density groups $(<25 \%, 25 \%-50 \%$, $50 \%$ $95 \%$ ) from 2013 to 2018. All birds were tagged off Cape Cod in July, except for 2017 when one was tagged in early August. Most birds (32 of 58) remained the Gulf of Maine during their tag lifespan.

density group was significantly different from both the $25 \%-50 \%$ group (Tukey's test, $P=0.004$ ) and the $50 \%-95 \%$ group (Tukey's test, $P=0.0002$ ). Hull shapes were rounder, indicating substantially more "area-restricted search" behavior within the $25 \%$ kernel density isopleth, where eccentricity was 0.82 vs. $0.85-0.87$ outside of it (Table 3 ). Thus, calculated behavioral traits that were measured conroborated more intense foraging activity within the aggregated $25 \%$ kemel UD.

Physical characteristics of the aggregate kernel density groups also showed significant differences in depth $(F=3024, d f=2$ and $71623, P<2.2 \mathrm{e}-16)$, sand content in the substrate $(F=1550$, $d f=2$ and $54653, P<2.2 \mathrm{e}-16$ ), and mean grain size of sediment ( $F=1597$, of $=2$ and $54653, P<22 \mathrm{e}-10$. None of the density groups overlapped (Tukey's test, $P=0.00$ ). The $<25 \%$ kernel density band had the shallowest depths (median $64 \mathrm{~m}$ ), highest sand content (median 62\%), and coarsest grain size (median $0.578 \mathrm{~mm}$ ). Hulls in the $50 \%$ and $95 \%$ kernel density groups were in deeper water $(>100 \mathrm{~m})$, where the substrate had lower sand content $(<45 \%)$ and finer grain sizes $(<0.287 \mathrm{~mm}$ ) (Table 3 ). There was no single surficial sediment data layer for the entire study area, so we used a single sediment layer for only the Gulf of Maine, Georges Bank, and the western Scotian Shelf including the Bay of Fundy (NOAA/NCCOS unpubl. data; Table 3). A separate sediment layer (Fader et al. 1982) was used for hulls located on the eastern Scotian Shelf (Browns Bank and Lurcher Shoals north to Laurentian Channel). Almost half of the Scotian Shelf hulls (44\% or 3892 of 8927 ) were located over gravel sediment with $>50 \%$ sand and an average depth of $67 \mathrm{~m}$. Seventy percent of these locations with sandier substrates occurred around Sable Island, Banquereau, Western Bank, and Browns Bank (see Fig. 1).

Since birds were tagged near Cape Cod, all individual movements across all years emanated from the same general area starting in July, except for 2017 when birds were tagged in early August (Fig. 4). Birds in the $<25 \%$ density group consistently stayed in the same general area centered at $42^{\circ},-70^{\circ}$ (southwest Gulf of

TABLE 4

Number of samples (count) and frequency of occurrence (FOO) of prey groups representing food habits of Great Shearwaters. Data were derived from

metagenomic analysis of 35 successfully sequenced fecal

samples collected in waters off Massachusetts, USA.

\begin{tabular}{llcc}
\hline \multicolumn{1}{c}{ Prey group } & \multicolumn{1}{c}{ Common name } & Count & FOO \\
\hline Ammodytes spp. & Sand lance & 25 & $71.4 \%$ \\
Brevoortia tyrannus & Atlantic menhaden & 17 & $48.6 \%$ \\
Scomber scombrus & Atlantic mackerel & 11 & $31.4 \%$ \\
Leucoraja erinacea & Little skate & 7 & $20.0 \%$ \\
Peprilus triacanthus & Atlantic butterfish & 3 & $8.6 \%$ \\
Clupea harengus & Atlantic herring & 2 & $5.7 \%$ \\
Squalus acanthias & Spiny dogfish & 2 & $5.7 \%$ \\
Morone saxatilis & Striped bass & 1 & $2.9 \%$ \\
Merluccius bilinearis & White hake & 1 & $2.9 \%$ \\
Pleuronectiformes & Flatfishes (flounders) & 1 & $2.9 \%$ \\
Tautogolabrus adspersus & Cunner & 1 & $2.9 \%$ \\
\hline
\end{tabular}

Marine Ornithology 48: 215-229 (2020) 
Maine) from July through November. Birds in the $25 \%$..50\% and $50 \%-95 \%$ density groups showed nothward progressions from July through September, a pattern that then shifted southward from October through December (Fig. 4A). Their monthly longitudinal progression was increasingly eastward while in the study area (Fig. $4 B$ ). The majority of the $25 \%-50 \%$ group was contained within the Gulf of Maine, with some reaching the Bay of Fundy to the north in September while staying west of Nova Seotia $<-65^{\circ}$ longitude) through October. In Novernber there was considerable movement east of Nova Scotia (> $-65^{\circ}$ 1ongitude) as tagged birds left the siudy area (Fig. 4B). The 50\% $95 \%$ group showed the largest latitudinal and longitudinal expansions over time. The not themnost points reached $48^{\circ}$ in Algust to September, after which the bitds progressively noved southwat to $41^{\circ}$ by December (Fig. 4A, also Fig. 2 for years 2014 and 2015). By September most of this group was east of Nova Scotia $>-65^{\circ}$ ), extending to the Grand Banks $\left(<-55^{\circ}\right)$ (Fig. 4B). In 2018, individuals (D number 112710 and 112717) moved east of the Grand Banks to $-30^{\circ}$ in Angust. The eastward trend continged into December while average latitude was decreasing southward. Thus, eastwatd movement ou of the study area occured over a wide latitudinal band $\left(40^{\circ}\right.$ to $47^{\circ}$ ) and was most pronounced in the outer parts of their collective UD ( $50 \%-95 \%$ kentel density) after September. Of the 58 birds in this sudy, 18 were tracked south of the Equator, 3 by the end of September and the remaining 15 in Nowember and December: Only two of the birds tracked south of the Equator wisited the Tristan da Cunha archipelago and neither stayed more that a single day before retutning to the Patagonian Shelf.

\section{Fecal DNA andasis}

Prey sequences were successfully obtained for 35 of the 47 fecal samples. Samples were collected in August and September 2017 and in July and Novenber 2018 . Average prey diversity was $2.11 \pm 0.28$ $($ mean $\pm 1 \mathrm{SE}$, min $=1$, max $=7)$. Most samples $(71.4 \%)$ contained sand lance; the next most frequent prey item was menhaden $448.6 \%$ Table 4). Only $14.3 \%$ ( 5 of 35 ) of samples lacked sand lanke or menhaden. Mackerel, Allantic butterfish Peprilus triacunhus, and herring were also identified in Great Shearwater diets at lowe frequemcies. Sand lance, menhaden, and mackerel were consumed in both years sampled ( 2017 and 2018)

In the remaining six prey groups, it is unclear whether shearwater were taking juvenile fish directly (which might explain the courrence of striped bass Morone saxatils, white hake Merlaceius bilinearis, cunner Tautogolabrus adspersus, and a plentonectiform flattish) of whether birds wete seavenging shum of bait from fishing vessels (which might explain the occurrence of little skate Letconja erinacea and spiny dogish Sonaltus acanihias). Altenatively, primaty prey may have contained bait or chum that we deteced indirectly (Sheppard et al. 2005).

\section{Depth, substrate, and prey distribution}

Foraging in $25 \%$ kenel UDs was linked to shallow depths and substrates with high sand content of coarser grain sizes (Table 3) Duting the 2013-2017 period, 88 of 2319 trawls conducted by NOAA's National Marine Fisheries Service (NMPS) captured sand lance, while $67 \%$ of trawls with sand lance were over the aggregate $25 \%$ kernel UD of Great Shearwaters (Fig. 5). The physical properties of these sand lance locations wete similat to those hull in the shearwater $25 \%$ kernel UD having a mean depth of $61.6 \mathrm{~m}$
$( \pm 48.5 \mathrm{III})$ with $71.1 \%( \pm 22.7 \%)$ sand content of relatively coarse grain size (median $0.685 \mathrm{~mm}$ ), In addition, $89 \%$ ( 78 of 88 ) of the NMFS trawls with sand lance also occutred within the $100-\mathrm{m}$ isobath. Of these 88 sand lance locations for which we could extract sand content and grain size information, we found that $64 \%$ (56 of 88 ) were found over substrates with $>50 \%$ sand and grain sizes in the range of $0.35-2.00 \mathrm{~mm}$. This range of grain sizes encompasses coarse to very coarse sand (Krumbein \& S10ss 1963; Fig. 5), of the Great Shearwater locations in the 25\% kenel UD, 63\% (26355 of 41637 ) were within the $100-\mathrm{m}$ isobath with > $50 \%$ sand content; $49 \%$ (20 562) wete also coincident within this inclusive tange of sedinent grain sizes.

Mackerel were calught at 688 locations on the northeastem US continental shelf, with greatest abundance near Race Point off Cape Cod, eastward along the 100-1n isobaih to northen Georges Bank. Among trawls with macketel, $38 \%$ (259 of 688 ) occurted within the sheatwater $25 \%$ kernel UD, and the fish were canght at an average depth of $111.1 \pm 68.2 \mathrm{~m}$ over substrate with $51.4 \% \pm 30.1 \%$ sand content and a median grain size of $0.238 \mathrm{~mm}$. Herring were caught in 1225 locations, with highest abundance over westetn Nantucket Shoals, notthern Georges Bank, and in Cape Cod Bay north to Jeffreys Ledge. Among trawls with herring, $36 \%$ (447 of 1225 ) occurred within the slearwater $25 \%$ kemel UD, and the fish were canght at an average depth of $125.7 \pm 72.1 \mathrm{~m}$ over substrate with $44.6 \% \pm 29.0 \%$ sand content and a median grain size of $0.174 \mathrm{~mm}$.

\section{Age analysis using bycatch}

Necropsies were performed on Great Shearwaters caught as bycatch near Gough Island in the Tristan da Cunha archipelago and neat Cape Cod. Solth stlantic specimens were landed in April 2018 $(n=23,16$ male and 7 female), December 2012 ( $n=1$, Inale), and October 2018 ( $n=1$, female), Northwest Allantic specinens were landed in August 2017 ( $n=71,27$ male and 44 female).

The median gonad area in males was $8 \mathrm{~mm}^{2}$ for Northwest Ailantic birds and $32 \mathrm{~mm}^{2}$ for South Aflantic birds. In females, median gonad area was $33 \mathrm{~mm}^{2}$ for Northwest Allantic birds and $100 \mathrm{~mm}^{2}$ for Sonth Altantic birds. Gonad size was significantly different in both sexes between tegions (nales: $W=429, P=1.248 \mathrm{e}-06$; females: $W=377, P=0.000136$. A tuth table was developed to sort age categories and determine maturity (Table 5). All birds from the South Allantic were mature, but only two males and six females from the Nothwest Atlantic region were mature. We then compared gonad size of young vs. mature birds for both sexes between the Nothwest Allanic and the Sonth Allantic groups. In terns of gonad size, young birds from the Northwest Atlantic were significantly different (smaller) from the South Atlantic birds (males? $W=424$, $F=4.411 \mathrm{e}-06$; females: $W=301, P=1.648 \mathrm{e}-05$ ), whereas there was no significant difference for mature birds (males: $W=5$, $P=0.1404$; fenales: $W=26, p=0.8461)$. Thus, $11 \%$ ( 8 of 71 ) of the Nothwest Atlantic bycatch specimens were sub-adults or adults who may have been possible breders, while $89 \%$ (63 of 71) were juvenile or immature individuals considered to be pre-breeders.

We analyzed 53 of the 71 Northwest Allantic birds and all 25 South Atiantic individuals for bureas. For the Northwest Allantic specinens, bursas were identified in 13 males and 24 females and deemed absent (iovolved) in 7 males and 9 females. Eursas were not foud in any South Allantic individuals, as they were all mature. Thus, of those 
birds examined for bursa, $70 \%$ of the pre-breeders from the Northwest Atlantic specimens were juvenile or hatch-year individuals.

We also compared primary molt score and head-bill length (mm) between the regional necropsy samples (Northwest vs. South
Atlantic) and 18 live shearwaters caught in July 2019 within the tagging area. The median primary molt scores among these areas (NwAtl-necrospy, NwAtl-live, SAtl-necropsy) were 50, 46.5 , and 0 , respectively. The average head-bill lengths for the same areas were $101.4 \pm 3.0 \mathrm{~mm}, 103.5 \pm 4.5 \mathrm{~mm}$, and

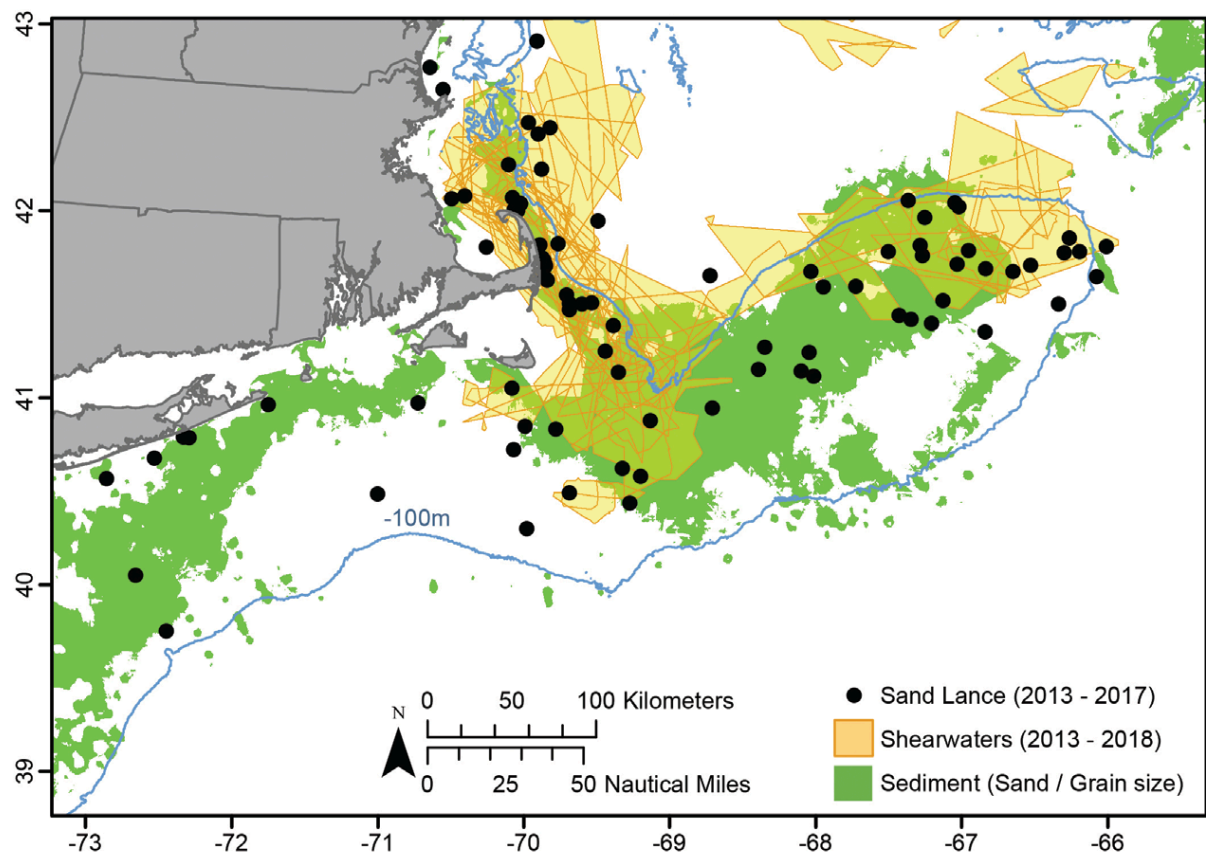

Fig. 5. Plot of aggregate 25\% kernel utilization distributions (UDs) of Great Shearwaters, sand lance trawl captures, and substrate properties common to both species in the southwestern Gulf of Maine and Georges Bank. Most sand lance (at least 64\%) caught in US NOAA/NMFS bottomfish trawl samples (2013-2017) occurred within the $100 \mathrm{~m}$ isobath over sediment with $>50 \%$ sand content and grain sizes ranging from $0.35-2.00 \mathrm{~mm}$ in diameter. Within the $25 \%$ kernel UD, $67 \%$ of sand lance captures occurred and $49 \%$ of shearwater hull locations intersected with these sediment properties.

TABLE 5

Parameters used to designate "mature" and "young" age categories of necropsied Great Shearwaters

\begin{tabular}{lll}
\hline \multicolumn{1}{c}{ Parameter } & Distinguishing features between ages & \multicolumn{1}{c}{ Notes } \\
\hline Molt score & $\begin{array}{l}\mathrm{HY}=50, \\
\text { non-HY }=<50\end{array}$ & $\begin{array}{l}\text { HY birds typically complete primary molt before arriving in the } \\
\text { southwestern Gulf of Maine; immature and sub-adult individuals } \\
\text { complete molt following migration }\end{array}$ \\
Gonad class & $\begin{array}{l}\text { Classes } 1 \& 2=\text { young } \\
\text { Classes } 3 \& 4=\text { mature }\end{array}$ & $\begin{array}{l}\text { Visual assessment of color and gonad size were used to define gonad } \\
\text { class }\end{array}$ \\
Gonad size comparison & $\begin{array}{l}\text { Within min-max range of suspected post- } \\
\text { birds considered "mature" if gonad area overlapped the range of } \\
\text { gonad area observed in suspected post-breeding adult/sub-adult } \\
\text { individuals from Gough Island }\end{array}$ \\
Bursa presence/size & Present, bulbous = HY & Bursa present in only HY individuals
\end{tabular}

$1 \mathrm{HY}=$ Hatch year

Marine Omithology 48: 215-229 (2020) 
$105.3 \pm 2.5 \mathrm{~mm}$, respecively. The necropsied birds from the Nothwest Atlantic had the smallest head-bill lengths and had predominantly completed molt, suggesting young individuals The live birds from the Northwest Alantic showed a mixture of younger and older birds, with most in active molt and larger headbill lengths. The necropsied birds from the South stlantic (Apri 2018 samples) were all older with the largest head-bill lengths and old primary feathers (molt score $=0$ )

\section{DISCUSSION}

We used individual tracks from 58 Great Shearwaters oxer a six-year period (2013-2018) to characterize the spatiotemporal utilization of Great Shearwaters over a latge portion of thei wintering range in the Northwest Atantic. This study improves our understanding of Great Shearwater use of the Gulf of Maine and Atlantic Canada by providing temporal and behavioral dynamics across the entire region, along with a mote comprehensive exarnination than available previonsly of how age is integrated into spatial use. In general, Great shearwaters begin to atrive on Georges Bank in late May (Powers 1983) and are abundan across the sonthern Gulf of Maine in June and July (Powers et al 2017). They undergo a rapid molt upon arrival, which does no render them flightess (Btown 1986, Brown 1988), Molt scores of birds in this study showed that primaty molt was $>90 \%$ complete (average score 45.4 of possible 50 ) in July (Table 1). Moli should have been completed in August, as has been noted in the Bay of Fundy (Powers et al. 2017), yet we found a preponderance of completed primary molt in July ( 20 of 48 scored). This indicated that Inany of our captures were hatch-year or, less likely, imiature pre-breeders that molted in the South Atlantic prior to coming notth (Watson 1971, Bugoni et al. 2015).

Our Inolt data were supported by our Iecropsy data on gonad size, which showed that only $11 \%$ had gonad sizes conparable to that of individuals from the Gough Island breeding colony and that $89 \%$ were non-adults. The presence of visually prominen bursa indicates that $70 \%$ of non-adult birds in the study area may be hatch-year birds. The non-breeding status of the birds was further supported by our tracking data. No birds that we tracked into the Sonth Allantic for this study spent any significant time near the breeding islands of Tristan da Cunha. As characterized by nape plumage, $89 \%$ of Great Shearwaters in the Gulf of Maine were thought to be less than three years of age (Powers et al. 2017). However, that methodology was derived from Pyle (2008) and has not been thoroughly vetted in the literature. In addition, bitds tagged in the Bay of Fundy by powers et al. (2017) were confirmed as non-breeders (Ronconi et ol 2018). An evaluation of mass strandings of Great Shearwater along the eastem coast of the United States noted that birds stranded in the southeastern US were primarily juveriles while those in the northeast were mix of adults and juveniles (Haman ef al 2013). The breeding population of Gteat Shearwaters has been estimated at 5-6 million pairs (10-12 million birds) (Ryan 2007) and the global population (inclusive of pre-breeding birds) is thought to be around 16.5 million (Fishpool \& Evans 2001), as noled by Ronconi et at (2018). These poplation estimates indicate that $27 \%-40 \%$ of the wotld population may be non-breeding. So, if ont Gulf of Maine sample consists of $89 \%$ non-breeders, it may be an important area used primaxily by younger non-breeding birds. Most adult bird may be winteting in areas further north on the Grand Banks, as suggested by Brown (1986).
The $95 \%$ kemel UD of birds in this study indicated that Great Shearwaters utilized much of the continetial shelf from southern New England actoss the Scotian Shelf to the Grand Banks after their initial artival to the southwestern Gulf of Maine and Georges Bank. Neatly half (45\%) of the birds moved to Alantic Canada by Angust, while the remainder (55\%) stayed within the Gulf of Maine until November. Wong et al. (2018) noted the highest densities of Great Shearwaters in the Bay of Fundy in early September, which is concident with the start of southern migration for 'early' migrants (Powers et al. 2017). The spatial spread over time was generally northward until October and eastwatd into November. The frequency of birds staying in the Gulf of Maine was consistent from year to year, but the number of birds with ranges that extended to Atlantic Canada varted annually

It's not entirely clear why some birds stayed in the Gulf of Maine and others undertook more extensive travelling, but it may be related to age and experience. Wib increasing maturity, individuals identify reew resonces across their wintering range. Cory's Shearwaters do not become familiar with main migratory routes until they reacb four years of age, when they broadly use the same routes as older immatures and adults (Campioni et al. 2020). Beyond this age their migratory behavior beconnes progressively adultlike until tine of first breeding (Campioni et al. 2020). This observation of older Great Shearwaters expanding their UD on the wintering range is supported by our data showing heavier bitds tending to move on the Gulf of Maine before lighter birds. While are at firt breeding is unknown for Great Shearwaters, the related Shot-tailed Shearwater breeds at six of seven years old (Bradley et al. 1999) and Cory's Shearwater at mine years (Campini at al. 2020); Great Shearwaters might be similar. The long duration of immaturity in Wandering Albatross Dionedea exulans relates to a long period of experiential learning about spatial, tehavioral, and resource consiraints (Riotte-Lambert \& Weimerskirch 2013). The ontogeny of long-distance migratory strategies and related paterns of spatial distribution in young Cory's Shearwaters progressively changes with age (Campioni et al. 2020).

Thus, most Gteat Shearwaters ( $55 \%$ ) staved within the Gulf of Maine from July to November instead of moving to Atlantic Canada. Individual $50 \%$ kemel UD sizes typically represented $22 \%$ of a bird's $95 \%$ kernel UD, and $84 \%$ of individual range interactions occutred ontside of the $50 \%$ kernel UD, indicating that foraging occuss over wide areas each year. When we aggregated all individuals across all years into a single dataset, the contrast of utilization reversed, and $82 \%$ of the holls were within the $50 \%$ kernel UD. The merge of all tangential $50 \%$ ketnel UDs from each year created a singular $25 \%$ kernel UD, which included $58 \%$ of all halls. Thus, birds were foraging in sirnilar areas each year with significant overlap among years. Behavioral trats in the high-densily region suppoted more intense daily foraging activity as birds remained in hulls for longer periods of time (duration), revisited the same hulls on differen days more often (revisiation), and showed more area-restricted search behavior (eccentricity). The aggregate $25 \%$ kemel UD also had commonality in its physical characteristics, as it was shallow (median $64 \mathrm{~m}$ ) with high sand content (median $62 \%$ ) of coarser grains (median $0.58 \mathrm{~mm}$ ) than the mote peripheral bands of the $50 \%$ or $95 \%$ kernel UDs.

The physical chatacteristics of shallow habitat with coarsily grained sand selected by Great. Shearwaters was also spatially aligned with that of sand lance, which ont fecal samples identified as their main 
prey ( $71 \%$ Frequency of Occurrence). The tabitat preferences (deeper, with less sand and smaller grains) of mackerel and herring were less aligned with that of shearwaters, and these species were identified as lesser prey in our fecal samples (mackerel $31 \%$, herring $6 \%$ ). Research trawl data showed that $67 \%$ of locations containing sand lance fell within the Great Shearwater $25 \%$ kenel UD, while far fewer locations containing mackerel and herring fell within this UD ( $38 \%$ and $36 \%$, respectively). Further, at least $64 \%$ of traws with sand lance ocurred in walers where deph was $<100 \mathrm{~m}$ sand content was $>50 \%$, and sediment grains were $0.35-2.00 \mathrm{~mm}$ in diameter. This combination of factors was also coincident with $49 \%$ of shearwater locations in their $25 \%$ kenel UD. This indicates that sand lance and the habitats that support them are key factors in shearwater habitat use. Depth $(<100 \mathrm{~m}$ ) was a significant predicto for the presencelabsence of Great Shearwaters in the Gulf of Maine and this may be related to their reliance on sand lance as forage (Powers et al. 2017)

Shearwater ntilization of the eastem Scotian Shelf was also associated with banks having shallow waters and high sand content. Sand lance on the Scotian shelf occur on most of the major banks, where the bottom consists of sand or fine gravel (Scott 1982) and in the shallower areas of the Grand Banks, paticularly in the notheastern and sontheasten areas (Winters 1983 . Prewions assessments of Great Shearwater diets in the sonthwestem Gulf of Maine noted sand lance as a common food item (Powers \& Backus 1987), while euphansids Megunyctiphanes nowegica, herritu mackerel, and shoti-finned squid lllex illecebrosus dominate shearwater diets in the Bay of Fundy (Brown et al. 1981, Roncont ef al 2010), Around Newfoundland, spawning aggregations of capelin Mallotts villosus and immature shot-tinned squid attract shearwaters (Brown et al. 1981, Davoren et al 2012 ).

Sand lance distributions are patchy with considerable annual fluctuations in abundance, and larval forms are prey to both herring and mackerel (Standinger et al. 2020). Populations increased 5- to 10-fold in the Gulf of Maine from 1976 to 1981, but abundance subsequently dropped between 1982 and 1987 (Nelson \& Ros 1991). 2016 to 2018 were poor years for herring (and likely mackerel), which may have contibuted to the high frequency of cenrence of sand lance found in shearwater fecal samples. Age-1 recruitrment of herring in the notheastern US has been below average since 2013 , and four of the six lowest annual tecruitment estimates have ocurred since that year (NEFSC 2018b). Since 2005, dies of Alantic Pufins Fratercula anctica and Razorbills Alca torda in the Gulf of Maine switched from herring to sand lance due to the decline in herring (Sconel et al. 2019). Based on fisherie model results and sensitivity, the mackerel stock in the nor theasten US was estimated to be at an all-time low in 2012, laving expetienced increasing exploitation (overfishing) through the eatly 2000 s to a high in 2010 (NEFSC 2018 a). We note that concurren with obtaining some fecal samples, there was an unusual event of macketel dtuing yeatling menhaden into the shoreline at Race Poin on Cape Cod, Massachusetts in August 2017. Menhaden were taken by shearwates in both 2017 and 2018, but in 2017 we observed hundreds of Great Shearwaters feeding on beached meniaden along the shoreline. Menhaden nursery grounds in New England have become significant contributors of are-1 fish i Anstead et $a$. 2016. Since 2015, abundance of both adult and juyenile menhaden bas increased in the Culf of Maine (ASMFC 2017). Menbaden and mackerel were previously unknown in diets of Great Shearwaters in the northeastem US, so their importance as prey may be increasing in this region. The availability of any suitable prey is inportant, but Great Shearwaters in the southwestern Gulf of Maine in 2013-2018 foraged most intensively over the same habitat used by sand lano.

Latitudinal differences in trophic levels of Great Shearwaters, as measured by stable isowpes (feathers and blood) between populations in Newfoundland, the Bay of Fundy, and southwestern Gulf of Maine, conld be attributable to preferences in primary prey (Ronconi ef al. 2010, Gulka et al. 2017, Hong et al. 2019). The dietary niche of shearwaters in coastal Newfoundland was found to natrow with increased availability of capelin, and shearwaters were miquely qualifed to forage over broader scales to continnously maintain contact with capelin patches (Gulka et al. 2017). Similarly, Great Shearwaters in the southwestern Gnif of Maine and not thern Georges Bank showed selectivity for habitats with higher availability of preferred prey (sand lance).

In surmary, a combination of PTT tracking and necropsy data indicate that the Gulf of Maine is a key wintering area for young 102 years) Great Shearwaters. The characteristics of identified foraging areas and DNA from fecal samples from birds indicate that shallow, sandy-bottomed marine habitat and sand lance are important aspects of their habitat use and survival. Since juveniles represent an iniportant demographic in the viability of any species (Hazen et az. 2012), we suggest that management actions in the Gulf of Maine that would impact their preferred habitat and prey should consider Great Shearwaters in their decision-making

\section{ACK NOWL EDGEMENTS}

We thank Peter Hong, Tammy Silva, Kent Hatch, Sara Williams, students from the Boston University Marine Program (especially Megan Feddem, Lindsay Smith, Montana Aitey, and Lucy MoGiunis), and the crew of NOAA RV Auk for assistance in collecting shearwater data. Labontory work was contributed by 1. Biagini of the College of Aits and Sciences at the University of New England. We are grateful to Peter Ryan and Vonica Perold at the Filzpatrick Institute of African Omithology and Maëlle Connan from Nelson Mandela University for sample collection in the South Aflantic, collaboration, and necropsy assistance. We thank Justin Snca for his critical review of an earlier version of the manuscript. NOAA's Northeast Fisheries Science Center provided botom fish trawl infotmation. NOA A s National Centers fot Coastal Ocean Science (NCCOS) provided surficial sediment datasets for the Northeast and Mid-Allantic aress of the US conthental shelf. Genetic analyses of blood and feather samples were done by Animal Cenetics, Inc, 1336 Timberlane Rd. Tallahassee, FL 32312-1766, USA. All applicable international, national, and institutional guidelines for the care and nse of animals were followed. Permits to capture, handle, and tag birds were obtained from the US Department of the Intetion (permit \$21963). Petmits to handle birds were obtained from the Massachusetts Division of Fisheries and Wildife (permit $\# 202-17 \mathrm{SCB}$ )

Funding. was provided by the Volgenau Foundation, Burean of Ocean Energy Management, Stellwagen Bank National Marine Sanctuary, National Marine Sanctuary Foundation, Mudge Foundation, Boston University Marine Program, and Pew Chartable Trusts. The NOA Nancy Foster Scholarship provided support to A. Robuck. Laboratory work was supported by a Summet Undergaduate Research Expetience fellowship throngh the College of Arts and Sciences at the University of New 
England. The conments of two anonymous reviewers greally helped us to improve the clarity of this paper.

\section{Diselaimer}

The findings and conclusions in this article are those of the author (s and do not necessarily represent the wiews of the US Fish and Wildife Service, NOA $A$, or the US Department of Commerce This publication does not constitule an endorsement of any commercial product or intend to be an opinion beyond scientific of other results obtained by these agencies.

\section{RENERENCES}

ANSTEAD, K.A., SCHAFFLER, J.J. \&ONES, C.M. 2016. Coastwide nursery contribution of thew recruits to the population of Atlantic Menhaden. Transactions of the American Fisherie Soctety 145: 627-636. doi:10.1080100028487.2016.1150345

ASMFC ATLANTIC MENHADEN STOCK ASSESSMENT SUBCOMMITTEE (ASMPC) 2017. 2017 Atlantic Menhaden Stock Assessment Update. Atington, USA: Atlantic States Marine Fisheries Commission.

BRADLEY, IS, GUNN, B.M., SKIRA, I. I, MEATHREL, C.E \& WOOLLER, R.D. 1999. Age-dependent prospecting and recruitment to a breeding colony of Shot-tailed Shearwaters Puffinus temairostris. Ibis 141:277-285.

BROUGHTON, J.M. 1994. Size of the bursa of Fabricius in relation to gonad size and age in Laysan and Black-footed Albatrosses. The Comior 96: 203-207.

BROWN, R.G.B. 1986. Revised Allas of Eastem Canadian Seabiras. Datmonth, Canada: Canadian Wildife Service Bedford Institute of Oceanography.

BROWN, R.G.B. 1988. The wing-monlt of fulmars and shearwaters (Frocellaridae) in Canadian Allantic waters. The Candian Field-Natiagallist 102: 203-208

BROWN, R.GB.BARKER, S.P, GASKIN, D.E \& SANDEMAN, M.R. 1981. The foods of Great and Sooty Shearwaters Putfinus gravis and $P$. grisets in eastern Canadian waters. Ibis 123: $19-30$.

BUGONI, L., NAVES, L.C. \& FURNESS, R.W. 2015. Moult of three Tristan da Cuma seabird species sampled at sea, Antarctic Science 27: 240-251, doi: $10.1017 / \$ 0954102014000583$

CAMPIONI, L. DIAS. MP. GRANADERKO, JP. 8 CATRY, 2020. An ontogenetic perspective on migratory strategy of a long-lived pelagic seabird: Timings and destinations change progtessively during maturation. Joumal of Animal Ecology 89 20-43, doi: $10.1111 / 1365-2656,13044$

CAPORASO, J.G. KUCZYNSKI, J, STOMBAUGH, J. ET AL. 2010. QIIME allows analysis of high-throughput community sequencing data. Nature Methods 7: 335-336.

CARVALHO, P.C. \& DAVOREN, G.K. 2019. Associations of tonbreeding shearwater species on the northeastem Newfoundland coast Marine Ecology Progress Series 627: 1-12, doi:10.3354 meps13112

CORTES, V, GARCIA-BARCELONA, S, \& GONZALEZ-SOLÍS, J. 2018. Sex- and age-biased mortality of three shearwate spectes in longline fisheties of the Meditetranean. Marine Ecology Progress Series 588: 220-241 doi:10.3354/meps 12427

CROXALL, J.P., BUTCHART, S.H.M, LASCELLES, B. ET AL. 2012. Seabird conservation status, threats and priority actions A global assessment. Bird Conservation International 22: 1-34. doi:10.1017/50959270912000020
DAVOREN, G.K., MONTEVECCHI, W.A. \& ANDERSON, JT. 2003. Search strategies of a pursuif-diving marine bird and the persistence of prey patches. Ecological Monographs 73 : 463-481. do1:10.1800102-0208

DAVOREN, G.K., PENTON, P., BURKE, C \& MONTEVECCHI, W.A. 2012. Water temperature and timing of capelin spawning deternine seabird diets. ICES Joumal of Morine Science 69: 1234-1241, doi: $10.1093 / \mathrm{cesins} / \mathrm{fs}=32$

DEAGLE, B.E. KIRKWOOD, R. \& JARMAN, SN. 2009. Analysis of Australian fur seal diet by pyrosequencing prey DNA in faeces. Molecular Ecology 18: 2022-2038.

DIAS, M.P. MARTIN, R., PEARMAIN, E.J. ET AL. 2019. Threats to seabirds: A global assessment. Biological Conservation 237 : $525-537$.

FADER, G.B, RING, L.H. \& JOSENHANS, H.W. 1982. Surficial Geologyol the Laurentian Channel and ine Western Grand Banks of Newfoundland. Paper 81-22. Ottawa, Canada: Geological Survey of Canada doi: 10.4095/119807

FAUCHALD, P. 2009. Spatial interaction between seabirds and prey: Rewiew and synthesis. Morine Ecology Progress Series 391: 139-151

FAUCHALD, P., ERIKSTAD, KE. \& SKARSFJORD, H. 2000. Scale-dependent predator-prey ineractions: The herarchical spatial distribution of seabirds and prey. Ecology 81, 773-783.

FSTPOOL, L DC. \& EVANS, MII EAs 2001 . Important Bird Areas in Arica and Associated Yshands: Pronty Sites for Conservation. Birdlife Intemational Conservation Series No. 11. Newbury and Cambridge, UK: Pisces Publications and BirdLife lnternational.

GETZ, WM., FORTMANN-ROE, S., CROSS, PC, LYONS, A.J. RYAN, S.I. \& WLMERS, C.C. 2007. LoCoH: Nonparanetric kemel methods for constructing home ranges and utilization distributions. PLOS One 2: e207. doi:10.1371/jounal. pone. 0000207

GLICK, B. 1983. Chapter 6: Bursa of Fabricius. In: FARNER, D.S., KING, JR \& PARKES, K. C. (Eds) Avian Biology, 1 st Edition. New Yotk, USA: Academic Press.

GINN, H.B. \& MELVILLE, D.S. 1983. Montt in Birds. BTO Guide 19. Bangor, Wales: British Trust for Ornithology

GONZALEZ-SOLS, I, CROXALL, IP, ORO, D. \& RUL, $\mathrm{X}$ 2007. Trans-equatorial migration and mixing in the wintering areas of a pelagie seabid. Frontien in Ecology and the Envionment 5: 297-301

GONZALEZ-SOLS, I SHAFFE S A 2000 . Introdiction and synthesis: Spatial ecology of seabirds at sea. Marine Ecology Progress Series 391: 117-120. doi:10.3354/meps08282

GULKA, J., CARVALHO, PC., JENKINS, E., JOHNSON, K., MAYNARD, L. \& DAVOREN, G.K. 2017. Dietary niche shifts of multiple marine predators under varving prey avalability on the northeast Newfoundland coast. Fronicers in Marine Science 4: 324

HAMAN, K.H, NOKTON, TM, RONCONI, R.A. ET AL. 2013 Great Shearwater (Puffinus grats) mortality events along the eastern coast of the United States. Joumal of Widilfe Diseases 49: $235-245$.

HAZEN, EL., MAXWELL, S.M., BALEX, H. ET AL. 2012. Ontogeny in marine tagging and tracking science: Technologies and data gaps. Marine Eoology Progress Series 457: 221-240. do: 10.3354 mepsog857

HECTOR, JA.L., CROXALL, J.P. \& FOLLETT, B.K. 1986. Reproductive endoctinology of the Wandering Albatross Diomedea exulans in relation to biennial breeding and deferred sexilat matutity lais $128: 9-22$ 
HEDD, A., MONTEVECCHI, W.A. OTLEY, H., PHILLIPS, R.A. \& FTIELD, D.A. 2012. Trans-equatorial migration and habitat use by sooty Shearwatets pulfinus grisens from the South Atlantic during the nonbreeding season. Marine Ecology Progress Series 449: $277-290$.

HONG, P., WILEY, DN, POWERS, K.D., MICHENER, R.H. KAUFMAN, L. \& HATCH, K.A. 2019. Stable isotope analyses of multiple tissues of Great Shearwaters (Avdema gravis teveals long-term dietary stability, short-iem changes in diet, and can be used as a tool to monitor food webs. Diversity 11: 163. doi: $10.3390 / \mathrm{d} 1109016$

HUETTMANN, F. \& DIAMOND, A.W. 2000. Seabird migration in the Canadian northwest Atlantic Ocean: Moulting locations and movement patterns of immature birds. Canodian Jownal of Zoology 78: 624-647.

JODICE, P.G.R., RONCONI, R.A., RUPP, E, WALLACE, G.E \& SATCE, Y, 2015. First satellite tracks of the endangered black-capped petrel. Endangered Species Reseatch 20:23-33. doi: $10.3354 / e s r 00697$

IONSEN, LD., FLEMMING, JM \& MYERS, R.A. 2005, Robus state-space modeling of animal movement data, Ecology 86 28742880 .

JONSEN, I.D., MYERS, R.A. \& JAMES, M.C. 2007. Identifying leatheback turtle foraging belaviour from satellite telemetry using a switching state-space model. Marine Ecology progres Series $337: 255264$

JOUVENTIN, P., CAPDEVILLE, D., CUENOT-CHAILLET, F. \& BOITEAU, C. 1994. Exploitation of pelagic tesources by a nonflying seabitd: Satellite tracking of the King Pengunin thron ghou the breeding cycle. Marine Eology Progress Series 106 : $11-19$

KRUMBEIN, W.C. SLOSS, L.L. 1963. Stratigraphy and Sedimentation, 2nd Ealtion. San Francisco, USA: WH. Freeman.

LYONS, A 2014. T-LoCoH for R: Tutorial and Users Gride Viema, Austia: R-Forge. [Accessed online at hitpi/tlocohr forge.t-projectorg/tocoh_tutorial_2014-08-17 pdf in Alig 2014.]

LYONS, A.J., TURNER, W.C. \& GETZ, W.M. 2013. Home range plus: $A$ space-time characterization of movement over real landscapes Movement Ecology 1:2 doi:10 1186/2051-3933-1-2

MACLEOD, C.I, ADAMS, J. \& LYVER, P. 2008. Al-se: distribntion of satellite-tracked Grey-faced Petrels, Pterodrom macroptera goildi, captured on the Ruamaahna (Aldernen) Islands, New Zealand. Papers and Proceedings of the Royal Society of Tasmania 142: 73.88

MANNOCCI, L., BOUSTANY, A.M, ROBERTS, IJ. ET AL. 2017. Temporal resolutions in species distribution models of highly moble marine animals: Recommendations for ecologist and managers. Diversily and Distributions 23: $1098-1109$ doi $10.1111 /$ da. 12609

MASE, Y. \& OISHI, T. 1991. Effects of castration and testosterone treatment on the development and involution of the bursa of Fabricius and the thymus in the Japanese Quail. General and Comparative tendocrinology $84: 426-433$.

MASELLA, A.P, BARTRAM, AK. TRUSZKOWSKI, JM. BROWN, D.G. \& NEUFELD, I.D. 2012. PANDAseg: Pairedend assembler for illumina sequenos. BMC Bictufonmalics 13: 31

MCMURDIE, PJ \& HOLMES, S. 2013, phyloeg: an R package for teproducible interactive analysis and graphics of mictobiome census data. PLOS One 8: e61217.

MOORE, J.K., FU, W, PRIMEAU, F. ET AL. 2018. Sustained climate warming drives declining matine biological productivity. Science 359: 1139-1143
NEFSC (NORTHEAST FISHERIES SCIENCE CENTER) 2018a. 64th Northeast Regional Stock, Assessment Workhop (6th SAW) Assersment Summary Report. Notheast Wisheries Science Center Reference Document 18-03. Woods Hole, USA: National Oceanic and Atmospheric Administration.

NEFSC (NORTHEAST FISHERIES SCIENCE CENTER) 2018b. 65th Northeast Regional Stock Assessment Workshop (65h SAW) Assessment Summany Report. Northeast Fisheries Science Center Reference Document 18-08. Woods Hole, USA: National Oceanic and Atmosphetic Administration.

NEFSC FSB (NEFSC FISHERIES SAMPLING BRANCH) 2016. Observer Dala Enury Monal. Woods Hole, USA: National Oceanic and Atmospheric Administration, Northeast Fisheries Science Center.

NELSON, G.A. \& ROSS, M.R. 1991 . Biology and population changes of Northern Sand Lance (Amnwdyles dubius) from the Gulf of Maine to the Middle Atlantic Bight. Joumat of Northwest Atlantic Fishery Science 11:11-27.

NYE, J.A., LINK. J.S., HARE, J.A. \& OVERHOLTZ, W.J. 2009 Changing spatial distribution of fish stocks in relation to climate change and population size on the northeast United States continental shelf. Marine Ecology Progress Series 393 : 111-129. doi: $10: 3354 / \mathrm{meps} 08220$

PALECZNY, M, HAMMLL, E, KARPOUZI, V, \& PAULY, D. 2015. Population trend of the world's monitored seabirds, 1950-2010. PLoS One 10: e0129342. doi:10.1371/jounal. pone. 0129342

PHILLIPS, R.A., XAVIER, JC \& CROXALL, J.P. 2003, Effects of satellite transmitters on albatrosses and petrels. The Auk 120: $1082-1090$

PINET, P., JAQUEMET, S., PINAUD, D., WEIMERSKIRCH, H., PHILIIPS, R.A. LE CORRE, M. 2011. Migration, winteting distribution and habitat use of an endangered tropical seabit, Barau's Petrel Plerodrona barahi. Marine Ecology Progress Sertes 423: 291-302, doi:10,3354/meps08971

POLITIS, P.J, GALBRAITH, JK., KOSTOVICK, P. \& BROWN, R.W. 2014. Northeast Fisheries Science Center Bottom Trawl Sutrey Protocols for the NOAA Ship Henry B. Bigelow. Northeast Fisheries Science Center Reference Document 14-06. Woods Hole, USA: National Oceanic and Atmospheric Administration.

POWERS, K.D. 1983. Pelagic Distributions of Marine Birds off the Northeastem United States. NOAA Technical Memorandum NMFS-F/NEC-27. Woods Hole, USA: National Oceanic and Amostheric Administration.

POWERS, K.D. \& BACKUS, E.H. 1987. Chapter 35: Energy transfer to seabifds. In: BACKUS E.H. (Bd.) Georges Bank. Cambridge, USA: MIT Press.

POWERS, K.D., WILEY, D.N., ALLYN, A.I, WELCH, LJ. \& RONCONI, R.A. 2017. Movements and foraging babitats of Great Shearwaters Puffous grows in the Gulf of Maine. Marine Ecology Progress Series 574: 211--226. doi:10.3354/ meps 12168

PYLE, P. 2008. Identification Gude to North American Binds. Part U. Anatidae to Alciade. Point Reyes Station, USA: Slate Creck Press.

$R$ DEVELOPMENT CORE TEAM 2018, $R$ : A language and emironment for statistical computims. Vienna, Austria: The $\mathrm{P}$ Foundation for Statistical Computing.

RIOTTE-LAMBERT, L. \& WEIMERSKIRCH, H. 2013. Do naive juvenile seabirds forage diferently from adults? Proceedings of the Royal Society B: Biological Sciences 280: 2013143. 
RONCONI, R.A., KOOPMAN, H.N., MCKINSTRY, C.A.E, WONG, S.N. \& WESTCATE, A.I. 2010 Intet-annual variability in diet of non-breeding pelagic seabirds Puffonus spp, at nigratory staging greas: Evidenoe from stable isotopes and fatty acdids. Marine Ecology Progress Series 419: 267 282 doi:10.3354/meps08860

RONCONI, R.A., SCHOOMBIE, S., WESTGATE, A.J., WONG, S.N.P., KOOPMAN, H.N. \& RYAN, P.C. 2018 . Effects of age sex, colony and breding phase on marite space use by Great Shearwaters Ardenta gravis in the South Allantic. Marine Biology 165: 58. doi:101007/s00227-018-3299-X

RYAN, PG. (Ed.) 2007. Field Guide to the Animals and Plonts of Tristan da Cunha and Gongh Island. Newbury, USA: Pisces Publications.

SCHAFTER, S.A, TREMBLAY, Y, WEMERSRIRCH, H, ET AL. 2006. Migratory shearwaters integrate oceanic resources across the Pacific Ocean in an endless summer. Proceedings of the National Academy of Sciences 103: 12799-12802.

SCHNEIDER D.C. \& PLATT, I F. 1986. Scale-dependent cortalation of seabirds with schooling fish in a coastal ecosystem. Marne Ecology Progress Sertes 32: 237-246.

SCOPEL, L, DLAMOND, A., KRESS, S. SHANNON, P. 2019 Varied breeding responses of seabirds to a regine shift in prey base in the Gulf of Maine. Marine Ecology Proopess Series 626 177-196, doi: $103354 / \mathrm{meps} 13048$

SCOTT, J.S. 1982 . Selection of bottom type by groundtishes of the Scotian Shelf. Conadian Joumal of Fisheries ond Aquatio Sciences 39: 943.947.

SHEPPARD, S.K., BELL, J., SUNDERLAND, K.D., FENLON, I. SKERVIN, D \& SYMONDSON, WOC 2005 . Detection of secondary predation by PCR awalyses of the gnt contents of invertebrate generalist predatots. Molecular Eoology 14 4461-4468. doi: 10.1111/j.1365-294X.2005.02742x

STAUDINGER, MD GOYERT H, SUCA, J J ET AL 2020. The role of sand lances (Ammodytes sp.) in the Northwest Allantic eccsystem: A synthesis of current knowledge with implication for conservation and management. Fish and Fisheries 21 52-556. doi: 10.1111 faf 12445

SURYAN, R.M., KULETZ, K.J, PARKER-STETTER, S.L. ET AL. 2016. Temporal shifts in sedbiti populations and spatial colerence with prey in the southeastern Bering Sea. Marn Ecology Progress Senes 549: 199-215, doi: 10.3354/meps 1165
THOMAS, A.C., JARMAN, S.N., HAMAN, K.H., TRITES, A.W. DEAGLE, B.E. 2014. Improwing aceuracy of DNA diet estimates using food tissue control materials and an evaluation of proxies for digestion bias. Molecular Ecology 23: $3706-3718$.

TREMBLAY, Y, BERTRAND, S., HENRY, RW, KAPPES, M.A. COSTA, D.P \& SHAFFER, S.A. 2009. Analytical approaches to investigating seabird-entironment interactions: A review Marine Ecology Progress Series 391: 153-163

VAN FRANEKER, I.A. 2004. Save the North Sea-FumarLitter-Ecooo Manhal Part 1: Collection and Dissection Procedures. Alerra-rapport 672. Wageningen, Nettenlands: Alterra.

WATSON, G.E. 1971. Molting Great Shearwaters (Puffums gravis) of Tierra del Fuego. The Auk 88: $440-442$.

WEIMERSKIRCH, H., SALAMOLARD, M., SARRAZIN, F. \& IOUVENTIN, P. 1993. Foraging strategy of Wandering Albatrosses through the breeding-season: a sudy using satellite telemetry. The A 14 . 110:325-342.

WEIMERSKIRCH, H, DONCASTER, CP, \& CUENOTCHAILLET, F. 1994. Pelagic seabirds and the marine environment: Foraging patterns of Wandering Albatrosses in relation to prey availability and distribution. Proceedings of the Royal Society B: Biological Sciences 255: 91 -.97.

WINTERS, G.H. 1983, Analysis of the biological and demographic parameters of the Northern Sand Lance. Ammodytes dubids, from the New fondland Gand Bank. Canadian foumal of Fisheries and Aquatics Sciences 40: $409-419$.

WONG, SN, RONCON, RA \& GERDRUM, C. 2018. Antuma at-sea distribution and abundance of phalaropes Phalaropus and other seabirds in the lower Bay of Pundy, Canada. Marine Omithology 46:1-10.

WINSHIP, A.I, KINLAN, B.P., WHITE, T.P, LEIRNESS, J.B. \& CHRISTENSEN, I. 2018. Modeling At-Sea Density of Marine Bitds to Suppont Atlantic Morine Renewable Energy Planning: Final Report. OCS Study: BOEM 2018-010. Sterling, USA: Bureau of Ocean Energy Management, Office of Renewable Energy Progtams. 


\section{Sensitivity of sand lance to shifting prey and hydrography indicates forthcoming change to the Northeast US shelf forage fish complex}

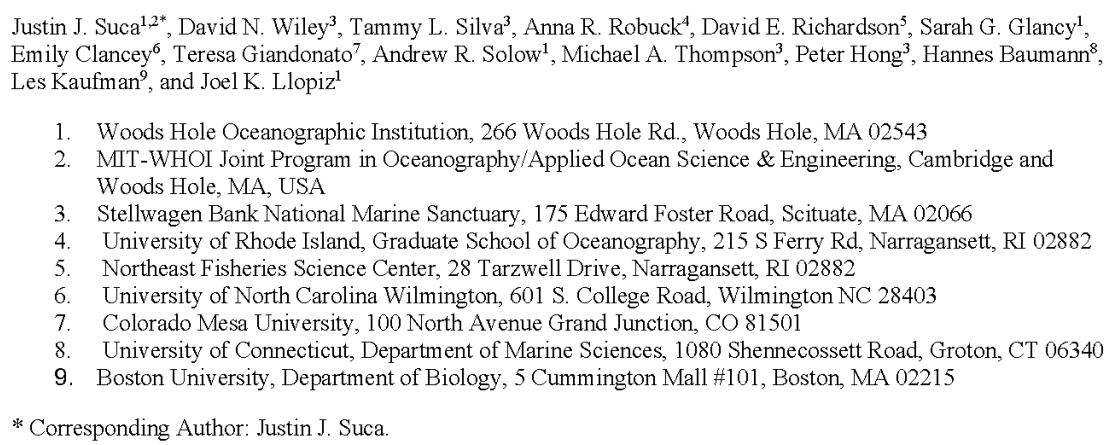

8. University of Connecticut, Department of Marine Sciences, 1080 Shennecossett Road, Groton, CT 06340

9. Boston University, Department of Biology, 5 Cummington Mall \#101, Boston, MA 02215

* Corresponding Author: Justin J. Suca.

Contact Information: jsuca $@$ whoi.edu, 941-713-9883

OrcID: J.J.S.: http://orcid.org/0000-0001-5532-4780

Author Email Addresses: Justin J. Suca (jsuca@whoi.edu); David N. Wiley (David.wilev@noaa.gov); Tammy L. Silva (tammy.silva(@) noaa.gov); Anna R. Robuck (anna robuck@uri.edu); David E. Richardson

(David.richardson(@noaa.gov); Sarah Glancy (sglancy@whoi.edu); Emily Clancey (Emily.clancey@noaa.gov);

Teresa Giandonato (giandonato16@gmail.com); Andrew R. Solow (asolow@whoi.edu); Michael A. Thompson

(Michael.a.thompson(a)noaa.gov); Peter Hong (peter.hong@onoaa.gov); Hannes Baumann

Hannes.baumann(auconn.edu); Les Kaufman (lesk(bu.edu); Joel K. Llopiz (1lopiz@whoi.edu 


\section{Abstract}

Northern sand lance (Ammodytes dubius) and Atlantic herring (Clupea harengus) represent the dominant lipid-rich forage fish species throughout the Northeast US shelf and are critical prey for numerous top predators. However, unlike Atlantic herring, there is little research on sand lance or information about drivers of their abundance. We use intra-annual measurements of sand lance diet, growth, and condition to explain annual variability in sand lance abundance on the Northeast US Shelf. Our observations indicate that northern sand lance feed, grow, and accumulate lipids in the late winter through summer, predominantly consuming the copepod Calamus finmarchicus. Sand lance then cease feeding, utilize lipids, and begin gonad development in the fall. We show that the abundance of Calanus finmarchicus strongly influences sand lance parental condition and recruitment. Atlantic herring can mute this effect through intra-guild predation. Hydrography further impacts sand lance abundance as increases in warm slope water decrease overwinter survival of reproductive adults. The predicted changes to these drivers indicate that sand lance will no longer be able to fill the role of lipid-rich forage during times of low Atlantic herring abundance - changing the Northeast US shelf forage fish complex by the end of the century.

Keywords: forage fish, trophic ecology, recruitment, climate change, Calanus, changepoint 


\section{Introduction}

Small pelagic fishes, or forage fishes, are critical components of both neritic and oceanic

2 food webs. They represent an important link between planktonic production and a diversity of

3 larger predators, including numerous endangered species. However, forage fish abundance and

4 recruitment are often sensitive to temperature and secondary production, leading to large

5 fluctuations in abundance, making this trophic link highly variable in space and time (Chavez et

6 al., 2003; Clausen et al., 2018; Thompson et al., 2019; Sydeman et al., 2020). Further, numerous

7 studies show that fluctuations in forage fish abundance can lead to marked effects on predator

8 condition and breeding success (Barrett et al., 2012; Kadin et al., 2012; Engelhard et al., 2013;

9 Church et al., 2019). Owing to the sensitivity of forage fish to climate regimes, and, in turn, the

10 breeding and condition of their predators, there is great interest in understanding climate-forage

11 fish relationships and using them to project forage fish abundances into the future (Fréon et al.,

12 2005; Alder et al., 2008; Checkley et al., 2017). Sand lances (family Ammodytidae) are one such group of quintessential forage fishes

14 throughout temperate and sub-polar shelf ecosystems of the Northern Hemisphere. This is

15 particularly true for northern sand lance (Ammodytes dubius) in the Northeast US Shelf

16 ecosystem (Figure 1a). Of 72 regional predators consuming sand lance, populations of humpback

17 whales (Megaptera novaeangilae) and numerous seabirds are particularly reliant on this resource

18 (Staudinger et al., 2020). However, the lack of a directed sand lance fishery and, therefore,

19 regular assessments, has hampered our understanding of basic sand lance ecology that is needed

20 to predict the species' characteristically large interannual fluctuations in abundance (Figure 1b,c;

21 (Nelson and Ross, 1991; Richardson et al., 2014; Staudinger et al., 2020). 
23 sensitive to prey availability and temperature regimes at multiple life stages throughout their

24 range (Winslade, 1974; Tomiyama and Yanagibashi, 2004; van Deurs et al., 2011, 2014, 2015;

25 Régnier et al., 2017, 2018; Clausen et al., 2018; Wright et al., 2017; Murray et al., 2019; Von

26 Biela et al., 2019). Larval prey availability and adult exposure to elevated overwintering

27 temperatures are hypothesized to drive variability in catch of the species $A$. marimus (T. Régnier

28 et al., 2017; Wright et al., 2017). Further, significant reductions in size-at-age were observed for

29 A. personatus off Alaska during the warm and zooplankton-poor "blob" event, and reductions in

30 catch of $A$. japonicus were correlated with decreasing zooplankton abundance off Japan (Von

31 Biela et al., 2019; Nishikawa et al., 2020). These relationships indicate that recruitment and

32 condition of sand lances are linked to prey availability, although existing studies have not

33 explicitly connected adult prey availability and condition to recruitment. Despite numerous

34 studies on congeners, little to no information currently exists on the drivers of northern sand

35 lance abundance throughout its range.

37 they often oscillate out-of-phase with one another, leading to one species replacing the role of the

38 other when environmental conditions change (Bakun and Broad, 2003; Möllmann et al., 2008;

39 Alheit et al., 2014). The Northeast US Shelf forage fish complex has historically exhibited an

40 oscillation between dominance of northern sand lance and Atlantic herring (Clupea harengus;

41 Sherman et al., 1981; Richardson et al., 2014). The causes of this oscillation remain unknown,

42 especially regarding sand lance. Intra-guild predation, zooplankton prey availability, and habitat

43 suitability may be contributing factors (Sherman et al., 1981; Fogarty et al., 1991; Richardson et

44 al., 2014). Intra-guild predation, when a species not only competes with (e.g. for food) but also 

the region also fluctuate on seasonal to decadal scales, resulting in temporally inconsistent prey availability for small pelagic fishes (Kane, 2007; Morse et al., 2017; Perretti et al., 2017).

\section{Materials and Methods}

67 Seasonal feeding and reproductive ecology of sand lance 
70 small-mesh $(0.63 \mathrm{~cm})$ beam trawl (Figure 1a). Stellwagen Bank, a sandy bank in Massachusetts

71 Bay within the Gulf of Maine, is a top predator hotspot and representative of typical sand lance

72 habitat throughout the Northeast US shelf (Hazen et al., 2009; Richardson et al., 2014). Thus,

73 our insights from Stellwagen Bank collections were used to better understand the ecology and

74 predict environmental drivers of this species across their range.

75 Collections occurred monthly in 2019 (except January, July, and December) and

76 opportunistically on cruises from 2016 through 2018 (Table S1). Trawl duration was 10 minutes

77 and distances ranged from $0.5-1 \mathrm{~km}$. Collected sand lance in 2019 were flash frozen in foil in

78 liquid nitrogen upon retrieval. Sand lance collected prior to 2019 were placed in a $-20^{\circ} \mathrm{C}$ freezer

79 and then stored in ice for transportation. All specimens were collected in accordance with the

80 guidelines and approval of Woods Hole Oceanographic Institution's Institutional Animal Care

81 and Use Committee (approval \#23453).

82 Stellwagen Bank can be split into three larger "regions", south, central, and north (Figure

83 1a). Collections aimed to cover both the northern and southern regions of Stellwagen Bank

84 where sand lance density is historically high, except for February when collections solely

85 occurred in the southern and central regions of the bank due to limited ship time.

Zooplankton collections 
in a cast from $3 \mathrm{~m}$ above the bottom to the surface, allowing for the calculation of water volume sampled. Zooplankton samples were immediately preserved in $95 \%$ ethanol upon retrieval.

93 with a modified box splitter. Zooplankton were sorted, counted, and identified to the lowest

94 possible taxa at the Polish Plankton Sorting and Identification Centre and in the lab at Woods

95 Hole Oceanographic Institution following Kane (2007).

96 Sand lance dissections

97 Adult sand lance were thawed to near room temperature in the laboratory for dissections.

98 Standard length, fork length, whole weight, eviscerated weight, liver weight, stomach content

99 weight, and gonad weight were recorded for each fish. Stomach contents were preserved in $95 \%$

100 ethanol after weighing. Heads of each fish were removed for otolith extraction and ageing by

101 making a vertical cut immediately posterior to the opercula. The remaining portion of each fish

102 (stomach contents and head removed) was weighed and freeze dried for subsequent lipid

103 extraction (see lipid extractions). Gonadosomatic index was calculated as the proportion of

104 gonad weight compared to somatic weight.

105 Stomach content identification

106 Stomach contents of each fish that contained prey were examined to identify the specific

107 zooplankton prey of sand lance and how consumption varies seasonally. Details of stomach

108 content identification methods can be found in the supplementary information. Feeding incidence

109 was defined as the proportion of stomachs inspected on each sampling date that contained prey

110 items. An overall proportion of prey taxa by number and biomass was also calculated for each

111 month of collection. 
113 in the stomach under a microscope after dissection. Samples collected before 2019 were not flash

114 frozen, and thus prey items were often degraded and thus may have biased results toward items

115 that were more robust to digestion. We therefore did not calculate diet by biomass or number for

116 pre-2019 collected sand lance.

117 Age and growth

118 A subset of fish from March, April, June, August and November of 2019 were

119 haphazardly selected for otolith-based ageing. Full details of ageing analysis can be found in the

120 supplementary information. For abundant age classes, a von-Bertalanffy growth curve was fit to

121 standard length $(S L)$ by month $(m)$ for each age class with sufficient number of individuals (n

$122>30$ ) following the equation:

$$
S L_{m}=S L_{\infty}\left(1-e^{-k\left(m-m_{0}\right)}\right)
$$

124 where $\mathrm{k}$ is the growth coefficient. Von-Bertalanffy growth curves were fit primarily to estimate

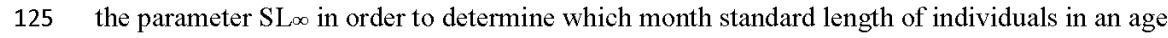
the standard von-Bertalanffy growth equation except for using month as the time scale. Vonand Wheeler, 2020).

Lipid extraction and content analysis 

described in the supplementary material.

Percent lipid values were then fit with a cyclic penalized cubic regression spline with day of collection as the predictor and region of collection on Stellwagen Bank (north, central, south) as a factor via the $m g c v$ package in $\mathrm{R}$ to determine the effect of day of year on sand lance lipid content while accounting for regional variability (Wood, 2017). Region of collection within Stellwagen Bank (north, central, south) was included due to observed differences in zooplankton and hydrography by region (Figure S1, S2). The model was written as follows:

Percent Lipid $=\beta_{0}+s($ DOY $)+$ Region

Where percent lipid represents percent lipid of whole sand lance, $s(D O Y)$ represents the fitted smoothing function for day of year (DOY), and region is fit as a categorical predictor. We report change in deviance explained $(\triangle \mathrm{DE})$ from the full model for each variable as an estimate of their relative importance.

Eighteen individuals were selected for duplicates, encompassing two individuals from each month of collection, to determine intra-fish variability in lipid content estimates. Duplicates indicated $<3 \%$ intra-fish difference.

Shelf-wide estimates and drivers of sand lance abundance

Northeast US shelf sand lance abundance

Estimations of Northeast US shelf sand lance abundance came from the spring Northeast Fisheries Science Center (NEFSC) bottom trawl survey, spanning from 1968 to present. During each survey, 300-400 stations are sampled in a random stratified design, ranging from the Western Scotian Shelf to Cape Hatteras (Figure 1b,c). At each station, all sand lance are weighed and individuals are measured, with sub-sampling occurring when catches were large. Further 
157

158

159

160

161

162

163

details of the survey and sampling gear are found within Politis et al. (2014). The log area

weighted stratified mean abundance in catch of each survey was calculated to estimate

interannual variability in sand lance abundance (Richardson et al., 2014). The spring survey

typically occurs in March and April, well within the feeding window of sand lance (see Results),

and has largely been consistent in timing, gear, and vessel_-allowing the survey to provide a

consistent index of northern sand lance abundance (Politis et al. 2014). This is opposed to the fall

survey which occurs at a transitional window in sand lance life history from feeding to preparing

for fall reproduction, leading to possible conflation of abundance trends with inter-annual

variability in behavior (see Results; Murray et al. 2019). The survey is also not explicitly

designed to sample sand lance habitat and thus there are rarely multiple trawls within a stratum

that represent equivalent sand lance habitat quality. We therefore focused on the log area

weighted stratified mean abundance for the entire survey region, assuming the broad spatial

range and large sample size mitigated the likelihood of spurious or small-scale behavior related

biases in abundances estimates. Sand lance abundance exhibits major fluctuations, ranging

orders of magnitude, allowing the survey to represent sand lance abundance trends despite being

imprecise.

In 2009 , the survey changed vessels and gear, notably switching the net liner from 1.27 to

$2.54 \mathrm{~cm}$, leading to a noticeable decrease in sand lance catchability during the calibration study

(Miller et al., 2010). The time series of sand lance abundance thus ends in 2008 to avoid

conflation of gear selectivity with abundance trends. We do not have age estimates for each year

of the survey, and thus all age classes of sand lance are included in the log area-weighted

stratified mean abundance. The most comprehensive ageing of sand lance from the NEFSC

spring trawl survey suggests $>90 \%$ of individuals collected in the survey are age- 2 or age- 3 
(Nelson and Ross, 1991). Due to this finding, we assumed that abundance of age- 2 and age-3 individuals were responsible for the changes in overall sand lance abundance in the survey.

While the survey provides a robust estimate of the abundance of age- 2 and age- 3 sand lance throughout the Northeast US Shelf, overall abundance of sand lance is likely underestimated due to low selectivity of age-1 individuals (Nelson and Ross, 1991).

Historical EcoMon zooplankton data Calanus abundance anomalies were calculated following Kane (2007) and Perretti et al., (2017) from collections of the NEFSC EcoMon sampling program spanning 1977 to present. Briefly, a mean abundance by date is computed by area for Calanus finmarchicus. This is accomplished by binning all samples for a given species to bi-monthly collection dates based on median cruise date, taking the mean abundance, then fitting a spline interpolation between mean bi-monthly abundance to give expected abundance on any given day of the year. Data were spatially constrained to the Gulf of Maine ecological production unit (Lucey and Fogarty, 2013). All years (1977-2008) were used because annual sampling effort included at least 35\% spatial coverage of the Gulf of Maine ecological production unit (Morse et al., 2017). The Gulf of Maine was chosen as opposed to the full Northeast US shelf as this region serves as the source of Calanus finmarchicus for much of the Northeast US shelf. The Gulf of Maine has deep basins that provide habitat for diapausing Calanus and the general southward flow of the region advects Calanus to the other regions of the Northeast US shelf (Kane, 2005, 2007; Runge et al., 2015; Ji et al., 2017).

Zooplankton data were constrained to cruises centering around bi-monthly dates from January to July to correspond with our observed feeding patterns for sand lance. Winter-summer Calanus abundance anomalies were then lagged to approximate an average Gulf of Maine 
Calanus abundance at $3-4$ years prior to the spring bottom trawl survey estimate of sand lance abundance. A 3-4 year lag was selected as this represents the years in which the parents of fish collected in the survey (age-2s and $3 \mathrm{~s}$ ) would be feeding on Calanus prior to spawning in the fall. The specific selection of this time lag was based upon our observations of sand lance feeding, growth, and lipid content being tightly coupled with their feeding on Calanus. The time lag used therefore represents a test of the parental condition hypothesis, whereby higher abundances of Calanus available to parents would result in either more or higher quality eggs, and thus higher abundances of sand lance in the survey three and four years later when they become catchable in the survey as age- 2 and age- 3 individuals. Atlantic herring index Atlantic herring are intra-guild predators of northern sand lance, meaning that adults of each species are competitors for prey but Atlantic herring also consume the larvae and juveniles of northern sand lance (Suca et al., 2018). Hence, Atlantic herring may exert a strong top-down influence on northern sand lance abundance by consuming the early life stages of sand lance (Polis et al., 1989). Similar to sand lance, Atlantic herring catchability in the NEFSC bottom trawl survey changed during the time series. Catchability of Atlantic herring substantially increased on the survey in the mid-1980s, possibly due to a change in trawl doors (which has been consistently noted; NEFSC, 2018; Miller et al., 2010). Richardson et al. (2014) thus developed a composite index of Atlantic herring abundance, from 1968 through 2010 , combining numerous state and federal fisheries independent surveys of Atlantic herring using a non-linear least-square optimization procedure. This composite index represents an estimation of the overall Atlantic herring abundance on the Northeast US Shelf. It is worth noting that Atlantic mackerel likely 
227 region and tendency to avoid gear used in standardized surveys precluded effective use of

228 Atlantic mackerel abundance as a predictor (Mbaye et al., 2020).

$2302-3$ years prior to the bottom trawl survey estimate of sand lance. This lag indicates the

231 abundance of Atlantic herring on the Northeast US shelf when survey-collected sand lance were

232 larvae and early juveniles and thus susceptible to predation by Atlantic herring.

233 Slope water proportions

234 We then further explored hydrographic drivers of variability in sand lance abundance on

235 the Northeast US Shelf in conjunction with trophic (prey availability and predation) effects. We

236 tested the hypothesis that increasing warm slope water proportion would have a direct

237 detrimental effect on adult northern sand lance abundance on the Northeast US shelf. Slope

238 waters enter the Gulf of Maine through the Northeast Channel (Figure 1) and are composed

239 largely of two sources, colder and fresher Arctic-influenced Labrador slope water (LSW) and

240 warmer, saltier Slope Sea and Gulf Stream-influenced "warm" slope waters (WSW) of which the

241 relative proportions of each source vary interannually (Mountain, 2012). These slope waters then

$242 \operatorname{mix}$ and circulate around the Gulf of Maine, becoming a substantial portion of the shelf water of

243 the Northeast US shelf (Mountain, 2012), the primary habitat of northern sand lance on the

244 Northeast US shelf. These warm slope waters have been shown to have deleterious effects to

245 wintertime populations of Calanus, the primary prey of sand lance (Record et al., 2019). This

246 deleterious effect of warm slope water on Calanus does not appear to carry through the spring,

247 with local production decoupling warm slope water proportions and integrated annual Calamus

248 abundance (Record et al., 2019). Thus the mechanism behind warm slope water's effect on sand 

observations suggest that the latter scenario is more likely as biological sampling showed that reproductive sand lance have their lowest energy reserves in winter, making mature individuals (age-2+) susceptible to mortality from increased metabolism and lack of prey availability from elevated warm slope water proportions. Thus, we hypothesized that increasing warm slope water proportions would have a deleterious effect at a 0 -year lag, given the slope water conditions that either favor age-2+ survival or mortality would occur in the winter prior to the spring trawl survey (i.e. in the same year as the survey). We also note that warm slope water may influence the catchability of sand lance in the survey due to its effect on bottom temperature. However, schooling behavior of congeners have been shown to be primarily linked to feeding and food availability (van Deurs et al. 2011), thus we believe this effect would be small relative the changes in true abundance given the survey overlaps with peak feeding for sand lance (see Results). Mountain, 2012). Slope water proportions were derived from bathythermograph data, CTD data, and water samples collected from Niskin bottles from Northeast Fisheries Science Center surveys from the 1977 to present between $150-200 \mathrm{~m}$ in the Northeast Channel $\left(42.2-42.6^{\circ} \mathrm{N}\right.$, 66-66.8 $8^{\circ} \mathrm{W}$, Figure 1). Data collected from $150-200 \mathrm{~m}$ are assumed to be waters sourced from three sources, Scotian Shelf water $\left(2^{\circ} \mathrm{C}, 32\right.$ salinity), Labrador slope water $\left(6^{\circ} \mathrm{C}, 34.7\right.$ salinity), and warm slope water from off the Northeast US Shelf $\left(10^{\circ} \mathrm{C}, 35\right.$ salinity). The relative proportion of each source was calculated via a 3-point mixing algorithm following Mountain, 

constant over time, though local heat fluxes and mixing likely alter these values. Despite these fluctuations, warm slope water proportion provides a reliable estimate of warmer waters entering the Gulf of Maine and provides an estimate of winter thermal conditions for sand lance over this period. While buoy data exist in the Northeast Channel from 2004-2018 (NERACOOS buoy N), annual estimates of slope water proportions were selected in order to extend the time series back to 1977 , thus matching the length of available zooplankton data. Sand lance abundance regression abundance, the composite Atlantic herring index, and warm slope water proportion using a multiple linear regression written as follows: $\log \left(S L_{t}\right)=\beta_{o}+\beta_{1} *$ Calanus $_{t-3,4}+\beta_{2} *$ Atlantic herring al-2,3 $+\beta_{3} * W S W_{t}$ Where SL represents stratified mean spring trawl survey abundance of sand lance in year $t$, Calanus represents winter-summer Calanus abundance anomaly at a 3-4 year lag, Atlantic herring represents the Atlantic herring index at a 2-3 year lag, and WSW represents warm slope water proportion in year $t$. Predictor variables were not strongly correlated $(\mathrm{r}<0.5)$. We inspected model residuals for temporal autocorrelation (see Figure S11) and used generalized additive models to confirm linear relationships and use of linear regression. We also considered a model including a population dynamics term either in the form of a transformed response variable $\log \left(S L_{t} / S L_{t-3}\right)$ and an autoregressive term $\left(S L_{t-3}\right)$ to allow the model to represent the current abundance relative to the parent population. regression within our dataset. This method iteratively trains the model using $n-1$ observations 
Change in slope water proportions with time

A changepoint beta regression was fit to the time series of warm slope water to determine if a changepoint occurred in warm slope water proportion after 2000 , concurrent with other changes in regional hydrography near this time (Andres, 2016; McCarthy et al., 2018;

Gangopadhyay et al., 2019; Friedland et al., 2020a). Beta distributions were used because warm slope water values are represented as proportions. The stepwise beta regression was written as follows:

$$
\log \left(\frac{W S W}{(1-W S W)}\right)=\beta_{o}+\beta_{1} t+\beta_{2}(t-\tau) I_{\tau}(t)
$$

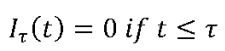

where WSW represents the proportion of warm slope water, $t$ is time in years, and $\tau$ is the changepoint. The parameters $\beta_{0}, \beta_{1}, \beta_{2}, \tau$, and $\theta$ (the dispersion parameter) were fit via maximum $\log$ likelihood using the fmincon function in Matlab. Significance $(\alpha=0.05)$ was determined through parametric bootstrap (1000 simulations). The p-value was defined as the proportion of times the simulated difference in maximized log likelihood of the changepoint beta regression and the beta regression without a changepoint exceeded the difference between these regressions using our observed values, as described in the following equation:

$$
\begin{gathered}
p=\frac{\sum_{i=1}^{1000} X(i)}{1000} \\
X(i)=1 \text { if }\left(L L(i)_{C P_{s i m}}-L L(i)_{\beta_{\text {sim }}}\right)>\left(L L_{C P_{o b s}}-L L_{\beta_{o b s}}\right)
\end{gathered}
$$




$$
=0 \text { if }\left(L L(i)_{C P_{s i m}}-L L(i)_{\beta_{s i m}}\right)<\left(L L_{C P_{o b s}}-L L_{\beta_{o b s}}\right)
$$

Where LL represents the maximum log likelihood, CP represents the changepoint regression, and $\beta$ represents the standard beta regression.

\section{Projections of sand lance abundance}

Log sand lance abundance was projected from 2020-2100 using multiple combinations of time-series predictor variables to represent several future scenarios. Eight scenarios were simulated, encompassed by four groupings ("current trajectory," "optimistic," "average herring," "reduced warm slope water"; Table 1; Figure S3). Owing to observed declines in Atlantic herring biomass and recruitment (NEFSC, 2018), predicted declines in Calanus abundance (Grieve et al., 2017), and our observation of increases in warm slope water proportion, we termed the predictors following this pattern as the "current trajectory" scenario. Long-term projections indicate suitable Calanus habitat throughout the Northeast US Shelf will decrease in the coming century, although there is regional variability in observations and projections of this trend (Reygondeau and Beaugrand, 2011; Grieve et al., 2017; Ji et al., 2017). Atlantic herring recruitment has been below average since 2013, with six of the eight worst recorded recruitment years occurring since 2013 (NEFSC, 2020). Herring spawning stock biomass is also below average and declining, despite stable to decreasing fishing mortality rates (NEFSC, 2020). Such declines, which are not easily attributable to fishing mortality, led to speculation that aspects of the rapidly changing Northeast US shelf ecosystem may be the cause of poor herring recruitment and result in continued declines in Atlantic herring (though egg predation by haddock may also be a cause; Richardson et al., 2011). While it is impossible to know if Atlantic herring populations will eventually recover, it will likely at least take multiple years for the stock to recover from the historically poor recruitment levels seen since 2013 . 
341 was represented by a normal random variable with a linearly decreasing abundance of Calanus

342 from the mean of the existing time series to $68 \%$ percent of its mean to represent the projected

343 decrease in Calanus under Representative Concentration Pathway (RCP) 4.5 or to $50 \%$ of its

344 mean, representing the projected decrease in Calanus abundance under RCP 8.5 (variance was

345 assumed to remain constant in time; rates of decline follow Grieve et al., 2017). Each predictor

346 was simulated independently owing to the lack of strong correlation among predictor variables.

347 Sand lance abundance was then estimated using our fitted multiple linear regression and these

348 simulated regressors. This was then repeated 1000 times to get a mean simulated $\log$ sand lance

349 abundance, along with interquartile range, for each year from 2020-2100.

350 Projection metrics

351 For each scenario, we calculated the percent decline in sand lance abundance from the

352 beginning of the simulation through the following equation:

$$
\text { Percent Decline }=\left(\frac{\overline{S L}_{x}-\overline{S L}_{2020}}{\overline{S L}_{2020}}\right) * 100
$$

354 Where $\overline{S L}_{\chi}$ represents the average projected sand lance abundance in year $x$.

355 We also calculated the proportion of years in each simulation of each scenario that had

356 below average sand lance abundance and proportion of years that had below average abundances

357 of both sand lance and Atlantic herring in the same year. This latter metric is perhaps most

358 informative as this represents an ecosystem with below average abundance of both dominant

359 lipid-rich forage fishes on the Northeast US shelf. 


\section{Results}

Sand lance feeding, lipid accumulation, and growth

Feeding incidence (the percentage of stomachs with prey) varied monthly for sand lance collected in 2019 , with highest values occurring in the winter and spring $(52-84 \% ; \mathrm{n}=212$; Figure 2a). Feeding incidence decreased to zero in August and remained at zero through October, followed by a reappearance of prey in the guts in November - consistent with patterns of monthly feeding incidence from previous years (Figure 2a; Figure S4). During months of feeding (other than November), the copepod Calanus finmarchicus (Calanus hereafter) dominated stomach contents, ranging from $30.4 \%$ of diet by biomass in February to $78 \%$ of the diet by biomass in April (Figure 2b).

Whole body lipid content of sand lance varied significantly by day of year and by region within Stellwagen Bank, peaking throughout the bank in August $(n=197$; deviance explained $=$ $35.2 \%$; Figure 2c; Figure S5). Notably, lipid content decreased rapidly between October and November in connection with spawning during this time window as evidenced by the sharp increase and subsequent decrease in gonadosomatic index (day of year $\Delta \mathrm{DE}=32.5 \%$, Figure 2e). Additionally, lipid content varied spatially within the bank, with highest lipid content values occurring on the northern portion of the bank in each month (region $\triangle \mathrm{DE}=3.0 \%$ ). Intra-annual size-at-age for both age- $2(n=54)$ and age- $3(n=45)$ fish approached their annual maximum in the summer, concurrent with the cessation of feeding and lipid accumulation (Figure 2d). Age-2 fish reached their annual maximum length in June (mean $\mathrm{SL}=168.3 \mathrm{~mm}$, median $\mathrm{SL}=171.0 \mathrm{~mm}, \mathrm{~L}_{\infty}=170.8 \mathrm{~mm}$ ) and age-3 fish reached their annual maximum length by August (mean SL $=184.3 \mathrm{~mm}$, median $\mathrm{SL}=182.5 \mathrm{~mm}, \mathrm{~L}_{\infty}=184.0 \mathrm{~mm}$ ). 

significantly correlated throughout the time series $\left(R^{2}=0.21, \mathrm{p}=0.015\right)$, and particularly well in the $1980 \mathrm{~s}$ and $1990 \mathrm{~s}\left(R^{2}=0.46, \mathrm{p}<0.01\right.$; Figure 3a). However, the relationship decouples later in the time series, concurrent with an increase in Atlantic herring in the region (Figure 3a). Lagged Atlantic herring index and sand lance abundance were negatively correlated $\left(R^{2}=0.28, \mathrm{p}\right.$ $<0.01)$ throughout the time series, with Atlantic herring in low abundance during the $1980 \mathrm{~s}$ "boom" of sand lance and in high abundance when the sand lance time series began to decouple from lagged Calanus abundances in the late 1990s and early 2000s (Figure 3a).

$392=0.52, \mathrm{p}<0.01 ;$ Figure $3 \mathrm{~b}$ ). A cross-correlation analysis indicated that only a 0 -year lag

393 between warm slope water proportion and sand lance abundance was significant (adjusted for

394 autocorrelation; Figure S6). Incorporating 3-4 year lagged Calanus abundance, 2-3 year lagged Atlantic herring index, and unlagged warm slope water proportion in a multiple linear regression explains a majority of the interannual variance in the northern sand lance spring trawl survey, indicating these three mechanisms are likely the dominant drivers of northern sand lance throughout the Northeast US shelf $\left(R^{2}=0.76\right.$; Figure 3; Table 2). Inclusion of population dynamics terms either as a transformed response variable or as an autoregressive term did not improve model fit and thus were not included. values (Figure S7). 
407 fold after $2009\left(\beta_{1}=0.01 ; \beta_{2}=0.26\right.$; Figure 3b; Table 3$)$. Slope water proportions showed no

408 significant mean change with time prior to this observed change point (i.e. from 1977-2008;

$409 \quad$ Figure 3b; Table 3).

410 Projections of sand lance abundance

411 Rates of sand lance decline were similar for each RCP projection in the four scenarios

412 (current trajectory, reduced warm slope water, average Atlantic herring, and optimistic). The

413 average decline in sand lance abundance was $45.6 \%$ and $74.6 \%$ by 2100 for RCP 4.5 and 8.5 ,

414 respectively (Figure 4). The minimum rate of decline for both RCP 4.5 was in the "reduced

415 warm slope water" scenario, with percent declines of $38.5 \%$ and in the "optimistic" scenarios for

416 RCP 8.5 at $71.4 \%$. The maximum percent decline for both RCP 4.5 was in the "average Atlantic

417 herring" scenario at $52.0 \%$ and in the "current trjectory" scenario at $76.9 \%$.

418 Scenarios varied drastically in both the proportion of years with below average sand

419 lance abundance and proportion of years with below average sand lance and Atlantic herring

420 abundance (Figure 4, Table 4). The "reduced warm slope water" scenario had the lowest average

421 proportion of years with below average sand lance abundance, with $34 \%$ and $47 \%$ of years

422 having below average sand lance abundance for RCP 4.5 and 8.5. The "average Atlantic herring"

423 scenario resulted in the highest number of years with below average sand lance abundance,

424 averaging $97 \%$ and $98 \%$ of years for RCP 4.5 and 8.5 , respectively.

425 When Atlantic herring are included in the calculation of below average years of

426 abundance, the "current trajectory" scenario represents the maximum amount of below average

427 years, while the "optimistic scenario" results in the lowest number of these years (Figure 5). An 
428 average of $20 \%$ of years in RCP 4.5 and $26 \%$ of years in RCP 8.5 of the "optimistic" scenario

429 had both negative sand lance and negative Atlantic herring anomalies. However, an average of

$43094 \%$ of years in RCP 4.5 and $96 \%$ of years in RCP 8.5 of the "current trajectory" scenario had

431 both negative sand lance and negative Atlantic herring anomalies indicating nearly all years

432 having below average sand lance and Atlantic herring in the future.

\section{Discussion}

434 Our work indicates that the future of adult northern sand lance in the Northeast US shelf

435 ecosystem is tenuous, owing to the observed and predicted changes in hydrography and prey

436 availability across the region. The sensitivity of sand lance to these changing factors are directly

437 linked to their capital breeding strategy, whereby the species has a defined feeding and growth

438 period, and individual lipid reserves undergo a two-fold change within one breeding season to

439 allocate energy for reproduction. Further, if ongoing declines in Atlantic herring recruitment and

440 biomass persist for decades to come (NEFSC, 2018), the Northeast US shelf will likely be in a

441 state of low adult abundances of its two most dominant lipid-rich forage fishes for much of the

$44221^{\text {st }}$ century.

443 Projections of long-term declines in sand lance abundance indicate marked change in the

444 forage fish complex of one of the world's most economically valuable shelf ecosystems

445 (Hoagland et al., 2005) as concurrent negative anomalies of northern sand lance and Atlantic

446 herring have only occurred in six years from 1968-2008. Such a shift may open niche space for

447 more warm, temperate small pelagic species such as Atlantic butterfish (Peprilus triacanthus)

448 and Atlantic menhaden (Brevoortia tyrannus), diversifying the forage fish complex (Friedland et

$449 a l ., 2020 \mathrm{~b}$ ). This will likely alter the food web of the Northeast US shelf and represent a

450 northward contraction of the region's "lipidscape" (Record et al., 2018). The timing and 
451

452

453

454

455

456

implications of a shift in forage fish community warrants continued, targeted estimates of forage fish abundances and concurrent measurements of the condition of top predator taxa throughout the Northeast US shelf. Tropicalization and shifts in forage community are likely to be detrimental to lipid-rich piscivores, such as humpback whales and bluefin tuna (Thunnus thynnus), while simultaneously ushering in piscivores commonly seen at lower latitudes such as black sea bass (Centropristis striata) and summer flounder (Paralichthys dentatus) (Henderson et al., 2017; Kleisner et al., 2017). This shift in community composition, from forage fish to top predators, would change much of the food web and fisheries of this region.

Declines in Ammodytes condition and catch have been documented in the Pacific, suggesting this key type of forage fish may be in global jeopardy due to shifting climate (Nishikawa et al., 2020; Von Biela et al., 2019). Suggestions of sensitivity of European congeners to temperature further this concern (Lindegren et al., 2018). Many protected species on the Northeast US shelf, such as humpback whales and seabirds, consistently target sand lance as their primary forage and may be in jeopardy from declining sand lance regardless of Atlantic herring's abundance (Staudinger et al., 2020). Populations of seabirds in the Northeast Atlantic suffer high chick mortality when local sand eel (A. marinus) abundances decline (Harris and Wanless, 1991; Rindorf et al., 2000). Recent reports indicate that declines in local sand lance populations even decrease adult survival of Arctic terns (Sterna paradisaea) in Iceland (Peterson et al., 2020). We may thus expect similar levels of repercussions to sand lance-reliant top predators on the Northeast US shelf within this century with recent data suggesting that declines in productivity of the endangered roseate tern (Sterna dongallii) in the Northeast US due to decreasing sand lance are already underway (J. Walsh, pers. comm.). 

consistent with observations of congeners around both the Pacific and Northeast Atlantic difference is timing of the dormancy period. Ammodytes in the Northeast Atlantic are largely

477 considered to be dormant in the winter, along with Ammodytes dubius populations in Greenland

478 (van Deurs et al., 2011; Danielsen et al., 2016; Wright et al., 2017). Our observations of late

479 summer dormancy indicate aestivation - a pattern observed in the warmer water Ammodytes

480 japonicus - as opposed to hibernation observed in Northeast Atlantic congeners (Kuzuhara et al.,

481 2019). Robards et al. (1999) also suggest that Ammodytes personatus in the Pacific undergo

482 winter dormancy, though they did not analyze stomach contents and their observations of

483 seasonal lipid accumulation match the pattern we observe. It is therefore possible Ammodytes

484 personatus also undergoes aestivation and feeds in the winter, contrary to current theory. 
indicate that northern sand lance condition and reproduction may be sensitive to bottom-up

forcing of prey availability. Indeed, even spatial differences in Calanus availability appear to drive differences in lipid accumulation in northern sand lance (Figure 2c). Elevated lipid content on the northern portion of Stellwagen bank corresponds with localized increases in seasonal Calanus abundance brought by the western Gulf of Maine Coastal Current that intercepts this region (Figure S1, S2). Such a link between sand lance condition and Calanus abundance indicates that projected decreases in Calanus on the Northeast US shelf will likely lead to a decrease in availability of high-quality prey for sand lance, and thus may decrease parental condition and spawning output (Grieve et al., 2017).

While consistent with other studies in indicating the importance of Calamus to Ammodytes, the mechanism suggested from our data differs from larval match-mismatch hypotheses proposed to drive Ammodytes recruitment in the Northeast Atlantic (T. Régnier et al., 2017) in that our data suggest Calanus abundance is linked to adult condition and growth. While the three year lag of sand lance behind Calanus abundance may represent both an effect of Calanus on parental condition of age- 2 fish and larval feeding for age- 3 fish, the strongest timelag correlations between sand lance abundance and Calanus anomalies occur at a 3-4 year lag as opposed to a 2-3 year lag, indicating the effect of parental condition is, at the very least, more pronounced than an effect of Calanus abundance on larval feeding (Figure S10). However, it is important to note that this does not exclude the possibility that Calanus abundance plays a role in the early life history of sand lance and further research is needed regarding this question. An effect of parental condition on offspring abundance and/or survival has been both theorized and observed in multiple systems (Green, 2008; Hare, 2014; Hixon et al., 2014, Saenz-Agudelo et al., 2015), including for haddock on Georges Bank (Friedland et al., 2008), and has even been 
520 noteworthy that this effect of Calanus on sand lance recruitment appears to be largely

521 independent of the sand lance spawning stock as population dynamics terms did not improve

522 model fit. This may be due to parental condition overwhelming an effect of abundance and/or the

523 per capita benefit of increased Calanus on parental condition being reduced in years and areas

524 with high densities of sand lance. Both scenarios could lead to present populations of sand lance

525 being largely independent of the size of their parents' population, as observed here. We are also

526 presently unsure if increased Calanus abundance only plays a role in lipid accumulation, or a

527 role in both lipid accumulation and growth. Future studies are needed to assess changes in

528 length-at-age and lipid accumulation for sand lance with changing zooplankton abundance and

529 regimes.

530 In addition to the capital breeding strategy driving recruitment through parental

531 condition, the large intra-annual change in lipid content for sand lance makes them susceptible to

532 overwinter conditions. Individuals are at their lowest energy reserves after spawning and are thus

533 sensitive to increased temperatures and shortages of prey. Such sensitivity to winter conditions

534 has been shown in Ammodytes marimus, indicating this scenario is not unique to northern sand

535 lance (van Deurs et al., 2011). This sensitivity becomes problematic for sand lance populations

536 with recent shifts in hydrography, particularly the changepoint in warm slope water proportion

537 entering the Northeast Channel (Figure 3). This changepoint in hydrography will likely lead to

538 northern sand lance enduring winter regimes with consistently high temperatures and low prey

539 (Record et al., 2019).

The changepoint in warm slope water proportion is noteworthy for the Northeast US

541 shelf ecosystem. Other changepoints have been identified in advective processes in the Northeast 

the Gulf Stream, a westward shift of the Gulf Stream destabilization point, and a rapid shift to an earlier spring transition date throughout the Northeast US shelf after 2008 (Friedland et al., 2015;

545 Andres, 2016; Gangopadhyay et al., 2019). The cause of this changepoint is still undetermined,

546 but hypotheses include a slowing of the Gulf Stream and warming of Slope Sea waters on the

547 coastal side of the Gulf Stream (Andres, 2016; Gangopadhyay et al., 2019; McCarthy et al.,

548 2018). While a change in survey gear precludes consistent estimates of northern sand lance

549 abundance after 2008 , this rapid increase in warm slope water proportion following 2009 is

550 likely to be detrimental to sand lance populations throughout the Northeast US shelf via

551 increasing overwinter mortality of this species.

552 Our work also indicates that sand lance populations may be heavily regulated by intra-

553 guild predation from Atlantic herring. This mechanism has been proposed before (Fogarty et al.,

554 1991; Polis et al., 1989), and subsequent diet studies indicate larval sand lance are important

555 prey for Atlantic herring and Atlantic mackerel (though the overall abundance of the former is

556 much greater; Suca et al., 2018). These dynamics largely allow either Atlantic herring or

557 northern sand lance to be the dominant forage fish throughout the Northeast US shelf in any

558 given year. However, when other drivers prevent a species from rebounding when their intra-

559 guild predator is low, concurrent low abundances of both species can occur. While this has not

560 happened often in the case of the Northeast US shelf, our projections indicate this situation of

561 low northern sand lance and Atlantic herring abundances will happen frequently in the coming decades, and thus the ecosystem level implications of this change require monitoring. 
warm slope water - indicates the worst outcome for the future of forage fish complex on the Northeast US shelf. These projections were also robust to the rate of decline of Calanus, with both RCP 4.5 and 8.5 rates of decline resulting in greater than $90 \%$ of years between $2020-2100$ having below average abundance of both sand lance and Atlantic herring. Given concurrent changepoints in warming rates and advection throughout the region (Andres, 2016; Gangopadhyay et al., 2019; Friedland et al., 2020a), and Calamis' sensitivity to changing temperature and salinity (Grieve et al., 2017), the least certain aspect of these projections is the future of Atlantic herring. However, even if Atlantic herring are to return to oscillating around their historic mean, it would take a drastic decline in warm slope water proportions to allow sand lance and Atlantic herring abundances on the Northeast US shelf to return to similar levels observed prior to 2008. Even this "optimistic" scenario results in a higher proportion of years with negative anomalies of sand lance and Atlantic herring than observed from 1968-2008, showing the projections of change to the forage fish complex on the Northeast US shelf - most notably a clear decline in sand lance - are consistent among all projections. However, the true disappearance of northern sand lance may take longer than the NEFSC trawl survey indicates. The deleterious effect of warm slope water occurs for post-spawning adults (age-2+) and thus it is likely age- 1 sand lance will be able to withstand this change in hydrography. These younger fish may not be sufficient for many predators though, as the ongoing decline in roseate tern productivity indicates (J. Walsh, pers. comm.). Further, if warm slope water affects catchability of sand lance in the NEFSC spring survey, even if the effect is small relative to changes in abundance, this would lead to sand lance disappearing at a slower rate than many of our projections suggest. Such an effect would result in a future more closely following either the "reduced warm slope water" or "optimistic" scenario. Sand lance focused surveys could better 
589 fish on the Northeast US shelf will certainly have a great degree of spatiotemporal complexity

590 not incorporated or assessed in our projections, large scale changes to sand lance abundance are

591 likely to have ecosystem-wide effects and need to be considered in management and protection

592 of top predator populations throughout the region.

593 Supplementary Materials: Supplementary methods, figures, and tables are available

594 at ICESJMS online.

\section{Acknowledgements}

596 We would like to thank Page Valentine and Dann Blackwood of USGS for assistance in

597 collecting data. We also thank the captain and crew of the $R V A u k$, particularly Amy Meloski

598 and David Slocum. Research was funded by the Bureau of Ocean Energy Management (IA

599 agreement M17PG0019; D.N.W, L.K., H.B., and J.K.L.), including a subaward via the National

600 Marine Sanctuary Foundation (18-11-B-203). Additional support came from the National

601 Oceanic and Atmospheric Administration Woods Hole Sea Grant Program (NA18OAR4170104,

602 Project No. R/O-57; J.K.L., H.B., D.N.W.) and a National Science Foundation Long-term

603 Ecological Research grant for the Northeast US Shelf Ecosystem (OCE 1655686; J.K.L.). J.J.S

604 was funded by the National Science Foundation Graduate Research Fellowship program. A.R.R

605 was funded by a NOAA Nancy Foster Scholarship. Manuscript drafts were improved from

606 suggestions by Jessica Perelman, Carin Ashjian, and two anonymous reviewers. No authors have

607 any conflict of interest to declare.

608 Data Availability Statement: Data are available to those with inquires upon request to the

609 corresponding author. 
610 Author Contribution Statement: J.J.S, D.N.W, T.L.S., H.B., L.K., and J.K.L designed

611 research, J.J.S, T.L.S, A.R.R., S.G., E.C., T.G., M.A.T., and P.H. performed the research; J.J.S.,

612 D.E.R., A.R.S., and J.K.L. contributed to data analysis and interpretation, and J.J.S wrote the

613 paper.

\section{References}

Alder, J., Campbell, B., Karpouzi, V., Kaschner, K., and Pauly, D. 2008. Forage fish: From ecosystems to markets. Annual Review of Environment and Resources, 33: 153-166.

Alheit, J., Licandro, P., Coombs, S., Garcia, A., Giráldez, A., Santamaría, M. T. G., Slotte, A., et al. 2014. Atlantic Multidecadal Oscillation (AMO) modulates dynamics of small pelagic fishes and ecosystem regime shifts in the eastern North and Central Atlantic. Journal of Marine Systems, 131: 21-35. Elsevier B.V.

http://dx.doi.org/10.1016/j.jmarsys.2013.11.002.

Andres, M. 2016. On the recent destabilization of the Gulf Stream path downstream of Cape Hatteras. Geophysical Research Letters, 43(18), 9836-9842.

Bakun, A., and Broad, K. 2003. Environmental 'loopholes' and fish population dynamics: Comparative pattern recognition with focus on El Niño effects in the Pacific. Fisheries Oceanography, 12: 458-473.

Barrett, R. T., Nilsen, E. B., and Anker-Nilssen, T. 2012. Long-term decline in egg size of Atlantic puffins Fratercula arctica is related to changes in forage fish stocks and climate conditions. Marine Ecology Progress Series, 457: 1-10.

Center, N. F. S. 2018. 57th Northeast Regional Stock Assessment Workshop (57th SAW)

Assessment Summary Report. 57(February), 12-22. 
Chavez, F. P., Ryan, J., Lluch-Cota, S. E., and Ñiquen, C. M. 2003. Climate: From anchovies to sardines and back: Multidecadal change in the Pacific Ocean. Science, 299: 217-221.

Checkley, D. M., Asch, R. G., and Rykaczewski, R. R. 2017. Climate, Anchovy, and Sardine. Annual Review of Marine Science, 9: 469-493.

Chen, Z., Kwon, Y. O., Chen, K., Fratantoni, P., and Gawarkiewicz, G. 2020. Long - Term SST Variability on the Northwest Atlantic Continental Shelf and Slope Geophysical Research Letters: $1-11$.

Church, G. E., Furness, R. W., Tyler, G., Gilbert, L., and Votier, S. C. 2019. Change in the North Sea ecosystem from the 1970s to the 2010s: Great skua diets reflect changing forage fish, seabirds, and fisheries. ICES Journal of Marine Science, 76: 925-937.

Clausen, L. W., Rindorf, A., van Deurs, M., Dickey-Collas, M., and Hintzen, N. T. 2018. Shifts in North Sea forage fish productivity and potential fisheries yield. Journal of Applied Ecology, 55: 1092-1101.

Danielsen, N. S. T., Hedeholm, R. B., and Grønkjær, P. 2016. Seasonal changes in diet and lipid content of northern sand lance Ammodytes dubius on fyllas bank, west Greenland. Marine Ecology Progress Series, 558: 97-113.

Derek, A., and Wheeler, P. 2020. Package ' FSA'.

Engelhard, G. H., Blanchard, J. L., Pinnegar, J. K., van der Kooij, J., Bell, E. D., Mackinson, S., \& Righton, D. A. 2013. Body condition of predatory fishes linked to the availability of sandeels. Marine Biology, 160(2), 299-308.

Fogarty, M. J., Sissenwine, M. P., and Cohen, E. B. 1991. Recruitment variability and the dynamics of exploited marine populations. Trends in Ecology and Evolution, 6: 241-246.

Fréon, P., Cury, P., Shannon, L., and Roy, C. 2005. Sustainable exploitation of small pelagic fish 
stocks challenged by environmental and ecosystem changes: A review. Bulletin of Marine Science, $76: 385-462$.

Friedland, K. D., Hare, J. A., Wood, G. B., Col, L. A., Buckley, L. J., Mountain, D. G., et al. 2008. Does the fall phytoplankton bloom control recruitment of Georges Bank haddock,

Melanogrammus aeglefinus, through parental condition?. Canadian Journal of Fisheries and Aquatic Sciences, 65(6), 1076-1086.

Friedland, K. D., Leaf, R. T., Kane, J., Tommasi, D., Asch, R. G., Rebuck, N., Ji, R., et al. 2015. Spring bloom dynamics and zooplankton biomass response on the US Northeast Continental Shelf. Continental Shelf Research, 102: 47-61. Elsevier. http://dx.doi.org/10.1016/j.csr.2015.04.005.

Friedland, K. D., Morse, R. E., Manning, J. P., Melrose, D. C., Miles, T., Goode, A. G., Brady, D. C., et al. 2020a. Trends and change points in surface and bottom thermal environments of the US Northeast Continental Shelf Ecosystem. Fisheries Oceanography: 1-19.

Friedland, K. D., Langan, J. A., Large, S. I., Selden, R. L., Link, J. S., Watson, R. A., and Collie, J. S. 2020b. Science of the Total Environment Changes in higher trophic level productivity, diversity and niche space in a rapidly warming continental shelf ecosystem. Science of the Total Environment, 704: 135270. Elsevier LTD. https://doi.org/10.1016/j.scitotenv.2019.135270.

Gangopadhyay, A., Gawarkiewicz, G., Silva, E. N. S., Monim, M., and Clark, J. 2019. An

Observed Regime Shift in the Formation of Warm Core Rings from the Gulf Stream.

Scientific Reports, 9: 1-9.

Green, B. S. 2008. Maternal effects in fish populations. Advances in marine biology, 54, 1-105.

Greene, C. H., and Pershing, A. J. 2007. Climate Drives Sea Change, Science 315: 1084-1086. 
Grieve, B. D., Hare, J. A., and Saba, V. S. 2017. Projecting the effects of climate change on Calanus finmarchicus distribution within the U. S. Northeast Continental Shelf. Scientific Reports: 1-12. Springer US. http://dx.doi.org/10.1038/s41598-017-06524-1.

Hare, J. A. 2014. The future of fisheries oceanography lies in the pursuit of multiple hypotheses. ICES Journal of Marine Science, 71(8), 2343-2356.

Harris, M. P., \& Wanless, S. 1991. The importance of the lesser sandeel Ammodytes marinus in the diet of the shag Phalacrocorax aristotelis. Ornis Scandinavica, 375-382.

Hazen, E. L., Friedlaender, A. S., Thompson, M. A., Ware, C. R., Weinrich, M. T., Halpin, P. N., and Wiley, D. N. 2009. Fine-scale prey aggregations and foraging ecology of humpback whales Megaptera novaeangliae. Marine Ecology Progress Series, 395: 75-89.

Henderson, M. E., Mills, K. E., Thomas, A. C., Pershing, A. J., and Nye, J. A. 2017. Effects of spring onset and summer duration on fish species distribution and biomass along the Northeast United States continental shelf. Reviews in Fish Biology and Fisheries, 27: 411 424. Springer International Publishing.

Hixon, M. A., Johnson, D. W., \& Sogard, S. M. 2014. BOFFFFs: on the importance of conserving old-growth age structure in fishery populations. ICES Journal of Marine Science, $71(8), 2171-2185$.

Hoagland, P., Jin, D., Thunberg, E., and Steinback, S. 2005. 7 Economic activity associated with the northeast shelf large marine ecosystem: Application of an input-output approach. Large Marine Ecosystems, 13: 157-179.

Ji, R., Feng, Z., Jones, B. T., Thompson, C., Chen, C., Record, N. R., and Runge, J. A. 2017. Coastal amplification of supply and transport (CAST): A new hypothesis about the persistence of Calanus finmarchicus in the Gulf of Maine. ICES Journal of Marine Science, 
74: $1865-1874$

Kadin, M., Österblom, H., Hentati-Sundberg, J., and Olsson, O. 2012. Contrasting effects of food quality and quantity on a marine top predator. Marine Ecology Progress Series, 444: 239249

Kane, J. 2005. The demography of Calanus finmarchicus (Copepoda: Calanoida) in the Middle Atlantic Bight, USA, 1977-2001. Journal of Plankton Research, 27: 401-414.

Kane, J. 2007. Zooplankton abundance trends on Georges Bank, 1977-2004. ICES Journal of Marine Science, 64: 909-919.

Kavanaugh, M. T., Rheuban, J. E., Luis, K. M. A., and Doney, S. C. 2017. Thirty-Three Years of Ocean Benthic Warming Along the U.S. Northeast Continental Shelf and Slope: Patterns, Drivers, and Ecological Consequences. Journal of Geophysical Research: Oceans, 122: 9399-9414.

Kleisner, K. M., Fogarty, M. J., McGee, S., Hare, J. A., Moret, S., Perretti, C. T., and Saba, V. S. 2017. Marine species distribution shifts on the U.S. Northeast Continental Shelf under continued ocean warming. Progress in Oceanography, 153: 24-36. http://dx.doi.org/10.1016/j.pocean.2017.04.001

Kuzuhara, H., Yoneda, M., Tsuzaki, T., Takahashi, M., Kono, N., and Tomiyama, T. 2019. Food availability before aestivation governs growth and winter reproductive potential in the capital breeding fish, Ammodytes japonicas. PLoS ONE, 14: 1-16.

Lindegren, M., Checkley Jr, D. M., Ohman, M. D., Koslow, J. A., \& Goericke, R. (2016). Resilience and stability of a pelagic marine ecosystem. Proceedings of the Royal Society B: Biological Sciences, 283(1822), 20151931.

Lindegren, M., Van Deurs, M., MacKenzie, B. R., Worsoe Clausen, L., Christensen, A., and 
Rindorf, A. 2018. Productivity and recovery of forage fish under climate change and fishing: North Sea sandeel as a case study. Fisheries Oceanography, 27: 212-221.

Lucey, S. M., and Fogarty, M. J. 2013. Operational fisheries in New England: Linking current fishing patterns to proposed ecological production units. Fisheries Research, 141: 3-12. Elsevier B.V. http://dx.doi.org/10.1016/j.fishres.2012.05.002.

Mbaye, B., Doniol-Valcroze, T., Brosset, P., Castonguay, M., Van Beveren, E., Smith, A., Lehoux, C., et al. 2020. Modelling Atlantic mackerel spawning habitat suitability and its future distribution in the north-west Atlantic. Fisheries Oceanography, 29: 84-99.

McCarthy, G. D., Joyce, T. M., and Josey, S. A. 2018. Gulf Stream Variability in the Context of Quasi-Decadal and Multidecadal Atlantic Climate Variability. Geophysical Research Letters, 45: 11,257-11,264.

Miller, T. J., Das, C., Politis, P. J., Miller, A. S., Lucey, S. M., Legault, C. M., Brown, R. W., et al. 2010. Estimation of Albatross IV to Henry B. Bigelow calibration factors. Northeast Fisheries Science Center Reference Document, 10-05: $233 \mathrm{p}$

Möllmann, C., Müller-Karulis, B., Kornilovs, G., and St John, M. A. 2008. Effects of climate and overfishing on zooplankton dynamics and ecosystem structure: Regime shifts, trophic cascade, and feedback loops in a simple ecosystem. ICES Journal of Marine Science, 65: $302-310$.

Morse, R. E., Friedland, K. D., Tommasi, D., Stock, C., and Nye, J. 2017. Distinct zooplankton regime shift patterns across ecoregions of the U.S. Northeast continental shelf Large Marine Ecosystem. Journal of Marine Systems, 165: 77-91. Elsevier B.V.

http://dx.doi.org/10.1016/j.jmarsys.2016.09.011.

Mountain, D. G. 2012. Labrador slope water entering the Gulf of Maine - response to the North 
Atlantic Oscillation. Continental Shelf Research, 47: 150-155. Elsevier.

http://dx.doi.org/10.1016/j.esr.2012.07.008

Murray, C. S., Wiley, D., and Baumann, H. 2019. High sensitivity of a keystone forage fish to elevated $\mathrm{CO} 2$ and temperature. Conservation Physiology, 7: 1-12.

Nelson, G. A., and Ross, M. R. 1991. Biology and population changes of northern sand lance (Ammodytes dubius) from the Gulf of Maine to the Middle Atlantic Bight. Journal of Northwest Atlantic Fishery Science, 11: 11-27.

Nishikawa, T., Nakamura, Y., Okamoto, S., and Ueda, H. 2020. Interannual decrease in condition factor of the western sand lance Ammodytes japonicus in Japan in the last decade: Evidence for food-limited decline of the catch. Fisheries Oceanography, 29: 52-55.

NEFSC (Northeast Fisheries Science Center). 2020. Atlantic herring 2020 management track assessment. US Dept. of Commerce. pp 9. https://appsnefsc.fisheries.noaa.gov/saw/sasi/sasi_report_options.php

Oskarsson, G. J., \& Taggart, C. T. 2010. Variation in reproductive potential and influence on Icelandic herring recruitment. Fisheries Oceanography, 19(5), 412-426.

Perretti, C. T., Fogarty, M. J., Friedland, K. D., Hare, J. A., Lucey, S. M., McBride, R. S., Miller, T. J., et al. 2017. Regime shifts in fish recruitment on the Northeast US Continental Shelf. Marine Ecology Progress Series, 574: 1-11.

Pershing, A. J., Alexander, M. A., Hernandez, C. M., Kerr, L. A., Le Bris, A., Mills, K. E., ... \& Sherwood, G. D. 2015. Slow adaptation in the face of rapid warming leads to collapse of the Gulf of Maine cod fishery. Science, 350(6262), 809-812.

Petersen, A., Robertson, G. J., Thorstensen, S., \& Mallory, M. L. 2020. Annual survival of Arctic terns in western Iceland. Polar Biology, 1-7. 
Polis, G. A., Myers, C. A., \& Holt, R. D. (1989). The ecology and evolution of intraguild predation: potential competitors that eat each other. Annual review of ecology and systematics, 20(1), 297-330.

Politis, P. J., Galbraith, J. K., Kostovick, P., and Brown, R. W. 2014. Northeast fisheries science center bottom trawl survey protocols for the NOAA ship Henry B. Bigelow: 138.

Record, N. R., Ji, R., Maps, F., Varpe, Ø., Runge, J. A., Petrik, C. M., and Johns, D. 2018. Copepod diapause and the biogeography of the marine lipidscape. Journal of Biogeography, 45: $2238-2251$

Record, N.R., Runge, J.A., Pendleton, D.E., Balch, W.M., Davies, K.T., Pershing, A.J., Johnson, C.L., Stamieszkin, K., Ji, R., Feng, Z. and Kraus, S.D., 2019. Rapid climate-driven circulation changes threaten conservation of endangered North Atlantic right whales. Oceanography, 32(2), pp.162-169.

Régnier, T., Gibb, F. M., and Wright, P. J. 2017. Importance of trophic mismatch in a winterhatching species: Evidence from lesser sandeel. Marine Ecology Progress Series, 567: $185-197$.

Régnier, T., Gibb, F. M., and Wright, P. J. 2018. Temperature effects on egg development and larval condition in the lesser sandeel, Ammodytes marimus. Journal of Sea Research, 134: 34-41. Elsevier. https://doi.org/10.1016/j.seares.2018.01.003.

Reygondeau, G., and Beaugrand, G. 2011. Future climate-driven shifts in distribution of Calanus finmarchicus. Global Change Biology, 17: 756-766.

Richardson, D. E., Hare, J. A., Fogarty, M. J., and Link, J. S. 2011. Role of egg predation by haddock in the decline of an atlantic herring population. Proceedings of the National Academy of Sciences of the United States of America, 108: 13606-13611. 
Richardson, D. E., Palmer, M. C., and Smith, B. E. 2014. The influence of forage fish abundance on the aggregation of Gulf of Maine Atlantic cod (Gadus morhua) and their catchability in the fishery. Canadian Journal of Fisheries and Aquatic Sciences, 71: 1349-1362.

Rindorf, A., Wanless, S., and Harris, M. P. 2000. Effects of changes in sandeel availability on the reproductive output of seabirds. Marine Ecology Progress Series, 202: 241-252.

Robards, M. D., Anthony, J. A., Rose, G. A., and Piatt, J. F. 1999. Changes in proximate composition and somatic energy content for Pacific sand lance (Ammodytes hexapterus) from Kachemak Bay, Alaska relative to maturity and season. Journal of Experimental Marine Biology and Ecology, 242: 245-258.

Runge, J. A., Ji, R., Thompson, C. R. S., Record, N. R., and Chen, C. 2015. Persistence of Calanus finmarchicus in the western Gulf of Maine during recent extreme warming, 37: $221-232$.

Saenz-Agudelo, P., Jones, G. P., Thorrold, S. R., \& Planes, S. (2015). Mothers matter: contribution to local replenishment is linked to female size, mate replacement and fecundity in a fish metapopulation. Marine Biology, 162(1), 3-14.

Sherman, K., Jones, C., Sullivan, L., Smith, W., Berrien, P., and Ejsymont, L. 1981. Congruent shifts in sand eel abundance in western and eastern North Atlantic ecosystems. Nature, 291: $486-489$.

Staudinger, M. D., Goyert, H., Suca, J. J., Coleman, K., Welch, L., Llopiz, J. K., Wiley, D., et al. 2020. The role of sand lances (Ammodytes sp .) in the Northwest Atlantic Ecosystem : A synthesis of current knowledge with implications for conservation and management: 1-34.

Suca, J. J., Pringle, J. W., Knorek, Z. R., Hamilton, S. L., Richardson, D. E., and Llopiz, J. K. 2018. Feeding dynamics of Northwest Atlantic small pelagic fishes. Progress in 
Oceanography, 165: 52-62. Elsevier. https://doi.org/10.1016/j.pocean.2018.04.014.

Sydeman, W. J., Dedman, S., García-Reyes, M., Thompson, S. A., Thayer, J. A., Bakun, A., and MacCall, A. D. 2020. Sixty-five years of northern anchovy population studies in the southern California Current: A review and suggestion for sensible management. ICES Journal of Marine Science, 77: 486-499.

Thompson, A. R., Harvey, C. J., Sydeman, W. J., Barceló, C., Bograd, S. J., Brodeur, R. D., Fiechter, J., et al. 2019. Indicators of pelagic forage community shifts in the California Current Large Marine Ecosystem, 1998-2016. Ecological Indicators, 105: 215-228. Elsevier. https://doi.org/10.1016/j.ecolind.2019.05.057.

Tomiyama, M., and Yanagibashi, S. 2004. Effect of temperature, age class, and growth on induction of aestivation in Japanese sandeel (Ammodytes personatus) in Ise Bay, central Japan. Fisheries Oceanography, 13: 81-90.

van Deurs, M., Hartvig, M., and Steffensen, J. F. 2011. Critical threshold size for overwintering sandeels (Ammodytes marimus). Marine Biology, 158: 2755-2764.

van Deurs, M., Jørgensen, C., \& Fiksen, Ø. 2015. Effects of copepod size on fish growth: A model based on data for North Sea sandeel. Marine Ecology Progress Series, 520, 235-243. van Deurs, M., Koski, M., \& Rindorf, A. 2015. Does copepod size determine food consumption of particulate feeding fish?. ICES Journal of Marine Science, 71(1), 35-43.

Von Biela, V. R., Arimitsu, M. L., Piatt, J. F., Heflin, B., Schoen, S. K., Trowbridge, J. L., and Clawson, C. M. 2019. Extreme reduction in nutritional value of a key forage fish during the pacific marine heatwave of 2014-2016. Marine Ecology Progress Series, 613: 171-182.

Winslade, P. 1974. Behavioural studies on the lesser sandeel Ammodytes marinus (Raitt) III. The effect of temperature on activity and the environmental control of the annual cycle of 
activity. Journal of Fish Biology, 6: 587-599.

Wood, S. N. 2017. Generalized additive models: An introduction with R, second edition. 1-476 pp.

Wright, P. J., Orpwood, J. E., and Scott, B. E. 2017. Impact of rising temperature on reproductive investment in a capital breeder: The lesser sandeel. Journal of Experimental Marine Biology and Ecology, 486: 52-58. Elsevier B.V.

http://dx.doi.org/10.1016/j.jembe.2016.09.014. 


\section{Figure Legends}

Figure 1. Hydrography of the Northeast US Shelf and sand lance abundance. (A) Overview of the Northeast US Shelf with blue arrows indicating prevailing surface currents of the region, red arrow indicating path of warm slope water entering the Northeast Channel (green box), and the purple arrow indicating flow of Labrador slope water entering the Northeast Channel. Inset displaying the $40 \mathrm{~m}$ isobath of Stellwagen Bank, and the three regions of the bank (North, Central, and South) including trawl locations referenced throughout the text. $(B)$ Mean interpolated cube-root catch of sand lance over the Northeast US shelf as part of the NEFSC spring bottom trawl survey from 1978-1982. (C) Same as (B), except for the period of 19911995.

Figure 2. Ecology and phenology of adult sand lance collected on Stellwagen Bank in 2019. (A) Feeding incidence by day of year for fish collected in $2019(\mathrm{n}=202)$. (B) Sand lance diet by proportion of biomass for each month $(n=70$ fish containing prey, $n=15,279$ prey). (C) Percent lipid of whole fish for sand lance separated by region and day of year on Stellwagen Bank $(n=$ 197). Solid lines represent fitted values from a generalized additive model including month and region with shading representing $95 \%$ confidence intervals (deviance explained $=38.5 \%$ ). $(D)$ Standard length by day of year for age- $2(n=54)$ and age- $3(n=45)$ and dashed lines represent fit von Bertalanffy curves for each age class. (E) Gonadosomatic index (GSI) by day of years for males $(\mathbf{n}=105)$ and females $(\mathbf{n}=107)$ along with mean $+/$ - SEM GSI for each sex by monthly collection. Horizontal axis ticks in each plot represent first of the respective month.

Figure 3. Time series of the environmental drivers of sand lance abundance. $(A)$ NEFSC spring bottom trawl sand lance abundance (log scale; blue), 3-4 year lagged winter-summer Calanus abundance anomaly in the Gulf of Maine (red), and 2-3 year lagged Atlantic herring index (green). ( $B$ ) NEFSC spring bottom trawl sand lance abundance (log scale; blue) and warm slope water proportion entering the Northeast Channel (gold). Dashed line indicates fitted changepoint beta regression (changepoint $=2009, \mathrm{p}<0.001$ )

Figure 4. Projections of sand lance abundance under various scenarios. Predicted sand lance abundance $(\log )$ under "reduced warm slope water" scenario (dotted lines), "optimistic" scenario (thin solid lines), "current trajectory" scenario (thick solid lines), and "average Atlantic herring" scenario (dashed lines). Color represents the projected decline of Calanus under RCP 4.5 (blue) or the projected decline of Calanus under RCP 8.5 (red). Shaded regions represent bounds for the upper (75\%) and lower (25\%) quantiles. Black dashed line represents mean sand lance abundance from $1968-2008$.

Figure 5. Histograms representing proportion of years between 2020 and 2100 that each simulation had negative sand lance abundance anomalies and negative Atlantic herring anomalies for $(A)$ the "current trajectory" scenario, $(B)$ "optimistic" scenario, $(C)$ "average herring" scenario, and $(D)$ "reduced warm slope water" scenario. Color represents RCP 4.5 (blue) and 8.5 (red) rates of Calanus decline. Thick dashed line represents proportion of years from 1968-2008 with both negative sand lance and Atlantic herring anomalies. 
Table 1. Description of scenarios used in predictions of sand lance abundance through the end of the $21^{\text {st }}$ century.

\begin{tabular}{|c|c|c|c|c|}
\hline Scenario & Description & Calanus & Atlantic herring & Warm slope water \\
\hline $\begin{array}{l}\text { Current } \\
\text { Trajectory }\end{array}$ & $\begin{array}{l}\text { Declining } \\
\text { Calanus } \\
\text { Low Atlantic } \\
\text { herring } \\
\text { Increasing } \\
\text { warm slope } \\
\text { water }\end{array}$ & $\begin{array}{c}\text { Normal random variable } \\
\text { with linearly decreasing } \\
\text { mean ( } 32 \% \text { decrease in } \\
\text { RCP } 4.5,50 \% \text { decrease in } \\
8.5) \text {. Constant variance } \\
\text { with time corresponding to } \\
\text { variance of used time series } \\
(1977-2008)\end{array}$ & $\begin{array}{l}\text { Normal random } \\
\text { variable with } \\
\text { mean and } \\
\quad \text { variance } \\
\text { corresponding to } \\
\text { previous declime } \\
(1973-1988)\end{array}$ & $\begin{array}{l}\text { Beta random variable } \\
\text { with mean and } \\
\text { dispersion parameter } \\
\text { following fitted } \\
\text { changepoint regression }\end{array}$ \\
\hline Optimistic & $\begin{array}{l}\text { Declining } \\
\text { Calanus } \\
\text { Average } \\
\text { Atlantic } \\
\text { herring } \\
\text { Pre-2009 } \\
\text { warm slope } \\
\text { water }\end{array}$ & $\begin{array}{l}\text { Normal random variable } \\
\text { with linearly decreasing } \\
\text { mean }(32 \% \text { decrease in } \\
\text { RCP } 4.5,50 \% \text { decrease in } \\
8.5) \text {. Constant variance } \\
\text { with time corresponding to } \\
\text { variance of used time series } \\
\text { (1977-2008) }\end{array}$ & $\begin{array}{l}\text { Normal random } \\
\text { variable where } \\
\qquad \mu=0, \sigma=1\end{array}$ & $\begin{array}{l}\text { Beta random variable } \\
\text { with mean and } \\
\text { dispersion parameter } \\
\text { corresponding to pre- } \\
2009 \text { time series (1977- } \\
\text { 2008) }\end{array}$ \\
\hline $\begin{array}{l}\text { Average } \\
\text { Herring }\end{array}$ & $\begin{array}{l}\text { Declining } \\
\text { Calanus } \\
\text { Average } \\
\text { Atlantic } \\
\text { herring } \\
\text { Increasing } \\
\text { warm slope } \\
\text { water }\end{array}$ & $\begin{array}{c}\text { Normal random variable } \\
\text { with linearly decreasing } \\
\text { mean ( } 32 \% \text { decrease in } \\
\text { RCP } 4.5,50 \% \text { decrease in } \\
8.5) \text {. Constant variance } \\
\text { with time corresponding to } \\
\text { variance of used time series } \\
\text { (1977-2008) }\end{array}$ & $\begin{array}{l}\text { Normal random } \\
\text { variable where } \\
\qquad \mu=0, \sigma=1\end{array}$ & $\begin{array}{l}\text { Beta random variable } \\
\text { with mean and } \\
\text { dispersion parameter } \\
\text { following fitted } \\
\text { changepoint regression }\end{array}$ \\
\hline $\begin{array}{c}\text { Reduced } \\
\text { Warm Slope } \\
\text { Water }\end{array}$ & $\begin{array}{l}\text { Declining } \\
\text { Calanus } \\
\text { Low Atlantic } \\
\text { herring } \\
\text { Pre-2009 } \\
\text { warm slope } \\
\text { water }\end{array}$ & $\begin{array}{c}\text { Normal random variable } \\
\text { with linearly decreasing } \\
\text { mean ( } 32 \% \text { decrease in } \\
\text { RCP } 4.5,50 \% \text { decrease in } \\
8.5) \text {. Constant variance } \\
\text { with time corresponding to } \\
\text { variance of used time series } \\
(1977-2008)\end{array}$ & $\begin{array}{l}\text { Normal random } \\
\text { variable with } \\
\text { mean and } \\
\text { variance } \\
\text { corresponding to } \\
\text { previous decline } \\
(1973-1988)\end{array}$ & $\begin{array}{l}\text { Beta random variable } \\
\text { with mean and } \\
\text { dispersion parameter } \\
\text { corresponding to pre- } \\
2009 \text { time series ( } 1977- \\
\text { 2008) }\end{array}$ \\
\hline
\end{tabular}


Table 2. Fitted parameter estimates for the multiple linear regression of sand lance abundance anomaly ( $\mathrm{n}=25 \mathrm{yrs}$ ).

\begin{tabular}{ccc} 
Regressor & $\begin{array}{c}\text { Slope } \\
\text { Estimate }\end{array}$ & p-value \\
\hline Gulf of Maine Calanus Anomaly & 2.48 & 0.009 \\
Atlantic herring index & -1.27 & $<0.001$ \\
Warm slope water proportion & -5.89 & $<0.001$
\end{tabular}


Table 3. Fitted parameter estimates for the changepoint beta regression of warm slope water proportion with time $(n=39 \mathrm{yrs})$. Model $\mathrm{p}$-value $<0.001$.

Parameter

Estimate

$\begin{array}{cc}\beta_{\circ} & -18.8 \\ \beta_{1} & 0.01 \\ \beta_{2} & 0.26 \\ \tau & 2009\end{array}$


Table 4. Percent sand lance decline under four scenarios and each RCP projection of Calanus decline and proportion of years from 2020-2100 with negative sand lance anomalies and both negative sand lance anomalies and negative Atlantic herring anomalies.

\begin{tabular}{ccccccc} 
Scenatio & $\begin{array}{c}\text { RCP 4.5 } \\
\text { Decline } \\
(\%)\end{array}$ & $\begin{array}{c}\text { Proportion of } \\
\text { Negative Sand } \\
\text { Lance Anomalies }\end{array}$ & $\begin{array}{c}\text { Proportion of } \\
\text { Negative Sand } \\
\text { lance \& Herring } \\
\text { Anomalies }\end{array}$ & $\begin{array}{c}\text { RCP 8.5 } \\
\text { Decline } \\
(\%)\end{array}$ & $\begin{array}{c}\text { Proportion of } \\
\text { Negative Sand } \\
\text { Lance Anomalies }\end{array}$ & $\begin{array}{c}\text { Proportion of } \\
\text { Negative Sand } \\
\text { lance \& Herring } \\
\text { Anomalies }\end{array}$ \\
\hline $\begin{array}{c}\text { Current } \\
\text { trajectory }\end{array}$ & -51.6 & 0.96 & 0.95 & -76.9 & 0.98 & 0.97 \\
$\begin{array}{c}\text { Reduced warm } \\
\text { slope water } \\
\text { Average }\end{array}$ & -38.5 & 0.34 & 0.33 & -74.8 & 0.47 & 0.46 \\
Atlantic herring & -52.0 & 0.97 & 0.47 & -75.1 & 0.98 & 0.48 \\
Optimistic & -40.4 & 0.64 & 0.20 & -71.4 & 0.72 & 0.26
\end{tabular}


AMILA DE SILVA ORCID iD: 0000-0002-5126-8854

Clifton Dassuncao ORCID iD: 0000-0001-7140-1344

Wendy Heiger-Bernays ～ORCID iD: 0000-0002-0091-9629

Barry Kelly ORCID iD: 0000-0002-3449-6310

Carla Ng ORCID iD: 0000-0001-5521-7862

Elsie Sunderland ORCID iD: 0000-0003-0386-9548

20-00597

Critical Review

A.O. De Silva et al.

PFAS Exposure Pathways

PFAS Exposure Pathways for Humans and Wildlife: A Synthesis of Current Knowledge and Key Gaps in Understanding

Amila O. De Silva ${ }^{a}$, James M. Armitage ${ }^{b}$, Thomas A. Bruton ${ }^{c}$, Clifton Dassuncao ${ }^{d}$,

Wendy Heiger-Bernays ${ }^{e}$, Xindi C. $\mathrm{Hu}^{\mathrm{f}}$, Anna Kärrman ${ }^{\mathrm{g}}$, Barry Kelly ${ }^{\mathrm{h}}$, Carla Ng${ }^{\mathrm{i}}$, Anna

Robuck $^{\mathrm{j}}$, Mei Sun ${ }^{\mathrm{k}}$, Thomas F. Webster ${ }^{\mathrm{e}}$, Elsie M. Sunderland ${ }^{1 *}$

This article has been accepted for publication and undergone full peer review but has not been through the copyediting, typesetting, pagination and proofreading process, which may lead to differences between this version and the Version of Record. Please cite this article as doi: $10.1002 /$ etc. 4935 .

This article is protected by copyright. All rights reserved. 


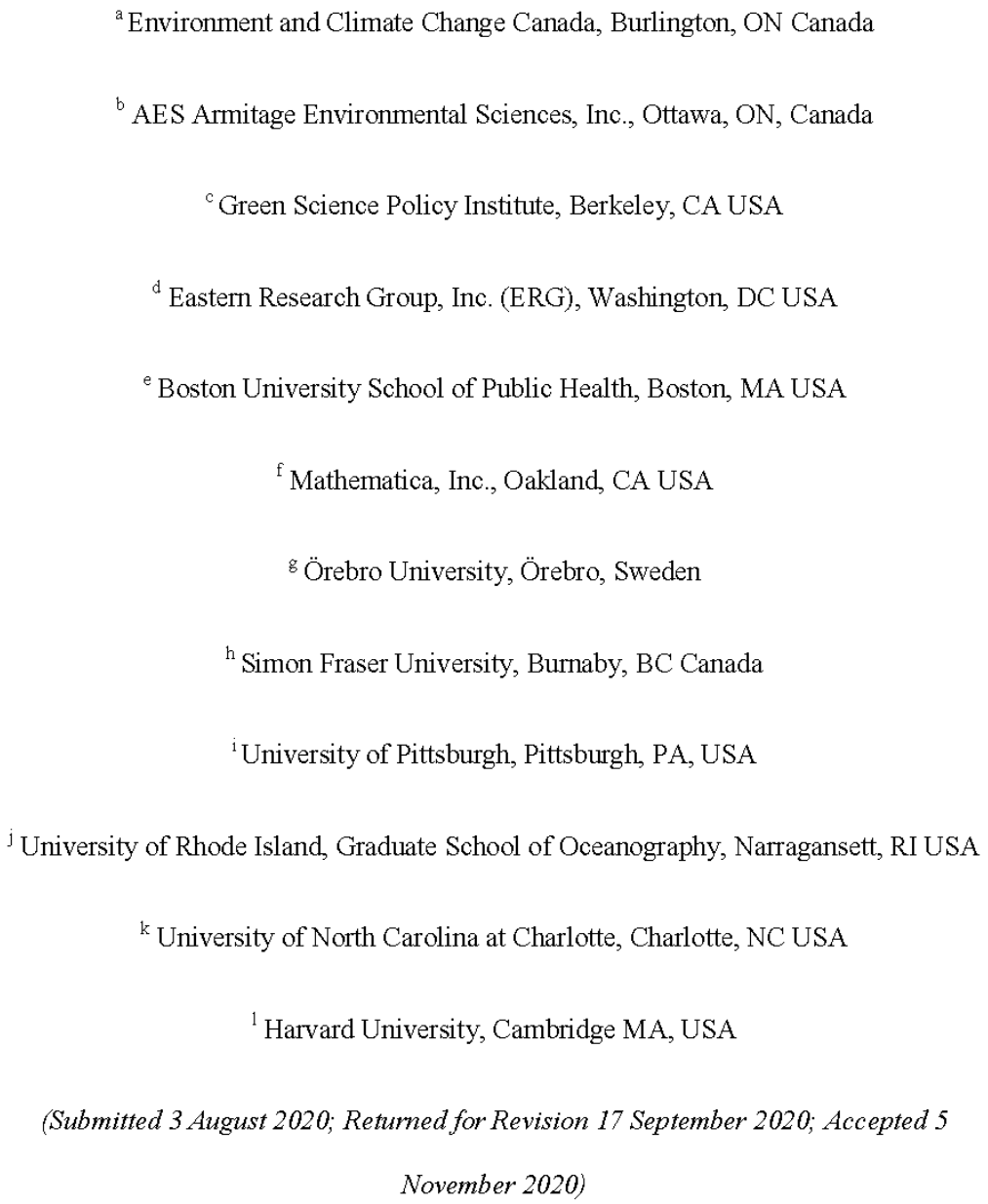

Abstract: Here we synthesize current understanding of the magnitudes and methods for assessing human and wildlife exposures to poly- and perfluoroalkyl substances (PFAS). Most human exposure assessments have focused on two to five legacy PFAS and wildlife assessments are typically limited to targeted PFAS (up to $\sim 30$ substances). However, This article is protected by copyright. All rights reserved. 
shifts in chemical production are occurring rapidly and targeted methods for detecting PFAS have not kept pace with these changes. Total fluorine (TF) measurements complemented by suspect screening using high resolution mass spectrometry are thus emerging as essential tools for PFAS exposure assessment. Such methods enable researchers to better understand contributions from precursor compounds that degrade into terminal perfluoroalkyl acids (PFAA). Available data suggest that diet is the major human exposure pathway for some PFAS but there is large variability across populations and PFAS compounds. Additional data on TF in exposure media and the fraction of unidentified organofluorine are needed. Drinking water has been established as the major exposure source in contaminated communities. As water supplies are remediated, and for the general population, exposures from dust, personal care products, indoor environments and other sources may be more important. A major challenge for exposure assessments is the lack of statistically representative population surveys. For wildlife, bioaccumulation processes differ substantially between PFAS and neutral lipophilic organic compounds, prompting a revaluation of traditional bioaccumulation metrics. There is evidence that both phospholipids and proteins are important for the tissue partitioning and accumulation of PFAS. New mechanistic models for PFAS bioaccumulation are being developed that will assist in wildlife risk evaluations.

Keywords: organofluorine, exposure assessment, bioaccumulation, drinking water, toxicants, wildlife

*Address correspondence to ems@seas.harvard.edu

Published online XXXX 2020 in Wiley Online Library (www.wileyonlinelibrary.com).

This article is protected by copyright. All rights reserved. 
DOI: $10.1002 /$ etc.xxxx

\section{INTRODUCTION}

Poly- and perfluoroalkyl substances (PFAS) are a class of thousands of anthropogenic substances containing an aliphatic fluorinated carbon chain (Buck 2011; OECD 2018). The PFAS family includes: (a) perfluoroalkyl substances, mainly perfluoroalkyl acids (PFAA) such as perfluoroalkyl carboxylates (PFCA) and perfluoroalkyl sulfonates (PFSA), as well as perfluoroalkane sulfonamide substances, and (b) polyfluoroalkyl substances such as fluorotelomer monomers, including fluorotelomer alcohols (FTOH), fluorotelomer olefins (FTO) and fluorotelomer iodides (FTI), and polyfluoroalkyl ether acids. Both perfluoroalkyl substances and polyfluoroalkyl substances can be polymers or non-polymers. The extraordinary strength of the C-F bond in the perfluoroalkyl moiety imparts unique properties (Smart 2001; Biffinger 2004) and has led to widespread industrial and commercial uses of PFAS. With over 7800 PFAS chemical structures identified to date (US EPA 2020a) and thousands registered for regulatory purposes (e.g., chemical inventories) (OECD 2018), they play a prominent role in modern society.

PFAS present a societal challenge. On one hand, PFAS represent some of the most innovative developments in materials chemistry and provide innumerable societal benefits (Johns 2000). However, following decades of widespread global use, and because many PFAS are highly persistent and mobile, concerns have been raised about the ecological and human health impacts of PFAS exposures. PFAS use categories include personal care products (PCP), cosmetics, ski wax, aqueous film forming foams

This article is protected by copyright. All rights reserved. 
(AFFF), textile treatments for stain and water repellency, food contact materials, medical devices, membranes in fuel cells, and membranes in chlor-alkali processes. This list of PFAS applications, though not encompassing of the full scale of PFAS use, captures their diversity. Prior work established the concept of "essential uses" of PFAS by first reviewing where they are abundantly used and when such uses can be replaced by safer alternatives (Cousins 2019).

Multiple strategies have been implemented to reduce emissions, production and use of specific PFAS. Manufacturers have phased out production of certain PFAS and in some cases replaced them with new PFAS or chemical substitutes. For example in textile treatments, many polymers containing long perfluoroalkyl side chains (more than seven perfluorinated carbons) were replaced by analogs containing short perfluoroalkyl side chains (six or four perfluorinated carbons) or fluorine-free moieties (e.g., siloxanes and hydrocarbon polymers) (Schellenberger 2019a). Further efforts are underway to constrain leachable content in fluoropolymers and side-chain fluorinated polymers. This leachable content consists of unbound monomers (such as fluorotelomer alcohols), oligomers, and other non-polymeric PFAS used during the polymer manufacturing process (such as surfactants and chain transfer reagents). Governments have implemented plans to restrict the usage, manufacture and import of certain PFAS, typically on a chemical-by-chemical basis and with certain exemptions. However, based on the recalcitrance of PFAS terminal products, their ubiquitous presence, and continued usage, PFAS exposure to humans and wildlife continues (Scheringer 2014).

This article is protected by copyright. All rights reserved. 
The volume of research publications over the past decade identifying PFAS in environmental media, humans and wildlife is staggering with several comprehensive review publications (Houde 2011b; Jian 2018; Sunderland 2019; Wang 2019). However, our ability to quantify how production and environmental releases of PFAS translate into PFAS tissue burdens in humans and wildlife is still limited. PFAS exposure science was a key area explored in the SETAC Focused Topic Meeting on Environmental Risk Assessment of PFAS held in Durham, NC, USA August 12-15, 2019.

Here we synthesize current understanding of PFAS exposure sources for humans and wildlife and identify key knowledge gaps that inhibit the development of informed regulatory decisions based on sound science. Specifically, we review: (a) PFAS sources and environmental transport pathways; (b) analytical methods used to assess PFAS exposures and their strengths and limitations; (c) methods for assessing human exposures to PFAS; (d) current understanding of the relative importance of different human exposure pathways; and (e) current understanding of PFAS bioaccumulation in wildlife.

\section{OVERVIEW OF PFAS SOURCES AND ENVIRONMENTAL PATHWAYS}

Figure 1 provides a schematic of the sources and spatial scales of PFAS transport and accumulation. Releases to the environment occur during the production, use, and disposal of materials containing PFAS. For example, legacy emissions of perfluorooctanoic acid (PFOA) were dominated by its manufacture and use to manufacture fluoropolymer products (Prevedouros 2006), whereas emissions of PFOS were dominated by its release during use of consumer and industrial products (e.g., surface treatments, AFFF, insecticides) (Armitage 2009b; Paul 2009; Wang 2017a).

This article is protected by copyright. All rights reserved. 
Existing chemical production and release inventories for PFAS have largely focused on PFCA, PFSA and their precursor compounds (e.g., FTOH and perfluorooctane- sulfonamides and -sulfonamidoethanols: FASA) (Armitage 2006; Prevedouros 2006; Yarwood 2007; Armitage 2009a; Armitage 2009b; Paul 2009; Wang 2014a; Wang 2014b; Kotthoff 2015; Shi 2015; Wang 2017b; Boucher 2019). A major shift in chemical production occurred between 2000-2002 with the voluntary phase-out of the base chemical POSF (perfluorooctane sulfonyl fluoride) to PFOS and perfluorooctane sulfonamide-based chemistry by $3 \mathrm{M}$, the major global manufacturer at the time ( $3 \mathrm{M}$ Company 1999). Stewardship programs for PFOA in the United States and Europe have similarly been very successful at phasing out releases of this compound. We now know PFOS, PFOA and other long-chain legacy PFAS compounds represent only a small proportion of the total number of PFAS (US EPA 2020a). Understanding of the global environmental production and distribution of the legacy PFAS has been greatly facilitated by academic partnerships with industry that allowed the development of global emissions inventories for some compounds (Prevedouros 2006; Armitage 2009a; Armitage 2009b; Paul 2009; Wang 2014a; Wang 2017a, 2017b). We thus recommend greater transparency and collaboration between academia, industry and government to prioritize and improve understanding of global environmental releases of the thousands of PFAS structures on US EPA Master List (US EPA 2020a).

The effects of the phase out in chemical production of PFOS and its precursors as well as the PFOA stewardship program are well documented and illustrate the potential benefits of coordinated action curbing chemical releases. For example, ocean modeling studies forced by changes in riverine discharges to the North Atlantic show a large

This article is protected by copyright. All rights reserved. 
decline in surface (0-10 $\mathrm{m}$ depth) seawater PFOS concentrations at their peak (median > $60 \mathrm{pg} \mathrm{L}^{-1}$ ) around the year 2000 to $<40 \mathrm{pg} / \mathrm{L}$ in 2020 (Zhang 2017). In juvenile North Atlantic pilot whales, perfluorooctane sulfonamide: FOSA (a precursor to PFOS) accounted for $84 \%$ of the 15 targeted PFAS measured in the year 2000 but declined to 34\% by 2013 (Dassuncao 2017). Human cohort studies in Denmark, Australia, Japan, Germany, and the Faroe Islands all indicate large declines in PFAS exposure for the general population outside of contaminated areas over this same time period (Olsen 2012; Okada 2013; Yeung 2013a; Toms 2014; Bjerregaard-Olesen 2016; Dassuncao 2018).

Sources of local scale contamination include fluorochemical manufacturing facilities, other manufacturing facilities where PFAS are used, PFAS-containing AFFF, wastewater treatment plants, and landfills. High environmental concentrations of PFAS result in exposure through consumption of contaminated drinking water, agricultural products, or fish and game. For more diffuse PFAS exposures driven predominantly by use of consumer products, population has been established as a good proxy for contamination of surface waters and coastal ecosystems (Paul 2012; Xie 2013; Li 2015; Zhang 2017).

Environmental concentrations and human and wildlife exposures to PFAS are typically highest at contaminated sites (Paustenbach 2007; Pistocchi 2009; Hoffman 2011; Shin 2011a; Shi 2015). Fluorochemical manufacturing sites are responsible for a large proportion of global emissions of certain types of PFAS such as PFCA, but there are relatively few of these sites. One study reported the presence of 16 fluorochemical manufacturing plants in the U.S. (Hu 2016) and another estimated that there were 33

This article is protected by copyright. All rights reserved. 
fluoropolymer production plants worldwide as of 2002 (Prevedouros 2006). High volume emissions from such facilities can impact large geographic areas and correspondingly large populations. For example, releases into the Ohio River from a fluoropolymer production plant in the U.S. state of West Virginia resulted in elevated level of PFAS in drinking water in communities hundreds of miles downstream (Herrick 2017).

Use of AFFF containing PFAS for fire suppression or training activities at military bases, commercial airports, and fire-training areas around the globe has contaminated many aquatic environments (Moody 1999; Barzen-Hanson 2017). Landscapes and water systems adjacent to areas of AFFF use often have high levels of PFAS in soil, sediment, groundwater, surface water, or drinking water (Karrman 2011; Houtz 2013; Anderson 2016; US DOD 2017). PFAS concentrations measured in different environmental media at ten active United States Air Force installations were highest for PFOS and included 4,300 $\mu \mathrm{g} / \mathrm{L}$ in groundwater, $8,970 \mu \mathrm{g} / \mathrm{L}$ in surface water, 190,000 $\mu \mathrm{g} / \mathrm{kg}$ in sediments, 9,700 $\mu \mathrm{g} / \mathrm{kg}$ in surface soil and $1,700 \mu \mathrm{g} / \mathrm{kg}$ in subsurface soil (Anderson 2016).

Incidents of localized contamination have been linked to facilities that employ PFAS to produce goods such as plastic and textile coating facilities (VTDEC 2016; NHDES 2020; NYDEC 2020), and leather tanneries (US EPA 2020b). Numerous other industries are users of PFAS, and all of these have the potential to cause localized contamination. For example, major sources of PFAS contamination in surface waters in New York State and Rhode Island, USA included mixed industrial sources that predominately release PFOS and PFOA, metal plating industry sites, and landfills (Zhang

This article is protected by copyright. All rights reserved. 
2016). PFAS enter landfills as components of residual materials and can be released to the environment in leachate, and may also contribute to elevated concentrations in wastewater (Huset 2011; Lang 2017; Masoner 2020). Concentrations of PFAS in municipal solid waste vary substantially depending on the waste source (Solo-Gabriele 2020).

Wastewater treatment facilities receive PFAS in influent and discharge PFAS in treated effluent and biosolids (Sinclair 2006; Coggan 2019). Treated effluent can be a source of PFAS exposure if it is discharged to a water body that is used as a drinking water source. For example, the probability of detecting PFAS in US public drinking supplies was significantly associated with higher numbers of wastewater treatment plants within a watershed ( $\mathrm{Hu} 2016$ ). Land application of biosolids or irrigation using reclaimed water can result in accumulation of PFAS in soils and underlying groundwater, and uptake into food or fodder crops (Choi 2019; Coggan 2019; Lazcano 2019; Letcher 2020). Concentrations of PFAS are highest in effluent and biosolids for treatment plants that receive wastewater from industrial plants that use PFAS or facilities that use AFFF (3M 2001; Houtz 2016).

Following decades of releases (ca. 1958 to present), PFAS are now ubiquitous in the global environment and the ocean is thought to be the final sink for the terminal products (PFAA) associated with most global production (Armitage 2009a; Armitage 2009b; Zhang 2017). The spatial distributions of PFAS in the environment following releases reflect their physical-chemical properties (propensity for sorption vs. transport in air and water) and types of releases (air, water, soil) during manufacturing, use, and

This article is protected by copyright. All rights reserved. 
disposal. The relative importance of the atmospheric transport/precursor degradation pathway versus the oceanic transport/terminal end product pathway has been assessed for some PFAA. Generally, aquatic discharges and oceanic transport are more relevant next to source regions in the United States, Europe, and Japan (Prevedouros 2006; Armitage 2009a; Zhang 2017) while atmospheric transport is important in remote regions such as the Arctic and Southern Ocean for many compounds (Wang 2015; Dassuncao 2017; MacInnis 2017; Yeung 2017; Pickard 2018). Accumulation of PFAS in the oceans reflects both contemporary and historic PFAS production because terminal PFAA are not known to appreciably degrade under environmental conditions and the timescales associated with PFAS removal through burial in coastal and deep-sea sediment are long (Yamashita 2008).

A major focus of PFAS research is better understanding the releases and environmental degradation pathways of precursor compounds that degrade into terminal PFAA. The majority of precursor compounds studied to date are neutral organics with appreciable vapor pressures. This means that they have a greater propensity for atmospheric transport, whereas most PFAA have low pKa values and exist as stable ions in solution (Cheng 2009). Significant effort is being dedicated to better understanding point source releases of atmospheric PFAS (both PFAA and precursor compounds), but stack testing methods and inventories are still limited. Ionizable precursors also exist but data are scarce. Surface deposition of atmospheric PFAA emissions followed by leaching of PFAS to groundwater has been demonstrated at multiple industrial sites (Guelfo 2018; NHDES 2020; NYDEC 2020). Integration of PFAS measurements into routine atmospheric monitoring for pollutants by programs like the North American Deposition This article is protected by copyright. All rights reserved. 
Program (ww.nadp.org) would thus be valuable for measuring changes in atmospheric

PFAS concentrations, assisting atmospheric PFAS modeling efforts (Thackray 2020), and identifying regions vulnerable to atmospheric PFAS contamination.

\section{ANALYTICAL TECHNIQUES FOR MEASURING PFAS EXPOSURES}

The ability of scientists and regulators to identify and rank the importance of different PFAS exposure sources is directly contingent on analytical methods available for measuring PFAS in a variety of environmental matrices and biological tissues. Analytical techniques for PFAS have advanced rapidly from an early focus on PFOA and PFOS to routine measurements of a suite of approximately 40 PFAS with commercially available analytical standards for detection using liquid chromatography-tandem mass spectrometry (LC-MS/MS). Recent methods have been evolving for detecting volatile species, new compounds, and total fluorine in environmental and human samples. These advances have been critical for keeping pace with the rapidly changing chemical landscape of PFAS exposure sources.

\subsection{Targeted PFAS analysis}

Most studies report concentrations of non-volatile PFAS measured using LCMS/MS. This is commonly referred to as "targeted" analysis because it is based on setting up the instrumental analysis to collect data on specific substances, while confirmation of these substances relies on specific mass-to-charge $(\mathrm{m} / \mathrm{z})$ ratios and retention times based on parameters determined using commercially available chemical standards. Nonetheless, this approach enables quantitative PFAS determination with high precision and high sensitivity. Targeted PFAS analysis is used for water (drinking water, This article is protected by copyright. All rights reserved. 
surface water, groundwater), air/ airborne particulate, food, solids (soil, sediment, house dust), and consumer products. The same techniques are also used to determine PFAS in diverse biological tissues including plasma, sera, whole blood, urine, breast milk, muscle, and other tissues. Over the past few decades, targeted PFAS measurements has improved substantially with improved instrument sensitivity and lower detection limits, numerous laboratory intercomparisons and standard operating procedures, and the addition of more PFAS that can be detected (Guelfo 2020). A major analytical challenge is that synthesis of analytical standards for newer PFAS has lagged their production and release and a lack of reference materials for the major PFAS in routine analysis (Xiao 2017; Land 2018).

3.2 Suspect screening and non-targeted analysis

While targeted analysis is limited to a finite number of PFAS analytes, a comprehensive understanding of PFAS exposure calls for innovative techniques. Specifically, techniques that reveal the presence of emerging PFAS produced intentionally and unintentionally in various industrial processes, as well as PFAS transformation products formed in natural and engineered systems. Suspect screening and nontargeted analysis using high-resolution mass spectrometry (HRMS) allow discovery and characterization of unidentified PFAS in the environment. HRMS provides highly accurate mass to charge ratio of the analytes $(< \pm 0.001 \mathrm{~m} / \mathrm{z})$, its fragments, and their isotopic patterns. Comparing such information with chemical databases that contain thousands of PFAS compounds enables identification of the molecular structure of analytes without analytical standards. Suspect screening and nontargeted analyses have led to the identification of emerging anionic, zwitterionic, cationic, and neutral PFAS in

This article is protected by copyright. All rights reserved. 
water (Strynar 2015; Barzen-Hanson 2017; Gebbink 2017; Newton 2017), sediment (Newton 2017), soil (Baduel 2017; Lin 2017), airborne particulate matter (Yu 2018), and biological samples (Rotander 2015b; Liu 2018).

A drawback of HRMS methods is that such analyses are typically qualitative, due to the absence of analytical standards. In addition, the PFAS congener can only be determined if the sample preparation has adequately recovered the analyte. Analyte spike and recovery validation is not possible without analytical standards. In addition, HRMS data analysis is labor intensive and require specialized analysts. However, it is increasingly recognized for its data-banking value, in which archived HRMS spectra for routine analyses can be re-analyzed when emerging analytes become a priority.

3.3 New methods for closing the fluorine mass balance in exposure studies

A major challenge in PFAS exposure assessment is that most studies employ targeted LC-MS/MS analyses and thus cannot assess the total burden of PFAS in environmental and biological samples. Even HRMS studies are limited by the sample preparation technique which can be discriminatory towards certain classes of PFAS. Also, because HRMS analyses are qualitative or semi-quantitative in nature, they cannot be used to develop mass budgets for total PFAS in the environment or the total burden of PFAS exposures. This has led to the development and use of several total fluorine (TF) measurements to better characterize the total burden of known and unknown PFAS in a sample.

TF methods rely on determinations of the concentration of atomic fluorine. Several strategies have been developed for the determination of the total organofluorine This article is protected by copyright. All rights reserved. 
(TOF), extractable organofluorine (EOF), or adsorbable organofluorine (AOF) content in order to assess the total PFAS content, as reviewed in prior work (Koch 2020). Known PFAS are typically determined using targeted LC-MS/MS techniques on the same sample extracts analyzed for EOF or AOF analyzed using combustion ion chromatography (CIC). The presence of organofluorine is indicative of anthropogenic substances, since organofluorines are very rare in nature as a consequence of the high energy bond between carbon and fluorine. By contrast, inorganic fluoride is the most abundant halogen on Earth and must be quantified to assess the organoflourine content. The portion of unidentified organofluorine in a sample is calculated by subtracting the concentration of target PFAS, converted to moles of fluorine, from the total organofluorine content in the same extract (Figure 2). Because the quantity of unidentified organofluorine is dependent on the targeted analysis, it is challenging to compare unidentified organofluorine among different studies. In Figure $2 \mathrm{~b}$, the unidentified organofluorine is shown relative to the targeted analysis of 50 PFAS congeners in human blood. In general, studies on TOF and $\mathrm{EOF} / \mathrm{AOF}$ indicate a significant portion of organofluorine in biota and the environment is not captured by monitoring of the typical suite of PFAA congeners.

Different methods have been developed for total fluorine or organofluorine measurements in environmental samples and consumer products, including defluorination with sodium biphenyl (Musijowski 2007), ${ }^{19} \mathrm{~F}$ Nuclear Magnetic Resonance (NMR) (Moody 2000), continuum source molecular absorption spectrometry (CS-MAS) (Qin 2012), inductively coupled plasma tandem mass spectrometry (ICP-MS/MS) (Jamari 2018), instrumental neutron activation analysis (INAA) (Schultes 2019), particle-induced gamma-ray emission spectrometry (PIGE) (Ritter 2017), CIC (Miyake 2007a), and X-ray

This article is protected by copyright. All rights reserved. 
photoelectron spectroscopy (XPS) (Tokranov 2019). While some methods can distinguish between organofluorine and inorganic fluoride (NMR, XPS), others give the total concentration of fluorine in a sample (for example PIGE and CIC), therefore a preextraction of inorganic fluorine is needed.

Assessing PFAS in consumer products presents an important challenge for fully understanding human exposure. Some studies have measured the fluorine concentration at the material surface or sub-surface (PIGE, XPS, and INAA), while others consider the average concentrations throughout the whole sample analyzed (CIC). The unknown organofluorine content is useful for understanding TF analysis in human tissues. Concentrations of organofluorine in human serum have been measured using the CIC method, both total fluorine before extraction (Miyake 2007b) and EOF after extraction (Yeung 2016). As an example of this application, one study targeted 52 PFAS congeners in human blood from Münster, Germany including PFSA, PFCA, FASA, fluorotelomer acids (FTUCA \& FTCA), and polyfluorinated phosphate esters (Yeung 2016). Through TF and EOF analysis, these PFAS accounted for approximately $80 \%$ of EOF from 19822006 and the unknown EOF increased to $50 \%$ from 2007 to 2009 , further emphasizing that humans are being increasingly exposed to new organofluorine substances. Caution must be applied in ascribing the unknown EOF to PFAS because many pharmaceuticals contain fluorine. It may be possible to adapt methods to avoid coextraction of non-PFAS organofluorine. For example, a recent study (Figure 2b) used acetonitrile extraction followed by a dispersive graphitized carbon clean-up for human plasma (Miaz 2020), which is likely to sorb the fluorine in pharmaceuticals that is typically in the form of a $-\mathrm{F}$ or $-\mathrm{CF}_{3}$ moiety on an aromatic ring.

This article is protected by copyright. All rights reserved. 
The Total Oxidizable Precursor Assay (TOP) assay provides another method for estimating unknown precursors. The method has been mainly applied to aqueous samples which are subjected to oxidation via hydroxyl radicals formed in a persulfate thermolysis, to transform PFAA precursors to their terminal products (Houtz 2013; Houtz 2016). The oxidized extracts and un-oxidized extracts are analyzed by LC-MS/MS for PFAS in order to quantify the concentrations of oxidizable precursors. The TOP assay highlights the relevance of PFAA precursors to PFAA concentrations in the environment. For example, one study determined that precursors in AFFF had significantly contributed to PFCA and PFSA in groundwater from a firefighting training area (Houtz 2013).

3.4 Strengths and limitations of analytical techniques for assessing exposures

Table 1 compares the strengths and limitations of different analytical methods used to assess PFAS exposures. Comprehensive human exposure assessments need to consider a wider range of PFAS than available from targeted LC-MS/MS measurements, including precursors that transform to terminal PFAA. Non-targeted screening for a variety of matrices and the TOP assay in aqueous samples have been proven useful for detecting additional PFAS. The utility of non-targeted analysis depends on identification strategies and available suspect lists. The TOP assay provides quantitative data on the contribution of oxidizable precursors and can provide some insight on the precursor structure (e.g. functional group and chain lengths). However, the TOP assay has only reliably been performed on aqueous samples due to matrix interference in soil and biota. Further, it is not able to oxidize certain emerging PFAS like per- and polyfluoroalkyl ether acids (PFEA) such as F-53B (6:2 Cl-PFESA) and GenX (hexafluoropropylene oxide dimer

This article is protected by copyright. All rights reserved. 
acid). Non-specific TF methods add information on the magnitude of unknown PFAS that are not quantifiable using targeted analysis. However, some of the detected total fluorine or organofluorine may include compounds outside of those PFAS according to the current definition by the Organization for Economic Cooperation and Development (OECD).

Pharmaceuticals and pesticides with a low atomic fraction of fluorine could result in moderate or high organofluorine content in water if present in high concentrations. Other PFAS classes could be excluded from the analysis during conventional sample extraction. For example, the non-polar PFAS like perfluorobutyl side-chain fluorinated co-polymers surfactant with molecular weight $>1600 \mathrm{~g} / \mathrm{mol}$ was only extracted from soil, wastewater sludge and sediment using 1:1 hexane/acetone, whereas much lower recoveries were obtained using methanol or acetonitrile (Chu 2017; Letcher 2020). The removal of inorganic fluoride also needs to be validated, especially for natural waters and drinking water where fluoride concentrations can be orders of magnitude higher than PFAS concentrations. Non-specific TF methods that target atomic fluorine thus risk overestimating the PFAS content of samples but nonetheless provide a useful screening metric for identifying the magnitude of unidentified organofluorine in an environmental sample.

\section{METHODS FOR ASSESSING HUMAN EXPOSURE TO PFAS}

Exposure pathways for PFAS can be examined as a chain of events shown in Figure 3, linking sources to media (via fate and transport) to external exposure (via behavioral factors) to concentrations in blood, the body's central compartment (via

This article is protected by copyright. All rights reserved. 
toxicokinetics). Exposure routes that are typically examined for PFAS include: dietary ingestion, water ingestion (particularly in contaminated communities), and inhalation of air and dust particles. Hand-to-mouth contact and dermal absorption can also be relevant pathways.

\subsection{Two approaches to PFAS exposure assessment}

Exposures are typically estimated using two complementary approaches. The exposure factor ("bottom up") approach relies on measured concentrations of certain PFAS in exposure media (e.g., food, water, air, dust) and uses estimates of exposure frequency and duration to estimate external exposure (mass $/ \mathrm{kg}$ body weight/time). PFAS levels in media (dietary items, drinking water, and the indoor environment) can also be estimated using multi-media modeling assessments of global or local sources, transport and accumulation. An alternate method is the epidemiologic ("top down") approach to exposure assessment, which typically involves regressing serum/blood levels against measured concentrations in different media (e.g., water, air, dust), and/or behavioral data (e.g., food consumption) to estimate the strength of association with one or more sources. With sufficient data regarding multiple pathways, either method can estimate the relative importance of different exposure routes.

Several studies have used the exposure factor approach to quantify the contribution of PFAS in the indoor environment, seafood, drinking water, and food packaging to total exposures in humans (Trudel 2008; Vestergren 2008; Harrad 2010; Gebbink 2015; Dassuncao 2018). Exposure to precursors has been linked to increased bioaccumulation in food webs (Kelly 2009; Dassuncao 2017; Boisvert 2019; Zhang 2019). Associations between serum PFAS concentrations and exposure behaviors such as

This article is protected by copyright. All rights reserved. 
water district of residence, consumption of tap water, and fish consumption have also been characterized in prior work (Christensen 2017; Herrick 2017; Dassuncao 2018; Barton 2020).

The two exposure assessment methods each have strengths and weaknesses and are complementary. In the exposure factor approach, one or both elements may be uncertain. For example, while measuring PFAS in food can be analytically challenging with many non-detects, average food consumption rates are better known. In contrast, measuring PFAS in house dust is easier, but dust ingestion rates are uncertain, particularly for adults. Carrying out statistically representative (and therefore extrapolatable) sampling and characterizing the fraction of exposure originating from precursors is challenging for many exposure routes. One strength of the exposure factor approach is the potential for a direct link to chemical production and environmental concentrations that drive exposures (Armitage 2009a). This information is critical for designing interventions that mitigate human and wildlife exposures.

The epidemiologic approach integrates both exposure and toxicokinetics, but often must take into account potential confounding between exposure routes as well as exposure measurement error. For example, the latter can arise when regressing serum concentrations of persistent PFAS with media measured at one point in time. The long half-life of many PFAS in humans means that serum concentrations reflect cumulative exposures over a relatively long time period, while external exposure measurements may often reflect a short exposure window. Hybrid models can be used to integrate and compare the two approaches. For example, one can use toxicokinetic models to estimate serum levels from exposure estimates (or vice versa), comparing the estimated and

This article is protected by copyright. All rights reserved. 


\subsection{Indoor exposure via inhalation and dust ingestion}

Most North Americans spend approximately $90 \%$ of their time in indoor environments. PFAS are used extensively in products designed for indoor use such as stain resistant coatings for carpet and furniture. They are found in indoor air and dust, although so far the connection to specific indoor sources has received little attention (Beesoon 2012). Thus, there is the potential for indoor exposures to PFAS via inhalation, ingestion of dust and dermally.

Investigation of indoor exposure to PFAS is more complicated than for many groups of compounds (e.g., polybrominated diphenyl ethers: PBDEs) due to the vast variety of physical-chemical properties for PFAS and the existence of precursors and polymers. Semivolatile organic compounds (SVOCs) will tend to partition between the vapor phase, suspended particulate, dust, and indoor surfaces (including skin and clothing), depending in part on their octanol/air partition coefficients (Weschler 2008). Some PFAS such as FTOH and FOSE are relatively volatile and are found in the vapor phase indoors. Other PFAS such as PFOA and PFOS are found at high concentrations in dust. There is little information available about the indoor presence and fate of fluorinated polymers (e.g., side-chain fluoropolymers used in some stain resistance formulations). In part this is due to analytical difficulties (Rankin 2015; Letcher 2020). They may be released from products to dust via physical abrasion—as has been shown for other low volatility compounds (Webster 2009) — or potentially gradually breakdown over time, releasing the fluorinated side chains as may occur in outdoor environments (Washington 2015; Letcher 2020). An important question is the amount of unidentified

This article is protected by copyright. All rights reserved. 
organic fluorine in air and dust. For example, TF analysis in conjunction with HRMS may be a useful first step in examining polymers or other unmeasured compounds in dust.

PFAS concentrations in indoor environments are usually measured through filtration or adsorption to a solid phase (filters or sorbents) using either active air pumping or passive samples, followed by extraction of the solid phase to recover PFAS for quantification (Martin 2002; Shoeib 2008; Padilla-Sánchez 2017; Guo 2018; Rauert 2018; Wong 2018; Yao 2018). In indoor air, concentrations can be an order of magnitude higher (ng/ $\mathrm{m}^{3}$ levels) than outdoor environments (Shoeib 2004; Shoeib 2005; Yao 2018). Indoor air sampling has tended to focus on the more volatile compounds such as the FTOHs and FASA.

PFAS concentrations in dust can be very high and have been reported at the $\mu \mathrm{g} / \mathrm{g}$ level (Moriwaki 2003; Kubwabo 2005; Shoeib 2005; Strynar 2008; Eriksson 2015a; Eriksson 2015b; Lankova 2015; Winkens 2018). Dust sampling has found PFAA and other PFAS, including relatively large amounts of polyfluorinated phosphate esters (diPAP) (De Silva 2012; Eriksson 2015a; Makey 2017). Methods for dust sampling and processing - e.g., where and how to sample dust in homes, sieving size — are less standardized than for air. Bigger issues are the variety of indoor environments-home, workplace, childcare facilities, vehicles, etc. (with homes being a main focus) - and the difficulty of doing representative sampling (Goosey 2012; Fraser 2013; Zheng 2020). Unlike the extraordinary efforts that have been made for representative biomonitoring (e.g., NHANES in the USA), most indoor sampling is convenience sampling that may not

This article is protected by copyright. All rights reserved. 
be representative of exposures across the general population. Exposure factors for inhalation are well known, but exposure factors for dust ingestion are quite uncertain (US EPA 2011). As a result, inhalation estimates are likely more reliable than those for dust ingestion. An additional source of uncertainty is the amount of conversion of precursors (e.g., FTOH, FASA) into terminal end products (e.g., PFCA, PFSA) found in serum/blood (Poothong 2020). This issue is particularly important when trying to compare inhalation with dust ingestion, or indoor routes of exposure to diet and other sources.

Two North American studies have found associations between serum levels of some PFAA and the precursors FTOH and FASA, respectively, in indoor air (Fraser 2012; Fraser 2013; Makey 2017), but little association with PFAS concentrations in dust A Norwegian study found associations between certain PFAS in whole blood and indoor air and/or dust (Poothong 2020). The latter study also estimated that diet was more important than indoor exposure on average but that inhalation and dust ingestion dominated for some study participants, particularly the people with the highest blood concentrations. Some studies have found that serum PFAS concentrations increase with socioeconomic status indicating that indoor and dietary exposure may be partly correlated due to purchasing decisions (Nelson 2012). Relatively few studies have used epidemiologic techniques to examine air and dust or other pathways simultaneously (Haug 2011; Fraser 2013; Makey 2017; Poothong 2020).

In summary, some epidemiologic evidence suggests indoor exposure is important enough to be empirically associated with serum/blood levels, and may be the dominant exposure route for some people (Fraser 2013; Makey 2017; Poothong 2020). More

This article is protected by copyright. All rights reserved. 
research is needed on the differences in indoor exposure patterns between people as well as differences between countries and time trends. Relatively little research has been conducted on the connection between indoor levels and putative sources, total organic fluorine, the contribution of fluorine-containing polymers, and exposure of children.

\subsection{Outdoor air exposures}

Reported PFAS concentrations in outdoor air range from non-detectable or sub $\mathrm{pg} / \mathrm{m}^{3}$ levels to hundreds of pg/m³ (Martin 2002; Stock 2004; Barber 2007; Jahnke 2007; Fromme 2009; Rauert 2018; Wong 2018). PFAS concentrations in urban areas are typically higher than in rural areas (Martin 2002; Stock 2004; Barber 2007; Jahnke 2007). A few studies of general populations report that outdoor air PFAS concentrations are one to two orders of magnitude lower than indoors (Shoeib 2011), presumably due to indoor sources. For such populations, we expect inhalation exposure indoors to exceed outdoor exposures.

Little is currently known about communities with major atmospheric point sources. In Parkersburg, West Virginia, USA, local drinking water sources were contaminated primarily by air emissions of PFOA emitted by the Washington Works facility followed by deposition and groundwater transport (Davis 2007). In Fayetteville, $\mathrm{NC}$, precipitation monitoring to assess the deposition of GenX via air emissions has been ongoing since 2018. Communities in Hoosick Falls, NY, Bennington, VT and Merrimack, NH have had varying extent and types of monitoring conducted in response to concerns or documentation of groundwater/drinking water contamination. An important question in these scenarios is the relative importance of exposure via inhalation This article is protected by copyright. All rights reserved. 
vs. water ingestion. Using a sophisticated fate and transport model, researchers estimated PFOA concentrations in ambient air and water in the communities surrounding the Washington Works facility in West Virginia, USA over time (Shin 2011b; Shin 2011c). Their results compared well with measured water values and indoor air was assumed to be $10 \%$ of outdoor air due to partial infiltration. Transport of PFAS in air is faster than in soil and groundwater. Thus, for people living in areas with contaminated air, estimated inhalation exposure exceeded that via water ingestion in the early time period but was less than water ingestion later on (Shin 2011b; Shin 2011c). These results suggest that inhalation exposure to PFAS might exceed water ingestion in areas with continuing air emissions but mitigation of drinking water (e.g., via filtration).

\subsection{Dermal exposures to PFAS}

Dermal exposure to PFAS can result from contact with house dust, PCP, and other consumer products. It has received relatively little attention, with two exceptions: dust and, more recently, cosmetics/personal care products (PCP). For example, one study estimated dermal exposure of children to PFAS in dust in childcare settings using measured dust concentrations and an exposure factor for the amount of dust adhering to skin (Zheng 2020). Poothong et al (2020) used an alternative method for skin contact: measuring PFAS on hands using handwipes. Both then used the fraction dermal absorption approach, a model commonly used in risk assessment (Kissel 2011).

One study examined selected PCP with product labeling indicating PFAS ingredients such as polyfluorinated phosphate esters (PAPs) or other fluorinated compounds and reported PFCAs in the ug/g range but did not determine the levels of

This article is protected by copyright. All rights reserved. 
PAPs (Fujii 2013). Another study that measured 39 PFAS, as well as EOF and total fluorine, detected PAPs at up to $470 \mathrm{ug} / \mathrm{g}$ in cosmetic products (Schultes 2018). The measured PFAA accounted for only a small fraction of the EOF and TF, implying the presence of unidentified compounds, potentially including polymers or inorganic fluorine. Skin contact with PCP can depend on a number of factors including the amount of the product applied per unit area (loading), the surface area of exposed skin, the duration of use and the frequency of washing the skin. Prior work has estimated dermal absorption of PFOA in foundation cosmetics, leading to an absorbed dose through dermal exposure of $<0.006-3.1 \mathrm{ng} / \mathrm{kg} /$ day, with the high end exceeding dietary exposure in Sweden (Schultes 2018). This dermal exposure estimate for PFOA does not include indirect exposure via PAP.

\section{RANKING SOURCES OF PFAS EXPOSURE FOR HUMAN POPULATIONS}

A major question discussed by the exposure assessment panel at the SETAC PFAS topic meeting was whether it is possible to rank the relative importance of PFAS exposure sources for different human populations at this time. Researchers and decisionmakers seek to understand the relative contributions of different exposure pathways to human exposure in order to inform risk assessments and prioritize interventions. The panel concluded that there were many gaps remaining in this area, especially for general populations that have diverse exposure pathways for PFAS. A summary of present understanding is provided below.

This article is protected by copyright. All rights reserved. 


\subsection{Occupational exposures}

Occupational health effects associated with human PFAS exposures have been reported for individuals who worked in fluorochemical production plants in the US (Olsen 1999), Italy (Girardi 2019) and China (Fu 2016). Occupational exposure to PFAS occurs mainly through inhalation and dermal contact (Franko 2012). Inhalation exposure to PFAS in a workplace situation can be important due to sublimation into the gaseous phase of volatile manufacturing intermediates that are hydrolyzed to end product PFAS (Kaiser 2010). Firefighters working and training with AFFF did not show a relationship between internal exposure of PFOS and the self-reported frequency of direct skin contact with AFFF, indicating that dermal contact may not be an important pathway of exposure (Rotander 2015a). However, they had elevated blood levels of PFOS and PFHxS, which is consistent with the composition of legacy electrochemical fluorination (ECF) AFFF. Elevated levels of PFNA and other long-chain PFCAs have been connected to occupational exposure for firefighters (Laitinen 2014; Trowbridge 2020). Professional ski wax technicians showed elevated blood levels of PFCAs, which was associated with number of working years. Ski wax contains both precursor semifluorinated n-alkanes and PFCA (Plassmann 2013; Carlson 2020) and is often applied by using heat $\left(130-220^{\circ} \mathrm{C}\right.$ ) which results in gaseous compounds and particles. An exposure study with internal dose measurement and personal and ambient air including airborne particles showed that inhalation of both precursor compounds and terminal end products contributed to the internal dose (Nilsson 2010).

5.2 Communities near contaminated sites

This article is protected by copyright. All rights reserved. 
PFAS have been detected in both surface water and groundwater sources near facilities that manufacture or process fluorochemicals. Concentrations in aqueous matrices in areas without point sources generally occur below detection limits or at the sub ng/L (parts per trillion) level but are commonly detected at the $\mu \mathrm{g} / \mathrm{L}$ (parts per billion) level or higher in contaminated areas (Chen 2017b; Cai 2018; Pan 2018; Park 2018; Janda 2019). These include sites with historical releases of AFFF, such as military bases and airports, and wastewater treatment plants that have received industrial wastes (Herrick 2017; Worley 2017; Barton 2020). These sites have been associated with contamination of drinking water across the U.S. with at least six million people estimated to have been exposed to levels above EPA health advisories for PFOS and PFOA of 70 $\mathrm{ng} / \mathrm{L}$ (Hu 2016). The total number of people exposed to PFAS in the U.S. is greater because this estimate does not include populations exposed through small drinking water systems or private wells or exposure to other PFAS.

Many biomonitoring studies have shown PFAS in drinking water near contaminated sites have led to population blood levels that are much greater than background levels (Daly 2018; Ingelido 2018; Li 2018). In such areas, serum levels are associated with concentrations in drinking water (Hoffman 2011). Drinking water has been estimated to contribute up to $75 \%$ of exposures near contaminated sites (Vestergren 2009). The U.S. Agency for Toxics Substances and Disease Registry (ATSDR) is currently conducting biological monitoring at over 15 sites with contaminated drinking water across the country following comparable methods for collecting blood and administering exposure questionnaires. This work will generate further information about

This article is protected by copyright. All rights reserved. 
the contributions of drinking water exposures across a diverse set of communities near contaminated sites.

Production and use of the most common legacy PFAS (PFOS, PFOA, PFHxS) have decreased in the U.S. but an increasing number of sites contaminated with these compounds have been identified over the past decade. Data compiled by the Northeastern University's Social Science Environmental Health Research Institute (SSEHRI) show that as of May 2020 there were 393 known contaminated public drinking water systems in the U.S. (SSEHRI 2020). As background levels of legacy PFAS decrease, the relative contribution of their exposure from drinking water sources near these contaminated sites will increase (Bao 2017). Furthermore, replacement compounds, such as PFEA, including GenX, have already been detected in drinking water near manufacturing facilities (Hopkins 2018). Newer methods that measure EOF and TF are beginning to be used on drinking water with the goal of quantifying the exposure of unidentified PFAS (Hu 2019). However, the large-scale implications of changes in production have yet to be fully understood.

\subsection{General population PFAS exposures}

The relative importance of different PFAS exposure sources varies dramatically across general populations with diverse PFAS exposure sources. Table 2 summarizes some prior literature estimates of source contributions to overall PFAA exposures for adult populations without occupational exposure and not living in close proximity to point sources of PFAS contamination. Large variability in the relative importance of different exposure sources across studies reflects variable concentrations in

This article is protected by copyright. All rights reserved. 
environmental media and differing assumptions regarding exposure sources, frequencies, duration, and consideration of precursors.

Several studies have shown that the exposure to PFAS in children differs from adults due to behavioral and dietary variability. Breast feeding is known to be an important source of early-life exposure to PFAS (Mogensen 2015; Kang 2016; Papadopoulou 2016). A study from the Faroe Islands showed hand-to-mouth contact with carpeting was an important exposure source for children but not adults based on the contrasting composition of PFAS measured in serum (Hu 2018). The 2020 European Food Safety Authority (EFSA) report found that toddlers/children had a two-fold higher exposure than adults, in part due to maternal exposure (European Food Safety Authority 2020).

There is general agreement that dietary exposure is the major contributor to population exposure for PFOS and PFOA (Table 2), but more limited evidence for PFHxA, PFHpA, PFNA, PFDA, and PFHxS. In 2018, EFSA estimated that the main contributors to adult dietary exposure to PFOS were fish, meat, eggs and products with these ingredients (European Food Safety Authority 2018). The greatest sources of exposure to PFOA were eggs and dairy and products containing these ingredients. The youngest population groups were estimated to have the highest dietary exposure to PFOA and PFOS. In 2020, EFSA expanded its analysis of exposures to PFAS in foods and reported that the sources contributing to PFAS exposure to adults and children were consistent with the findings from 2018. They concluded that PFOA contributed $21 \%$, PFNA $4 \%$, PFHxS $10 \%$ and PFOS $66 \%$ to the sum of PFAS exposures, based on the

This article is protected by copyright. All rights reserved. 
Reiner 2015; Penland 2020). Several comprehensive reviews (Houde 2011a; Reiner 2015; Muir 2019) have synthesized data from available biomonitoring studies.

The highest PFAS concentrations in wildlife tend to be associated with proximity to contaminated sites. For example, one of the highest reported fish PFOS concentrations (maximum $9349 \mathrm{ng} / \mathrm{g}$ dry weight in whole fish tissue) was from an AFFF-impacted site downstream from Barksdale Air Force Base in Louisiana (Lanza 2017). Many biomonitoring studies have identified elevated exposures to legacy and emerging PFAS as the result of industrial activities (Custer 2012; Custer 2014; Liu 2017; Groffen 2019; Lopez-Antia 2019; Guillette 2020). Legacy PFAS such as PFOS are still abundant at many contaminated sites and novel PFAS are increasingly being detected. For example, one study reported PFOS was the predominant compound in fish (mean 263-348 ng/g wet weight (ww) in muscle) adjacent to a major fluorochemical production facility in Wuhan, China (Zhou 2013). Suspect and non-target screening subsequently revealed a suite of 330 novel fluorinated structures belonging to 10 different chemical classes in fish liver from the same region (Liu 2018). The profile of specific PFAS released at each contaminated site affects the accumulation of different compounds in biota and may also be relevant for determining exposure risks such as near AFFF contaminated regions (Yeung 2013c; Custer 2014; Munoz 2017; Larson 2018; Salice 2018; Langberg 2019; Munoz 2020).

Biological time series data for specific ecosystems suggest variable temporal changes in PFAS across compounds and ecosystems. In the Arctic, there is sustained or increasing (post-2010) PFAA levels in some wildlife (Muir et al. 2019). Following the

This article is protected by copyright. All rights reserved. 
phase out of the parent chemical to PFOS and its precursors ca. 2000-2002, several studies have noted rapid declines in perfluorooctane sulfonamide (FOSA), an atmospheric precursor to PFOS that is biotransformed by most mammals, but less consistent declines or even increases in PFOS (Smithwick 2006; Ahrens 2009a; Dassuncao 2017; Sun 2019; Schultes 2020). This likely reflects the rapid response of atmospheric concentrations to changes in chemical production but a lagged response of most aquatic ecosystems. Most time series studies have focused on targeted PFAS but recent work has shown that trends in TF indicated by EOF and other methods differ from the legacy compounds (Schultes 2020). Understanding temporal and spatial trends in emerging PFAS compounds in biota is thus an important research need (Spaan 2020).

\subsection{PFAS bioaccumulation metrics}

The bioaccumulation potential of persistent organic pollutants (POPs) is commonly reported based on several metrics: bioconcentration factor (BCF: the direct uptake of a chemical by an organism from water or air, i.e., $\mathrm{BCF}=\mathrm{C}_{\text {fish }} / \mathrm{C}_{\mathrm{water}}$ in a controlled laboratory experiment with no dietary intake); biomagnification factor (BMF: the concentration of an organism relative to their diet, i.e., $\mathrm{C}_{\text {fish }} / \mathrm{C}_{\text {prey }}$ ); bioaccumulation factor (BAF: the combined effects of all uptake pathways). BCFs are commonly measured in laboratory experiments, while BMFs and BAFs are typically field-based measurements. The trophic magnification factor (TMF) is an indicatory of dietary biomagnification and is generally established empirically using slope of the relationship between trophic position in a food web based on stable nitrogen isotopes and chemical concentrations in organisms from a field-based food web. A large body of work has established that for neutral,

This article is protected by copyright. All rights reserved. 
hydrophobic POPs, simple partitioning between lipid and water indicated by their octanolwater partition coefficient $\left(\mathrm{K}_{\mathrm{ow}}\right)$ or octanol-air partition coefficient $\left(\mathrm{K}_{\mathrm{oa}}\right)$ provides a reasonable proxy for bioaccumulation propensity.

By contrast, processes governing the uptake of PFAS into organisms and partitioning across tissues are less well-understood, even for commonly studied PFAA (Figure 4). The pKa of long-chain PFAA are thought to range from less than zero to 1, indicating that they are almost completely ionized at environmentally and biologically relevant pH (Goss 2008a, 2008b). Despite being ionized, PFAS are bioavailable, and long-chain PFAA can accumulate in specific biological media to levels equivalent to lipid accumulation of neutral POPs. Given these observations, the question emerges of whether and how existing metrics for chemical accumulation in wildlife can be used to describe diverse PFAS. This formidable data gap hampers efforts to develop mechanistic models for exposure and risk assessment of PFAS, and stands as a major limitation to ecological exposure assessment of PFAS.

A synthesis of 513 laboratory-based and 931 field-based measurements indicates long-chain PFCA with 12-14 carbon-chain length generally exhibit the highest bioaccumulation potential, with whole-body $\mathrm{BCF}$ values ranging between $18,000-40,000$ L/kg (Gobas 2020). Laboratory-based whole-body BCFs of PFCA with 8 to 11 carbonchain lengths are generally much lower (BCF range: 4.0-4,900 L/kg). Similarly, PFOS exhibits relatively low laboratory-based whole-body BCFs, generally in the range of 100 to $1,000 \mathrm{~L} / \mathrm{kg}$. Field-based BAFs are generally in agreement with the laboratory-derived BCF values, but in some cases are somewhat higher, reflecting dietary accumulation.

This article is protected by copyright. All rights reserved. 
Generally, BCFs and BAFs ( $\mathrm{L} / \mathrm{kg})$ of individual PFAAs in plankton, aquatic gillventilating invertebrates, and fish increase with increasing perfluoroalkyl chain length and hydrophobicity (Condor 2008), though exceptions have been identified (Munoz 2017; Zhang 2019).

Avian and marine mammalian food webs exhibit the highest reported TMFs for PFAA (Kelly 2009; Tomy 2009). For example, the TMF for PFOS in these relatively long food webs containing air-breathing wildlife (e.g., marine birds and mammals) is approximately 20. TMFs in aquatic piscivorous food webs tend to be much lower. For example, TMFs of PFOS in the Lake Ontario aquatic piscivorous food webs range between 1.9 to 5.9 (Martin 2004; Houde 2008). Other studies have reported negligible biomagnification of PFOS in aquatic piscivorous food webs, with TMFs not substantially different than one (Loi 2011; Penland 2020). This behavior mirrors previous observations of food web-specific biomagnification of low $K_{\mathrm{OW}}$-high $K_{\mathrm{OA}}$ moderately hydrophobic organic chemicals (Kelly 2007). In particular, PFOS and several other PFAS of concern, which are likewise moderately hydrophobic and poorly metabolizable substances, may not biomagnify extensively in aquatic food webs due to efficient respiratory elimination to water via gills. Conversely, these substances can biomagnify to a high degree in food webs containing air-breathing animals because elimination of these substances via lungair exchange is negligible. More data are needed to refine these hypotheses and address variability across current data sets. Observed variability in TMFs for PFAA is likely due to selection of species, tissues, concentration normalization techniques, as well as the influence of site-specific conditions, life history stage, trophic condition of sampled individuals, and tissues and techniques used for stable isotope analyses.

This article is protected by copyright. All rights reserved. 
The contribution of PFAA precursors to field-based measurements of BAFs represents a major gap in understanding of PFAS bioaccumulation. For example, one study noted higher than expected accumulation of PFCA with five and six carbons in marine plankton from the Northwestern Atlantic and posited this reflects the accumulation of degraded precursor compounds (Zhang 2019). Another study that included liver tissues from marine mammals from the same region found a large fraction (30-75\%) of unidentified organofluorine (Spaan 2020). Shrimp from a subtropical food-web in Hong Kong were similarly noted to have a high fraction of unknown fluorinated compounds (Loi 2011). Some precursor compounds behave more similarly to traditional POPs and may thus have enhanced bioaccumulation propensity (Dassuncao 2017). Additional data on bioaccumulation potential and health risks associated with unidentified organofluorine are thus needed, particularly as chemical manufacturing has shifted away from the legacy PFAS typically detected in biota (Section 2).

\subsection{Modifications to bioaccumulation metrics for POPs needed for PFAS}

Table 3 illustrates some potential modifications to key bioaccumulation metrics that better reflect the behavior of PFAS in biological systems. In deciding the appropriate metric to use and how it is to be defined (in what tissue, with what type of normalization) a key guiding question is: "For what purpose?" If a TMF is being calculated to understand the exposure of a predator organism to PFAS in its prey, it makes the most sense to use the concentration in the portion of the prey consumed by that organism to define the denominator. For example, one study calculated the BMF for PFAA in polar bears using the ratio of polar bear liver to ringed seal blubber concentrations (Boisvert 2019).

This article is protected by copyright. All rights reserved. 
Similarly, when considering BCFs in sport fish, PFAS concentrations in muscle tissue may be most relevant in establishing dietary guidelines for humans, but liver-specific BCFs may provide better insight for potential health consequences to the fish population itself.

Refining metrics to better capture the behavior of PFAS in biological systems requires a better understanding of how PFAS are transferred from external exposure sources to the internal environment, and how, once internalized, they distribute to different tissues and are eliminated (Figure 5). Presently there is little consensus on the tissue type (e.g., liver, kidney, muscle) that best represents PFAS bioaccumulation in wildlife and whether normalization of concentrations to protein or phospholipid is needed. For example, using tissue-specific measurements in fish, there were large variations in blood BCF and blood BAF compared to the analogous whole-body BAF and BCF whereas liver-based BCF and BAF were in the same order of magnitude for the whole body accumulation parameters (Martin 2003; Shi 2018).

PFAA are acidic compounds with low $\mathrm{pKa}$, which means they are often associated with proteins (e.g., serum albumin) and phospholipids rather than storage lipids (Armitage 2012; Armitage 2013; Ng 2013, 2014; Chen 2016; Armitage 2017; Dassuncao 2019). As a result, PFAA accumulate primarily in the blood, liver, kidney, and brain of organisms rather than adipose tissue (Martin 2003; Ahrens 2009b; Borg 2010; Kowalczyk 2013; Dassuncao 2019). The affinity of different PFAA for proteins varies widely, suggesting binding-site-specific interactions and facilitated transport of some compounds. PFAA interact with protein molecules through a combination of polar, non-polar, and

This article is protected by copyright. All rights reserved. 
electrostatic interactions and not through covalent binding. Hence, sorption and desorption are dependent on thermodynamic gradients (i.e., chemical activity differences) in biological systems (Bischel 2010). Distribution of PFAA to structural (muscle) proteins is generally low, while binding to specific proteins (e.g. serum albumin, liver fatty acid binding protein) leads to tissue-specific accumulation patterns (Luebker 2002; Jones 2003; Martin 2003; Ahrens 2009b; Ng 2014). Phospholipids also play an important role in tissue specific partitioning (Armitage 2012). This is particularly true for the long chain PFAS and brain tissue, which is very high in phospholipid content (Dassuncao 2019). Thus, more refined approaches to account for tissue-specific uptake in bioaccumulation assessments are needed.

\subsection{PFAS bioaccumulation modeling}

Mechanistic bioaccumulation (i.e., toxicokinetic) models developed for neutral lipophilic contaminants such as polychlorinated biphenyls (PCBs) and organochlorine pesticides have been widely used by academics, risk assessment professionals, and regulatory authorities (Arnot 2004). Applications of these models include substance prioritization, screening-level risk assessments, development of environmental quality guidelines, total maximum daily loads, and other purposes. They use information on the physical-chemical properties of the compound of interest, as well as environmental and biological properties of the site and organisms. The success of these existing mechanistic approaches relies on good data quality on the partitioning properties of neutral compounds in biological media and tissues. Thus, a major challenge for PFAS is suitable data on their partitioning in biological compartments.

This article is protected by copyright. All rights reserved. 
One available approach for modeling fish bioaccumulation of ionizable organic compounds (IOCs, including PFAA) takes into account the role of thermodynamic gradients in tissue partitioning for PFAS (Armitage 2013). Specifically, the model is based on $\mathrm{pH}$-dependent distribution ratios, including the chemical's octanol-water distribution ratio $\left(D_{\mathrm{OW}}\right)$ and membrane-water distribution ratio $\left(D_{\mathrm{MW}}\right)$, as well as the estimated distribution ratio between non-lipid organic matter (NLOM) and water. It provides a simple partitioning-based equation to predict steady-state concentrations of PFAA and other IOCs in aquatic organisms.

Strong relationships between empirically derived bioaccumulation metrics (BCF, $\mathrm{BAF}, \mathrm{TMF})$ and distribution ratios for protein-water $\left(\mathrm{D}_{\mathrm{PW}}\right)$ and membrane-water $\left(\mathrm{D}_{\mathrm{MW}}\right)$ of individual PFAA have been demonstrated for some ecosystems (Kelly 2009; Chen 2016). The relatively high degree of bioaccumulation of long-chain PFCA with 12-14 carbons in fish is likely attributable to high $\mathrm{D}_{\mathrm{MW}}$ and $\mathrm{D}_{\mathrm{PW}}$ (estimated values of $10^{5}$ to $10^{6} \mathrm{D}_{\mathrm{MW}}$ and $10^{4}$ to $\left.10^{6} \mathrm{D}_{\mathrm{PW}}\right)$. Thus, $\mathrm{D}_{\mathrm{PW}}$ and $\mathrm{D}_{\mathrm{MW}}$ are two key parameters that may be useful for predicting PFAS bioaccumulation potential (Table 3).

The NLOM component of the IOC model represents endogenous protein (Armitage 2013). While it performs well for many IOCs, it tends to underestimate the bioaccumulation capacity of some PFAA. This is likely due to the complex association of PFAS with proteins. Thus, a key to parameterizing this model is the difference in distribution ratios between neutral and ionized forms of the specific PFAS congener, which may require challenging experimental determinations. Further, different protein types can exhibit different sorptive capacities for IOCs (Henneberger 2016). For some

This article is protected by copyright. All rights reserved. 
compounds, protein-water distribution ratios $\left(D_{\mathrm{pw}}\right)$ in plasma protein (e.g., albumin) can be orders of magnitude greater than those in structural proteins (e.g., muscle protein). Thus, application of simple equilibrium partitioning-based models may require utilizing a series of distribution coefficients for different proteins (e.g., transporter protein-water distribution ratios $\left(D_{\text {TP-W }}\right)$ and structural protein-water distribution ratios $\left(D_{\text {SP-W }}\right)$. This approach was used to assess tissue-specific bioaccumulation of PFAA and other IOCs in laboratory exposed fish (Chen 2016, 2017a).

A chemical activity-based approach to ecological risk assessment bridges some gaps between traditional empirical modeling efforts and mechanistic models (Gobas 2017; Gobas 2018). This approach was used to assess bioaccumulation and exposure risks of several PFAS in wildlife at AFFF-impacted sites (Gobas 2020). The chemical activities of PFOS and other PFAA indicated that these compounds tend to primarily biomagnify in food webs composed of air-breathing wildlife (birds, mammals, terrestrial reptiles), compared to those comprising only aquatic organisms. For example, activitybased biomagnification factors in upper trophic wildlife (bird and mammals) were found to range between 10 and 20 and in aquatic organisms were close to 1, indicating negligible biomagnification. An advantage of this approach that is particularly relevant to PFAS is that it can be used effectively for both neutral and ionic substances, including anionic, cationic and zwitterionic compounds. A limitation of this approach is that solubility estimates and hence calculated activities are based on numerous assumptions regarding physicochemical properties, phase partitioning, protein-binding and toxicokinetics.

This article is protected by copyright. All rights reserved. 
Beyond simple partitioning-based models for substance screening, more sophisticated approaches may be required for higher resolution modeling. For example, IOC binding to intra- and extracellular protein (serum albumin, liver fatty acid binding protein), as well as membrane-associated organic anion transporters, may act to provide both enhanced sorption capacity and advective transport across biological membranes ( $\mathrm{Ng}$ 2013). This affects uptake and elimination rates as well as tissue distribution, and helps explain the long elimination half-lives of PFAA in organisms.

Physiologically-based toxicokinetic (PBTK) models incorporating absorption, distribution, metabolism, and excretion (ADME) metrics have been developed to assess toxicokinetics of PFOS and PFOA in various animal models, including fish and mammals (Andersen 2006; Tan 2008; Consoer 2014; Fabrega 2014; Consoer 2016; Fabrega 2016; Cheng 2017; Khazaee 2018). PBTK models are particularly useful for assessing the influence of membrane transporters, and revealing important challenges in equilibrium modeling of PFAS bioaccumulation. For example, laboratory-based evaluations of bioconcentration (e.g. the OECD 305 test), a kinetic BCF can be defined as the ratio of uptake and elimination rates $\left(\mathrm{k}_{1} / \mathrm{k}_{2}\right)$. However, the elimination rate for PFAS can be affected by saturable transporter-mediated renal uptake and clearance, as has been observed in rats (Han 2012) and monkeys (Andersen 2006), so that the $\mathrm{k}_{2}$ rate becomes dose-dependent. Therefore, observations both in the laboratory and in the field (e.g. at highly contaminated sites) may be concentration dependent and thus not representable by first-order kinetics.

This article is protected by copyright. All rights reserved. 


\subsection{In vitro and in silico methods to support modeling and assessment}

As more focus is placed on molecular interactions between PFAS and biomolecules including proteins, transporters, and phospholipids, a variety of in vitro and analytical methods have been developed to support both laboratory assessments and the parameterization of mechanistic models. In vitro methods include cell- and vesicle-based assays to estimate and compare uptake rates into cells via passive diffusion and active transport (Luebker 2002; Katakura 2007; Nakagawa 2008; Weaver 2010; Herédi-Szabó 2012), and protein-binding assays using equilibrium dialysis (Bischel 2010; Bischel 2011), fluorescence displacement (Chen 2009; MacManus-Spencer 2010; Yang 2020), and other methods (MacManus-Spencer 2010). A key challenge of these approaches is high variability across studies, which can lead to order of magnitude differences in estimated binding affinities for the same PFAS-protein combinations (MacManusSpencer 2010; Ng 2014). Recently, a hybrid approach used size exclusion column coelution (SECC) with non-target mass spectrometry to identify L-FABP (liver fatty acid binding protein) ligands from complex PFAS mixtures (Yang 2020). The method identified 31 new L-FABP ligands from AFFF. Given the known importance of this protein to liver accumulation of PFAS in diverse organisms, this method illustrates the potential for combining non-target analysis with bioaccumulation potential screening.

In silico methods have been developed to predict the behavior of PFAS at the molecular level in biological systems. These methods have an advantage of high throughput testing and are not hindered by the lack of available standards and samples. Molecular docking and molecular dynamics are computational approaches originally

This article is protected by copyright. All rights reserved. 
developed for drug discovery, and are powerful tools for the prediction of protein-ligand interactions.

They have now been used to screen a number of legacy and emerging PFAS for binding with serum albumin (Salvalaglio 2010; Ng 2015), L-FABP (Zhang 2013; Ng 2015; Cheng 2018; Yang 2020), and peroxisome proliferator activated receptors (PPARs), which are thought to be linked to some of the toxic effects of PFAS (Li 2019). Predicted binding affinities from these kinds of studies are highly correlated with experimental observations and can be used to help parameterize mechanistic PBTK models. However, we note the predicted binding affinities are overestimated for perfluoroalkylsulfonamide and may still require anchoring to some experimental binding data.

The role of phospholipids and related application of $\mathrm{D}_{\mathrm{MW}}$ in PFAS toxicokinetics and tissue has not been fully realized. It vitro assay protocols to assess phospholipid partitioning using laboratory-prepared liposomes (Escher 2000) are applicable to determining $\mathrm{D}_{\mathrm{MW}}$ for PFAS. Solid-supported phosphatidylcholine (PC) membranes can also be applied for generating empirical data for $\mathrm{D}_{\mathrm{MW}}$ for PFAS such as TRANSIL-XL membrane affinity assay kits (Droge 2019).

Finally, more empirical computational methods attempt to build predictive relationships between PFAS structure and their bioaccumulation potential or toxicity based on available data rather than mechanistic process-based approaches. These include quantitative structure activity relationships (QSARs) for predicting key physicochemical properties for PFAS, as recently reviewed (Lampic 2020), and a machine learning-based This article is protected by copyright. All rights reserved. 
approach to classifying the potential bioactivity of thousands of PFAS (Cheng 2019).

Hybrid in vitro/in silico approaches that generate data with which to develop a QSAR

have also been used, for example to tackle the important subject of PFAS mixture

toxicity (Hoover 2019).

\section{SUMMARY AND KEY RESEARCH GAPS}

In this manuscript, we reviewed current understanding of: (a) PFAS sources and environmental transport pathways, (b) analytical methods used to assess PFAS exposures and their strengths and limitations, (c) methods for assessing human exposures and the relative importance of different sources and pathways, and (d) PFAS bioaccumulation in wildlife. Changing geographic locations of PFAS manufacturing and the shifting chemical landscape poses a number of challenges for exposure assessments for humans and wildlife. Synthesis of analytical standards for newer PFAS has lagged their production and release and thus non-targeted methods, suspect screening and total fluorine assessments have all emerged as essential tools for understanding the total burden of PFAS in the environment, humans and wildlife. Although such data are now appearing across all media, more data are needed, particularly for food items, PCPs, dust, drinking water, and wildlife tissues that contain PFAS. For both humans and wildlife, additional research is needed to characterize the fraction of exposure originating from precursors that degrade into terminal PFAA that have been linked to adverse health effects. Existing literature on time trends in PFAS in biological tissues suggest that while some legacy PFAS have decreased over the past two decades, the percent of unidentifiable EOF has increased. There is a need for greater constraints on residual

This article is protected by copyright. All rights reserved. 
content (i.e. unbound fluorinated surfactants, monomers) in fluoropolymers, side-chain polymers and ether-based polymers, and for more data on their contribution to PFAS exposure. Measurement of polymers in environmental media remains an important challenge.

A major focus of past research has been contaminated sites where the highest concentrations in the environment and biota are often found. Moving forward, a better understanding of atmospheric PFAS sources and potential resulting exposures is needed, particularly as treatment systems are introduced for contaminated drinking water supplies. Integration of PFAS measurements into long-term atmospheric monitoring for pollutants would be valuable for measuring changes in atmospheric PFAS concentrations, aiding model development, and identifying vulnerable regions

Occupational health effects associated with human PFAS exposures have been reported for individuals who worked in fluorochemical production plants. There is a reasonable consensus that near contaminated sites, drinking water is often the main pathway for human exposure to PFAS. However, data are still sparse on exposure sources for the general population and for communities where drinking water contamination has been remediated. Presently, there is a paucity of data on the indoor environment where many PFAS-containing products are found. Dermal exposures to PFAS from dust and PCP warrant additional consideration given the extremely high concentrations reported for some PCP and dust. There is general agreement that dietary exposure is the major contributor to population exposure for PFOS and PFOA, but more limited evidence for other PFAS, including that contributed by food processing and packaging. More

This article is protected by copyright. All rights reserved. 
information is needed about the contribution of food processing and packaging to PFAS

in food.

Most prior work on human exposure pathways has not included statistically representative population surveys. Future PFAS researchers may benefit from establishing collaborations with existing representative populations. The field needs total exposure studies seeking to characterize all pathways and routes of exposure to PFAS including precursors. Empirical TK data needed to describe PFAS partitioning among tissues, facilitated transport and elimination half-lives are still limited or non-existent for many PFAS and represent an important research need. As the focus of PFAS research shifts toward issues associated with emerging compounds, additional data for parameterizing TK models represent a critical research need.

For wildlife exposures, a major challenge is adapting existing metrics for chemical accumulation to describe diverse PFAS. This data gap hampers efforts to develop mechanistic models for exposure and risk assessment of PFAS. Early PFAS bioaccumulation modeling suggested that some PFAS are efficiently eliminated though the gills of water breathing organisms but biomagnify to a greater degree in air-breathing organisms due to limited lung-air exchange (Kelly 2007). Mechanistic bioaccumulation models for PFAS are needed to understand the tissue accumulation patterns for novel PFAS, including neutral precursors and intermediates. Non-traditional approaches to probing molecular mechanisms of bioaccumulation such as protein association have important impacts on predicting tissue distribution and internal dose but also can have organismal effects if PFAS are able to perturb endogenous functions. Tissue and cellular

This article is protected by copyright. All rights reserved. 
partitioning of PFAS can be used to inform models; current wildlife monitoring is primarily limited to liver or muscle or blood.

Early research on PFAS releases and global transport was conducted as a partnership between industry and academia. We thus recommend greater transparency and collaboration between academia, industry and government to prioritize and then improve understanding of global environmental releases of the thousands of PFAS structures present in global commerce. One example of improved transparency could be a commitment by industry to release information on the chemical structures and analytical standards for new PFAS used in commerce. Better characterizing PFAS exposure sources for humans and wildlife is an essential first step in the design of effective risk mitigation strategies.

Acknowledgment - This manuscript was developed by participants in the Exposure Assessment Panel as part of the SETAC North America Focused Topic Meeting on Environmental Risk Assessment of PFAS held August 12-15, 2019. WH-B acknowledges funding from the NIEHS Superfund Research Program (P42ES007381). CN acknowledges support from the U.S. National Science Foundation (NSF: 1845336). AR acknowledges financial support from the U.S. National Oceanic and Atmospheric Administration (NOAA) Dr. Nancy Foster Scholarship program (NA17NOS4290028) and the NIEHS Superfund Research Program (P42ES027706). EMS acknowledges support from NIEHS (P42ES027706) and the Strategic Environmental Research and Development Program (SERDP, ER18-1280). TFW was supported in part by NIEHS (R01ES027813, R01ES028800). MS acknowledges financial support from the North

This article is protected by copyright. All rights reserved. 
Carolina PFAS Testing Network through the North Carolina Policy Collaboratory.

Positions or statements expressed herein do not necessarily reflect organizational views of the authors. All coauthors contributing to synthesizing information and writing this review. AOD and EMS led the synthesis team.

Data availability statement —Data, associated metadata, and calculation tools are available from the corresponding author (ems@seas.harvard.edu).

\section{REFERENCES}

3M. 2001. Environmental Monitoring-Multi-City Study (Water, Sludge, Sediment, POTW Effluent and Landfill Leachate Samples). U.S. Environmental Protection Agency, Office of Pollution and Prevention and Toxic Substances, Washington, DC. Docket AR-226-1030a.

3M Company. 1999. Fluorochemical Use, Distribution and Release Overview. US EPA Public Docket AR226-0550, 3M Company: St Paul, MN.

Ahrens L. 2011. Polyfluoroalkyl compounds in the aquatic environment: a review of their occurrence and fate. Journal of Environmental Monitoring 13:20-31. DOI: 10.1039/c0em00373e.

Ahrens L, Siebert U, Ebinghaus R. 2009a. Temporal trends of polyfluoroalkyl compounds in harbor seals (Phoca vitulina) from the German Bight, 1999-2008. Chemosphere 76:151-158. DOI: 10.1016/j.chemosphere.2009.03.053.

This article is protected by copyright. All rights reserved.

\section{6 pages of references omitted for brevity - available upon request}


China. Environmental Science \& Technology 47:9249-9257. DOI:

10.1021/es402120y.

Figure 1. Conceptual representation of key emission sources and global transport pathways of perfluoroalkyl acids (PFAA) and their polyfluorinated precursors.

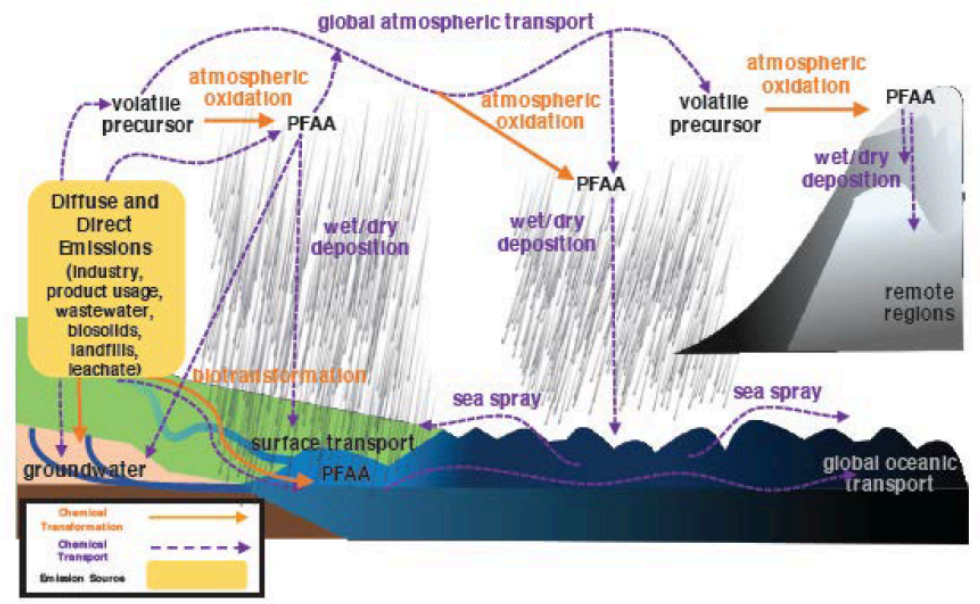

This article is protected by copyright. All rights reserved. 
Figure 2. Components of the fluorine mass balance. Panel a) shows a conceptual diagram representing the relative fractions of total fluorine (TF) including fluoride, unextracted organofluorine, extractable organofluorine (EOF), and targeted PFAS. Panel b) shows actual data where unknown EOF was determined using targeted analysis of 50 PFAS congeners in blood plasma of first time mothers from Uppsala, Sweden (data from Miaz 2020).
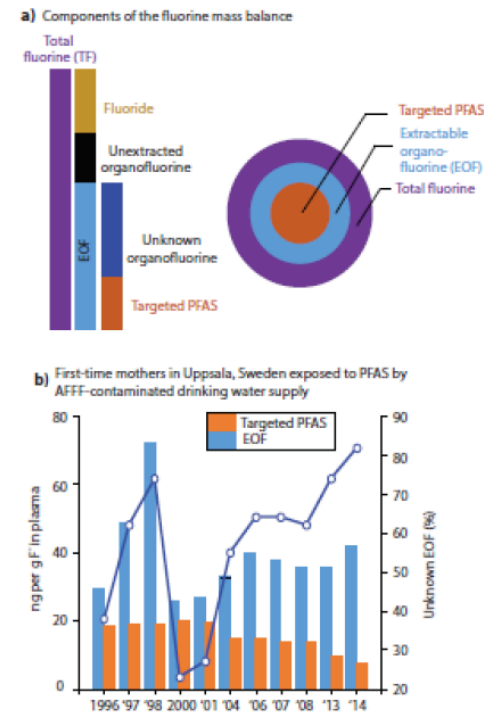

This article is protected by copyright. All rights reserved. 
Figure 3. Schematic of exposure assessment steps for humans that relates PFAS sources to exposure media, and internal concentrations of PFAS in blood. Not all possible exposure routes (e.g., outdoor air) or arrows are shown. $\mathrm{ADME}=$ Absorption, distribution, metabolism and excretion.
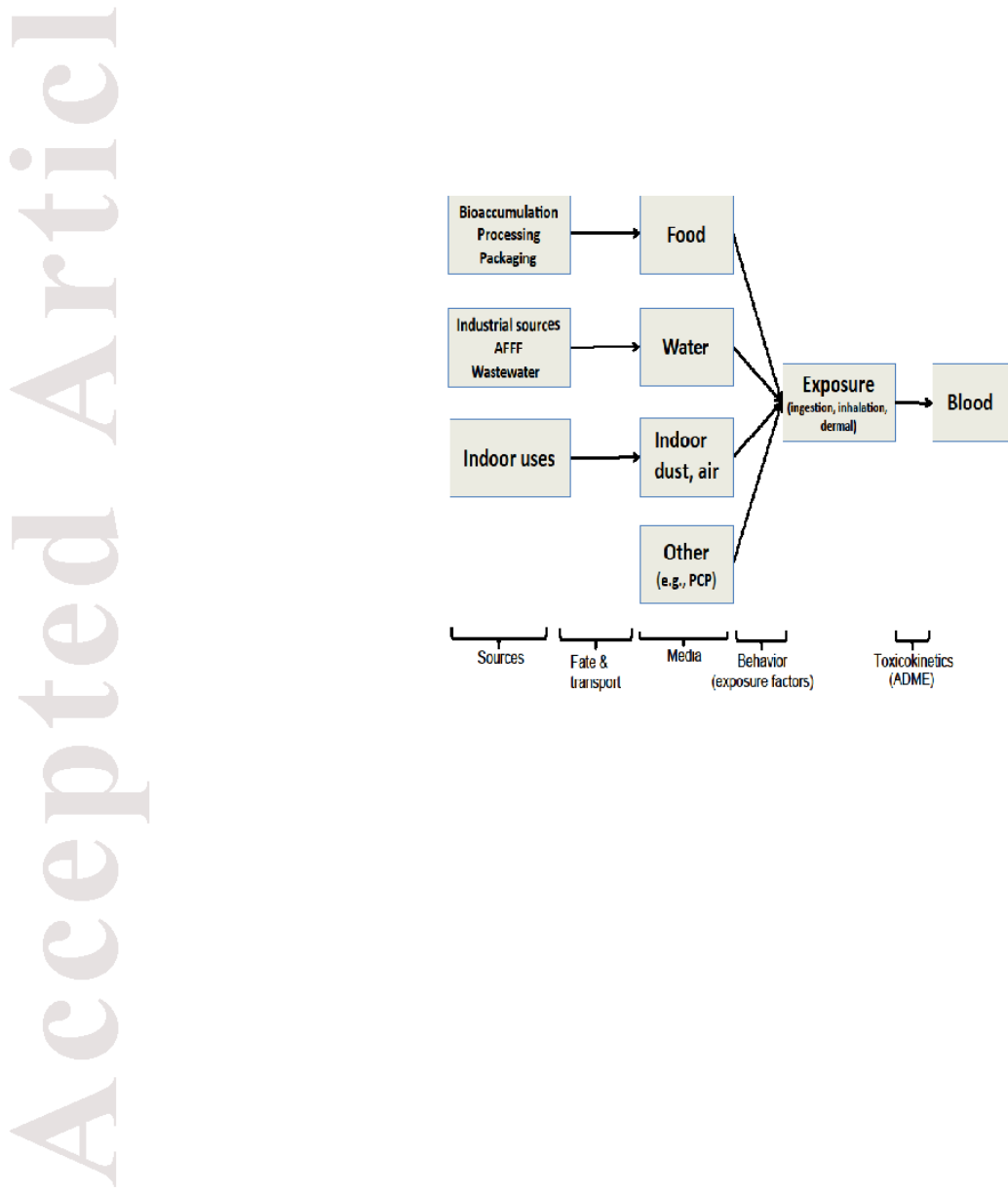

This article is protected by copyright. All rights reserved. 
Figure 4. Key bioaccumulation processes, metrics and gaps associated with PFAS in wildlife. $\mathrm{BMR}=$ basal metabolic rate; $\mathrm{BAF}=$ bioaccumulation factor; $\mathrm{BMF}=$ biomagnification factor; $\mathrm{TMF}=$ trophic magnification factor; $\mathrm{BCF}=$ bioconcentration factor; $\mathrm{BSAF}=$ biota-sediment accumulation factor.

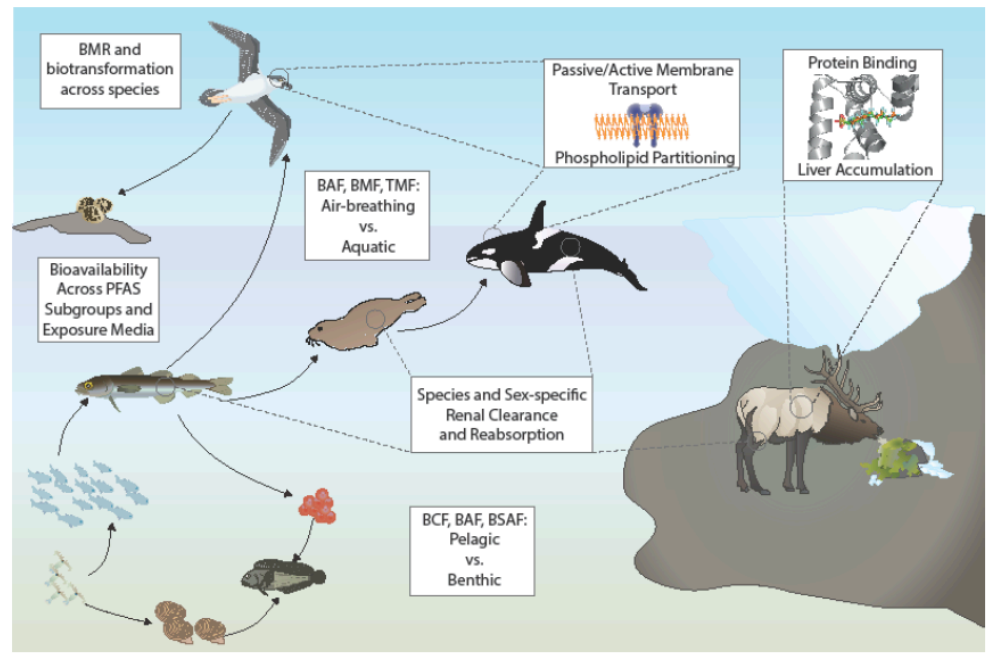

This article is protected by copyright. All rights reserved. 
Figure 5. Key elements for predicting the relationship between external exposure and internal dose of wildlife and human to PFAS. The internal distribution and dose are driven by the balance of absorption, distribution, metabolism, and elimination (ADME). For PFAS, unlike for neutral organic chemicals, internal distribution is substantially influenced by protein binding and transporter uptake and efflux, leading to accumulation in the liver and blood and species and sex-specific elimination half-lives.
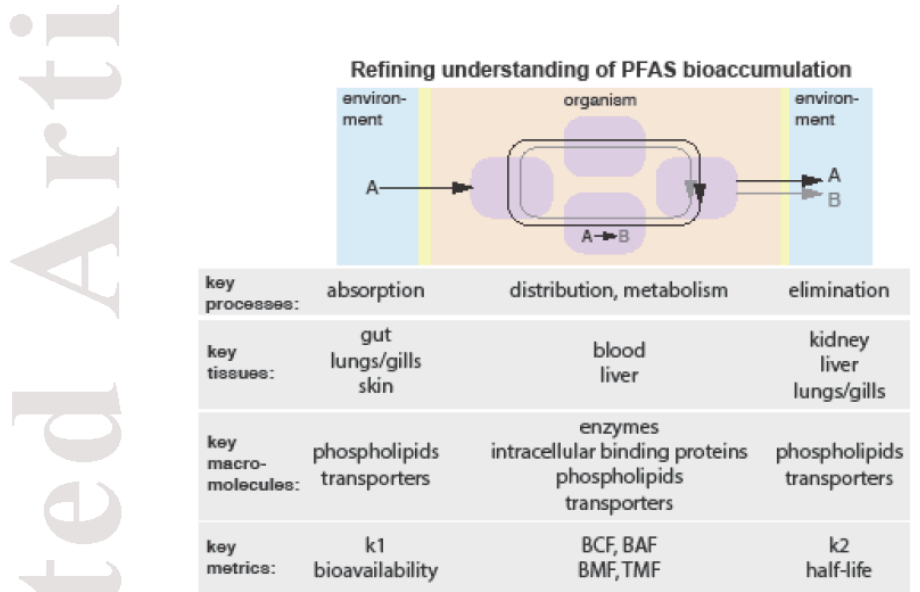

This article is protected by copyright. All rights reserved. 
Graphical Abstract: Methods for assessing human and wildlife exposures to per- and polyfluoroalkyl substances are reviewed along with current understanding of exposure sources and pathways

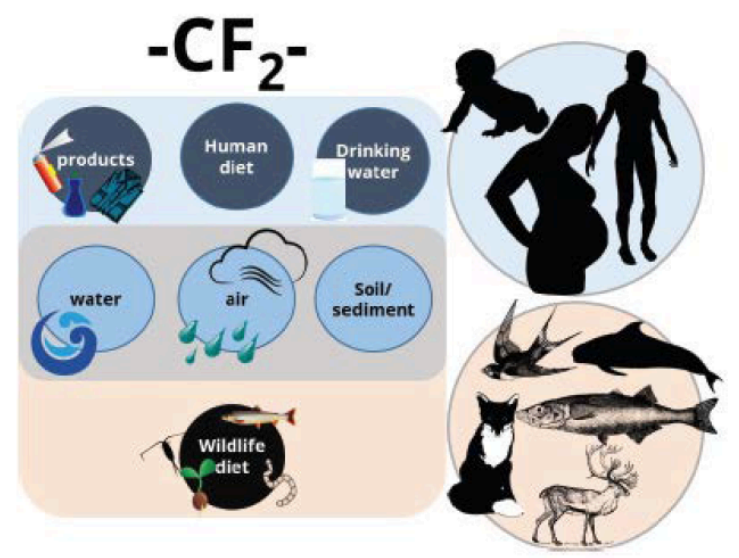

This article is protected by copyright. All rights reserved. 
Tables

Table 1. Comparison of analytical techniques for assessing PFAS exposure. ${ }^{a}$

\begin{tabular}{|c|c|c|c|}
\hline Methods & $\begin{array}{l}\text { Applicable } \\
\text { matrices }\end{array}$ & Advantages & Limitations \\
\hline $\begin{array}{l}\text { Targeted } \\
\text { analysis }\end{array}$ & $\begin{array}{l}\text { All } \\
\text { matrices }\end{array}$ & $\begin{array}{l}\text { - Sensitive }(0.1-1 \\
\text { ng/L) } \\
\text { - High selectivity to } \\
\text { the analysis targets }\end{array}$ & $\begin{array}{l}\text { - Limited inclusivity } \\
\text { - Variable recoveries } \\
\text { - Potential bias from sample } \\
\text { extraction }\end{array}$ \\
\hline $\begin{array}{l}\text { Non-targeted } \\
\text { analysis }\end{array}$ & $\begin{array}{l}\text { All } \\
\text { matrices }\end{array}$ & $\begin{array}{l}\text { - High inclusivity } \\
\text { - Elucidate unknown } \\
\text { structures }\end{array}$ & $\begin{array}{l}\text { - Not quantitative } \\
\text { - Potential bias from sample } \\
\text { extraction } \\
\text { - Need expensive } \\
\text { instruments and highly } \\
\text { skilled users }\end{array}$ \\
\hline TOP & $\begin{array}{l}\text { Mainly } \\
\text { aqueous } \\
\text { samples }\end{array}$ & $\begin{array}{l}\text { - Sensitive }(0.1-1 \\
\text { ng/L) } \\
\text { - High selectivity to } \\
\text { PFAS }\end{array}$ & $\begin{array}{l}\text { - Limited inclusivity } \\
\text { - Variable recoveries }\end{array}$ \\
\hline EOF (CIC) & $\begin{array}{l}\text { All } \\
\text { matrices }\end{array}$ & $\begin{array}{l}\text { - High selectivity to } \\
\text { organofluorine }\end{array}$ & $\begin{array}{l}\text { - Less sensitive }(0.1-0.5 \mathrm{ppm} \\
\text { F) than targeted analyses } \\
\text { - Potential bias from sample } \\
\text { extraction }\end{array}$ \\
\hline AOF (CIC) & Water & $\begin{array}{l}\text { - High selectivity to } \\
\text { organofluorine }\end{array}$ & $\begin{array}{l}\text { - Not as sensitive (0.1-0.5 } \\
\text { ppm F) }\end{array}$ \\
\hline PIGE & Solids & $\begin{array}{l}\text { - High-throughput } \\
\text { - Non-destructive }\end{array}$ & $\begin{array}{l}\text { - Not as sensitive (50 nmol } \\
\mathrm{F} / \mathrm{cm}^{2} \text { ) }\end{array}$ \\
\hline
\end{tabular}

This article is protected by copyright. All rights reserved. 


\begin{tabular}{|c|c|c|c|}
\hline & & $\begin{array}{l}\text { - No matrix effects } \\
\text { - Surface measurement } \\
(100-250 \mu \mathrm{m})\end{array}$ & - Interference by fluoride \\
\hline XPS & Solids & $\begin{array}{l}\text { - High-throughput } \\
\text { - Widely-available } \\
\text { instrumentation } \\
\text { - Identification of } \\
\text { perfluoroalkyl } \\
\text { moiety } \\
\text { - Etching of surface } \\
\text { possible to create } \\
\text { depth profiles } \\
\text { - Surface measurement } \\
(\sim 10 \mathrm{~nm})\end{array}$ & $\begin{array}{l}\text { - High detection limits }(\sim 1 \% \\
\text { F) } \\
\text { - Small area measurement } \\
\text { - Can be affected by surface } \\
\text { roughness and } \\
\text { inhomogeneity }\end{array}$ \\
\hline
\end{tabular}

${ }^{a} \mathrm{~A}$ more comprehensive discussion of the strengths and limitations of different analytical techniques is reviewed elsewhere (Guelfo 2020).

${ }^{b}$ For a more detailed discussion of the strengths and limitations of non-targeted analysis please see the following viewpoints and response (Hites 2018, 2019; Samanipour 2019).

Table 2. Literature estimates of source contributions (\%) to adult exposures to PFAS ${ }^{\text {a }}$

\begin{tabular}{|c|c|c|c|c|c|c|c|c|c|c|}
\hline \multirow[t]{2}{*}{ PFAS } & \multirow{2}{*}{$\begin{array}{l}\text { Carbon } \\
\text { length }\end{array}$} & \multicolumn{4}{|c|}{ Exposure Medium ${ }^{b}$} & \multicolumn{3}{|c|}{ Exposure Route $^{b}$} & \multirow{2}{*}{$\begin{array}{c}\text { Study } \\
\text { Location }\end{array}$} & \multirow[b]{2}{*}{ Ref } \\
\hline & & Diet & Dust & Water & $\begin{array}{c}\text { Consumer } \\
\text { goods }\end{array}$ & Inhalation & Dermal & Indirect & & \\
\hline PFBA & 4 & & 4 & 96 & & & & & NA & c \\
\hline PFHXA & 6 & 38 & 4 & 38 & & 8 & & 12 & NA & c \\
\hline PFHXA & 6 & 87 & 4 & & & 2 & & & Norway & d \\
\hline PFHxS & 6 & 57 & 38 & & & 5 & & & Finland & e \\
\hline PFHxS & 6 & 94 & 1 & & & & & & Norway & d \\
\hline PFHрA & 7 & 93 & 1 & & & & & & Norway & d \\
\hline PFHpS & 7 & & & & 100 & & & & Norway & d \\
\hline
\end{tabular}

This article is protected by copyright. All rights reserved. 


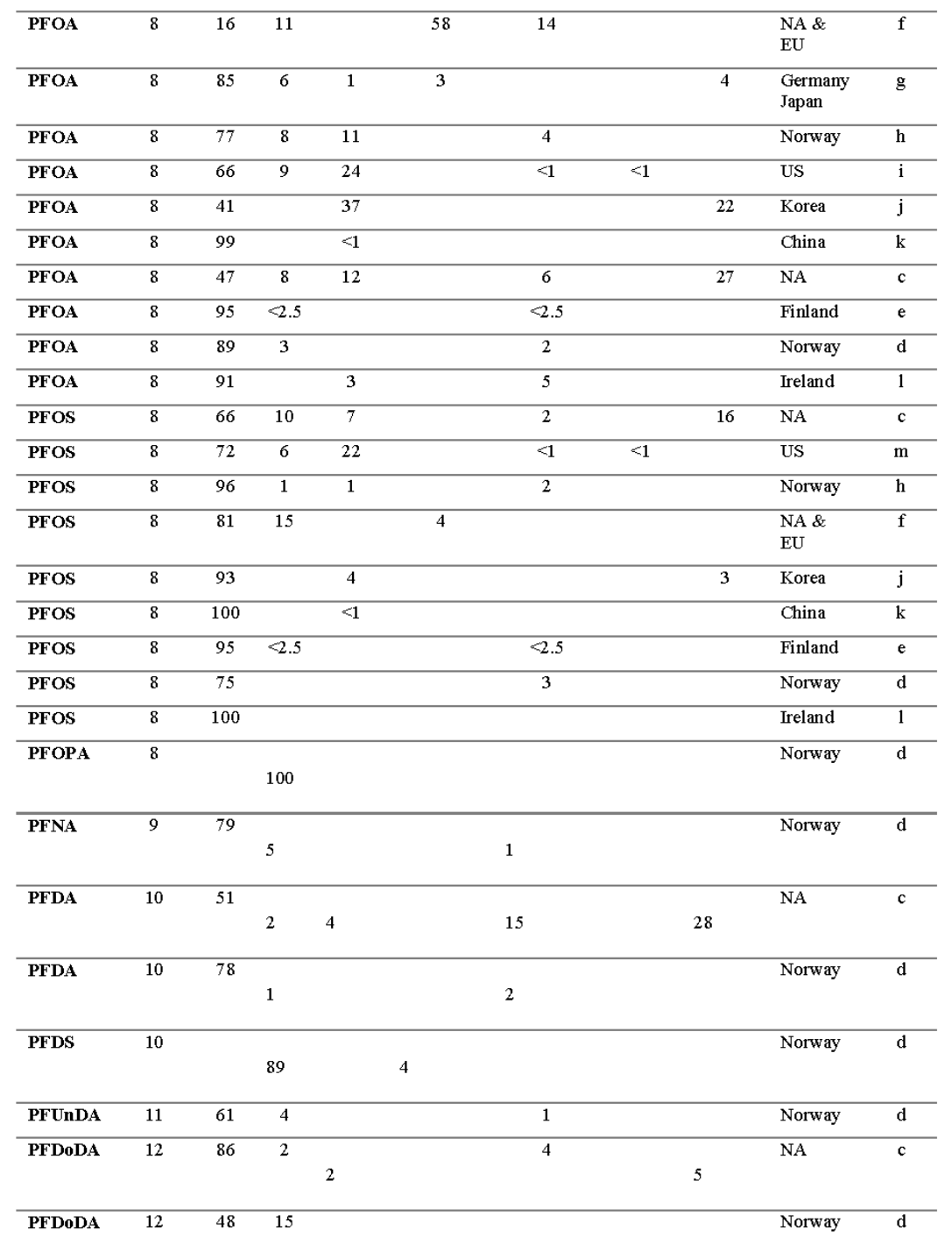

This article is protected by copyright. All rights reserved. 


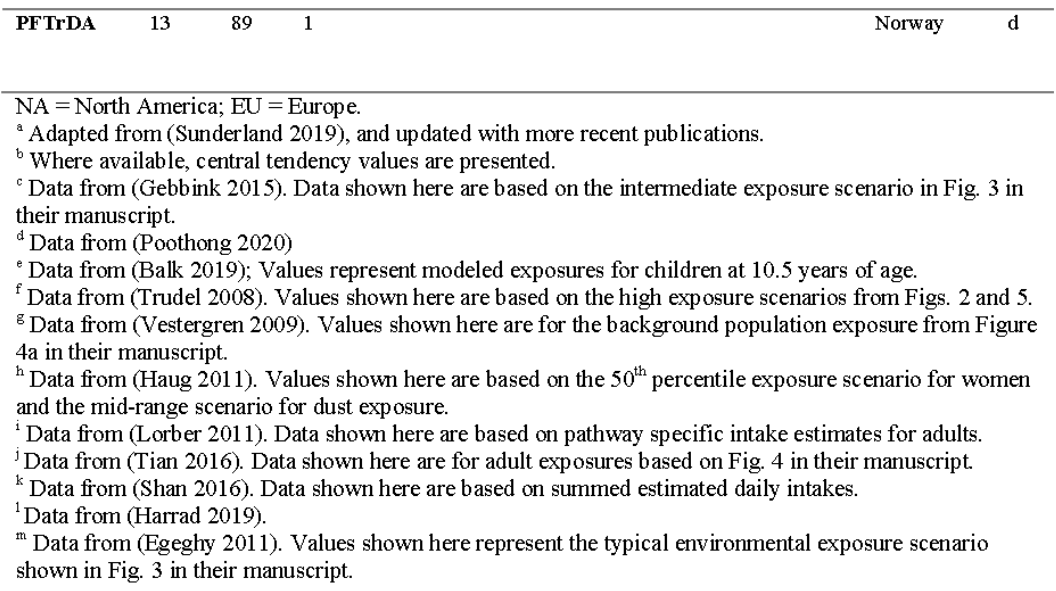

Table 3. Modifications to persistent organic pollutant bioaccumulation metrics for PFAS.

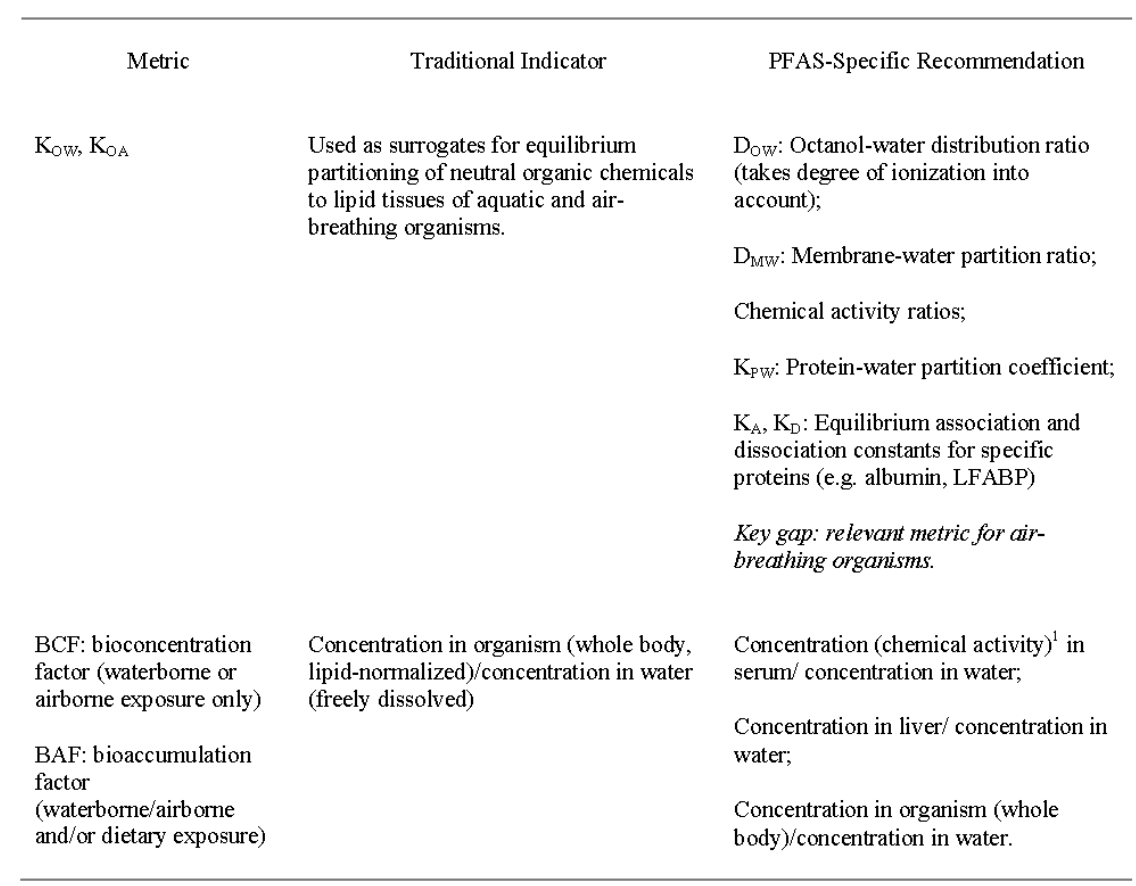

This article is protected by copyright. All rights reserved. 


\begin{tabular}{|c|c|c|}
\hline & & $\begin{array}{l}\text { Key gap: (1) Selecting appropriate } \\
\text { tissue to represent accumulation in } \\
\text { organism. (2) Accounting for } \\
\text { contributions of precursors to field } \\
\text { based BAFs. }\end{array}$ \\
\hline $\begin{array}{l}\text { BMF: biomagnification } \\
\text { factor }\end{array}$ & $\begin{array}{l}\text { Concentration in predator (whole body, } \\
\text { lipid-normalized)/concentration in prey } \\
\text { (whole body, lipid-normalized) }\end{array}$ & $\begin{array}{l}\text { Concentration in predator liver } \\
\text { /concentration in prey liver; }\end{array}$ \\
\hline \multirow[t]{2}{*}{$\begin{array}{l}\text { TMF: trophic } \\
\text { magnification factor }\end{array}$} & & $\begin{array}{l}\text { Concentration in predator (whole body) } \\
\text { / concentration in prey (whole body); }\end{array}$ \\
\hline & & $\begin{array}{l}\text { Key gaps: Selecting appropriate } \\
\text { predator and prey tissues across food } \\
\text { webs. }\end{array}$ \\
\hline
\end{tabular}

${ }^{1}$ Emerging approach: activity-based metrics. See section 6.4 .

This article is protected by copyright. All rights reserved. 


\section{APPENDIX B}

\section{TABLE OF PFAS ACRONYMS}

This appendix contains the full name, abbreviations, molecular structure, CAS number, and potential uses of PFAS measured within dissertation. 


\begin{tabular}{|c|c|c|c|c|c|}
\hline Compound & Abbreviation & Chemical Formula & CAS \# & $\begin{array}{l}\text { PFAS Family } \\
\text { after Buck et } \\
\text { al. } 2011 \\
\end{array}$ & Some Potential Sources or Uses \\
\hline Perfluorobutyric acid & PFBA & $\mathrm{C} 4 \mathrm{HF} 7 \mathrm{O} 2$ & $375-22-4$ & PFCA & $\begin{array}{l}\text { Chromatographic reagent, manufacturing of } \\
\text { photographic film, breakdown product of } \\
\text { precursors or other PFCAs }\end{array}$ \\
\hline Perfluoropentanoic acid & PFPeA & C5HF9O2 & $2706-90-3$ & PFCA & $\begin{array}{l}\text { Stain and grease proof coatings on food } \\
\text { packaging, couches, and carpets, breakdown } \\
\text { product of precursors and other PFCAs }\end{array}$ \\
\hline Perfluorobutanesulfonic acid & PFBS & C4HF9O3S & $375-73-5$ & PFSA & Stain repellent; Replacement for PFOS \\
\hline Perfluorohexanoic acid & PFHxA & C6HF11O2 & $307-24-4$ & PFCA & Breakdown product of PFHxS \\
\hline $\begin{array}{c}\text { 1-Octanesulfonic acid, } \\
3,3,4,4,5,5,6,6,7,7,8,8,8 \text {-tridecafluoro- }\end{array}$ & 4:2-FTS & C8H5F13O3S & $27619-97-2$ & FTS & Potential impurity of 6:2 FTS creation \\
\hline $\begin{array}{c}\text { 1-Pentanesulfonic acid, } \\
1,1,2,2,3,3,4,4,5,5,5 \text {-undecafluoro- }\end{array}$ & PFPeS & C5HF11O3S & $2706-91-4$ & PFSA & $\begin{array}{l}\text { Firefighting foams, stain and grease proof } \\
\text { coatings on carpets and other textiles }\end{array}$ \\
\hline Perfluoroheptanoic acid & PFHpA & $\mathrm{C} 7 \mathrm{HF} 13 \mathrm{O} 2$ & $375-85-9$ & PFCA & Surfactant \\
\hline Perfluorohexanesulfonic acid & PFHxS & C6HF13O3S & $355-46-4$ & PFSA & Firefighting foam \\
\hline Perfluorooctanoic acid & PFOA & $\mathrm{C} 8 \mathrm{HF} 15 \mathrm{O} 2$ & $335-67-1$ & PFCA & $\begin{array}{c}\text { Nonstick coatings, general surfactant and } \\
\text { polymerization/processing aid }\end{array}$ \\
\hline $\begin{array}{c}\text { 1-Octanesulfonic acid, } \\
3,3,4,4,5,5,6,6,7,7,8,8,8 \text {-tridecafluoro- }\end{array}$ & 6:2-FTS & C8H5F13O3S & $27619-97-2$ & FTS & Firefighting foam, mist suppressant \\
\hline Perfluoroheptanesulfonic acid & PFHpS & C7HF15O3S & $375-92-8$ & PFSA & $\begin{array}{l}\text { Firefighting foams, stain and grease proof } \\
\text { coatings on carpets and other textiles }\end{array}$ \\
\hline Perfluorononanoic acid & PFNA & C9HF17O2 & $375-95-1$ & PFCA & $\begin{array}{l}\text { Performance plastic production, general } \\
\text { surfactant and processing aid }\end{array}$ \\
\hline Perfluorooctanesulfoamide & FOSA & $\mathrm{C} 8 \mathrm{H} 2 \mathrm{~F} 17 \mathrm{NO} 2 \mathrm{~S}$ & $754-91-6$ & FASA & $\begin{array}{c}\text { Used as raw material in the electrochemical } \\
\text { fluorination process to make substances that are } \\
\text { used for surfactants and surface treatments. } \\
\text { FASAs can degrade to form PFAAs such as } \\
\text { PFOS }\end{array}$ \\
\hline Perfluorooctanesulfonic acid & PFOS & C8HF17O3S & $1763-23-1$ & PFSA & $\begin{array}{c}\begin{array}{c}\text { Firefighting foams, stain and grease proof } \\
\text { coatings on carpets and other textiles }\end{array} \\
\end{array}$ \\
\hline Perfluorodecanoic acid & PFDA & $\mathrm{C} 10 \mathrm{HF} 19 \mathrm{O} 2$ & $335-76-2$ & PFCA & $\begin{array}{l}\text { Rarely used in commercial products directly. } \\
\text { Breakdown or process byproduct of stain and } \\
\text { grease proof coatings, other PFCA surfactant } \\
\text { uses }\end{array}$ \\
\hline $\begin{array}{c}3,3,4,4,5,5,6,6,7,7,8,8,9,9,10,10,10- \\
\text { Heptadecafluorodecanesulphonic } \\
\text { acid }\end{array}$ & 8:2-FTS & $\mathrm{C} 10 \mathrm{H} 5 \mathrm{~F} 17 \mathrm{O} 3 \mathrm{~S}$ & $39108-34-4$ & FTS & Mist suppression, metal plating \\
\hline Perfluorononanesulfonic acid & PFNS & C9HF19O3S & $68259-12-1$ & PFSA & Impurity of production \\
\hline Perfluoroundecanoic acid & PFUnDA & $\mathrm{C} 11 \mathrm{HF} 21 \mathrm{O} 2$ & $2058-94-8$ & PFCA & $\begin{array}{c}\text { Rarely used in commercial products directly. } \\
\text { Breakdown or process byproduct of stain and } \\
\text { grease proof coatings, other PFCA surfactant } \\
\text { uses }\end{array}$ \\
\hline $\begin{array}{l}\text { N-methylperfluoro-1- } \\
\text { octanesulfonamidoacetic acid }\end{array}$ & N-MeFOSAA & C11H6F17NO4S & $2355-31-9$ & FASAA & \multirow{2}{*}{ Food packaging, coatings } \\
\hline $\begin{array}{l}\text { N-ethylperfluoro-1- } \\
\text { octanesulfonamidoacetic acid }\end{array}$ & $\mathrm{N}-\mathrm{EtFOSAA}$ & $\mathrm{C} 12 \mathrm{H} 8 \mathrm{~F} 17 \mathrm{NO} 4 \mathrm{~S}$ & $2991-50-6$ & FASAA & \\
\hline Perfluorodecanesulfonic acid & PFDS & $\mathrm{C} 10 \mathrm{HF} 21 \mathrm{O} 3 \mathrm{~S}$ & $335-77-3$ & PFSA & Impurity of production \\
\hline Perfluorododecanoic acid & PFDoA & $\mathrm{C} 12 \mathrm{HF} 23 \mathrm{O} 2$ & $307-55-1$ & PFCA & \multirow{3}{*}{$\begin{array}{c}\text { Rarely used in commercial products directly. } \\
\text { Breakdown or process byproduct of stain and } \\
\text { grease proof coatings, other PFCA surfactant } \\
\text { uses }\end{array}$} \\
\hline Perfluorotridecanoic acid & PFTrDA & $\mathrm{C} 13 \mathrm{HF} 25 \mathrm{O} 2$ & $72629-94-8$ & PFCA & \\
\hline Perfluorotetradecanoic acid & PFTeDA & $\mathrm{C} 14 \mathrm{HF} 27 \mathrm{O} 2$ & $376-06-7$ & PFCA & \\
\hline $\begin{array}{l}\text { 2,3,3,3-Tetrafluoro-2-(1,1,2,2,3,3,3- } \\
\text { heptafluoropropoxy)propanoic acid }\end{array}$ & HFPO-DA & C6HF11O3 & $13252-13-6$ & PFEA & $\begin{array}{c}\text { Replacement for PFOA. Surfactant and } \\
\text { polymerization aid used in the producion of } \\
\text { PTFE. }\end{array}$ \\
\hline Perfluoro-2-ethoxypropanoic acid & PEPA & C5HF9O3 & $267239-61-2$ & PFEA & \multirow{6}{*}{$\begin{array}{l}\text { Replacement for PFOA. Nonstick coatings, } \\
\text { general surfactant and processing aid }\end{array}$} \\
\hline Perfluoro-2-methoxypropanoic acid & PMPA & $\mathrm{C} 4 \mathrm{HF} 7 \mathrm{O} 3$ & $13140-29-9$ & PFEA & \\
\hline Perfluoro-3,5-dioxahexanoic acid & $\mathrm{PFO} 2 \mathrm{H} \times \mathrm{A}$ & $\mathrm{C} 4 \mathrm{HF} 7 \mathrm{O} 4$ & $39492-88-1$ & PFEA & \\
\hline Perfluoro(3,5,7-trioxaoctanoic) acid & PFO3OA & C5HF9O5 & $39492-89-2$ & PFEA & \\
\hline $\begin{array}{c}\text { Perfluoro-3,5,7,9-tetraoxadecanoic } \\
\text { acid }\end{array}$ & PFO4DA & C6HF11O6 & $39492-90-5$ & PFEA & \\
\hline $\begin{array}{c}\text { Perfluoro(3,5,7,9,11- } \\
\text { pentaoxadodecanoic) acid }\end{array}$ & PFO5DoDA & $\mathrm{C} 7 \mathrm{HF} 13 \mathrm{O} 7$ & $39492-91-6$ & PFEA & \\
\hline $\begin{array}{c}\text { 2-[1-[difluoro[(1,2,2- } \\
\text { trifluoroethenyl)oxy]methyl]-1,2,2,2- } \\
\text { tetrafluoroethoxy]-1,1,2,2-tetrafluoro- } \\
\text { Ethanesulfonic acid, }\end{array}$ & $\begin{array}{c}\text { Nafion by-product } \\
1 \text { (BP1) }\end{array}$ & C7HF1305S & 29311-67-9 & PFEA & \multirow{3}{*}{$\begin{array}{l}\text { Byproduct of the production of the Nafion } \\
\text { ionomer }\end{array}$} \\
\hline $\begin{array}{c}\text { 2-[1-[difluoro(1,2,2,2- } \\
\text { tetrafluoroethoxy)methyl]-1,2,2,2- } \\
\text { tetrafluoroethoxy]-1,1,2,2-tetrafluoro- } \\
\text { ethanesulfonic acid }\end{array}$ & $\begin{array}{c}\text { Nafion byproduct } 2 \\
\text { (BP2) }\end{array}$ & $\mathrm{C} 7 \mathrm{H} 2 \mathrm{~F} 14 \mathrm{O} 5 \mathrm{~S}$ & $749836-20-2$ & PFEA & \\
\hline $\begin{array}{c}\text { 2,2,3,3,4,5,5,5-4-(1,1,2,2-tetrafluoro- } \\
\text { 2- sulfoethoxy)pentanoic acid }\end{array}$ & $\begin{array}{c}\text { Nafion byproduct } 4 \\
\text { (BP4) }\end{array}$ & $\mathrm{C} 7 \mathrm{H} 2 \mathrm{~F} 12 \mathrm{O} 6 \mathrm{~S}$ & na & PFEA & \\
\hline $\begin{array}{l}\text { 1,1,2,2-tetrafluoro-2-(1,2,2,2- } \\
\text { tetrafluoro-ethoxy)ethane sulfonate }\end{array}$ & NVHOS & $\mathrm{C} 4 \mathrm{H} 2 \mathrm{~F} 8 \mathrm{O} 4 \mathrm{~S}$ & $\begin{array}{l}\text { 801209-99-4 } \\
\text { (free acid) }\end{array}$ & PFEA & \\
\hline $\begin{array}{c}1,2,2,3,3,4,5,5,6,6 \text {-decafluoro-4- } \\
\text { (1,1,2,2,2-pentafluoroethyl)- } \\
\text { cyclohexanesulfonicacid, }\end{array}$ & PFECHS & C8HF15O3S & $646-83-3$ & cyclic PFSA & Erosion inhibitor in aircraft hydraulic fluids \\
\hline $\begin{array}{l}4,4,5,5,6,6,7,7,8,8,9,9,10,10,10- \\
\text { Pentadecafluorodecanoic Acid }\end{array}$ & $7: 3$ FTCA & $\mathrm{C} 10 \mathrm{H} 5 \mathrm{~F} 15 \mathrm{O} 2$ & $812-70-4$ & FTCA & $\begin{array}{l}\text { Formed via oxidation of } 8: 2 \mathrm{FTOH}, \text { a } \\
\text { fluorotelomer alcohol commonly used in textile } \\
\text { production }\end{array}$ \\
\hline
\end{tabular}

